July 2004 • NREL/TP-500-35816

\title{
Modern Control Design for Flexible Wind Turbines
}

\author{
Alan D. Wright
}

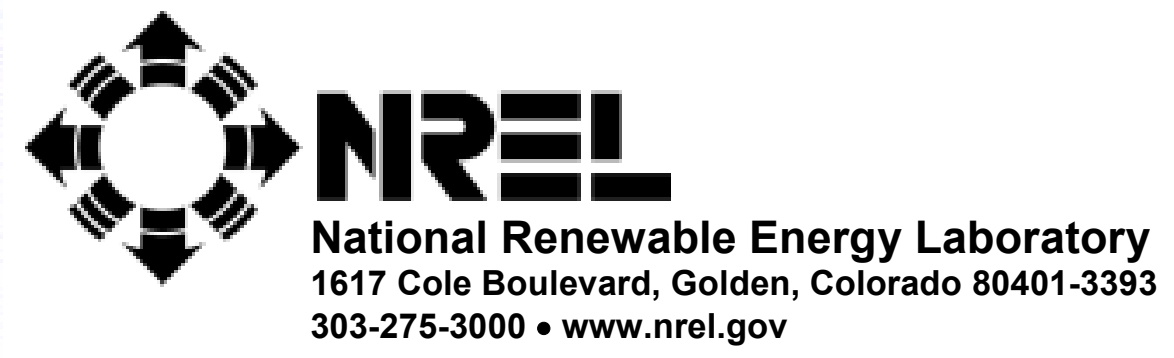

Operated for the U.S. Department of Energy

Office of Energy Efficiency and Renewable Energy

by Midwest Research Institute • Battelle

Contract No. DE-AC36-99-G010337 


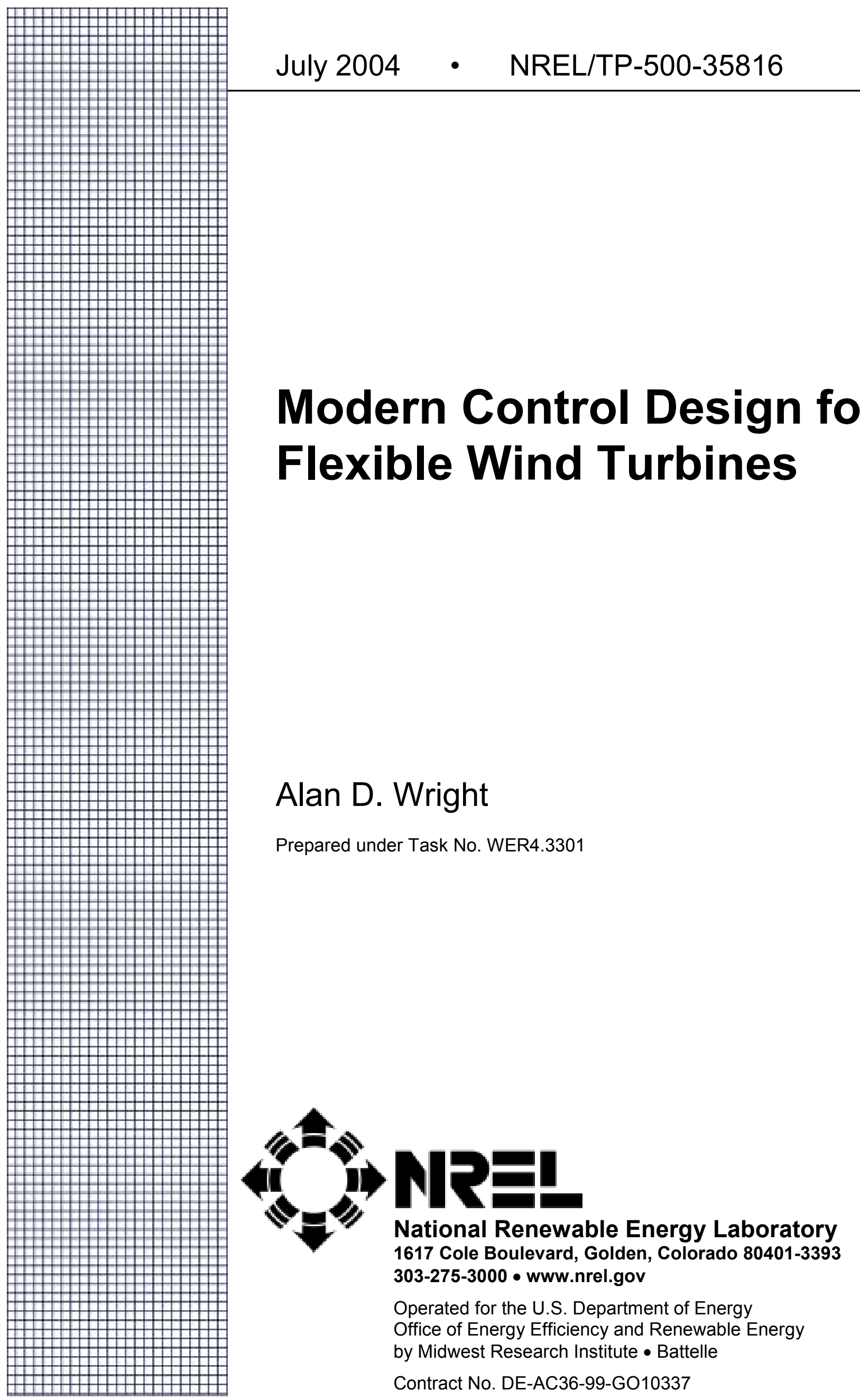




\section{NOTICE}

This report was prepared as an account of work sponsored by an agency of the United States government. Neither the United States government nor any agency thereof, nor any of their employees, makes any warranty, express or implied, or assumes any legal liability or responsibility for the accuracy, completeness, or usefulness of any information, apparatus, product, or process disclosed, or represents that its use would not infringe privately owned rights. Reference herein to any specific commercial product, process, or service by trade name, trademark, manufacturer, or otherwise does not necessarily constitute or imply its endorsement, recommendation, or favoring by the United States government or any agency thereof. The views and opinions of authors expressed herein do not necessarily state or reflect those of the United States government or any agency thereof.

Available electronically at http://www.osti.gov/bridge

Available for a processing fee to U.S. Department of Energy and its contractors, in paper, from:

U.S. Department of Energy

Office of Scientific and Technical Information

P.O. Box 62

Oak Ridge, TN 37831-0062

phone: 865.576.8401

fax: 865.576.5728

email: mailto:reports@adonis.osti.gov

Available for sale to the public, in paper, from:

U.S. Department of Commerce

National Technical Information Service

5285 Port Royal Road

Springfield, VA 22161

phone: 800.553 .6847

fax: 703.605.6900

email: orders@ntis.fedworld.gov

online ordering: http://www.ntis.gov/ordering.htm 


\section{Summary}

Control can improve energy capture and reduce dynamic loads in wind turbines. In the 1970s and 1980s, wind turbines used classical control designs to regulate power and speed. The methods used, however, were not always successful. These systems often had bandwidths large enough to destabilize low-damped flexible modes leading to high dynamic load fatigue failures. Modern turbines are larger, mounted on taller towers, and more dynamically active than their predecessors. Control systems to regulate turbine power and maintain stable, closed-loop behavior in the presence of turbulent wind inflow will be critical for these designs. New, advanced control approaches and paradigms must account for low-damped flexible modes in order to reduce structural dynamic loading and achieve the 20- to 25-year operational life required of today's machines.

This report applies modern state-space control design methods to a two-bladed teetering hub upwind machine at the National Wind Technology Center (NWTC), which is managed by the U.S. Department of Energy's National Renewable Energy Laboratory (NREL) in Golden, Colorado. The design objective is to regulate turbine speed and enhance damping in several low-damped flexible modes of the turbine. Starting with simple control algorithms based on linear models, complexity is added incrementally until the desired performance is firmly established.

The controls approach is based on the Disturbance Accommodating Control (DAC) method and provides accountability for wind-speed fluctuations. First, controls are designed using the single control input rotor collective pitch to stabilize the first drive-train torsion as well as the tower first fore-aft bending modes. Generator torque is then incorporated as an additional control input. This reduces some of the demand placed on the rotor collective pitch control system and enhances first drive train torsion mode damping. Individual blade pitch control is then used to attenuate wind disturbances having spatial variation over the rotor, and it effectively reduces blade flap deflections due to wind shear.

Finally, we compare the results for these modern controls with results for simpler, classical controls in order to assess modern controller performance. These modern controls are shown to more effectively mitigate tower fore-aft motion, drive-train shaft torsion moments, and blade root flap bending moments when compared with classical control approaches. 


\section{Acknowledgments}

I gratefully thank my doctoral thesis advisor, Professor Mark Balas of the University of Colorado, for his invaluable encouragement and support. I also thank Mike Robinson and Bob Thresher of the NWTC at NREL for their great support and interest. Bob has been my mentor for many years in the wind turbine field, and I have learned a great deal from him. Mike made it possible for me to perform the work described here. In addition, I would like to thank my colleagues Maureen Hand and Karl Stol for their technical feedback on important issues.

This work was funded under the U.S. Department of Energy's Wind and Hydropower Technologies Program in the Office of Energy Efficiency and Renewable Energy. 


\section{Contents}

Chapter

$\underline{\text { Page }}$

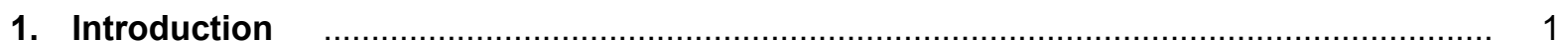

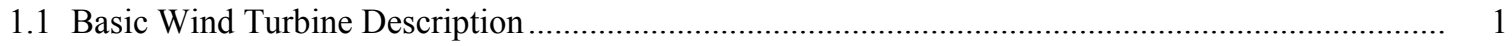

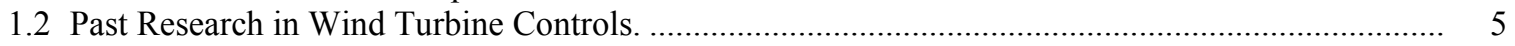

1.2.1 Classical Control Designs...................................................................................................... 5

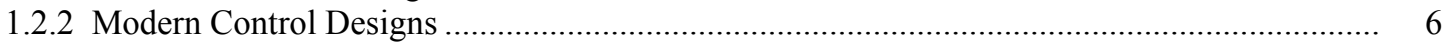

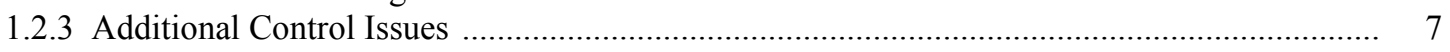

1.3 Analytical Tool Development and Validation............................................................................ 8

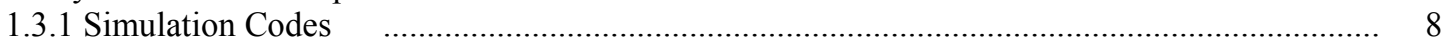

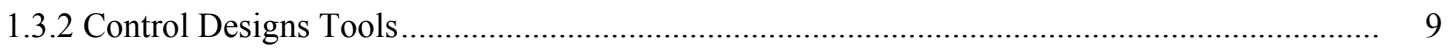

1.4 Control Needs for Future Wind Turbine Designs ...................................................................... 9

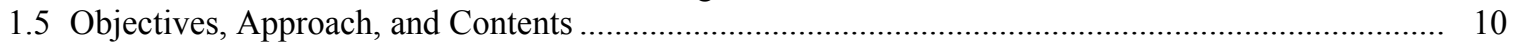

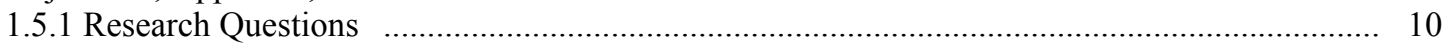

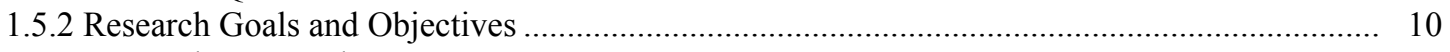

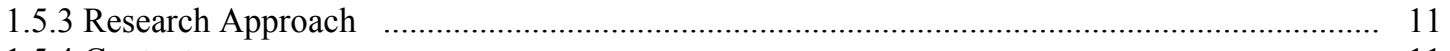

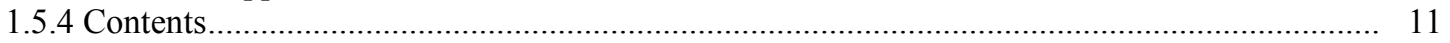

\section{Analytical Models and Simulation Tools}

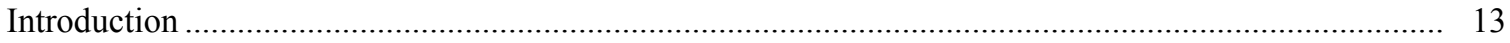

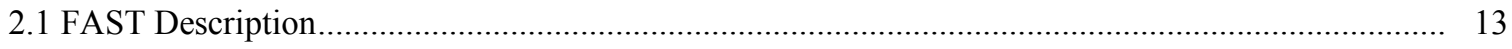

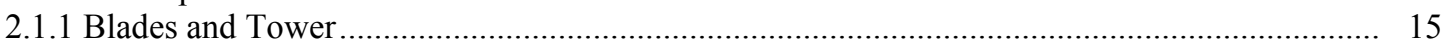

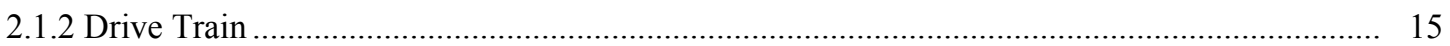

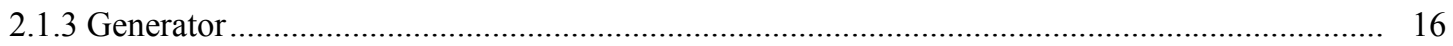

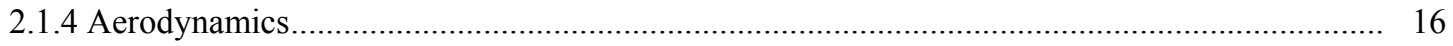

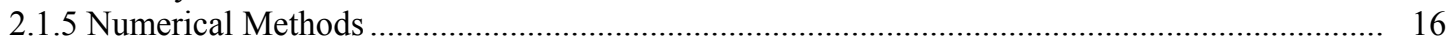

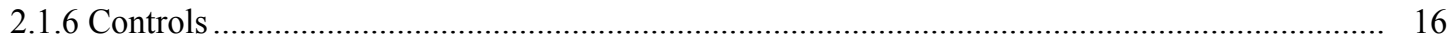

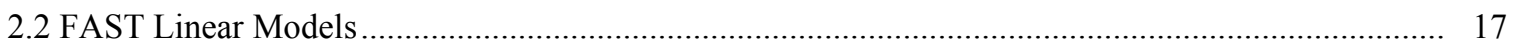

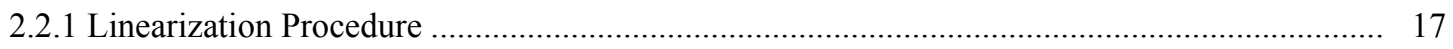

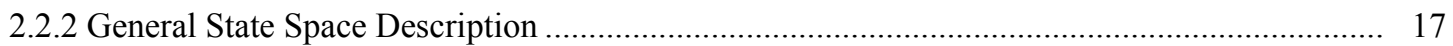

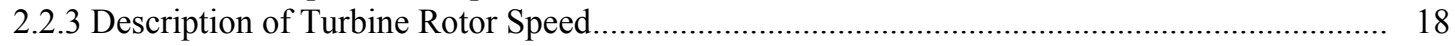

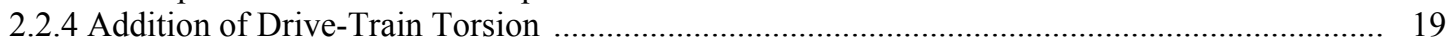

2.2.5 Addition of Blade Flap ............................................................................................. 21

2.2.6 Addition of First Tower Fore-Aft Mode.............................................................................. 23

2.3 Linear Model Evaluations for the Controls Advanced Research Turbine ............................................ 24

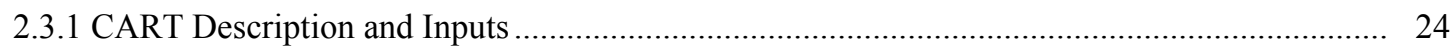

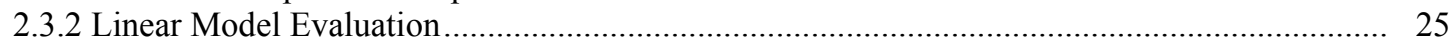

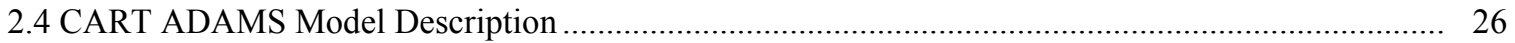

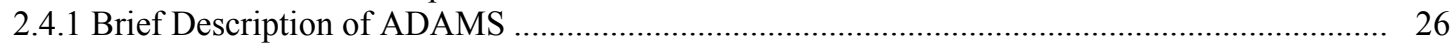

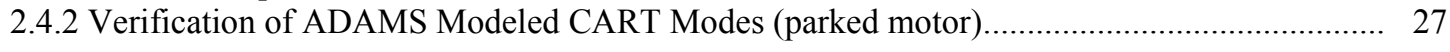

3. Modeling and Simulation of CART Classical Control .................................................. 29

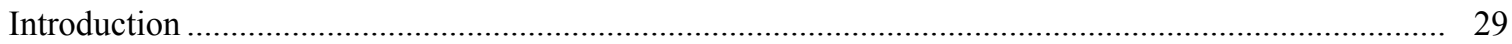

3.1 Description of the CART PI Control System ………................................................................. 29

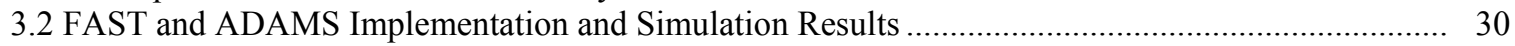

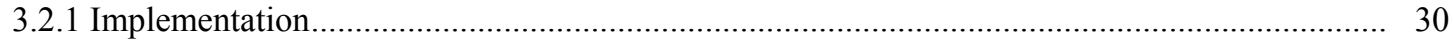

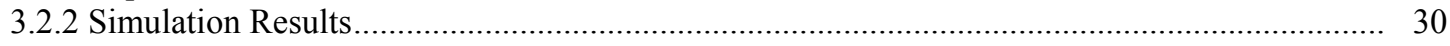

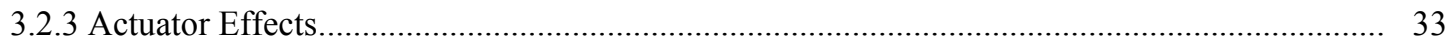




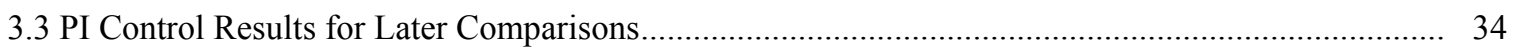

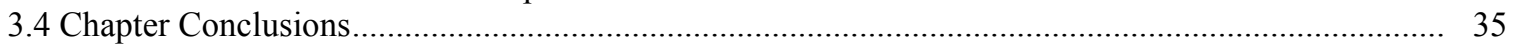

4. Illustration of Modern Control Design Steps …................................................................. 36

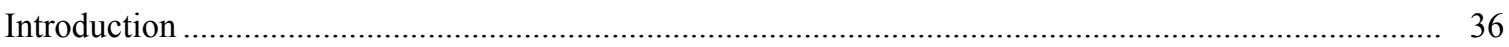

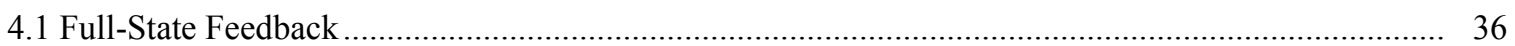

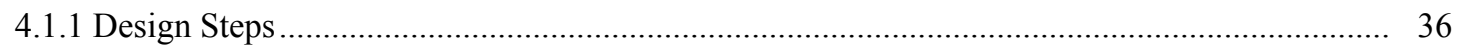

4.1.2 Full-State Feedback Design Using the 1-State Linear Model ................................................. 37

4.1.3 Full-State Feedback Design from the 3-State Linear Model .................................................. 39

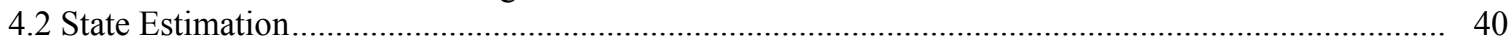

4.2.1 State Estimator Design from the 3-State Linear Mode ......................................................... 41

4.3 Disturbance Accommodating Control ....................................................................................... 43

4.3.1 DAC Design from the 3-State Linear Model....................................................................... 44

4.4 Linear Quadratic Regulator Design ....................................................................................... 47

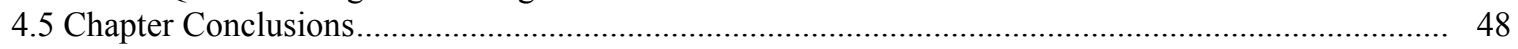

5. Control Designs for Speed Regulation Using Rotor Collective Pitch Control ...................... 50

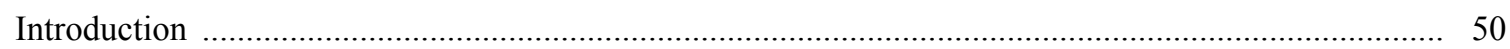

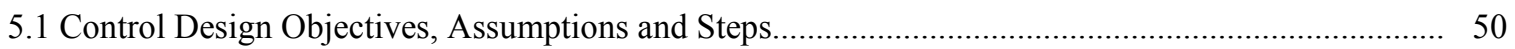

5.2 DAC Controls Designed from the 1-State Linear Model ……....................................................... 50

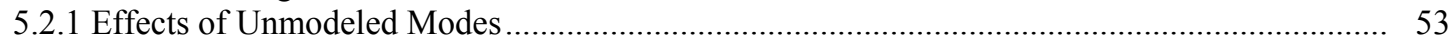

5.3 DAC Control Design from the 3-State Model ........................................................................... 54

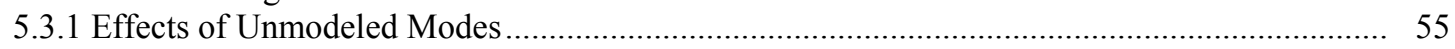

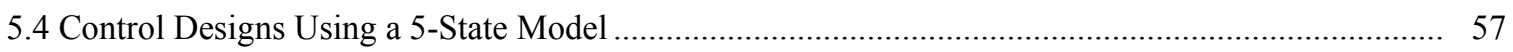

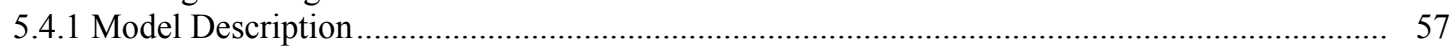

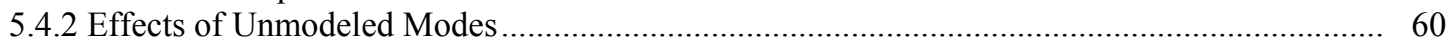

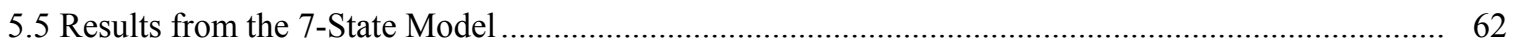

5.5.1 Addition of Tower-Top Fore-Aft Deflection Measurement ……….......................................... 63

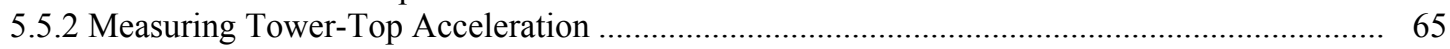

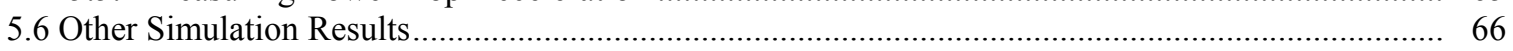

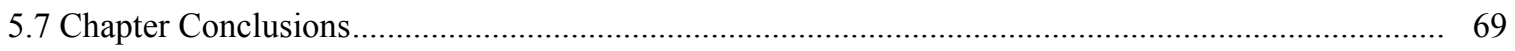

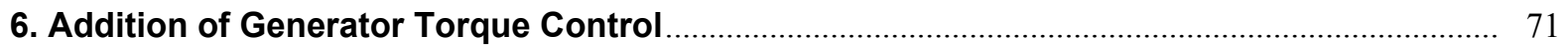

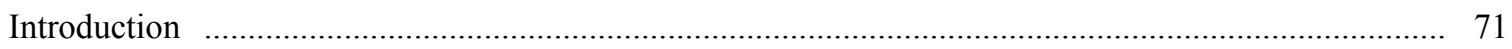

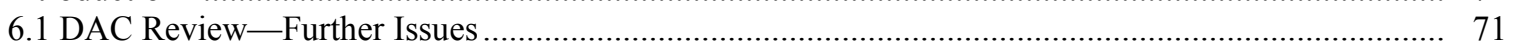

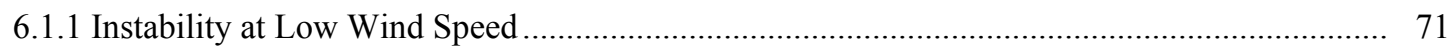

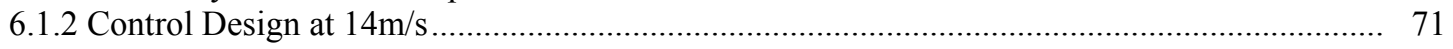

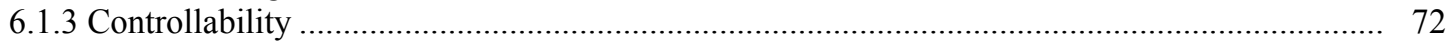

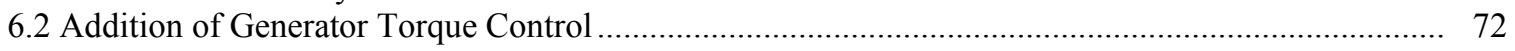

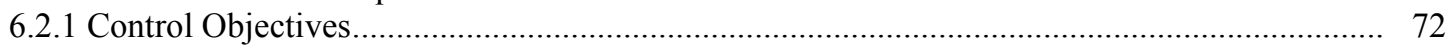

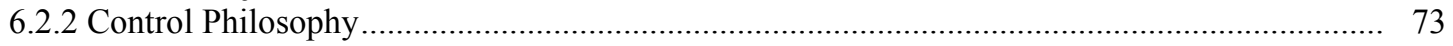

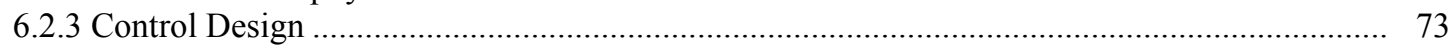

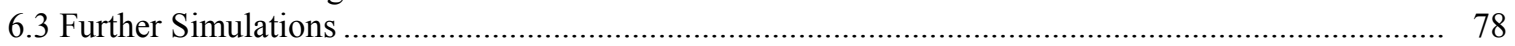

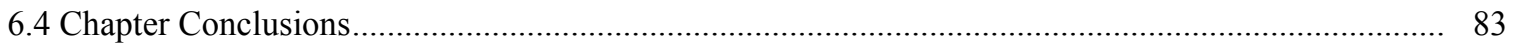

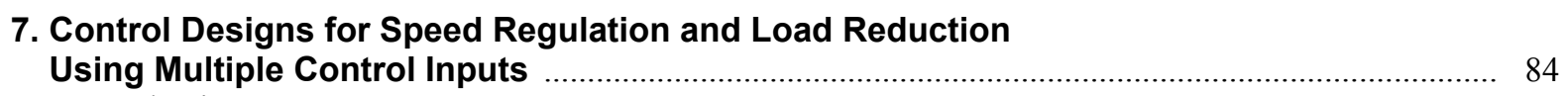

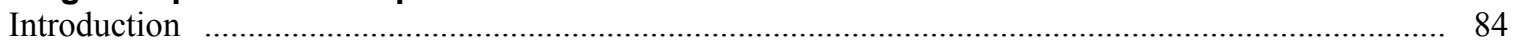

7.1 Independent Blade Pitch Control................................................................................... 84

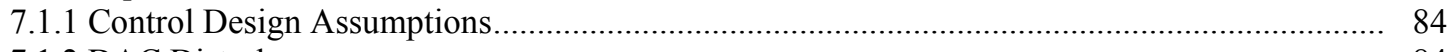

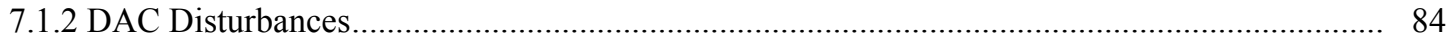




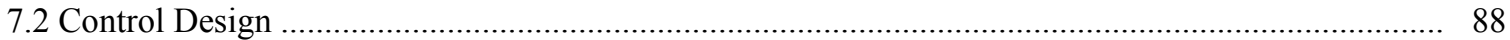

7.2.1 Results from a Model with 5 States.......................................................................... 88

7.2.2 Control Designs including First Drive-Train Torsion and Tower First Fore-Aft Motion .......... 96

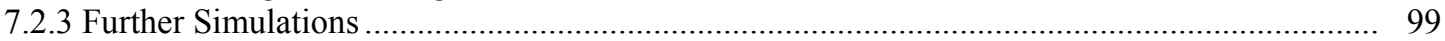

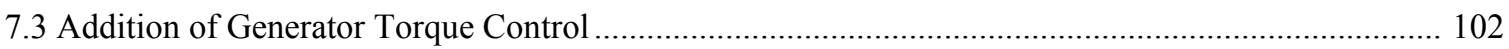

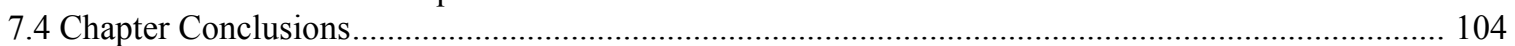

8. Comparisons of Modern and Classical Control Results ….............................................................. 106

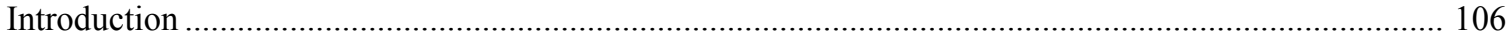

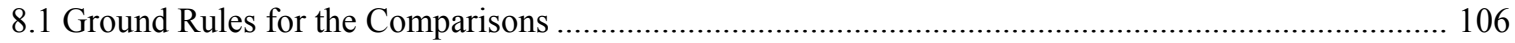

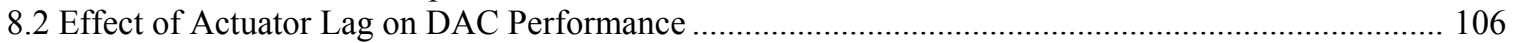

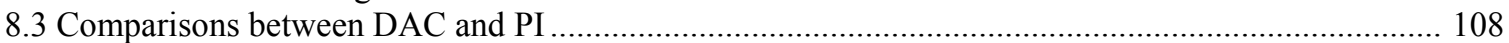

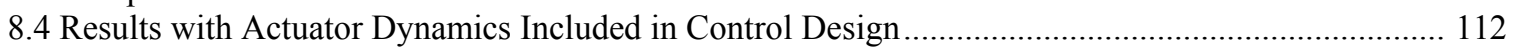

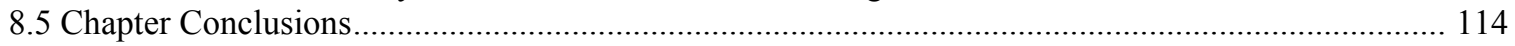

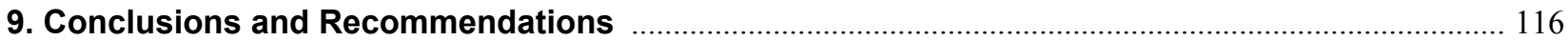

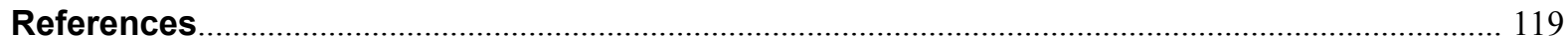

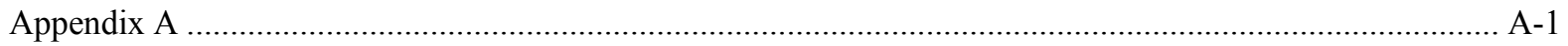

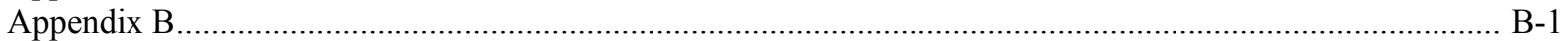

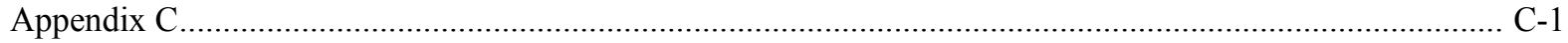

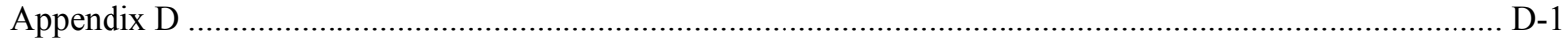




\title{
Nomenclature
}

\author{
$\hat{A} \quad$ area of the rotor disk \\ $\operatorname{Act}(s) \quad$ actuator transfer function \\ $A \quad$ state matrix \\ $\bar{A} \quad$ augmented state matrix \\ $A_{A} \quad$ state matrix in actuator dynamics model \\ $B \quad$ control input gain matrix \\ $B_{A} \quad$ control input gain matrix in actuator dynamics model \\ $\bar{B} \quad$ augmented control input gain matrix \\ $\mathrm{C}_{\mathrm{rs}} \quad$ element of the FAST mass matrix in the rth row and sth column \\ $\mathrm{C}_{\mathrm{ij}} \quad$ coefficients of damping matrix for various values of $\mathrm{i}$ and $\mathrm{j}$ \\ $C_{d} \quad$ drive-train torsional damping coefficient \\ Cont $(s) \quad$ controller transfer function \\ $C_{p} \quad$ power coefficient \\ C relates plant output to states \\ $C_{A} \quad$ relates plant output to states in actuator dynamics model \\ $\bar{C} \quad$ augmented matrix $\mathrm{C}$ \\ $D \quad$ relates the output to the control input in state-space equation \\ $E \quad$ relates the output to the disturbance input in state-space equation \\ $\mathrm{E}_{\mathrm{iy}} \quad$ blade or tower stiffness about the $\mathrm{y}$-axis (blade edgewise, tower longitudinal) \\ $\mathrm{E}_{\mathrm{iz}} \quad$ blade or tower stiffness about the y-axis (blade flapwise, tower latitudinal) \\ $\underline{e} \quad$ state estimator error: difference between estimated state vector $\underline{\hat{x}}$ and state vector $\underline{x}$ \\ $\underline{e}_{x} \quad$ plant state estimator error \\ $\underline{e}_{D} \quad$ difference between estimated disturbance state vector $\hat{z}_{D}$ and disturbance \\ state vector $\underline{z}_{D}$ \\ $\mathrm{f}_{\mathrm{r}}(q, \dot{q}) \quad$ applied force terms in FAST equations of motion, containing aerodynamic forces, \\ gravity, spring and damping forces, as well as the effects of inertia forces caused by \\ centrifugal and coriolis terms \\ $F \quad$ state matrix for disturbance state-space model (Section 4.3) \\ $G \quad$ gain in full state feedback law \\ $\bar{G} \quad$ augmented gain in full state feedback law-using $\underline{u}_{*}$ (Section 4.3) \\ $G_{D} \quad$ gain in full state feedback law associated with disturbance state \\ G J blade or tower torsional stiffness \\ $\mathrm{E}_{\mathrm{A}} \quad$ blade or tower tensile stiffness \\ $h \quad$ hub height \\ I identity matrix \\ $\mathrm{I}_{\text {gen }} \quad$ generator mass moment of inertia \\ $\mathrm{I}_{\text {rot }} \quad$ rotor mass moment of inertia \\ $\mathrm{I}_{\text {tot }}=\mathrm{I}_{\mathrm{rot}}+\mathrm{I}_{\mathrm{gen}}$ total turbine rotational inertia, sum of rotor inertia and generator inertia \\ $\mathrm{I}_{\mathrm{y}} / \mathrm{L} \quad$ blade or tower distributed mass moment of inertia about the $y$-axis
}




\begin{tabular}{|c|c|}
\hline $\mathrm{I}_{\mathrm{z}} / \mathrm{L}$ & blade or tower distributed mass moment of inertia about the z-axis \\
\hline$J$ & \\
\hline$K$ & state estimator gain matrix \\
\hline $\bar{K}$ & augmented state estimator gain matrix \\
\hline$K_{d}$ & drive-train torsional spring stiffness \\
\hline$K_{x}$ & plant state estimator gain matrix \\
\hline$K_{D}$ & disturbance state estimator gain matrix \\
\hline $\mathrm{K}_{\mathrm{ij}}$ & coefficients of stiffness matrix for various values of $i$ and $j$ \\
\hline$K_{p}$ & classical controller proportional gain \\
\hline$K_{i}$ & classical controller integral gain \\
\hline Obs & observability matrix \\
\hline$m$ & power law wind-shear coefficient \\
\hline $\mathrm{M}_{\mathrm{ij}}$ & coefficients of mass matrix for various values of $i$ and $j$ \\
\hline $\mathrm{m} / \mathrm{L}$ & blade or tower mass per unit length \\
\hline $\mathrm{N}$ & dimension of state matrix $A$ \\
\hline $\mathrm{P}$ & power \\
\hline$P(s)$ & plant transfer function \\
\hline$Q$ & symmetric, positive semi-definite weighting on the states $\underline{x}$ \\
\hline$q_{1}$ & blade 1 flapwise tip displacement for mode 1 \\
\hline$\delta q_{1}$ & perturbed blade-1 first flap mode displacement \\
\hline$\delta \dot{q}_{1}$ & perturbed blade-1 first flap mode velocity \\
\hline$q_{2}$ & blade 2 flapwise tip displacement for mode 1 \\
\hline$\delta q_{2}$ & perturbed blade- 2 first flap mode displacement \\
\hline$\delta \dot{q}_{2}$ & perturbed blade- 2 first flap mode velocity \\
\hline$q_{3}$ & teeter angle \\
\hline$q_{4}$ & rotor azimuth angle \\
\hline$\delta q_{4}$ & perturbed rotor azimuth angle \\
\hline$\delta \dot{q}_{4}$ & perturbed rotor speed \\
\hline$q_{5}$ & nacelle tilt angle \\
\hline$q_{6}$ & nacelle yaw angle \\
\hline$q_{7}$ & longitudinal tower top displacement for first tower fore-aft mode \\
\hline$\delta q_{7}$ & perturbed tower first fore-aft mode displacement \\
\hline$\delta \dot{q}_{7}$ & perturbed tower first fore-aft mode velocity \\
\hline$q_{8}$ & lateral tower top displacement for first tower side-side mode \\
\hline$q_{9}$ & longitudinal tower top displacement for second tower fore-aft mode \\
\hline$q_{10}$ & longitudinal tower top displacement for second tower side-side mode \\
\hline
\end{tabular}




\begin{tabular}{|c|c|}
\hline$q_{11}$ & blade 1 flapwise tip displacement for mode 2 \\
\hline$q_{12}$ & blade 2 flapwise tip displacement for mode 2 \\
\hline$q_{13}$ & blade 1 edgewise tip displacement for mode 1 \\
\hline$q_{14}$ & blade 2 edgewise tip displacement for mode 1 \\
\hline$q_{15}$ & generator rotor azimuth angle \\
\hline$\delta q_{15}$ & perturbed generator rotor azimuth angle \\
\hline$\delta \dot{q}_{15}$ & perturbed generator rotational speed. \\
\hline \multicolumn{2}{|c|}{$\mathrm{K}_{\mathrm{d}}\left(\delta q_{4}-\delta q_{15}\right)$ perturbed drive-train torsional spring force } \\
\hline$R$ & symmetric, positive definite weighting on the input $\underline{u}$ \\
\hline $\begin{array}{l}r \\
R\end{array}$ & $\begin{array}{l}\text { radial position along blade from center of hub } \\
\text { rotor radius }\end{array}$ \\
\hline$R^{N}$ & vector space of all real vectors of dimension $\mathrm{N}$ \\
\hline$R^{N x N}$ & vector space of all real $\mathrm{NxN}$ matrices \\
\hline & time \\
\hline $\mathrm{T}_{\text {aero }}$ & rotor aerodynamic torque \\
\hline$\delta \mathrm{T}_{\text {aero }}$ & perturbed rotor aerodynamic torque \\
\hline$T_{\text {shaft }}$ & torque at generator end of low-speed shaft \\
\hline$T_{\text {shaft }_{0}}$ & torque at generator end of low-speed shaft at equilibrium \\
\hline$\delta \mathrm{T}_{\text {shaft }}$ & perturbed torque at generator end of low-speed shaft \\
\hline$T(s)$ & transfer function \\
\hline$u$ & blade or tower deflection \\
\hline$\underline{u}$ & control input \\
\hline$\underline{u_{D}}$ & disturbance state-space model output \\
\hline$\underline{\hat{u}_{D}}$ & estimated disturbance state-space model output \\
\hline$\underline{u}_{*}$ & superposition of plant state feedback law and disturbance feedback law \\
\hline$u_{D_{1}}$ & specific disturbance state-space model output \\
\hline$u_{D_{2}}$ & specific disturbance state-space model output \\
\hline$V_{w}$ & free-stream wind speed \\
\hline $\mathrm{V}_{\text {hub }}$ & wind speed at hub height \\
\hline$V(z)$ & wind speed as a function of height $z$ \\
\hline $\mathrm{W}$ & wind disturbance (uniform over rotor disk) \\
\hline$w_{0}$ & wind speed at control design point (uniform over rotor disk) \\
\hline$\delta \mathrm{w}$ & perturbed wind disturbance (uniform over rotor disk) \\
\hline$\delta \mathrm{w}_{1}$ & perturbed wind disturbance acting on blade-1 \\
\hline$\delta \mathrm{w}_{2}$ & perturbed wind disturbance acting on blade- 2 \\
\hline$x_{i}-i^{t h}$ & state in state vector $\underline{x}$ \\
\hline$\dot{x}_{i}$ & time derivative of $i^{\text {th }}$ state \\
\hline
\end{tabular}




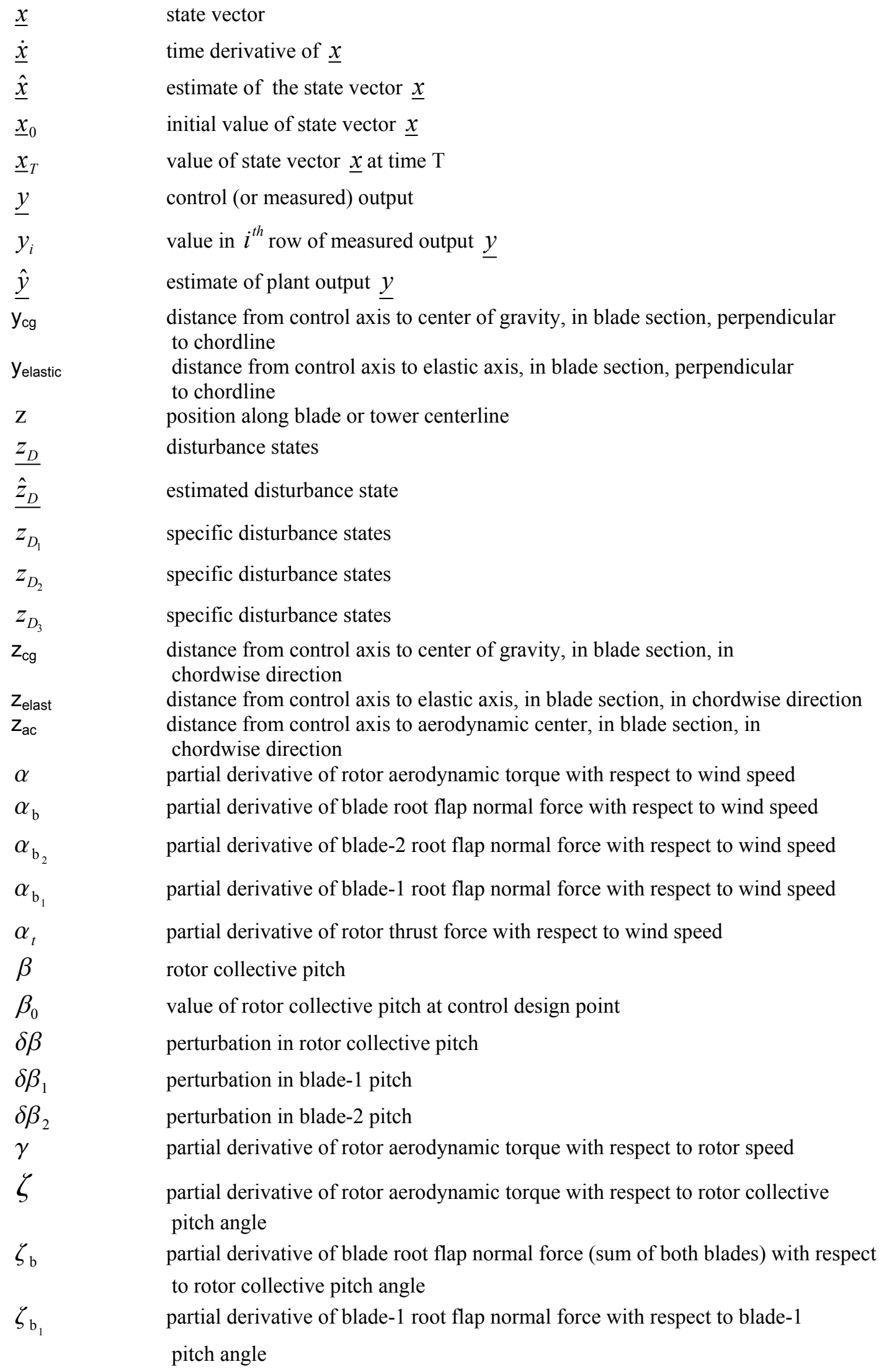




$\begin{array}{ll}\zeta_{\mathrm{b}_{2}} & \text { partial derivative of blade-2 root flap normal force with respect to blade-2 } \\ \zeta_{t} & \text { pitch angle } \\ \Gamma & \text { partial derivative of rotor thrust force with respect to rotor collective pitch angle } \\ \theta & \text { disturbance input gain matrix } \\ \rho & \text { relates disturbance model output to disturbance states } \\ \lambda & \text { air density } \\ \Omega & \text { tip-speed ratio } \\ \Omega_{0} & \text { rotor speed } \\ \tau & \text { value of rotor speed at control design point } \\ \phi_{i} & \text { actuator time constant } \\ \Psi & \text { blade or tower it } \\ \delta \psi_{\text {rot }} & \text { blade azimuth angle shape } \\ \delta \psi_{\text {gen }} & \text { perturbed rotor azimuth angle }\end{array}$




\section{List of Tables}

$\underline{\text { Page }}$

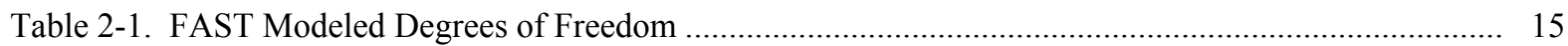

Table 2-2. General Specifications of the CART Machine ……................................................................... 24

Table 2-3. Comparison of Measured and Predicted Turbine Natural Frequencies with

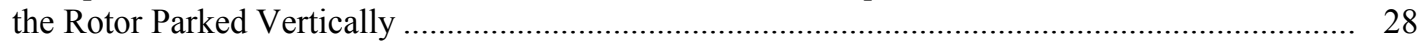

Table 4-1. CART State Matrix Values for the 1-State Model ................................................................... 38

Table 4-2. A, B, and $\Gamma$ matrices for the CART 3-State Linear Model....................................................... 39

Table 5-1. A, B, and $\Gamma$ matrices for the CART 5-State Linear Model....................................................... 58

Table 6-1. Variation of Pitch and Wind-Speed Gains with Design Point ........................................................ 71

Table 6-2. A and B Matrices for the CART 3-State Linear Model................................................................ 75

Table 7-1. A-Matrix for the 5-State Linear Model with Independent Pitch .................................................. 89

Table 7-2. $\mathrm{B}$ and $\Gamma$ Matrices for the 5-State Linear Model.................................................................... 89

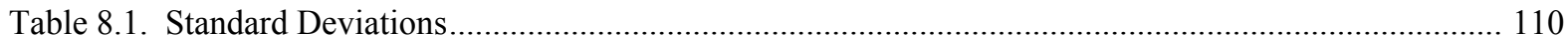

Table B-1.1. CART Tower Distributed Properties ........................................................................................... B-1

Table B-1.2. CART Blade Distributed Properties …….......................................................................................... B-4 


\section{List of Figures}

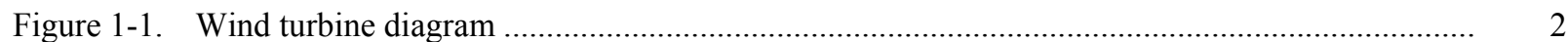

Figure 1-2. Typical turbine degrees of freedom and motions ..................................................................

Figure 1-3. Plot of rotor $\mathrm{C}_{\mathrm{p}}$ versus tip-speed ratio for various pitch angles ..................................................

Figure 1-4. Typical wind turbine power curve .......................................................................................

Figure 2-1. Degrees of freedom modeled with FAST ...........................................................................

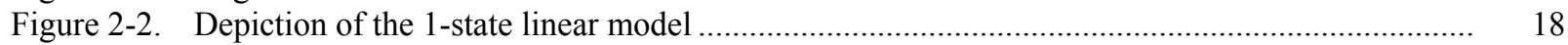

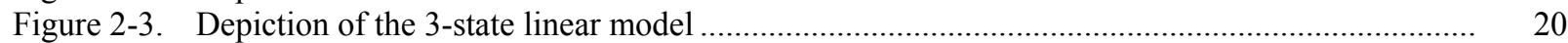

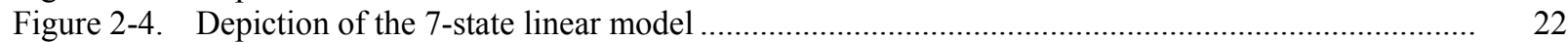

Figure 2-5. Depiction of the 9-state linear model ............................................................................... 23

Figure 2-6. The Controls Advanced Research Turbine (CART) ............................................................. 25

Figure 3-1. Diagram of CART speed regulation control system ............................................................. 29

Figure 3-2. Test wind applied to CART FAST models ......................................................................... 30

Figure 3-3. FAST simulated rotor speed and pitch, using PI control, for rigid blades, tower, and drive train $\left(\mathrm{K}_{\mathrm{p}}=1.2217, \mathrm{~K}_{\mathrm{i}}=0.2618\right)$

Figure 3-4. FAST simulated rotor speed, using classical controller with increased integral $\backslash$ control gain $\left(\mathrm{K}_{\mathrm{p}}=1.2217, \mathrm{~K}_{\mathrm{i}}=1.0\right)$

Figure 3-5. FAST simulated generator speed and pitch, using PI control, simulated with first drive-train torsion $\left(\mathrm{K}_{\mathrm{p}}=1.2217, \mathrm{~K}_{\mathrm{i}}=0.2618\right)$

Figure 3-6. FAST simulated generator speed and pitch, using PI control, simulated with drive-train first torsion and blade first flap $\left(\mathrm{K}_{\mathrm{p}}=1.2217, \mathrm{~K}_{\mathrm{i}}=0.2618\right)$.

Figure 3-7. FAST simulated generator speed and pitch, using PI control, simulated with all flexible modes on $\left(\mathrm{K}_{\mathrm{p}}=1.2217, \mathrm{~K}_{\mathrm{i}}=0.2618\right)$, with actuator.

Figure 3-8. Comparison between FAST simulated generator speed and measured results, using PI control, simulated with all flexible modes on $\left(\mathrm{K}_{\mathrm{p}}=1.2217, \mathrm{~K}_{\mathrm{i}}=0.2618\right)$,

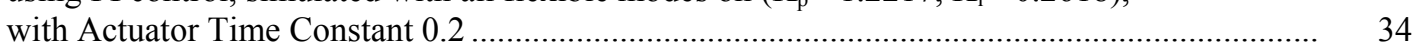

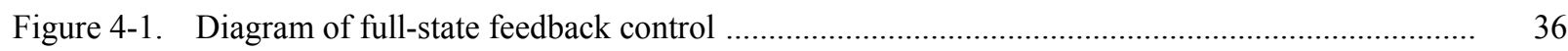

Figure 4-2. Test wind applied to CART FAST models ........................................................................ 37

Figure 4-3. FAST simulated CART rotor speed and pitch during full-state feedback control based on 1-state controller model...

Figure 4-4. FAST predicted CART rotor speed using full-state feedback based on 1-state controller for various values of $\mathrm{G}$......

Figure 4-5. Plot of FAST simulated CART generator speed using 3-state full-state feedback controller ...... $\quad 40$

Figure 4-6. Plot of FAST simulated CART generator speed using 3-state full-state feedback controller for two pole selections to give low- and high drive-train damping...

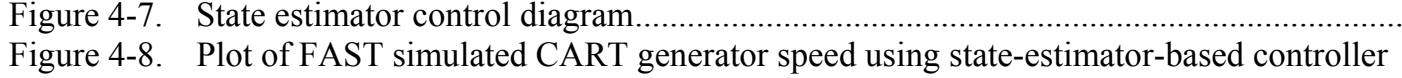
designed from 3-state model

Figure 4-9. Diagram of DAC controller.

Figure 4-10. Plot of FAST simulated CART generator speed using DAC-based controller based on 3-state model

Figure 4-11. Plot of FAST simulated CART generator speed using DAC controller designed

from 3-state model, for different pole locations ....................................................

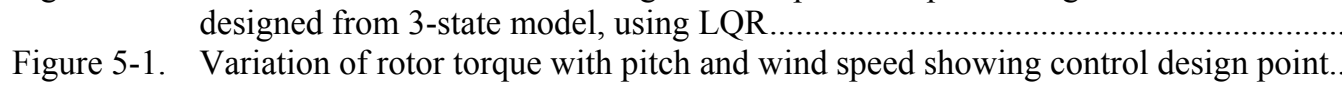

Figure 5-2. Test wind applied to CART FAST models ...

Figure 5-3. FAST simulated CART rotor speed and pitch during DAC control designed from 1-state model 
Figure 5-4 Comparison of estimated and actual wind speed as simulated by FAST for the

CART using DAC control based on 1-state model .....

Figure 5-5. Comparisons of ADAMS and FAST predicted rotor speed using 1-state controller

for the CART ....

Figure 5-6. Plot of CART generator speed as simulated with FAST and ADAMS with DAC control designed from 1-state model, with first drive-train torsion mode switched on

Figure 5-7. Pole plot of the closed-loop system, with first drive-train torsion mode switched on but not accounted for in control design

Figure 5-8. Plot of FAST simulated CART generator speed with drive-train torsion using 1-state controller, for increased drive-train torsional stiffness .

Figure 5-9. Comparison of estimated and actual wind speed as simulated by FAST for the CART using DAC control based on 3-state model

Figure 5-10. Comparisons of ADAMS and FAST simulated CART generator speed using DAC control designed from 3 -state model.

Figure 5-11. FAST and ADAMS simulated CART generator speed using DAC designed from 3 -state model with first flap turned on

Figure 5-12. Pole plot of the closed-loop system, with blade first flap mode switched on but not accounted for in control design

Figure 5-13. FAST simulated CART generator speed using DAC 3-state controller for various blade flap stiffness values

Figure 5-14. Plot of FAST simulated CART generator speed usingDAC-based controller based on 5-state model

Figure 5-15. Comparison of estimated and actual wind speed as simulated by FAST for the CART using DAC control based on 5-state model.

Figure 5-16. Comparisons of ADAMS blade-hinged and FAST simulated CART generator

Figure 5-17. Close-up of FAST and ADAMS simulated CART generator speed with 5-state

Figure 5-18. FAST simulated CART generator speed with 5-state controller with all blade modes turned on in FAST with undamped and damped blade edge mode

Figure 5-19. ADAMS and FAST simulated CART generator speed with DAC controller designed from 5 -state model with tower first fore-aft switched on in FAST, and several tower modes modeled in ADAMS

Figure 5-20. ADAMS and FAST simulated CART tower-top fore-aft deflections with DAC controller designed from 5 -state model

Figure 5-21. Plot of FAST simulated CART tower-top deflection using DAC based on 7-state model with generator speed and tower-top fore-aft deflection measurements

Figure 5-22. Comparing FAST simulated CART tower-top fore-aft deflections using both the 5 - and 7 -state controller.

Figure 5-23. Comparisons of ADAMS (flex-blade) and FAST simulated CART generator speed using DAC control designed from 7 -state controller.

Figure 5-24. ADAMS and FAST simulated CART tower-top fore-aft deflections with DAC controller designed from 7-state model

Figure 5-25. FAST simulated CART tower-top fore-aft deflections with DAC controller designed from 7-state model, when measuring tower-top acceleration.

Figure 5-26. Comparisons of ADAMS blade-flex and FAST simulated CART generator speed using DAC control designed from 5-state controller with turbulent inflow.....

Figure 5-27. Comparison of FAST estimated and actual wind speed using DAC control based on 5-state linear model, with turbulent inflow

Figure 5-28. FAST simulated CART tower-top fore-aft deflection with turbulent inflow using DAC control designed from 5- and 7-state models

Figure 5-29. Comparisons of ADAMS blade-flex and FAST simulated CART tower-top fore-aft deflection using DAC control designed from 7-state controller with turbulent inflow 
Figure 5-30. FAST-simulated pitch rates using DAC control designed from 7-state model with turbulent inflow, for high and low tower damping.....

Figure 6-1. Plot of FAST simulated CART generator speed using DAC controller based on 5 -state model, designed at $14-\mathrm{m} / \mathrm{s}$ and $18-\mathrm{m} / \mathrm{s}$ design points

Figure 6-2. Control diagram

Figure 6-3. Plot of FAST simulated CART generator speed using DAC controller based on 5-state model, with drive-train damping from two different controllers .

Figure 6-4. Close-up of FAST simulated CART generator speed using DAC controller based

Figure 6-5. Plot of FAST simulated CART pitch rate using DAC controller based

on 5-state model, with drive-train damping from pitch control compared with drive-train damping from generator torque control.

Figure 6-6. Plot of FAST simulated CART generator torque using DAC controller based on 5-state model, with drive-train damping from pitch control compared with drive-train damping from generator torque control.

Figure 6-7. Plot of FAST simulated CART generator speed using DAC controller based on 7-state model, with drive-train damping from pitch control compared with drive-train damping from generator torque control.

Figure 6-8. Plot of FAST simulated CART generator torque using DAC controller based on 7-state model, with drive-train damping from pitch control compared with drive-train damping from generator torque control.

Figure 6-9. Plot of FAST and ADAMS simulated generator speed, using generator torque to add damping to drive-train torsion

Figure 6-10. Plot of FAST and ADAMS simulated tower-top fore-aft motion, using generator torque to add damping to drive-train torsion

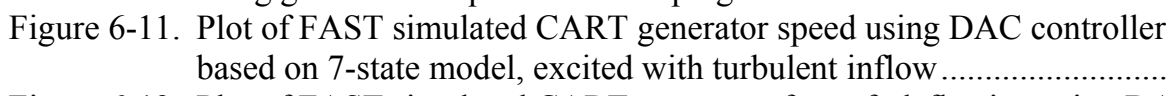

Figure 6-12. Plot of FAST simulated CART tower-top fore-aft deflection using DAC controller based on 7-state model, excited with turbulent inflow

Figure 6-13. Plot of FAST simulated CART tower-top fore-aft deflection using DAC controller based on 7-state model, excited with turbulent inflow.

Figure 6-14. Plot of FAST simulated CART blade pitch rate using DAC controller based on 7-state model, excited with turbulent inflow.

Figure 6-15. Plot of FAST simulated CART electrical power, using DAC controller

based on 7-state model, excited with turbulent inflow...

Figure 6-16. Plot of FAST and ADAMS simulated generator speed, using generator torque to add damping to drive-train torsion, with turbulent inflow .....

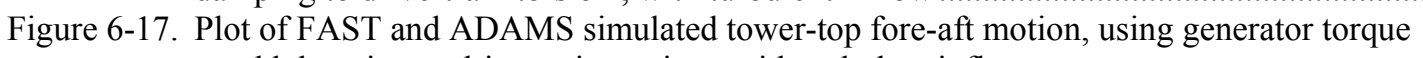

to add damping to drive-train torsion, with turbulent inflow ..................................

to add damping to drive-train torsion, with turbulent inflow ..........................

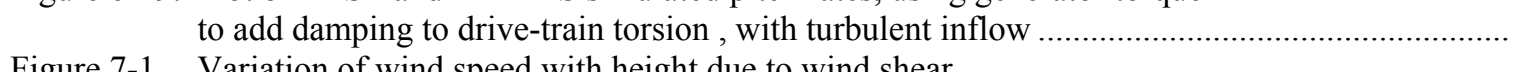

Figure 7-2. Polar coordintes

Figure 7-2. Polar coordinates

Figure 7-3. Diagram of DAC controller........

Figure 7-4. Test winds .....

Figure 7-5. FAST simulated generator speed and pitch using full-state feedback controller

Figure 7-6. Plot of CART generator speed and blade1 pitch as simulated with FAST using

Figure 7-7. Comparison of FAST simulated blade-tip flap deflections with full-state feedback for three pole locations, designed from 5 -state model 
Figure 7-8. Comparison of FAST simulated blade-1 pitch with full-state feedback for three pole locations, designed from 5 -state model

Figure 7-9. FAST simulated blade-1 and blade-2 pitch, using full-state feedback with high flap damping...

Figure 7-10. FAST simulated generator speed for various measured turbine parameters

Figure 7-11. FAST simulated blade tip flap deflections using different controllers designed from

Figure 7-12. FAST simulated generator speed and pitch using DAC controller designed

Figure 7-13. FAST simulated blade

Figure 7-13. FAST simulated blade tip flap deflections using full-state feedback and DAC controllers designed from 5-state model

Figure 7-14. FAST simulated blade pitch using full-state feedback and DAC controllers designed from 5-state model

Figure 7-15. FAST simulated CART tower-top fore-aft deflection using DAC based on 5-state model

Figure 7-16. Plot of CART generator speed and blade-1 pitch as simulated with FAST using DAC control designed from 9-state model

FAST simulated blade-tip flap deflections showing effect of setting wind-shear

Figure 7-17. FAST simulated blade-tip
disturbance gain to zero.

Figure 7-18. FAST simulated tower-top fore-aft deflections using two controllers

providing high and low values of damping in the tower's first fore-aft mode
Figure 7-19. Comparisons of ADAMS (flexible blade and tower) and FAST simulated generator speed using DAC controller designed from 9-state model.

Figure 7-20. Comparisons of ADAMS (flexible blade and tower) and FAST simulated tower-top fore-aft deflection using DAC controller based on 9-state model.

Figure 7-21. Comparisons of ADAMS (flexible blade and tower) and FAST simulated blade-tip flap deflection using DAC controller based on 9-state model

Figure 7-22. Comparisons of ADAMS (flexible blade and tower) and FAST simulated generator speed, using DAC controller based on 9-state model tested with turbulent wind inflow....

Figure 7-23. Comparisons of ADAMS (flexible blade and tower) and FAST simulated tower-top fore-aft deflection using DAC controller based on 9-state model tested with turbulent wind inflow.

Figure 7-24. Comparisons of ADAMS (flexible blade and tower) and FAST simulated blade-tip flap deflection using DAC controller based on 9-state model tested with turbulent wind inflow

Figure 7-25. Comparisons of ADAMS (flexible blade and tower) and FAST simulated blade pitch rate using DAC controller based on 9-state model tested with turbulent wind inflow.

Figure 7-26. FAST and ADAMS simulated generator speed, using generator torque control to add damping to drive-train torsion, independent pitch control designed from 9-state model

Figure 7-27. FAST and ADAMS simulated generator torque, using generator torque control to add damping to drive-train torsion, independent pitch control designed from 9-state model

Figure 7-28. ADAMS (flexible blade and tower) simulated blade pitch rate using DAC controller based on 9-state model, with and without generator torque control

Figure 7-29. FAST simulated blade pitch rate using DAC controller based on 9-state model, with and without generator torque control.

Figure 8-1. FAST simulated generator speed, showing effects of actuator time constants, DAC designed from 9-state model, with generator torque control.

Figure 8-2. FAST simulated tower-top fore-aft deflection, showing effects of actuator time constants, DAC designed from 9-state model, with generator torque control. 
Figure 8-3. FAST simulated blade-tip flap deflection, showing effects of actuator time constants, DAC designed from 9-state model, with generator torque control............................. 108

Figure 8-4. FAST simulated generator speed, comparing DAC and PI control results for slow and fast actuator

Figure 8-5. FAST simulated low-speed shaft torque, comparing DAC and PI control results for $\mathrm{Tau}=0.2$

Figure 8-6. FAST simulated tower-top fore-aft deflection, comparing DAC and PI control results for a fast and slow actuator

Figure 8-7. FAST simulated blade-tip flap deflection, comparing DAC and PI control results for a slow and fast actuator

Figure 8-8. FAST simulated generator speed, comparing DAC and PI control results for a slow and fast actuator .

Figure 8-9. FAST simulated low-speed shaft torque, comparing DAC and PI control results for a slow and fast actuator

Figure 8-10. FAST simulated tower-top fore-aft deflection, comparing DAC and PI control results for a slow and fast actuator

Figure 8-11. FAST simulated blade-tip flap deflection, comparing DAC and PI control results for a slow and fast actuator

Figure 8-12. FAST simulated blade pitch rate, comparing DAC and PI control results for a slow and fast actuator

Figure 8-13. FAST simulated tower-top fore-aft motion, showing effects of including actuator in design

Figure 8-14. FAST simulated tower-top fore-aft deflection, comparing DAC and PI control results, including actuator dynamics in control design for step and turbulent winds 


\section{Chapter 1. Introduction}

Wind turbines are relatively simple machines when compared with complex electrical power plants. However, the stochastic nature of the wind introduces a degree of complexity not usually associated with other regulated power-producing services. In this chapter, a general overview of wind turbine technology is presented, and the major issues associated with advanced control are introduced.

We start with a general description of the horizontal-axis wind turbine. Some of the basic wind turbine control problems and an overview of previous wind turbine controls research and technology are presented. Past work in the development and validation of wind turbine simulation codes is also given. Finally, the objectives of this work, as well as the approach used to answer the questions posed in this research, are described.

\subsection{Basic Wind Turbine Description}

A wind turbine is a device for extracting energy from the wind. Figure 1-1 shows the basic components, including the blades, hub, low- and high-speed shaft, gearbox, generator, nacelle, and tower.

The generator is coupled to the utility grid either directly or via a frequency-conversion system. In the latter case, the rotor speed is variable; in the former case, it is fixed by the frequency of the grid. The aerodynamic torque is transferred to the generator via the drive train. This transmission consists of a low-speed and highspeed shaft, separated by the gearbox. The rotational speed of the generator is typically 1500 revolutions per minute (rpm), whereas the rotor rotates at 20-40 rpm.

A wind turbine may exhibit various motions as a result of rotor and generator rotation, nacelle yaw and tilt, rotor teeter; and blade, drive train, and tower elastic deflections. Figures 1-2a, 1-2b, 1-2c, and 1-2d depict the most important degrees of freedom (DOF).

In a "free-yaw" wind turbine, the yaw motion is unrestrained; in the "yaw-driven" machine, the yaw position is regulated with a motor to orient the turbine into the wind. Wind turbines can be designed as either upwind or downwind machines. In a downwind machine, the wind passes the tower first, before impinging upon the rotor (as in Figure 1-2a); just the opposite occurs for the upwind machine. In a downwind machine, the yaw motion is stable and can be unrestrained (free yaw), meaning that the turbine will track wind direction changes without being yaw-driven. This is not true for the upwind machine, and a yaw drive must be employed to maintain the turbine's orientation into the wind. This gives the downwind machine a definite advantage in terms of simplification.

The wind inflow to the rotor varies both spatially and with time and can usually be separated into a deterministic part and a stochastic part. The deterministic part is constant with time but varies spatially as a result of wind shear, yaw misalignment, and tower shadow (tower shadow is the obstruction of wind flow to the rotor by the tower, as a blade passes behind the tower for a downwind machine). The stochastic part of the wind varies both with time and spatially. The disadvantage for the downwind machine is the effect of tower shadow, which causes a sudden change in aerodynamic loading on the blades. This can cause excitation of flexible blade modes and blade fatigue loading. 


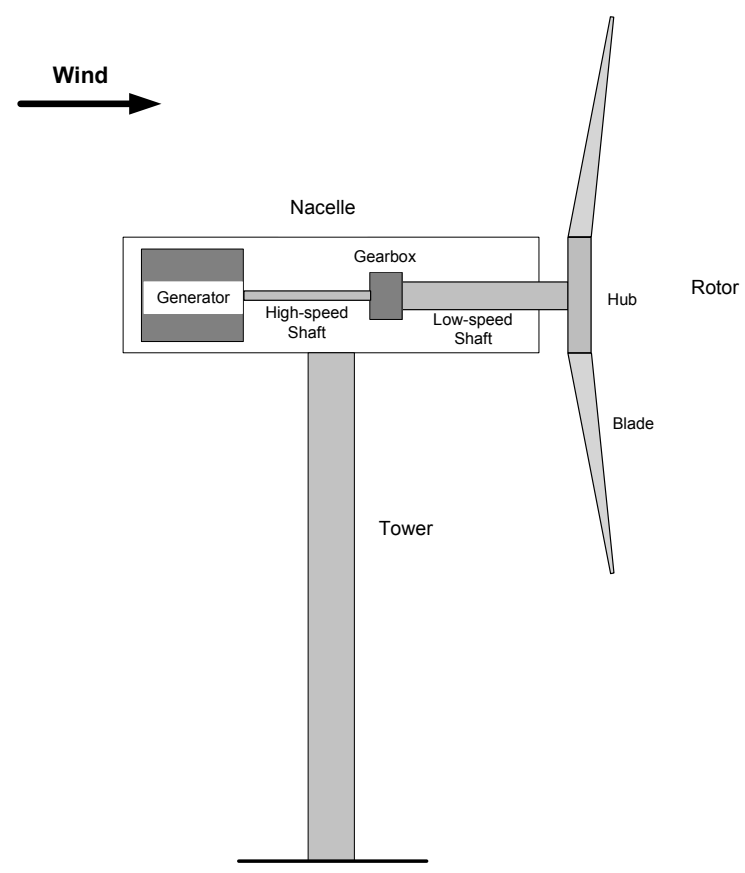

Figure 1-1. Wind turbine diagram

Wind turbines can be classified as fixed speed (the rotor and generator rotational speeds are held constant) or variable speed. Controls are incorporated to hold or adjust rotational speed, and one of the main objectives is to maximize power. The amount of power produced can be expressed as

$$
\mathrm{P}=\frac{1}{2} \rho \hat{A} C_{p} V_{w}{ }^{3}
$$

where $\mathrm{P}$ is power, $\hat{A}$ is area of the rotor disk, $\rho$ is the air density, and $V_{w}$ is the free-stream wind velocity (Wilson and Lissaman 1974). 


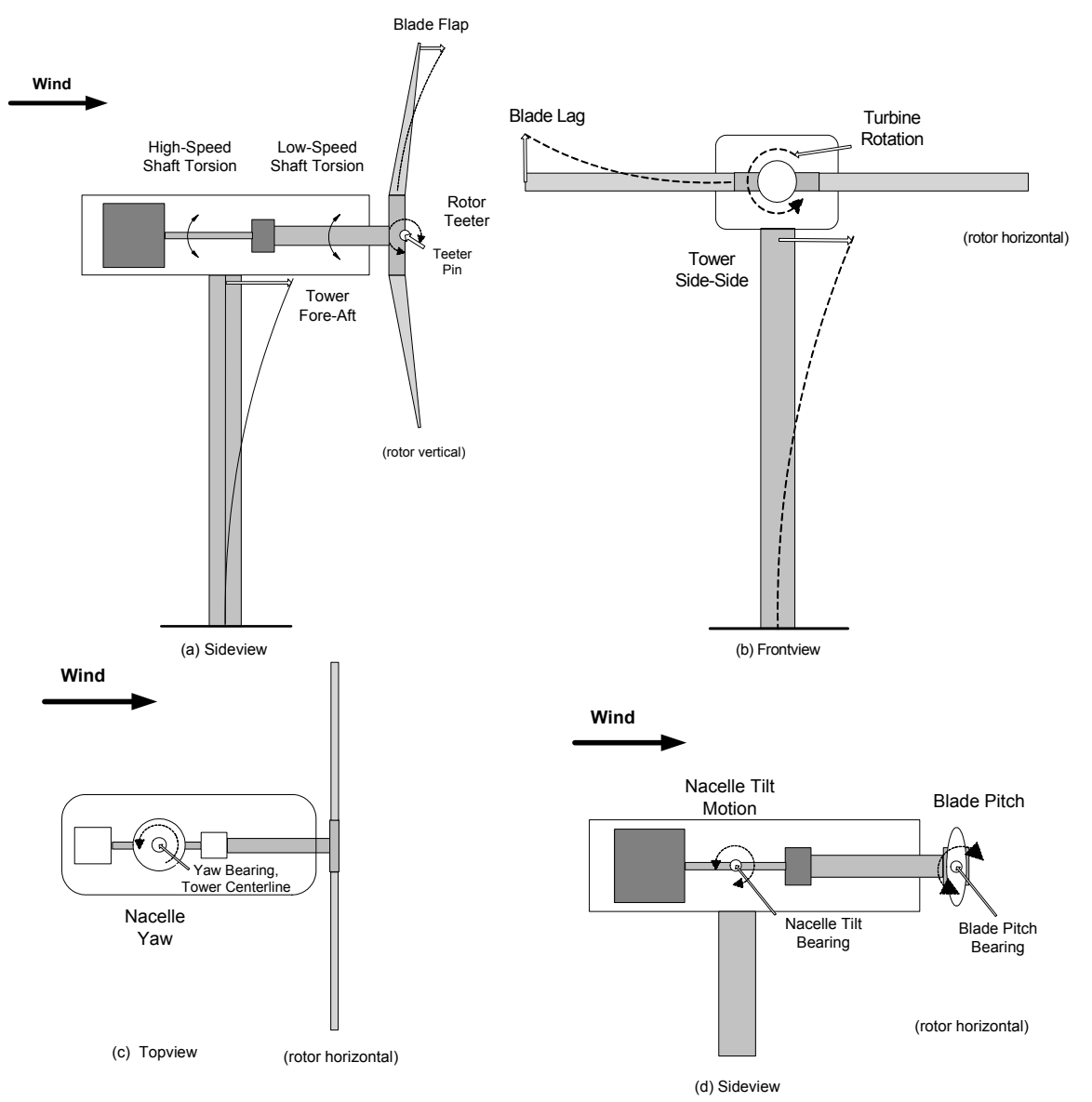

Figure 1-2. Typical turbine degrees of freedom and motions

The power coefficient $C_{p}$ is a function of the tip-speed ratio $\lambda=\frac{\Omega R}{V_{w}}$ and the blade pitch angle $\beta$, where $\Omega$ is the rotor speed and $R$ is the rotor radius. Figure 1-3 shows a typical plot of wind turbine $C_{p}$ as a function of the tip-speed ratio, for various pitch angles. As can be seen from the figure, each curve has one maximum at a certain tip-speed ratio. For an optimum energy production strategy, the tip-speed ratio and pitch angle should be chosen to give an optimum $C_{p}$. The turbine should operate at this tip-speed ratio, regardless of the wind speed. Since the tip-speed ratio is a function of rotor speed and wind speed, the rotor speed must be varied as the wind speed varies. For the fixed-speed turbine, this is not possible, although, some attempt is made to optimize energy by changing blade pitch to adjust aerodynamic torque as the wind speed varies. In the variable-speed machine, rotor speed can be changed by controlling the generator torque (Bossanyi 2000). 


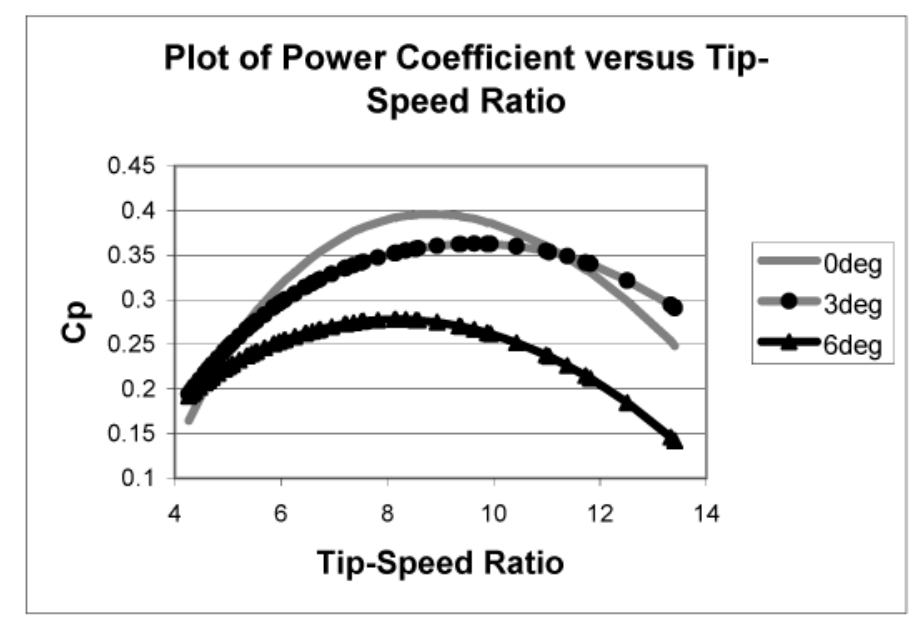

Figure 1-3. Plot of rotor $C_{p}$ versus tip-speed ratio for various pitch angles

Operating wind turbines at the optimum $C_{p}$ over a large range of wind speeds is not practical. Forces on the machine increase as the square of the mean wind speed. Thus, at higher wind speeds, the turbine must be designed to withstand higher forces, which increases machine weight and cost.

Rated wind speed is the velocity at which maximum power output ("rated power") is achieved. For wind speeds above this "rated wind speed" power must be held constant by the use of wind turbine controls. Uncontrolled, the power would increase in proportion to the cube of the wind speed and overheat the generator and power electronic system. Figure 1-4 shows a typical power curve for a wind turbine. The velocity where $C_{p_{\max }}$ (maximum $C_{p}$ ) is maintained by varying the turbine speed is called "region 2" (or "partial load"). The area in which power is held constant, at and above the rated power point, is called "region 3" (or "full load").

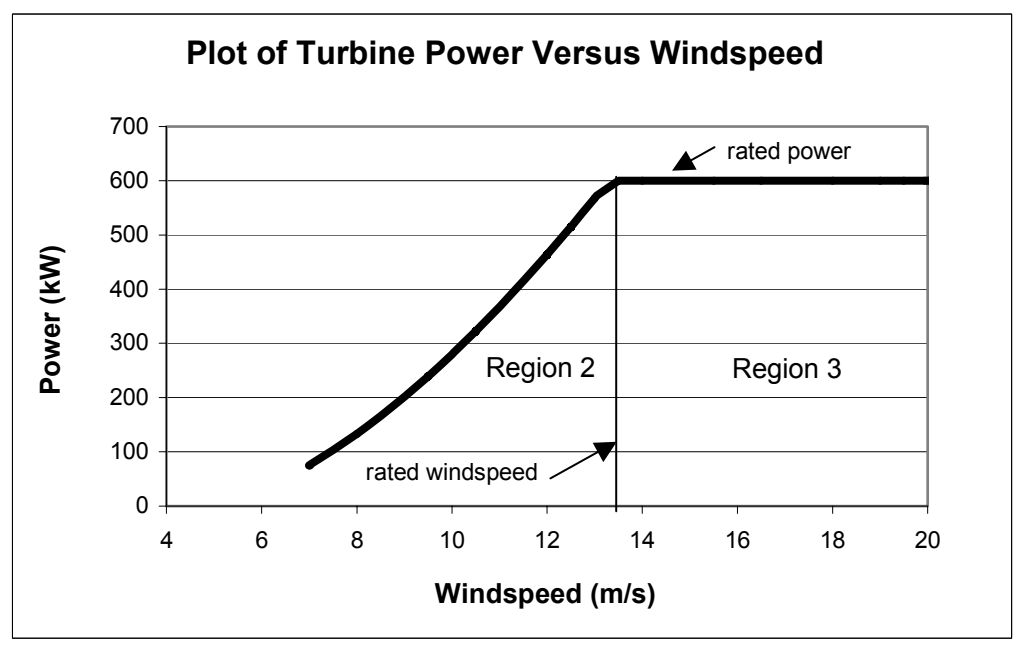

Figure 1-4. Typical wind turbine power curve

In region 3, turbine power is maintained at "rated power." For the variable-speed machine, a constant torque is applied at the generator, and the turbine rotational speed is maintained at the desired value through the use of blade pitch. For the fixed-speed turbine, aerodynamic design and/or blade pitch is used to maintain constant ("rated") aerodynamic torque. One great advantage of the variable-speed machine is its ability to produce constant power in region 3. By commanding constant generator torque, and setting blade pitch, the variable- 
speed turbine's rotor inertia prohibits rapid speed variations, and a slower bandwidth pitch system can be used. In contrast, constant-speed machines either suffer power spikes caused by rapid inflow velocity changes or pitch at high rates to cancel inflow changes. As a result, all modern machines are incorporating variable speed and pitch control.

Wind turbines usually have at least three different possible control actuators: blade pitch, generator torque, and machine yaw. Blade pitch is the most effective method of controlling aerodynamic loads. In some machines, the blade pitch is adjusted independently of the other blades (independent blade pitch control); in others, the pitch angle of each blade is adjusted identically (rotor collective pitch control). Generator torque is most often used in region 2 to maintain turbine operation at maximum $\mathrm{C}_{\mathrm{p}}$. It can also be used to add damping to the drivetrain torsion modes of the turbine in region 3. In addition, the power output of the turbine can be limited by yawing the machine out of the wind, thereby decreasing the projected rotor area and reducing power. Most often, yaw control is used only to respond to changes in wind direction in an attempt to reduce the yaw error (the angle between the mean wind direction and the direction of orientation of the turbine) and thereby maximize power.

In order to be cost-effective, future wind turbines must be designed with lighter, more flexible components (blades, drive train, tower, etc.) in order to reduce their weight and cost. As wind turbines become larger and more flexible, structural dynamic loads and instabilities will increase unless control systems are specifically designed to mitigate these loads and dynamic interactions. Modern control paradigms must be developed that adequately treat the flexibilities of the turbine system, using one or all of the control actuators just mentioned. These control paradigms must be thoroughly tested both analytically and experimentally.

What prior work has been done in controls research? How have controls been designed to account for the flexible nature of wind turbines? What has been done in controls explicitly for load and response alleviation, especially for flexible turbines? What analytical tools have been developed for these control designs? To address these questions, we now review some pertinent past wind turbine controls and analytical code development work.

\subsection{Past Research in Wind Turbine Controls}

To review previous wind turbine controls research, we begin with a look at classical controls for large turbines designed in the 1970s and 1980s.

\subsubsection{Classical Control Designs}

Classical control design techniques based on the proportional-integral-derivative (PID) approach were used for several large wind turbines built in the 1970s and 1980s. Most of the fixed-speed machines of that era had stiff drive trains and a large rotor with high inertia. It was found that wind turbulence easily excited the machine's first drive-train torsion mode. For turbines with synchronous generators, the damping in this mode was very low (Kos 1978). The objectives of the control system were to regulate power while also adding damping to this mode using blade pitch (Svensson and Ulen 1982; Rothman 1978; Hinrichsen 1984; Hinrichsen and Nolan 1984; Kos 1978; Wasynczuk et al. 1981).

Often, it was found that a large controller bandwidth was needed to properly control the machine in the presence of turbulent wind inflow, as reported in Rothman (1978) and Kos (1978). Rothman compared rotor gust load responses under what was termed "a moderately fast pitch rate," a loop bandwidth of 2 radians/second $(\mathrm{r} / \mathrm{s})$, against a slower system with a bandwidth of $0.2 \mathrm{r} / \mathrm{s}$. He concluded that, although loads due to lowfrequency wind variations were satisfactorily attenuated, a much more responsive control system was needed to reduce structural loading due to high-frequency wind inputs. Fast pitch control has been discussed as helping to alleviate machine loads (Johnson and Smith 1976). None of the studies explored the effect of fast pitch rates on blade loads.

In these studies, system dynamic response was intimately related to the choice of controller bandwidth (Kos 1978). It was determined that a large bandwidth introduced the possibility of exciting various modes of the 
turbine, because of their low natural frequencies and low damping. One such mode is the tower first fore-aft bending mode. Svensson and Ulen (1982) used tower motion feedback in the controller to add damping to this mode. Hamilton Standard also implemented this method in the WTS-4 turbine (Hamilton Standard 1985).

In these various applications, the use of classical controls to address more than one control objective was not straightforward. Often, multiple control loops were used, which added complexity to the control design and system behavior. It was difficult to properly address control-structure interaction issues using classical control methods, since the controller used only a single measured turbine output as the basis of its control and did not have direct knowledge of the system dynamics of the turbine. Modern control designs using state-space methods more adequately address these issues, since the controller uses a model to determine system states. Controllers can be designed not only to maximize power or regulate speed but also to add damping to important flexible modes, through full-state feedback.

\subsubsection{Modern Control Designs}

Liebst (1985) developed a pitch control system for the KaMeWa wind turbine using the Linear Quadratic Regulator (LQR) design. The objective of this controller was to alleviate blade loads due to wind shear, gravity, and tower reflection using individual blade pitch control (the pitch of each blade can be controlled independently). The wind turbine DOF modeled in this study were blade flap, lag, and pitch; drive-train torsion; generator rotation; and tower bending. Results of this work included a reduction of blade and tower cyclic responses as well as the reduction of a large 2-per-revolution (2P) variation in power, as demonstrated through simulation.

The practical application of this technique is limited by problems in obtaining measurements of the states needed in the controller. Extra sensors and measurements can add considerable cost and complexity to a wind turbine. Errors in the measurement of these states can cause poor controller behavior. To avoid these problems, a state estimator must be employed in any practical implementation.

Mattson (1984) used a state estimator in combination with LQR. He described the regulation of power for a fixed-speed machine using blade pitch. Both synchronous and induction generator cases were analyzed. He based this control design on linear models containing drive-train torsion and tower fore-aft bending DOF. The blade elastic DOF were ignored. Controllers were developed for such large wind turbines as the Mod-2 and WTS-3 machines, with a view not only to regulating power but also to adding damping to the drive-train torsion mode. He advocated the use of a torsionally soft drive train in combination with pitch control to attenuate $2 \mathrm{P}$ variations in shaft torque caused by wind shear, tower shadow, and turbulence.

Mattson (1984) also described the use of state estimation to estimate wind speed. He reported good results except in frequency intervals close to the natural frequency of the first drive-train torsional mode. This was due to the amplified effects of measurement noise, caused by the controller attempting to compensate for phase lag at this natural frequency. This resulted in excessive pitch servo motions at frequencies close to the natural frequency of the first drive-train torsional mode.

The nonlinear behavior of a wind turbine can make control design difficult. For example, the aerodynamics is highly nonlinear. In pitch control, the control input gains are usually the partial derivative of the rotor aerodynamic torque with respect to blade pitch angle. These input gains vary with wind speed, rotor speed, and pitch angle. A controller designed for a turbine at one operating point may give poor results at other operating points. In fact, the controller may result in unstable closed-loop behavior for some operating conditions.

To address this issue, multiple controllers can be designed at different turbine operating points. As the turbine's operating point varies from one region to another, the controllers can be switched. This usually requires a "scheduling" or "switching" parameter, such as wind speed or pitch. Wind speed can be a poor scheduling parameter, however, because the wind speed at the rotor disk is not known. Usually, anemometers are located upwind of the turbine, giving only an approximation of the wind speed at the rotor. The wind speed at a point is usually poorly correlated with the wind speed actually experienced by the turbine. The turbine may also induce 
local disturbances, so that an anemometer gives bad measurements. This makes practical implementation of controller scheduling (or switching) difficult.

Some work has been performed to study switching between controllers for wind turbines (Kraan 1992). Often, switching between different controllers can be problematic, in that if one simply switches between one controller and the next controller, undesirable switching transients can occur. Bongers (1994) describes the use of controller conditioning, in which the next controller to be activated is prepared for this task, so that switching transients are minimized.

Another modern control technique to account for changing gains is adaptive control, in which the control gains "adapt" to changing conditions. Bossanyi (1987) researched an adaptive scheme, consisting of a time-varying state estimator using optimal control, applied to take varying gains into account. The author reported satisfactory simulation results for schemes based on combined power and shaft-speed measurements. More recently, an adaptive control method was studied by Freeman and Balas (1999) for a three-bladed turbine.

Control systems must be developed that maintain the stable closed-loop behavior of the turbine. It is particularly important to design control systems that properly account for the flexible modes of the turbine. In modern state-space methods, linear models of the turbine are generated for use in control design. These linear models must contain the states necessary to model the most important flexible modes of the turbine. If certain modes of the turbine are ignored in the control design, then the controller can destabilize these modes. In Stol et al. (2000), Disturbance Accommodating Control (DAC) was developed for a two-bladed teetering hub turbine from a linear model containing only rotor rotation as the DOF. It was shown that this DAC adequately controlled a turbine as modeled in SymDyn with just the rotor rotation DOF. This system became unstable when more DOF were turned on in SymDyn than were included in the linear model for controller design.

These investigations show that consideration must be given to unmodeled structural DOF when designing a controller. Less aggressive control gains could probably be chosen for the lowest-order controllers, resulting in stable behavior of the complete wind turbine. However, there are always components in a wind turbine that are difficult to model or have uncertain properties. It is important to begin to assess the importance of these unmodeled effects in the design of controllers for wind turbines.

\subsubsection{Additional Control Issues}

In other studies, research has been conducted to design improved controls for operation in region 2. To maximize energy capture, a variable-speed wind turbine should operate continuously at the tip-speed ratio that results in the maximum power coefficient. This goal is only partially achieved, because of rapid variations in wind speed and the inertia of the wind turbine rotor. Although it is not possible for a turbine to operate continuously at maximum efficiency, improvements in energy capture during variable-speed operation can be gained by improved tracking of $C_{p}$. Pierce (1999) reported that the aerodynamic torque, estimated by an observer, and rotor speed can be used to improve the energy capture of a variable-speed turbine. Small improvements of energy capture were shown in that paper through the use of the control methods described.

As Holley et al. (1999) have shown, control that optimizes energy capture in region 2 can also cause undesirable torque fluctuations. These fluctuations result from the inertia of the rotor as the torque control attempts to follow the wind. They also showed that variable blade pitch is not effective in improving the energy capture in region 2 while the turbine is operating at maximum $\mathrm{Cp}$, because the system is locally uncontrollable using blade pitch at this operating point. A simple modification to the square-law controller allowed a modest increase in energy capture, but at the expense of increased torque fluctuations.

Other work has focused on controls explicitly for load reduction. The effects of tower flexibility were included in a control study by Eggers et al. (1998). In this study, it was found that rotor/tower coupling had a negligible influence on control system effectiveness in reducing blade root moments in closed-loop operations. This same control had no influence, however, in reducing tower base moments. In order to reduce these moments, the system bandwidth had to be increased, which increased the control activity. 
Eggers et al. further extended this study (1999) to include effects of actuator lag, error sensing, and nonuniform turbulence on blade pitch control of fluctuating aerodynamic loads. In this work, they found that nonuniform turbulence required the use of individual blade pitch control for a rigid rotor, mainly to remove the effects of once-per-revolution (1P) variations in wind speed across the rotor disk. For the teetered rotor case, they found that the teeter DOF removed this variation, and collective pitch could be used.

This work was extended by Moriarty et al. (2001) to show the effects of control system lag. They showed that actuator lag reduced the effectiveness of the control system. However, load reductions were possible even when implementing a relatively slow actuator with a 1-second time constant. They also showed that more aggressive control modes may be limited by excessively high control accelerations.

Another issue in wind turbine control design is the periodicity of the wind turbine dynamic system. Stol developed periodic control gains using time-varying LQR techniques for a two-bladed teetering hub turbine operating in region 3 (Stol 2001; Stol and Balas 2001; Stol and Balas 2002). Stol concluded that, if speed regulation is the only objective, then periodic control is not the most appropriate method. He also concluded that, when blade load reduction is the primary goal, periodic control gives the best results when full-state feedback is used. Ekelund (1997) designed controls for attenuating structural-dynamic load oscillations by means of the yaw actuator. In three design examples, various structural modes were studied. The tower sideside bending mode showed the best potential for active load reduction. The results also indicated the importance of considering the periodic dependence on time of the system in those control designs.

Another issue in control design is thorough testing of the designed controller through simulation and field tests. For simulation, adequate simulation codes, which include the effects of the controls, must be available. Next, we review progress in the development of analytical codes.

\subsection{Analytical Tool Development and Validation}

\subsubsection{Simulation Codes}

Most large turbines built in the 1970s and 1980s were designed using very simple analytical methods and simulation codes that neglected important turbine dynamics as well as the effects of turbulent wind inflow and control systems. Since that time we have learned that (1) system dynamic loads are the principal design drivers for wind turbines, (2) measured loads on turbines produced in the 1970s and early 1980s were often two or three times higher than predictions, and (3) simulation codes must account for the complex statistical nature of turbulent fluctuations in the wind as well as the complete nonlinear coupled system, including the effects of the controls. Controls must be designed to account for these complex processes. The full turbine system, including control system effects, must be simulated for a wide variety of turbine operating conditions in order to test the closed-loop system.

In recent years, progress has been made in developing and validating analytical codes for wind-turbine loads and response prediction. A thorough review of several wind turbine design codes developed both in the United States and other countries can be found in Molenaar (2003).

Code development activities in the United States have taken a two-pronged approach: (1) developing a general multi-purpose code that can be used to analyze a wide variety of wind turbines in great detail, and (2) developing specialized codes with minimal sets of degrees of freedom (DOF) for predicting critical design loads in two- and three-bladed horizontal-axis wind turbines. The general multi-purpose code is very useful because analyzing a new wind turbine configuration does not necessitate developing a new code from scratch, whereas the specialized codes are very useful for rapidly calculating critical design loads.

In the first approach, a commercial general-purpose code was selected for adaptation to wind turbine use. This package is named Automatic Dynamic Analysis of Mechanical Systems (ADAMS ${ }^{1}$ ) and is produced by

${ }^{1}$ ADAMS is a registered trademark of Mechanical Dynamics, Inc. 
Mechanical Dynamics, Inc., of Ann Arbor, Michigan (Elliott and McConville 1990). This code automatically generates numerical equations of motion at runtime, removing the major modeling task of developing and validating large numbers of equations of motion. Validation of ADAMS for a very flexible turbine was performed by Wright (1999).

In the second approach, streamlined codes such as FAST (Fatigue, Aerodynamics, Structures, and Turbulence) are being developed and refined. This code was first developed at Oregon State University (Wilson et al. 1999) and validated and refined at the National Renewable Energy Laboratory (NREL). Validation of the FAST code has been done by Buhl et al. (2000).

In addition, an aerodynamic subroutine package named AeroDyn (Laino and Hansen 2003) was developed at the University of Utah. This subroutine package is used in ADAMS and FAST. Specifically, this subroutine generates the aerodynamic forces on a wind-turbine blade, which are then used by each of these codes to calculate the dynamic response of the wind-turbine system. Calculating the aerodynamic forces on the blades is really the core of any wind-turbine simulation code, and it must be included as a core element of any structural analysis code for wind turbines.

Validation of AeroDyn has been done by Hansen (1998) using data from the Unsteady Aerodynamics Experiment (UAE) turbine (Simms 1996). More extensive validation of AeroDyn has been performed using data obtained from wind tunnel tests of the UAE turbine (Jonkman 2001). In these studies, 3-D flow effects along the blade led to higher lift coefficients than predicted by AeroDyn (Laino 2002). In order to improve these results, refinements have been made to the Generalized Dynamic Wake (GDW) model in AeroDyn (Laino 2003).

\subsubsection{Control Designs Tools}

More recently, codes have been developed specifically to assist wind turbine designers in the design of control systems. DUWECS, a nonlinear wind turbine simulation tool, was developed at the Delft University of Technology (Bongers 1994). It uses Kane Dynamics to formulate the equations of motion. The model includes the effects of blade and tower flexibility as well as nacelle yaw and rotor teeter motion. It also models a flexible drive train. The blade and tower flexibility is modeled by assuming the blade (or tower) is connected to the hub (or foundation) with a discrete spring and damper at the blade root (foundation). The stiffness of the spring is adjusted to give a natural frequency of motion equal to the first flap frequency of the blade (or first fore-aft frequency of the tower). This approach (called the rigid-blade/hinge or rigid-tower/hinge approach) neglects higher bending modes of the blade or tower. The code can be used to generate a linear model of a turbine for control design or to simulate the complete system once the controller has been designed. This has all been incorporated into an integrated wind turbine control design and simulation package named DUWECS. DUWECS can also obtain a linear model of a nonlinear wind turbine for control design purposes.

Another code, SymDyn, has been developed at the University of Colorado (Stol et al. 2000). Blade and tower flexibility are also modeled using the rigid blade (tower)/hinge approach. The code generates linear models for control design and simulates system behavior once the control system has been designed.

The utility of SymDyn is the modal approach to wind turbine modeling, which results in models having fewer DOF than large finite element or multibody dynamics codes. Linear models can be generated that account for the relevant turbine dynamics with only a few DOF. This makes interpretation of results much easier than with multi-degree-of-freedom models.

All these are current tools to aid in designing wind turbines and controls. What is needed to design controls for future wind turbines?

\subsection{Control Needs for Future Wind Turbine Designs}

Future goals for wind turbine technology include the design of large, flexible rotors attached to tall towers. These machines will be easily excited by turbulent wind inflow. Wind turbine controls must play an important 
role in adding damping to the flexible modes of these machines, to stabilize the machine and reduce dynamic response and loads. A control design approach must be applied that accounts properly for the flexibility of the turbine, so that stable closed-loop behavior can be assured.

The codes and models we use to design and simulate controls for future wind turbines should be complex enough to model additional modes not modeled by the rigid-blade/hinge approach. They should be simple enough, though, to allow careful checkout and testing of the controls in closed-loop operation. It is of great importance to begin with simple control models and simulation tools and add complexity in steps, after thorough checkout and debugging of the simple cases. The simulation code and control design models (linear models used for control design) should be flexible, allowing one to model just a few of the most important turbine DOF and then add complexity in steps. This objective cannot be met if the modeling platform (both the linear models used for control design, as well as the nonlinear simulation code) results in models with a large number of DOF. Thus, codes that allow one to switch on and off turbine DOF are desirable. They would allow the designer to begin simply and work up in steps, adding just a few new DOF at each step. In this report, we begin with simple models and tools and add complexity in steps, only after the need to add this complexity has been firmly established.

The modeling approach used here is based on the FAST code (Wilson et al. 1999). This code is based on an assumed-modes approach, which models higher modes of the blades and tower. FAST, which is described further in Chapter 2, is intermediate in complexity, having more sophistication than codes that use a simple rigid-blade/hinge approach. But it is much less complex than codes that use a multibody or finite-element approach. In this code, DOF can be switched on and off. One can simulate with a subset of the total DOF included in the modeling formulation of FAST. This makes debugging and interpreting results very straightforward.

One weakness of FAST is that this code was not originally developed to provide a linear model for control design. As shown later in this report, methods have been developed to extract a linear model from FAST, both symbolically and numerically. FAST will be used for both the extraction of the linear model for control design and for closed-loop simulation. Linear models can be extracted from FAST based on just a subset of the total modeling DOF contained in FAST. The same DOF can be used in closed-loop simulation, or additional DOF can be switched on. The effects of unmodeled modes can be studied by switching on those DOF in simulation, which are neglected in the linear model used for control design.

It is also important to incorporate the control system into a simulation code and test whether additional flexible modes, not modeled with FAST, become unstable during closed-loop operation. A more thorough cross-check with a code that models the turbine in great detail will be made to see if additional flexible modes become unstable. The ADAMS multibody dynamics code, which models a turbine with a large number of DOF will be used for these cross-check simulations. These questions are particularly important in designing control systems for future large, flexible wind turbines.

\subsection{Objectives, Approach, and Contents}

\subsubsection{Research Questions}

Several significant issues must be addressed regarding the applicability of modern control approaches to advanced wind turbine designs. Research questions to be answered in this work include these: (1) Can control systems for flexible wind turbines be designed to both regulate rotor speed and stabilize the turbine structure (and thus reduce dynamic loads and response) in the presence of turbulent wind inflow? (2) Can state estimation be used to reduce the required number of turbine measurements and make actual implementation of such control systems more practical? (3) Which flexible modes are the most important to include in the linear model used for control design, i.e., which modes are most likely to become unstable during closed-loop operation? 


\subsubsection{Research Goals and Objectives}

The goal of this work is to develop the controls methodology, paradigms, and simulation tools required for advanced, large-scale flexible wind turbine designs. Specifically, the objectives are to (1) understand the flexibility of the integrated turbine system and identify the most critical modes of the turbine, as well as to design controls to regulate rotor speed and/or maximize energy extraction while properly accounting for these modes; (2) determine the number of states in linear models needed for control design and simulation fidelity; and (3) study the effect of meeting multiple control design objectives (such as load mitigation and enhanced mode damping) on the primary objective of speed regulation.

Contributions to the current state of the art in wind turbine controls technology include (1) the systematic application of modern control theory to the design of controls for turbine speed regulation and structural dynamic load reduction in flexible wind turbines; (2) development of turbine linear models with the minimum number of states needed to design control systems that result in stable closed-loop behavior; (3) identification of the most critical flexible modes that need to be included in linear models used for control design; (4) use of state estimation to reduce the number of necessary turbine measurements in order to simplify the implementation of these control algorithms; and (5) application of these control paradigms and methodologies to the CART (Controls Advanced Research Turbine) at the National Wind Technology Center (NWTC).

An important part of designing a control system for wind turbines is verification through field tests after implementation in the real machine. This report focuses on analytical verification through simulation.

\subsubsection{Research Approach}

Controls will be designed for the CART to regulate turbine speed in region 3. These controls will also be designed to stabilize important flexible modes of the machine and to minimize the effects of wind-speed disturbances. An approach to this work is to begin with simple control design models and to add complexity in steps, after careful checkout and verification of the simpler control design algorithms.

Another important part of this work is the simulation of the closed-loop system in order to test control algorithm performance. Two simulation tools are used: the FAST code, which uses a modal approach to model a limited number of the most important turbine DOFs, and the comprehensive ADAMS code, which allows us to model a turbine in great detail using a large number of DOFs. This permits simulation with a code of intermediate complexity (FAST) and high complexity (ADAMS). Simulating the closed-loop system with a detailed model such as ADAMS is important in order to check whether additional DOF, not modeled by FAST, become unstable during closed-loop operation.

\subsubsection{Contents of the Report}

In this chapter, we reviewed basic wind turbine technology as well as the major issues associated with advanced wind turbine control. In Chapter 2, the control design and simulation tools are described. A very limited verification of the models, through comparison to modal test data for the CART, is given.

In Chapter 3, a brief description is given of typical results obtained in simulation of classical ProportionalIntegral (PI) control for the CART. The chapter shows the deficiency of using simple PI control for a flexible system such as the CART. It presents reasons why more sophisticated control design methods are needed, methods that properly account for the turbine's flexibility.

In Chapter 4, modern control design steps are presented and illustrated with example cases. The chapter describes and applies methods that will be used in the remaining chapters.

In Chapter 5, controls are designed and simulated for speed regulation in region 3 using rotor collective pitch. These controls are also designed to stabilize and enhance damping in several flexible modes of the machine. 
They are also designed to attenuate (and in some cases cancel) wind disturbances, which are uniform over the rotor disk (have no azimuthal or spatial variation).

Chapter 6 describes the addition of generator torque as a control input to enhance damping of the drive-train torsion mode and relieve some of the requirements placed on the rotor collective pitch control system.

Chapter 7 describes the design and performance of controls using independent blade pitch as the control input. The main emphasis in the chapter is attenuation of wind disturbance components, which vary azimuthally, such as wind shear. These controls are also shown to satisfy the main control objective, regulation of rotor speed in region 3.

Chapter 8 compares results for these modern control designs with results from simple PI control for the CART. In this comparison, the actual PI gains implemented in the CART are used in the PI controller. This provides a measure of the performance of the newly designed modern controls.

Conclusions are presented in Chapter 9. 


\section{Chapter 2. Analytical Models and Simulation Tools}

\section{Introduction}

Various analytical tools are needed for control design and simulation. A linear model of the nonlinear wind turbine is needed for the application of modern control design theory. After the control system has been designed, simulations of the closed-loop system must be performed in order to test controller performance. In this chapter, a brief description is given of the modeling framework that forms the basis of these linear models and the simulation tool. A more comprehensive simulation tool, used to cross-check results, is also discussed.

\subsection{FAST Description}

The FAST code (Wilson et al. 1999) can model the dynamic response of both two- and three-bladed, horizontal-axis wind turbines. For two-bladed turbines, $15 \mathrm{DOF}$ are used to describe the turbine dynamics. Accounted for in the DOF are tower flexibility (4 DOF), rotor teetering (1 DOF), blade flexibility (6 DOF, 3 for each blade), nacelle yaw (1 DOF), nacelle tilt (1 DOF), and variable generator speed (2 DOF). The DOF for the two-bladed rotor case are listed in Table 2-1 and illustrated in Figure 2-1. FAST can be run with any subset of these DOF switched on during simulation. Further details are described in Wilson et al. (1999).

FAST models rigid bodies (earth, base plate, nacelle, generator, and hub) and flexible bodies (blades, low-speed shaft, and tower). It uses an assumed-modes approach to model blade and tower flexibility. The tower is assumed cantilevered to the earth and can bend in two directions, producing fore-aft and side-side motion. The bending flexibility is modeled by two modes in each direction. The top of the tower has a fixed base plate supporting a yaw bearing and nacelle. The yaw bearing allows everything atop the tower to rotate as the wind direction changes.

The nacelle houses the drive-train assembly, including the generator, gearbox, and low-speed shaft. As indicated in Figure 2-1, the nacelle can be allowed to tilt. The high-speed shaft connects the generator to the gearbox, and the low-speed shaft connects the gearbox to the rotor.

The rotor consists of a hub, blade pitch system, and rotor blades. A teeter hinge (for two-bladed designs) may be included between the rotor and the low-speed shaft, and can be offset by a delta- 3 angle. The hub supports two or three blades, each of which can be coned and can have aerodynamic pitch and twist. The blades are flexible, and their properties can vary along their length. Each blade can be structurally pretwisted, but no torsional motion is allowed in the current model. Bending can occur in the rotor plane (defined using one vibration mode), or out of the rotor plane (defined with two vibration modes). 

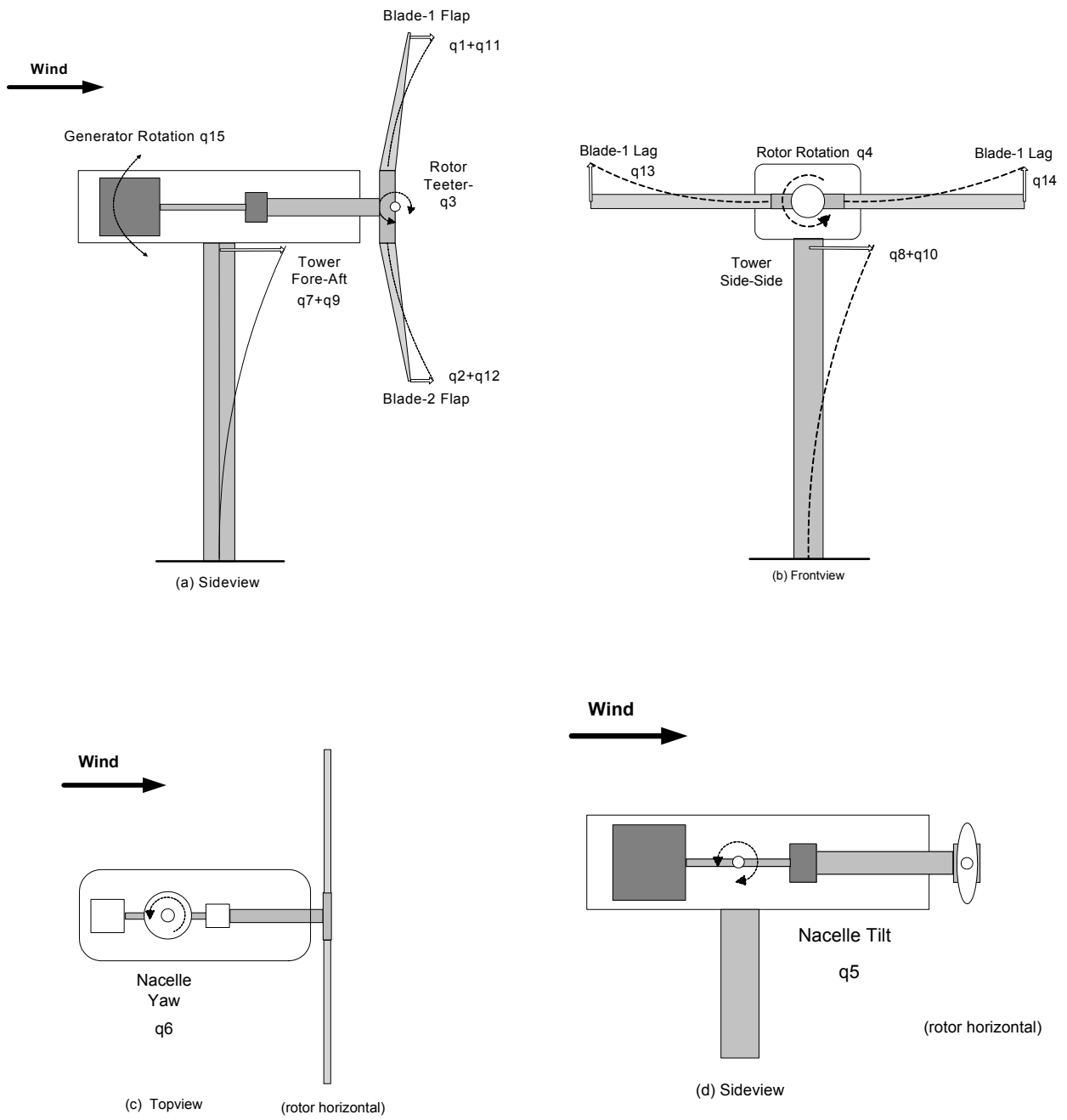

Figure 2-1. Degrees of freedom modeled with FAST 
Table 2-1. FAST Modeled Degrees of Freedom

\begin{tabular}{c|l}
\hline Variable & Description \\
\hline$q_{1}$ & Blade 1 flapwise tip displacement for mode 1 \\
\hline$q_{2}$ & Blade 2 flapwise tip displacement for mode 1 \\
\hline$q_{3}$ & Teeter angle \\
\hline$q_{4}$ & Azimuth angle, rotor side \\
\hline$q_{5}$ & Nacelle tilt angle \\
\hline$q_{6}$ & Nacelle yaw angle \\
\hline$q_{7}$ & Fore-aft tower top displacement for mode 1 \\
\hline$q_{8}$ & Side-side tower top displacement for mode 1 \\
\hline$q_{9}$ & Fore-aft tower top displacement for mode 2 \\
\hline$q_{10}$ & Side-side tower top displacement for mode 2 \\
\hline$q_{11}$ & Blade 1 flapwise tip displacement for mode 2 \\
\hline$q_{12}$ & Blade 2 flapwise tip displacement for mode 2 \\
\hline$q_{13}$ & Blade 1 edgewise tip displacement for mode 1 \\
\hline$q_{14}$ & Blade 2 edgewise tip displacement for mode 1 \\
\hline$q_{15}$ & Azimuth angle, generator side \\
\hline
\end{tabular}

Coordinate systems formed by orthogonal sets of unit vectors define reference frames that are fixed to each rigid body. Most of the transformation matrices represent a rigid body rotation about one of the local coordinate axes, thus representing a change in angular orientation from one body to the next. Once a complete set of coordinate systems has been defined, the displacements and motions of any point on the turbine can be written, and these expressions can be translated to any system by coordinate transformations. These expressions are then used in the formulation of the kinematics and kinetics for the turbine. The coordinate systems and transformations used in FAST are described in detail in Wilson et al. (1999).

Kane's method (Kane and Levinson 1985) is used to set up equations of motion in FAST that are solved numerically at run-time. No explicit symbolic equations of motion are derived in this code. The following sections describe the method of modeling specific subsystems in FAST.

\subsubsection{Blades and Tower}

The treatment of flexible bodies in rigid-body dynamics requires an approximation so that the general deflection can be represented by only a few DOF. In FAST, both the blades and the tower are treated as cantilever beams attached to the rotor hub or ground. An assumed modes approach is used to formulate the dynamics of these blade and tower models. The deflection $u(\mathrm{z}, \mathrm{t})$ of any point on the blade or tower at any time $t$ can be expressed as

$$
u(\mathrm{z}, t)=\sum_{i=1}^{n} q_{i}(t) \phi_{i}(\mathrm{z}), \quad i=1, \ldots, n
$$

where $\mathrm{Z}$ is position along the blade or tower centerline, $t$ is time, $\phi_{\mathrm{i}}(\mathrm{z})$ is an appropriate blade or tower modeshape, and $q_{i}(t)$ is the tower-top or blade-tip generalized coordinate, which is a function of time. Further details are contained in Wilson et al. (1999).

\subsubsection{Drive Train}

The drive train is modeled as an equivalent torsional spring separating the generator from the hub. The shaft can have a linear torsional spring and a linear torsional damper. The equation for the shaft may be expressed as

$$
T_{\text {shaft }}=K_{d}\left(q_{4}-q_{15}\right)+C_{d}\left(\dot{q}_{4}-\dot{q}_{15}\right)
$$


where $T_{\text {shaft }}$ is the torque at the generator end of the low-speed shaft, $K_{d}$ is the drive-train torsional spring stiffness coefficient, and $C_{d}$ is the drive-train torsional damping coefficient. $q_{4}, q_{15}, \dot{q}_{4}$, and $\dot{q}_{15}$ are the rotor and generator azimuth angles and their derivatives, respectively (see Table 2-1).

\subsubsection{Generator}

Four options are included in FAST for modeling variations in shaft rotational speed: constant rotational speed, induction generator, machine start-up, and machine shutdown. Further details for the drive-train and generator models are given in Wilson et al. (1999). For much of the work described here, we model the generator as a constant torque applied to the generator side of the low-speed shaft. The turbine rotational speed is allowed to vary, but it will be regulated to a desired value through the use of blade pitch. This is consistent with modeling turbine operation in region 3 , where generator torque is assumed to be constant.

\subsubsection{Aerodynamics}

Blade aerodynamic force calculations are based on blade element momentum theory, modeled in the AeroDyn subroutines (Laino and Hansen 2003) linked to FAST. Blade aerodynamic forces are calculated in AeroDyn and then passed to FAST's dynamics subroutine. In FAST, deflections and velocities are calculated at several points along each blade from root to tip and passed into AeroDyn. AeroDyn uses these deflections and velocities in calculating blade section angle of attack, which in turn alters the calculation of resulting blade section aerodynamic forces and moments.

\subsubsection{Numerical Methods}

Using Kane's method (Kane and Levinson 1985), we solve the resulting equations of motion for the acceleration using Gauss elimination.

The $\mathrm{r}^{\text {th }}$ equation has the form

$$
\sum_{s} \mathrm{C}_{\mathrm{rs}} \ddot{q}_{\mathrm{s}}=\mathrm{f}_{\mathrm{r}}(q, \dot{q})
$$

where $\mathrm{C}_{\mathrm{rs}}$ is the element of the mass matrix in the $\mathrm{r}^{\text {th }}$ row and $\mathrm{s}^{\text {th }}$ column. The $\ddot{q}_{\mathrm{s}}$ are the accelerations of each generalized coordinate. The term $\mathrm{f}_{\mathrm{r}}(q, \dot{q})$ contains all of the effects of applied forces (aerodynamic forces, gravity, spring and damping forces) as well as the effects of inertia forces caused by centrifugal and coriolis terms.

The equations of motion in FAST are solved numerically using a fourth-order Adams-Bashforth predictor and an Adams-Moulton corrector (Press et al. 1989). Since this method is not self-starting, a fourth-order RungeKutta method (Press et al. 1989) is used for the first four time steps at the beginning of a simulation (Wilson et al. 1999). The numerical integration time-step, which is constant throughout the simulation, is chosen and entered into the FAST input file.

\subsubsection{Controls}

To accommodate this work, a controls simulation capability has been added to FAST in the form of a set of subroutines to model the controllers of interest. Virtually any turbine DOF modeled in FAST, or turbine output, can be passed into these subroutines. Here, all turbine states are passed into these subroutines as well as turbine measurements such as generator or rotor speed, rotor and generator power and torque. The output of the subroutines is commanded blade pitch angle, or generator torque, depending upon the control actuators being used. 
These subroutines are implemented as an auxiliary set of first-order linear differential equations, representing the state-space equations for the controller, and are solved along with the other nonlinear equations of motion in FAST. Inputs are contained in a separate file for these subroutines. The input file values consist of several items such as desired rotor or generator speed, integrator time step (for the control subroutines), and other constants. The transfer function numerator and denominator coefficients are entered through this supplemental input file. Any number of transfer functions can be input to these subroutines in this manner. The control subroutines use the coefficients to convert the transfer function into a set of state-space equations in canonical form. As long as the controller can be expressed as a proper transfer function, the coefficients can easily be entered as inputs to these subroutines. As shown in later chapters, most of the control systems designed here can be expressed in this form, making incorporation of the control algorithm into FAST very straightforward.

\subsection{FAST Linear Models}

\subsubsection{Linearization Procedure}

A method for extracting a linear systems model from FAST has been developed using two methods: a symbolic method and a numerical perturbation method. The symbolic method is used as a tool for cross-checking simple cases and providing the mass, damping, and stiffness matrices without the effects of aerodynamics. The numerical method extracts mass, damping, and stiffness matrices and includes aerodynamic effects.

In the first method, the equations of motion used in FAST, based on Kane's method, are set up and derived symbolically using MATHEMATICA (Wolfram Research 2003). The system mass, damping, and stiffness matrices are computed in symbolic form by taking derivatives of the equations of motion with respect to small perturbations in the symbolic DOF and their derivatives. Once the symbolic coefficients have been derived, they can be evaluated numerically for a particular turbine by substituting numerical input property values for the symbolic values. The example shows the resulting symbolic coefficients in the mass, damping, and stiffness matrices. In the example, the blade first flap mode, first drive-train torsion mode, first tower fore-aft mode, and rotor and generator azimuth were modeled. Aerodynamic forces were ignored.

The symbolic method can be used to model any subset of DOF used in FAST. However, if all DOF are enabled, the symbolic calculations become very slow because the equations contain numerous terms. This method can't be used to model aerodynamic forces on the blades, since a good linear model of the highly nonlinear aerodynamics has yet to be developed. For this reason the symbolic method is used only as a crosscheck for the numerical method results, without aerodynamics.

In the numerical method, values for the mass, damping and stiffness matrices are calculated directly in FAST, during runtime. The code is first run for steady winds, i.e., no turbulence. Once a steady-state solution has been reached, the equations of motion are numerically perturbed with respect to each DOF and its derivative. Partial derivatives are formed by taking the difference between the perturbed right-hand side (see Equation 2.3) and the original right-hand side of the equations. This difference is then divided by the value of the perturbation in the DOF (or it's derivative). In this manner, numerical derivatives are obtained; giving numerical coefficients contained in the damping, and stiffness matrices. Numerical values for the mass matrix are already available, having been calculated from the left-hand side of Equation 2.3. In this work, simple linear models containing just a few DOF have been developed first. Complexity has been added in steps, after checkout of the simpler models.

\subsubsection{General State-Space Description}

Linear models for a wind turbine system can be expressed as

$$
\begin{aligned}
& \underline{\dot{x}}=A \underline{x}+B \underline{u}+\Gamma \underline{u_{D}} \\
& \underline{y}=C \underline{x}+D \underline{u}+E \underline{u}_{D} .
\end{aligned}
$$


where $\underline{x} \in R^{N}$ is the state vector, $\underline{u} \in R^{M}$ is the control input vector, and $\underline{u_{D}} \in R^{O}$ is the disturbance input vector, and $\underline{y} \in R^{P}$ is the control (or measured) output. $A \in R^{N x N}$ represents the state matrix, $B \in R^{N x M}$ the control input gain matrix, and $\Gamma \in R^{N x O}$ the disturbance input gain matrix. $C \in R^{P x N}$ relates the measured output $\underline{y}$ to the turbine states. $D \in R^{P x M}$ relates the output to the control input. $E \in R^{P x O}$ relates the measured output to the disturbance states. In most cases in this report, $D$ and $E$ are zero. In this notation, $\underline{\dot{x}}$ represents the time derivative of $\underline{x}$.

For modern conventional wind turbines, the principal control input is rotor blade collective pitch. The disturbance is the wind input and the primary system states include rotor or generator speed (or both), blade flap displacement and velocity, tower displacement and velocity, etc. Measured control signals include several possibilities: generator or rotor speed (or both), blade displacement as well as velocity and acceleration, tower displacement, velocity and acceleration, etc. The objective of an optimal system is to provide control capability with a minimum number of required measurements. Adding complexity to the controlled system translates into higher operating or O\&M costs, or both.

\subsubsection{Description of Turbine Rotor Speed}

\section{1-State Model}

The simplest linear model examined contains only the rotor speed state. For this model, it is assumed that the control input is perturbation in rotor collective pitch angle $\delta \beta$ (in rotor collective pitch, the pitch angle of each blade is identical), and the disturbance input is perturbation in the uniform component of wind speed over the rotor disk $\delta \mathrm{W}$. It is also assumed that rotor speed is the measured control signal. Figure 2-2 depicts this model.

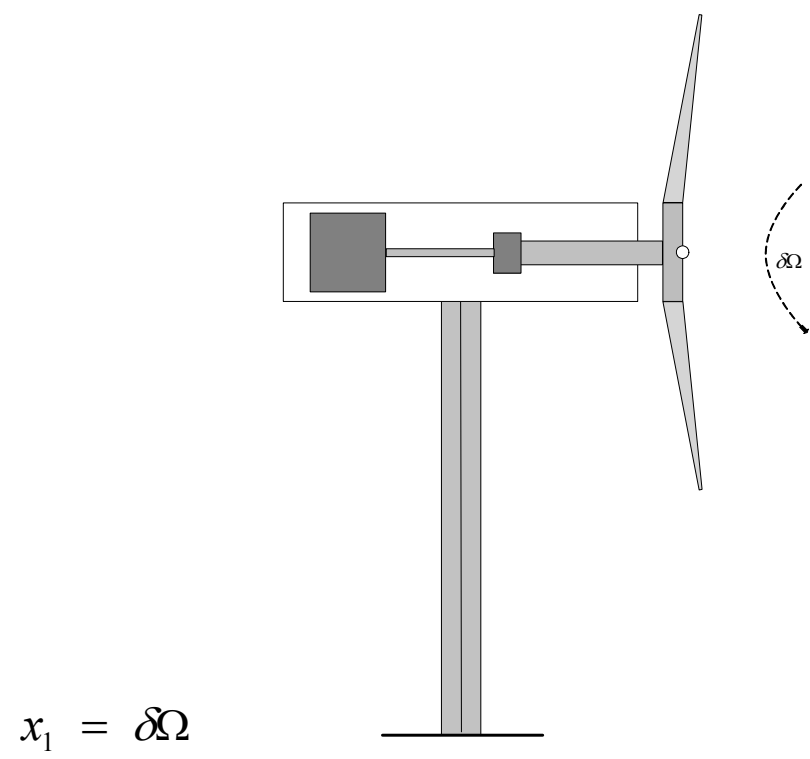

Figure 2-2. Depiction of the 1-state linear model

As derived in Appendix A-1, the state-space equation for this model with perturbed rotor rotational speed $x_{1}$ is 


$$
\begin{aligned}
& \dot{x}_{1}=\frac{\gamma}{\mathrm{I}_{\text {rot }}} x_{1}+\frac{\zeta}{\mathrm{I}_{\text {rot }}} \delta \beta+\frac{\alpha}{\mathrm{I}_{\text {rot }}} \delta \mathrm{w} \\
& \mathrm{y}=x_{1} .
\end{aligned}
$$

Comparing this with the general state-space form in Equation 2.4, $\underline{x}=x_{1}$-perturbed rotor speed, $\underline{y}=x_{1}$ measured perturbed rotor speed, $\underline{u}=\delta \beta$-perturbed rotor collective pitch, $\underline{u_{D}}=\delta \mathrm{w}$-perturbed wind disturbance (the uniform component over the rotor disk), and $A=\frac{\gamma}{\mathrm{I}_{\text {rot }}}$ is the state matrix. $\mathrm{I}_{\text {rot }}$ is the total rotor rotational inertia about the spin axis.

The parameter $\gamma$ is the partial derivative of rotor aerodynamic torque with respect to rotor speed $\frac{\partial \mathrm{T}_{\text {aero }}}{\partial x_{1}}$ (or $\frac{\partial \mathrm{T}_{\text {aero }}}{\partial \Omega}$ ). The control input gain matrix is $B=\frac{\zeta}{\mathrm{I}_{\mathrm{rot}}}$, in which $\zeta$ is the partial derivative of rotor aerodynamic torque with respect to rotor collective pitch angle, $\frac{\partial \mathrm{T}_{\text {aero }}}{\partial \beta}$. The disturbance input gain matrix is $\Gamma=\frac{\alpha}{\mathrm{I}_{\text {rot }}}$, in which $\alpha$ represents the partial derivative of rotor aerodynamic torque with respect to wind speed, $\frac{\partial \mathrm{T}_{\mathrm{aero}}}{\partial \mathrm{w}}$.

This simple first-order state-space model is an excellent place to begin with control design to regulate turbine speed in region 3. As shown in Chapter 4, this model is not adequate if there is torsional flexibility in the drive train. To account for this flexibility, a linear model that accounts for drive-train torsion will be needed, as described below.

\subsubsection{Addition of Drive-Train Torsion}

\section{3-State Model}

The addition of two states to the previous model allows modeling of the first drive-train torsion mode as well as rotor and generator speeds. As shown in Chapters 4 and 5, this is an important mode to include in the control design (Figure 2-3). The states in this updated model include the following: 


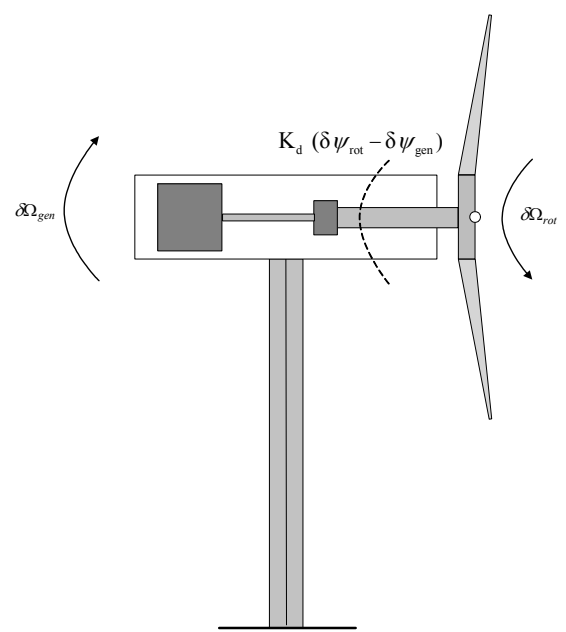

Figure 2-3. Depiction of the 3-state linear model

$\mathrm{x}_{1}=\delta \dot{q}_{4}$, perturbed rotor speed,

$\mathrm{x}_{2}=\mathrm{K}_{\mathrm{d}}\left(\delta q_{4}-\delta q_{15}\right)$, perturbed drive-train torsional spring force,

$\mathrm{X}_{3}=\delta \dot{q}_{15}$, perturbed generator speed.

The state-space equation as derived in Appendix A is

$$
\begin{aligned}
& {\left[\begin{array}{l}
\dot{\mathrm{x}}_{1} \\
\dot{\mathrm{x}}_{2} \\
\dot{\mathrm{x}}_{3}
\end{array}\right]=\left[\begin{array}{ccc}
\frac{\left(\gamma-\mathrm{C}_{\mathrm{d}}\right)}{\mathrm{I}_{\mathrm{rot}}} & \frac{-1}{\mathrm{I}_{\mathrm{rot}}} & \frac{\mathrm{C}_{\mathrm{d}}}{\mathrm{I}_{\mathrm{rot}}} \\
\mathrm{K}_{\mathrm{d}} & 0 & -\mathrm{K}_{\mathrm{d}} \\
\frac{\mathrm{C}_{\mathrm{d}}}{\mathrm{I}_{\text {gen }}} & \frac{1}{\mathrm{I}_{\text {gen }}} & \frac{-\mathrm{C}_{\mathrm{d}}}{\mathrm{I}_{\text {gen }}}
\end{array}\right]\left[\begin{array}{c}
\mathrm{x}_{1} \\
\mathrm{x}_{2} \\
\mathrm{x}_{3}
\end{array}\right]+\left[\begin{array}{c}
\frac{\zeta}{\mathrm{I}_{\mathrm{rot}}} \\
0 \\
0
\end{array}\right] \delta \beta+\left[\begin{array}{c}
\frac{\alpha}{\mathrm{I}_{\mathrm{rot}}} \\
0 \\
0
\end{array}\right] \delta \mathrm{w}} \\
& \mathrm{y}=\left[\begin{array}{lll}
0 & 0 & 1
\end{array}\right]\left[\begin{array}{l}
\mathrm{x}_{1} \\
\mathrm{x}_{2} \\
\mathrm{x}_{3}
\end{array}\right] .
\end{aligned}
$$

This is a first-order state-space equation, with $\underline{x}=\left[\begin{array}{l}\mathrm{x}_{1} \\ \mathrm{x}_{2} \\ \mathrm{x}_{3}\end{array}\right]$ and $\underline{y}=x_{3}$. We have the same control input, $\underline{u}=\delta \beta$, and disturbance input, $\underline{u_{D}}=\delta \mathrm{w}$. For this state-space equation, 


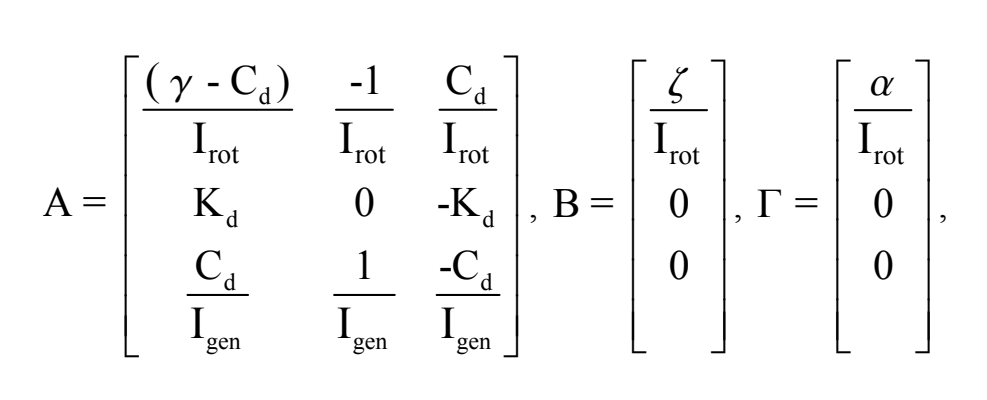

while $C=\left[\begin{array}{lll}0 & 0 & 1\end{array}\right]$.

Here, $\mathrm{K}_{\mathrm{d}}$ is the drive-train torsional spring constant, while $\mathrm{C}_{\mathrm{d}}$ is the torsional damping constant (described in Equation 2.2). $I_{\text {rot }}$ and $I_{\text {gen }}$ represent the rotational inertia of the rotor and generator about the spin axis, respectively. The torsional spring/damper connects the rotor to the generator, modeled as lumped mass rotational inertia, as described in Section 2.1.2.

It is interesting to note that the rotor speed and the generator speed are coupled because of terms such as $\frac{\mathrm{C}_{\mathrm{d}}}{\mathrm{I}_{\text {rot }}}$, $\mathrm{K}_{\mathrm{d}}$, $\frac{\mathrm{C}_{\mathrm{d}}}{\mathrm{I}_{\text {gen }}}$, etc. In addition, the pitch input and wind disturbance gain matrices for this system now have three rows, instead of just one. The fact that only the first row elements of $B$ and $\Gamma$ are nonzero indicates that the control input (rotor collective pitch) and wind disturbance input enter the system via the rotor speed state, because they directly influence the rotor aerodynamic torque. The other states of the system are influenced because they are coupled to the rotor speed state.

As shown in Chapters 5 and 7, a linear model will be needed that also describes the first flap mode for each blade. This model is shown in the following section.

\subsubsection{Addition of Blade Flap}

\section{7-State Model}

For a two-bladed rotor, four extra states (two for each blade) are needed in order to add the first flap mode for each blade (Figure 2-4). This results in a model with the seven states:

$\mathrm{x}_{1}=\delta q_{1}$, perturbed blade-1 first flap mode displacement,

$\mathrm{x}_{2}=\delta \dot{q}_{1}$, perturbed blade-1 first flap mode velocity,

$\mathrm{x}_{3}=\delta q_{2}$, perturbed blade-2 first flap mode displacement,

$\mathrm{x}_{4}=\delta \dot{q}_{2}$, perturbed blade-2 first flap mode velocity,

$\mathrm{x}_{5}=\delta \dot{q}_{4}$, perturbed rotor rotational speed,

$\mathrm{X}_{6}=\mathrm{K}_{\mathrm{d}}\left(\delta q_{4}-\delta q_{15}\right)$, perturbed drive-train torsional spring force,

$\mathrm{x}_{7}=\delta \dot{q}_{15}$, perturbed generator rotational speed. 


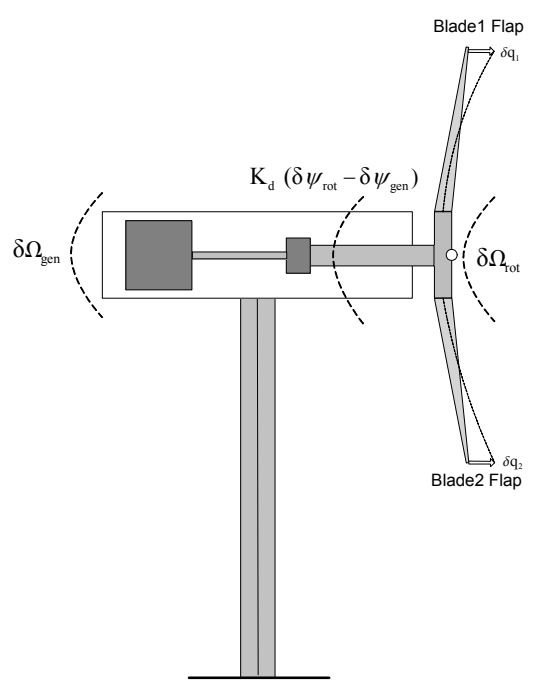

Figure 2-4. Depiction of the 7-state linear model

As derived in Appendix A, the final state equation for this model is:

$$
\begin{aligned}
& {\left[\begin{array}{ccccccc}
1 & 0 & 0 & 0 & 0 & 0 & 0 \\
0 & \mathrm{M}_{11} & 0 & 0 & \mathrm{M}_{14} & 0 & 0 \\
0 & 0 & 1 & 0 & 0 & 0 & 0 \\
0 & 0 & 0 & \mathrm{M}_{22} & \mathrm{M}_{24} & 0 & 0 \\
0 & \mathrm{M}_{41} & 0 & \mathrm{M}_{42} & \mathrm{I}_{\mathrm{rot}} & 0 & 0 \\
0 & 0 & 0 & 0 & 0 & 1 & 0 \\
0 & 0 & 0 & 0 & 0 & 0 & \mathrm{I}_{\mathrm{gen}}
\end{array}\right]\left[\begin{array}{l}
\dot{\mathrm{x}}_{1} \\
\dot{\mathrm{x}}_{2} \\
\dot{\mathrm{x}}_{3} \\
\dot{\mathrm{x}}_{4} \\
\dot{\mathrm{x}}_{5} \\
\dot{\mathrm{x}}_{6} \\
\dot{\mathrm{x}}_{7}
\end{array}\right]=\left[\begin{array}{ccccccc}
0 & 1 & 0 & 0 & 0 & 0 & 0 \\
-\mathrm{K}_{11} & -\mathrm{C}_{11} & 0 & 0 & -\mathrm{C}_{14} & 0 & 0 \\
0 & 0 & 0 & 1 & 0 & 0 & 0 \\
0 & 0 & -\mathrm{K}_{22} & -\mathrm{C}_{22} & -\mathrm{C}_{24} & 0 & 0 \\
0 & -\mathrm{C}_{41} & 0 & -\mathrm{C}_{42} & \gamma-\mathrm{C}_{\mathrm{d}} & -1 & \mathrm{C}_{\mathrm{d}} \\
0 & 0 & 0 & 0 & \mathrm{~K}_{\mathrm{d}} & 0 & -\mathrm{K}_{\mathrm{d}} \\
0 & 0 & 0 & 0 & \mathrm{C}_{\mathrm{d}} & 1 & -\mathrm{C}_{\mathrm{d}}
\end{array}\right]\left[\begin{array}{c}
\mathrm{x}_{1} \\
\mathrm{x}_{2} \\
\mathrm{x}_{3} \\
\mathrm{x}_{4} \\
\mathrm{x}_{5} \\
\mathrm{x}_{6} \\
\mathrm{x}_{7}
\end{array}\right]+\left[\begin{array}{cc}
0 & 0 \\
\zeta_{\mathrm{b}} & 0 \\
0 & 0 \\
0 & \zeta_{\mathrm{b}} \\
\zeta & \zeta \\
0 & 0 \\
0 & 0
\end{array}\right]\left[\begin{array}{l}
\delta \beta_{1} \\
\delta \beta_{2}
\end{array}\right]+\left[\begin{array}{cc}
0 & 0 \\
\alpha_{\mathrm{b}} & 0 \\
0 & 0 \\
0 & \alpha_{\mathrm{b}} \\
\alpha & \alpha \\
0 & 0 \\
0 & 0
\end{array}\right]\left[\begin{array}{l}
\delta \mathrm{w}_{1} \\
\delta \mathrm{w}_{2}
\end{array}\right]} \\
& {\left[\begin{array}{l}
\mathrm{y}_{1} \\
\mathrm{y}_{2} \\
\mathrm{y}_{3}
\end{array}\right]=\left[\begin{array}{lllllll}
0 & 0 & 0 & 0 & 0 & 0 & 1 \\
1 & 0 & 0 & 0 & 0 & 0 & 0 \\
0 & 0 & 1 & 0 & 0 & 0 & 0
\end{array}\right]\left[\begin{array}{l}
\mathrm{x}_{1} \\
\mathrm{x}_{2} \\
\mathrm{x}_{3} \\
\mathrm{x}_{4} \\
\mathrm{x}_{5} \\
\mathrm{x}_{6} \\
\mathrm{x}_{7}
\end{array}\right] .}
\end{aligned}
$$

Note that individual blade pitch inputs are now allowed: $\delta \beta_{1}$ and $\delta \beta_{2}$, which are the perturbations in blade-1 and -2 pitch. It is assumed that blade-1 is identical to blade-2, so that the gains $\zeta_{\mathrm{b}}, \zeta, \alpha_{\mathrm{b}}$, and $\alpha$ are identical for each blade. It is also assumed that generator speed is measured, as well as the flap deflections of each blade. We also allow two wind disturbance inputs, one for each blade.

The coefficients $I_{\text {rot }}, I_{\text {gen }}, K_{d}$, and $C_{d}$ are the rotor and generator rotational inertias, and the drive-train stiffness and damping constants that also appeared in the 3-state model. The other terms $\mathrm{M}_{11}, \mathrm{M}_{14}, \mathrm{M}_{41}$, $\mathrm{K}_{11}, \mathrm{C}_{11}, \mathrm{C}_{14}, \mathrm{C}_{41}$, etc., are more complicated mass, stiffness, and damping terms. These terms are composed of expressions involving the turbine geometry. As the number of states in the linear model increases, these terms become more complicated and will not be shown. It is important to note that the damping terms contain the effects of aerodynamics, when using the numerical perturbation technique in FAST (if aerodynamics is switched on in FAST during linearization). This is not the case when the symbolic method is used to derive these coefficients. 
Note in Equation 2.7 that the blade-1 and -2 flap velocity states couple with the rotor-speed state $\left(\mathrm{X}_{5}\right)$ through terms such as $\mathrm{M}_{14}, \mathrm{M}_{24}, \mathrm{M}_{41}, \mathrm{M}_{42}, \mathrm{C}_{14}, \mathrm{C}_{24}, \mathrm{C}_{41}$, and $\mathrm{C}_{42}$. This shows that the blade flap modes are coupled to the rotational speed states through these terms.

In Chapter 5, control systems are designed using rotor collective pitch. As shown, only the rotor first symmetric flap mode is controllable using rotor collective pitch. This necessitates transforming the blade flap states into rotor symmetric and asymmetric flap (see Appendix A). In Chapter 5 we also show that the addition of states to model the tower's first fore-aft mode allows us to add damping to this mode using control. The required model formulation for this addition is indicated below.

\subsubsection{Addition of first Tower Fore-Aft Mode}

\section{9-State Model}

Figure 2-5 depicts this model. The 9-state model is described with the following states:

$$
\begin{aligned}
& x_{1}=\delta q_{1}, \text { blade-1 perturbed flap tip displacement } \\
& x_{2}=\delta \dot{q}_{1}, \text { blade-1 perturbed flap tip velocity } \\
& x_{3}=\delta q_{2}, \text { blade-2 perturbed flap tip displacement } \\
& x_{4}=\delta \dot{q}_{2}, \text { blade-2 perturbed flap tip velocity } \\
& x_{5}=\delta \dot{q}_{4}, \text { perturbed rotor rotational speed } \\
& x_{6}=\mathrm{K}_{\mathrm{d}}\left(\delta q_{4}-\delta q_{15}\right), \text { perturbed drive-train torsional spring force } \\
& x_{7}=\delta \dot{q}_{15}, \text { perturbed generator rotational speed } \\
& x_{8}=\delta q_{7}, \text { perturbed tower-top first fore-aft mode deflection } \\
& x_{9}=\delta \dot{q}_{7}, \text { perturbed tower-top first fore-aft mode velocity. }
\end{aligned}
$$

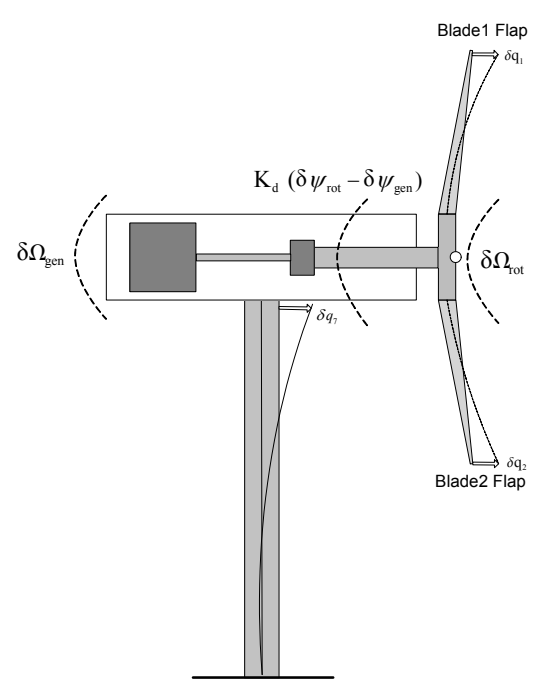

Figure 2-5. Depiction of the 9-state linear model 
The linear model corresponding to these states is

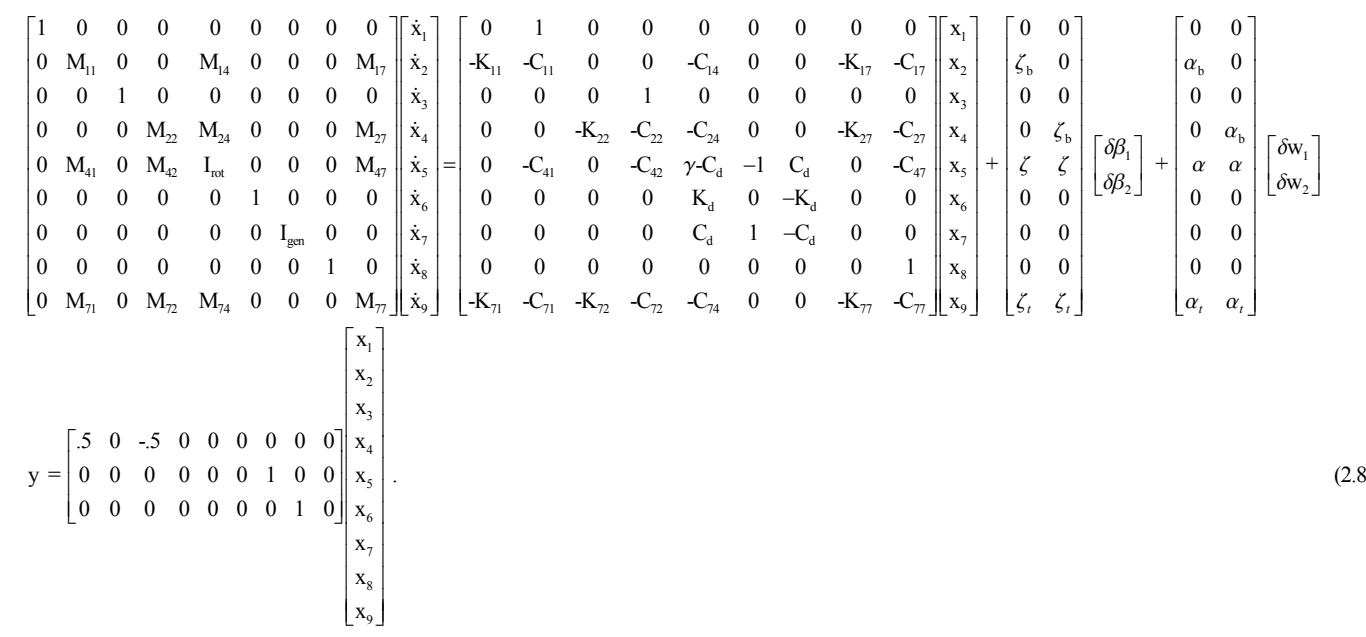

In this equation, several mechanisms for coupling can be observed. Looking at the second and fourth row, the blade flap modes couple with the rotor speed state $\left(\mathrm{x}_{5}\right)$, through various coupling terms, described earlier. Blade flap also couples with the tower first fore-aft state $\left(x_{9}\right)$, through the mass terms $M_{17}$ and $M_{27}$, the damping terms $\mathrm{C}_{17}$ and $\mathrm{C}_{27}$, and the stiffness terms $\mathrm{K}_{17}$ and $\mathrm{K}_{27}$. Other couplings can be seen from these equations as well. The selection of measurements (y), shown in Equation 2-8, is described in Chapter 7.

\subsection{Linear Model Evaluations for the Controls Advanced Research Turbine}

\subsubsection{CART Description and Inputs}

The Controls Advanced Research Turbine (CART), shown in Figure 2-6, is a two-bladed, teetered, upwind, active-yaw wind turbine. This machine is used as a test bed for studying a number of aspects of wind turbine controls technology on medium- to large-scale machines (see Figure 2-6 and Table 2-2).

Table 2-2. General Specifications of the CART Machine

\begin{tabular}{|c|}
\hline 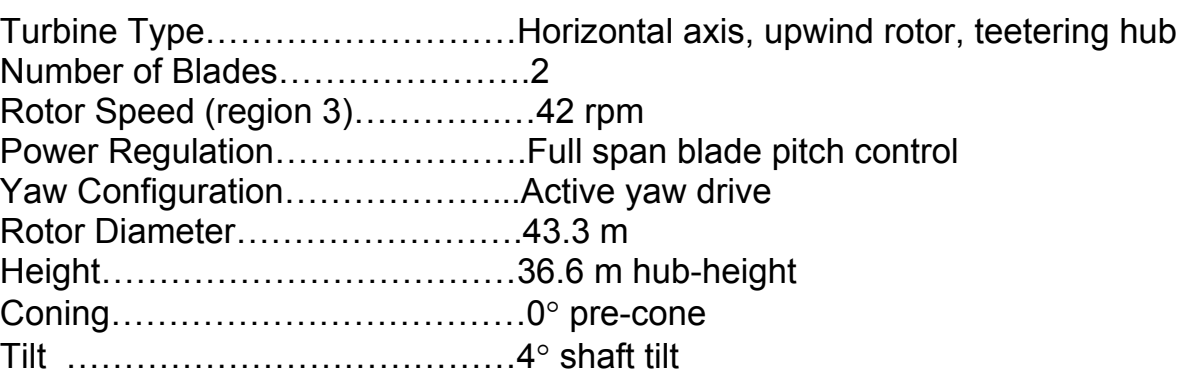 \\
\hline
\end{tabular}

The two-bladed teetering upwind turbine operates at variable speed; each blade is capable of being independently pitched with its own electromechanical drive. Rated electrical power $(600 \mathrm{~kW}$ at $42 \mathrm{rpm})$ is maintained in region 3 in a conventional variable-speed approach. Power electronics are used to command constant torque from the generator and blade pitch controls the rotor speed. In region 2, the machine torque is 
varied to produce variable rotor speed in order to maintain optimum $C_{p}$. Detailed inputs to FAST for the CART are shown in Appendix B.

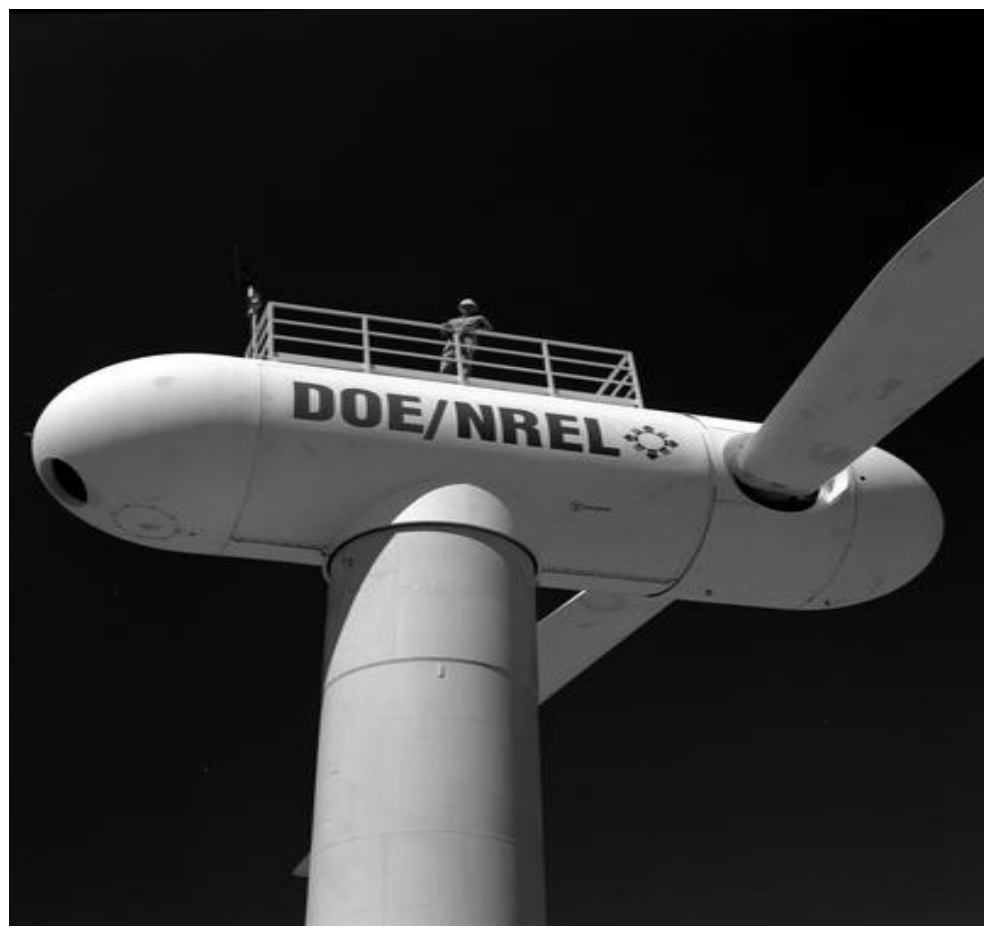

Figure 2-6. The Controls Advanced Research Turbine (CART)

\subsubsection{Linear Model Evaluation}

The 9-state model just described was used to describe a linear model of the CART. This model includes the first flap mode for blades 1 and 2, rotor and generator speeds, first drive-train torsion mode, and tower first foreaft mode.

To produce numerical values for the coefficients in this linear model, the FAST code is run until the motion reaches steady state, in nonturbulent winds, with wind shear and tower shadow set to zero. State matrices for this turbine are shown in Appendix D (Section D-4.3), at a rotor speed of $42 \mathrm{rpm}$, a blade pitch angle of 12 deg., and a wind speed of $18 \mathrm{~m} / \mathrm{s}$.

An eigen-analysis of the A matrix for this system gives the open loop poles: $-0.12 \pm 5.9 \mathrm{j}$ radians/second $(\mathrm{r} / \mathrm{s})$, $0.04 \pm 22.6 \mathrm{j} \mathrm{r} / \mathrm{s},-4.45 \pm 13.5 \mathrm{j} \mathrm{r} / \mathrm{s},-4.54 \pm 13.0 \mathrm{j} \mathrm{r} / \mathrm{s}$, and $-0.121 \mathrm{r} / \mathrm{s}$. The first pole pair represents the very lightly damped tower first fore-aft mode. The second pole pair represents the very lightly damped first drive-train torsional mode. The next pole pair represents the rotor first asymmetric flap mode. The second pole pair represents the rotor first symmetric flap mode. The 7th pole represents the generator speed.

It is interesting to note that the tower first fore-aft mode and the first drive-train torsion mode are very lightly damped. Since all structural damping has been set to zero, this damping is mostly due to aerodynamics, with some damping from coriolis and other effects. The rotor flap modes are highly damped due to aerodynamics.

The natural frequency of the rotor flap modes are higher while rotating than when the rotor is parked, as a result of centrifugal stiffening of the blades. Later, we will examine a linear model extracted from ADAMS for the parked rotor. In this case, the rotor first symmetric flap mode has a lower natural frequency than it does in the case in which the rotor is spinning. 
The designed controller from this model addresses several objectives: regulation of generator speed, stabilization and enhanced damping of the first drive-train torsional mode, enhanced damping of the tower first fore-aft mode, stabilization of the rotor first symmetric and asymmetric flap modes, and attenuation of blade flap response due to wind shear, using individual blade pitch control.

An ADAMS model of a wind turbine contains many more modes than we can model using FAST. Thus, simulations with ADAMS will indicate whether additional flexible modes, not modeled with FAST, become unstable during closed-loop control. A brief description of ADAMS and its application is presented below.

\subsection{CART ADAMS Model Description}

\subsubsection{Brief Description of ADAMS}

The Automatic Dynamic Analysis of Mechanical Systems (ADAMS) code is a general-purpose, multi-body systems analysis code developed by Mechanical Dynamics, Inc. (MDI 2003). ADAMS automatically generates numerical equations of motion at runtime, removing the major modeling task of developing and validating large numbers of equations of motion. It makes no restrictions on the size of displacements, angles, or angular rates, and includes the effects of geometric nonlinearities.

ADAMS models systems as groups of rigid masses connected by joints and forces that correspond to physical components (Elliott and Wright 1994). A variety of linearly elastic elements are available, ranging from simple spring dampers to BEAMs and FIELDs (Elliot and McConville 1990). ADAMS also includes a general force definition statement, which allows forces and moments to be defined as arbitrary functions of the state variables.

Wind turbine blades can be modeled in ADAMS using two methods. For relatively rigid blades, in which higher flexible modes of the blade can be neglected, the blade can be modeled as a rigid body attached to the hub with a revolute joint. A spring-damper is placed at this joint and the spring stiffness is adjusted to give the correct value for the frequency of the blade first flap mode. In this method, higher bending modes of the blade are neglected. A similar procedure can be used to model the first fore-aft or side-to-side mode of the tower.

Flexible blades can be modeled in ADAMS as a series of rigid-body parts (which have mass and inertia) connected together with elastic beam elements (BEAMs or FIELDs). In this way, many higher bending modes of the blade are captured. The same approach is used to model the tower and other flexible wind turbine components.

ADAMS has the capability to model control systems in the same way as FAST. The same control subroutines used with FAST are compiled and linked to ADAMS. ADAMS allows one to pass to these subroutines such turbine measurements as generator and rotor speed, and generator and rotor torque. The inputs to this subroutine are the same as those entered into the subroutine used with FAST, such as desired rotor or generator speed, controls simulation time-step, and the numerator and denominator coefficients to a set of transfer functions. The auxiliary state-space equations that are formed from these transfer functions are solved along with the other nonlinear equations of motion contained in ADAMS. The output from these subroutines is also passed back to ADAMS.

In the CART ADAMS models, the generator torque was assumed to be constant, and set at the same value as used in FAST. All blade, tower, and drive-train structural damping was set to zero, unless otherwise noted. For most simulations, the teeter DOF was locked. In some cases, the blades were modeled as a rigid body connected to the hub with a revolute joint (hinged blade) and a spring. In other cases, the blades and tower were modeled as a series of rigid body parts connected by elastic elements (fully flexible blade and tower). In the ADAMS simulations in later chapters, the differentiations of the different models will be discussed.

The model inputs to ADAMS for the CART are shown in Appendix B. Our goal in using ADAMS is to determine whether the control systems that we design cause flexible modes of the turbine to become unstable in closed-loop operation. 


\subsubsection{Verification of ADAMS Modeled CART Modes (parked rotor)}

As Bir and Robinson (1999) found, predicting the modal characteristics of the operating turbine is not possible with ADAMS. Formulation of the equations of motion in ADAMS prohibits correct linearization in rotating frames. ADAMS can faithfully predict the modal characteristics of only the nonrotating ("parked") turbine. A comparison of ADAMS modal predictions with measured results for the CART is given for the parked case. Model verification of modal results for the parked turbine is useful to validate the structural model alone, without aerodynamics.

A modal test of the CART machine was conducted for the blades parked vertically. In this modal survey, the blades were pitched to the position that they would be in if the machine were operating. The drive-train brake was applied in order to maintain the rotor in the parked position. In addition, both the teeter and yaw brakes were engaged. The modal survey was designed to obtain test data that could be correlated with the CART simulation models.

In ADAMS, the teeter and yaw DOF were locked. The rotor was maintained in the parked position by increasing the generator inertia to a very high number to prevent generator rotation. The rotor was restrained from free rotation via the low-speed shaft torsional spring.

For the experimental modal analysis, nine system modes were measured. Table 2-3 shows a comparison of ADAMS predicted natural frequencies to test data. The ADAMS/Linear module (MDI 2003) was used to linearize the nonlinear ADAMS model and perform an eigen-analysis for the parked turbine.

For the first tower fore-aft and side-side modes (modes 1 and 2), ADAMS slightly overpredicts these frequencies. These modes contain mostly tower motion and some motion from other components like the blades. In the tower first fore-aft mode, there is some contribution from rotor first symmetric flap, showing that these motions are coupled.

The next predicted mode is the first drive-train torsion and represents the first drive-train torsion natural frequency when the generator rotor is locked. When the turbine is in normal operation, the generator is free to rotate, a very different boundary condition than when the generator rotation is locked. When rotating, the first drive-train torsion natural frequency increases to approximately $3.58 \mathrm{~Hz}$.

The next mode (4) couples rotor first asymmetric flap with elastic pitch bending of the drive-train. In ADAMS, the low-speed shaft was modeled with a single beam element, having both bending and torsional flexibility. When the rotor is in the vertical position, flap asymmetric motion couples with bending of the drive train.

The next mode (5) represents the first symmetric flap mode, with good agreement between ADAMS and the test result. This mode consists mainly of blade motion, with very little motion from the other turbine components, such as the tower.

The next mode (6) represents the rotor first symmetric lag mode, with participation from rotor asymmetric flap and tower torsion. One can visualize that symmetric lag motion causes the rotor center of gravity location to shift back and forth from side to side. This motion couples with the tower elastic torsional DOF, since the nacelle yaw DOF is locked (ordinarily, this mode would couple with nacelle yaw if that DOF is unrestrained).

The next mode (7) represents rotor second asymmetric flap, coupled with rotor first symmetric lag and drivetrain pitch bending (vertical motion). The main contribution to this mode is from rotor second asymmetric flap with a very small amount of rotor first symmetric lag bending. In addition, there is a small component of drivetrain pitch bending in this mode.

The next mode (8) represents first symmetric lag, coupled to second asymmetric flap, tower first lateral (sideside) bending, and tower torsion). 
The next mode (9) is the rotor second symmetric flap. This mode is dominated by rotor symmetric flap motion with little contribution from any other motion.

Table 2-3. Comparison of Measured and Predicted Turbine Natural Frequencies with the Rotor Parked Vertically

\begin{tabular}{|c|c|c|c|c|}
\hline Mode \# & Mode & $\begin{array}{l}\text { Measured } \\
\text { Frequency } \\
(\mathrm{Hz})\end{array}$ & $\begin{array}{l}\text { Predicted } \\
\text { Frequency } \\
\text { ADAMS } \\
(\mathrm{Hz})\end{array}$ & $\begin{array}{l}\text { Error } \\
(\%)\end{array}$ \\
\hline 1 & first Tower fore-aft & 0.858 & 0.872 & 1.6 \\
\hline 2 & first Tower side-side & 0.877 & 0.881 & 0.5 \\
\hline 3 & first Drive-train torsion & - & 1.38 & - \\
\hline 4 & $\begin{array}{l}\text { first Asymmetric Flap and Drive- } \\
\text { train Pitch Bending }\end{array}$ & 1.58 & 1.58 & 0.0 \\
\hline 5 & first Symmetric Flap & 2.06 & 2.07 & 0.5 \\
\hline 6 & $\begin{array}{l}\text { first Symmetric Lag coupled } \\
\text { with } 2^{\text {nd }} \text { asymmetric flap + tower } \\
\text { torsion }\end{array}$ & 3.94 & 3.87 & -1.8 \\
\hline 7 & $\begin{array}{l}2^{\text {nd }} \text { asymmetric flap }+ \text { first } \\
\text { symmetric lag w/drive-train pitch } \\
\text { bending }\end{array}$ & 4.09 & 4.36 & 6.6 \\
\hline 8 & $\begin{array}{l}\text { first symmetric lag }+2^{\text {nd }} \\
\text { asymmetric flap }+ \text { first tower } \\
\text { lateral bending }+ \text { tower torsion } \\
\text { w/drive-train yaw }\end{array}$ & 5.05 & 5.05 & 0.0 \\
\hline 9 & $2^{\text {nd }}$ symmetric flap & 5.35 & 5.83 & 9.0 \\
\hline
\end{tabular}

Modal damping values were also obtained experimentally. All modal damping values were very low (generally below $1 \%$ modal damping) with the exception of the first tower side-side mode, with a damping value of $2 \%$. All structural damping in ADAMS was set to zero, except for the first tower side-side mode, which was set at $2 \%$.

These comparisons show that ADAMS faithfully represents several of the most important coupled modes of the CART when the rotor is parked. It will be important to see if controls designed from the simple linear models we described in Section 2.2 excite any of these coupled modes modeled by ADAMS. With close agreement between the ADAMS predicted modes and the measured results for the parked turbine, we can use the CART ADAMS model with confidence to check if higher modes of the machine become unstable during closed-loop operation. There is ample opportunity for excitation of these modes. It is hoped that the controllers NREL has designed, as described in this report, will result in stable closed-loop behavior as simulated in ADAMS. 


\section{Chapter 3. Modeling and Simulation of CART Classical Control}

\section{Introduction}

Simulation results are obtained from FAST and ADAMS when implementing the CART classical proportional-integral (PI) control system. Rather than model this control system in great detail, these simulations model only the most important aspects of this control system. Results when simulating this PI control will be obtained for use in comparing them with modern control design results. These PI control results will be used as a baseline to judge the performance of a modern control system design described in later chapters.

\subsection{Description of the CART PI Control System}

A simple classical control system has been implemented and tested in the CART. The goal of the control is to regulate turbine speed in region 3, using rotor collective pitch and holding generator torque constant. The CART controller is a simple PI controller. Figure 3-1 shows a diagram of this control system in transfer function form. The wind turbine is represented by the plant with transfer function $P(s)$. Turbine speed is measured and fed back to the control system with transfer function

$$
\operatorname{Cont}(s)=K_{p}+\frac{K_{i}}{s}
$$

Here, $\operatorname{Cont}(s)$ is the controller transfer function. $K_{p}$ and $K_{i}$ are the proportional and integral gains.

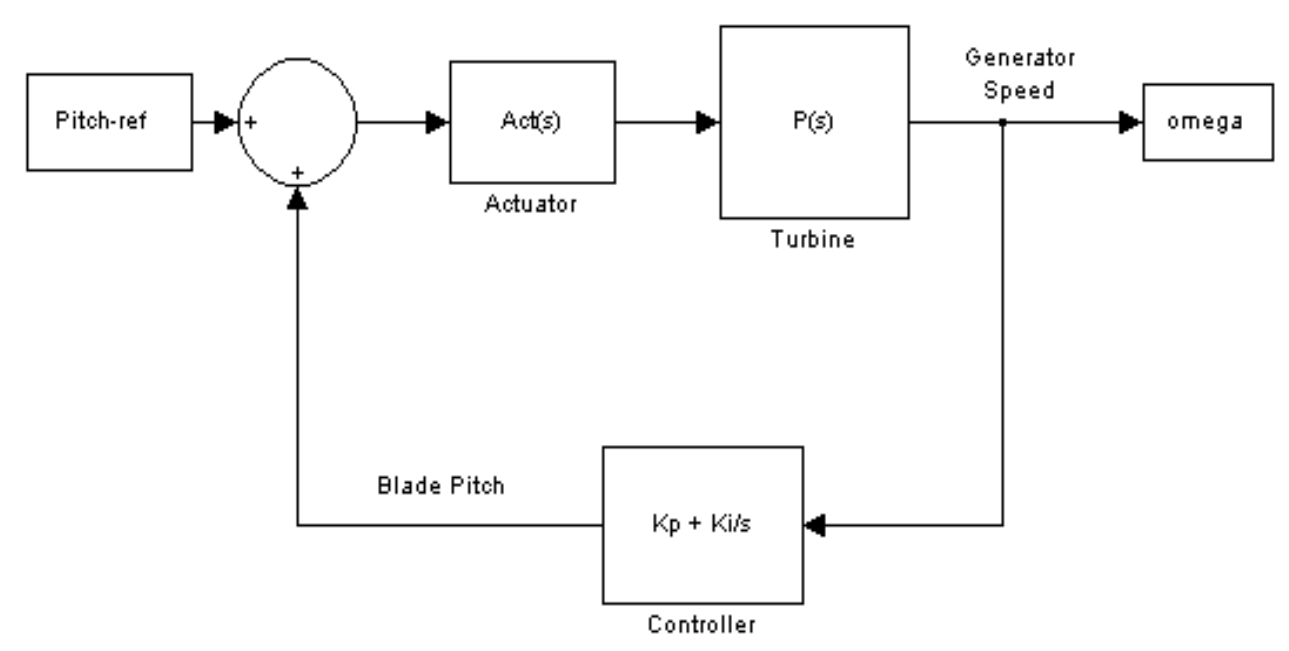

Figure 3-1. Diagram of CART speed regulation control system

The pitch signal from the controller passes through a summing point where a reference pitch value is added and passed to the pitch actuator $\operatorname{Act}(s)$. It takes a finite time for the pitch actuator to respond and produce the final pitch needed by the turbine. This actuator lag is modeled with a first-order model, ignoring pitching inertia. 
This control system is a simplification of the control actually implemented in the CART. Some additional complexity in the actual controller exists because of methods of measuring turbine speed. Turbine speed is obtained by measuring turbine azimuth position and taking its derivative. The resulting signal is then filtered to remove high-frequency noise. These details will not be included in the controller implemented in the FAST simulator. The results provide an understanding of the overall controller behavior and provide a basis for comparison with new, modern control designs described in later chapters. The generator speed signal is fed back to both this PI controller and the modern control designs. Thus, a direct comparison can be made between PI results and modern control design results.

\subsection{FAST and ADAMS Implementation and Simulation Results}

\subsubsection{Implementation}

The CART PI control system is now implemented into FAST and ADAMS. It is assumed that the generator torque is constant. The goal is to regulate turbine speed in region 3. We assume that we measure generator speed.

First, the effects of the actuator will be neglected. For the control implemented and field-tested in the CART, the values for the proportional and integral gains are $\mathrm{K}_{\mathrm{i}}=0.2618$ and $\mathrm{K}_{\mathrm{p}}=1.2217$. It is easy to implement this controller into FAST and ADAMS. The numerator and denominator coefficients of the controller transfer function are input to the control subroutines:

$$
\operatorname{Cont}(s)=1.2217+\frac{0.2618}{s}
$$

\subsubsection{Simulation Results}

This controller is first tested with FAST, for the case in which there are no flexible modes, i.e., all flexible-blade, drivetrain, and tower modes are switched off. The closed-loop system is tested with the simple test winds shown in Figure 32. After implementing this transfer function into the FAST control subroutines and simulating for the step winds, we obtain the results shown in Figure 3-3.

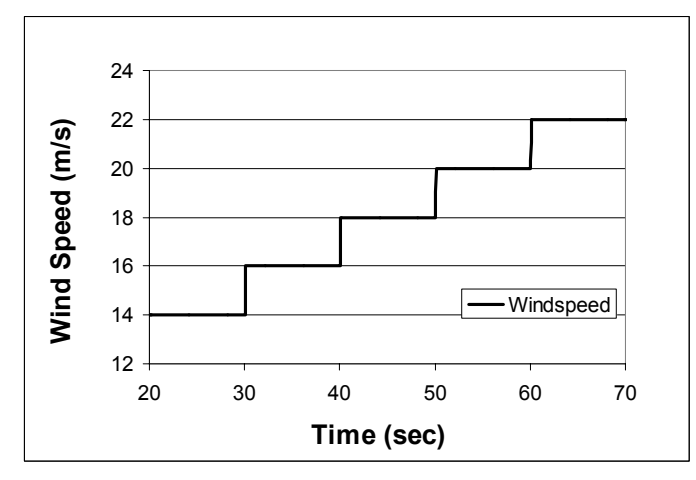

Figure 3-2. Test wind applied to CART FAST models 
The performance of this system can be studied by examining the closed-loop transfer function. The actuator is ignored, which means that $\operatorname{Act}(s)=1$. The resulting closed-loop transfer function is

$$
T(s)=\frac{P(s)}{1+P(s) \operatorname{Cont}(s)} .
$$

For stability, the poles of the closed-loop transfer function $T(s)$ must all have negative real parts (Theorem C-.1, Appendix C, Section C-1.2.2). This system can be examined by inserting a transfer function to represent the wind turbine linear model for $P(s)$ into Equation 3.3.

Since all DOF except turbine rotation are switched off during simulation, the 1-state linear turbine model described in Section 2.2.3 can be used. A transfer function representation of this model can be found (see Appendix C, section C1.2.3) by

$$
P(s)=C(s I-A)^{-1} B .
$$

Here $A, B$, and $C$ are the coefficients in the general state-space equation shown in Section 2.2.3 (see Equation 2.4). For the 1-state model for the CART, evaluated at a turbine operating point:

$$
\begin{gathered}
\qquad \begin{array}{l}
\mathrm{w}_{0}=18 \mathrm{~m} / \mathrm{s}, \\
\Omega_{0}=42 \mathrm{RPM}, \text { and } \\
\beta_{0}=12 \mathrm{deg} .
\end{array} \\
C=1, B=-2.8818, \text { and } A=-0.12053 ; \text { thus, } \\
P(s)=\frac{-2.8818}{(s+0.12053)} .
\end{gathered}
$$

When Equations 3.5 and 3.2 are inserted into Equation 3.3, the closed-loop transfer function becomes

$$
T(s)=\frac{-2.8818 s}{s^{2}+\left(2.8818 K_{p}+0.12053\right) s+2.8818 K_{i}} .
$$

The characteristic equation for this transfer function is found by setting the denominator of Equation 3.6 to zero and is

$$
s^{2}+\left(2.8818 K_{p}+0.12053\right) s+2.8818 K_{i}=0 \text {. }
$$

Inserting the values $\mathrm{K}_{\mathrm{i}}=0.2618$ and $\mathrm{K}_{\mathrm{p}}=1.2217$ and solving for the roots gives us the two poles:

$$
\begin{aligned}
& s_{1}=-3.421, \\
& s_{2}=-0.221
\end{aligned}
$$

This helps explain the characteristics of the response observed in Figure 3-3. The fast pole (-3.421) provides overall speed regulation. The slow pole $(-0.221)$ is repsonsible for the decay immediately after application of a step change in 
wind speed. The response is improved by increasing the integral gain $\mathrm{K}_{\mathrm{i}}$. At a value of $\mathrm{K}_{\mathrm{i}}=1.0$, Figure 3.4 shows the improved regulation of generator (rotor) speed with poles located at

$$
\begin{aligned}
& s_{1}=-2.479, \\
& s_{2}=-1.163
\end{aligned}
$$

Increasing the integral gain helps improve the regulation of speed.

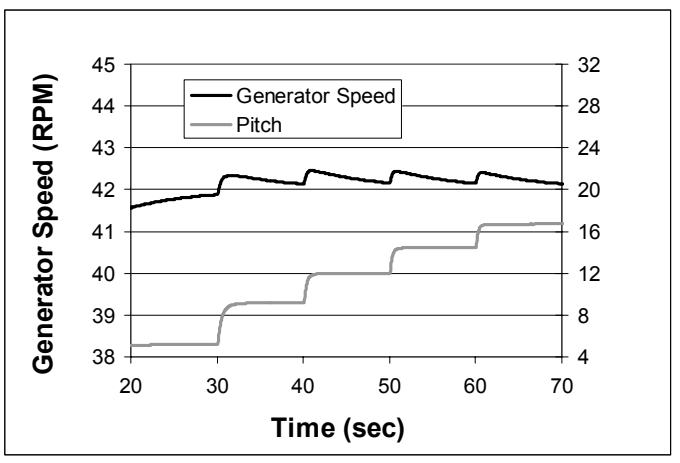

Figure 3-3. FAST simulated rotor speed and pitch, using PI control, for rigid blades, tower, and drive train $\left(K_{p}=1.2217, K_{i}=0.2618\right)$

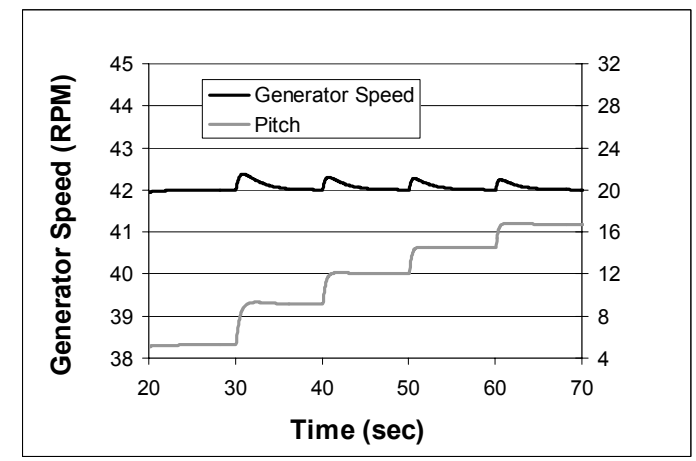

Figure 3-4. FAST simulated rotor speed, using classical controller with increased integral control gain $\left(K_{p}=1.2217, K_{i}=1.0\right)$

These results were all simulated in FAST with the flexible modes switched off, representative of a rigid version of the CART with infinitely stiff drive train, blades, and tower.

Now, the first drive-train torsion mode is switched on. The integral control gain is set back to $\mathrm{K}_{\mathrm{i}}=0.2618$. Figure 3-5 shows the simulated response, indicating instability. Figure 3-6 shows the simulated response when both first drive-train torsion, and blade first flap are switched on, again showing undesirable results. The unstable mode is the first drive-train torsion mode. 


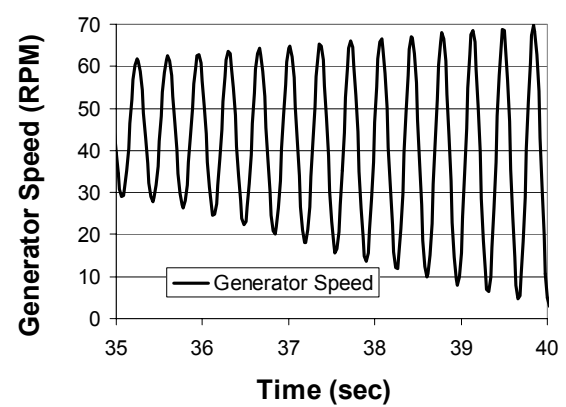

Figure 3-5. FAST simulated generator speed and pitch, using PI control, simulated with first drivetrain torsion $\left(K_{p}=1.2217, K_{i}=0.2618\right)$

Designing a stable PI control system for this flexible system is difficult using classical control design techniques. This difficulty increases with turbine flexibility. A control design technique that assures stability when flexible modes of the turbine are included in the linear model is required. The appropriate theories and methods are applied in Chapter 4.

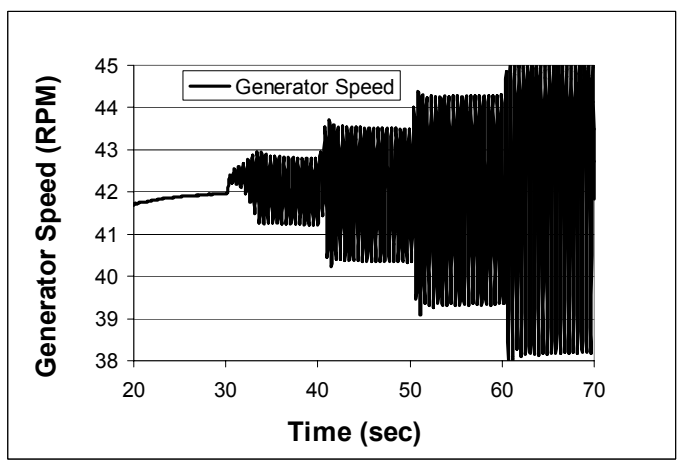

Figure 3-6. FAST simulated generator speed and pitch, using PI control, simulated with drive train first torsion and blade first flap $\left(K_{p}=1.2217, K_{i}=0.2618\right)$

\subsubsection{Actuator Effects}

An important component omitted in the previous simulations is the pitch actuator. There is a lag time between what the controller requests for the "commanded pitch" and the actual pitch delivered to the blade by the actuator. This pitch actuator lag must be accounted for.

The pitch actuator can be modeled with a simple first-order model. CART blade pitching inertia is very small in comparison with other terms and can be neglected. The actuator transfer function is described by

$$
\operatorname{Act}(s)=\frac{1}{\tau s+1} .
$$

Typical values for the time constant $\tau$ for the CART are 0.20 . This gives

$$
\operatorname{Act}(s)=\frac{1}{0.2 s+1} .
$$


Implementation of this actuator into FAST (or ADAMS) is accomplished by adding another transfer function to the control subroutines. The input to this transfer function is the commanded pitch from the PI transfer function. The output from this transfer function is actuator pitch passed to the turbine (in this case, the simulator).

All flexible blade and tower modes are switched on in FAST for this simulation. Figure 3-7 shows that both the simulated generator speed and blade pitch are stable with the addition of the pitch actuator.

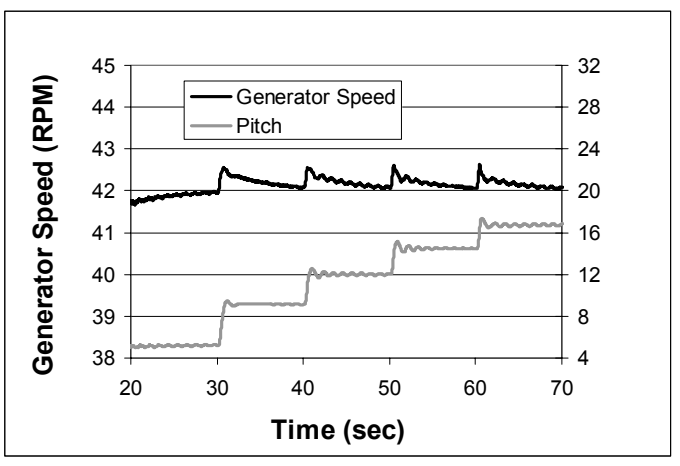

Figure 3-7. FAST simulated generator speed and pitch, using PI control, simulated with all flexible modes on $\left(K_{p}=1.2217, K_{i}=0.2618\right)$, with actuator

The actuator limits the frequency content of the pitch input to the turbine, acting like a low-pass filter. This removes the frequencies that can destabilize flexible modes. As the actuator time constant $\tau$ is decreased (and actuator speed increases), pitch inputs at higher frequencies are introduced. As shown in Chapter 8, as the actuator speed is increased, the PI results become less stable. This agrees with the limiting no actuator case (infinitely fast actuator speed), where the results become unstable when flexible modes are switched on.

\subsection{PI Control Results for Later Comparisons}

An in-depth validation of the modeled PI controller to replicate the CART is beyond the scope of this work. For a quick verification, machine test data were analyzed and prepared for comparison with simulation results. The field data selected were obtained with the CART operating in region 3. Hub-height anemometer data were extracted from the field data and used as the wind input to excite the turbine in the simulations.

When FAST is used (Figure 3-8), the simulated generator speed follows trends seen in the measured data. The generator speed is regulated "tightly" between 41 and $43 \mathrm{rpm}$ in both the simulation results and the measured results.

The simulated generator speed does not replicate the measured generator speed exactly. Much of this is the result of a single hub-height wind being input to the simulator. The real turbine "sees" a complex field of turbulent wind inflow, with spatial variations of turbulence across the rotor disk. These complex wind components are neglected when only hub-height wind is used to excite the turbine simulation. Even with this simplification, the agreement between simulated and measured results are excellent and follow the general low-frequency variations of generator speed. 


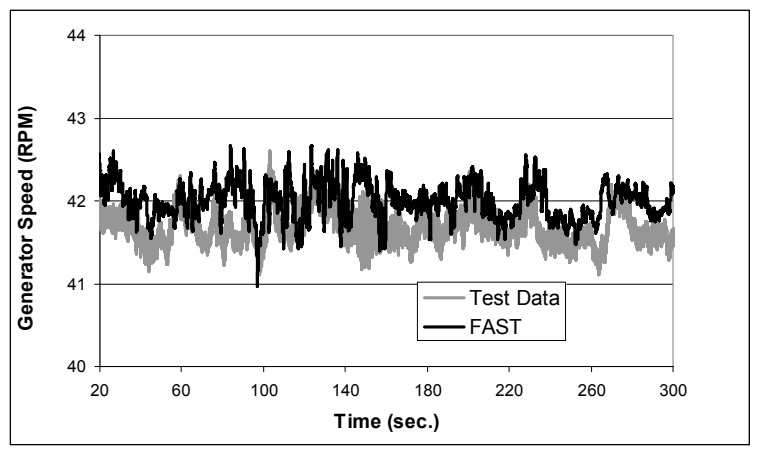

Figure 3-8. Comparison between FAST simulated generator speed and measured results, using PI control, simulated with all flexible modes on $\left(K_{p}=1.2217, K_{i}=0.2618\right)$, with actuator time constant 0.2

In later chapters, results from the modern control designs will be compared with results from the PI controller. Both step function winds (as seen in Figure 3-2) as well as more complex turbulent wind inflow generated by the SNLWIND3-D code (Kelley 1992) will be used to drive the simulators. This approach provides more realistic inflow excitation to the turbine.

\subsection{Chapter Conclusions}

FAST and ADAMS have been shown to be useful tools for implementing and simulating the CART PI control system response. If the actuator is neglected, use of this PI control causes unstable closed-loop behavior in the flexible machine. Given the increase in flexibility with increasing machine size, flexibility must be properly accounted for in any modern control design. For safety and other considerations, controls must also be designed to maintain stable closed-loop behavior without an actuator. These constraints require modern control design methods that can properly account for the integrated flexibilities.

In this chapter, the CART system was simulated using classical PI control to regulate turbine speed in region 3 . When using the gains actually implemented in the CART, the response was unstable when the actuator was neglected. The addition of an actuator stabilized the flexible system by preventing higher frequency pitch control inputs from destabilizing flexible modes. It is clear that control design techniques are needed to account for the system flexibility and to enhance damping in the flexible modes. 


\section{Chapter 4. Illustration of Modern Control Design Steps}

\section{Introduction}

In this chapter, a set of control design steps are applied to stabilize flexible turbine modes. The theory forming the basis of these control design steps is described in detail in Appendix C. Application of the theory to control designs for speed regulation in region 3 is shown here. In later chapters, these methods will be used to stabilize additional flexible modes.

\subsection{Full-State Feedback}

\subsubsection{Design Steps}

The design method used to develop modern turbine controls is based on full-state feedback. A detailed description of this theory is given in Appendix C, Section C-1.4. This theory provides a set of control design steps. With full-state feedback, control systems can be designed to add damping to selected flexible turbine modes.

Recall that for the general state-space system:

$$
\begin{aligned}
& \underline{\dot{x}}=A \underline{x}+B \underline{u}+\Gamma \underline{u_{D}} \\
& \underline{y}=C \underline{x}+D \underline{u} .
\end{aligned}
$$

Figure 4-1 shows a full-state feedback controller.

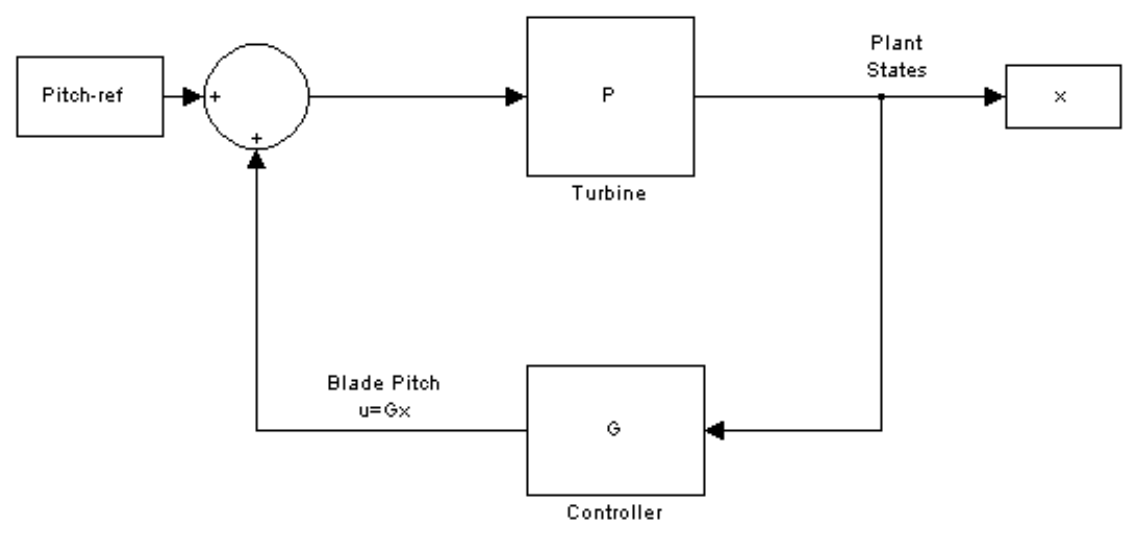

Figure 4-1. Diagram of full-state feedback control 
Designing control systems using full-state feedback includes these steps:

1. Generate an appropriate linear model of the turbine at a specific operating point (the control design point) in the form of Equation 4.1. Evaluate the state matrices $A$ and $B$.

2. Assess the controllability of the system $(A, B)$. If the system is controllable, then the closed-loop poles can be placed arbitrarily and damping can be enhanced through pole placement.

3. Calculate gains $G$ to give the desired poles selected in Step 2 .

4. Form the feedback control law $\underline{u}(t)=G \underline{x}(t)$ that will be fed back to the wind turbine (or the nonlinear wind turbine simulator in this work).

5. Simulate this control law response using FAST and/or ADAMS to test.

\subsubsection{Full-State Feedback Design Using the 1-State Linear Model}

Full-state feedback is demonstrated for a simple controller to regulate turbine speed in region 3 (using rotor collective pitch). This control is designed using the 1-state linear turbine model described in Section 2.2.3 at a control design point:

$$
\begin{aligned}
& \mathrm{w}_{0}=18 \mathrm{~m} / \mathrm{s}, \\
& \Omega_{0}=42 \mathrm{RPM}, \text { and } \\
& \beta_{0}=12 \mathrm{deg} .
\end{aligned}
$$

The step function wind input shown in Figure 4-2 will be used to excite the turbine response. As can be seen in Figure 4-2, the control design point occurs only in the interval from 40 to 50 seconds. To test controller stability at other operating points, it is important to test this control at other wind speeds around the control design point.

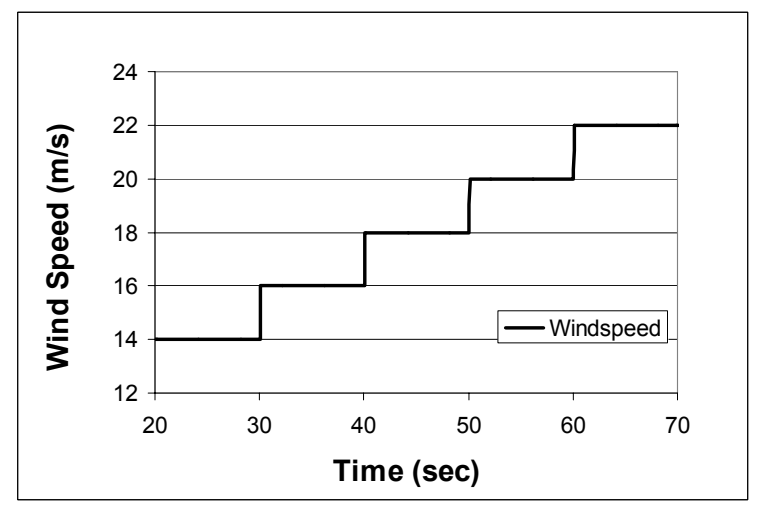

Figure 4-2. Test wind applied to CART FAST models

The first step is to evaluate the linear model at this point. Table 4-1 lists the numerical state matrix coefficients for the CART for this 1-state model. 
Table 4-1. CART State Matrix Values for the 1-State Model

\begin{tabular}{|c|c|c|}
\hline A & B & $\Gamma$ \\
\hline-0.1205 & -2.882 & 0.0658 \\
\hline
\end{tabular}

In the next step, controllability of $(A, B)$ requires only that $B$ be nonzero. At the control design point selected, the control gain has the value -2.88 .

Next, the plant pole location is selected. Better response of the rotor speed state (and hence better speed regulation) can be obtained by locating this pole to the left of its open-loop value in the complex plane. This pole is placed at -2 and can be contrasted with the open-loop value of -0.1205 . Since the system is controllable, this pole can be placed arbitrarily. The gain required to give us this pole location is $G=0.652$.

This feedback control law: $u(t)=0.652 x_{1}(t)$ is implemented in FAST and driven with a wind step function change (Figure 4-3). In this case, FAST was run with a completely rigid turbine and all flexible blade, drive train and tower DOF switched off.

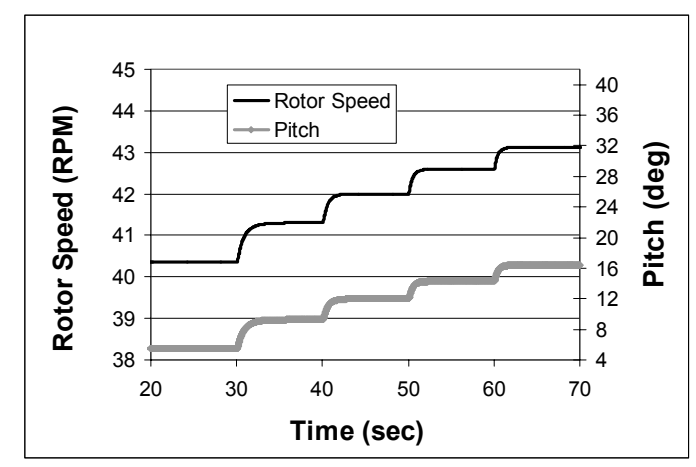

Figure 4-3. FAST simulated CART rotor speed and pitch during full-state feedback control based on 1 -state controller model

This response merits additional attention. The control design point is at a wind speed of $18 \mathrm{~m} / \mathrm{s}$ and pitch of 12 degrees. For wind speeds above and below the design point, the control and disturbance input gains do not match the values used in the control design. Also, wind-speed disturbances are not accounted for. As a result, generator speed is not regulated exactly to the desired value (42 rpm).

Now, we investigate behavior for a different pole location. The speed pole is placed at -1 with $\mathrm{G}=0.305$. This gain increases settling time and causes greater deviation between regulated speed and the $42 \mathrm{rpm}$ desired value (Figure 4-4), at operating points that do not match the control design point. The placement of the speed pole has a direct impact on the speed regulation. 


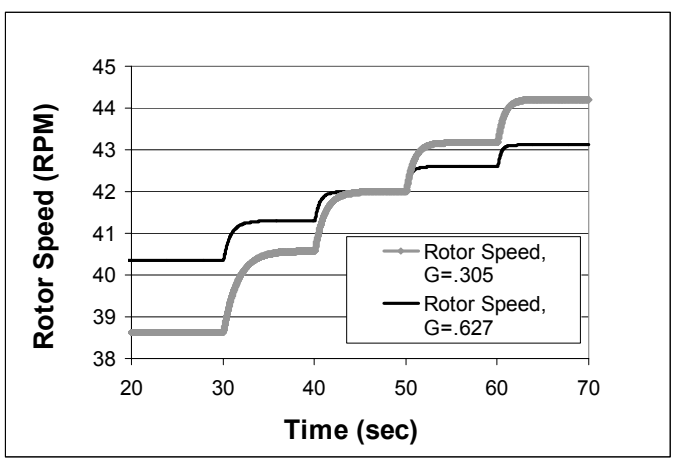

Figure 4-4. FAST predicted CART rotor speed using full-state feedback based on 1-state controller for various values of $\mathbf{G}$

\subsubsection{Full-State Feedback Design from the 3-State Linear Model}

Modeling the first drive-train torsion mode requires the addition of two states, as shown in Section 2.2.4. Using the same turbine operating point as the 1-state model produces the state matrix coefficients listed in Table 4-2. The controller is designed at the same turbine operating point as in the 1-state model. Table 4-2 shows the state matrices evaluated at this control design point.

Table 4-2. A, B, and $\Gamma$ Matrices for the CART 3-State Linear Model

\begin{tabular}{|l|l|l|l|l|}
\hline \multicolumn{2}{|c|}{ A } & \multicolumn{1}{c|}{ B } & \multicolumn{1}{|c|}{$\Gamma$} \\
\hline-.145 & $-3.108 \times 10^{-6}$ & 0. & -3.456 & 0.079 \\
\hline $2.691 \times 10^{7}$ & 0. & $-2.691 \times 10^{7}$ & 0. & 0. \\
\hline 0. & $1.56 \times 10^{-5}$ & 0. & 0. & 0. \\
\hline
\end{tabular}

An eigen-analysis of the A matrix for this system gives the open loop poles: $-0.012 \pm 22.4 \mathrm{j}$ and -0.121 . The first pole pair represents the very lightly damped first drive-train torsion mode. The third pole represents the generator rotational speed.

The calculated controllability matrix (see Appendix C) is defined by

$$
\text { Cont }=\left[\begin{array}{lll}
B & A B & A^{2} B
\end{array}\right]=\left[\begin{array}{ccc}
-3.456 & .500 & 288.95 \\
0 . & -9.300 \times 10^{7} & 1.344 \times 10^{7} \\
0 . & 0 . & -1450.82
\end{array}\right] \text {. }
$$

This matrix has full rank, thus the system is controllable, and poles can be placed arbitrarily.

Where should the poles of this model be placed? An objective is to increase first drive-train torsion mode damping. Another objective is to place the generator speed pole further to the left, as for the design from the 1-state model. The poles are placed at $-2 \pm 22.5 \mathrm{j}$, and -2 .

The gains corresponding to these pole locations are calculated as

$$
G=\left[\begin{array}{lll}
1.694 & 1.109 \times 10^{-7} & -1.039
\end{array}\right]^{1} .
$$

\footnotetext{
${ }^{1}$ This was done in MATLAB, using the PLACE command.
} 
The feedback control law: $u(t)=\left[\begin{array}{lll}1.694 & 1.109 \times 10^{-7} & -1.039\end{array}\right] \underline{x}(t)$ is implemented into FAST and simulated, inputting the same step winds. Figure 4-5 shows the speed regulation when the control law is simulated using FAST. These results are comparable to the results obtained using the 1-state model simulation. In this simulation, only the drive-train torsional mode was turned on in FAST. All other components, such as the blade and tower, were assumed to be rigid.

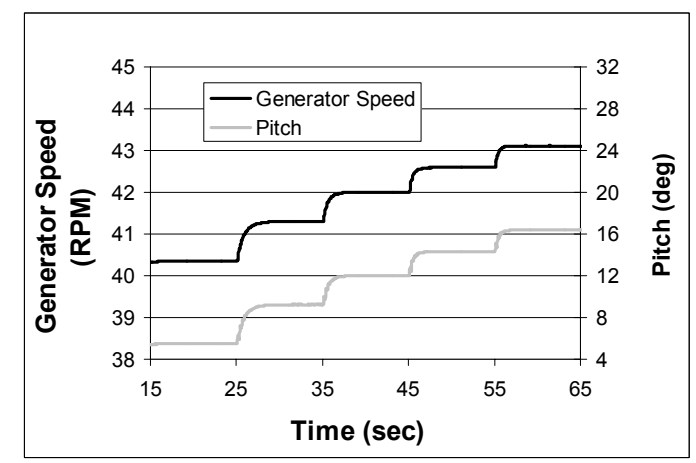

Figure 4-5. Plot of FAST simulated CART generator speed using 3-state full-statefeedback controller

Figure 4-6 shows how direct control over the first drive-train torsional mode can be achieved using full-state feedback for high and low damping. The wind step change excites the first drive-train torsional mode, and oscillations die out more slowly for the low damping case (Figure 4-6).

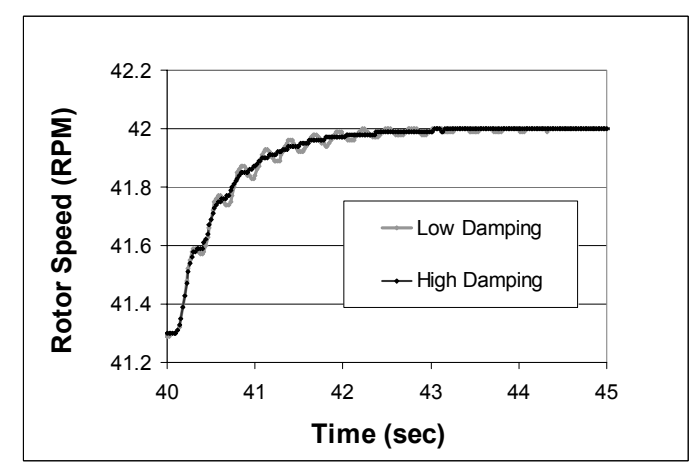

\section{Figure 4-6. Plot of FAST simulated CART generator speed using 3-state full-state feedback controller for two pole selections to give low- and high-drive-train damping}

An assumption in the use of full-state feedback is that the controller has all the state information contained in the linear model. Thus, accurate measurement of all of these states is required. This problem is trivial for the simple 1-state model, since the plant output measured is the only state in the model. As the number of states is increased, more measurements are required. A method is needed that permits the use of full-state feedback but with a reduced number of measurements.

\subsection{State Estimation}

A detailed discussion of state estimation is given in Appendix C, Section C-1.5. The steps can be summarized to include these:

1. Generate an appropriate linear model of the turbine at a specific operating point (the control design point). Evaluate the state matrices $A, B, C$. 
2. Assess controllability of the system $(A, B)$ for pole placement. If controllable, choose system poles to enhance damping and improve system response as desired.

3. Calculate gains ( $G$ ) required to give the desired pole placement.

4. Assess the observability of the system $(A, C)$ in order to place state estimator poles. If observable, choose state estimator poles to give quick decay of the error $\underline{e}(t)=\underline{\hat{x}}(t)-\underline{x}(t)$.

5. Calculate the values in the state estimator gain matrix $K$ that give the desired state estimator poles chosen in step 4.

6. Form the feedback law $\underline{u}(t)=G \underline{\hat{x}}(t)$. This is the control law that will be fed back to the wind turbine simulator.

Note that, now, the states that are fed back are not the actual wind turbine states, but the estimated states. Figure 4-7 shows a state estimator controller.

The implementation of a state estimator controller into the simulation codes can be simplified, through the use of an equivalent transfer function. As shown in Appendix C, the transfer function for the controller alone can be expressed as

$$
\operatorname{Cont}(s)=G(s I-L)^{-1} K
$$

where $G$ and $K$ have been calculated from these control design steps. The matrix $L$ is derived in Appendix C, Section C-1.5. As seen in Chapter 3, implementation of a controller expressed as a transfer function is straightforward. In that chapter, a simple PI controller was implemented into FAST and ADAMS.

\subsubsection{State Estimator Design from the 3-State Linear Model}

We now apply these steps to the design of a turbine controller. The first three steps in designing a state estimator are exactly the same steps used when designing full-state feedback control.

Hence, the appropriate linear model has already been generated and numerically evaluated in Section 4.1.3.

Controllability of the system $(A, B)$ was also checked in section 4.1.3. The goal is to again place the plant poles at $-2 \pm$ $22.4 \mathrm{j}$, and -2 . This gives good damping to the first drive-train torsional mode, as well as good speed response. The gains for this control have already been calculated as

$$
G=\left[\begin{array}{lll}
1.694 & 1.109 \times 10^{-7} & -1.039
\end{array}\right]^{1} .
$$

\footnotetext{
${ }^{1}$ This was done in MATLAB, using the PLACE command.
} 


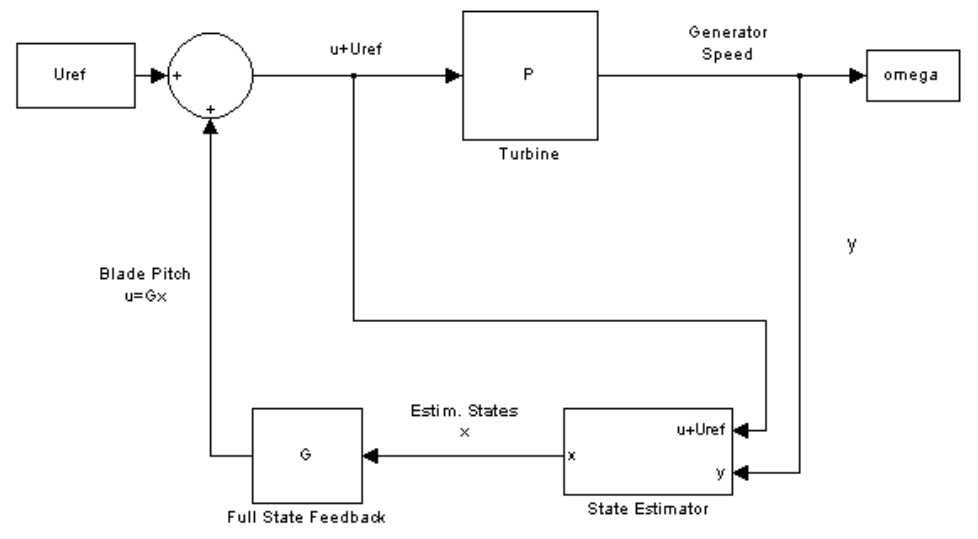

Figure 4-7. State estimator control diagram

The observability matrix (see Section C-1.3) is:

$$
O b s=\left[\begin{array}{c}
C \\
C A \\
C A^{2}
\end{array}\right]=\left[\begin{array}{ccc}
0 & 0 & 1 \\
0 . & 1.56 \times 10^{-5} & 0 . \\
419.813 & 0 . & -419.813
\end{array}\right]
$$

which has full rank. This system is thus observable, and state estimator poles can be arbitrarily placed. For the analyses, poles are placed at $-14,-15$, and -16 , and the required state estimator gains are calculated as

$$
K=\left[\begin{array}{c}
-1.1332 \\
1.0517 \times 10^{7} \\
44.8555
\end{array}\right]
$$

The feedback law $\underline{u}(t)=G \underline{\hat{x}}(t)$ is fed back to the FAST simulator utilizing the equivalent controller transfer function:

$$
\operatorname{Cont}(s)=\left(2102.76-369.69 \mathrm{~s}-47.357 \mathrm{~s}^{2}+0 \mathrm{~s}^{3}\right) /\left(5734.286+946.965 \mathrm{~s}+50.856 \mathrm{~s}^{2}+\mathrm{s}^{3}\right)
$$

This control is implemented in FAST by inputting the numerator and denominator coefficients of this transfer function into the input file for the control subroutines.

Figure 4-8 shows the simulated generator speed and blade pitch angle for this controller. These results look similar to the case for full-state feedback shown in Figure 4-5, but with greater deviation between regulated and desired generator speed (42 rpm). Now three plant states are being estimated from one turbine measurement, generator speed. This approach has several consequences, including a reduction in the robustness of speed regulation owing to the neglect of wind disturbance effects and additional nonlinear effects.

\footnotetext{
${ }^{1}$ This was done in MATLAB, using the PLACE command.
} 


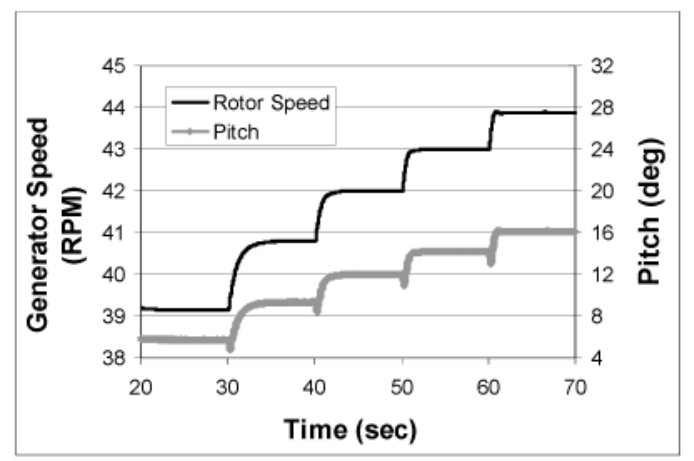

\section{Figure 4-8. Plot of FAST simulated CART generator speed using state estimator based controller designed from 3-state model}

We now investigate whether controller performance can be improved by properly accounting for the wind disturbance effects.

\subsection{Disturbance Accommodating Control}

One way to account for this disturbance is to add states to the linear model representing the disturbance. Disturbance Accommodating Control (DAC), the approach of reducing or counteracting persistent disturbances, was developed by Johnson (1976) for lumped parameter systems. Balas $(1980,1990)$ applied the method to large-scale and distributed parameter systems via model reduction and residual mode filters. Balas recently extended this theory to wind turbine control (Balas et al. 1998) as well. DAC is the augmentation of the usual state-estimator-based controller to recreate disturbance states via an assumed-waveform model; these disturbance states are used as part of the feedback control to reduce ("accommodate") or counteract any persistent disturbance effects.

These are the steps for designing controls using DAC:

Generate an appropriate linear model of the turbine at a specific operating point (the control design point). Evaluate the state matrices $A, B, C$, and $\Gamma$. Determine the open loop eigenvalues of the $A$ matrix.

1. Assess controllability of the system $(A, B)$ in order to allow pole placement. If the system is controllable, choose plant poles to enhance damping and improve system response as desired.

2. Calculate gains $G$ to give the desired poles chosen in Step 2 .

3. Form the feedback law $\underline{u}_{*}(t)=G \underline{x}(t)+G_{D} \underline{z}_{D}(t)$. Choose the wind disturbance state gain $G_{D}$ to exactly cancel wind-speed disturbances, if possible. This can be done by choosing $G_{D}$ so that $\left\|B G_{D}+\Gamma \theta\right\|=0^{1}$. If this is not possible, then choose $G_{D}$ to minimize the norm $\left\|B G_{D}+\Gamma \theta\right\|$. Once $G_{D}$ has been calculated, we have numerical values for the vector $\bar{G} \equiv\left[\begin{array}{ll}G & G_{D}\end{array}\right]$.

4. Calculate the augmented state matrices $(\bar{A}, \bar{B}, \bar{C})$ and assess the observability of $(\bar{A}, \bar{C})$. From Appendix C:

\footnotetext{
$1\|$.$\| indicates the standard 2-norm.$
} 


$$
\begin{aligned}
& \bar{A}=\left[\begin{array}{cc}
A & \Gamma \theta \\
0 & F
\end{array}\right], \bar{C}=\left[\begin{array}{ll}
C & 0
\end{array}\right], \text { and } \\
& \bar{B}=\left[\begin{array}{l}
B \\
0
\end{array}\right] .
\end{aligned}
$$

5. If observability is achieved, choose the poles of the state estimators (including the wind disturbance state estimator) to achieve the desired behavior (high damping for state estimator poles). We now have numerical values for the elements of the vector $\bar{K} \equiv\left[\begin{array}{c}K \\ K_{D}\end{array}\right]$.

6. Now that $\bar{G} \equiv\left[\begin{array}{ll}G & G_{D}\end{array}\right]$ and $\bar{K} \equiv\left[\begin{array}{c}K \\ K_{D}\end{array}\right]$ have been calculated, calculate the equivalent controller transfer function $\operatorname{Cont}(s)=\bar{G}(s I-\bar{L})^{-1} \bar{K}$ (see Appendix C), where $I$ is the identity matrix and $\bar{L}$ is shown in Appendix C. This transfer function is input to the control subroutines used in FAST and ADAMS. Simulate the closed-loop system for various conditions to assess controller performance.

\subsubsection{DAC Design from the 3-State Linear Model http://www.eere.energy.gov/femp/program/procuring_services.cfm}

The DAC design steps, for control based on the 3-state model are now shown. The linear model has already been developed and evaluated at the control design point, as discussed in Section 4.1.3. Controllability of the system $(A, B)$ has also been checked, and the gains calculated to place the plant poles at $-2 \pm 22.5 \mathrm{j} r / \mathrm{s}$, and $-2 \mathrm{r} / \mathrm{s}$, shown in Section 4.2.1.

Assume that the DAC waveform takes the form of a step function, which is a good representation of sudden uniform wind velocity fluctuations over the rotor disk. Referring to the general form in Equation C-1.32, Appendix C, the statespace model for the DAC step waveform can be expressed (Stol et al. 2000) as

$$
\begin{aligned}
& u_{D}(t)=z_{D}(t) \\
& \dot{z}_{D}(t)=0 z_{D}(t)
\end{aligned}
$$

Note that $\theta=1$ and $F=0$ in Equation C-1.32. Since the wind disturbance is represented by only one state, only one disturbance pole must be placed, and $G_{D}$ consists of only a single element. $G_{D}$ can be calculated to exactly cancel the wind-speed disturbance, using

$$
\left\|B G_{D}+\Gamma \theta\right\|=0 .
$$

This gives 


$$
\left\|\left[\begin{array}{c}
\zeta \\
\mathrm{I}_{\text {rot }} \\
0 \\
0
\end{array}\right] G_{D}+\left[\begin{array}{c}
\frac{\alpha}{\mathrm{I}_{\text {rot }}} \\
0 \\
0
\end{array}\right]\right\|=0
$$

which reduces to

$$
\sqrt{\left(\frac{\zeta G_{d}+\alpha}{\mathrm{I}_{\mathrm{rot}}}\right)^{2}}=0
$$

or $G_{D}=-\frac{\alpha}{\zeta}$. Substituting the appropriate values for $\alpha$ and $\zeta$ gives $G_{D}=.0228$, and wind-speed disturbances can be cancelled exactly.

The complete gain matrix is $\bar{G}=\left[\begin{array}{llll}0.8263 & 6.0866 \times 10^{-9} & -0.1761 & 0.0228\end{array}\right]$.

$\bar{G}$ contains 4 elements; 3 are due to the states in the linear plant model, and 1 state corresponds to the wind disturbance.

To assess observability for DAC, the augmented state matrices $\bar{A}$ and $\bar{C}$ are calculated, and the observability of the pair $(\bar{A}, \bar{C})$ is checked. The DAC step waveform is used; thus, $\theta=1$, and $F=0$. This gives

$$
\bar{A}=\left[\begin{array}{cccc}
-.145 & -3.108 \times 10^{-6} & 0 . & 0.079 \\
2.691 \times 10^{7} & 0 . & -2.691 \times 10^{7} & 0 . \\
0 . & 0.000016 & 0 . & 0 . \\
0 . & 0 . & 0 . & 0 .
\end{array}\right] \text {, and } \bar{C}=\left[\begin{array}{llll}
0 & 0 & 1 & 0
\end{array}\right] \text {. }
$$

The observability matrix is

$$
\left[\begin{array}{c}
\bar{C} \\
\overline{C A} \\
\overline{C A}^{2} \\
\overline{C A}^{3}
\end{array}\right]=\left[\begin{array}{cccc}
0 . & 0 . & 1 . & 0 . \\
.0 & 1.56 \times 10^{-5} & 0 . & 0 . \\
419.813 & 0 . & -419.813 & 0 . \\
-60.6815 & -0.0079 & 0 . & 33.1393
\end{array}\right]
$$

which has full rank. The system is thus observable, and state estimator poles can be placed as desired.

For disturbance state estimator poles placed at $-14,-15,-16$, and -17 , the gains are calculated using the PLACE command in MATLAB (The Mathworks, Inc., 2003): 


$$
\bar{K}=\left[\begin{array}{c}
22.511 \\
5.940 \times 10^{7} \\
61.856 \\
1723.64
\end{array}\right] .
$$

Figure 4-9 is a diagram of the DAC. The equivalent transfer function for the controller is calculated and incorporated into the FAST control subroutines as

$$
\operatorname{Cont}(s)=\frac{39824.5+29645.2 \mathrm{~s}-1165.05 \mathrm{~s}^{2}+19.8352 \mathrm{~s}^{3}}{0 .+21832.8 \mathrm{~s}+1811.51 \mathrm{~s}^{2}+67.8555 \mathrm{~s}^{3}+\mathrm{s}^{4}}
$$

Figure 4-10 shows the FAST simulated generator speed and blade pitch using this DAC controller. The speed regulation is robust over the entire range of wind speeds tested, as contrasted to the state estimator (neglecting the disturbance) results shown in Figure 4-8. This is a definite improvement over the full-state feedback and state estimation results obtained when neglecting the wind disturbance. The only measurement we are assuming is generator speed (equivalent to rotor speed for the 1-state model with rigid drive train, ignoring gearbox effects).

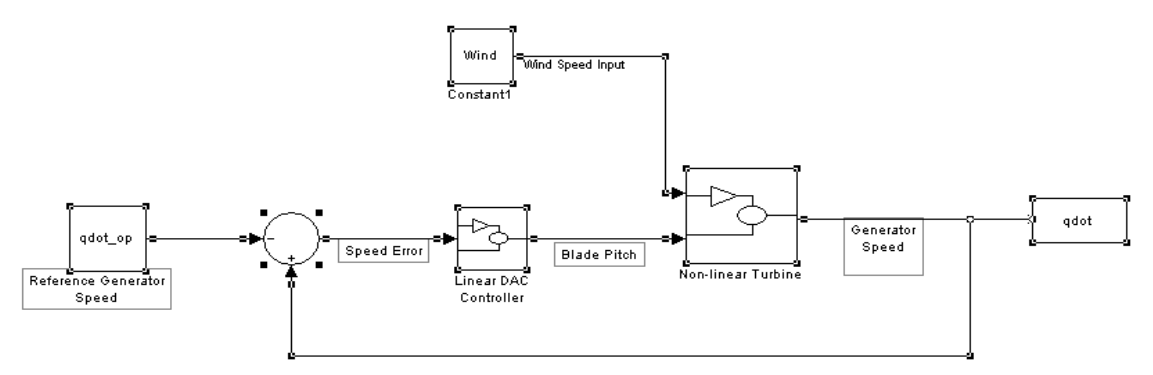

Figure 4-9. Diagram of DAC controller

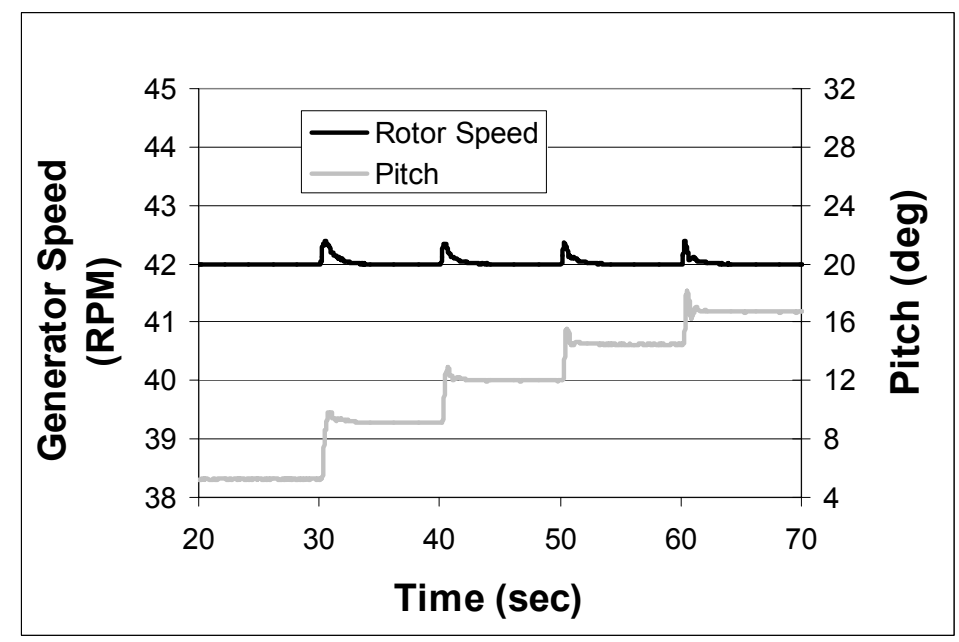

Figure 4-10. Plot of FAST simulated CART generator speed using DAC based controller based on 3-state model. 
This DAC controller provides the benefit of full-state feedback, while requiring only measurement of the generator speed. The plant poles can be placed arbitrarily, because of controllability. Figure 4-11 shows the effect of changing the control design to move the poles corresponding to the first drive-train torsional mode closer to the imaginary axis, for lower damping. Following a step change in wind speed, drive-train torsional oscillations die out more slowly, especially at the low-wind-speed end of region $3(14 \mathrm{~m} / \mathrm{s})$, which is undesirable.

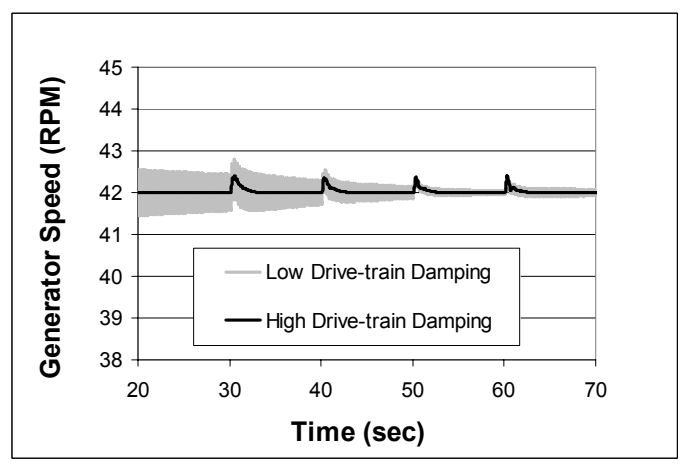

\section{Figure 4-11. Plot of FAST simulated CART generator speed using DAC controller} designed from 3-state model, for different pole locations

\subsection{Linear Quadratic Regulator Design}

In Chapter 7, Linear Quadratic Regulator design, or LQR, is used to form feedback laws. A brief discussion of LQR is given in Appendix C, Section C-1.7.

The state matrices for this state-space model for the CART are input to MATLAB (The Mathworks, Inc., 2003). Q is set to have the following values:

$$
Q=\left[\begin{array}{ccc}
0.1 & 0 & 0 \\
0 . & 1.0 \times 10^{-12} & 0 . \\
0 & 0 & 1
\end{array}\right]
$$

This resulting gain matrix $\mathrm{G}$ is:

$$
G=\left[\begin{array}{lll}
1.88452 & 0.000000235 & -1.18355
\end{array}\right]
$$

which gives the closed-loop plant poles: $-2.284+22.595 \mathrm{i},-2.284-22.595 \mathrm{i}$, and -2.090 . The first pole pair corresponds to the first drive-train torsion mode, and the last pole corresponds to generator speed. The poles are placed close to the values that have been previously selected. Note that a value for the wind disturbance gain $G_{D}$ is not calculated with LQR. Calculation of this gain is performed separately (as it was in the previous DAC design) by finding $G_{D}$, which minimizes $\left\|B G_{D}+\Gamma \theta\right\|$. This again gives $G_{D}=.023$.

Next, the state estimator gain matrix $\bar{K} \equiv\left[\begin{array}{c}K \\ K_{D}\end{array}\right]$ is calculated and the state estimator weight matrix set equal to 


$$
Q e=\left[\begin{array}{cccc}
100 & 0 & 0 & 0 \\
0 & 1 & 0 & 0 \\
0 & 0 & 1000 & 0 \\
0 & 0 & 0 & 0.1
\end{array}\right]
$$

which gives the state estimator gain matrix:

$$
K=\left[\begin{array}{c}
30.042 \\
47627947.15 \\
49.858 \\
3161.149
\end{array}\right] \text {. }
$$

Note that this includes the state estimator gain corresponding to the wind disturbance (last element). The corresponding poles are located at $-9.89+16.828 \mathrm{i},-9.89-16.828 \mathrm{i},-15.12+6.84 \mathrm{i}$, and $-15.12-6.84 \mathrm{i}$. Two poles correspond to the state estimator for the first drive-train torsional mode. The other two poles correspond to the state estimator for the generator speed and the wind disturbance.

All of this information is required to calculate an equivalent controller transfer function. After computing the equivalent transfer function and simulating in FAST, the simulation results shown in Figure 4-12 are obtained. The results are stable, as expected, with robust speed regulation. As used here, LQR is just another method of calculating gain matrices for the plant and estimator states, and it provides little additional value. In Chapter 7, the utility of LQR is demonstrated when there are several control inputs and several flexible modes in the models.

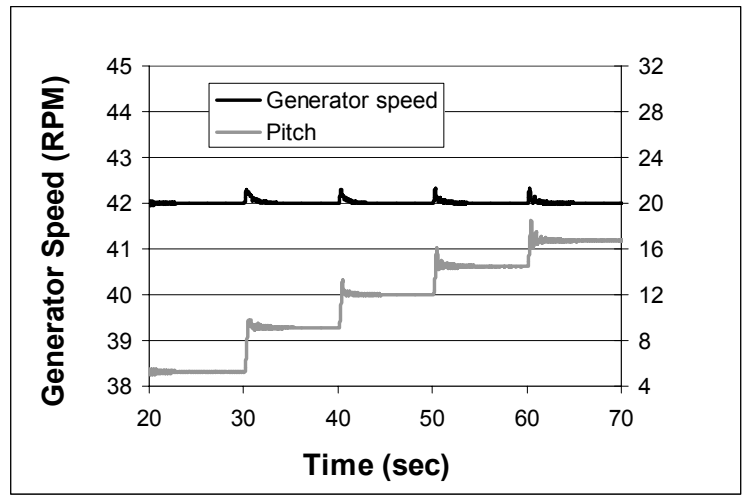

\section{Figure 4-12. Plot of FAST simulated CART generator speed and pitch using DAC controller designed from 3-state model, using LQR}

\subsection{Chapter Conclusions}

In this chapter, the control design steps for full-state feedback, state estimation, DAC, and LQR have been illustrated. These modern control design methods have been applied to speed regulation of the CART in region 3. It was shown that the use of full-state feedback permits controls to be designed that enhance damping for flexible turbine modes. State estimation, a technique used to estimate plant states from a limited number of measurements was also demonstrated. With state estimation, full-state feedback can still be used by employing the separation principle (see Appendix C).

Disturbance Accommodating Control (to account for wind disturbance effects) was also illustrated. For the case shown, we were able to exactly cancel wind disturbances and robustly regulate turbine speed over a wide range of wind speeds. Finally, Linear Quadratic Regulator design was also illustrated. These tools can be used to design controls for additional 
speed regulation and disturbance attenuation, as shown in the next few chapters. The results of these control designs are shown without describing the calculations in each design step. Those details are listed in Appendix D. 


\section{Chapter 5. Control Designs for Turbine Speed Regulation Using Rotor Collective Pitch}

\section{Introduction}

This chapter presents further control design results for the regulation of turbine speed in region 3 using rotor collective pitch. These controls are applied to the Controls Advanced Research Turbine (CART). A key objective is to apply advanced control techniques to flexible wind turbines. The word "flexible" is important; as shown in Chapter 4, these control design steps give us tools to account for the flexibility of the wind turbine.

In this chapter, controls are designed from various linear models. Starting with a simple 1-state model and systematically increasing the number of states, we establish the minimum number of states required to ensure system stability. Additional results include: expected controller performance for speed regulation, minimum number of turbine states needed in the linear model for control design, identification of the critical flexible modes, and the minimum number of turbine measurements needed to successfully use state estimation.

\subsection{Control Design Objectives and Assumptions}

This chapter shows how turbine speed in region 3 is regulated using only the rotor collective pitch. With the generator torque held constant, changes in aerodynamic torque from wind-speed variation would result in the rpm "drifting" and turbine power fluctuating. Rotor collective pitch essentially regulates the rotor aerodynamic torque, thus regulating rotor and generator speed and producing constant power. At first, it is assumed that the only measurement available for control is generator speed (or rotor speed with no drive-train torsional flexibility). This limitation will be reexamined later. The control objective, in addition to constant speed regulation, is to maintain stable closed-loop behavior over a wide range of turbine operating parameters and enhance damping in several flexible modes. In order to enhance damping in a particular mode, the linear model must contain the states necessary to describe this mode.

Using only rotor collective pitch, we show that only the symmetric rotor modes can be controlled. These modes are defined in a later section. The uniform wind components over the rotor disk excite the symmetric modes. Wind components varying spatially across the rotor disk, such as wind shear, excite asymmetric modes, as shown later in Chapter 7.

\subsection{DAC Controls Designed from the 1-State Linear Model}

We first design a DAC controller from the 1-state model described in Section 2.2.3. Referring to Equation 2.4, the effects of variations in the pitch input gain coefficient $\frac{\zeta}{\mathrm{I}_{\text {rot }}}$, and the wind disturbance gain coefficient $\frac{\alpha}{\mathrm{I}_{\text {rot }}}$ with turbine operating point must be established.

The aerodynamic response is highly nonlinear, causing the gain coefficients to vary with pitch and wind speed. Figure 5-1 shows the rotor aerodynamic torque versus blade pitch angle and wind speed for the CART. As can readily be seen in the top graph of the figure, the change in torque with pitch angle (seen from the slopes of these curves) varies as we move along any constant wind-speed curve. To a lesser degree, the change in torque with wind speed varies as we move along any constant pitch curve, as seen in the bottom graph of the figure. Thus, pitch and wind disturbance input gains vary as the turbine operating point changes. The controller performance will be evaluated as the turbine operating point deviates from the control design point indicated in Figure 5-1. 

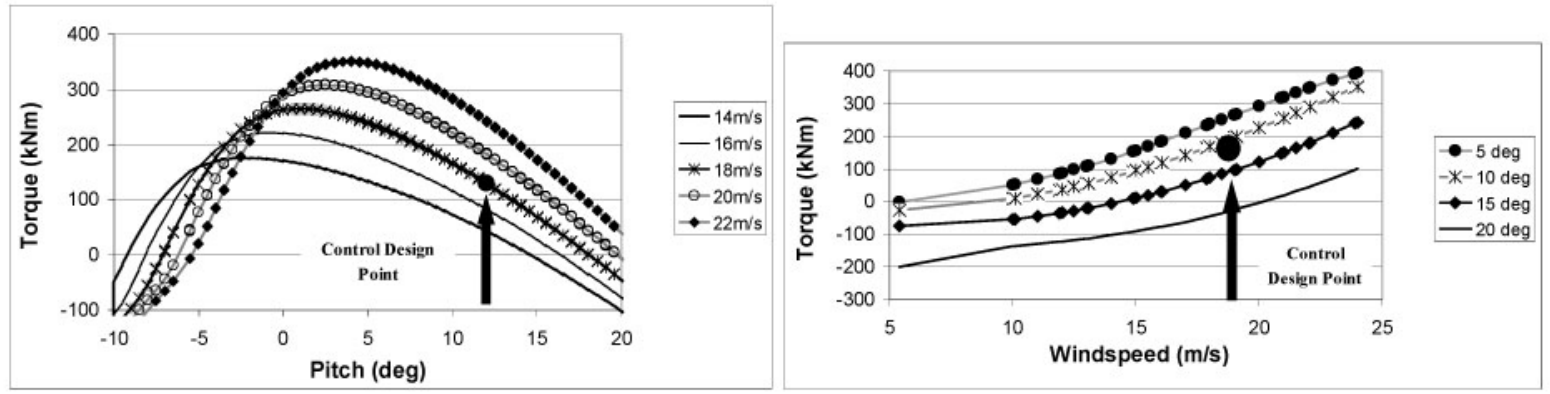

Figure 5-1. Variation of rotor torque with pitch and wind speed, showing the control design point

The operating point shown in Figure 5-1 was chosen midway between the lowest $(12 \mathrm{~m} / \mathrm{s})$ and highest $(24 \mathrm{~m} / \mathrm{s})$ wind speeds of region 3 and is

$$
\begin{aligned}
& \mathrm{w}_{0}=18 \mathrm{~m} / \mathrm{s}, \\
& \Omega_{0}=42 \mathrm{RPM}, \text { and } \\
& \beta_{0}=12 \mathrm{deg} .
\end{aligned}
$$

As can be seen in Figure 5-2, the test and design wind speeds coincide only in the interval from 40 to 50 seconds. Table 4-1 (Section 4.1.2) showed the model evaluation at this design point.

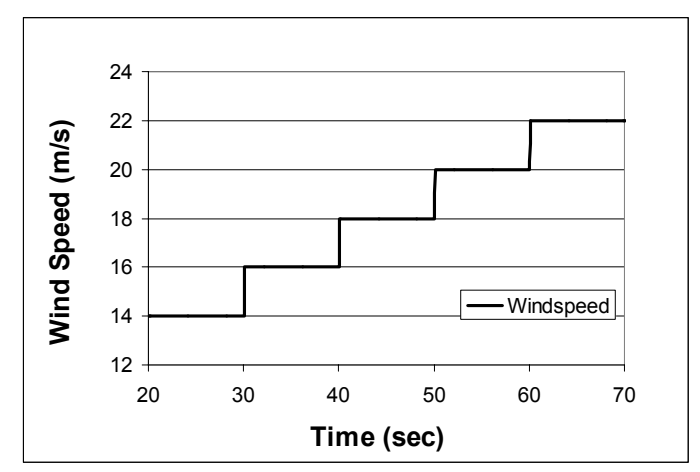

Figure 5-2. Test wind applied to CART FAST models

For the DAC controller (using the steps explained in Section 4.3), the equivalent controller transfer function is defined by

$$
\operatorname{Cont}(s)=(145.742+91.706 \mathrm{~s}) /\left(0 .+30.880 \mathrm{~s}+\mathrm{s}^{2}\right)
$$

Figure 5-3 shows the resulting speed regulation and blade pitch using this DAC controller. Rotor speed is regulated precisely to the 42-rpm set point over a wide range of wind speeds. Blade pitch varied from 5 to 17 degrees to reduce aerodynamic torque with increasing wind speed. In this simulation, only the rotor rotational speed DOF was simulated.

Precise rpm control is obtained for step wind disturbances. This is similar to the PI design result obtained in Section 3.2 by increasing the integrator gain. Note that the first term in the denominator of the DAC controller transfer function in Equation 5.2 is zero, indicating a pole at $s=0$. The controller will robustly cancel step disturbances, for any turbine operating point. 


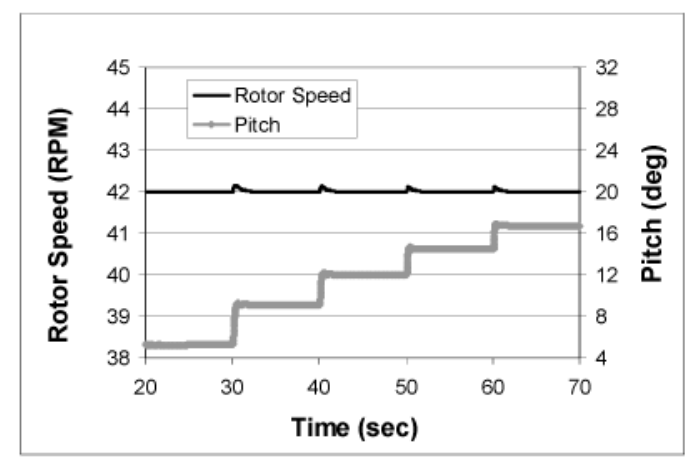

Figure 5-3. FAST simulated CART rotor speed and pitch during DAC control designed from 1state model

Constant gains were used in the design of this controller, even though the true gains vary with wind speed and pitch, as we have seen. As the turbine's operating point deviates significantly from the control design point, the controller estimates the wind speed less accurately. Figure 5-4 illustrates this discrepancy. The "actual" wind in this plot is the test wind that was input to FAST. The "estimated" wind was the value calculated by the disturbance state estimator in DAC. In general, the wind disturbance state estimator does a good job of estimating wind speed over a broad range of operating conditions. The largest deviation occurs when the wind speed drops to $14 \mathrm{~m} / \mathrm{s}$, and the DAC underestimates the wind speed by about $1.2 \mathrm{~m} / \mathrm{s}$. This delta is related to the large change in the slopes of the torque versus wind speed and pitch curves as the turbine operating point deviates from the control design point $(18 \mathrm{~m} / \mathrm{s}$ wind speed and 12 degree pitch). These slopes have large variation when wind speed and pitch decrease relative to the control design point; they have less variation when wind speed and pitch increase relative to this control design point.

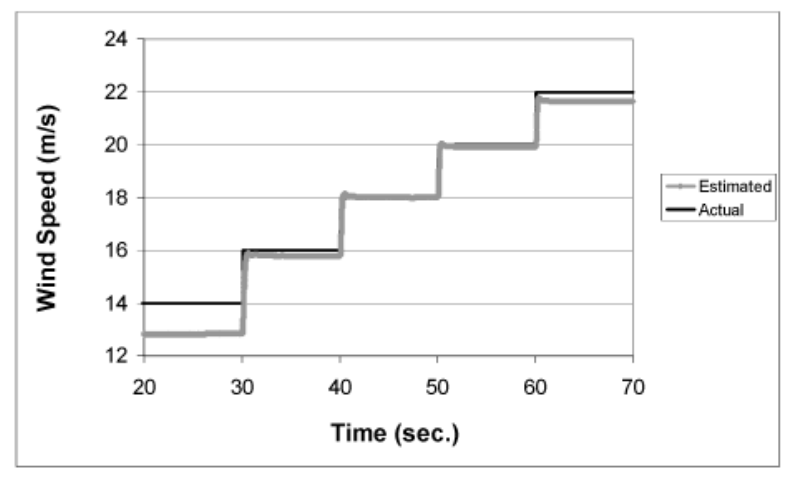

Figure 5-4. Comparison of estimated and actual wind speed as simulated by FAST for the CART using DAC control based on 1-state model

In these results, flexible modes are ignored, both in the linear model and in the simulations. Figure 5-5 shows a baseline comparison between ADAMS and FAST with no flexibility. As described in Chapter 2, the same turbine property inputs have been used for both codes to provide equivalent model fidelity, and the responses are virtually identical. 


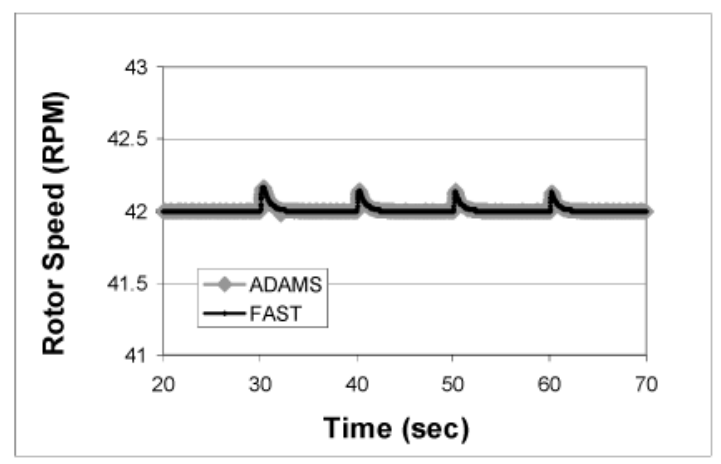

Figure 5-5. Comparisons of ADAMS and FAST predicted rotor speed using 1-state controller for the CART

\subsubsection{Effects of Unmodeled Modes}

The first drive-train torsion mode is now enabled during simulation with both ADAMS and FAST. In the CART ADAMS model, the blades and tower are held rigid, and the only flexibility is drive-train torsion. This DOF is modeled with an equivalent spring damper system equivalent to FAST. The models are now simulated using the controller designed from the 1-state linear model, but with first drive-train torsion switched on and excited by the same step winds. As can be seen in Figure 5-6, the generator speed becomes unstable, oscillating at the first drive-train torsion frequency. Even adding a large amount of mechanical damping to the torsional spring fails to stabilize this response. The degree of instability appears to be less in ADAMS than in FAST, which could be the result of differences in modeling or numerical integration. The general trend from both models is the same, giving confidence that both codes are simulating similar behavior. Figure 5-7 shows a pole plot of the closed-loop system. Using the 1-state linear model described in the last section, we calculated a gain corresponding to the generator speed state. When first drive-train torsion is switched on, this gain causes this mode to be driven to the right of the imaginary axis in the complex plane, resulting in instability.

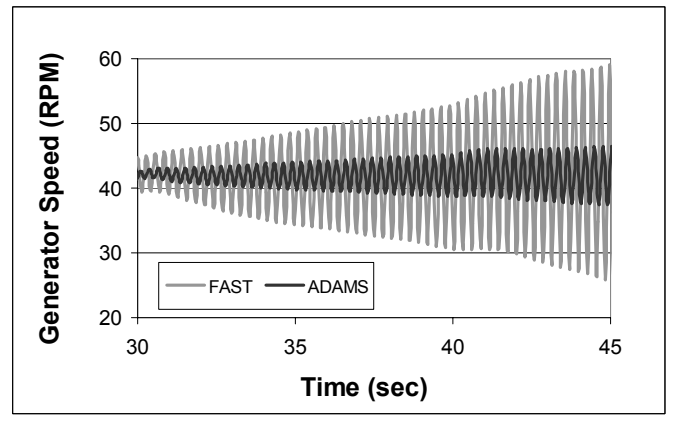

Figure 5-6. Plot of CART generator speed as simulated with FAST and ADAMS with DAC control designed from 1-state model, with first drive-train torsion mode switched on 


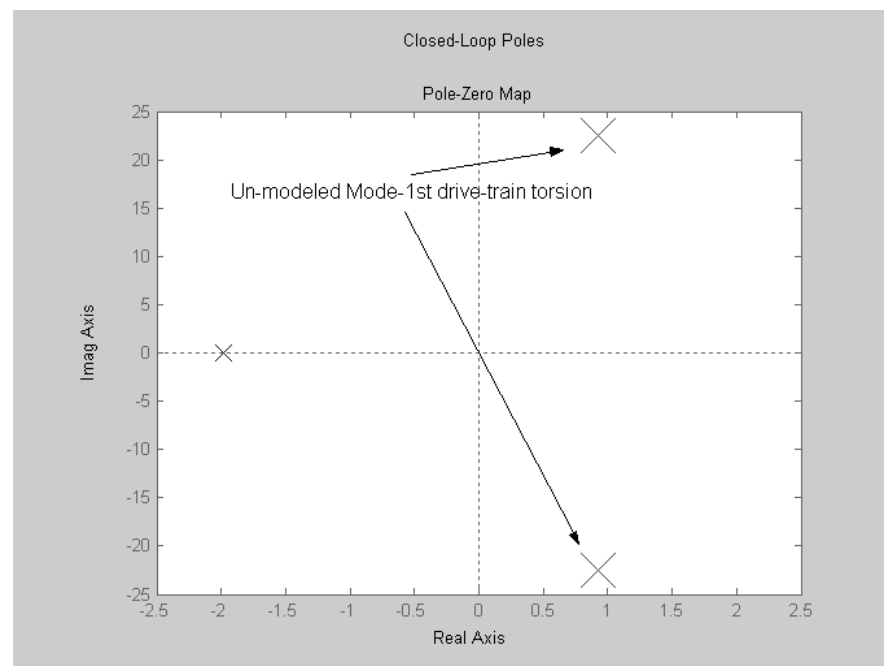

Figure 5-7. Pole plot of the closed-loop system, with first drive-train torsion mode switched on but not accounted for in control design

Presumably, placement of the rotor speed pole closer to the imaginary axis (less aggressive control gains) would result in stable behavior. To test this hypothesis, another controller was designed with the rotor speed pole at -0.5 radians/second $(\mathrm{r} / \mathrm{s})$, instead of $-2 \mathrm{r} / \mathrm{s}$. This model also resulted in unstable behavior.

An additional consideration is the effect of increasing the torsional stiffness of the drive train. As this stiffness increases, we might expect that the degree of interaction between the controller and the first drive-train torsional mode would decrease. Figure 5-8 shows these results using the same controller with the pole also placed at $-2 \mathrm{r} / \mathrm{s}$. As the stiffness is increased, the degree of instability decreases, until, at 5x the baseline CART stiffness, this controller exhibits stable closed-loop behavior. Thus, for a very stiff turbine, a controller designed from this simple 1-state linear model is sufficient.

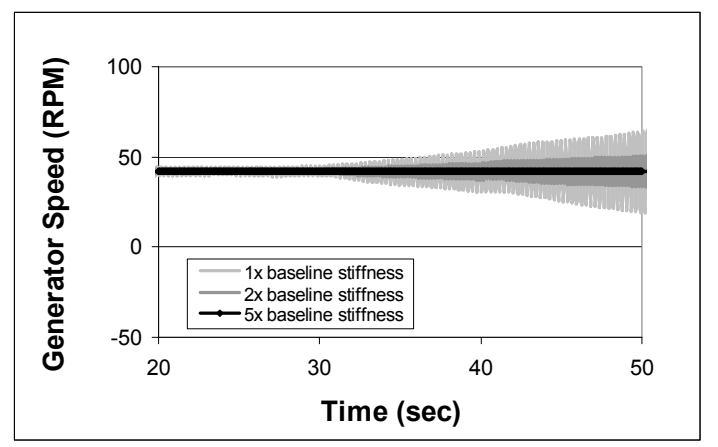

Figure 5-8. Plot of FAST simulated CART generator speed with drive-train torsion using 1-state controller, for increased drive-train torsional stiffness

This study shows that controls designed from the 1-state model are inadequate for the CART. The potential for the control system to excite the first drive-train torsional mode is high. The controller should be designed from a model that includes states necessary to model this mode.

\subsection{DAC Control Design from the 3-State Model}

Figure 4-10 (Chapter 4) reviews FAST simulated generator speed and pitch using DAC designed from the 3-state model described in Section 4.3.1, confirming stable results. In this simulation, first drive-train torsion is switched on. Unlike 
the results obtained for DAC designed from the 1-state model, the results remain stable for the 3-state model, because the states needed to describe the first drive-train torsion mode are included in the linear model.

Figure 5-9 shows a comparison between the estimated wind and wind input to FAST. In general, the magnitude of the deviation between input and estimated wind speed is greatest when the turbine operating point deviates significantly from the control design point. The magnitude is greatest at the lowest wind speed tested $(14 \mathrm{~m} / \mathrm{s})$, similar to the estimation results presented from the DAC designed from the 1-state model.

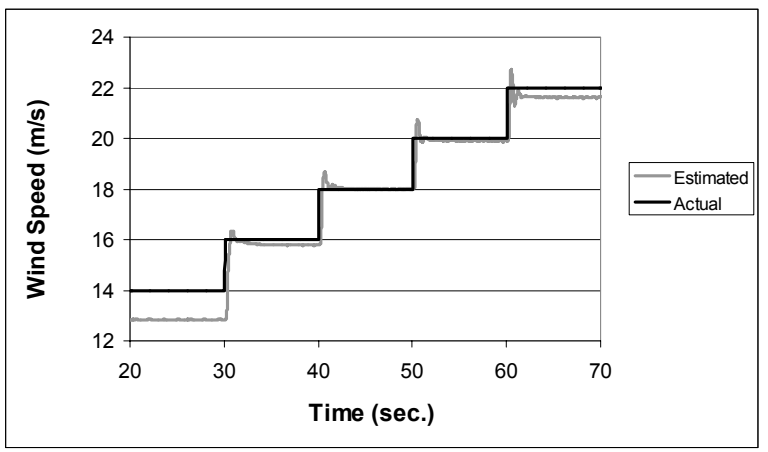

\section{Figure 5-9. Comparison of estimated and actual wind speed as simulated by FAST for the CARTusing DAC control based on 3-state model}

We again investigate the closed-loop response when additional DOF are switched on during simulation.

\subsubsection{Effects of Unmodeled Modes}

The DAC controller is now incorporated into ADAMS. The ADAMS model consists of rigid blades and tower, with a torsionally flexible drive train equivalent to the FAST model. In FAST, all blade and tower modes are switched off. Figure 5-10 shows the simulated generator rotational speed from FAST and ADAMS, confirming that the system is stable in both simulations. Next, this same case is simulated with the blade first flap mode switched on in FAST and ADAMS. In ADAMS, the blade flapping motion is simulated by modeling the rigid blade attachment to the hub with a revolute joint. A rotational spring, placed at this joint, models the blade flapwise flexibility. The spring stiffness is adjusted until the blade flap frequency matches the blade first flap frequency in FAST.

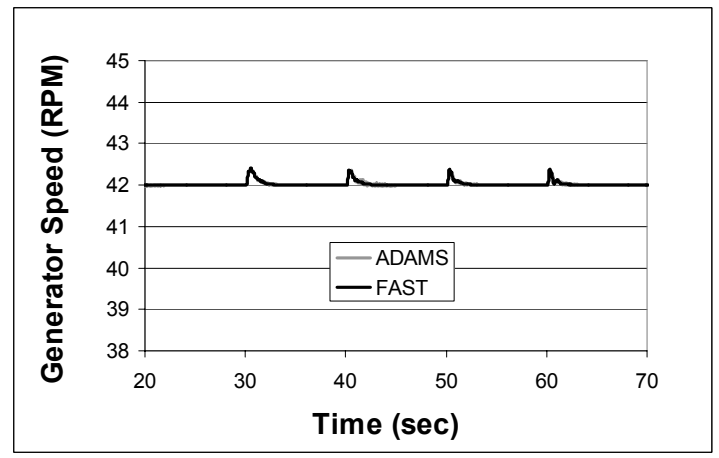

Figure 5-10. Comparisons of ADAMS and FAST simulated cart generator speed using DAC control designed from 3-state model

Figure 5-11 shows simulation of the generator rotational speed, with the DAC designed from the 3-state model and with blade first flap switched on. Undesirable fluctuations in generator speed can be seen from both codes. These fluctuations are at the frequency of the first drive-train torsion mode. As it turns out, the blade first flap mode is highly damped, as a result of aerodynamic damping. 


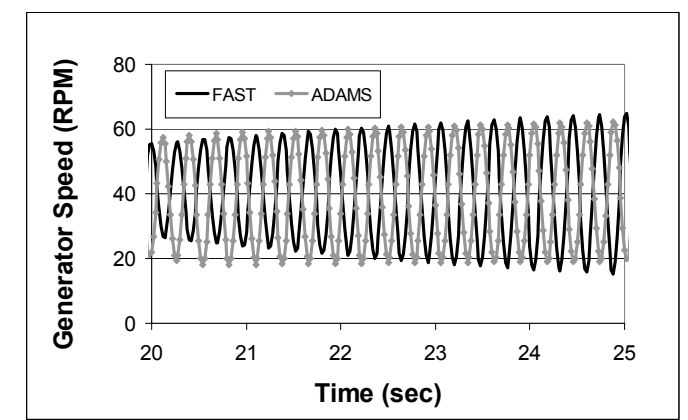

\section{Figure 5-11. FAST and ADAMS simulated CART generator speed using DAC designed from 3-state model with first flap turned on}

Figure 5-12 shows a pole plot of the closed-loop system with the blade first flap mode switched on, but not accounted for in the linear model used for control design. The gains calculated in the last section are used to calculate the closed-loop eigenvalues. Even though the first flap mode itself is stable (with poles to the left of the imaginary axis in the complex plane), the presence of the first flap mode has destabilized the first drive train mode. This is due to various terms which couple the first drive-train torsion mode to the blade first flap mode.

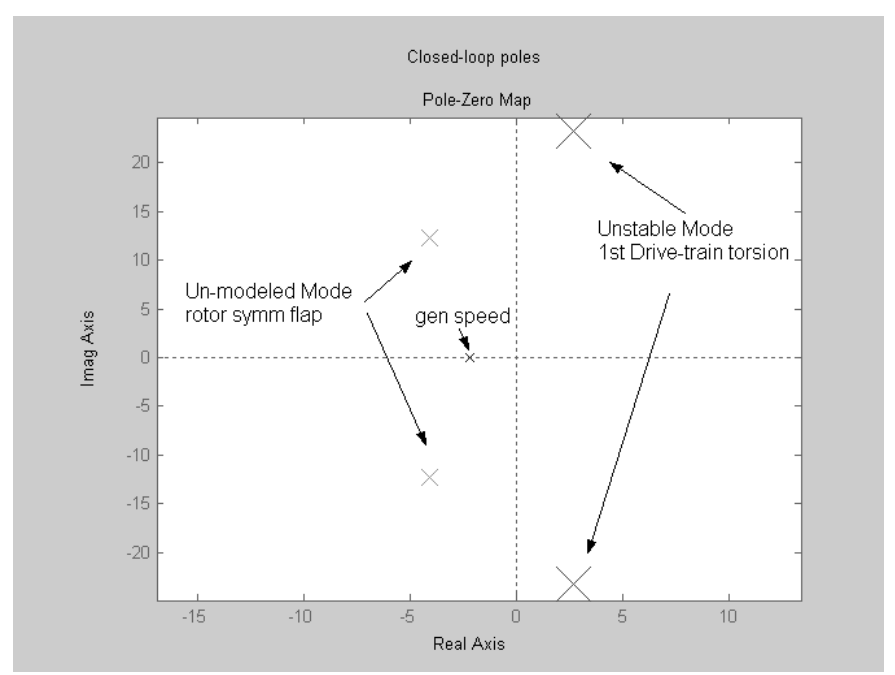

\section{Figure 5-12. Pole plot of the closed-loop system, with blade first flap mode switched on but not accounted for in control design}

An additional consideration is the effect of increasing the blade flapwise stiffness. As this stiffness increases, we might expect that the degree of interaction between the controller and the first drive-train torsion mode might decrease. Figure 5-13 shows the results of increasing this stiffness. The same controller is used. Indeed, as the blade flapwise stiffness is increased, the degree of instability decreases until, at 10x the baseline flapwise stiffness, this controller seems to result in stable closed-loop behavior. This shows that, for a very stiff turbine, a controller designed from this simple 3-state linear model is sufficient.

It is clear that control for the CART, designed from the 3-state model, excites the first drive-train torsion mode when the blade first flap mode is switched on during simulation. This is caused by the high degree of coupling between the blade first flap mode and the first drive-train torsion mode. The blade first flap mode must be accounted for in the control design, since switching it on during simulation destabilizes the first drive-train torsion mode. The linear model with 3 states must be modified to include the first flap mode of each blade. 


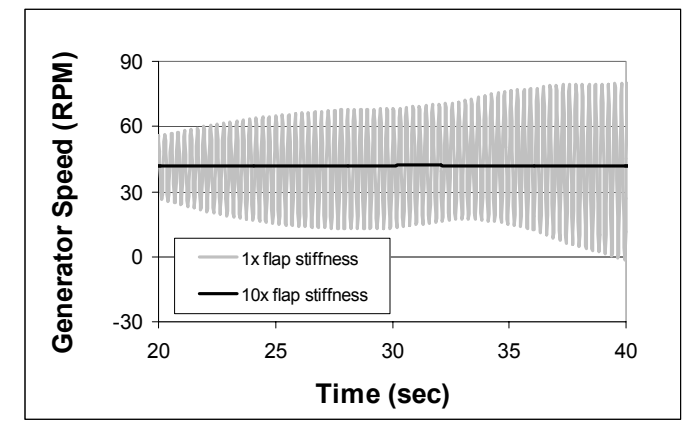

\section{Figure 5-13. FAST simulated CART generator speed using DAC 3-state controller for various blade flap stiffness values}

Additional DOF are switched on in FAST and ADAMS to test the effects of other unmodeled modes. Simulation using this controller with the blade first edge mode shows some instability, but these results are dramatically improved with the addition of a small amount of drive train structural damping. Simulation with other modes switched on shows little difference in these results. For the CART, the most critical mode to add to the linear model is blade first flap, which is described below.

\subsection{Control Designs Using a 5-State Model}

\subsubsection{Model Description}

Section 2.2.5 described a linear model including the blade-tip flap deflection and velocity of each blade. This model adds the first flap mode of each blade. Recall that we are using rotor collective pitch, in which the pitch of each blade is identical.

Because there is only one control input, the flap mode of each blade cannot be controlled individually. Since the pitch of each blade is identical in rotor collective pitch, only symmetric modes can be controlled with this control input. In a symmetric rotor mode, the flap deflection of each blade is identical. It can be shown that addition of two states for each blade (a flap mode displacement state and a flap mode velocity state) results in an uncontrollable system with rotor collective pitch.

To obtain a controllable system, one must transform the flap states of the two blades to the rotor first symmetric flap mode, and the rotor first asymmetric flap mode, as explained in Appendix A, Section A1.4. We discard the states corresponding to the rotor first asymmetric flap mode, since it cannot be controlled with rotor collective pitch. The final transformed model can be described with the following states and state-space equation:

$$
\begin{aligned}
& x_{1}=\frac{1}{2}\left(\delta q_{1}+\delta q_{2}\right), \text { perturbed rotor first symmetric flap displacement, } \\
& x_{2}=\frac{1}{2}\left(\delta \dot{q}_{1}+\delta \dot{q}_{2}\right), \text { perturbed rotor first symmetric flap velocity, } \\
& x_{3}=\delta \dot{q}_{4}, \text { perturbed rotor rotational speed, } \\
& x_{4}=\mathrm{K}_{\mathrm{d}}\left(\delta q_{4}-\delta q_{15}\right), \text { perturbed drive-train torsional spring force, and } \\
& x_{5}=\delta \dot{q}_{15}, \text { perturbed generator rotational speed. }
\end{aligned}
$$




$$
\begin{aligned}
& {\left[\begin{array}{ccccc}
1 & 0 & 0 & 0 & 0 \\
0 & \mathrm{M}_{11} & \mathrm{M}_{14} & 0 & 0 \\
0 & 2 \mathrm{M}_{14} & \mathrm{I}_{\text {rot }} & 0 & 0 \\
0 & 0 & 0 & 1 & 0 \\
0 & 0 & 0 & 0 & \mathrm{I}_{\text {gen }}
\end{array}\right]\left[\begin{array}{c}
\dot{x}_{1} \\
\dot{x}_{2} \\
\dot{x}_{3} \\
\dot{x}_{4} \\
\dot{x}_{5}
\end{array}\right]=\left[\begin{array}{ccccc}
0 & 1 & 0 & 0 & 0 \\
-\mathrm{K}_{11} & -\mathrm{C}_{11} & -\mathrm{C}_{14} & 0 & 0 \\
0 & -2 \mathrm{C}_{41} & \gamma-\mathrm{C}_{\mathrm{d}} & -1 & \mathrm{C}_{\mathrm{d}} \\
0 & 0 & \mathrm{~K}_{\mathrm{d}} & 0 & -\mathrm{K}_{\mathrm{d}} \\
0 & 0 & \mathrm{C}_{\mathrm{d}} & 1 & -\mathrm{C}_{\mathrm{d}}
\end{array}\right]\left[\begin{array}{c}
x_{1} \\
x_{2} \\
x_{3} \\
x_{4} \\
x_{5}
\end{array}\right]+\left[\begin{array}{c}
0 \\
\zeta_{\mathrm{b}} \\
\zeta \\
0 \\
0
\end{array}\right] \delta \beta+\left[\begin{array}{c}
0 \\
\alpha_{\mathrm{b}} \\
\alpha \\
0 \\
0
\end{array}\right] \delta \mathrm{w}}
\end{aligned}
$$

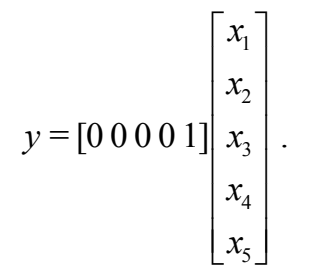

This system presents some differences when compared with the previous two linear models described in the preceding sections. Note that now the gain and wind disturbance input matrices have nonzero entries in both the second and third rows. Any perturbation in either collective pitch or wind speed perturbs the flap-wise aerodynamic blade force as well as the rotor aerodynamic torque. This means that the rotor collective pitch input enters the system in two rows of the pitch input gain matrix instead of just one, as in the previous models. The uniform wind-speed disturbance also enters the system through these same two rows. As we will see, this complicates the selection of the wind disturbance gain $G_{D}$.

Of course, we must evaluate the state matrices for the CART at the control design point for this 5 -state model. Table 5-1 shows the numerical values.

Table 5-1. A, B, and $\Gamma$ Matrices for the CART 5-State Linear Model

\begin{tabular}{|c|c|c|c|c|c|c|}
\hline \multicolumn{5}{|l|}{ A } & \multirow{2}{*}{$\begin{array}{l}\text { B } \\
0 .\end{array}$} & \multirow{2}{*}{$\begin{array}{l}\Gamma \\
0 .\end{array}$} \\
\hline 0. & 1. & 0. & 0. & 0. & & \\
\hline-200.185 & -8.990 & -20.053 & 0.000029 & 0. & -1120.9 & 14.13 \\
\hline 1.033 & 0.008 & -0.041 & $-3.258 \times 10^{-6}$ & 0. & 2.327 & 0.006 \\
\hline 0. & 0. & $2.691 \times 10^{7}$ & 0. & $-2.691 \times 10^{7}$ & 0. & 0. \\
\hline 0. & 0. & 0. & 0.000016 & 0 . & 0. & 0 . \\
\hline
\end{tabular}

An eigen-analysis of the A matrix for this system, from Table 5-1, gives the following open loop poles: $-0.033 \pm 22.6 \mathrm{j}$ $\mathrm{r} / \mathrm{s},-4.42 \pm 13.3 \mathrm{j} \mathrm{r} / \mathrm{s}$, and $-0.121 \mathrm{r} / \mathrm{s}$. The first pole pair represents the very lightly damped first drive-train torsion mode, as we have seen before. The second pole pair represents the highly damped rotor first symmetric flap mode. The third pole represents the generator rotational speed. We increase the damping in the drive-train torsion mode, stabilize the rotor first symmetric flap mode, and add damping to the generator rotational speed by placing the poles at $-2 \pm 22.6 \mathrm{j} r / \mathrm{s}$, $4.4 \pm 13.4 \mathrm{j} \mathrm{r} / \mathrm{s}$, and $-2 \mathrm{r} / \mathrm{s}$.

Detailed steps shown in Appendix D are the same as those described for the design example presented in Section 4.3. One major difference in designing DAC from the 5-state linear model is in calculating the wind disturbance gain $G_{D}$. For this system, it is not possible to choose this gain to cancel exactly the wind-speed disturbance. This can be seen by examining the norm: $\left\|B G_{D}+\Gamma \theta\right\|$. For this model this becomes (with $\theta=1$ ), 


$$
\left\|B G_{D}+\Gamma \theta\right\|=\left\|\left[\begin{array}{c}
0 \\
\zeta_{\mathrm{b}} \\
\zeta \\
0 \\
0
\end{array}\right] G_{D}+\left[\begin{array}{c}
0 \\
\alpha_{\mathrm{b}} \\
\alpha \\
0 \\
0
\end{array}\right]\right\|
$$

There are now two nonzero entries in $B$ and $\Gamma$, which prevents the determination of a value that exactly cancels the wind-speed disturbance. However, $G_{D}$ can be selected to minimize this norm and thereby attenuate wind-speed disturbance effects.

After the DAC design steps are completed, simulation results of the closed-loop system with this controller, shown in Figure 5-14, now demonstrate stable generator speed and blade pitch. As seen in this figure, generator speed is not regulated as precisely to the $42 \mathrm{rpm}$ desired value as it was with the controls designed from the 1-and 3-state linear models. The figure shows exact regulation to $42 \mathrm{rpm}$ only in the interval between 40 and 50 seconds, corresponding to the control design point. About the design point, the regulated speed deviates from the $42 \mathrm{rpm}$ set point. One factor causing this deviation is the inability to choose a value of $G_{D}$ to exactly cancel wind-speed disturbances.

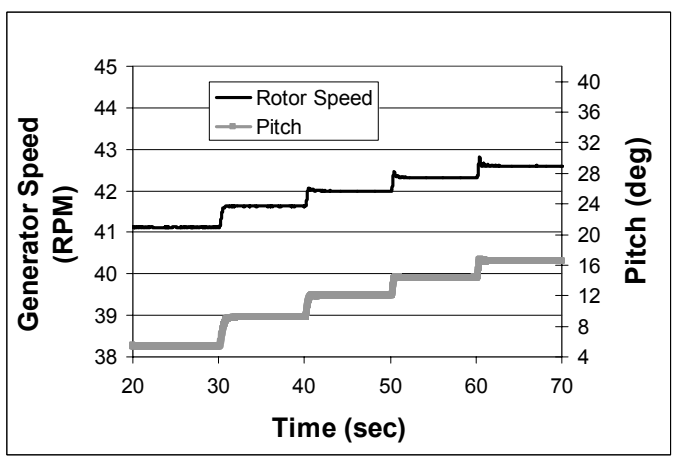

Figure 5-14. Plot of FAST simulated CART generator speed using DAC based controller based on 5state model

Figure 5-15 shows a comparison between actual and estimated winds from FAST. In general, there is a larger deviation between actual and estimated wind speed, in comparison to the DAC designed from the 1- and 3-state models. Again, the variation of control gains with turbine operating point is evident. In addition, the fact that the wind-speed disturbance cannot be cancelled exactly causes additional deviation. In order to improve results, one would have to design controllers at several operating points and switch between controllers based on some type of switching parameter. This "gain scheduling" or "controller switching" problem is not addressed here.

Figure 5-16 shows that this controller stabilizes the response in both FAST and ADAMS. In both simulations, the only flexible modes are the first drive-train torsion, and the blade first flap modes (ADAMS was run with the rigid blade flapping hinge/spring). The effects of additional modes switched on in FAST and ADAMS are discussed below. 


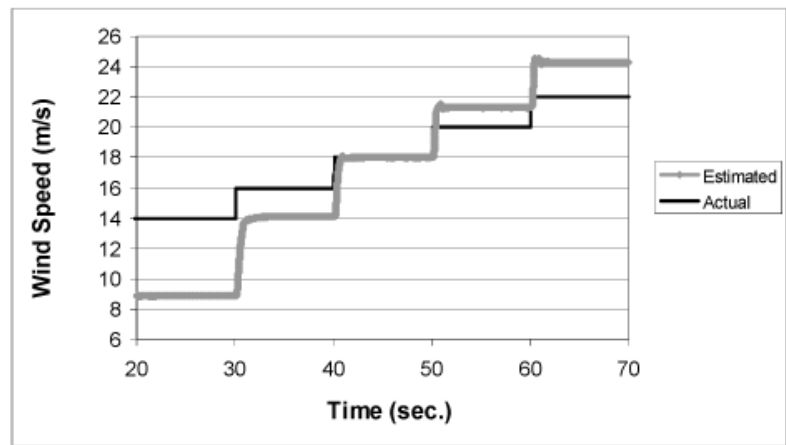

Figure 5-15. Comparison of estimated and actual wind speed as simulated by FAST for the CART using DAC control based on 5-state model

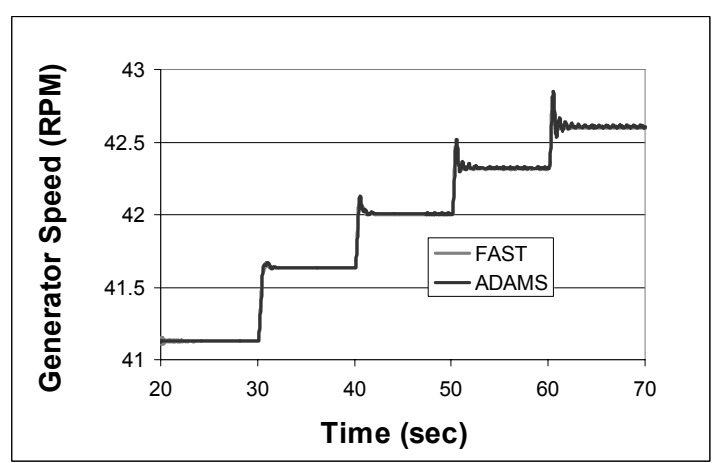

Figure 5-16. Comparisons of ADAMS blade-hinged and FAST simulated CART generator speed using DAC control designed from 5-state controller

\subsubsection{Effects of Unmodeled Modes}

The effect of simulating additional DOF are now investigated. Additional blade modes are turned on in FAST. To model higher blade modes in ADAMS, the blade flapping hinge model is replaced with a blade model that divides the blade into several rigid body parts connected by elastic elements. This means that several elastic blade modes are modeled (several flap and edge modes, as well as torsion modes). The tower is still rigid.

Figure 5-17 shows FAST and ADAMS simulations with these additional blade modes. The FAST simulation indicates a small degree of instability at the lowest wind speed $(14 \mathrm{~m} / \mathrm{s})$, between 20 and 30 seconds. This behavior is not shown in the ADAMS simulation, which probably reflects small modeling differences. Although every effort was made to produce "equivalent" ADAMS and FAST models, the modeling approach in ADAMS is different from that used by FAST. Recall that ADAMS uses a multi-body-dynamics approach, whereas FAST uses an assumed-modes approach. The difference could also be due to differences in the numerical integration schemes used in the two codes. 


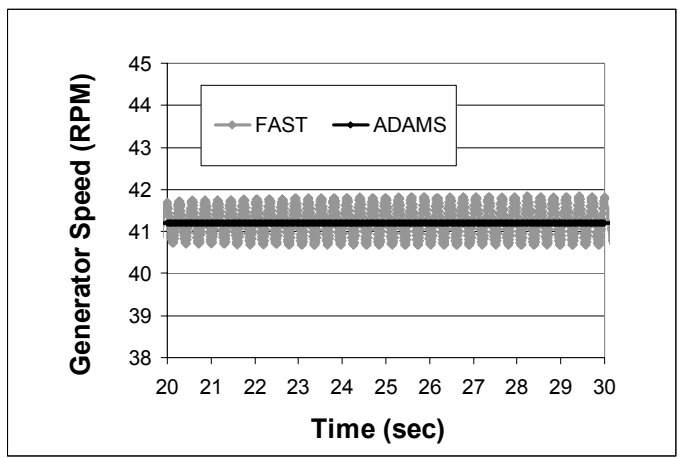

Figure 5-17. Close-up of FAST and ADAMS simulated CART generator speed with 5-state controller with all blade modes in FAST and ADAMS

The unstable oscillation from 20 to 30 seconds seen in the FAST simulation corresponds to the first drive-train torsion mode. This mode is coupled to the blade's first edgewise-bending mode. When the blade first edge mode is switched on during simulation, the damping in the first drive-train torsion mode probably decreases, leading to instability at low wind speeds. The blade edgewise mode receives some aerodynamic damping as a result of coupling with the blade first flap mode, during normal operation. This coupling between the blade flap mode and the blade edge mode occurs for nonzero blade pitch, decreasing as the pitch angle decreases.

For low wind speeds in region 3 (at $14 \mathrm{~m} / \mathrm{s}$ ), the blade pitch is small (about 5 degrees), which causes the damping in the blade first edge mode to decrease, relative to a higher wind speed (such as the control design point). This decreases the damping in the first drive-train torsion mode, leading to the behavior seen in Figure 5-17. This response can be stabilized either by adding structural damping to the blade first edge mode (about $4 \%$ modal damping) or the first drivetrain torsion mode. Figure 5-18 shows the effect of adding damping to the blade first edge mode. In Chapter 6, a method of adding damping with an additional control input is shown. For the remaining simulations in this section (unless otherwise noted), $4 \%$ critical damping in the blade first edgewise mode is included.

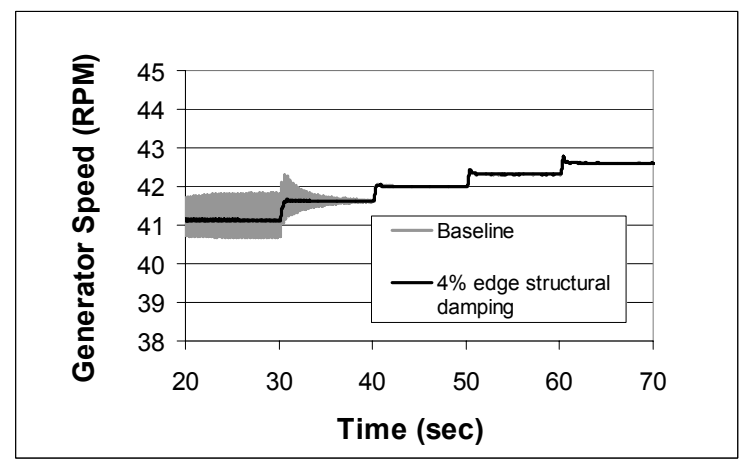

Figure 5-18. FAST simulated CART generator speed with 5-state controller with all blade modes turned on in FAST with undamped and damped blade edge mode

Next, simulations are run with different tower DOF switched on. In ADAMS, the tower is modeled as a series of rigid body parts connected by elastic beam elements, so many modes are modeled. In FAST, different tower modes are switched on systematically. Figure 5-19 shows simulated generator speed using the controller based on the 5-state model, with the tower's first fore-aft mode turned on. A slight fluctuation in generator rotational speed at the tower's first fore-aft natural frequency can be seen in these plots, but the response is stable over the entire range of wind speeds. When the remaining tower modes were switched on during simulation, the results were almost identical to the results obtained with only the tower's first fore-aft mode switched on. Hence, these additional tower modes seem to contribute little to the closed-loop behavior of this system. 


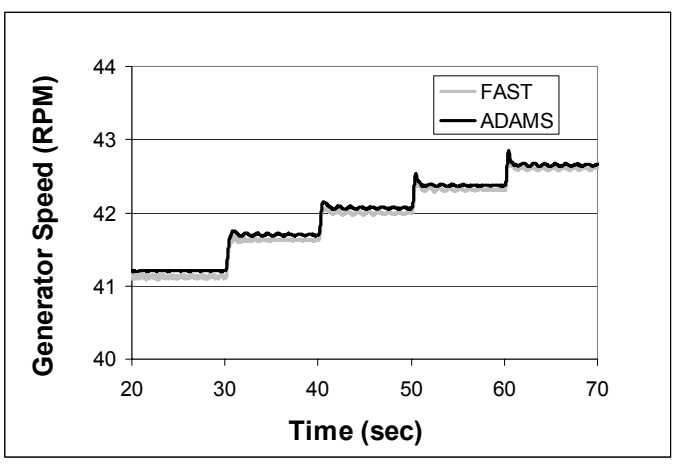

Figure 5-19. ADAMS and FAST simulated CART generator speed with DAC controller designed from 5-state model with tower first fore-aft switched on in FAST, and several tower modes modeled in ADAMS

Figure 5-20 shows a plot of the tower-top fore-aft deflection from FAST and ADAMS. The tower first fore-aft mode is very lightly damped. Although the controlled response is stable without including states in the linear model to describe the tower modes, it would be good to add damping to this mode with the controller. The next linear model contains two extra states to model the tower's first fore-aft mode. We now show the advantage of including this mode in the control design.

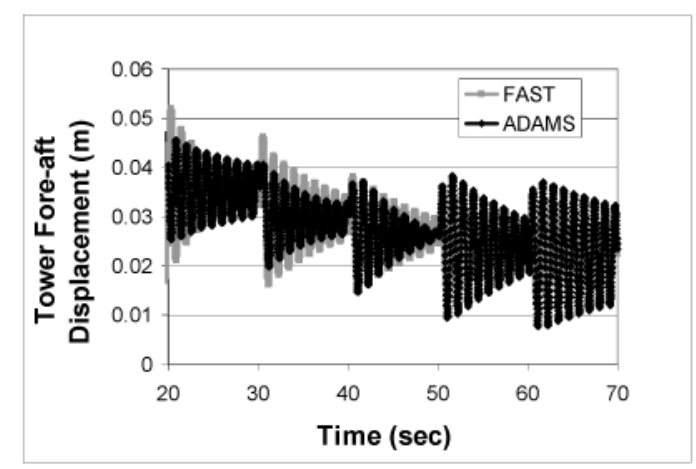
Figure 5-20. ADAMS and FAST simulated CART tower-top fore-aft deflections with DAC controller
designed from 5-state model

\subsection{Results from the 7-State Model}

Two additional states are added to the 5-state model presented in the last section, in order to add the tower first fore-aft mode to the linear model. The derivation of this model is described in detail in Appendix A and yields the following model:

$x_{1}=\frac{1}{2}\left(\delta q_{1}+\delta q_{2}\right)$, perturbed rotor first symmetric flap displacement,

$x_{2}=\frac{1}{2}\left(\delta \dot{q}_{1}+\delta \dot{q}_{2}\right)$, perturbed rotor first symmetric flap velocity,

$x_{3}=\delta \dot{q}_{4}$, perturbed rotor rotational speed,

$x_{4}=\mathrm{K}_{\mathrm{d}}\left(\delta q_{4}-\delta q_{15}\right)$, perturbed drive-train torsional spring force,

$x_{5}=\delta \dot{q}_{15}$, perturbed generator rotational speed, 
$x_{6}=\delta q_{7}$, perturbed tower first mode fore-aft deflection, and

$x_{7}=\delta \dot{q}_{7}$, perturbed tower first mode fore-aft velocity.

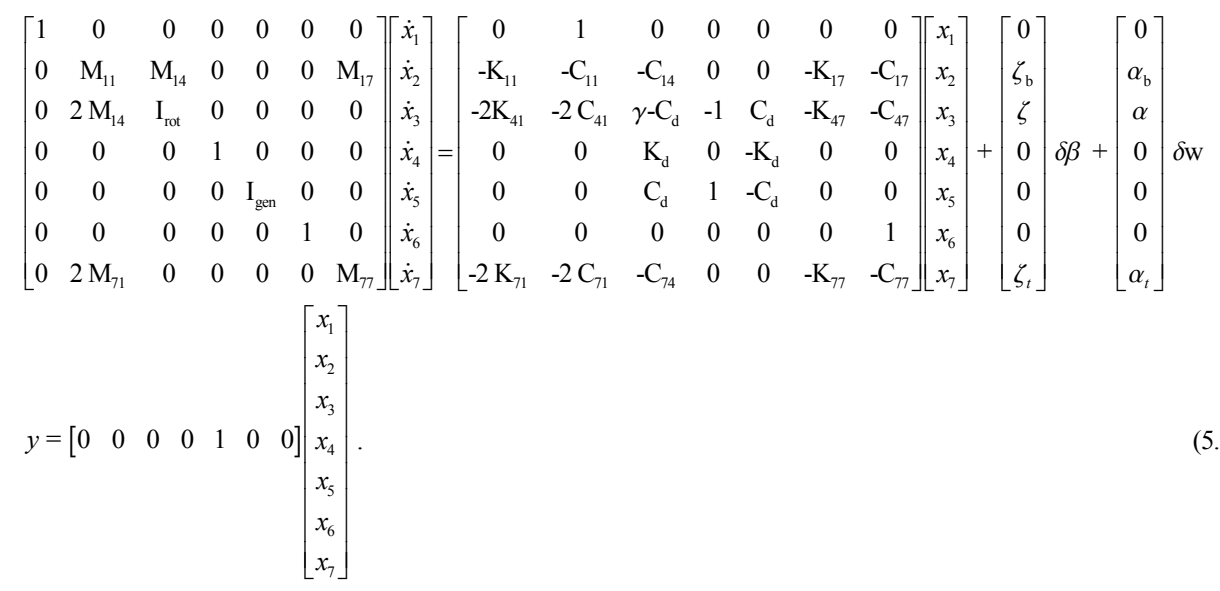

This system again presents some differences when compared with previous models. One should note that now the gain and wind disturbance input matrices have another nonzero entry, in the seventh row. Any perturbation in either collective pitch or wind speed (the uniform component across the rotor disk) perturbs the tower fore-aft bending DOF. This happens because rotor pitch affects the aerodynamic forces acting on the blades, which affects the resulting aerodynamic thrust force. This thrust force, acting at the tower-top, affects the tower fore-aft bending motion. This means that the tower fore-aft bending mode can be controlled using rotor collective pitch. It also means that wind-speed disturbances perturb this mode.

Evaluation of the state matrices for the CART gives the values shown in Appendix D, Section D-2.4. An eigen-analysis of the A matrix for this system gives the open loop poles: $-0.038 \pm 22.6 \mathrm{j} \mathrm{r} / \mathrm{s},-4.4 \pm 13.5 \mathrm{j} \mathrm{r} / \mathrm{s},-0.073 \pm 5.7 \mathrm{j} \mathrm{r} / \mathrm{s}$, and -0.121 $\mathrm{r} / \mathrm{s}$. The first pole pair represents the very lightly damped first drive-train torsion mode. The second pole pair represents the highly damped rotor first symmetric flap mode. The third pole pair represents the lightly damped tower first fore-aft mode. The seventh pole represents the generator speed. As usual, a control objective is to add damping to these flexible modes, and place the generator speed pole further to the left (of its open-loop value) in order to improve speed regulation.

\subsubsection{Addition of Tower-Top Fore-Aft Deflection Measurement}

Problems occur when simulating with DAC from this 7-state model with only a generator speed measurement. The tower first fore-aft mode is only weakly observable using the generator speed measurement, producing poor simulation results. A new measurement must be added: either the tower-top fore-aft deflection, or the tower-top fore-aft acceleration. For now, the tower-top fore-aft deflection will be used. This measurement is expressed as

$$
\left[\begin{array}{l}
\mathrm{y}_{1} \\
\mathrm{y}_{2}
\end{array}\right]=\left[\begin{array}{lllllll}
0 & 0 & 0 & 0 & 1 & 0 & 0 \\
0 & 0 & 0 & 0 & 0 & 1 & 0
\end{array}\right]\left[\begin{array}{l}
\mathrm{x}_{1} \\
\mathrm{x}_{2} \\
\mathrm{x}_{3} \\
\mathrm{x}_{4} \\
\mathrm{x}_{5} \\
\mathrm{x}_{6} \\
\mathrm{x}_{7}
\end{array}\right]
$$


After designing DAC based on this model, we simulate with blade second flap, blade first edge, and tower side-side DOF turned off. Figure 5-21 shows the FAST regulated generator rotational speed and pitch from this simulation, indicating stable response.

Figure 5-22 shows tower top fore-aft deflections using the 5- and 7-state controllers, showing the marked improvement in tower damping added by the DAC controller designed from the 7-state model (which includes 2 extra states to model tower first fore-aft). Recall that the 5-state model ignores tower fore-aft motion, while the 7-state model includes it. After an initial peak in response at the application of each step change in wind speed, the deflections are highly damped. Including these 2 extra states in the linear model allows us to place the poles corresponding to the tower's first fore-aft mode, to add significant damping. These improved results are obtained at the expense of adding an extra measurement: tower top fore-aft deflection. In the next section, results using tower-top acceleration are shown.

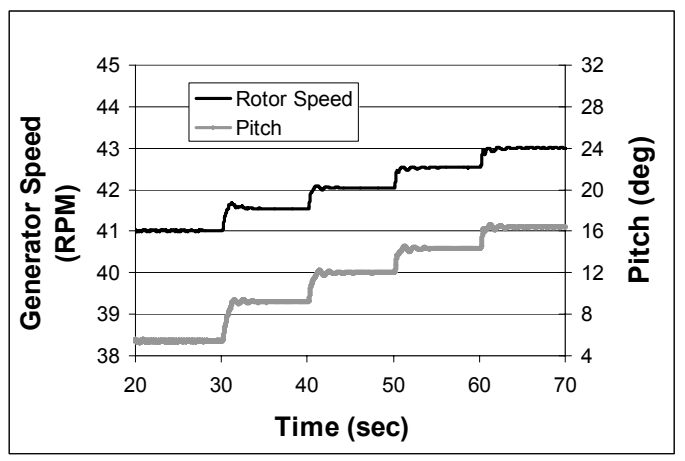

Figure 5-21. Plot of FAST simulated CART tower-top deflection using DAC based on 7-state model with generator speed and tower-top fore-aft deflection measurements

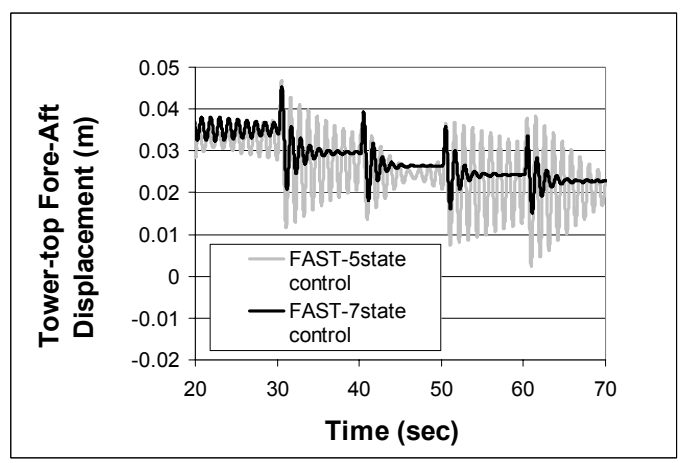

Figure 5-22. Comparing FAST simulated CART tower-top fore-aft deflections using both the 5- and 7state controller 


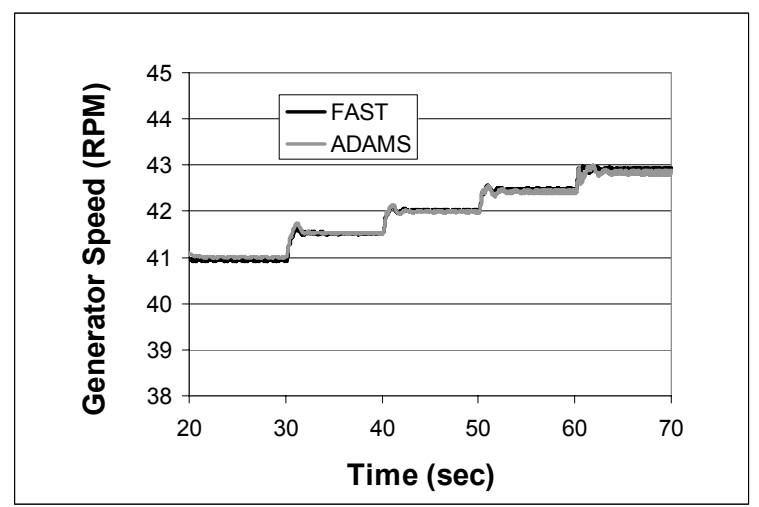

\section{Figure 5-23. Comparisons of ADAMS (flex-blade) and FAST simulated CART generator speed using DAC control designed from 7-state controller}

Further simulations using FAST and ADAMS are performed, with additional modes not included in the previous simulations. Figure 5-23 shows the simulated generator speed using FAST and ADAMS with additional blade and tower modes. It is evident that the results are stable over the entire range of wind speeds. In ADAMS, the rotor teeter DOF was also switched on. Another difference between this ADAMS model and the FAST model is that now ADAMS uses a beam to model flexibility in the low-speed shaft. (In previous sections, the drive-train torsion was modeled in ADAMS with an equivalent torsional spring. Now it is modeled with a beam, which allows shaft bending.) This beam allows flexibility in both bending and torsion. FAST assumes rigid drive-train bending. Figure 5-24 shows good agreement between FAST and ADAMS for simulated tower-top fore-aft deflection. Furthermore, even with rotor teeter and drive train bending, the ADAMS simulation is stable.

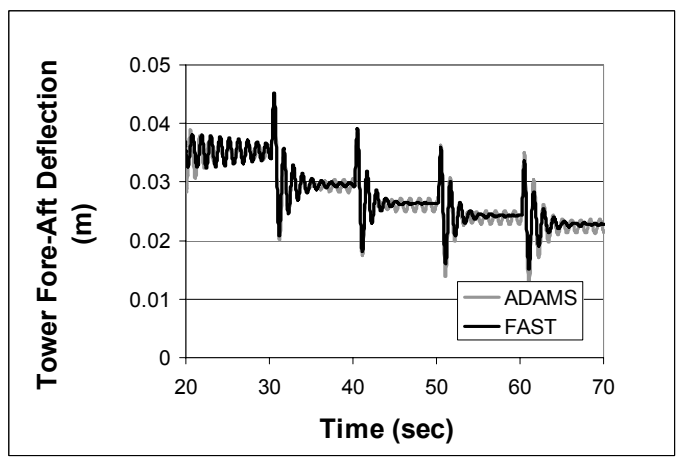

Figure 5-24. ADAMS and FAST simulated CART tower-top fore-aft deflections with DAC controller designed from 7-state model

For the test cases considered, no additional flexible modes become unstable during closed-loop operation, using the DAC controllers based on the 7-state linear model. The linear model with 7 states seems to be adequate for design of these control systems. ADAMS includes many additional flexible modes for the blades and tower and seemed to remain stable during control.

The use of tower-top acceleration as the measured variable, instead of tower-top deflection is now explored.

\subsubsection{Measuring Tower-Top Acceleration}

Recall from state-space Equation 2.4 that

$$
\underline{y}=C \underline{x}+D \underline{u}+E \underline{u}_{D}
$$


In this expression for $\underline{y}$, we included the effect of $\underline{u}$ and added the effect of the disturbance input $\underline{u}_{D}$ on the measured control signal $\underline{y}$.

When deflection is measured, then $D$ and $E$ are zero in Equation 5.6, since the measurement is just a linear combination of the turbine states. From Equation 5.5, we can see that the expression for tower-top fore-aft acceleration is

$$
\begin{aligned}
& \dot{\mathrm{x}}_{7}=\frac{1}{\mathrm{M}_{77}}\left(-2 \mathrm{M}_{71} \dot{\mathrm{x}}_{2}-2 \mathrm{~K}_{71} \mathrm{x}_{1}-2 \mathrm{C}_{71} \mathrm{x}_{2}-\mathrm{C}_{74} \mathrm{x}_{3}\right. \\
& \left.-\mathrm{K}_{77} \mathrm{x}_{6}-\mathrm{C}_{77} \mathrm{x}_{7}+\zeta_{t} \delta \beta+\alpha_{t} \delta \mathrm{w}\right)
\end{aligned}
$$

From Equation 5-7, the measurement is a function of the states, the rotor collective pitch input, and the disturbance input. Thus, $D$ and $E$ are both nonzero. Because of these extra terms in $\underline{y}$, the $\overline{\mathrm{L}}$ matrix in $T_{c}(s)=\overline{\mathrm{G}}(\mathrm{sI}-\overline{\mathrm{L}})^{-1} \bar{K}($ the equivalent transfer function) now contains extra terms. Please see Appendix C (Section C-1.6.3) for details.

The design steps for DAC based on the 7-state model, using measured tower-top fore-aft acceleration rather than deflection, are exactly the same. Figure 5-25 shows simulated tower-top fore-aft deflection when tower-top acceleration is measured instead of deflection. The results are very similar to measuring tower-top deflection, as can be seen by comparing Figures 5-24 and 5-25. We will continue to measure tower-top deflection, just to reduce complexity in the matrices and equations, since it seems that measuring tower-top acceleration does not improve the results.

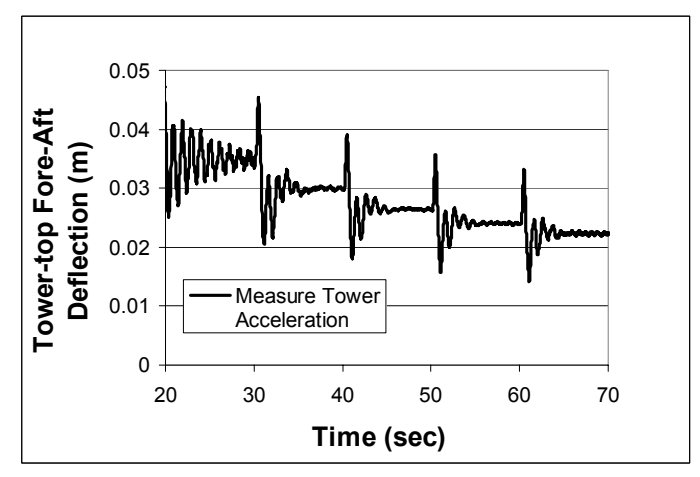

Figure 5-25. FAST simulated CART tower-top fore-aft deflections with DAC controller designed from 7-state model, when measuring tower-top acceleration

\subsection{Other Simulation Results}

In order to test the robustness of the designed controller, simulations driven by turbulent winds were performed. It is important to test the controlled nonlinear turbine when excited by these un-modeled effects. Turbulent winds are generated using the SNLWIND3D code (Kelley 1992). Simulations are run with FAST and ADAMS for 300 seconds of turbine operation. We use the controllers already designed from the 5- and 7-state models (measuring tower-top deflection). 
Results of interest include these:

- System stability when excited by turbulent wind inflow, and

- Ability of the DAC controller to estimate turbulent winds.

Figure 5-26 shows that the FAST- and ADAMS-simulated generator speed are in good agreement. In ADAMS, the blades and tower are modeled by connecting a series of rigid bodies by elastic beam elements. This modeling approach captures the effects of higher blade and tower modes not present in FAST. It is evident that these higher blade and tower modes remain stable with this controller. In the figure, the generator speed is closely regulated to the 42-rpm set point, deviating by approximately $\pm 1.5 \mathrm{rpm}$.

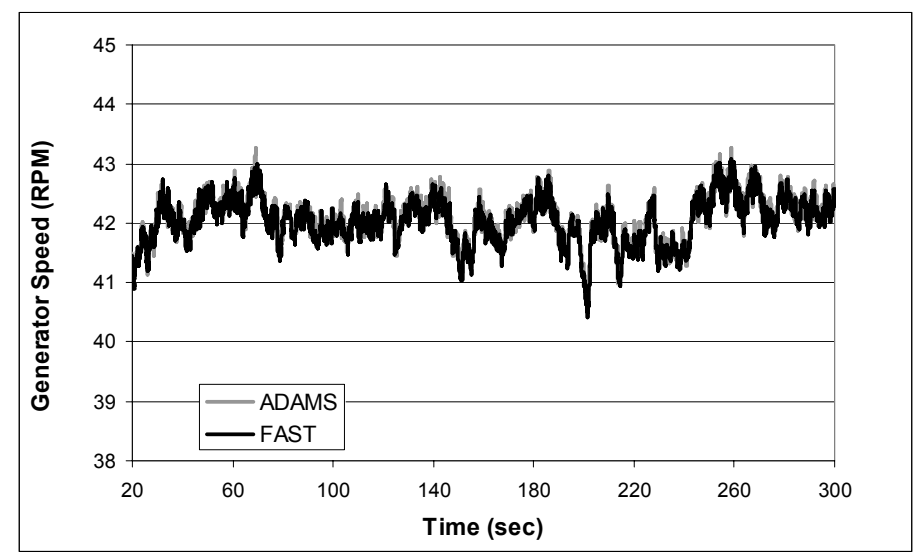

Figure 5-26. Comparisons of ADAMS blade-flex and FAST simulated CART generator speed using DAC control designed from 5-state controller with turbulent inflow

Figure 5-27 shows the actual and estimated hub-height wind speed normal to the rotor disk from FAST for this case. In general, the estimator does a fair job of estimating the wind speed. When the wind speed is above $18 \mathrm{~m} / \mathrm{s}$, the model estimates higher wind speeds than actual values, while estimating lower than actual values for wind speeds below 18 $\mathrm{m} / \mathrm{s}$. Large differences in the actual and estimated wind speeds are evident when the wind speed drops down below 15 $\mathrm{m} / \mathrm{s}$. This is because the turbine operating point deviates significantly from the control design point. These results could be improved with the use of gain scheduling, or switching between different controllers as the wind speeds change.

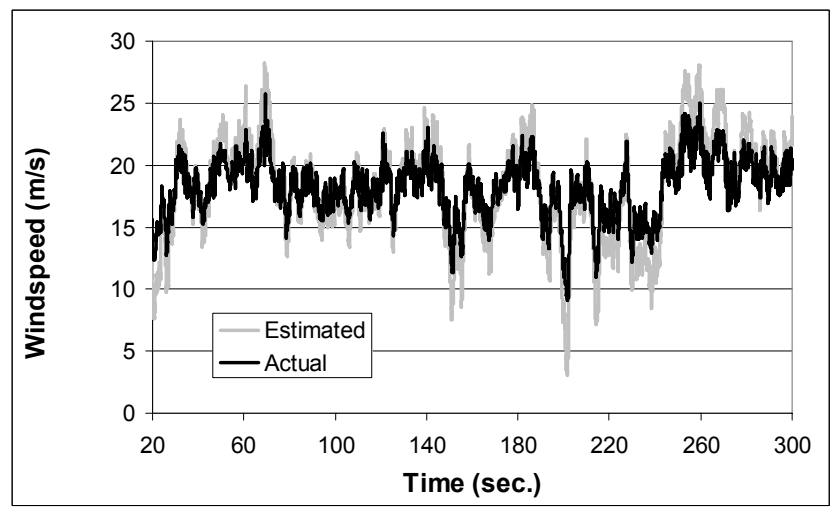

Figure 5-27. Comparison of FAST estimated and actual wind speed using DAC control based on 5state linear model, with turbulent inflow 
Figure 5-28 shows FAST simulated tower-top fore-aft deflection, comparing results from the DAC designed from the 5state model with results from the DAC designed from the 7-state model. The reduction in motion when the DAC is designed from the 7-state model is readily apparent. Figure 5-29 shows the FAST- and ADAMS-predicted tower-top fore-aft displacement, using the DAC designed from the 7-state controller. The ADAMS response is very similar to the FAST simulated response, showing good agreement. This also shows that additional flexible modes remain stable in ADAMS using this controller.

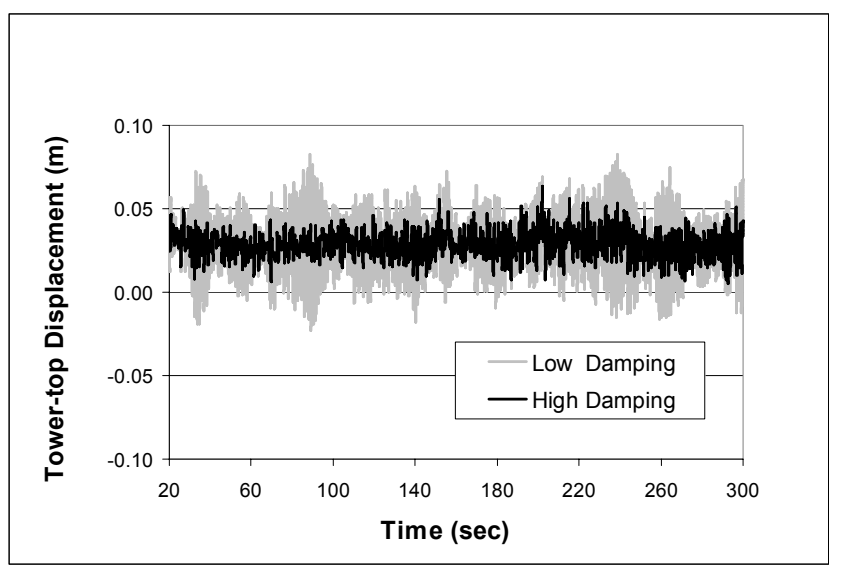

Figure 5-28. FAST simulated CART tower-top fore-aft deflection with turbulent inflow using DAC control designed from 5- and 7-state models

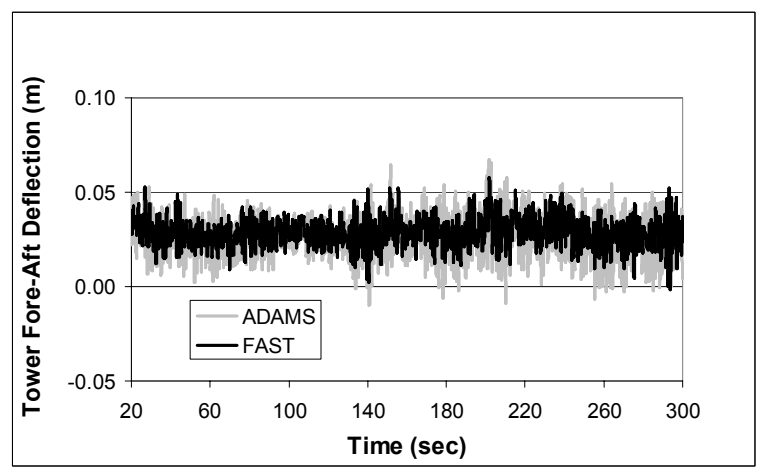

\section{Figure 5-29. Comparisons of ADAMS blade-flex and FAST simulated CART tower-top fore-aft deflection using DAC control designed from 7-state controller with turbulent inflow}

Figure 5-30 shows the effects of pole placement on pitch rates from FAST, for the controller designed from the 7-state linear model. In the high damped case, the real parts of the poles corresponding to the tower first fore-aft mode are placed at $-2 \mathrm{r} / \mathrm{s}$. For the low-damped case, they are placed at $-0.5 \mathrm{r} / \mathrm{s}$. As expected, the high-damped case results in higher pitch rates; the magnitude of the highest pitch rate is approximately 21 degrees/second. 


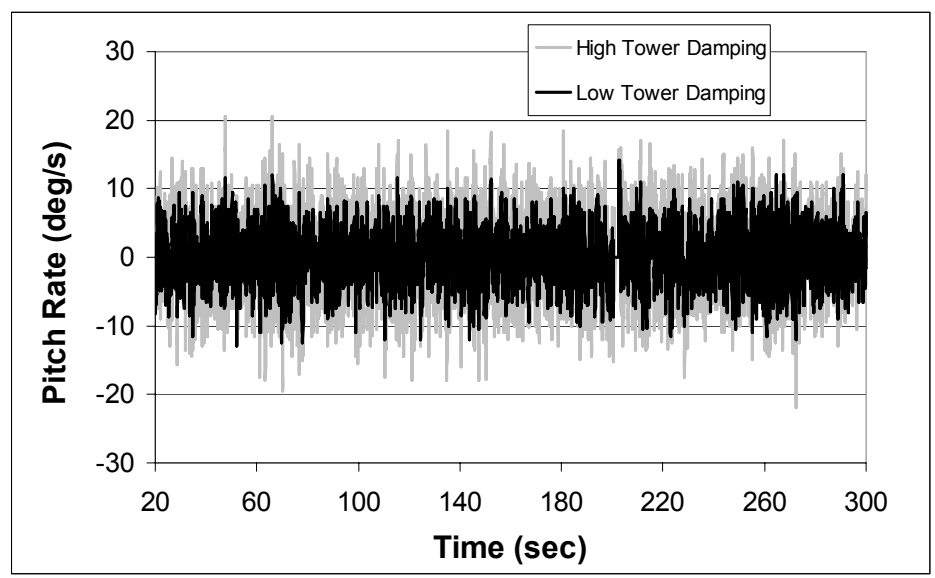

Figure 5-30. FAST-simulated pitch rates using DAC control designed from 7-state model with turbulent inflow, for high and low tower damping

It seems as if designing the controller to add damping to this mode can significantly reduce the tower base bending moment excursions. To perform this control, either tower-top deflection or acceleration must be measured in addition to generator rotational speed. Adding damping to this mode results in higher pitch rates, however.

\subsection{Chapter Conclusions}

In this chapter, DAC control design results were shown, based on a progression of linear models, starting with a simple 1-state linear model and progressing up to a model containing 7 states. The use of DAC controls based on the 1- and 3state linear models resulted in very precise regulation of speed over a wide range, using stepwise changes in wind to test the models.

The DAC controller designed from the 1-state model resulted in unstable response when the first drive-train torsion mode was switched on in FAST and ADAMS. This necessitated the addition of states to describe this mode, resulting in a 3-state linear model. The DAC controller designed from the 3-state linear model stabilized this response when the first drive-train torsion mode was switched on during simulation. When the blade first flap mode was switched on, however, the results became unstable. As shown, the rotor first symmetric flap mode is highly coupled with drive-train torsion. Controllers designed from models which neglect these modes can destabilize the system, when these modes are switched on during simulation. States were added to the linear model to describe the rotor first symmetric flap mode, resulting in a linear model with 5 states. DAC control designed from this 5-state model resulted in stable behavior, with the blade's first flap mode switched on during simulation. Further tests were performed in FAST with all modes switched on and in ADAMS, with multiple blade and tower modes. Tests using step winds with these comprehensive models showed continued stable behavior; no additional modes became unstable using the DAC controller designed from the 5-state linear model.

In terms of control model complexity, we concluded that a DAC controller designed from the 5 -state model is adequate to meet the stated control design objectives. It was also shown that as the drive-train torsional and blade flapwise stiffness is increased, simpler linear models can be used. For a very stiff turbine (with stiff blades and drive train), the DAC designed from the 1-state linear model would probably be sufficient to maintain stable closed-loop behavior. For the CART, however, the linear model with 5 states was needed in order to design controls, to maintain stable closed-loop behavior.

It was also shown that state estimation was successfully used to reduce the required number of turbine measurements. For controls designed from the 1-, 3-, and 5-state models, successful control performance was obtained by measuring only the generator speed. When the tower first fore-aft mode was added to the linear model, tower-top fore-aft deflection or acceleration was also needed. 
The effect of the nonlinear behavior of the controlled turbine was also shown. It was found that the nonlinear aerodynamics of the turbine caused variations of the control and disturbance input gains with wind speed and pitch angle. The controller was designed using one set of gains appropriate for a particular wind speed and blade pitch angle. These DAC controls also provided an estimate of the uniform component of wind speed over the rotor disk. As the turbine's operating point deviated significantly from this design point, the wind-speed estimator became less accurate. Designing the controller at the chosen wind speed and pitch resulted in stable behavior over the range of wind speeds tested. It was found that the use of gain scheduling (or controller switching) was not necessary to design controls, which maintain stable behavior for the CART. These techniques could improve the wind-speed estimation, however.

The speed regulation performance of these controls was examined. It was noted that DAC controls designed from the 1and 3-state models gave precise speed regulation over a broad range of wind speeds in region 3. As additional states were added to the linear model to describe additional flexible modes of the turbine, the speed regulation was less precise. This was a result of extra terms contained in the pitch input and disturbance input matrices, corresponding to these extra states. There is a trade-off here in the complexity of the linear model and resulting controller, and the precision with which turbine speed is regulated. For a very stiff turbine, controls could probably be designed just using the 1- or 3-state linear models. Speed regulation with such a controller would be very precise. As the flexibility of the turbine increases, and the need for additional states in the linear model increases, speed regulation becomes less precise.

In this control, a single control input has been used. We now investigate the addition of another control input, generator torque control. The objective of this control input is to remove some of the task of enhancing damping of the first drivetrain torsion mode from the pitch control systems. In the next chapter, we investigate this control. 


\section{Chapter 6. Addition of Generator Torque Control}

\section{Introduction}

Generator torque can also be used as a control input to add damping to the drive-train torsional mode. Simulated closedloop response using this control, as demonstrated below, reduces the requirements on the pitch control system for performing this damping function.

\subsection{DAC Review-Further Issues}

\subsubsection{Instability at Low Wind Speed}

Section 5.4.2 examined the effects of un-modeled modes when using the DAC designed from the 5-state model. When the blade's first edgewise-bending mode was enabled, the results became unstable at the low-wind-speed end of region 3 (at $14 \mathrm{~m} / \mathrm{s}$ ). Examination of the cyclic content in close detail showed instability of the first drive-train torsion mode.

The DAC pitch control system had been designed to add sufficient damping to the first drive-train torsion mode at the control design point $(18 \mathrm{~m} / \mathrm{s}, 12 \mathrm{deg}$. pitch). The controller was not performing as designed at the low-wind-speed end of region 3, because the turbine operating point deviates significantly from the control design point. A possible solution is to redesign the control system at $14 \mathrm{~m} / \mathrm{s}$.

\subsubsection{Control Design at $14 \mathrm{~m} / \mathrm{s}$}

The DAC based on the 5-state model (Section 5.4) is designed at this point:

$$
\begin{aligned}
& \mathrm{w}_{0}=14 \mathrm{~m} / \mathrm{s}, \\
& \Omega_{0}=42 \mathrm{RPM}, \text { and } \\
& \beta_{0}=5.3 \mathrm{deg} .
\end{aligned}
$$

Table 6-1 shows the values for the pitch input gain and the disturbance input gain for the 14 and $18 \mathrm{~m} / \mathrm{s}$ control design points, respectively. As the wind speed changes, these gains also vary, with the greatest change occurring in B. These same trends are observed in the rotor aerodynamic torque versus pitch and wind-speed curves (Figure 5-1).

Table 6-1. Variation of Pitch and Wind-Speed Gains with Design Point

\begin{tabular}{|l|l|l|l|l|}
\hline $\begin{array}{l}\text { Control Design } \\
\text { Point }\end{array}$ & $\begin{array}{l}\text { Wind speed } \\
\text { (m/s) }\end{array}$ & $\begin{array}{l}\text { Pitch } \\
\text { (deg.) }\end{array}$ & B & $\Gamma$ \\
\hline 1 & 14 & 5.25 & -1.554 & 0.063 \\
\hline 2 & 18 & 12.0 & -2.882 & 0.066 \\
\hline
\end{tabular}

Figure 6-1 shows the simulated generator speed after designing DAC at the two design points in Table 6-1. Improved response is seen at the low-wind-speed end of region 3 (between 20 and 30 seconds, $14 \mathrm{~m} / \mathrm{s}$ wind speed) with the DAC designed at point 1 , although the response is just marginally stable. It is clear that designing at this alternative turbine operating point does not completely solve the stability issue. This could be because of the issue of controllability using rotor collective pitch at the low-wind-speed end of region 3, which we investigate below. 


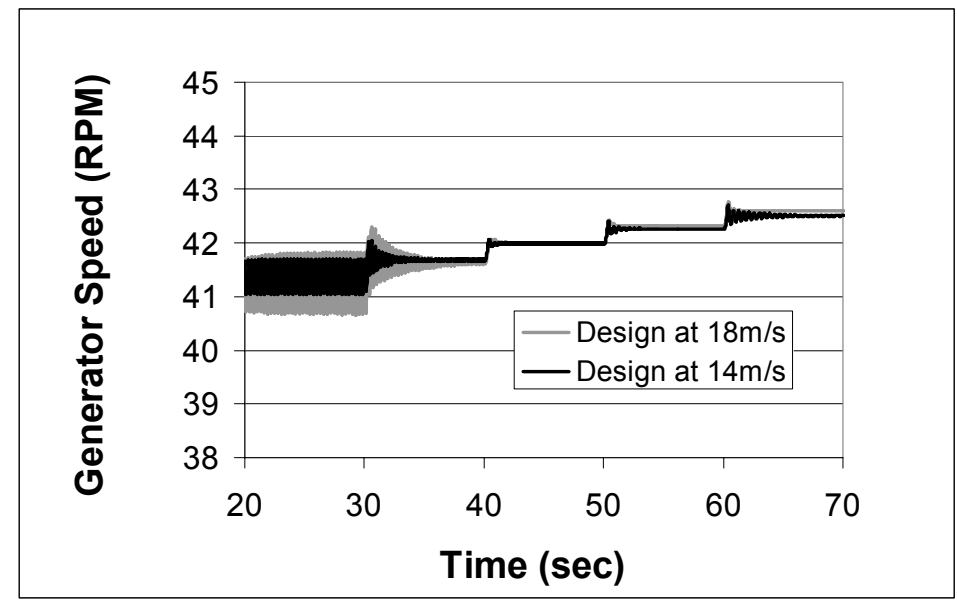

Figure 6-1. Plot of FAST simulated CART generator speed using DAC controller based on 5state model, designed at $14 \mathrm{~m} / \mathrm{s}$ and $18 \mathrm{~m} / \mathrm{s}$ design points

\subsubsection{Controllability}

Controllability in region 3 using rotor collective pitch is another issue to be examined in more detail. Detailed expressions for controllability of the 3- and 5-state models are given in Appendix A, Section A-2. For the 3-state model (described in Section 2.3.4), the determinant of the controllability matrix is

$$
\frac{\mathrm{K}_{\mathrm{d}}{ }^{2} \zeta^{3}}{\mathrm{I}_{\mathrm{gen}}}
$$

This gives us a means of examining the effect of various parameters on controllability. If this determinant becomes zero, then the system is uncontrollable. From this expression, the controllability for this 3-state model depends upon the drive-train torsional spring stiffness $\mathrm{K}_{\mathrm{d}}$, the pitch input distribution gain $\zeta$, and the generator inertia $\mathrm{I}_{\mathrm{gen}} \cdot \mathrm{K}_{\mathrm{d}}$ and $I_{\text {gen }}$ are constant. From Table 6-1, we see that, as wind speed decreases, the magnitude of $\zeta$ decreases. This can also be observed from the slope of the aerodynamic torque versus pitch angle plots shown in Chapter 5, Figure 5-1. Thus controllability of the first drive-train torsion mode decreases as the wind speed in region 3 decreases.

What is really needed is another method of control to add damping to the first drive-train torsion mode, one in which gains are not sensitive to changes in wind speed. This can be accomplished using generator torque as a control actuator, as shown in the following sections.

\subsection{Addition of Generator Torque Control}

An added benefit of this approach is the reduction in demand placed on the rotor collective pitch control, which is seen as a reduction in pitch rates. Next, we discuss the control objectives for generator torque.

\subsubsection{Control Objectives}

The primary goal in region 3 is to maintain rated power. This is done by maintaining constant generator torque (by commanding constant generator torque through the power electronics) and using rotor collective pitch to regulate aerodynamic torque, which regulates speed. If generator torque and generator speed are held constant, then generator power will also be constant. 
If generator torque is allowed to vary, there will be increased variation in generator power. Only small variations in generator torque can be allowed. The only function of the generator torque control is to add damping to the first drivetrain torsion mode. Generator torque is not used to regulate speed or perform any other function. In this chapter, the generator is used as a control actuator to add damping to the first drive-train torsion mode. It is hoped that this will take some of the requirement away from the blade pitch control system, thus reducing pitch rates while allowing only small perturbations in generator torque.

In the next section, we look at the design of such a control system.

\subsubsection{Control Philosophy}

The objectives of the pitch control system are to regulate generator speed and enhance damping in the tower first fore-aft mode (later using the 7-state model). We remove the requirement of enhancing damping in the first drive-train torsion mode from the rotor collective pitch control system and assign that task to the generator torque control system.

Two separate controllers are designed: the generator torque controller and the rotor collective pitch controller. The generator torque controller design is based on a reduced state-space model containing only the states needed to describe the first drive-train torsion mode. The poles of these states (pending controllability) are placed to add damping to this mode.

The rotor collective pitch controller will be designed using the 5- and 7-state linear models described in Chapter 5. The poles in this control design are placed to regulate speed and enhance damping in the tower first fore-aft mode. Even though the rotor collective pitch controller accounts for the states involved with the first drive-train torsion mode, the poles for this mode are placed at their open-loop values. This removes the task of adding damping to the first drive-train torsion mode from the pitch controller.

\subsubsection{Control Design}

A generator torque feedback law is formed based only on these states:

$\mathrm{x}_{1}=\delta \dot{q}_{4}$, perturbed rotor rotational speed

$\mathrm{x}_{2}=\mathrm{K}_{\mathrm{d}}\left(\delta q_{4}-\delta q_{15}\right)$, perturbed drive-train torsional spring force

$\mathrm{x}_{3}=\delta \dot{q}_{15}$, perturbed generator rotational speed.

These are the states needed to describe the first drive-train torsion mode.

From Appendix A (Section A-1.2), it is shown that the linear model for this system, with generator torque perturbation as the only control input (we ignore the pitch control input and wind disturbance) is as follows:

$$
\begin{aligned}
& {\left[\begin{array}{l}
\dot{\mathrm{x}}_{1} \\
\dot{\mathrm{x}}_{2} \\
\dot{\mathrm{x}}_{3}
\end{array}\right]=\left[\begin{array}{ccc}
\frac{\left(\gamma-\mathrm{C}_{\mathrm{d}}\right)}{\mathrm{I}_{\mathrm{rot}}} & \frac{-1}{\mathrm{I}_{\mathrm{rot}}} & \frac{\mathrm{C}_{\mathrm{d}}}{\mathrm{I}_{\mathrm{rot}}} \\
\mathrm{K}_{\mathrm{d}} & 0 & -\mathrm{K}_{\mathrm{d}} \\
\frac{\mathrm{C}_{\mathrm{d}}}{\mathrm{I}_{\text {gen }}} & \frac{1}{\mathrm{I}_{\text {gen }}} & \frac{-\mathrm{C}_{\mathrm{d}}}{\mathrm{I}_{\text {gen }}}
\end{array}\right]\left[\begin{array}{c}
\mathrm{x}_{1} \\
\mathrm{x}_{2} \\
\mathrm{x}_{3}
\end{array}\right]+\left[\begin{array}{c}
0 \\
0 \\
\frac{-1}{\mathrm{I}_{\text {gen }}}
\end{array}\right] \delta T_{\text {gen }}} \\
& \mathrm{y}=\left[\begin{array}{lll}
0 & 0 & 1
\end{array}\right]\left[\begin{array}{c}
\mathrm{x}_{1} \\
\mathrm{x}_{2} \\
\mathrm{x}_{3}
\end{array}\right] .
\end{aligned}
$$


This system is much like the 3-state model shown in Section 5.3, except of course that now the control input is $\delta T_{\text {gen }}$. Notice the control input gain $\frac{-1}{I_{\text {gen }}}$, in the third row of B. This shows us that control input enters the system through the generator speed state $x_{3}$. This gain is constant (we assume that $I_{\text {gen }}$ is constant) and does not change with wind speed or pitch. It is again assumed that generator speed is the only measurement in this model.

Wind-speed disturbances are ignored in this model. State estimation is performed of only the plant states. The only goal for this controller is to enhance damping of the first drive-train torsion mode. These control objectives can be achieved by appropriate pole placement using the design steps shown in Chapter 4.

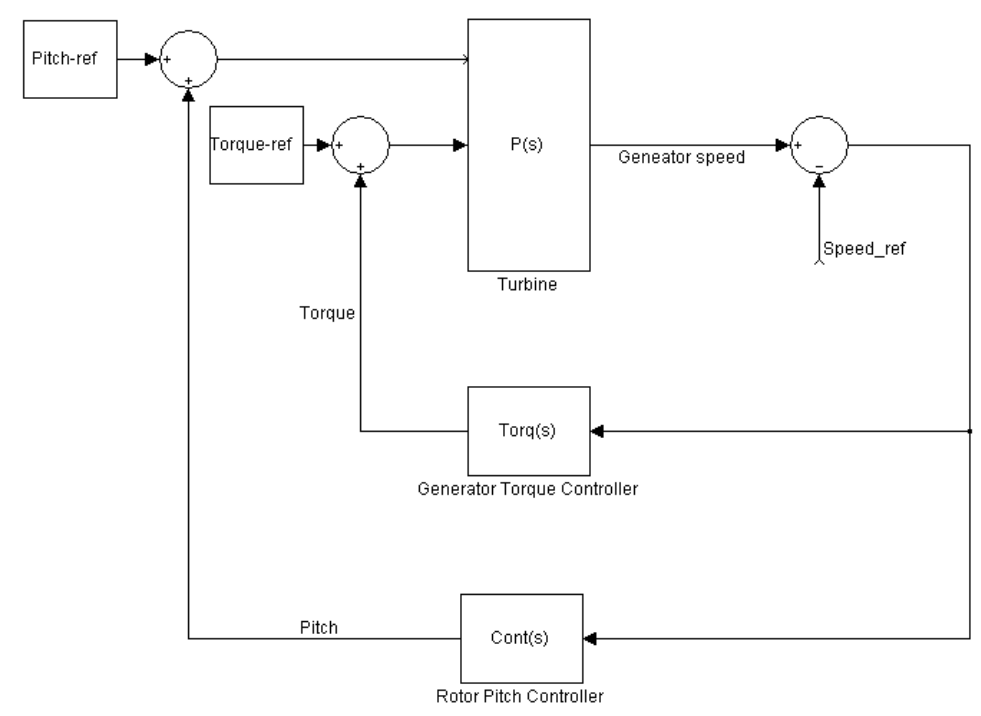

Figure 6-2. Control diagram

The state matrices in Equation 6.1 for the CART at the control design point,

$$
\begin{aligned}
& \mathrm{w}_{0}=18 \mathrm{~m} / \mathrm{s}, \\
& \Omega_{0}=42 \mathrm{RPM}, \\
& \beta_{0}=12 \mathrm{deg} .
\end{aligned}
$$

are summarized in Table 6-2. 
Table 6-2. A and B Matrices for the CART 3-State Linear Model

\begin{tabular}{|l|l|l|l|}
\hline A & \multicolumn{3}{|l|}{ B } \\
\hline-.145 & $-3.108 \times 10^{-6}$ & 0. & 0. \\
\hline $2.691 \times 10^{7}$ & 0. & $-2.691 \times 10^{7}$ & 0. \\
\hline 0. & 0.000016 & 0. & -0.000016 \\
\hline
\end{tabular}

The eigenvalues of the A matrix here are the same as those reported in Section 4.1.3. The only difference between this and the previous 3-state model is the matrix B. Placing the poles at $-2 \pm 22.5 \mathrm{i} \mathrm{r} / \mathrm{s}$, and $-0.121 \mathrm{r} / \mathrm{s}$ results in significant damping in the first drive-train torsion mode and maintains the speed pole at the open-loop value. This prevents the generator torque controller from attempting to regulate generator speed in response to wind-speed fluctuations. We design the pitch controller to perform this task. Design details for the torque controller are shown in Appendix D.

The rotor collective pitch control system is first designed based on the 5-state model, described in Section 5.4.1. The poles for this controller are placed at $-0.033 \pm 22.6 \mathrm{i} \mathrm{r} / \mathrm{s},-4.4 \pm 13.3 \mathrm{i} \mathrm{r} / \mathrm{s}$, and $-2 \mathrm{r} / \mathrm{s}$. Notice that the poles corresponding to the first drive-train torsion mode are placed at their open-loop values. This removes the task of adding damping to this mode from the pitch controller. The other poles are placed at their previous values. Design details are given in Appendix D.

Step test winds are used to excite the FAST models containing these controllers. Figure 6-3 shows these results in comparison to the old results when using all pitch control. Notice that the generator speed is stable over the entire range of wind speeds. This is a direct result of the improved controllability of the first drive-train torsion mode, using generator torque control instead of pitch control. Figure 6-4 shows the blade pitch for the DAC control case when drivetrain damping is added by rotor collective pitch, in comparison to using generator torque control. There is a big reduction in cyclic pitch activity for the generator torque control case at the low-wind-speed end of region 3 . This results in reduced pitch rates, as shown in Figure 6-5. Figure 6-5 also shows realistic pitch rate limits for this machine. The pitch rates for the drive-train damping pitch case are high at the low-wind-speed end of region 3 , due to the drive train instability. Figure 6-6 shows the generator torque for this same wind-speed range, exhibiting stable behavior. The generator torque shows a well-damped response after application of the step change in wind speed (at 30 seconds), exhibiting good controllability of the first drive-train torsion mode.

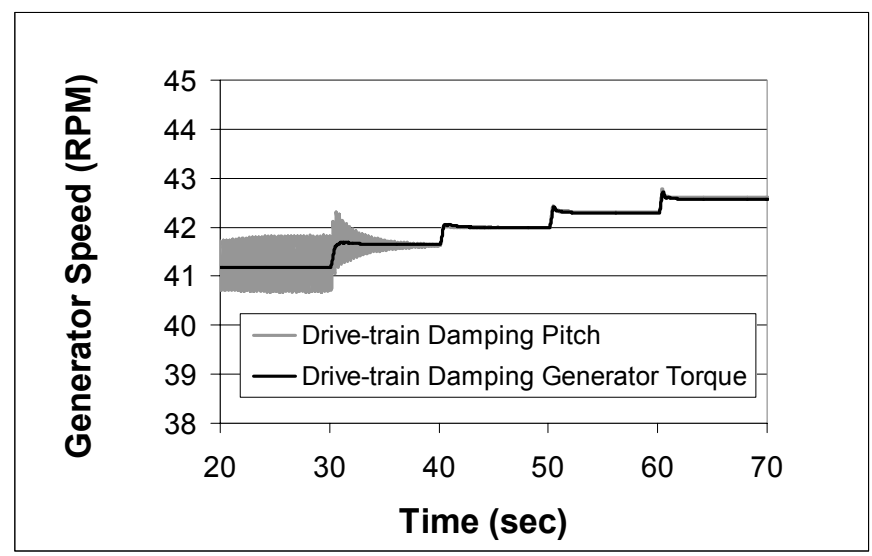

Figure 6-3. Plot of FAST simulated CART generator speed using DAC controller based on 5-state model, with drive-train damping from two different controllers 


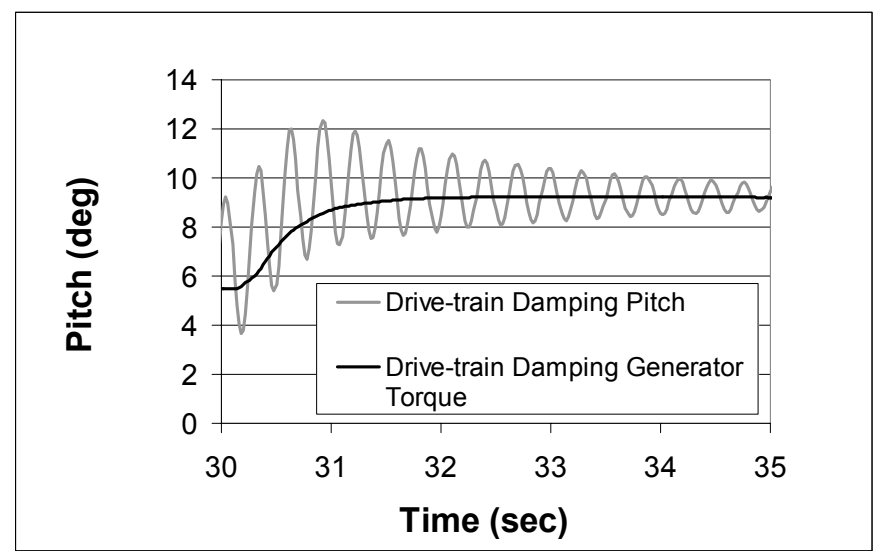

Figure 6-4. Close-up of FAST simulated CART generator speed using DAC controller based on 5-state model, with drive-train damping from two different controllers

The rotor collective pitch controller can also be designed from the 7-state model, allowing damping to be added to the first tower-fore-aft mode. Recall that the 7-state model, described in Section 5.4, used pitch as the control input, instead of generator torque. The design of the generator torque control remains the same, based on the 3-state model shown previously. For the pitch control, the poles of the first drive-train torsion mode are again placed at their open-loop values.

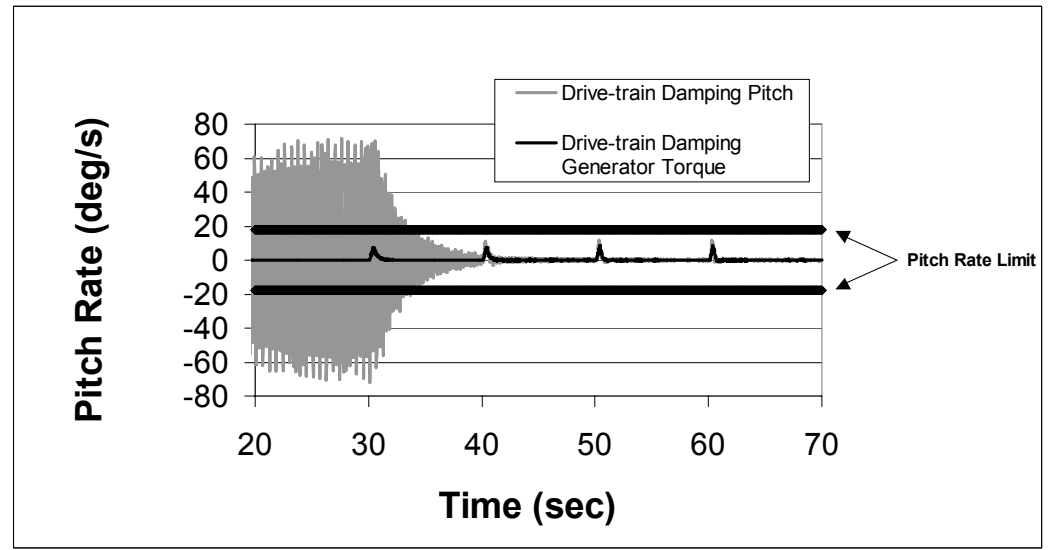

Figure 6-5. Plot of FAST simulated CART pitch rate using DAC controller based on 5-state model, with drive-train damping from pitch control compared to drive-train damping from generator torque control 


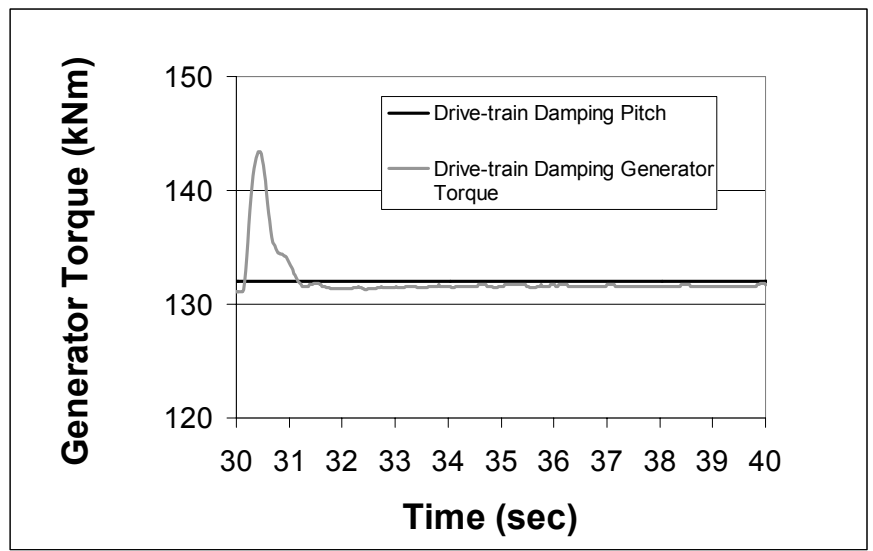

Figure 6-6. Plot of FAST simulated CART generator torque using DAC controller based on 5state model, with drive-train damping from pitch control compared with drive-train damping from generator torque control

Figure 6-7 shows the regulated generator speed from two controllers; one is all-rotor collective pitch control, and the other is generator torque control to add damping to the first drive-train torsion mode. The pitch control is based on the 7state model. Improvement is again seen in regulated speed at the low-wind-speed end of region 3 (between 20 and 30 seconds), due to stability with the generator torque control. Figure 6-8 shows the generator torque, in the interval from 40 to 50 seconds. This response shows some oscillation at the frequency of the first tower fore-aft mode. This shows that there is some interaction (although stable) between the generator torque control and the tower fore-aft motion, even though the tower states are not contained in the linear model from which the generator torque controller is designed. This is because the first tower fore-aft mode is observable in the generator speed signal. Recall that we are assuming measurement of generator speed for the generator torque controller. 


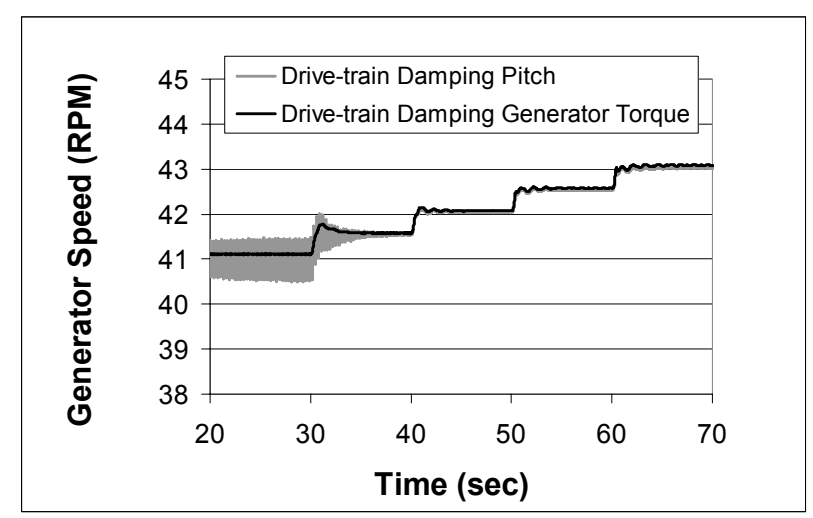

Figure 6-7. Plot of FAST simulated CART generator speed using DAC controller based on 7-state model, with drive-train damping from pitch control compared with drive-train damping from generator torque control

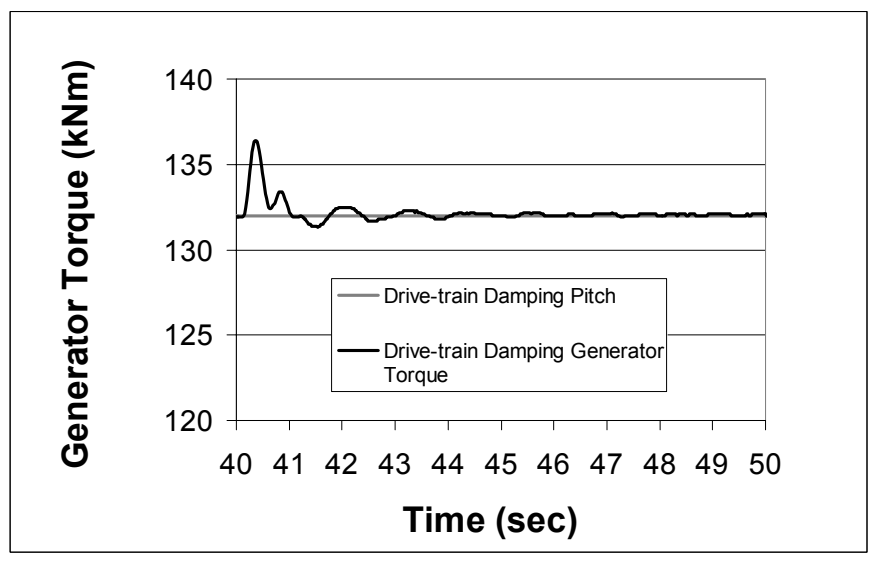

Figure 6-8. Plot of FAST simulated CART generator torque using DAC controller based on 7-state model, with drive-train damping from pitch control compared with drivetrain damping from generator torque control

\subsection{Further Simulations}

Further simulations were made with ADAMS. The pitch designed from the 7-state model and the generator torque control designed from the 3-state model just shown were both incorporated into ADAMS. This ADAMS model had fully flexible blades and tower. Figure 6-9 is a comparison between FAST and ADAMS for simulated generator speed, showing good agreement. Figure 6-10 is a comparison for simulated tower-top fore-aft motion, also showing excellent agreement. In ADAMS, this control also resulted in stable closed-loop behavior, in the presence of many flexible modes.

Further simulations were performed with FAST using this controller inputting turbulent inflow. Figure 6-11 shows a comparison of FAST simulated generator speed, when damping of the first drive-train torsion mode is provided by generator torque control versus rotor collective pitch control. The regulated generator rotational speed is about the same in both cases.

Figure 6-12 shows tower-top fore-aft deflection. In the case of turbulent wind inflow, both controllers giving about the same excursions in tower fore-aft motion. Figure 6-13 shows generator torque for both cases. Of course, the generator torque is constant when adding damping to the drive train through all rotor collective pitch. The figure indicates the variations of generator torque about the mean value, for the generator torque control case. 


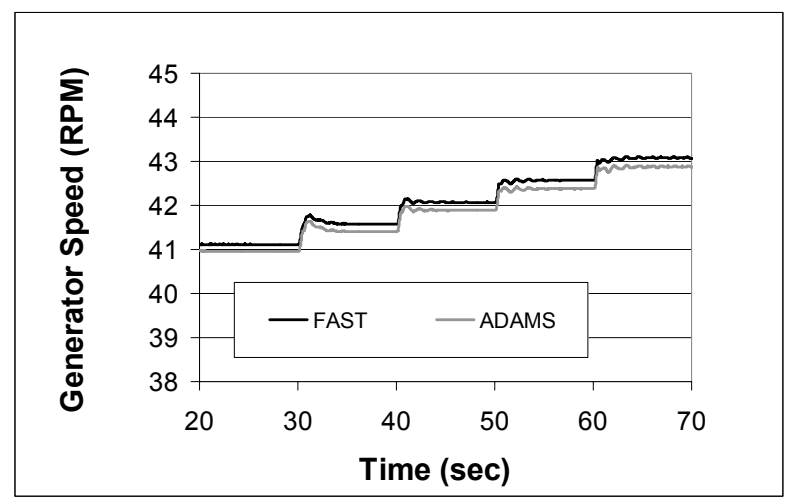

Figure 6-9. Plot of FAST and ADAMS simulated generator speed, using generator torque to add damping to drive-train torsion

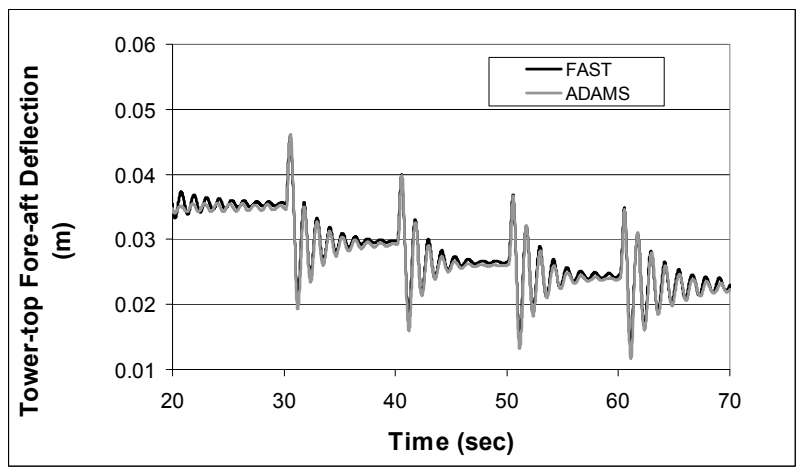

Figure 6-10. Plot of FAST and ADAMS simulated tower-top fore-aft motion, using generator torque to add damping to drive-train torsion

Figure 6-14 shows FAST simulated pitch rates from both control methods. In general, pitch rates are reduced when the generator torque is used as the actuator to add damping to the drive train. This removes some of the requirement from the rotor collective pitch control system, as desired.

One penalty for using generator torque as a control actuator is higher electrical power excursions, when compared with all pitch control. Figure 6-15 shows a plot of electrical power for the case of using generator torque to add damping to the first drive-train torsion mode, compared with using rotor collective pitch to perform this function. The higher electrical power excursions when using generator torque as the control input is evident. A trade-off must be made between allowable excursions in electrical power versus the amount of damping added to the first drive-train torsion mode (through pole placement).

Further simulations were also performed using ADAMS, with turbulent inflow, for the same control design. Figure 6-16 is a comparison of ADAMS and FAST for generator speed, showing good agreement. This shows that generator speed remains stable in ADAMS.

Figure 6-17 shows simulated tower-top fore-aft deflections. These results also show good agreement. There is some indication of a slight instability in ADAMS at 260 seconds. This instability is probably not due to a flexible mode, but it could probably be reduced by placing the state estimator poles for the rotor collective pitch controller closer to the imaginary axis, thus reducing the controller gains. 


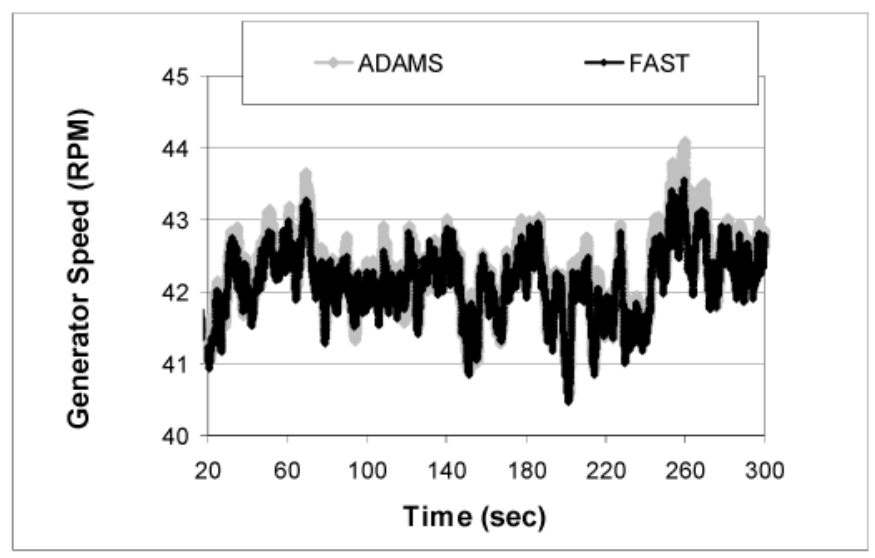

Figure 6-11. Plot of FAST simulated CART generator speed using DAC controller based on 7-state model, excited with turbulent inflow

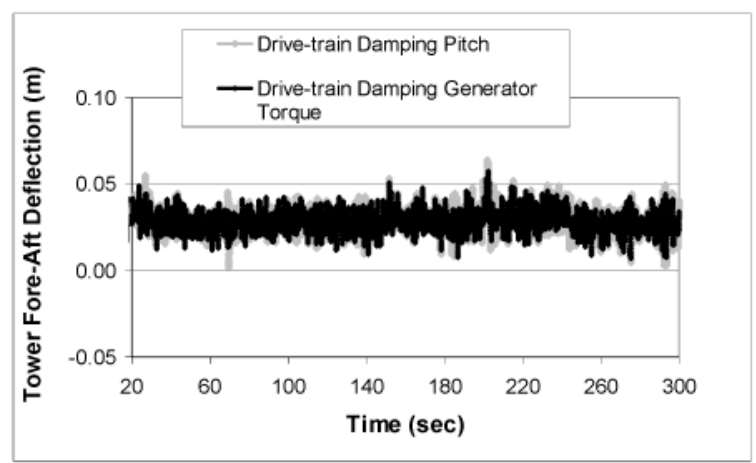

Figure 6-12. Plot of FAST simulated CART tower-top fore-aft deflection using DAC controller based on 7-state model, excited with turbulent inflow

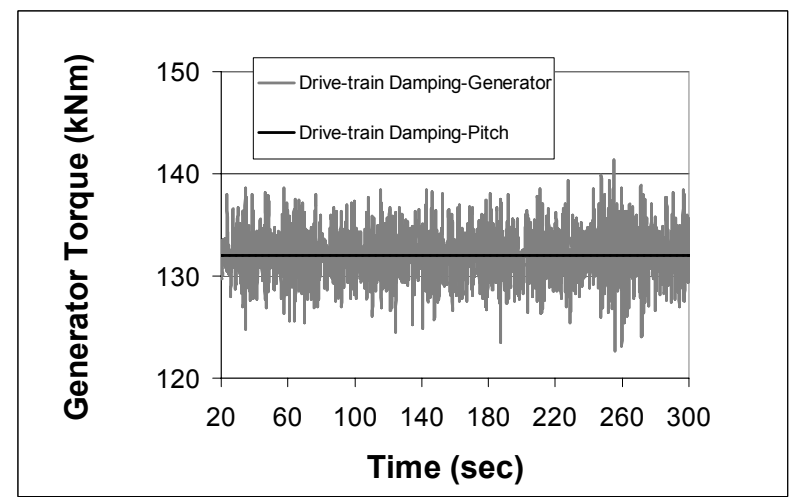

Figure 6-13. Plot of FAST simulated CART tower-top fore-aft deflection using DAC controller based on 7 -state model, excited with turbulent inflow 


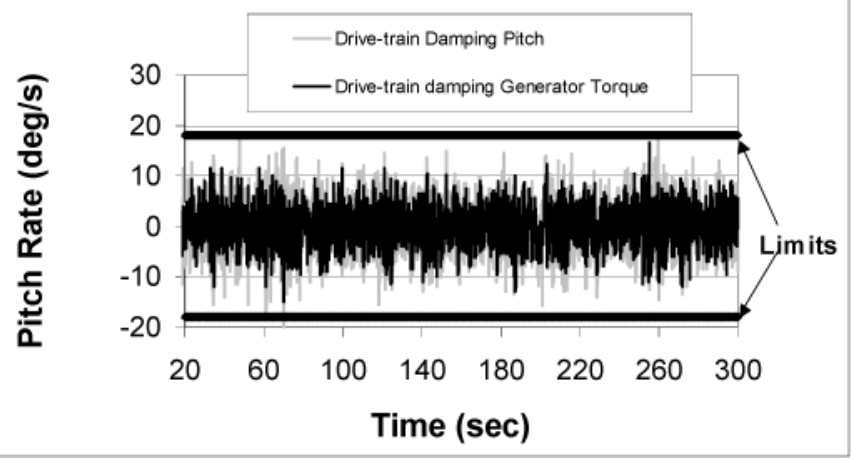

Figure 6-14. Plot of FAST simulated CART blade pitch rate using DAC controller based on 7-state model, excited with turbulent inflow

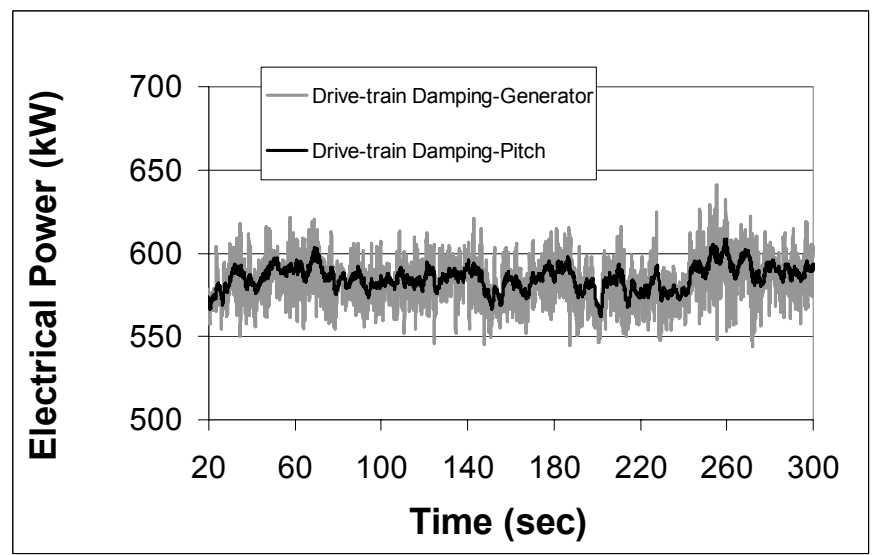

Figure 6-15. Plot of FAST simulated CART electrical power, using DAC controller based on 7-state model, excited with turbulent inflow

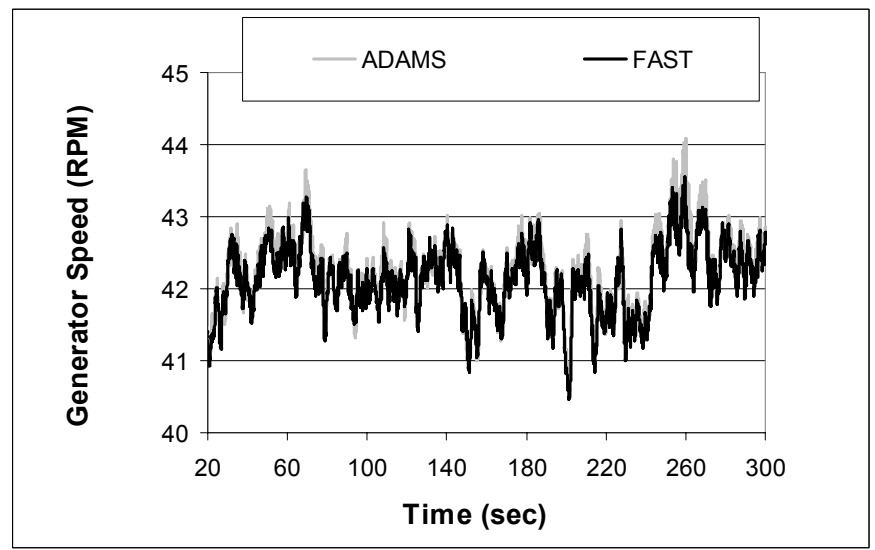

Figure 6-16. Plot of FAST and ADAMS simulated generator speed, using generator torque to add damping to drive-train torsion, with turbulent inflow 
Figure 6-18 shows simulated generator torque, showing the generator torque excursions when using generator torque control. As can be seen, both the ADAMS and FAST simulations are in good agreement. Figure 6-19 shows simulated pitch rates from FAST and ADAMS, also showing good agreement. The ADAMS pitch rates are slightly higher than the FAST pitch rates. This is an expected result, since the ADAMS model contains many more flexible modes than FAST. The FAST pitch rates are all below $20 \mathrm{deg} / \mathrm{s}$; the ADAMS pitch rates exceed this value, especially at 260 seconds, where the ADAMS results tend to be slightly unstable. Overall, however, the ADAMS results are in very close agreement with FAST. This shows that additional flexible modes remain stable during closed-loop operation with this control. It also shows that this control system, with two separate controllers, remains stable for these simulation tests.

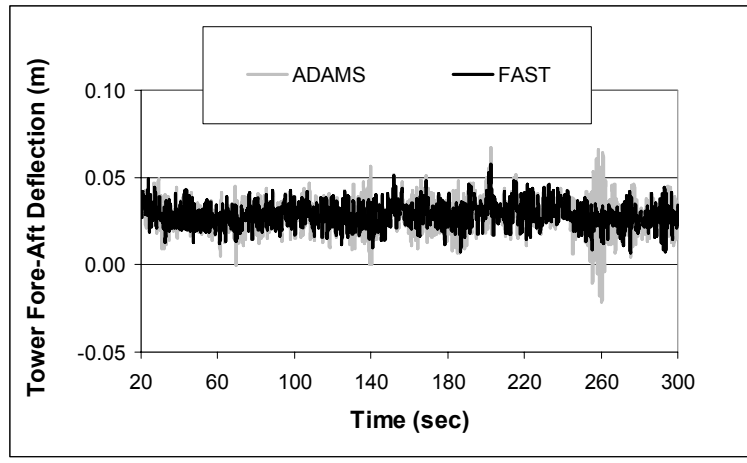

Figure 6-17. Plot of FAST and ADAMS simulated tower-top fore-aft motion, using generator torque to add damping to drive-train torsion, with turbulent inflow

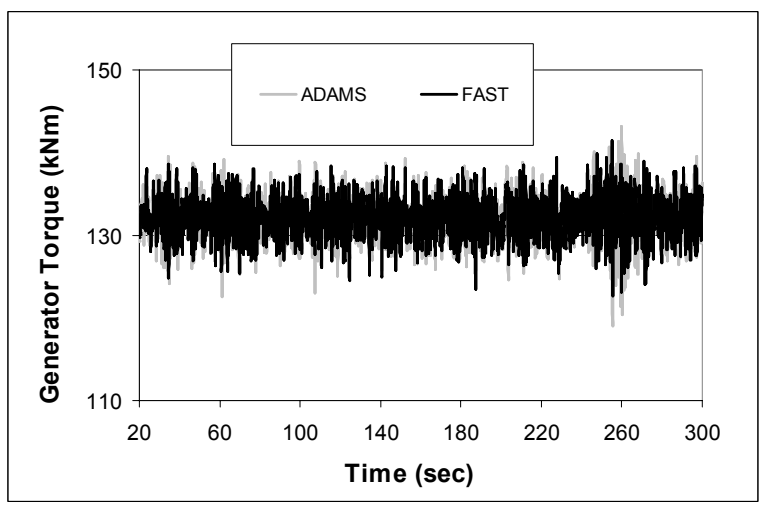

Figure 6-18. Plot of FAST and ADAMS simulated generator torque, using generator torque to add damping to drive-train torsion, with turbulent inflow 


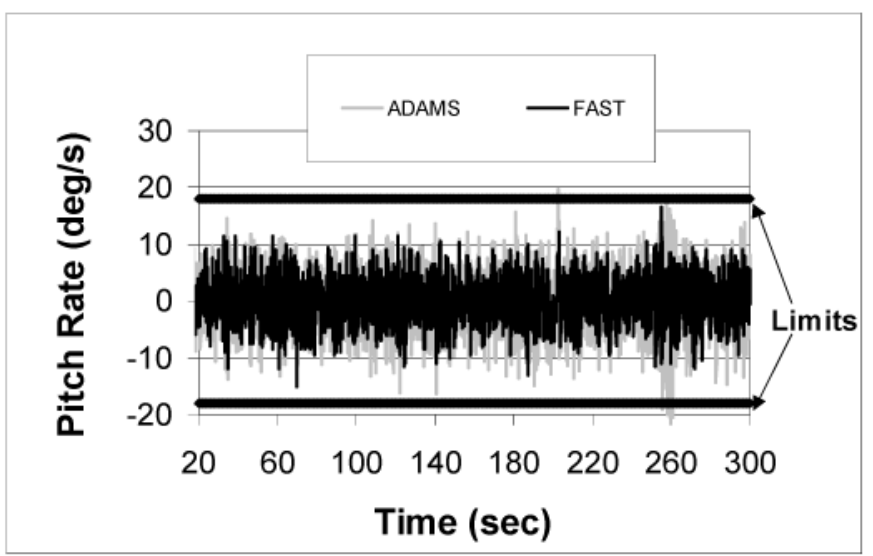

Figure 6-19. Plot of FAST and ADAMS simulated pitch rates, using generator torque to add damping to drive-train torsion, with turbulent inflow

\subsection{Chapter Conclusions}

We studied an instability involving the first drive-train torsion mode, coupled with the blade first edge mode, for the low-wind-speed side of region $3(14 \mathrm{~m} / \mathrm{s})$. Design of the rotor collective pitch control system at $14 \mathrm{~m} / \mathrm{s}$ failed to completely stabilize this response. The cause was low controllability of this mode at the low-wind-speed side of region 3 when using rotor collective pitch control. We then investigated the design of a torque controller to add damping to the first drive-train torsion mode.

In this control scheme, two separate controllers were used: generator torque control and rotor collective pitch control. The objective of the generator torque control was to stabilize the first drive-train torsion mode. This controller was designed from a state-space model having 3 states, to describe the first drive-train torsion mode. Using state estimation required only generator speed as the measurement for the generator torque control.

The objective of the rotor collective pitch controller was to regulate turbine speed and enhance damping in the first tower fore-aft mode. The pitch control system was designed using the 5- and 7-state linear models described in Chapter 5. Using the generator torque control stabilized the response at the low-wind-speed end of region 3, because controllability of the first drive-train torsion mode was increased using this control.

Simulation with turbulent wind inflow showed that the use of generator torque control reduced the blade pitch rates, as desired. This control was also introduced into the ADAMS code, and simulations with the same inflow conditions were performed. The response was stable, indicating that higher modes remain stable using this control. It also indicated that this control system, with two separate control loops, remains stable as desired. 


\section{Chapter 7. Control Designs for Speed Regulation and Load Reduction Using Multiple Control Inputs}

\section{Introduction}

The last two chapters described control systems to regulate turbine speed using rotor collective pitch and generator torque. Wind disturbances uniform over the rotor disk were accommodated using Disturbance Accommodating Control or DAC. It was also shown that only the rotor symmetric modes could be controlled with rotor collective pitch and generator torque.

In this chapter, separate pitch control inputs for each blade are allowed. This permits nonuniform wind components to be attenuated and asymmetric rotor modes to be controlled. In this chapter, controller performance is investigated for speed regulation and load reduction using independent blade pitch control. The effectiveness of independent blade pitch control in attenuating wind shear effects is examined. The minimum number of turbine measurements needed to successfully use state estimation is also shown. First, control is designed using only independent blade pitch control, holding generator torque constant. Later, generator torque is added as a control input to reduce demand on the pitch control.

\subsection{Independent Blade Pitch Control}

\subsubsection{Control Design Assumptions}

The first control objective is to regulate turbine speed in region 3, using independent blade pitch control, with generator torque assumed to be constant. The next control objective is to maintain stable closed-loop behavior over a wide range of turbine operating parameters, which includes enhancing the damping in several flexible modes to reduce dynamic response and structural loads. The final control objective is to reduce the effects of spatial variations of wind speed over the rotor disk.

In this work it is assumed that the teeter DOF is locked in the CART. Ordinarily, teeter motion assists in attenuating the effects of spatial wind-speed variations over the rotor disk (Eggers et al. 1999). The problem with this method of reducing loads and response is that the teeter motion is often limited by a teeter restraint, which can cause high teeter impact loads. As an alternative to teeter, independent blade pitch is investigated for attenuating these spatial wind variation responses. To attenuate these responses, states are added to the disturbance model to include wind-speed disturbances that depend on rotor azimuth angle.

\subsubsection{DAC Disturbances}

Figure 7-1 shows typical wind-speed variation with height, which can be expressed as

$$
V(z)=\mathrm{V}_{\text {hub }}(1+z / h)^{m}
$$

where $z$ is height above the hub, $\mathrm{V}_{\text {hub }}$ is the wind speed at hub-height $h$, and the coefficient $m$ is the power law wind-shear coefficient.

Equation 7.1 can be expanded as a binomial series in $\frac{z}{h}$ :

$$
\left(1+\frac{z}{h}\right)^{m}=1+m\left(\frac{z}{h}\right)+\frac{m(m-1)}{2}\left(\frac{z}{h}\right)^{2}+\frac{m(m-1)(m-2)}{6}\left(\frac{z}{h}\right)^{3}+\text { hots, }
$$


where hots refers to higher order terms.

We convert to polar coordinates $r$ and $\Psi$ by

$$
z=-r \sin \Psi
$$

as shown in Figure 7-2. Here, $r$ is the radial position along the blade from the center of the hub; $\Psi$ is the blade azimuth angle.

Substituting Equation 7.3 into 7.2, and neglecting the hots, results in the following expression involving $V_{h u b}, h, r$, $m$, and $\Psi$ :

$$
\begin{aligned}
& V(r, \Psi) \cong \frac{V_{h u b}}{24 h^{3}}\left[\left(24 h^{3}-6 h m r^{2}+6 h m^{2} r^{2}\right)+\left(-24 h^{2} m r-6 m r^{3}+9 m^{2} r^{3}-3 m^{3} r^{3}\right) \sin (\Psi)\right. \\
& \left.+\left(6 h m r^{2}-6 h m^{2} r^{2}\right) \cos (2 \Psi)+\left(2 m r^{3}-3 m^{2} r^{3}+m^{3} r^{3}\right) \sin (3 \Psi)\right]
\end{aligned}
$$

It is important to note that Equation 7.4 expresses the wind-shear distribution in terms of rotor azimuth $\Psi$.

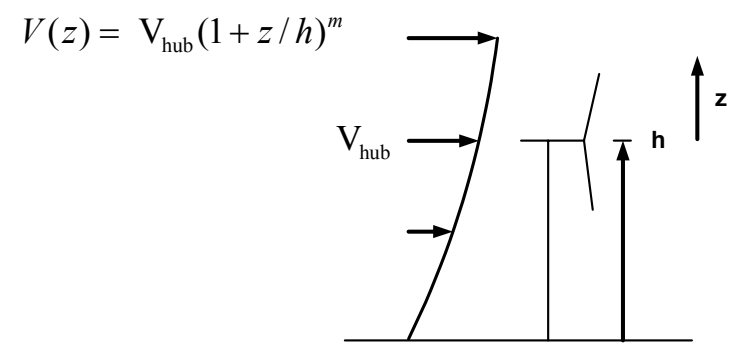

Figure 7-1. Variation of wind speed with height due to wind shear

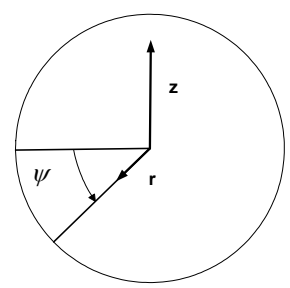

Figure 7-2. Polar coordinates 
For the CART, hub-height $h$ is approximately $35 \mathrm{~m}$, and at the blade tip, $r$ is approximately $21 \mathrm{~m}$. A shear coefficient of $m=0.4$ represents a severe wind shear profile relative to $m \approx 0.14$, which represents the standard 1/7 power law. Equation 7.4 (with $m=0.4$ ) becomes

$$
\begin{aligned}
& V(r, \Psi) \cong V_{h u b}[0.978-0.250 \sin (\Psi) \\
& +0.022 \cos (2 \Psi)+0.004 \sin (3 \Psi)]
\end{aligned}
$$

The dominant term that depends upon $\Psi$ is the term $-0.250 \sin (\Psi)$, and remaining terms can be neglected.

The disturbances are chosen to model the two terms in

$$
\frac{V_{h u b}}{24 h^{3}}\left[\left(24 h^{3}-6 h m r^{2}+6 h m^{2} r^{2}\right)-\left(24 h^{2} m r+6 m r^{3}-9 m^{2} r^{3}+3 m^{3} r^{3}\right) \sin (\Psi)\right]
$$

The first term in Equation 7.6 is the wind component, which is independent of azimuth position $\Psi$ and is modeled with the DAC step waveform, as in Chapters 4, 5, and 6. The second term represents the azimuth-dependent component. To counteract this disturbance component, each blade is pitched independently, and the pitch angle varies with azimuth angle. This additional term must be represented with a new DAC waveform.

To represent this new term, recall that the state-space equation for a general disturbance takes the form (see Appendix C):

$$
\begin{aligned}
& \underline{u_{D}}(t)=\theta \underline{z_{D}}(t) \\
& \underline{\dot{z}_{D}}(t)=F \underline{z_{D}}(t) ; \quad \underline{z_{D}}(0)=\underline{z_{D}^{0}} .
\end{aligned}
$$

The new term is periodic; the frequency is equal to the rotor speed $\Omega$ and is written as:

$$
u_{D_{1}}(t)=A_{D} \sin (\Omega t), \text { where } \psi=\Omega t .
$$

As we will show, a disturbance model with 2 states will define this part of the disturbance. Let

$$
z_{D_{1}}(t)=A_{\mathrm{D}} \sin (\Omega t), \text { then } \dot{z}_{D_{1}}(t)=A_{\mathrm{D}} \Omega \cos (\Omega t)
$$

Define the second disturbance state as

$$
z_{D_{2}}(t)=A_{\mathrm{D}} \Omega \cos (\Omega t)
$$

then,

$$
\dot{z}_{D_{2}}(t)=-\Omega^{2} A_{D} \sin (\Omega t)
$$

and thus, the state-space model for the azimuth-dependent part of the disturbance is 


$$
\begin{aligned}
& {\left[\begin{array}{c}
\dot{z}_{D_{1}}(t) \\
\dot{z}_{D_{2}}(t)
\end{array}\right]=\left[\begin{array}{cc}
0 & 1 \\
-\Omega^{2} & 0
\end{array}\right]\left[\begin{array}{l}
z_{D_{1}}(t) \\
z_{D_{2}}(t)
\end{array}\right]} \\
& u_{D_{1}}(t)=\left[\begin{array}{ll}
1 & 0
\end{array}\right]\left[\begin{array}{l}
z_{D_{1}} \\
z_{D_{2}}
\end{array}\right] .
\end{aligned}
$$

This model neglects the uniform term modeled in previous chapters. To model this term, recall that for the step waveform,

$$
\begin{aligned}
& u_{D_{2}}(t)=z_{D_{3}}(t) \\
& \dot{z}_{D_{3}}(t)=0 z_{D_{3}}(t)
\end{aligned} .
$$

This third state is added to the model.

The resulting state-space model for these disturbances then becomes

$$
\begin{aligned}
& {\left[\begin{array}{l}
\dot{z}_{D_{1}}(t) \\
\dot{z}_{D_{2}}(t) \\
\dot{z}_{D_{3}}(t)
\end{array}\right]=\left[\begin{array}{ccc}
0 & 1 & 0 \\
-\Omega^{2} & 0 & 0 \\
0 & 0 & 0
\end{array}\right]\left[\begin{array}{c}
z_{D_{1}}(t) \\
z_{D_{2}}(t) \\
z_{D_{3}}(t)
\end{array}\right]} \\
& {\left[\begin{array}{l}
u_{D_{1}}(t) \\
u_{D_{2}}(t)
\end{array}\right]=\left[\begin{array}{lll}
1 & 0 & 0 \\
0 & 0 & 1
\end{array}\right]\left[\begin{array}{c}
z_{D_{1}}(t) \\
z_{D_{2}}(t) \\
z_{D_{3}}(t)
\end{array}\right] .}
\end{aligned}
$$

Consistent with Equation 7.7, one can see that $\theta=\left[\begin{array}{lll}1 & 0 & 0 \\ 0 & 0 & 1\end{array}\right]$ and $F=\left[\begin{array}{ccc}0 & 1 & 0 \\ -\Omega^{2} & 0 & 0 \\ 0 & 0 & 0\end{array}\right]$.

This gives a disturbance model having 3 states. Figure 7-3 shows a diagram of the DAC controller.

We first design controls using full-state feedback and state estimation, ignoring disturbances. We then assess improvements in controller performance when the disturbances just described are included using DAC. Controls are initially designed using a simple 5-state model, and further complexity is subsequently added. 


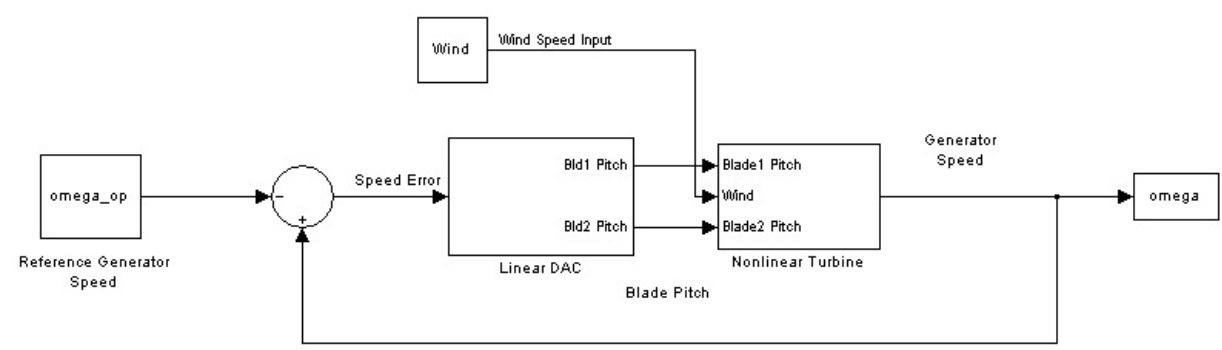

Figure 7-3. Diagram of DAC controller

\subsection{Control Design}

\subsubsection{Results from a Model with 5 States}

Let

$x_{1}=\delta q_{1}$, blade 1 perturbed flap tip displacement

$x_{2}=\delta \dot{q}_{1}$, blade 1 perturbed flap tip velocity

$x_{3}=\delta q_{2}$, blade 2 perturbed flap tip displacement

$x_{4}=\delta \dot{q}_{2}$, blade 2 perturbed flap tip velocity

$x_{5}=\delta \dot{q}_{4}$, perturbed rotor speed.

The linear model corresponding to these states (as shown in Appendix A) is

$$
\begin{aligned}
& {\left[\begin{array}{ccccc}
1 & 0 & 0 & 0 & 0 \\
0 & \mathrm{M}_{11} & 0 & 0 & \mathrm{M}_{14} \\
0 & 0 & 1 & 0 & 0 \\
0 & 0 & 0 & \mathrm{M}_{22} & \mathrm{M}_{24} \\
0 & \mathrm{M}_{41} & 0 & \mathrm{M}_{42} & \mathrm{I}_{\mathrm{rot}}+\mathrm{I}_{\mathrm{gen}}
\end{array}\right]\left[\begin{array}{c}
\dot{x}_{1} \\
\dot{x}_{2} \\
\dot{x}_{3} \\
\dot{x}_{4} \\
\dot{x}_{5}
\end{array}\right]=\left[\begin{array}{ccccc}
0 & 1 & 0 & 0 & 0 \\
-\mathrm{K}_{11} & -\mathrm{C}_{11} & 0 & 0 & -\mathrm{C}_{14} \\
0 & 0 & 0 & 1 & 0 \\
0 & 0 & -\mathrm{K}_{22} & -\mathrm{C}_{22} & -\mathrm{C}_{24} \\
0 & -\mathrm{C}_{41} & 0 & -\mathrm{C}_{42} & \gamma
\end{array}\right]\left[\begin{array}{c}
x_{1} \\
x_{2} \\
x_{3} \\
x_{4} \\
x_{5}
\end{array}\right]+\left[\begin{array}{cc}
0 & 0 \\
\zeta_{\mathrm{b}} & 0 \\
0 & 0 \\
0 & \zeta_{\mathrm{b}} \\
\zeta & \zeta
\end{array}\right]\left[\begin{array}{c}
\delta \beta_{1} \\
\delta \beta_{2}
\end{array}\right]+\left[\begin{array}{cc}
0 & 0 \\
\alpha_{\mathrm{b}} & 0 \\
0 & 0 \\
0 & \alpha_{\mathrm{b}} \\
\alpha & \alpha
\end{array}\right]\left[\begin{array}{l}
\delta \mathrm{w}_{1} \\
\delta \mathrm{w}_{2}
\end{array}\right]} \\
& y=\left[\begin{array}{lllll}
0 & 0 & 0 & 0 & 1
\end{array}\right]\left[\begin{array}{l}
x_{2} \\
x_{3} \\
x_{4} \\
x_{5}
\end{array}\right]
\end{aligned}
$$

Note that states $x_{2}$ and $x_{4}$, corresponding to blade-1 and blade-2 flap velocity $\left(x_{2}, x_{4}\right)$, couple with rotor speed $\left(x_{5}\right)$ in this model. 
It is important to note that this system is controllable, unlike the linear system in which the first flap mode of each blade was uncontrollable using rotor collective pitch. Since two control inputs are now available (the pitch of each blade), this system is controllable.

The control design point was chosen as follows:

$$
\begin{aligned}
& w_{0}=18 \mathrm{~m} / \mathrm{s} \\
& \beta_{0}=12 \mathrm{deg} . \\
& \Omega_{0}=42 \mathrm{RPM} .
\end{aligned}
$$

Tables 7-1 and 7-2 show evaluation of the state matrices at this point.

Table 7-1. A-Matrix for the 5-State Linear Model

\begin{tabular}{|l|l|l|l|l|}
\hline A & \multicolumn{5}{|l|}{} \\
\hline 0. & 1. & 0. & 0. & 0. \\
\hline-194.783 & -8.949 & -3.810 & -0.029 & -20.116 \\
\hline 0. & 0. & 0. & 1. & 0. \\
\hline-3.810 & -0.029 & -194.783 & -8.949 & -20.116 \\
\hline 0.4272 & 0.0032 & 0.427 & 0.0032 & -0.034 \\
\hline
\end{tabular}

Table 7-2. B and $\Gamma$ Matrices for the 5-State Linear Model

\begin{tabular}{|l|l|l|l|}
\hline B & \multicolumn{1}{|l|}{$\Gamma$} \\
\hline 0. & 0. & 0. & 0. \\
\hline-1108.75 & -8.585 & 14.186 & 14.141 \\
\hline 0. & 0. & 0. & 0. \\
\hline-8.585 & -1108.75 & -14.186 & 14.141 \\
\hline 0.963 & 0.963 & 0. & 0.005 \\
\hline
\end{tabular}

An eigen-analysis of the A matrix for this system gives the open loop poles: $-4.4 \pm 13.3 \mathrm{j}(\mathrm{r} / \mathrm{s}),-4.46 \pm 13.1 \mathrm{j} \mathrm{r} / \mathrm{s}$, and $0.121 \mathrm{r} / \mathrm{s}$, corresponding to the rotor first asymmetric flap mode, the rotor first symmetric flap mode, and the rotor speed.

At this stage, goals are rotor speed regulation and reduction of the effects of spatial variations of wind speed across the rotor disk. Tests were performed with the same step winds used in Chapters 4 and 5, except that a spatial variation of wind speed over the rotor disk was added as a linear vertical wind shear, with coefficient $0.4{ }^{1}$.

Since the system was controllable, the plant poles were placed as desired, at $-4.4 \pm 13.3 \mathrm{j} \mathrm{r} / \mathrm{s},-4.46 \pm 13.1 \mathrm{j} \mathrm{r} / \mathrm{s}$, and $-2 \mathrm{r} / \mathrm{s}$. The calculated gains for these poles are listed in Appendix D. This full-state feedback design is implemented into FAST and simulated in response to the step winds shown in Figure 7-4, yielding the results shown in Figure 7-5. These results show stable closed-loop behavior over the full range of wind speeds tested.

${ }^{1}$ This coefficient represents the wind speed at the blade tip at the top of the rotor minus the wind speed at the blade tip at the bottom of the rotor, divided by the hub-height wind speed. 


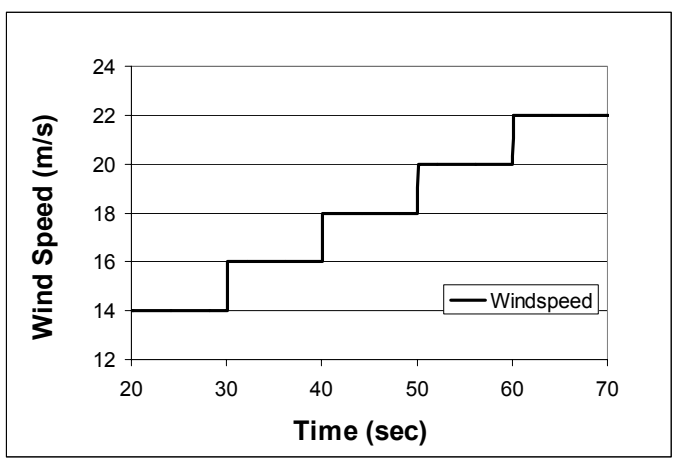

Figure 7-4. Test winds

Figure 7-5 also shows the blade pitch angle, which after a step change in wind speed, changes in order to regulate rotor speed to the $42 \mathrm{rpm}$ setpoint. This result is almost identical to previous results obtained using rotor collective pitch, in Chapters 4 and 5, showing that this controller achieves the primary objective of speed regulation in response to fluctuations in wind, uniform over the rotor disk.

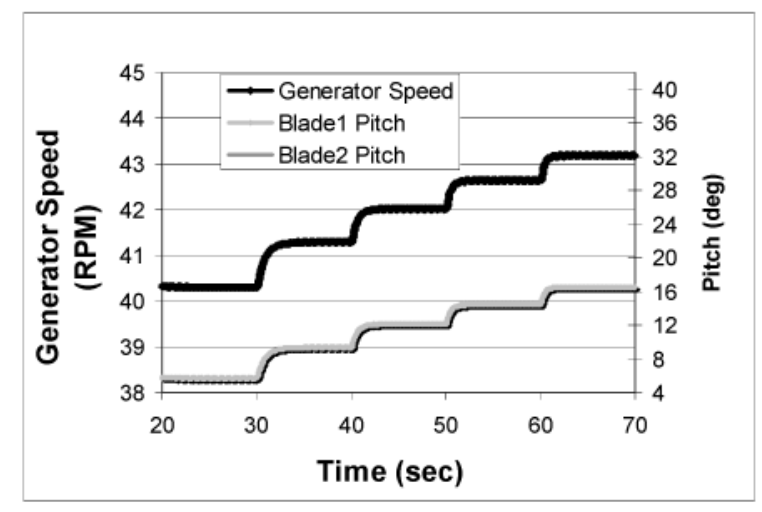

\section{Figure 7-5. FAST simulated generator speed and pitch using full-state feedback controller designed from 5 -state model}

The input step winds also contain azimuthal wind-speed variation, since a linear vertical wind-shear profile is superimposed onto these winds. Figure 7-5 shows that the pitch angle of the two blades is nearly identical, indicating that the control system is not attenuating this disturbance as desired.

A much more evident response to the wind shear appears in Figure 7-6, as the flap deflections of both blade tips show strong periodic response to the wind-shear component. Note also that the response of blade 2 is 180 degrees out of phase with that of blade 1, confirming the periodic nature of this response. 


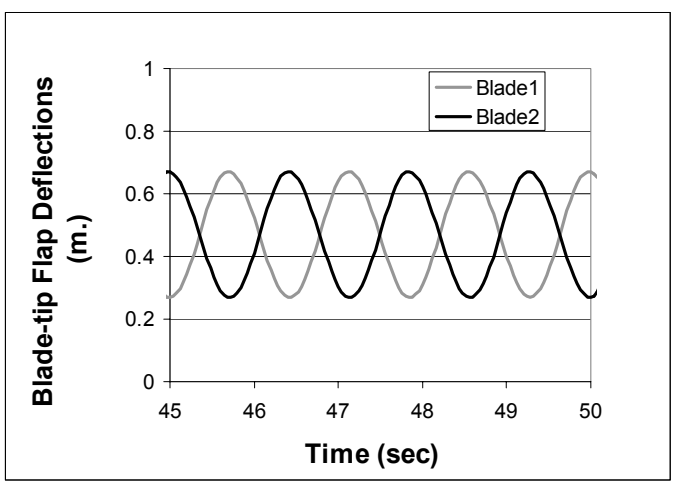

\section{Figure 7-6. FAST simulated blade tip flap deflections using full-state feedback controller designed from 5-state model}

It is evident that with the gains chosen in this full-state feedback design, the wind shear disturbance is not attenuated. In this design, the poles of the first flap mode of each blade have been maintained at their open-loop values, implying that the controller adds no damping to these modes. To enhance flap damping, these poles were moved to the left in the complex plane.

Figure 7-7 shows flap deflections for three different full-state feedback controllers, for blade 1. In the first case, flap mode damping is equal to the open-loop value. In the second case, these poles were placed at $-12.1 \pm 13.4 \mathrm{i}$ and $11.9 \pm 13.1 \mathrm{i}$, and in the third case, the poles were placed at $-15.9 \pm 13.5 \mathrm{i}$ and $-15.1 \pm 13.1 \mathrm{i}$. The decrease in amplitude of the flap response is apparent as the damping is increased.

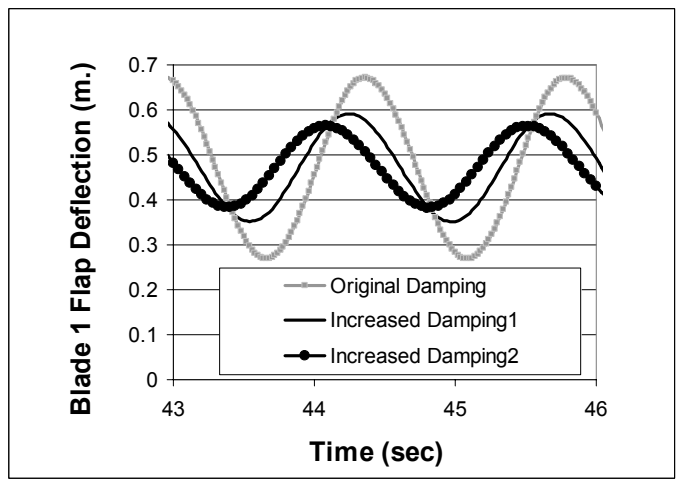

Figure 7-7. Comparison of FAST simulated blade-tip flap deflections with full-state feedback for three pole locations, designed from 5-state model

Figure 7-8 shows the pitch angle of blade 1 for each of these cases. As flap damping is increased, the cyclic pitch variations increase. Figure 7-9 shows the pitch of blades 1 and 2 for the case with highest damping (Damping 2), showing that the pitch of blade 2 is 180 degrees out of phase with blade 1 because of the azimuthally varying wind component. This shows that increasing the blade flap damping, through control, results in increased pitch variation to attenuate the effects of wind shear. 


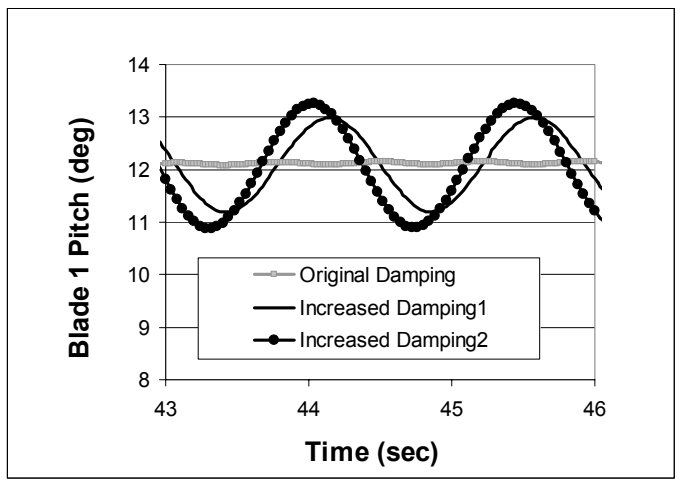

Figure 7-8. Comparison of FAST simulated blade-1 pitch with full-state feedback for three pole locations, designed from 5-state model

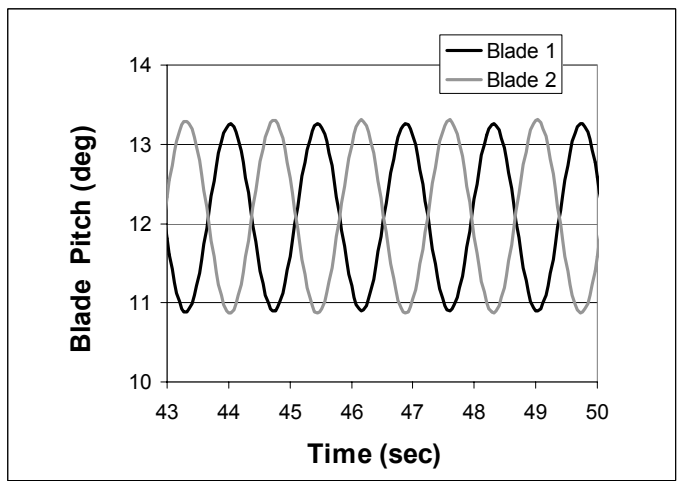

Figure 7-9. FAST simulated blade-1 and -2 pitch, using full-state feedback with high flap damping

The problem with full-state feedback is that every state in the linear model must be accurately measured. As always, this control should be performed with a minimum number of turbine measurements, which requires state estimation. First, though, observability must be assessed.

Observability. Which turbine measurements are needed in order to successfully perform state estimation for this system? First, control will be designed based only on plant states, omitting the disturbance states. Later, the disturbance states described in Section 7.1.2 will be added to the model.

In Chapters 4 and 5, control systems were successfully designed when only generator speed was measured. Only uniform wind disturbances over the rotor disk were modeled, and only symmetric rotor modes could be controlled.

Now that the rotor first asymmetric mode is included in the model, the flap displacement of at least one blade will require measurement. If we assume that generator speed is the only measurement (and do not measure blade-tip flap displacement), this system is found to be unobservable, as the observability matrix is rank-deficient. This is because the rotor first asymmetric mode is present in this model and is unobservable when only generator speed is measured.

Next, measurement of the flap deflection of a single blade (blade 1) is added. The measurements can now be expressed as 


$$
y=\left[\begin{array}{lllll}
0 & 0 & 0 & 0 & 1 \\
1 & 0 & 0 & 0 & 0
\end{array}\right]\left[\begin{array}{l}
\mathrm{x}_{1} \\
\mathrm{x}_{2} \\
\mathrm{x}_{3} \\
\mathrm{x}_{4} \\
\mathrm{x}_{5}
\end{array}\right]
$$

Calculation of the rank of the observability matrix now reveals that this system is observable. A controller using this measurement gives the results shown in Figure 7-10 (Measure Flap1), which shows significant undesirable cyclic content in the generator speed simulation.

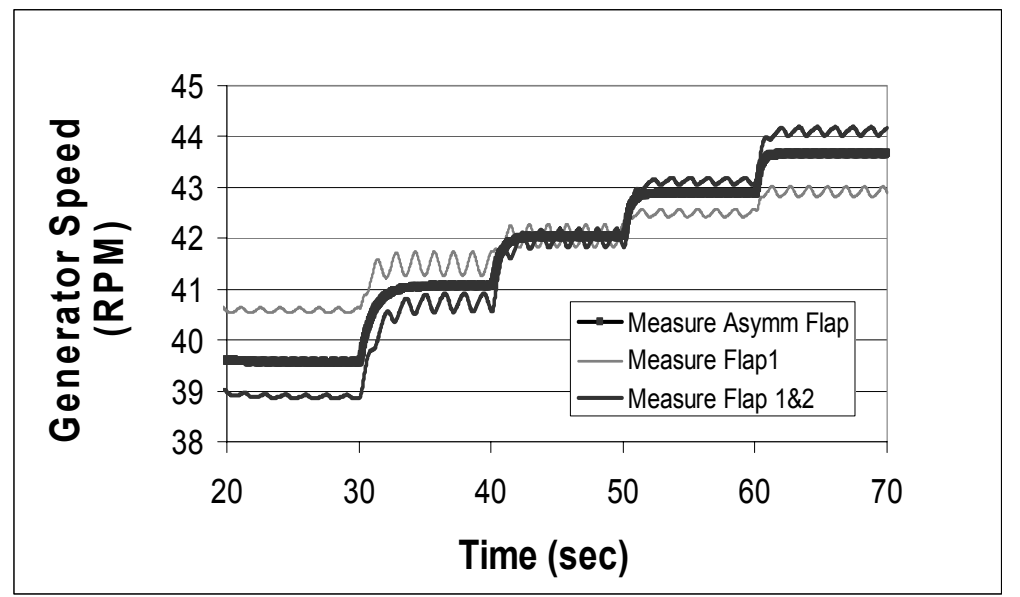

Figure 7-10. FAST simulated generator speed for various measured turbine parameters while using state estimation without disturbances based on 5 -state model

Adding another measured state, the flap deflection of blade 2 gives

$$
y=\left[\begin{array}{lllll}
0 & 0 & 0 & 0 & 1 \\
1 & 0 & 0 & 0 & 0 \\
0 & 0 & 1 & 0 & 0
\end{array}\right]\left[\begin{array}{l}
\mathrm{x}_{1} \\
\mathrm{x}_{2} \\
\mathrm{x}_{3} \\
\mathrm{x}_{4} \\
\mathrm{x}_{5}
\end{array}\right]
$$

This system is observable, and a controller using this measurement produces the results shown in Figure 7-10 (Measure Flap 1\&2). Here, simulated generator speed again contains significant undesirable cyclic content. The cause of these undesirable fluctuations may be weak observability of the rotor first asymmetric flap mode, which is not directly measured when the flap displacement of one or both blades is measured.

Now, the rotor first asymmetric flap mode deflection is measured, by forming a linear combination of the blade 1 and 2 flap deflection measurements: 


$$
y=\left[\begin{array}{ccccc}
0 & 0 & 0 & 0 & 1 \\
\frac{1}{2} & 0 & -\frac{1}{2} & 0 & 0
\end{array}\right]\left[\begin{array}{l}
x_{1} \\
x_{2} \\
x_{3} \\
x_{4} \\
x_{5}
\end{array}\right] .
$$

This system is observable, and a controller designed from this system is simulated and the results shown in Figure 7-10 (Measure Asymm Flap). System observability has been greatly improved, and this yields improved simulation results. Rotor first asymmetric flap mode measurement is assumed in all further work in this chapter.

State Estimation. A controller using state estimation of only plant states was designed, ignoring all disturbance effects and placing both plant poles and state estimator poles. The plant poles were placed at $-12.1 \pm 13.4 \mathrm{i}$, $11.9 \pm 13.1 \mathrm{i}$, and -2 , resulting in reduced flap response from wind shear using full-state feedback, as shown in the previous section.

State estimator poles were first placed at $-14,-15,-14,-15$, and -16 and then at $-34,-35,-34,-35$, and -36 . Figure 7-11 is a comparison of the simulated blade-tip flap deflections from these two controllers and the full-state feedback controller. The first state estimator produces larger flap deflections, compared with the full-state feedback case, while the second state estimator yields closer agreement with full-state feedback results. This shows that designing a controller based on plant state estimation alone results in some attenuation of the wind-shear disturbance effects. However, the state estimator poles must be placed far to the left in the complex plane, which may result in large pitch actuation rates.

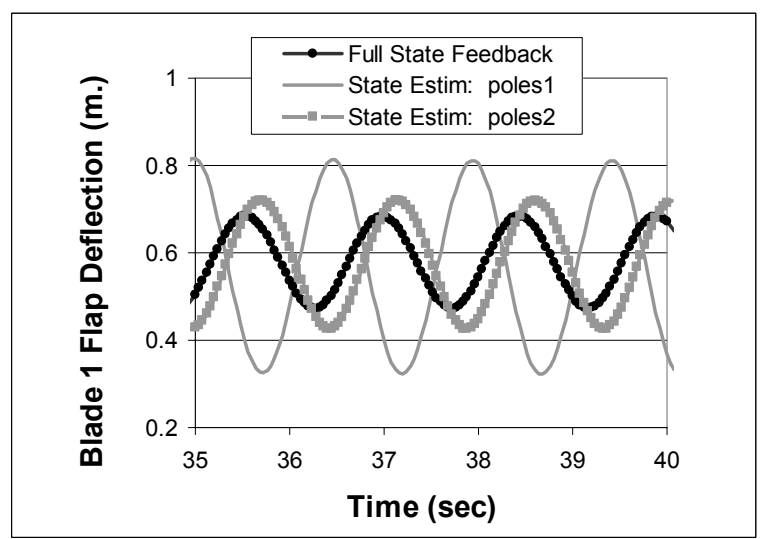

Figure 7-11. FAST simulated blade tip flap deflections using different controllers designed from 5state model, measuring first asymmetric flap

Design of DAC. The advantages of including disturbance effects, especially the azimuthal variation of wind speed due to wind shear, are now shown by designing Disturbance Accommodating Control or DAC, including the disturbances described in Section 7.1.2.

The first step in designing DAC is to confirm the controllability of $(A, B)$ and the observability of the augmented system $(\bar{A}, \bar{C})$. Controllability is automatically satisfied, since this is the same system used for the full-state feedback and state estimator just designed, in the absence of disturbances. To confirm observability, note that the original system ( $A, C$ ) is augmented with 3 states to account for the disturbances, 2 states for the azimuthally varying disturbance, and 1 state for the step disturbance. As shown in Appendix D (Section D-4.2), this augmented system is observable, when measuring generator speed and rotor first asymmetric flap; thus, state estimator pole placement can be achieved. 
If the azimuthally varying disturbance is effective in attenuating wind-shear response, plant and state estimator poles need not be placed as far to the left in the complex plane as in the previous section. The plant poles corresponding to the rotor flap modes are placed at the open-loop values, $-4.4 \pm 13.3 \mathrm{i} \mathrm{r} / \mathrm{s}$, and $-4.5 \pm 13.1 \mathrm{i} \mathrm{r} / \mathrm{s}$, and the rotor-speed pole is placed at -2 . Plant and disturbance state estimator poles are placed at $-10 \mathrm{r} / \mathrm{s},-11 \mathrm{r} / \mathrm{s},-10 \mathrm{r} / \mathrm{s},-11 \mathrm{r} / \mathrm{s},-12 \mathrm{r} / \mathrm{s},-12 \mathrm{r} / \mathrm{s},-13 \mathrm{r} / \mathrm{s}$, and $-13 \mathrm{r} / \mathrm{s}$.

This controller design uses the same design steps for DAC that were used in Chapter 4. The final step is to calculate an equivalent transfer function and implement it into FAST. Figure 7-12 shows rotor speed and blade pitch simulation results from FAST. The generator speed is much more closely regulated to the $42 \mathrm{rpm}$ set point than the full-state feedback or state estimator controllers. This improvement in speed regulation was also noted in Chapter 5 using rotor collective pitch to design DAC control with the step disturbance. Here, the improvement in speed regulation is also a direct result of including the step disturbance state in the model. Figure 7-12 shows that blade 1 pitch contains significant cyclic variation, corresponding to the azimuthally varying shear disturbance.

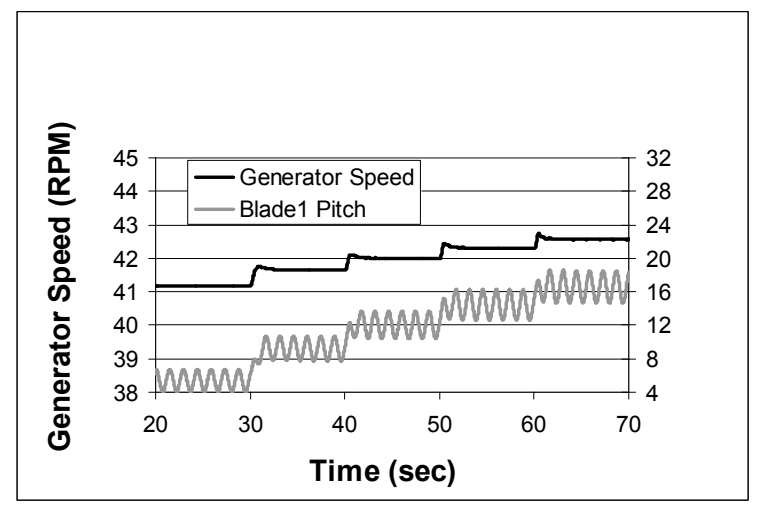

Figure 7-12. FAST simulated generator speed and pitch using DAC controller designed from 5-state model

Figure 7-13 shows the blade-tip flap response for this DAC, compared to the full-state feedback results, demonstrating a large reduction in blade flap deflection. This is due to the new disturbance in the model, and accommodation of the 1P disturbance component.

Figure 7-14 documents blade pitch, showing the dramatic increase in activity for the DAC. This controller simultaneously meets the control objectives of speed regulation, first symmetric and asymmetric flap mode stabilization, and reduction of wind-shear response.

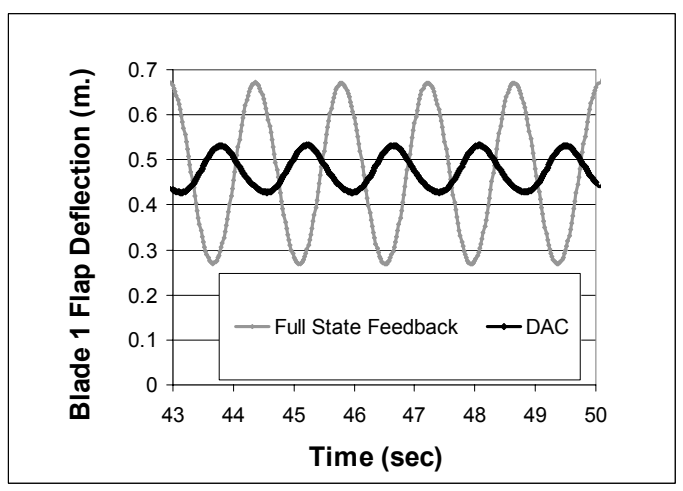

Figure 7-13. FAST simulated blade tip flap deflections using full-state feedback and DAC controllers designed from 5 -state model 


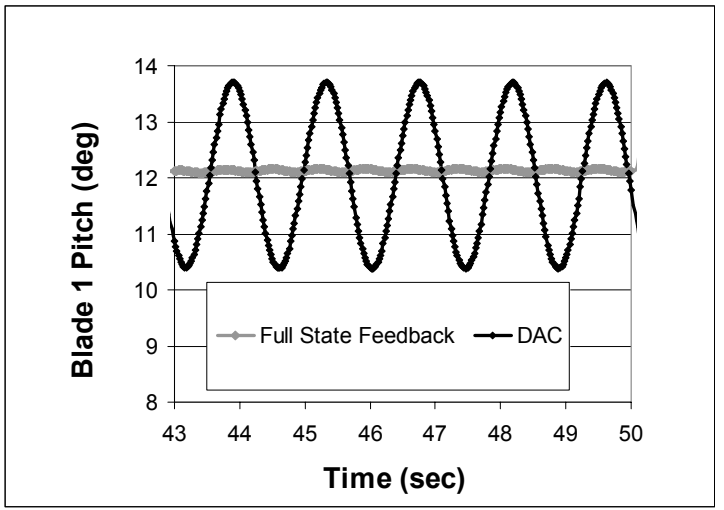

Figure 7-14. FAST simulated blade pitch using full-state feedback and DAC controllers designed from 5-state model

\subsubsection{Control Designs Including First Drive-Train Torsion and Tower First Fore-Aft Motion}

All previous controls in this chapter have been designed using a model ignoring drive-train torsion and tower fore-aft motion. Chapter 5 showed that the drive train mode must be included in a linear model for CART control design to prevent instability. Figure 7-15 shows lightly damped tower first fore-aft mode response from the previous controller, which requires added damping. To meet these objectives, a model with first drive-train torsion and tower first fore-aft is formulated.

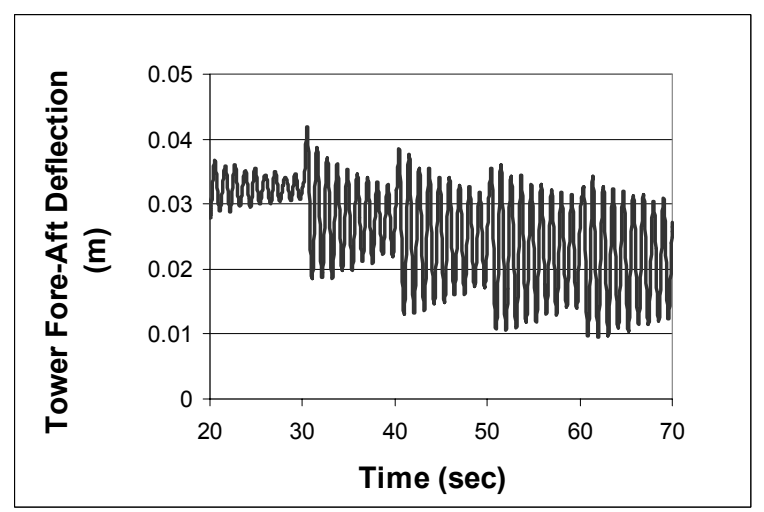

Figure 7-15. FAST simulated CART tower-top fore-aft deflection using DAC based on 5-state model

States are added to model the first drive-train torsion as well as the tower's first fore-aft mode to the previous model.

Now,

$x_{1}=\delta q_{1}$, blade 1 perturbed flap tip displacement

$x_{2}=\delta \dot{q}_{1}$, blade 1 perturbed flap tip velocity

$x_{3}=\delta q_{2}$, blade 1 perturbed flap tip displacement

$x_{4}=\delta \dot{q}_{2}$, blade 1 perturbed flap tip velocity

$x_{5}=\delta \dot{q}_{4}$, perturbed rotor rotational speed 
$x_{6}=\mathrm{K}_{\mathrm{d}}\left(\delta q_{4}-\delta q_{15}\right)$, perturbed Drive-train torsional spring force

$x_{7}=\delta \dot{q}_{15}$, perturbed generator rotational speed

$x_{8}=\delta q_{7}$, perturbed tower-top first fore-aft mode deflection

$x_{9}=\delta \dot{q}_{7}$, perturbed tower-top first fore-aft mode velocity.

The linear model corresponding to these states is as follows:
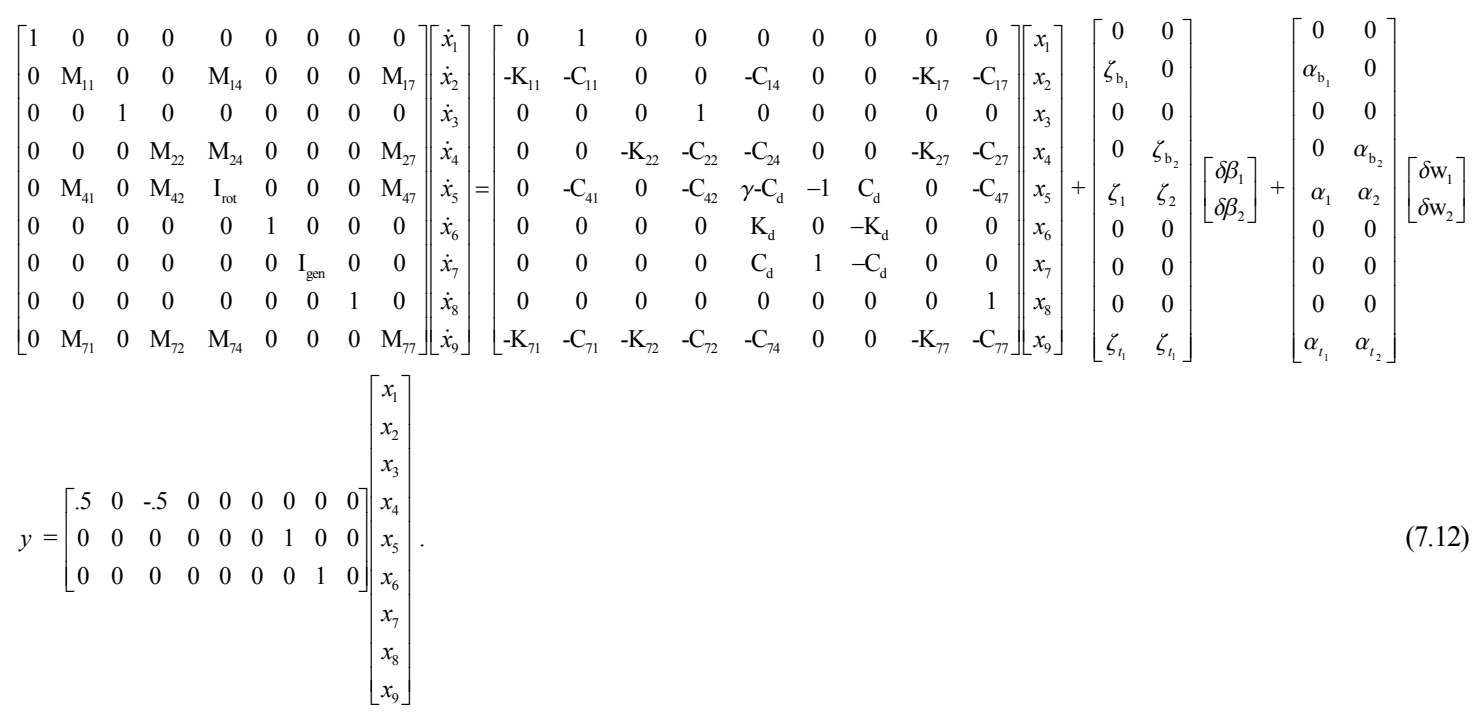

The control design point is

$$
\begin{aligned}
& w_{0}=18 \mathrm{~m} / \mathrm{s} \\
& \beta_{0}=12 \mathrm{deg} . \\
& \Omega_{0}=42 \mathrm{RPM} .
\end{aligned}
$$

The state matrices for this equation are evaluated and shown in Appendix D (Section D-4.3).

An eigen-analysis of the A matrix for this system gives the open loop poles: $-0.08 \pm 5.9 \mathrm{j} \mathrm{r} / \mathrm{s},-0.03 \pm 22.6 \mathrm{j} \mathrm{r} / \mathrm{s},-4.4 \pm$ $13.6 \mathrm{j} \mathrm{r} / \mathrm{s},-4.5 \pm 13.1 \mathrm{j} \mathrm{r} / \mathrm{s}$, and $-0.121 \mathrm{r} / \mathrm{s}$. The first pole pair represents the lightly damped tower first fore-aft mode, while the second pole pair represents the very lightly damped first drive-train torsional mode. The third pole pair represents the rotor first symmetric flap mode. The second pole pair represents the rotor first asymmetric flap mode. The final pole represents generator speed.

This represents the most complex model used in these control studies and the resulting controller has several objectives. These are generator speed regulation, first drive-train torsional mode stabilization, enhanced damping of the tower first fore-aft mode, stabilization of rotor first symmetric and asymmetric flap mode, and attenuation of blade flap wind shear response. Let's now be sure that all goals are being achieved with this controller. Model and controller detailed formulations are described in Appendix D, Section D-4.3; model and controller validations are presented below.

With this model and controller, FAST simulations were carried out to quantify generator speed and blade pitch in response to step wind disturbances, and results appear in Figure 7-16. The generator speed is again closely regulated to the $42 \mathrm{rpm}$ set point. 


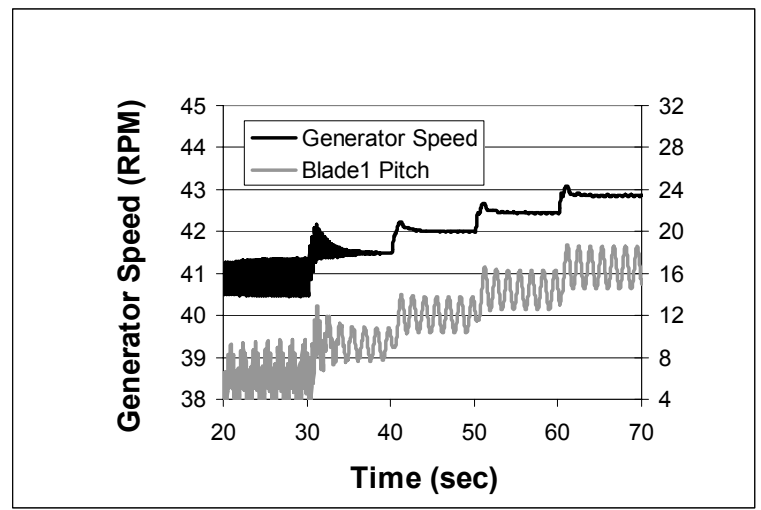

\section{Figure 7-16. Plot of CART generator speed and blade-1 pitch as simulated with FAST using DAC control designed from 9-state model}

Figure 7-17 shows FAST simulated blade flap deflection, for two different cases. In the first case, the linear wind shear disturbance gain is set for maximum attenuation of the azimuthally varying disturbance component, and in the second, the gain is set to zero. The large decrease in flap response when setting the disturbance gain for maximum attenuation shows that the DAC controller is mitigating the wind-shear response as desired.

This control results from the use of step and periodic disturbances in DAC. The mean pitch angle variation is due to mean wind-speed variations; the $1 \mathrm{P}$ cyclic variation is due to the linear wind shear variation across the rotor disk.

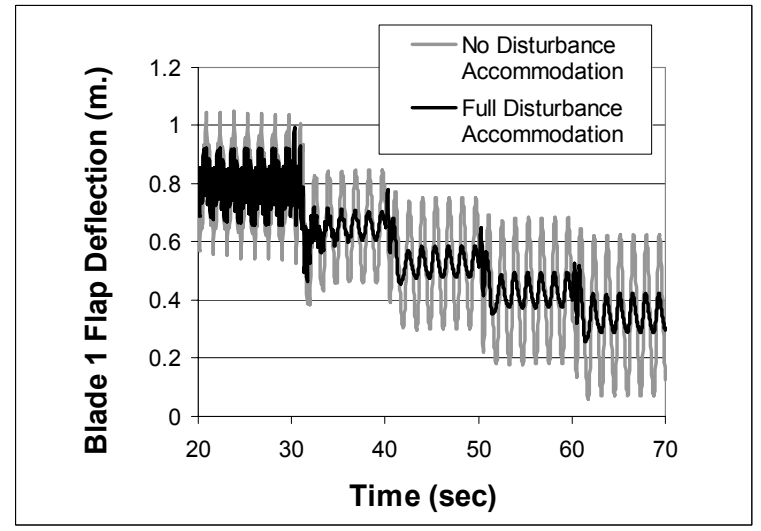

\section{Figure 7-17. FAST simulated blade-tip flap deflections showing effect of setting wind-shear disturbance gain to zero}

Figure 7-18 shows the FAST simulated tower-top fore-aft deflection. The dramatic decrease in tower fore-aft motion is readily evident when compared with Figure 7-15, showing that the controller is performing as intended. 


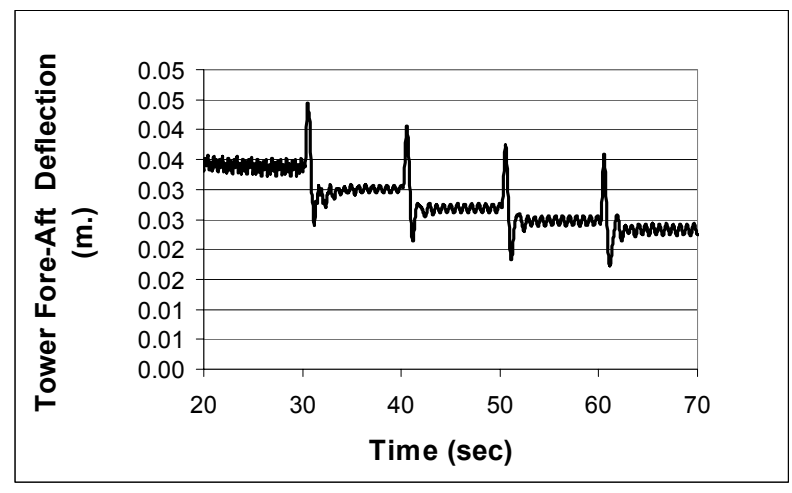

Figure 7-18. FAST simulated tower-top fore-aft deflections using two controllers providing high and low values of damping in the tower's first fore-aft mode

\subsubsection{Further Simulations}

To determine whether higher structural flexure modes degrade controller performance, selected simulations were repeated using ADAMS. The ADAMS model of the CART had fully flexible blades and tower. The drive train flexibility in ADAMS was modeled with a beam that allows both torsion and bending. In addition, the control subroutines for ADAMS were modified to perform individual blade pitch control. In FAST, all blade and tower modes were enabled.

First, identical step winds are used to excite both FAST and ADAMS models. FAST and ADAMS simulated generator speed, tower-top fore-aft deflection, and blade tip flap deflection, all show good agreement, as documented in the Figures 7-19 to 7-21. This shows that no unmodeled modes become unstable during control using these test winds.

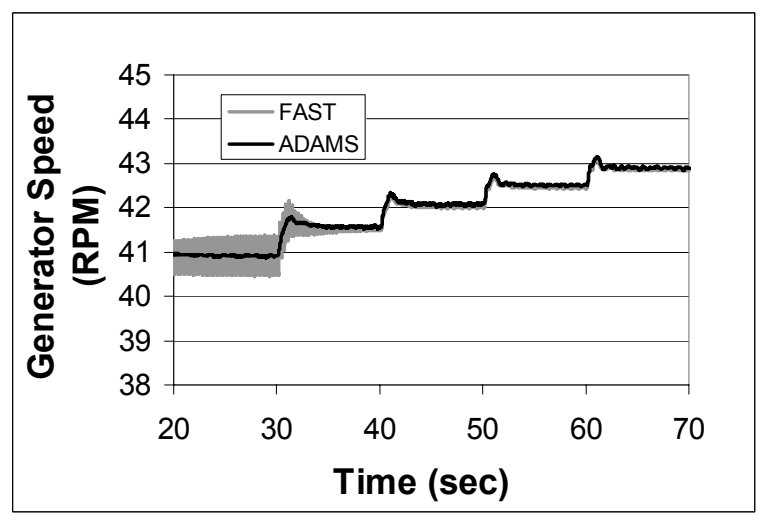

Figure 7-19. Comparisons of ADAMS (flexible blade and tower) and FAST simulated generator speed using DAC controller designed from 9-state model 


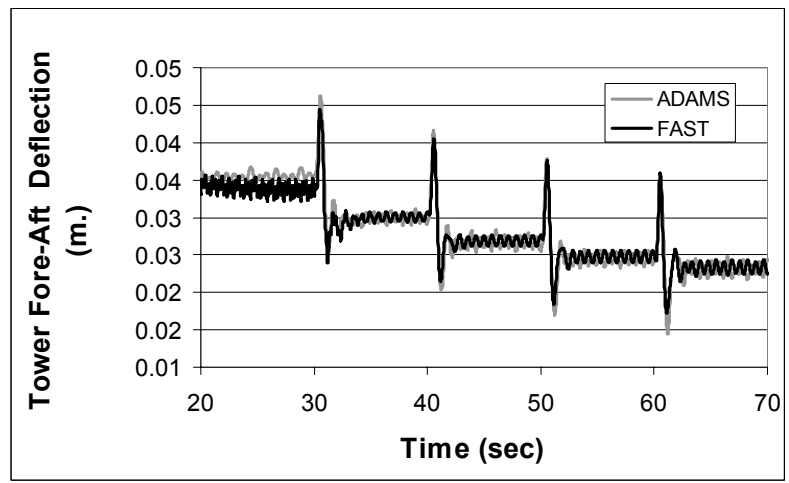

Figure 7-20. Comparisons of ADAMS (flexible blade and tower) and FAST simulated tower-top fore-aft deflection using DAC controller based on 9-state model

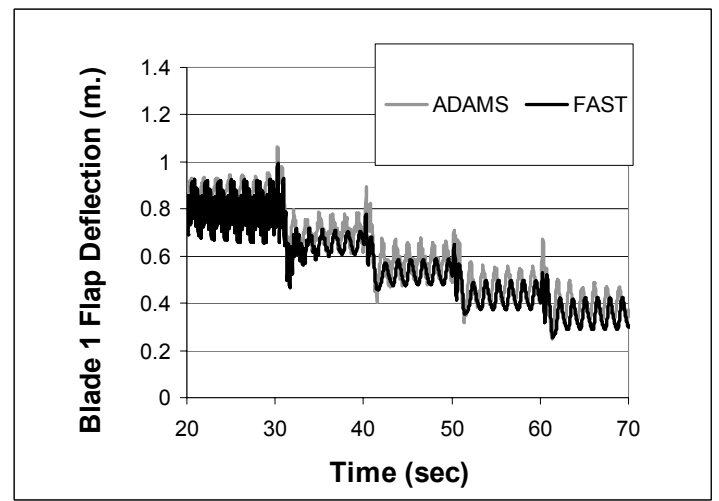

Figure 7-21. Comparisons of ADAMS (flexible blade and tower) and FAST simulated blade-tip flap deflection using DAC controller based on 9-state model

Subsequently, FAST and ADAMS models were excited with the same turbulent wind inflow case as used in Chapters 5 and 6. Figures 7-22 through 7-25 compare FAST and ADAMS simulated generator speed, tower-top fore-aft deflection, blade-tip flap displacement, and pitch rates. Pitch rates from ADAMS are higher than those from FAST, which could be caused by the greater number of flexible modes in the ADAMS simulation. As shown in Figure 7-25, these pitch rates exceed the $18 \mathrm{deg} / \mathrm{s}$ pitch rate limit imposed in the CART. 


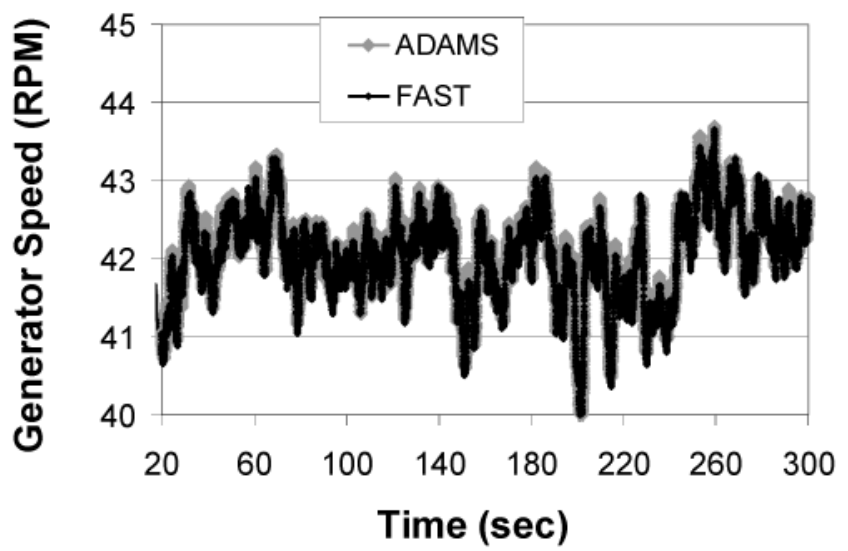

Figure 7-22. Comparisons of ADAMS (flexible blade and tower) and FAST simulated generator speed, using DAC controller based on 9-state model tested with turbulent wind inflow

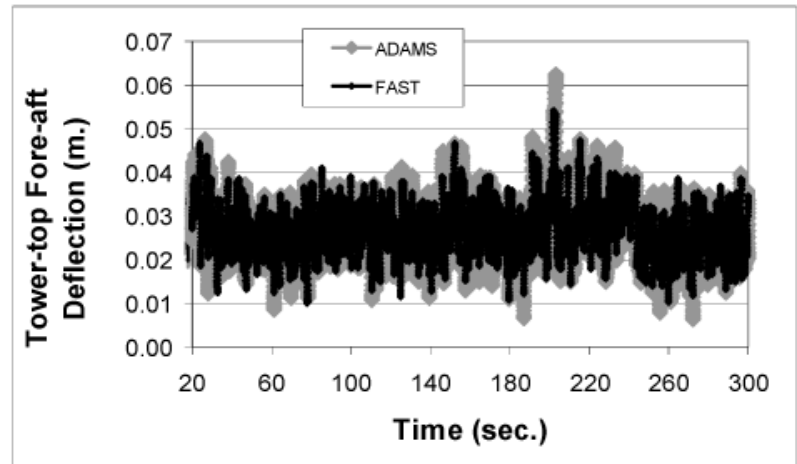

Figure 7-23. Comparisons of ADAMS (flexible blade and tower) and FAST simulated tower-top fore-aft Deflection using DAC controller based on 9-state model tested with turbulent wind inflow 


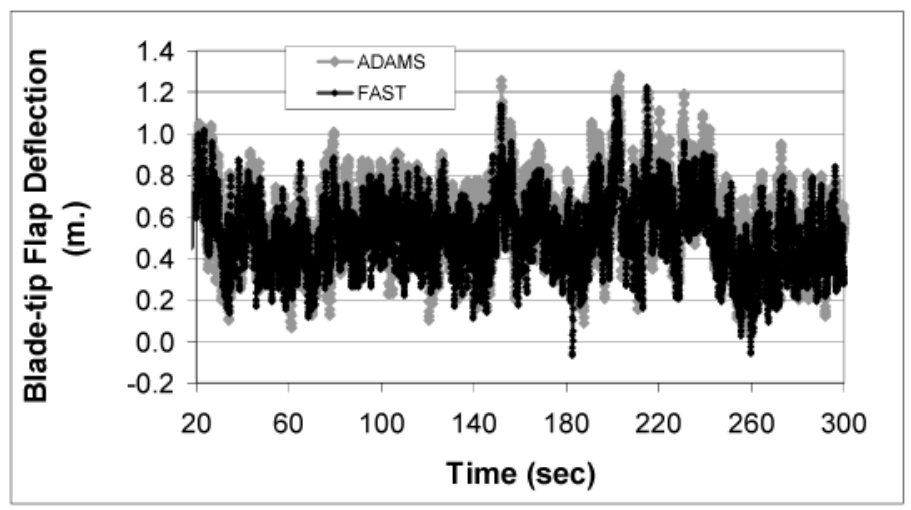

Figure 7-24. Comparisons of ADAMS (flexible blade and tower) and FAST simulated blade-tip flap deflection using DAC controller based on 9-state model tested with turbulent wind inflow

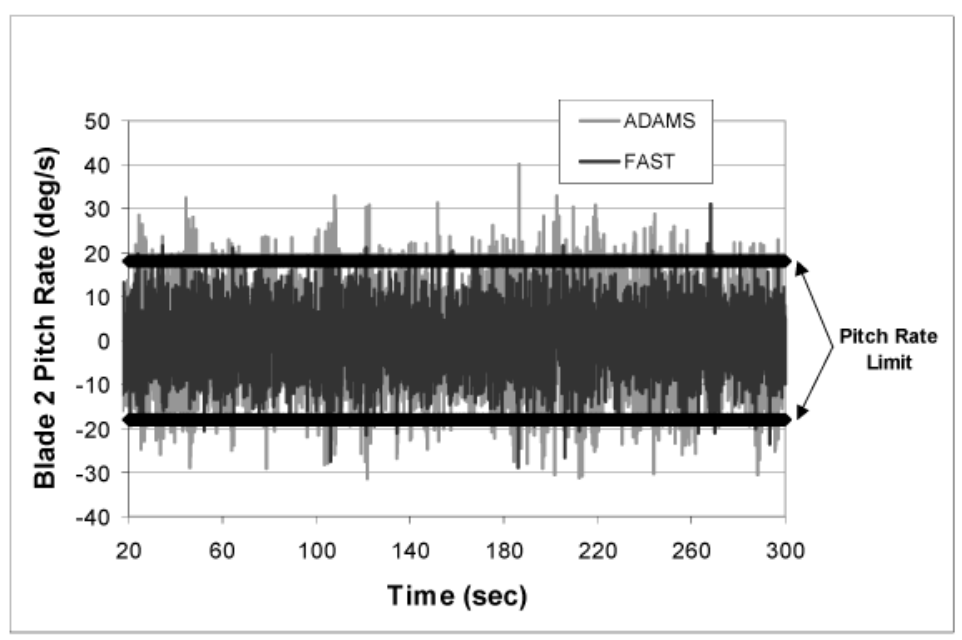

Figure 7-25. Comparisons of ADAMS (flexible blade and tower) and FAST simulated blade pitch rate using DAC controller based on 9-state model tested with turbulent wind inflow

\subsection{Addition of Generator Torque Control}

In the previous section, blade pitch rates predicted by ADAMS and FAST exceeded the CART actuator limit of 18 deg/s. To reduce these pitch rates and concurrently add damping to the first drive-train torsion mode, generator torque was investigated as a control input.

The generator torque control is designed based on the 3-state model shown in Section 6.2.3. The poles for the first drivetrain torsion mode are placed at $-2 \pm 22.6 \mathrm{j} \mathrm{r} / \mathrm{s}$. The generator speed pole is maintained at its open-loop value of -0.121 $\mathrm{r} / \mathrm{s}$, so that generator torque control will not conflict with speed regulation. The blade independent pitch control is based on the 9-state model given in Section 7.1.4. Poles for this control are placed at $-2 \pm 5.9 \mathrm{j} \mathrm{r} / \mathrm{s},-0.03 \pm 22.6 \mathrm{j} \mathrm{r} / \mathrm{s},-4.4 \pm 13.6 \mathrm{j}$ $\mathrm{r} / \mathrm{s},-4.5 \pm 13.1 \mathrm{j} \mathrm{r} / \mathrm{s}$, and $-2 \mathrm{r} / \mathrm{s}$. After incorporating the generator torque control and blade independent pitch control into FAST, the simulation was conducted using turbulent wind inflow.

Figure 7-26 shows a comparison between FAST and ADAMS, with generator torque control enabled in both simulations.

The generator speed results from both codes are virtually identical. Figure 7-27 shows simulated generator torque. The torque varies approximately $+/-7 \%$ of the mean torque value for this control. Figure $7-28$ shows the blade pitch rate 
from ADAMS for two cases in which drive-train damping is augmented via generator torque and blade pitch. The same comparison from FAST is shown in Figure 7-29. Both codes show a reduction in pitch rates when using generator torque control to add damping to the drive train. This shows that generator torque control can be used with independent blade pitch control to add damping to the first drive-train torsion mode while reducing blade pitch rates.

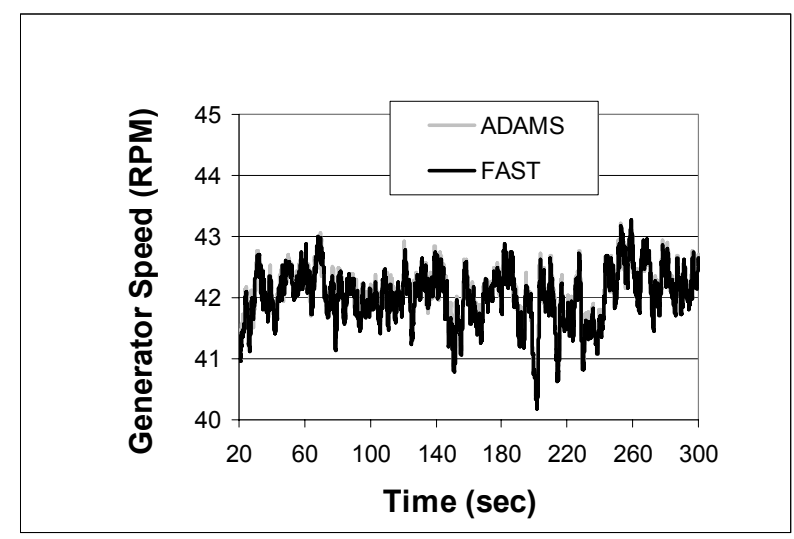

Figure 7-26. FAST and ADAMS simulated generator speed, using generator torque control to add damping to drive-train torsion, independent pitch control designed from 9-state model

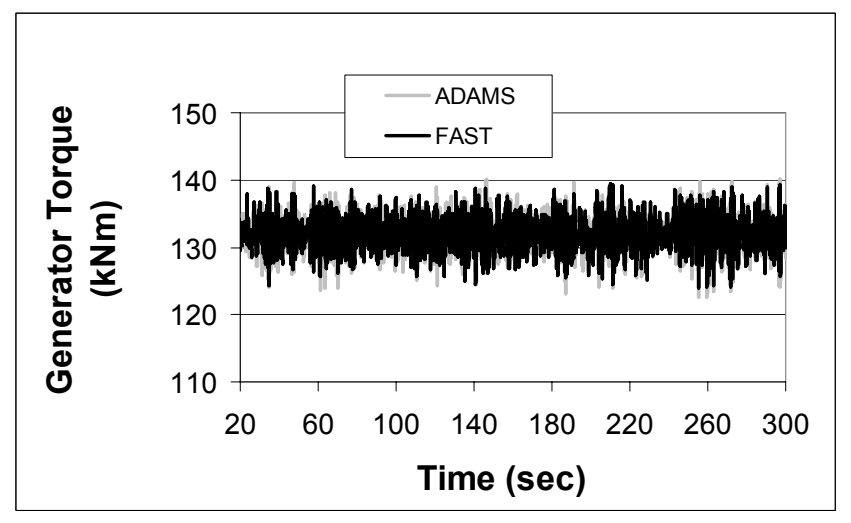

Figure 7-27. FAST and ADAMS simulated generator torque, using generator torque control to add damping to drive-train torsion, independent pitch control designed from 9-state model 


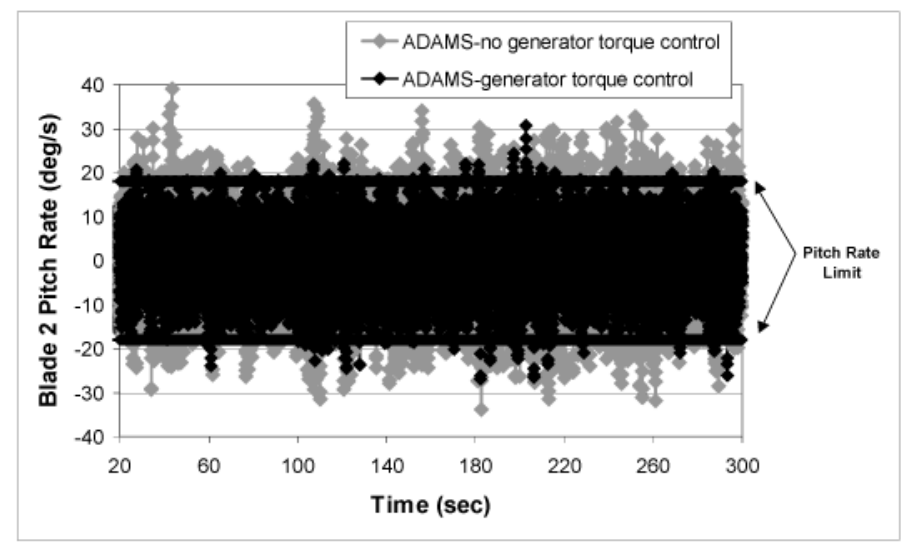

Figure 7-28. ADAMS (flexible blade and tower) simulated blade pitch rate using DAC controller based on 9-state model, with and without generator torque control

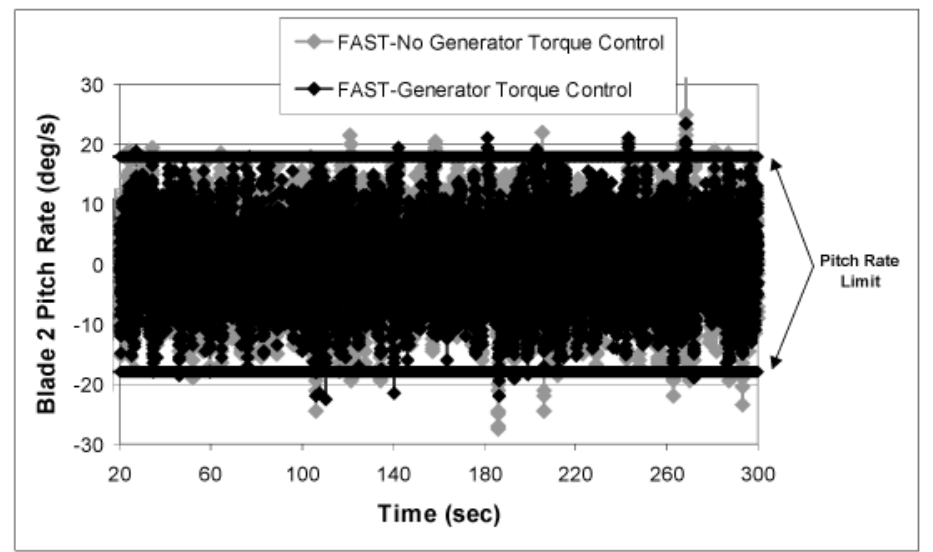

Figure 7-29. FAST simulated blade pitch rate using DAC controller based on 9-state model, with and without generator torque control

\subsection{Chapter Conclusions}

In this chapter, blade-independent pitch control has been used to regulate speed in region 3. Adding an extra control input stabilized the rotor first asymmetric flap mode and attenuated the effects of spatial wind speed variations over the rotor disk. The best attenuation of wind shear occurred when extra disturbance states were added to DAC to model azimuthal wind-speed variation. A large reduction in blade flap response to wind shear was achieved by using controls designed from DAC, including extra disturbance states.

In addition, control was designed from a 9-state model which included blade-1 and -2 flap, first drive-train torsion, generator and rotor speed, and tower first fore-aft. The DAC designed from this model regulated generator speed as desired and stabilized rotor first symmetric and asymmetric flap modes. It also stabilized the first drive-train torsion mode and enhanced tower first fore-aft mode damping. Finally, independent blade ptich control attenuated response to wind shear.

To achieve this control using state estimation, measurement of rotor first asymmetric flap deflection was needed, to effectively use blade independent pitch. The DAC based on the 9-state model required measurement of generator speed, tower-top fore-aft deflection, and rotor first asymmetric flap deflection. Adding generator torque as a control input increased damping to the first drive-train torsion mode. This mitigated demands on the blade independent pitch control system by reducing pitch rates to modest levels. 
Simulations in FAST, with all flexible modes switched on, and in ADAMS, with fully flexible blades and tower, indicated that no additional flexible modes became unstable with the designed controls. These simulations were performed using independent blade pitch and generator torque as control inputs. In simulations performed using turbulent wind inflow, good performance of the designed controls was also shown. 


\section{Chapter 8. Comparisons between Modern and Classical Control Results}

\section{Introduction}

In this chapter, results for the modern controls designed in previous chapters are compared with results for simple PI controls for the CART. These comparisons will provide a measure of the performance of the modern controls.

\subsection{Ground Rules for the Comparisons}

Section 3.2.2 highlighted a problem with the use of classical control for CART turbine speed regulation. Implementing the actual CART PI control gains resulted in unstable closed-loop operation, as simulated with FAST. The PI controller yielded stable operation for a stiff turbine, as shown by simulating the CART with no flexible modes enabled. However, simulations with the first drive-train torsion mode produced unstable operation.

When the pitch actuator was implemented in FAST, operation was stable with PI control (when simulating the flexible turbine), revealing the central role of the actuator in closed-loop dynamics. Apparently, the pitch actuator behaves like a low-pass filter, attenuating higher frequency flexible modes previously destabilized by PI pitch control. It is also possible that this actuator degrades DAC, which has been designed to add damping to various turbine flexible modes. Pitch inputs must be allowed to enter at machine flexible mode frequencies in order for the DAC to perform as intended. The effects of the actuator on DAC performance must be assessed. Thus, the effects of including the actuator in both DAC and PI control will be shown.

The same DOF are simulated in both the DAC and the PI control cases, including all blade, tower, and first drive-train torsion modes. In both the PI and DAC cases, the structural dynamic damping is set to zero, to represent the limiting condition. Both simulations use the same integrator step size, and the same wind inflow is used to excite both simulations. These cases are simulated only with FAST.

\subsection{Effect of Actuator Lag on DAC Performance}

The influence of actuator dynamics on the DAC and PI results is shown by implementing the actuator transfer function in the control subroutines. The actuator model is

$$
\operatorname{Act}(s)=\frac{1}{\tau s+1}
$$

The effects of actuator lag are studied using the DAC developed in Section 7.3 using the 9-state model. The results from this control are compared to PI results.

In this DAC, independent blade pitch was used to attenuate wind-shear response and to enhance damping in flexible modes. In addition, generator torque was used to add damping to the first drive-train torsion mode. Simulation inputs were step winds, as used in Chapter 7.

Figure 8-1 shows FAST simulated generator speed for two actuator speeds in response to successive step increases in wind speed. Actuator speed has only a small effect on regulated speed; the slow actuator $(\operatorname{tau}=0.2)$ response and fast actuator $(\operatorname{tau}=0.05)$ response are nearly identical. 


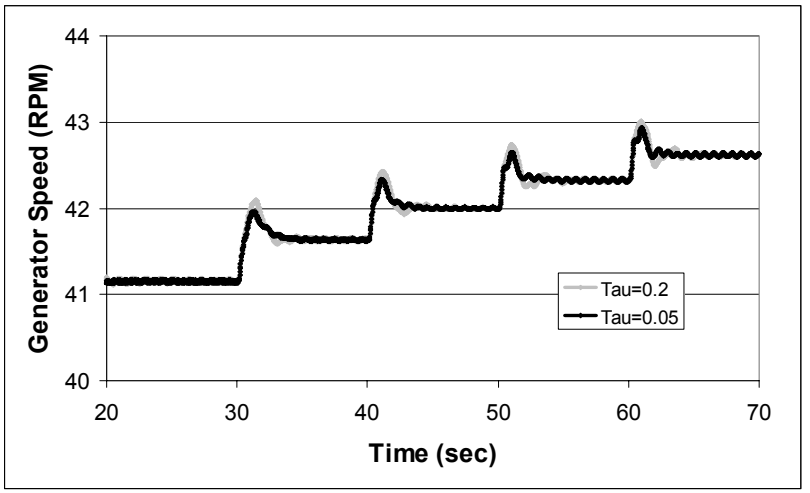

Figure 8-1. FAST simulated generator speed, showing effects of actuator time constants, DAC designed from 9-state model, with generator torque control

Actuator speed has a much larger effect on tower fore-aft deflection, as seen in Figure 8-2. Slow pitch actuation prevents sufficient pitch input from entering the system to add damping to the tower motion. As the actuator time constant decreases (speed becomes faster), the controller's ability to add damping to this mode improves.

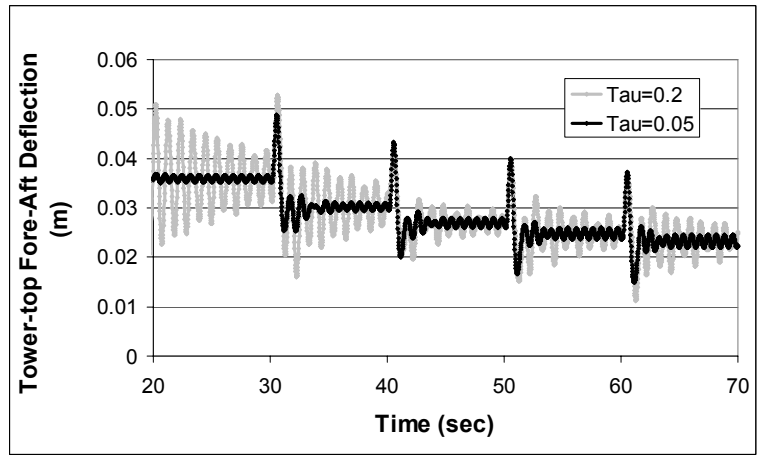

Figure 8-2. FAST simulated tower-top fore-aft deflection, showing effects of actuator time constants, DAC designed from 9-state model, with generator torque control

Figure 8-3 shows the effects of the pitch actuator on blade-tip flap deflections. The actuator lag has a small effect on the attenuation of the effects of wind shear. As the actuator time constant decreases, the blade flap deflections decrease slightly, showing that the controller is attenuating wind-shear effects with slightly better performance. 


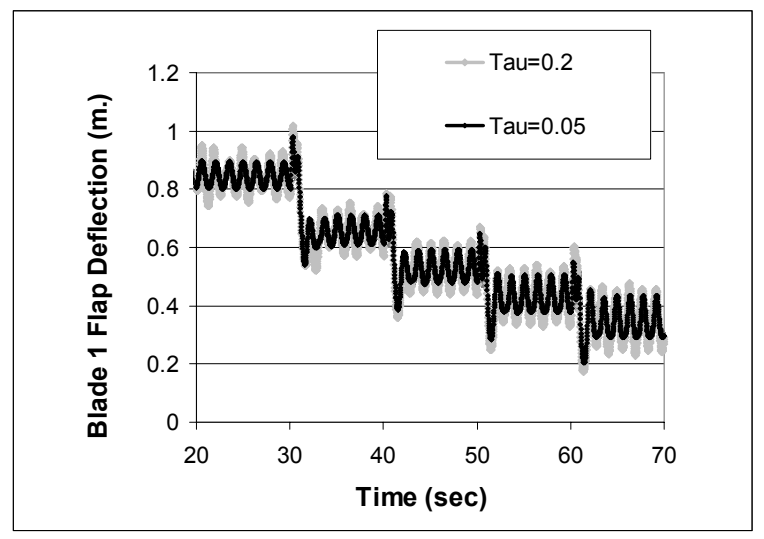

Figure 8-3. FAST simulated blade-tip flap deflection, showing effects of actuator time constants, DAC designed from 9-state model, with generator torque control

\subsection{Comparisons between DAC and PI}

DAC results are now compared to PI results. Again, the DAC designed from the 9-state model in Section 7.3 is used, and the step winds are used as inputs to both the DAC and PI control simulations. The PI control was simulated in FAST as in Chapter 3, with the PI gains set to $\mathrm{K}_{\mathrm{i}}=0.2618$ and $\mathrm{K}_{\mathrm{p}}=1.2217$.

It was shown in Chapter 3 that PI control regulates generator speed within a narrow limit. In order to obtain similar speed regulation with DAC, the gain corresponding to the step disturbance state $\left(G_{D}\right)$ was increased (compared with previous cases), which resulted in improved speed regulation. Another way to improve speed regulation would be to place the pole corresponding to the generator speed state further to the left in the complex plane than it appears in previous designs. Figure 8-4 shows simulated generator speed from FAST, comparing DAC with PI, for the actuator time constant 0.2 and 0.05 . Speed regulation for DAC and PI using the slow actuator $(\tau=0.2)$ is comparable, although DAC still does not regulate speed as robustly as PI control.
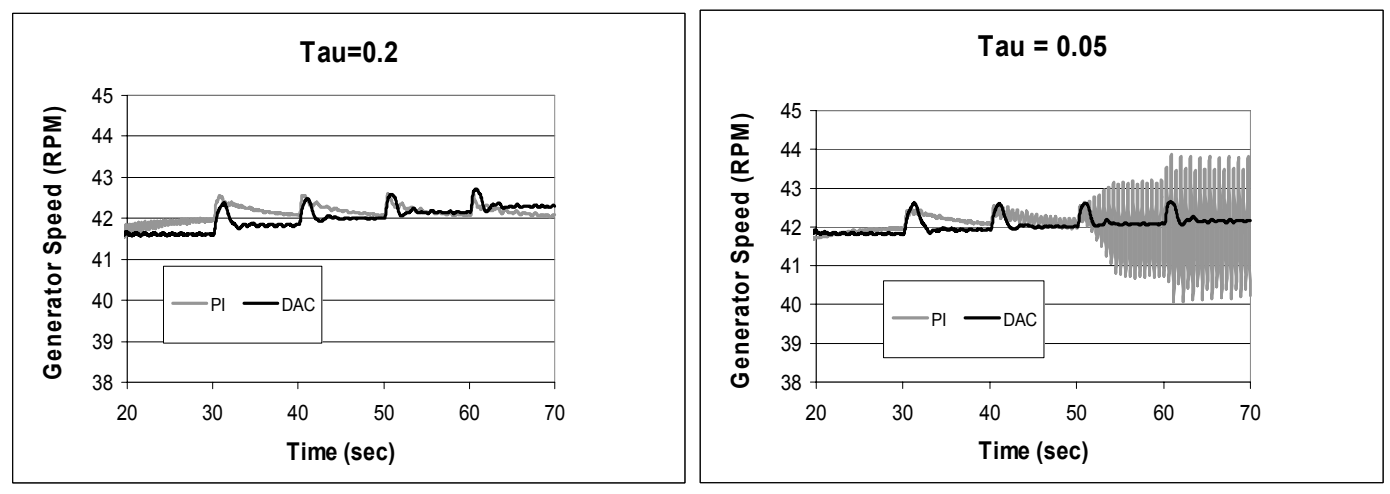

Figure 8-4. FAST simulated generator speed, comparing DAC and PI control results for slow and fast actuator

Figure 8-4 also shows a comparison of DAC with PI for a faster actuator $(\tau=0.05)$. Here, it can be seen that the fast actuator has an adverse effect on the PI speed regulation, with response becoming unstable as wind speed increases. This is similar to the results in Chapter 3, in which we showed the PI control simulated with first drive-train torsion and blade first flap for the case of no actuator. In the limit, the "no actuator" case represents an infinitely fast actuator, which allows pitch inputs to enter the system at high frequencies and destabilize machine flexible modes using PI control. The 
DAC results remain stable or improve with the fast actuator because the DAC has been specifically designed to add damping to these modes, ensuring closed-loop stability.

Figures 8-5 shows comparisons for the low-speed shaft torque for the same actuator time constants. Again we see that the fast actuator adversely effects PI control, but the system remains stable under DAC.
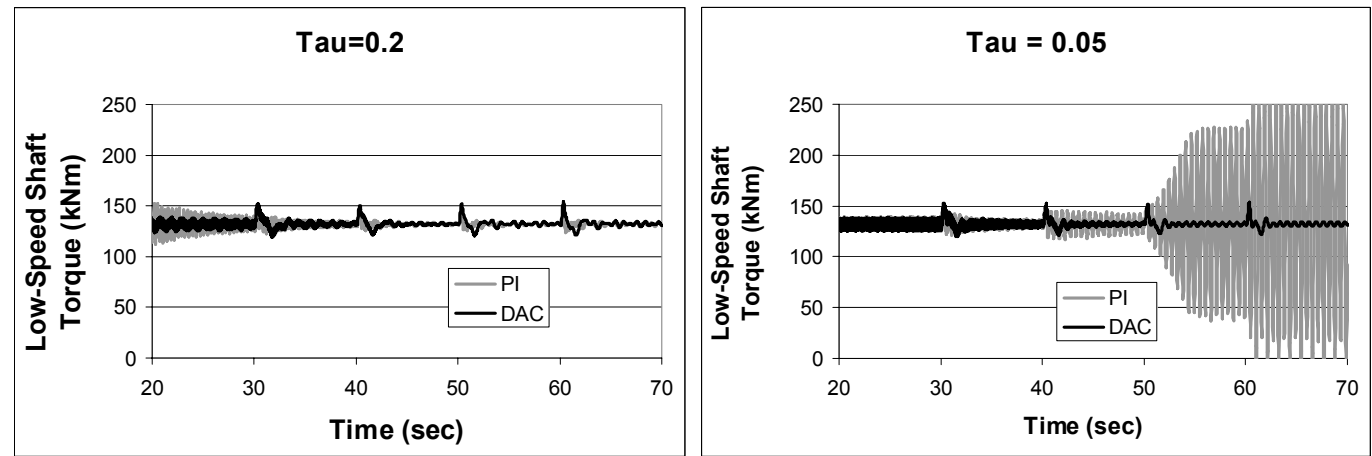

Figure 8-5. FAST simulated low-speed shaft torque, comparing DAC and PI control results for tau $=0.2$

Figure 8-6 compares simulated tower-top fore-aft deflection for PI and DAC, for the slow actuator. The slow actuator hinders the DAC from adding damping to the tower first fore-aft mode. However, DAC performance still surpasses PI control, because PI control has not been designed to add damping to the tower first fore-aft mode. The DAC benefits from a faster actuator, as shown in Figure 8.6, with tower-top fore-aft deflections showing much improved damping. Figure 8-6 again shows the adverse effect of a faster actuator rate on the PI control, with tower-top motion becoming unstable at higher wind speeds.
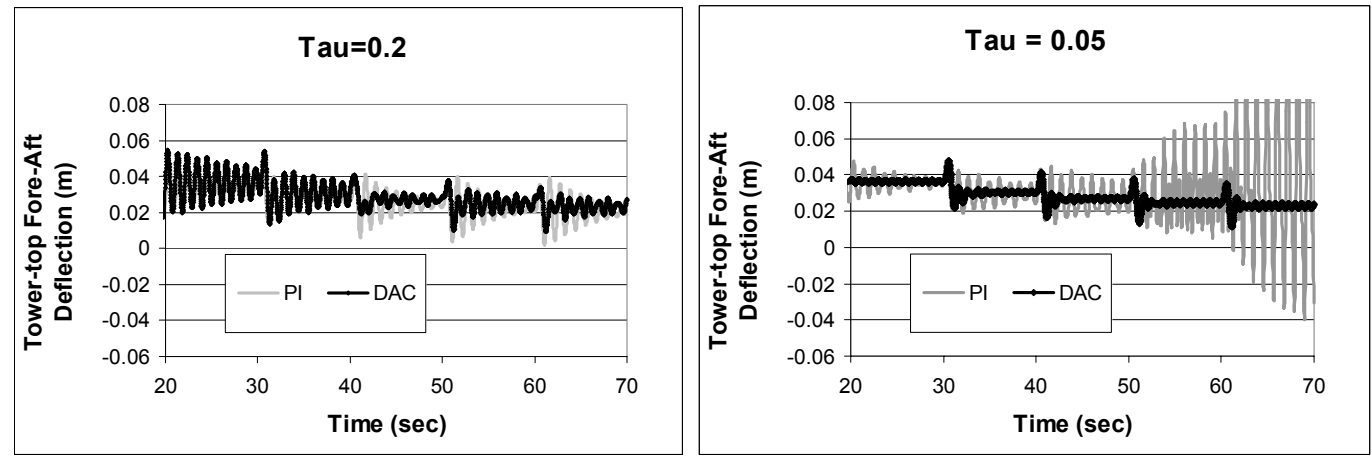

\section{Figure 8-6. FAST simulated tower-top fore-aft deflection, comparing DAC and PI control results for a fast and slow actuator}

Figures 8-7 shows comparisons between DAC and PI, for the slow and fast actuator, for blade-tip flap displacement. DAC outperforms PI in both cases, with DAC performance improving slightly for the faster actuator. The PI control has not been designed to attenuate the effects of wind shear. It can be seen that the fast actuator destabilizes PI control. 

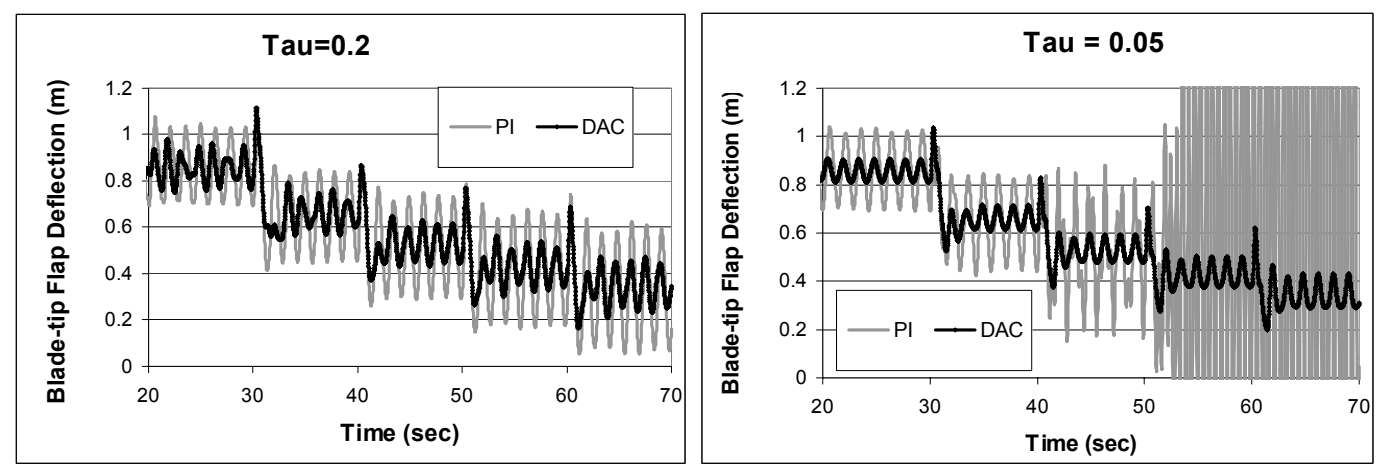

Figure 8-7. FAST simulated blade-tip flap deflection, comparing DAC and PI control results
for a slow and fast actuator

The previous comparisons are repeated, this time exciting the turbine with turbulent inflow instead of step winds. Figure 8-8 shows regulated generator speed for DAC and PI for the actuator time constant 0.2 . The DAC results show slightly greater variation, indicating that DAC is not regulating speed as precisely as PI control. This is a direct result of the decreases in speed regulation robustness as the number of flexible modes that must be stabilized with DAC increases. A simpler controller such as PI can be designed to perform very robust speed regulation. Table 8-1 shows the standard deviations (sd) of the results from the turbulent wind inflow simulations; greater standard deviation is shown for the DAC results in comparison to the PI results for the slow actuator. Figure 8-8 also shows this comparison for the faster actuator. The fast actuator causes the PI control to become unstable. The DAC results for the fast actuator show slightly improved speed regulation $(\mathrm{sd}=0.325)$, compared with the slow actuator $(\mathrm{sd}=0.371)$.

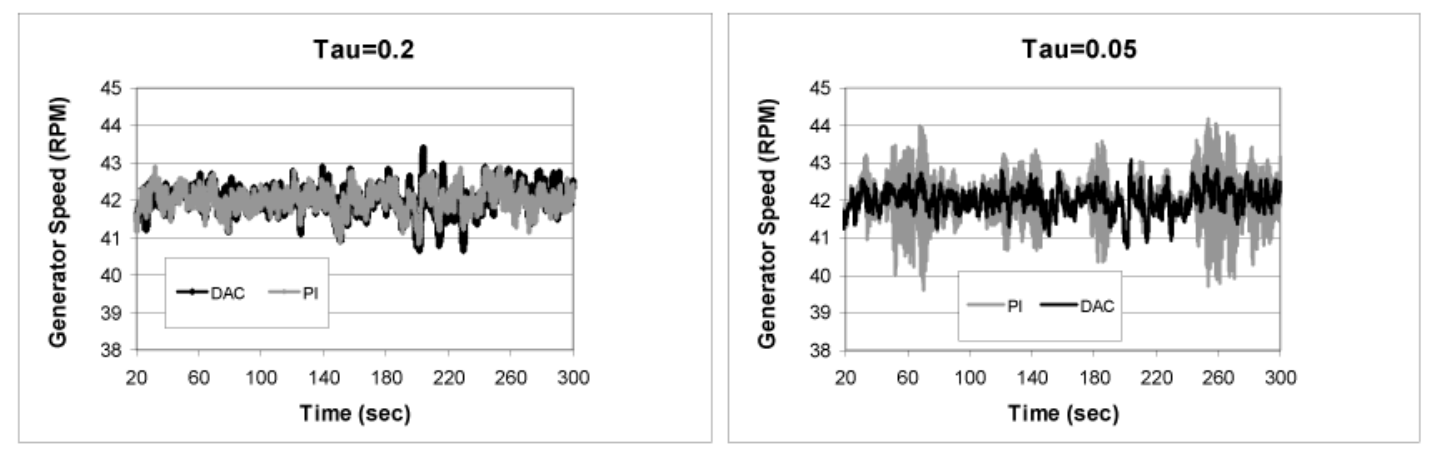

Figure 8-8. FAST simulated generator speed, comparing DAC and PI control results for a slow and fast actuator

Table 8-1. Standard Deviations

\begin{tabular}{|l|l|l|l|l|}
\hline \multicolumn{3}{|c|}{ Slow Actuator $(\tau=0.2)$} & \multicolumn{2}{l|}{ Fast Actuator $(\tau=0.05)$} \\
\hline & DAC & PI & DAC & PI \\
\hline Generator Speed & 0.371 & 0.314 & 0.325 & 0.584 \\
\hline $\begin{array}{l}\text { Tower-top Fore-aft } \\
\text { Deflection }\end{array}$ & .0136 & .0119 & .0081 & .0161 \\
\hline Drive-train Torsion & 9.37 & 9.60 & 9.91 & 40.6 \\
\hline $\begin{array}{l}\text { Blade Root Flap } \\
\text { Moment }\end{array}$ & 77.1 & 82.6 & 72.9 & 235. \\
\hline
\end{tabular}

The low-speed shaft torque is shown in Figure 8-9. For the slow actuator, the low-speed shaft torque with DAC has a smaller variation $(\mathrm{sd}=9.37)$ in comparison to that of $\mathrm{PI}(\mathrm{sd}=9.60)$. This is a direct result of designing DAC to enhance damping in the first drive-train torsion mode using generator torque control. It is interesting to note that the slow pitch actuator does not hinder the ability of DAC to enhance damping in this mode, since generator torque is adding damping to this mode instead of blade pitch. From Figure 8-9, it is again seen that the faster actuator has a very adverse effect on 
the PI control $(\mathrm{sd}=40.6)$ but causes negligible degradation of the DAC. Again, this is because the faster actuator allows pitch inputs to enter the system, destabilizing flexible modes of the turbine, especially the first drive-train torsion mode.
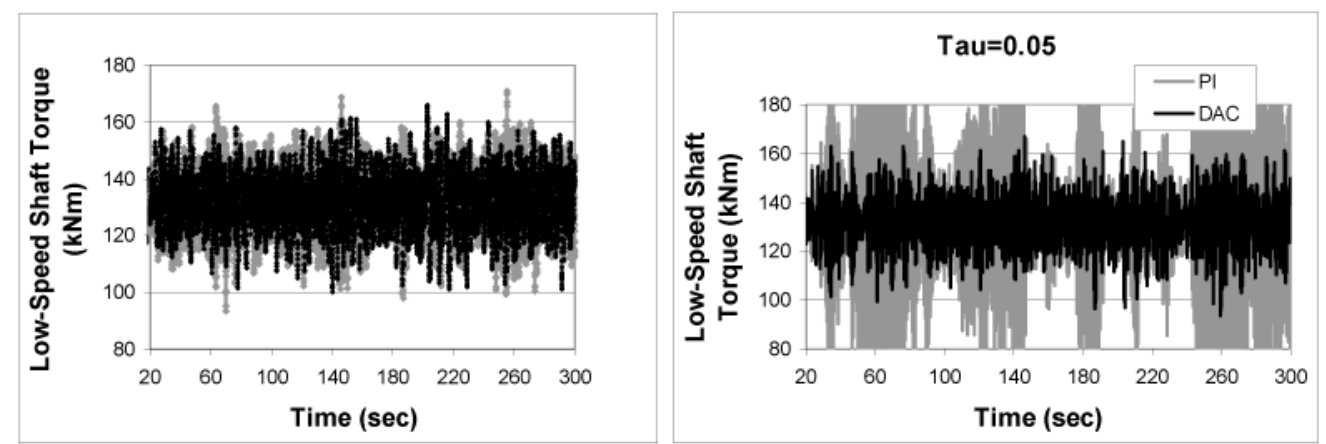

Figure 8-9. FAST simulated low-speed shaft torque, comparing DAC and PI control results for a slow and fast actuator

Tower-top fore-aft deflections are shown in Figure 8-10. For the slow actuator $(\tau=0.2)$, the DAC simulated towertop deflections have greater variation $(\mathrm{sd}=0.0136)$ than those for the PI control $(\mathrm{sd}=0.0119)$. This result is caused by the slow actuation hindering DAC's ability to add damping to the tower first fore-aft mode. The faster actuator seen in Figure 8-10 improves the DAC results $(\mathrm{sd}=0.0081)$ compared with results for the slow actuator for $\mathrm{DAC}(\mathrm{sd}=0.0136)$, whereas it destabilizes the PI control $(\mathrm{sd}=0.0161)$.
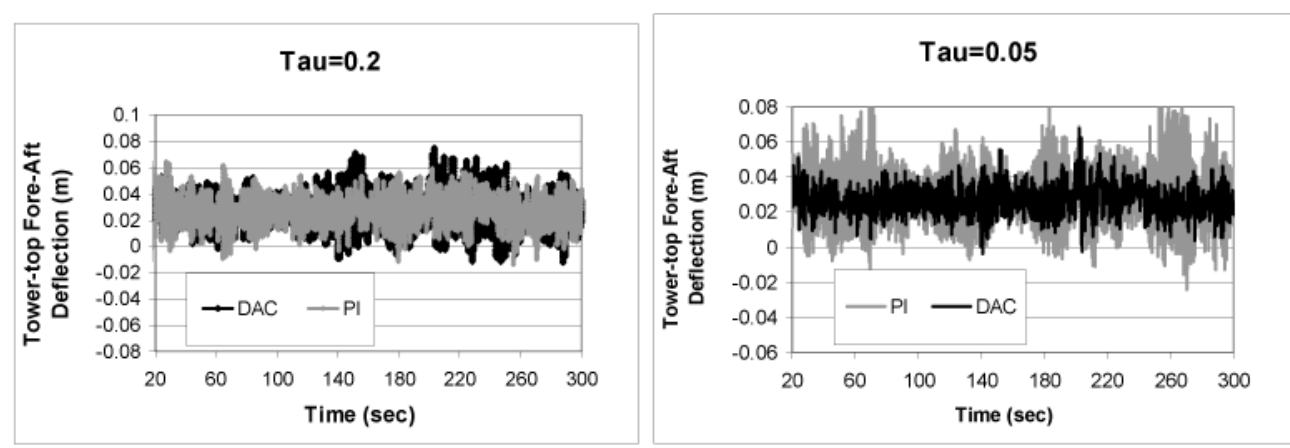

\section{Figure 8-10. FAST simulated tower-top fore-aft deflection, comparing DAC and PI control results for a slow and fast actuator}

Figure 8-11 shows simulated blade-tip flap deflection. For the slow actuator, the DAC results have smaller variation $(\mathrm{sd}=7.1)$ compared with the results from PI control $(\mathrm{sd}=82.6)$. This is because DAC has been designed to attenuate the effects of spatial variations of wind speed across the rotor disk (namely, the wind-shear component at 1P). Again, it can be seen in Figure 8-11 that the fast actuator destabilizes the PI results $(\mathrm{sd}=235)$, with large flap deflections. The faster actuator slightly improves the ability of the DAC to attenuate wind-shear effects $(\mathrm{sd}=72.9)$ in comparison to the slow actuator $(\mathrm{sd}=77.1)$. 

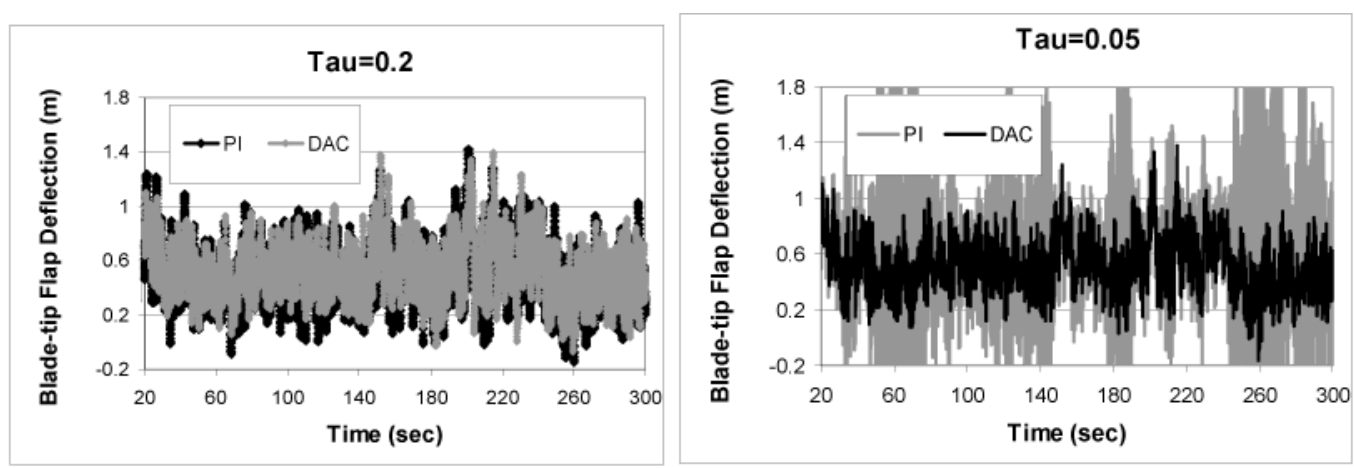

\section{Figure 8-11. FAST simulated blade-tip flap deflection, comparing DAC and PI control results for a slow and fast actuator}

Figure 8-12 shows pitch rates for the two controllers for the slow and fast actuators. For the slow actuator, the pitch rates using DAC are higher than those using PI control. This figure also shows realistic CART pitch rate limits $(18 \mathrm{deg} / \mathrm{s})$. For the fast actuator, the pitch rates from both controllers increase. The greatest increase is for the PI control, in which pitch rates have greatly increased because of the unstable behavior of this system. The DAC pitch rates have also slightly increased with the fast actuator. For both actuators, the pitch rates using DAC sometimes exceed the $18 \mathrm{deg} / \mathrm{s}$ pitch rate limit. These pitch rates could be reduced by decreasing the gains corresponding to the step and shear disturbances. The penalty for these decreased gains would be less precise speed regulation and a reduced ability to attenuate the effects of the $1 \mathrm{P}$ component of wind shear.
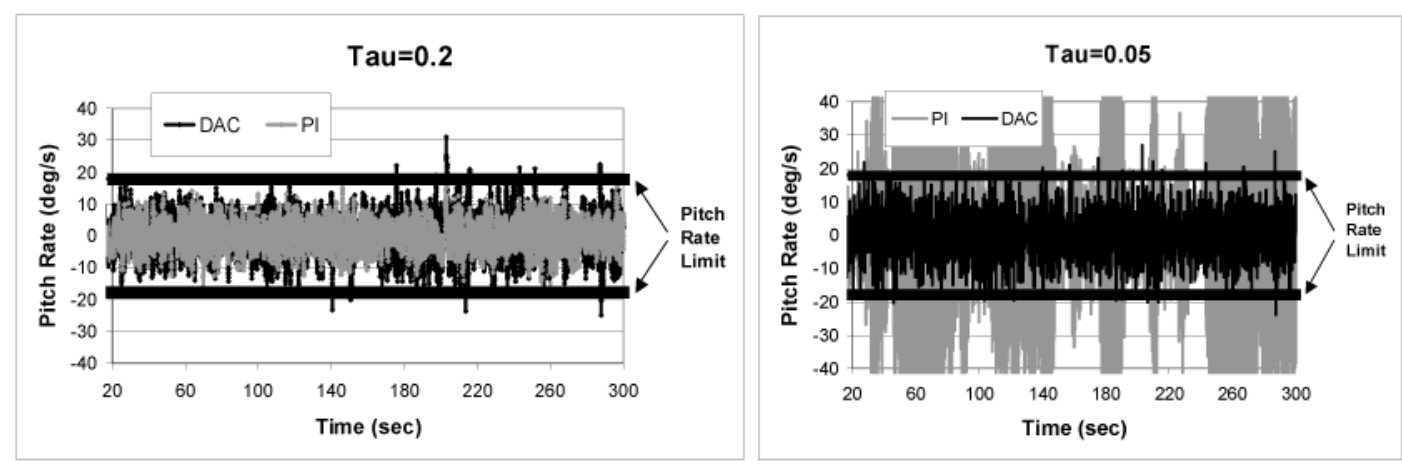

Figure 8-12. FAST simulated blade pitch rate, comparing DAC and PI control results for a slow and fast actuator

In general, DAC performance improves as actuation speed increases, while PI control is adversely affected. The PI control has not been designed to properly account for the flexible modes of the turbine. The faster actuator allows pitch inputs to enter the system, which destabilize the flexible modes with PI control.

The slow actuator has an adverse effect on the DAC performance. The DAC has been designed to add stability to various flexible modes of the turbine. Slow actuators prevent pitch inputs from entering the system, which enhance damping of these flexible modes, especially the tower first fore-aft mode. If the speed of realistic actuators cannot be increased, then these modern controls need to be designed to properly account for these slow actuators. A method to account for these actuators is shown below.

\subsection{Results with Actuator Dynamics Included in Control Design}

One way to account for actuators in DAC design is to include a description of actuator dynamics in the linear model used for DAC design. The linear model is revised to include a description of the linear actuator dynamics. If the resulting system is controllable, then pole placement can be used to increase actuator speed. The pole corresponding to the actuator state is placed to the left of its open-loop value in the complex plane. This results in faster actuator speed. 
The actuator modeled in Equation 8.1 can be represented by just one state. This technique is now illustrated using a DAC designed from the 7-state model of Section 5.5, using rotor collective pitch to control speed and add damping to the tower first fore-aft mode. The derivation of a more general case for including actuator dynamics is shown in Appendix C, Section C-1.8.

For the actuator represented by the transfer function shown in Equation 8.1, $A_{A}=\frac{-1}{\tau}$, where $A_{A}$ represents the state matrix for the actuator model. The linear model including the actuator state is shown in Equation 8.2. Note that the previous pitch input matrix B (see Equation 5.5) now appears in the A matrix, in the right-hand column and the first 7 rows. The new $\mathrm{B}$ matrix contains all zeros except for the last row.

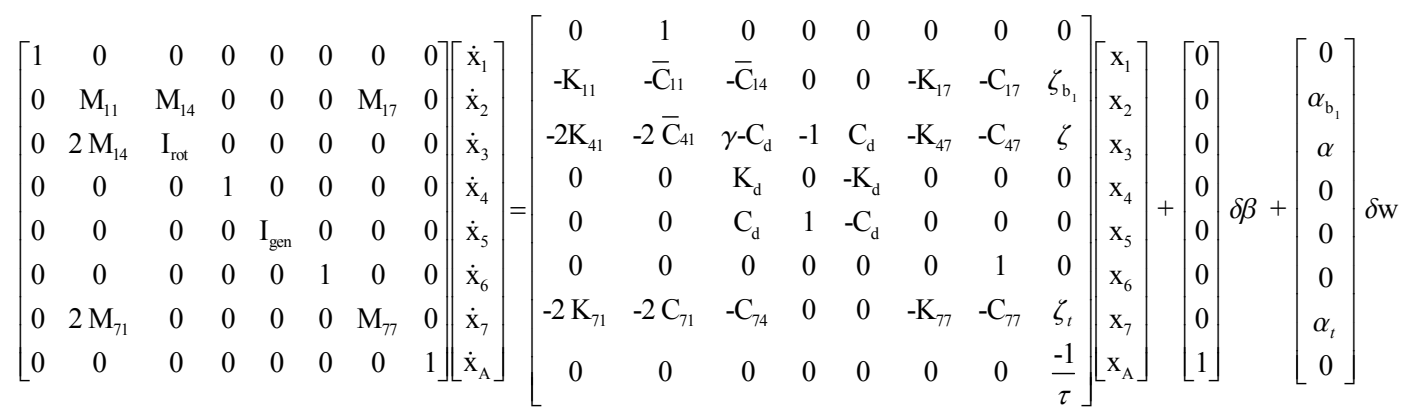

$$
\begin{aligned}
& y=\left[\begin{array}{llllllll}
0 & 0 & 0 & 0 & 1 & 0 & 0 & 0 \\
0 & 0 & 0 & 0 & 0 & 1 & 0 & 0
\end{array}\right]\left[\begin{array}{c}
\mathrm{x}_{1} \\
\mathrm{x}_{2} \\
\mathrm{x}_{3} \\
\mathrm{x}_{4} \\
\mathrm{x}_{5} \\
\mathrm{x}_{6} \\
\mathrm{x}_{7} \\
\mathrm{x}_{\mathrm{A}}
\end{array}\right] .
\end{aligned}
$$

This system is controllable, and closed-loop poles can be placed arbitrarily. For the actuator with a time constant of $\tau=0.2$, the pole of the actuator state is located at -5 . To increase the actuator speed, this pole should be placed far to the left in the complex plane, perhaps at -50 . This can be accomplished with full-state feedback, assuming that all of states in this model are measured, including the actuator state.

Instead of using full-state feedback, the goal is to use state estimation, measuring only generator speed and tower-top fore-aft deflection. This requires observability of the system. As shown in Appendix D, this system is observable and state estimation can be applied.

After completing this DAC design, simulating with step winds gives the tower-top fore-aft response shown in Figure 813 (Actuator Incorporated in Design). This figure shows the effect on tower response when including the actuator model in the control design and using full-state feedback to improve actuator speed (through pole placement). Including the actuator model in the control design enhances the actuator response and improves the damping added by the control system to the tower first fore-aft mode. The improvement in tower damping is very evident when the actuator model is included in the linear model for control design, and full-state feedback is used to place the actuator pole to increase actuation speed. Note that only generator speed and tower-top fore-aft deflection are measured, using state estimation of the states in the model, including the actuator state. 


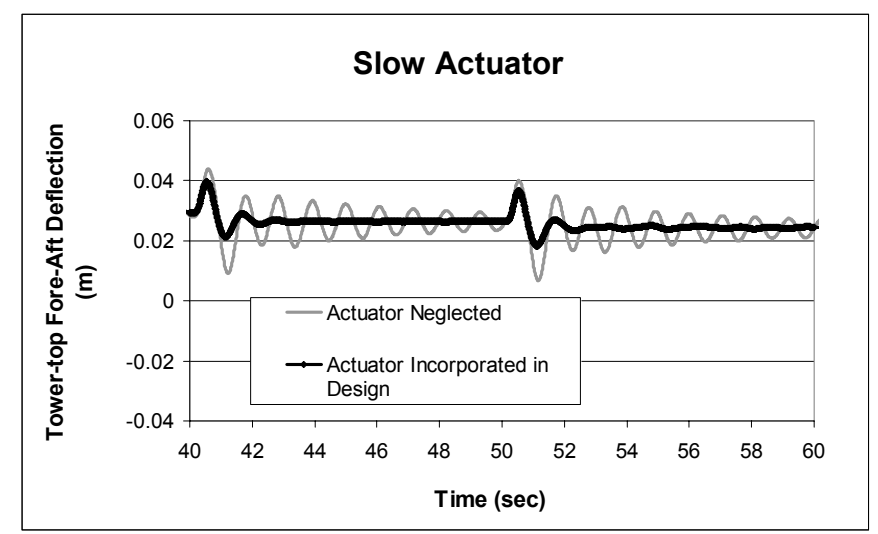

\section{Figure 8-13. FAST simulated tower-top fore-aft motion, showing effects of including actuator in design}

In Figure 8-14, DAC results are again compared with PI results, and now the speed of actuation is increased; this state is included in the linear model and used for control design. The figure shows results for two cases: one using step winds to excite the model, and the other using turbulent winds. In both cases, DAC performance significantly exceeds that of the PI control.
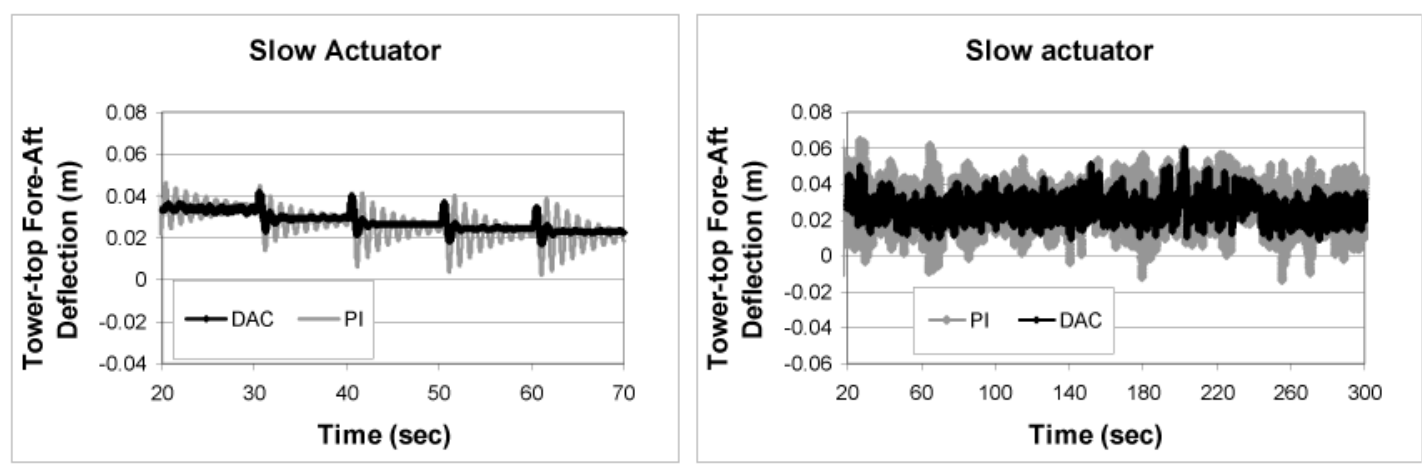

Figure 8-14. FAST simulated tower-top fore-aft deflection, comparing DAC and PI control
results, including actuator dynamics in control design for step and turbulent winds

The pitch control system now delivers pitch at the frequencies required to achieve the desired damping of the tower first fore-aft mode. The results for this DAC are comparable to the results for the fast actuator (without including the actuator dynamics in the linear model) when excited by step and turbulent winds. This shows that accounting for actuator dynamics in the control design is feasible. Further work must be performed to include the actuator model for independent blade pitch control as well as both step and wind-shear disturbance effects.

\subsection{Chapter Conclusions}

In this chapter, results for the modern control design of Chapter 7 were compared with results for PI control for the CART. Actuator speed exercised a significant impact; modern control designs using DAC and state estimation benefited from a fast actuator, and PI control benefited from a slow actuator. The fast actuator injects high-frequency pitch inputs into the turbine system. Since the PI control has not been designed to stabilize flexible modes, faster actuators and associated higher frequency pitch inputs destabilize flexible modes.

The slow actuator hampers DAC enhancement of tower first fore-aft mode damping. This is because of the low-pass filtering effects of the actuator, blocking pitch inputs at the frequencies needed to enhance damping in this mode. For the slow actuator, the actuator dynamics can be included in the control design, allowing a control design that effectively increases the actuator speed, through full-state feedback and pole placement. 
The benefit of including the actuator state in the control design is that damping of the tower first fore-aft mode is improved, approaching the performance of a similar DAC control when pitch actuation is assumed to be infinitely fast.

Overall, the DAC regulated turbine speed less robustly than the simple PI controller. This is caused by the effects shown in Chapter 5 when progressing from a linear model with few states to a model with several states. As increased demand is placed on the DAC to enhance damping in more flexible modes, the speed regulation performance of DAC is degraded. When a larger number of states are present in the linear model used for control design, the gain corresponding to the step wind disturbance can be selected only to minimize wind-speed disturbance effects, instead of exact cancellation. This degrades the controller's ability to regulate turbine speed. 


\section{Chapter 9. Conclusions and Recommendations}

Controls have been used to mitigate the adverse dynamic response of wind turbines for the last 30 years. Most commercial efforts targeted the relatively small machines of that era, in the 50 - to $100-\mathrm{kW}$ range. Over the last 30 years, utility-class machines have literally grown with time. Today's commercial machines are rated at more than $1.5 \mathrm{MW}$, and offshore designs are moving toward 5-10 MW. These mammoth pieces of rotating machinery, weighing hundreds of tons and suspended on slender $150-\mathrm{m}$ towers, are challenging engineers to incorporate active monitoring and control to alleviate loads. Because future applications will include offshore turbine deployment on both fixed and floating platforms, the addition of combined wind and wave loading doubles the complexity of today's applications. Advanced designs are extremely flexible and dynamically active, requiring new approaches for integrated control strategies. Therefore, this report investigated the design of controls using modern state-space methods that account for the flexibility of the turbine.

Chapter 2 reviewed the simulation and control design basis for the development of advanced controls. Using the FAST model's assumed-modes approach allowed a continuum of linear models of varying complexity ranging from 1 to 9 states. The most complex linear model included rotor and generator speed, the first flap mode of each blade, the first drive-train torsion mode, and tower first fore-aft mode. These linear models were evaluated for the Controls Advanced Research Turbine, or CART, an existing 600-kW machine at the National Wind Technology Center with many of the flexible-mode characteristics of large machines.

Simulation of simple classical PI control for the CART resulted in unstable closed-loop behavior in this flexible system (Chapter 3). Including the effects of actuator dynamics stabilized the results, because it prevented higher frequency pitch control inputs from destabilizing the system. These results highlighted the need for modern control methods that properly account for system flexibilities and allow controls to be designed to stabilize the flexible modes of the turbine.

Chapter 4 reviewed and applied the modern control design procedures used in the remaining chapters. These control design steps were illustrated in a few example cases for the CART, highlighting the design of controls to enhance damping in flexible modes of the turbine.

Chapter 5 showed Disturbance Accommodating Control, or DAC, results for designs based on a progression of linear models, starting with the simplest and concluding with the most complex. The control objective in this chapter was the regulation of turbine speed in region 3, using rotor collective pitch. Other objectives included maintaining stable closedloop behavior as well as enhancement of damping in various flexible modes of the turbine. Uniform wind disturbances over the rotor disk were accommodated with DAC.

Another objective was to determine the complexity of the linear model needed to assure the stable closed-loop performance of the machine. A model containing 5 states was needed, including rotor and generator speed, the first drive-train torsion mode, and the rotor first symmetric flap mode. These flexible modes were the most critical ones for control design using rotor collective pitch and the most likely modes to be destabilized if the control algorithm is not properly designed.

It was also shown that, as the drive-train torsional stiffness and blade flapwise stiffness are increased, simpler linear models could be used. For very stiff blades and drive train, the DAC designed from a linear model describing only turbine speed would be sufficient to maintain stable closed-loop behavior. For the CART, however, the linear model with 5 states is necessary because of the drive-train torsion and flapwise blade flexibility. We also found that using control to enhance tower first fore-aft mode damping is desirable, although not critical. Controls designed without accounting for this mode still result in stable behavior. When the tower first fore-aft mode is added to the linear model, significant damping can be added to the tower fore-aft motion with DAC. Including this additional mode, however, necessitates adding a tower-top fore-aft deflection or acceleration measurement to the design.

Speed regulation using DAC designed from the 1- and 3-state models is robust over a wide range of wind speeds when stepwise changes in wind speed are used to excite the simulation. DAC designed from the 5- and 7-state linear models results in less robust speed regulation. In general, as the linear model complexity used for control design increases, 
speed regulation becomes less robust. In controls designed from the 1- and 3-state models, the wind disturbance gain could be selected to exactly cancel step wind disturbances. For the 5- and 7-state models, this gain could only be selected to minimize these disturbances, resulting in less robust speed regulation. There is a trade-off in model complexity and the ability to design controls that robustly regulate speed. When additional flexible modes must be stabilized by the controller, the robustness of speed regulation is degraded.

Controllers designed at a turbine operating point midway between the lowest and highest wind speed in region 3 (at 18 $\mathrm{m} / \mathrm{s}$ and 12 degree pitch) results in stable operation over a broad range of wind speeds spanning region 3. Gain scheduling or controller switching, in order to improve performance, has not been firmly established. The performance obtained from these control designs is adequate without the use of gain scheduling. The use of gain scheduling or controller switching might improve the estimation of wind speed, especially for DAC designed from the models containing 5 and 7 states.

Chapter 6 extended the results of Chapter 5 by adding generator torque as a control input to drive-train torsion mode damping. This was shown to reduce some of the demand of the rotor collective pitch control system, because the requirement to enhance damping in the first drive-train torsion mode is removed from the pitch control system and added to the generator torque control system. Use of generator torque control has been shown to improve controllability of the first drive-train torsion mode, especially at the low-wind-speed end of region $3(14 \mathrm{~m} / \mathrm{s})$.

Independent blade pitch control, introduced in Chapter 7, uses this control to not only regulate turbine speed but also attenuate the effects of spatial variations of wind speed over the rotor disk (such as wind shear). A new disturbance was added to the DAC model to describe the dominant component of wind shear, the 1P component that has spatial variation over the rotor disk. A large reduction in blade-tip flap deflections results from using this method. In addition, these control inputs stabilize the rotor first asymmetric flap mode, the rotor first symmetric flap mode, and the first drive-train torsion mode.

Independent pitch control required the rotor first asymmetric flap mode displacement (in addition to generator speed) to be measured. For the controls designed using rotor collective pitch control, blade flap displacement is not needed because the rotor first symmetric flap mode was observable in the generator speed measurement. When blade independent pitch is used, the rotor first asymmetric mode is contained in the linear model for control design. It was found that this mode is not observable by measuring only generator speed. The best controller performance occurred when the flap deflection of both blades was measured and transformed to obtain a measurement of the rotor first asymmetric mode. The improved control results are the result of improved observability when measuring rotor first asymmetric flap.

Extra disturbance terms in DAC were used to model the uniform and shear component of wind across the rotor disk. When these disturbance states are included in the model, the controller performs as expected, regulating turbine speed in response to mean fluctuations in wind speed (modeled with the step disturbance) and attenuating the effects of wind shear (modeled with the 1P disturbance component). Using only full-state feedback or state estimation (without these disturbance states) results in less than desired performance. When these disturbance states are included in the control model, the speed regulation is greatly improved, as well as attenuation of the effects of wind shear.

In the last part of Chapter 7, DAC is designed from a model with 9 states describing rotor and generator speed, the flap modes of each blade, the first drive-train torsion mode, and the tower first fore-aft mode. This DAC control also accounts for the wind step disturbance and shear disturbances previously described. The resulting DAC is shown to meet several control objectives simultaneously: regulation of turbine speed; enhanced damping and stabilization of several flexible modes, such as rotor first symmetric and asymmetric flap; and first drive-train torsion and tower first fore-aft modes. It also successfully attenuates the $1 \mathrm{P}$ component of wind shear. Generator torque control was also added to enhance damping of the first drive-train torsion mode, thereby removing this task from the blade pitch control. This DAC represents the most complex control designed in this work, and it meets all of the desired control objectives.

Chapter 8 compared results for these modern control designs with results for simple PI control for the CART. In this comparison, the effects of the actuator lag were included in both the DAC and the PI results. It was shown that slow actuators degrade some of the DAC results, especially damping of the tower fore-aft motion. The slow actuator prevents DAC pitch inputs from entering the system at the frequencies required to add damping to the tower first fore-aft mode. It was also shown that fast actuation greatly degrades the PI control results, because fast actuation allowed high-frequency 
pitch inputs to enter the system, causing destabilization of the flexible modes of the turbine (the PI control was not designed to stabilize these flexible modes).

In general, speed regulation with DAC is less robust than with the PI control. For the slow actuator, and exciting the simulation with turbulent wind, the standard deviation of the generator speed using DAC is larger than in the PI control results. The fast actuator improved the generator speed regulation for DAC while greatly destabilizing the results for PI control. The DAC results could be further improved by placing the pole corresponding to the generator speed further to the left in the complex plane in comparison to its place in designs shown in this chapter.

The slow actuator hinders the ability of DAC to increase the damping in the first tower fore-aft mode, in comparison to the fast actuator case. It was shown that DAC results could be improved for the slow actuator by properly accounting for the actuator dynamics in the control design model. This is demonstrated by designing a DAC control that improves the actuator speed through pole placement. This improved DAC restores the damping to the tower first fore-aft mode. The PI control was not designed to account for the tower first fore-aft mode. For the fast actuator, the PI results show unstable fore-aft tower motion.

The DAC outperforms the PI control in reducing drive-train shaft torsional moments and blade root flapwise-bending moments. This was a direct result of designing DAC to enhance damping in the first drive-train torsional mode and to include a state representing the disturbance corresponding to the wind-shear variation over the rotor disk. The PI control was not designed to account for these flexible modes or wind disturbances.

Although the analytic results obtained using DAC appear extremely promising, the ultimate test of the DAC controller developed here will occur with planned field testing on the actual CART machine over 12 to 18 months. These tests should provide substantial insight into the practical applicability of DAC for control of flexible structures. As wind turbines continue to grow in size and flexibility and are deployed in more hostile environments, the need to develop advanced control paradigms will be essential to deliver the lowest possible energy costs using wind systems.

Directions for future work include the implementation and field-testing of these controls in the CART. Important issues may include the effects of measurement noise as well as the need to convert the control algorithms into digital form. The effects of modeling and turbine property uncertainties must also be accounted for.

Further studies need to be conducted to investigate controls for very flexible machines. As machines become much more flexible than the CART, periodic control design methods will probably be needed. Other important issues include the attenuation of more complex turbulent wind inflow structures, which may require additional model complexity as well as turbine measurements. 


\section{References}

Balas, M.J., 1980, "Disturbance Accommodating Controllers for Distributed Parameter Systems: An Introduction,” J. of Interdisciplinary Modeling and Simulation, January.

Balas, M.J., 1990, “Active Control of Persistent Disturbances in Large Precision Aerospace Structures”, SPIE Cont., Orlando Fl, April.

Balas, M.J., Lee, Y.J. and Kendall, L., 1998, "Disturbance Tracking Control Theory with Application to Horizontal Axis Wind Turbines," Proceeding of the 1998 ASME Wind Energy Symposium, Reno, Nevada, 12-15 January, pp. 95-99.

Barton, R.S., Bowler, C.E.J. and Piwko, R.J., 1979, Control and Stabilization of the NASA/DOE MOD-1 Two Megawatt Wind Turbine Generator, American Chemical Society, pp. 325-330.

Bir, G.S., and Robinson, M.C., 1999, “Code Development for Control Design Applications," Proceedings of the 1999 ASME Wind Energy Symposium, 37 $7^{\text {th }}$ AIAA Aerospace Sciences Meeting and Exhibit, Reno, Nevada, 11-14 January, pp. 114-123.

Bongers, P.M., 1994, Modeling and Identification of Flexible Wind Turbines and a Factorizational Approach to Robust Control, Ph.D. Thesis, Department of Mechanical Engineering and Marine Technology, Delft University of Technology.

Bossanyi, E.A, 1987, “Adaptive Pitch Control for a $250 \mathrm{~kW}$ Wind Turbine,” Proceedings of the $9^{\text {th }}$ BWEA Wind Energy Conference, Edinburgh, Scotland, pp. 85-92.

Bossanyi, E.A., 1997, BLADED for Windows Theory Manual, Bristol, England: Garrad Hassan and Partners Limited.

Bossanyi, E.A., 2000, “Developments in Closed-loop Controller Design for Wind Turbines," Proceedings of the 2000 ASME Wind Energy Symposium, Reno, Nevada, pp. 64-74.

Buhl Jr., M.L., Wright, A.D. and Pierce, K.G, 2000, "Wind Turbine Design Codes: A Comparison of the Structural Response," Proceedings of the 2000 ASME Wind Energy Symposium, $38^{\text {th }}$ AIAA Aerospace Sciences Meeting and Exhibit, Reno, Nevada, pp. 12-22.

Buhl, M.L., Wright, A.D., and Pierce, K.G., 2001, "FAST_AD Code Verification: A Comparison to ADAMS," Proceeding of the 2001 ASME Wind Energy Symposium, Reno, Nevada, pp. 368-377.

Eggers, A., Jr., Ashley, H., Chaney, K., Rock, S. and Digumarthi, R., 1998, "Effects of Coupled Rotor-Tower Motions on Aerodynamic Control of Fluctuating Loads on Light-Weight HAWTS," Proceedings of the $36^{\text {th }}$ AIAA Aerospace Sciences Meeting and Exhibit, Reno, Nevada, pp. 113-122.

Eggers, A., Jr., Holley, W., Chaney, K. and Jennings, C., 1999, "Some Effects of Actuator Lag, Error Sensing, and Nonuniform Turbulence on Blade Pitch Control of Fluctuating Aerodynamic Loads on HAWTS," Proceedings of the $37^{\text {th }}$ AIAA Aerospace Sciences Meeting and Exhibit, Reno, Nevada, pp. 104-113.

Ekelund, T., 1997, Modeling and Linear Quadratic Optimal Control of Wind Turbines, Technical Report No. 306, Chalmers University of Technology, Goteborg, Sweden.

Elliott, A.S., and Wright, A.D., 1994, “ADAMS/WT: An Industry-Specific Interactive Modeling Interface for Wind Turbine Analysis," in Wind Energy 1994, edited by W.D. Musial, S.M. Hock, and D.E. Berg, SED-Vol. 14, New York: American Society of Mechanical Engineers; pp. 111-122.

Elliott, A.S., and McConville, J.B., 1990, “Analyzing Rotor Dynamics with A General-Purpose Code,” in Mechanical Engineering Magazine, Vol. 112, No. 12, pp. 21-25. 
Freeman, J.B., and Balas, M.J., 1999, “An Investigation of Variable-Speed Horizontal-Axis Wind Turbines Using Direct Model-Reference Adaptive Control," Proceedings of the 37 th AIAA Aerospace Sciences Meeting and Exhibit, Reno, Nevada, pp. 66-76.

Hamilton Standard, 1985, WTS-4 Wind Turbine Test Program, EPRI report \# AP-4054.

Hansen, A.C., and Laino, D.J., March 1998, Validation Study of YawDyn and AeroDyn as Dynamic Loads Predictors for the Combined Experiment Rotor (Phase 3), Report on Subcontract No. XAF-5-14076-02, National Renewable Energy Laboratory.

Hinrichsen, E.N., 1984, "Controls for Variable Pitch Wind Turbine Generators," I.E.E.E. Transactions on Power Apparatus and Systems, 103, pp. 886-892.

Hinrichsen, E.N., and Nolen, P.J., 1984, "Dynamics and Stability of Wind Turbine Generators," I.E.E.E. Transactions on Power Apparatus and Systems, 101, pp. 2640-2648.

Holley, W.E., Rock, S.M. and Chaney, K., 1999, "Control of Variable Speed Wind Turbines Below-Rated Wind Speed," Proceedings of the $3^{\text {rd }}$ ASME/JSME Joint Fluids Engineering Conference, July 18-23, San Francisco, California.

Johnson, C.D., 1976, "Theory of Disturbance Accommodating Controllers," in Advances in control and Dynamic Systems, 12, ed. C. T. Leondes, 1976.

Johnson, C.J., and Smith, R.T., 1976, Dynamics of Wind Generators on Electric Utility Networks. I.E.E.E. Transactions on Aerospace and Electronic Systems, 12, pp. 483-492.

Jonkman, J., 2001, Modeling of the UAE Wind Turbine for Refinement of FAST_AD, M.S. thesis, Department of Mechanical Engineering, Colorado State University.

Kane, T.R., and Levinson, D.A., 1985, Dynamics: Theory and Applications, McGraw-Hill Book Company, New York, New York, 1985.

Kelley, N.D., 1993, Full Vector (3-D) Inflow Simulation in Natural and Wind Farm Environments Using an Expanded Version of the SNLWIND (Veers) Turbulence Code, NREL/TP-442-5225, Golden, Colorado, the National Renewable Energy Laboratory.

Kos, J.M., 1978, "On Line Control of a Large Horizontal Axis Energy Conversion System and its Performance in a Turbulent Wind Environment," Proceedings of the $13^{\text {th }}$ Intersociety Energy Conversion Engineering Conference, U.S.A., pp. 2064-2073.

Kraan, I., 1992, Control Design for a Flexible Wind Turbine (in Dutch), Deflt University of Technology, TUD-WBMRA-613, 1992.

Kwakernaak, H., and Sivan, R., 1972, Linear Optimal Control Systems, Wiley Interscience, New York, NY.

Laino, D., Hansen, A. and Minnema, J., 2002, "Validation of the AeroDyn Subroutines Using NREL Unsteady Aerodynamics Experimental Data," Proceeding of the 2002 ASME Wind Energy Symposium, Reno, Nevada, pp. 179189.

Laino, D., and Hansen, A., 2003, "Continued Validation of the AeroDyn Subroutines Using NREL Unsteady Aerodynamics Experiment Data," Proceeding of the 2003 ASME Wind Energy Symposium, Reno, Nevada, pp. 84-93.

Laino, D., and Hansen, A., 2003, "YawDyn User's Guide-Version 12.14," Prepared for the National Renewable Energy laboratory under subcontract No. TCX-9-29209-01, January.

Liebst, B. S., 1983, “Pitch Control System for Large Scale Wind Turbines,” J. Energy, 7(2), pp. 182-192. 
Liebst, B.S., 1985, “A Pitch Control System for the KaMeWa Wind Turbine," Journal of Dynamic Systems and Control, $107,47$.

Lindenburg, C., and Schepers, J.G., 1996, Status of Phatas-III Release "Mar-1995”, Sun and Dos Version, ECN-R-95015, Petten.

Malcolm, D.J., and Wright, A.D., 1994, "The Use of ADAMS to Model the AWT-26 Prototype," Proceedings of the 1994 ASME Wind Energy Symposium, New Orleans, Louisiana, pp. 125-131.

The Mathworks, Inc., MATLAB, The Language of Technical Computing, Matlab User's Manual, Version 6.5.

Mattson, S.E., 1984, Modeling and Control of Large Horizontal Axis Wind Power Plants, Ph.D. Thesis, Department of Automatic Control, Lund Institute of Technology, Lund, Sweden.

Mechanical Dynamics, Inc., 2003, "Using ADAMS Solver," Automatic Dynamic Analysis of Mechanical Systems User's Manual, Version 12.

Molenaar, D.P., 2003, Cost-effective Design and Operation of Variable Speed Wind Turbines, Ph.D. Thesis, Mechanical Engineering and Systems and Controls Group, Delft University of Technology, Delft, The Netherlands.

Moriarty, P.J., Eggers, A.J., Jr., Chaney, K. and Holley, W.E., "Scale and Lag Effects on Control of Aerodynamic Power and Loads on a HAWT Rotor," Proceeding of the 2001 ASME Wind Energy Symposium, Reno, Nevada, pp. 306-314.

Pierce, K., 1999, “Control Method for Improved Energy Capture below Rated Power," Presented at the $3^{\text {rd }}$ ASME/JSME Joint Fluids Engineering Conference, San Francisco, California, July 18-23, 1999, NREL/CP-500-26322.

Press, W.H., Flannery, B.P., Teukolsky, S.A. and Vetterling, W.T., 1989, Numerical Recipes, The Art of Scientific Computing, Cambridge University Press, New York, New York.

Rock, S.M., Eggers, A.J., Jr., Moriarty, P.J. and Chaney, K., 2000, "Tradeoffs in Active Control of Aerodynamic Power and Loads on a HAWT Rotor," Proceeding of the 2000 ASME Wind Energy Symposium, Reno, Nevada, pp. 75-83.

Rothman, E.A., 1978, "The Effects of Control Modes on Rotor Loads," $2^{\text {nd }}$ International Symposium of Wind Energy Systems, Amsterdam, The Netherlands, pp. 107- 117.

Simms, D.A., Robinson, M.C., Hand, M.M. and Fingersh, L.J., 1996, "Characterization and Comparison of Baseline Aerodynamic Performance of Optimally Twisted Versus Non-Twisted HAWT Blades," in Energy Week, Houston, Texas, pp. 143-148.

Stol, K. and Bir, G., 2000, "Validation of a Symbolic Wind Turbine Structural Dynamics Model," Proceedings of the 2000 AIAA-ASME Wind Energy Symposium, Reno, Nevada, pp.41-48.

Stol, K., Rigney, B. and Balas, M.J., 2000, "Disturbance Accommodating Control of a Variable-Speed Turbine using a Symbolic Dynamics Structural Model," Proceeding of the 2000 ASME Wind Energy Symposium, Reno, Nevada, pp. 8490.

Stol, K. and Balas, M.J., 2001, "Full-State Feedback Control of a Variable-Speed Wind Turbine: A Comparison of Periodic and Constant Gains," Journal of Solar Energy Engineering.

Stol, K., 2001, "Dynamics Modeling and Periodic Control of Horzontal-Axis Wind Turbines," Ph.D thesis, University of Colorado.

Stol, K. and Balas, M.J., 2002, "Periodic Disturbance Accommodating Control for Speed Regulation of Wind Turbines," Proceeding of the 2002 ASME Wind Energy Symposium, Reno, Nevada, pp. 310-320. 
Stol, K. and Bir, G., 2003, Geometry and Structural Properties for the Controls Advanced Research Turbine (CART), NREL/TP-32087, Golden, Colorado, the National Renewable Energy Laboratory.

Svensson, J.E. and Ulen, E., 1982, "The Control of the WTS-3, Instrumentation and Testing," $4^{\text {th }}$ International Symposium on Wind Energy Systems, Stockholm, Sweden, pp. 195-215.

Wasynczuk, O., Man, D.T. and Sullivan, J.P., 1981, "Dynamic Behavior of a Class of Wind Turbine Generators During Random Wind Fluctuations, ”I.E.E.E. Transactions on Power Apparatus and Systems, 100, pp. 2837-2845.

Wilson, R. E and Lissaman, P., 1974, Applied Aerodynamics of Wind Power Machines, Corvallis, Oregon: Oregon State University.

Wilson, R.E., Walker, S.N. and Heh, P., 1999, Technical and User's Manual for the FAST_AD Advanced Dynamics Code, OSU/NREL Report 99-01, Corvallis, Oregon: Oregon State University.

Wolfram Research, Inc., 2003, The MATHEMATICA Book, Cambridge University Press, New York, New York, 1999.

Wonham, W.M., 1967, “On Pole Assignment in Multi-input Controllable Linear Systems,” IEEE Trans. Autom. Control, 12, pp. 660-665.

Wright, A.D., Kelley, N.D. and Osgood, R.M., 1999, "Validation of a Model for a Two-Bladed Flexible Rotor System: Progress to Date," Proceedings of the 37 $7^{\text {th }}$ AIAA Aerospace Sciences Meeting and Exhibit, Reno, Nevada, pp. 293-307.

Wright, A.D., and Balas, M. 2002, "Design of State-Space-Based Control Algorithms for Wind Turbine Speed Regulation," Proceeding of the 2002 ASME Wind Energy Symposium, Reno, Nevada, pp. 299-309. 


\section{Appendix A. FAST Linearization}

\section{A-1. Derivation of Linear Models}

\section{A-1.1. Rotor rotation 1-state model}

The first linear model contains just the rotor rotational speed as the single degree of freedom (DOF). We assume in this model that we are able to measure this state.

Borrowing from the FAST code nomenclature: $\mathrm{q}_{4}$ is the rotor azimuth angle.

It can be shown that for the rotor rotational degree of freedom, the equation of motion is:

$$
\mathrm{I}_{\text {tot }} \ddot{\mathrm{q}}_{4}=\mathrm{T}_{\text {aero }}-\mathrm{T}_{\text {gen }}
$$

where $\mathrm{I}_{\text {tot }}$ is the total rotational inertia of the turbine, including the rotational inertia of the rotor, and generator. Thus $I_{\text {tot }}=I_{\text {rot }}+I_{\text {gen }}$, where, $I_{\text {rot }}$ is the rotor rotational inertia, and $I_{\text {gen }}$ is the generator rotational inertia. Please note that we lump the inertia of the low-speed shaft, high-speed shaft, gearbox, and generator into $I_{\text {gen }} \cdot T_{\text {aero }}$ and $T_{\text {gen }}$ are the rotor aerodynamic and generator torque respectively.

The only control input to this model will be rotor collective pitch. This means that the pitch angle $\beta$ of each blade is identical. Since $\mathrm{T}_{\text {aero }}$ is a continuous function of wind-speed $\mathrm{W}$, rotor-speed $\Omega$, and pitch angle $\beta$, it can be expanded as a Taylor series in terms of $\mathbf{W}, \Omega$, and $\beta$ as:

$$
\mathrm{T}_{\text {aero }}=\mathrm{T}_{\text {aero }}\left(\mathrm{w}_{0}, \Omega_{0}, \beta_{0}\right)+\frac{\partial \mathrm{T}_{\mathrm{aero}}}{\partial \mathrm{w}} \delta \mathrm{w}+\frac{\partial \mathrm{T}_{\mathrm{aero}}}{\partial \Omega} \delta \Omega+\frac{\partial \mathrm{T}_{\mathrm{aero}}}{\partial \beta} \delta \beta+\text { hots, }
$$

where $\mathrm{w}_{0}, \Omega_{0}, \beta_{0}$ are the nominal wind-speed, rotor speed and blade pitch angle at equilibrium. $\delta \mathrm{w}, \delta \Omega$, and $\delta \beta$ are perturbations in $\mathrm{w}, \Omega$, and $\beta$ respectively. The term "hots" stands for "higher order terms," which we neglect.

Let $\mathrm{x}_{1}=\delta \dot{\mathrm{q}}_{4}$, the perturbed rotor rotational speed.

If we let

$$
\begin{aligned}
& \delta \mathrm{T}_{\text {aero }}=\mathrm{T}_{\mathrm{aero}}(\mathrm{w}, \Omega, \beta)-\mathrm{T}_{\text {aero }}\left(\mathrm{w}_{0}, \Omega_{0}, \beta_{0}\right) \\
& \text { then, using }
\end{aligned}
$$




$$
\begin{gathered}
\delta \Omega=\delta \dot{\mathrm{q}}_{4}=\mathrm{x}_{1}: \\
\delta \mathrm{T}_{\text {aero }}=\frac{\partial \mathrm{T}_{\text {aero }}}{\partial \mathrm{w}} \delta \mathrm{w}+\frac{\partial \mathrm{T}_{\text {aero }}}{\partial \Omega} \delta \Omega+\frac{\partial \mathrm{T}_{\text {aero }}}{\partial \beta} \delta \beta \\
=\frac{\partial \mathrm{T}_{\text {aero }}}{\partial \mathrm{w}} \delta \mathrm{w}+\frac{\partial \mathrm{T}_{\text {aero }}}{\partial \Omega} \mathrm{x}_{1}+\frac{\partial \mathrm{T}_{\text {aero }}}{\partial \beta} \delta \beta . \\
\frac{\partial \mathrm{T}_{\text {aero }}}{\partial \mathrm{w}}=\alpha, \frac{\partial \mathrm{T}_{\text {aero }}}{\partial \Omega}=\gamma, \text { and } \frac{\partial \mathrm{T}_{\text {aero }}}{\partial \beta}=\zeta,
\end{gathered}
$$

Then

$$
\delta \mathrm{T}_{\mathrm{aero}}=\alpha \delta \mathrm{w}+\gamma \mathrm{x}_{1}+\zeta \delta \beta
$$

Now

$$
\mathrm{I}_{\text {tot }} \ddot{\mathrm{q}}_{4}=\mathrm{T}_{\text {aero }}-\mathrm{T}_{\text {gen }}
$$

In this work we assume that the generator torque is constant. We also assume that the rotor rotational acceleration is zero at equilibrium, $\ddot{\mathrm{q}}_{4}=0$.

Thus, we can write:

$$
\mathrm{I}_{\text {tot }} \delta \ddot{\mathrm{q}}_{4}=\mathrm{T}_{\text {aero }}\left(\mathrm{w}_{0}, \Omega_{0}, \beta_{0}\right)+\alpha \delta \mathrm{w}+\gamma \delta \Omega+\zeta \delta \beta-\mathrm{T}_{\text {gen }}
$$

Since at equilibrium, the aerodynamic torque and generator torque must be equal (or acceleration would occur):

$$
\mathrm{T}_{\text {aero }}\left(\mathrm{w}_{0}, \Omega_{0}, \beta_{0}\right)-\mathrm{T}_{\text {gen }}=0
$$

Thus we have:

$$
\mathrm{I}_{\mathrm{tot}} \delta \ddot{\mathrm{q}}_{4}=\alpha \delta \mathrm{w}+\gamma \delta \Omega+\zeta \delta \beta,
$$

Or finally:

$$
\dot{\mathrm{x}}_{1}=\frac{\gamma}{\mathrm{I}_{\text {tot }}} \mathrm{x}_{1}+\frac{\zeta}{\mathrm{I}_{\text {tot }}} \delta \beta+\frac{\alpha}{\mathrm{I}_{\text {tot }}} \delta \mathrm{W} .
$$

If we assume that we measure the rotor rotational speed, then:

$$
\mathrm{y}=\mathrm{x}_{1} .
$$

This concludes derivation of the simple 1-state model. 


\section{A-1.2. Rotor and generator rotation, drive-train torsion 3-state model}

The next linear model contains the generator and rotor speed states and drive-train torsional spring force state. These states were chosen in order to add the drive-train torsion mode to the previous 1-state model, and to allow modeling the generator rotational degree of freedom (DOF). We assume in this model that we are able to measure generator speed.

The reason for choosing 3 states to model this configuration is that this model results in a controllable and observable system (minimal system). It is not hard to show that if 4 states are chosen in this model (such as generator and rotor azimuth angle, and rotor and generator speed) then the resulting model is not minimal (not controllable and observable).

Referring to Chapter $2, \mathrm{q}_{4}$ and $\mathrm{q}_{15}$ are the rotor and generator azimuth angle, while $\dot{\mathrm{q}}_{4}$ and $\dot{\mathrm{q}}_{15}$ are the rotor and generator rotational speed states. $\mathrm{K}_{\mathrm{d}}$ is the drive-train torsional stiffness, and $\mathrm{C}_{\mathrm{d}}$ is the drive-train torsional damping.

It can be shown that for the rotor rotational degree of freedom, as in the 1-state model:

$$
\mathrm{I}_{\text {rot }} \ddot{\mathrm{q}}_{4}=\mathrm{T}_{\text {aero }}-\mathrm{T}_{\text {shaft }}
$$

Here, $\mathrm{T}_{\text {aero }}$ is the rotor aerodynamic torque, and $\mathrm{T}_{\text {shaft }}$ is the reaction torque from the shaft. Note that the rotational inertia in this equation is $\mathrm{I}_{\text {rot }}$, the rotor rotational inertia, instead of the total turbine rotational inertia. Note that:

$$
\mathrm{T}_{\text {shaft }}=\mathrm{K}_{\mathrm{d}}\left(\mathrm{q}_{4}-\mathrm{q}_{15}\right)+\mathrm{C}_{\mathrm{d}}\left(\dot{\mathrm{q}}_{4}-\dot{\mathrm{q}}_{15}\right)
$$

is the reaction torque in the low-speed shaft.

Let's now obtain a linear model for these equations.

We can now expand $\mathrm{T}_{\text {aero }}$ exactly as we did in the last section, Eq. (A1.2).

$$
\mathrm{T}_{\mathrm{aero}}=\mathrm{T}_{\mathrm{aero}}\left(\mathrm{w}_{0}, \Omega_{0}, \beta_{0}\right)+\alpha \delta \mathrm{w}+\gamma \mathrm{x}_{1}+\zeta \delta \beta
$$

where $\mathrm{w}_{0}, \Omega_{0}, \beta_{0}$ are the nominal wind-speed, rotor rotational speed and blade pitch angle respectively. $\delta \mathrm{w}, \delta \Omega$, and $\delta \beta$ are again the perturbations in $\mathrm{w}, \Omega$, and $\beta$ respectively.

Now the perturbation in the shaft torque is:

$$
\delta \mathrm{T}_{\text {shaft }}=\mathrm{K}_{\mathrm{d}}\left(\delta \mathrm{q}_{4}-\delta \mathrm{q}_{15}\right)+\mathrm{C}_{\mathrm{d}}\left(\delta \dot{\mathrm{q}}_{4}-\delta \dot{\mathrm{q}}_{15}\right) .
$$

Now

$$
\begin{aligned}
& \mathrm{I}_{\text {rot }} \ddot{\mathrm{q}}_{4}=\mathrm{T}_{\text {aero }}-\mathrm{T}_{\text {shaft }} \\
& =\mathrm{T}_{\text {aero }}\left(\mathrm{w}_{0}, \Omega_{0}, \beta_{0}\right)+\delta \mathrm{T}_{\text {aero }}-\mathrm{T}_{\text {shaft }_{0}}-\delta \mathrm{T}_{\text {shaft }},
\end{aligned}
$$


where $\mathrm{T}_{\text {shaft }_{0}}$ is the shaft torque at equilibrium. Since at equilibrium, rotor acceleration is zero, and perturbations in aerodynamic torque and shaft torque are zero,

$$
\mathrm{T}_{\text {aero }}\left(\mathrm{w}_{0}, \Omega_{0}, \beta_{0}\right)=\mathrm{T}_{\text {shaft }_{0}} .
$$

Thus

$$
\mathrm{I}_{\text {rot }} \delta \ddot{\mathrm{q}}_{4}=\delta \mathrm{T}_{\text {aero }}-\delta \mathrm{T}_{\text {shaft }}
$$

Now

$$
\delta \mathrm{T}_{\text {aero }}-\delta \mathrm{T}_{\text {shaft }}=\alpha \delta \mathrm{w}+\gamma \delta \dot{\mathrm{q}}_{4}+\zeta \delta \beta-\mathrm{K}_{\mathrm{d}}\left(\delta \mathrm{q}_{4}-\delta \mathrm{q}_{15}\right)-\mathrm{C}_{\mathrm{d}}\left(\delta \dot{\mathrm{q}}_{4}-\delta \dot{\mathrm{q}}_{15}\right)
$$

Let

$$
\begin{aligned}
& x_{1}=\delta \dot{\mathrm{q}}_{4} \\
& \mathrm{x}_{2}=\mathrm{K}_{\mathrm{d}}\left(\delta \mathrm{q}_{4}-\delta \mathrm{q}_{15}\right) \\
& \mathrm{x}_{3}=\delta \dot{\mathrm{q}}_{15}
\end{aligned}
$$

then

$$
\begin{aligned}
& I_{\mathrm{rot}} \delta \ddot{\mathrm{q}}_{4}=\alpha \delta \mathrm{w}+\gamma \mathrm{x}_{1}+\zeta \delta \beta-\mathrm{K}_{\mathrm{d}}\left(\delta \mathrm{q}_{4}-\delta \mathrm{q}_{15}\right)-\mathrm{C}_{\mathrm{d}}\left(\delta \dot{\mathrm{q}}_{4}-\delta \dot{\mathrm{q}}_{15}\right) \\
& =\alpha \delta \mathrm{w}+\gamma \mathrm{x}_{1}+\zeta \delta \beta-\mathrm{x}_{2}-\mathrm{C}_{\mathrm{d}}\left(\mathrm{x}_{1}-\mathrm{x}_{3}\right) \\
& \text { thus } \\
& I_{\text {rot }} \dot{\mathrm{x}}_{1}=\left(\gamma-\mathrm{C}_{\mathrm{d}}\right) \mathrm{x}_{1}-\mathrm{x}_{2}+\mathrm{C}_{\mathrm{d}} \mathrm{x}_{3}+\zeta \delta \beta+\alpha \delta \mathrm{w} .
\end{aligned}
$$

which is the linear equation of motion for rotor rotation.

Now:

$$
\dot{\mathrm{x}}_{2}=\mathrm{K}_{\mathrm{d}}\left(\delta \dot{\mathrm{q}}_{4}-\delta \dot{\mathrm{q}}_{15}\right)=\mathrm{K}_{\mathrm{d}}\left(\mathrm{x}_{1}-\mathrm{x}_{3}\right)
$$

This is the linear state equation for $\mathrm{x}_{2}$.

The equation for the generator can be written:

$$
\mathrm{I}_{\text {gen }} \ddot{\mathrm{q}}_{15}=\mathrm{T}_{\text {shaft }}-\mathrm{T}_{\text {gen }} .
$$

Again, at equilibrium, the generator acceleration is zero. We can use a similar argument as before to show that at equilibrium, the shaft torque and generator torque are equal. Thus: 


$$
\begin{aligned}
& \mathrm{I}_{\text {gen }} \delta \ddot{\mathrm{q}}_{15}=\delta \mathrm{T}_{\text {shaft }}-\delta \mathrm{T}_{\text {gen }} \\
& =\mathrm{K}_{\mathrm{d}}\left(\delta \mathrm{q}_{4}-\delta \mathrm{q}_{15}\right)+\mathrm{C}_{\mathrm{d}}\left(\delta \dot{\mathrm{q}}_{4}-\delta \dot{\mathrm{q}}_{15}\right)-\delta \mathrm{T}_{\text {gen }}
\end{aligned}
$$

thus

$$
\mathrm{I}_{\text {gen }} \dot{\mathrm{x}}_{3}=\mathrm{C}_{\mathrm{d}} \mathrm{x}_{1}+\mathrm{x}_{2}-\mathrm{C}_{\mathrm{d}} \mathrm{x}_{3}-\delta \mathrm{T}_{\mathrm{gen}} \text {. }
$$

where $\mathrm{I}_{\text {gen }}$ is the inertia of the generator, and $\delta \mathrm{T}_{\text {gen }}$ is the perturbation in the generator torque. Let's assume that we have constant generator torque for now, which means that

$$
\delta \mathrm{T}_{\text {gen }}=0
$$

In addition, we assume that we measure generator speed $\mathrm{X}_{3}$. We can now write the linear state-space equation for this system, with

$$
\begin{aligned}
& \mathrm{x}_{1}=\delta \dot{\mathrm{q}}_{4}, \text { perturbed rotor rotational speed } \\
& \mathrm{x}_{2}=\mathrm{K}_{\mathrm{d}}\left(\delta \mathrm{q}_{4}-\delta \mathrm{q}_{15}\right), \text { perturbed drive-train torsional spring force }
\end{aligned}
$$

(one could also define $\mathrm{x}_{2}=\delta \mathrm{q}_{4}-\delta \mathrm{q}_{15}$, just the torsional deflection in shaft),

and

$\mathrm{x}_{3}=\delta \dot{\mathrm{q}}_{15}$, perturbed generator rotational speed.

$$
\begin{aligned}
& {\left[\begin{array}{c}
\dot{\mathrm{x}}_{1} \\
\dot{\mathrm{x}}_{2} \\
\dot{\mathrm{x}}_{3}
\end{array}\right]=\left[\begin{array}{ccc}
\frac{\left(\gamma-\mathrm{C}_{\mathrm{d}}\right)}{\mathrm{I}_{\mathrm{rot}}} & \frac{-1}{\mathrm{I}_{\mathrm{rot}}} & \frac{\mathrm{C}_{\mathrm{d}}}{\mathrm{I}_{\mathrm{rot}}} \\
\mathrm{K}_{\mathrm{d}} & 0 & -\mathrm{K}_{\mathrm{d}} \\
\frac{\mathrm{C}_{\mathrm{d}}}{\mathrm{I}_{\mathrm{gen}}} & \frac{1}{\mathrm{I}_{\text {gen }}} & \frac{-\mathrm{C}_{\mathrm{d}}}{\mathrm{I}_{\mathrm{gen}}}
\end{array}\right]\left[\begin{array}{c}
\mathrm{x}_{1} \\
\mathrm{x}_{2} \\
\mathrm{x}_{3}
\end{array}\right]+\left[\begin{array}{c}
\frac{\zeta}{\mathrm{I}_{\text {rot }}} \\
0 \\
0
\end{array}\right] \delta \beta+\left[\begin{array}{c}
\frac{\alpha}{\mathrm{I}_{\mathrm{rot}}} \\
0 \\
0
\end{array}\right] \delta \mathrm{W}} \\
& y=\left[\begin{array}{lll}
0 & 0 & 1
\end{array}\right]\left[\begin{array}{l}
x_{1} \\
x_{2} \\
x_{3}
\end{array}\right]
\end{aligned}
$$

What if we use generator torque as the control actuator instead of rotor collective pitch? Then we ignore the rotor collective pitch control input. Our control input is now $\delta \mathrm{T}_{\text {gen }}$. From Eq. (A1.18) we have:

$$
\mathrm{I}_{\text {gen }} \dot{\mathrm{x}}_{3}=\mathrm{C}_{\mathrm{d}} \mathrm{x}_{1}+\mathrm{x}_{2}-\mathrm{C}_{\mathrm{d}} \mathrm{x}_{3}-\delta \mathrm{T}_{\text {gen }} .
$$

The state space model thus becomes: 


$$
\begin{aligned}
& {\left[\begin{array}{c}
\dot{\mathrm{x}}_{1} \\
\dot{\mathrm{x}}_{2} \\
\dot{\mathrm{x}}_{3}
\end{array}\right]=\left[\begin{array}{ccc}
\frac{\left(\gamma-\mathrm{C}_{\mathrm{d}}\right)}{\mathrm{I}_{\mathrm{rot}}} & \frac{-1}{\mathrm{I}_{\mathrm{rot}}} & \frac{\mathrm{C}_{\mathrm{d}}}{\mathrm{I}_{\mathrm{rot}}} \\
\mathrm{K}_{\mathrm{d}} & 0 & -\frac{\mathrm{K}_{\mathrm{d}}}{\mathrm{C}_{\mathrm{d}}} \\
\frac{1}{\mathrm{I}_{\mathrm{gen}}} & \frac{-\mathrm{C}_{\mathrm{d}}}{\mathrm{I}_{\mathrm{gen}}} & \frac{\mathrm{I}_{\mathrm{gen}}}{\mathrm{x}_{1}}
\end{array}\right]+\left[\begin{array}{c}
0 \\
\mathrm{x}_{2} \\
\mathrm{x}_{3}
\end{array}\right]+\left[\begin{array}{c}
0 \\
0 \\
\frac{-1}{\mathrm{I}_{\mathrm{gen}}}
\end{array}\right] \delta \mathrm{T}_{\mathrm{gen}}+\left[\begin{array}{c}
\frac{\alpha}{\mathrm{I}_{\mathrm{rot}}} \\
0 \\
0
\end{array}\right] \delta \mathrm{W}} \\
& \mathrm{y}=\left[\begin{array}{lll}
0 & 0 & 1
\end{array}\right]\left[\begin{array}{c}
\mathrm{x}_{1} \\
\mathrm{x}_{2} \\
\mathrm{x}_{3}
\end{array}\right],
\end{aligned}
$$

\section{A-1.3. Rotor and generator rotation, drive-train torsion model, blade-1 and-2 first flap mode, tower first fore-aft mode}

From Chapter 2, $\mathrm{q}_{4}$ and $\mathrm{q}_{15}$ are defined as before. $\mathrm{q}_{1}$ and $\mathrm{q}_{2}$ represent blade-tip flap displacement for blade 1 and 2 (the displacement of the blade tip normal to the section chord-line at the tip). $q_{7}$ represents the tower-top fore-aft bending displacement. All other states are the same as in previous sections. First, the equation of motion for blade 1 flap now becomes:

$$
\begin{aligned}
& \mathrm{M}_{11} \delta \ddot{\mathrm{q}}_{1}+\mathrm{M}_{14} \delta \ddot{\mathrm{q}}_{4}+\mathrm{M}_{17} \delta \ddot{\mathrm{q}}_{7}+\mathrm{C}_{11} \delta \dot{\mathrm{q}}_{1}+\mathrm{C}_{14} \delta \dot{\mathrm{q}}_{4}+ \\
& \mathrm{C}_{17} \delta \dot{\mathrm{q}}_{7}+\mathrm{K}_{11} \delta \mathrm{q}_{1}+\mathrm{K}_{17} \delta \mathrm{q}_{7}=\delta \mathrm{F}_{\text {aero }}
\end{aligned}
$$

where $M_{11}$ =blade-1 first mode mass coefficient, $M_{14}$ = mass coefficient which couples blade-1 first flap mode to rotor rotation, $\mathrm{M}_{17}$ = mass coefficient which couples blade-1 first flap mode to tower first fore-aft mode, $\mathrm{C}_{11}=$ blade- 1 first mode damping coefficient, $\mathrm{C}_{14}=$ damping coefficient which couples blade-1 first flap mode to rotor rotation, $\mathrm{C}_{17}=$ damping coefficient which couples blade-1 first flap mode to tower first fore-aft mode, $\mathrm{K}_{11}=$ blade-1 first mode stiffness coefficient, $\mathrm{K}_{17}=$ stiffness coefficient which couples blade-1 first flap mode to tower first fore-aft mode and $\delta \mathrm{F}_{\text {aero }_{1}}$ is the perturbed distributed aerodynamic flap force on blade 1. The terms shown in Eq. (A1.21) are known to be the dominant terms for a turbine such as the CART. Other coupling terms might exist but are small for the CART. These extra terms could become important for other machine configurations, such as very soft, flexible rotors.

The equation of motion for blade 2 flap is:

$$
\begin{aligned}
& \mathrm{M}_{22} \delta \ddot{\mathrm{q}}_{2}+\mathrm{M}_{24} \delta \ddot{\mathrm{q}}_{4}+\mathrm{M}_{27} \delta \ddot{\mathrm{q}}_{7}+\mathrm{C}_{22} \delta \dot{\mathrm{q}}_{2}+\mathrm{C}_{24} \delta \dot{\mathrm{q}}_{4}+ \\
& \mathrm{C}_{27} \delta \dot{\mathrm{q}}_{7}+\mathrm{K}_{22} \delta \mathrm{q}_{2}+\mathrm{K}_{27} \delta \mathrm{q}_{7}=\delta \mathrm{F}_{\mathrm{aero}_{2}} .
\end{aligned}
$$

where $\mathrm{M}_{22}$ = blade-2 first mode mass coefficient, $\mathrm{M}_{24}$ = mass coefficient which couples blade-2 first flap mode to rotor rotation, $\mathrm{M}_{27}=$ mass coefficient which couples blade- 2 first flap mode to tower first fore-aft mode, $\mathrm{C}_{22}=$ blade2 first mode damping coefficient, $\mathrm{C}_{24}=$ damping coefficient which couples blade- 2 first flap mode to rotor rotation, $\mathrm{C}_{27}=$ damping coefficient which couples blade-2 first flap mode to tower first fore-aft mode, $\mathrm{K}_{22}=$ blade- 2 first 
mode stiffness coefficient, $\mathrm{K}_{27}=$ stiffness coefficient which couples blade-2 first flap mode to tower first fore-aft mode, and $\delta \mathrm{F}_{\text {aero }_{2}}$ is the perturbed distributed aerodynamic flap force on blade 2.

The equation for rotor rotation is very similar to Eq. (A1.10), except that there are extra terms coupling the rotor rotation with blade- 1 and -2 flap and tower fore-aft motion:

$$
\begin{aligned}
& \mathrm{I}_{\text {rot }} \delta \ddot{\mathrm{q}}_{4}+\mathrm{M}_{41} \delta \ddot{\mathrm{q}}_{1}+\mathrm{M}_{42} \delta \ddot{\mathrm{q}}_{2}+\mathrm{M}_{47} \delta \ddot{\mathrm{q}}_{7}+ \\
& \mathrm{C}_{41} \delta \dot{\mathrm{q}}_{1}+\mathrm{C}_{42} \delta \dot{\mathrm{q}}_{2}+\mathrm{C}_{47} \delta \dot{\mathrm{q}}_{7}=\delta \mathrm{T}_{\text {aero }}-\delta \mathrm{T}_{\text {shaft }}
\end{aligned}
$$

where $\mathrm{M}_{41}=$ mass coefficient which couples rotor rotation to blade-1 first flap, $\mathrm{M}_{42}=$ mass coefficient which couples rotor rotation to blade-2 first flap, $\mathrm{C}_{41}=$ damping coefficient which couples rotor rotation to blade-1 first flap, $\mathrm{C}_{42}=$ damping coefficient which couples rotor rotation to blade-2 first flap. $\mathrm{M}_{47}=$ mass coefficient which couples rotor rotation to tower first fore-aft mode, while $\mathrm{C}_{47}=$ damping coefficient which couples rotor rotation to tower first fore-aft mode.

Now in Eq. (A1.21), the aerodynamic force on blade 1 is a function of blade-1 pitch $\beta_{1}$, and the wind speed on blade-1 $\mathrm{W}_{1}$, as well as the rotor speed $\Omega$ :

$$
\mathrm{F}_{\text {aero }_{1}}=\mathrm{F}_{\text {aero }_{1}}\left(\beta_{1}, \mathrm{w}_{1}, \Omega\right) \text {. }
$$

Likewise, in (A1.22), the aerodynamic force on blade 2 can be written,

$$
\mathrm{F}_{\text {aero }_{2}}=\mathrm{F}_{\text {aero }_{2}}\left(\beta_{2}, \mathrm{w}_{2}, \Omega\right),
$$

where $\beta_{2}$ is blade- 2 pitch, and $\mathrm{w}_{2}$ is the wind speed impinging upon blade- 2 .

In Eq. (A1.23), $\mathrm{T}_{\text {aero }}$ is a function of blade-1 and -2 pitch, blade-1 and -2 wind speed, as well as rotor speed: $\mathrm{T}_{\text {aero }}=\mathrm{T}_{\text {aero }}\left(\beta_{1}, \beta_{2} \mathrm{w}_{1}, \mathrm{w}_{2}, \Omega\right)$.

These functions can be expanded in a Taylor Series as:

$$
\mathrm{F}_{\text {aero }_{1}}=\mathrm{F}_{\text {aero }_{1}}\left(\beta_{1_{0}}, \mathrm{w}_{1_{0}}, \Omega_{0}\right)+\frac{\partial \mathrm{F}_{\text {aero }_{1}}}{\partial \beta_{1}} \delta \beta_{1}+\frac{\partial \mathrm{F}_{\text {aero }_{1}}}{\partial \mathrm{w}_{1}} \delta \mathrm{w}_{1}+\frac{\partial \mathrm{F}_{\text {aero }_{1}}}{\partial \Omega} \delta \Omega
$$

where, $\beta_{1_{0}}$ and $\mathrm{w}_{1_{0}}$ are blade-1 pitch and blade-1 wind speed at equilibrium.

Similarly,

$$
\mathrm{F}_{\mathrm{aero}_{2}}=\mathrm{F}_{\text {aero }_{2}}\left(\beta_{2_{0}}, \mathrm{w}_{2_{0}}, \Omega_{0}\right)+\frac{\partial \mathrm{F}_{\mathrm{aero}_{2}}}{\partial \beta_{2}} \delta \beta_{2}+\frac{\partial \mathrm{F}_{\mathrm{aero}_{2}}}{\partial \mathrm{w}_{2}} \delta \mathrm{w}_{2}+\frac{\partial \mathrm{F}_{\mathrm{aero}_{2}}}{\partial \Omega} \delta \Omega
$$

and 


$$
\begin{aligned}
& \mathrm{T}_{\text {aero }}=\mathrm{T}_{\text {aero }}\left(\beta_{1_{0}}, \beta_{2_{0}} \mathrm{w}_{1_{0}}, \mathrm{w}_{2_{0}}, \Omega_{0}\right)+\frac{\partial \mathrm{T}_{\text {aero }}}{\partial \beta_{1}} \delta \beta_{1} \\
& +\frac{\partial \mathrm{T}_{\text {aero }}}{\partial \mathrm{w}_{1}} \delta \mathrm{w}_{1}+\frac{\partial \mathrm{T}_{\text {aero }}}{\partial \beta_{2}} \delta \beta_{2}+\frac{\partial \mathrm{T}_{\text {aero }}}{\partial \mathrm{w}_{2}} \delta \mathrm{w}_{2}+\frac{\partial \mathrm{T}_{\text {aero }}}{\partial \Omega} \delta \Omega
\end{aligned}
$$

We finally obtain equations containing only perturbation values of the states:

$$
\begin{aligned}
& \mathrm{M}_{11} \delta \ddot{\mathrm{q}}_{1}+\mathrm{M}_{14} \delta \ddot{\mathrm{q}}_{4}+\mathrm{M}_{17} \delta \ddot{\mathrm{q}}_{7}+\mathrm{C}_{11} \delta \dot{\mathrm{q}}_{1}+\mathrm{C}_{14} \delta \dot{\mathrm{q}}_{4}+\mathrm{C}_{17} \delta \dot{\mathrm{q}}_{7}+\mathrm{K}_{11} \delta \mathrm{q}_{1} \\
& +\mathrm{K}_{17} \delta \mathrm{q}_{7}=\frac{\partial \mathrm{F}_{\text {aero }_{1}}}{\partial \beta_{1}} \delta \beta_{1}+\frac{\partial \mathrm{F}_{\text {aero }_{1}}}{\partial \mathrm{w}_{1}} \delta \mathrm{w}_{1}+\frac{\partial \mathrm{F}_{\text {aero }_{1}}}{\partial \Omega} \delta \Omega .
\end{aligned}
$$

We will assume that the term $\frac{\partial \mathrm{F}_{\text {aero }}}{\partial \Omega} \delta \Omega$ is contained in $\mathrm{C}_{14} \delta \dot{\mathrm{q}}_{4}$, which now includes aerodynamic damping.

Let

$$
\frac{\partial \mathrm{F}_{\mathrm{aero}_{1}}}{\partial \beta_{1}}=\zeta_{\mathrm{b}_{1}}, \frac{\partial \mathrm{F}_{\mathrm{aero}_{1}}}{\partial \mathrm{w}_{1}}=\alpha_{\mathrm{b}_{1}},
$$

then

$$
\begin{aligned}
& \mathrm{M}_{11} \delta \ddot{\mathrm{q}}_{1}+\mathrm{M}_{14} \delta \ddot{\mathrm{q}}_{4}+\mathrm{M}_{17} \delta \ddot{\mathrm{q}}_{7}=-\mathrm{C}_{11} \delta \dot{\mathrm{q}}_{1}-\mathrm{C}_{14} \delta \dot{\mathrm{q}}_{4}-\mathrm{C}_{17} \delta \dot{\mathrm{q}}_{7}-\mathrm{K}_{11} \delta \mathrm{q}_{1} \\
& -\mathrm{K}_{17} \delta \mathrm{q}_{7}+\zeta_{\mathrm{b}_{1}} \delta \beta_{1}+\alpha_{\mathrm{b}_{1}} \delta \mathrm{w}_{1} .
\end{aligned}
$$

Similarly, for blade 2:

$$
\begin{aligned}
& \mathrm{M}_{22} \delta \ddot{\mathrm{q}}_{2}+\mathrm{M}_{24} \delta \ddot{\mathrm{q}}_{4}+\mathrm{M}_{27} \delta \ddot{\mathrm{q}}_{7}=-\mathrm{C}_{22} \delta \dot{\mathrm{q}}_{2}-\mathrm{C}_{24} \delta \dot{\mathrm{q}}_{4}-\mathrm{C}_{27} \delta \dot{\mathrm{q}}_{7}-\mathrm{K}_{22} \delta \mathrm{q}_{2} \\
& -\mathrm{K}_{27} \delta \mathrm{q}_{7}+\zeta_{\mathrm{b}_{2}} \delta \beta_{2}+\alpha_{\mathrm{b}_{2}} \delta \mathrm{w}_{2} .
\end{aligned}
$$

with

$$
\frac{\partial \mathrm{F}_{\mathrm{aero}_{2}}}{\partial \beta_{2}}=\zeta_{\mathrm{b}_{2}} \text {, and } \frac{\partial \mathrm{F}_{\mathrm{aero}_{2}}}{\partial \mathrm{w}_{2}}=\alpha_{\mathrm{b}_{2}} .
$$

Now the rotor torque equation becomes:

$$
\begin{aligned}
& \mathrm{I}_{\text {rot }} \delta \ddot{\mathrm{q}}_{4}+\mathrm{M}_{41} \delta \ddot{\mathrm{q}}_{1}+\mathrm{M}_{42} \delta \ddot{\mathrm{q}}_{2}+\mathrm{M}_{47} \delta \ddot{\mathrm{q}}_{7}+ \\
& \mathrm{C}_{41} \delta \dot{\mathrm{q}}_{1}+\mathrm{C}_{42} \delta \dot{\mathrm{q}}_{2}+\mathrm{C}_{47} \delta \dot{\mathrm{q}}_{7}=\delta \mathrm{T}_{\text {aero }}-\delta \mathrm{T}_{\text {shaft }}
\end{aligned}
$$

Now 


$$
\begin{aligned}
& \delta \mathrm{T}_{\text {aero }}=\mathrm{T}_{\text {aero }}-\mathrm{T}_{\text {aero }}\left(\beta_{1_{0}}, \beta_{2_{0}} \mathrm{w}_{1_{0}}, \mathrm{w}_{2_{0}}, \Omega_{0}\right)=\frac{\partial \mathrm{T}_{\text {aero }}}{\partial \beta_{1}} \delta \beta_{1} \\
& +\frac{\partial \mathrm{T}_{\text {aero }}}{\partial \mathrm{w}_{1}} \delta \mathrm{w}_{1}+\frac{\partial \mathrm{T}_{\text {aero }}}{\partial \beta_{2}} \delta \beta_{2}+\frac{\partial \mathrm{T}_{\text {aero }}}{\partial \mathrm{w}_{2}} \delta \mathrm{w}_{2}+\frac{\partial \mathrm{T}_{\text {aero }}}{\partial \Omega} \delta \Omega .
\end{aligned}
$$

Let

$$
\begin{aligned}
& \frac{\partial \mathrm{T}_{\text {aero }}}{\partial \beta_{1}}=\zeta_{1}, \frac{\partial \mathrm{T}_{\text {aero }}}{\partial \beta_{2}}=\zeta_{2}, \frac{\partial \mathrm{T}_{\text {aero }}}{\partial \mathrm{w}_{1}}=\alpha_{1}, \frac{\partial \mathrm{T}_{\text {aero }}}{\partial \mathrm{w}_{2}}=\alpha_{2} \text {, and } \frac{\partial \mathrm{T}_{\text {aero }}}{\partial \Omega}=\gamma . \text { Then } \\
& \delta \mathrm{T}_{\text {aero }}-\delta \mathrm{T}_{\text {shaft }}=\zeta_{1} \delta \beta_{1}+\alpha_{1} \delta \mathrm{w}_{1}+\zeta_{2} \delta \beta_{2}+\alpha_{2} \delta \mathrm{w}_{2} \\
& +\gamma \delta \Omega-\mathrm{K}_{\mathrm{d}}\left(\delta \mathrm{q}_{4}-\delta \mathrm{q}_{15}\right)-\mathrm{C}_{\mathrm{d}}\left(\delta \dot{\mathrm{q}}_{4}-\delta \dot{\mathrm{q}}_{15}\right)
\end{aligned}
$$

thus

$$
\begin{aligned}
& \mathrm{I}_{\text {rot }} \delta \ddot{\mathrm{q}}_{4}+\mathrm{M}_{41} \delta \ddot{\mathrm{q}}_{1}+\mathrm{M}_{42} \delta \ddot{\mathrm{q}}_{2}+\mathrm{M}_{47} \delta \ddot{\mathrm{q}}_{7}=-\mathrm{C}_{41} \delta \dot{\mathrm{q}}_{1}-\mathrm{C}_{42} \delta \dot{\mathrm{q}}_{2}-\mathrm{C}_{47} \delta \dot{\mathrm{q}}_{7} \\
& \left.+\gamma \delta \dot{\mathrm{q}}_{4}-\mathrm{K}_{\mathrm{d}} \delta \mathrm{q}_{4}-\delta \mathrm{q}_{15}\right)-\mathrm{C}_{\mathrm{d}}\left(\delta \dot{\mathrm{q}}_{4}-\delta \dot{\mathrm{q}}_{15}\right) \\
& +\zeta_{1} \delta \beta_{1}+\alpha_{1} \delta \mathrm{w}_{1}+\zeta_{2} \delta \beta_{2}+\alpha_{2} \delta \mathrm{w}_{2} .
\end{aligned}
$$

Now examine the generator torque equation:

$$
\mathrm{I}_{\text {gen }} \ddot{\mathrm{q}}_{15}=\mathrm{T}_{\text {shaft }}-\mathrm{T}_{\text {gen }} .
$$

Since the blade flap states do not couple with the generator states, the equation for the generator is exactly the same as in the last section.

$$
\begin{aligned}
& \mathrm{I}_{\text {gen }} \delta \ddot{\mathrm{q}}_{15}=\delta \mathrm{T}_{\text {shaft }}-\delta \mathrm{T}_{\text {gen }} \\
& =\mathrm{K}_{\mathrm{d}}\left(\delta \mathrm{q}_{4}-\delta \mathrm{q}_{15}\right)+\mathrm{C}_{\mathrm{d}}\left(\delta \dot{\mathrm{q}}_{4}-\delta \dot{\mathrm{q}}_{15}\right)-\delta \mathrm{T}_{\text {gen }}
\end{aligned}
$$

Again, we assume that the generator torque is constant (Region 3 operation), thus

$$
\delta \mathrm{T}_{\text {gen }}=0,
$$

so that finally:

$$
\mathrm{I}_{\mathrm{gen}} \delta \ddot{\mathrm{q}}_{15}=\mathrm{K}_{\mathrm{d}}\left(\delta \mathrm{q}_{4}-\delta \mathrm{q}_{15}\right)+\mathrm{C}_{\mathrm{d}}\left(\delta \dot{\mathrm{q}}_{4}-\delta \dot{\mathrm{q}}_{15}\right)
$$

The equation for the tower first fore-aft mode motion can be written:

$$
\begin{aligned}
& \mathrm{M}_{77} \delta \ddot{\mathrm{q}}_{7}+\mathrm{M}_{74} \delta \ddot{\mathrm{q}}_{4}+\mathrm{M}_{71} \delta \ddot{\mathrm{q}}_{1}+\mathrm{M}_{72} \delta \ddot{\mathrm{q}}_{2}+\mathrm{C}_{77} \delta \dot{\mathrm{q}}_{7}+\mathrm{C}_{74} \delta \dot{\mathrm{q}}_{4}+\mathrm{C}_{71} \delta \dot{\mathrm{q}}_{1}+\mathrm{C}_{72} \delta \dot{\mathrm{q}}_{2} \\
& +\mathrm{K}_{77} \delta \mathrm{q}_{7}+\mathrm{K}_{71} \delta \mathrm{q}_{1}+\mathrm{K}_{72} \delta \mathrm{q}_{2}=\delta \text { Thrust }{ }_{\text {aero }}
\end{aligned}
$$


where $\delta$ Thrust $_{\text {aero }}$ is the perturbation in rotor aerodynamic thrust force.

Now we can show that the aerodynamic thrust is a function of wind speed, rotor speed, and the pitch angle of each blade.

$$
\begin{aligned}
& \delta \text { Thrust }_{\text {aero }}=\text { Thrust }_{\text {aero }}-\text { Thrust }_{\text {aero }}\left(\beta_{1_{0}}, \beta_{2_{0}} \mathrm{w}_{1_{0}}, \mathrm{w}_{2_{0}}, \Omega_{0}\right)=\frac{\partial \mathrm{Thrust}_{\text {aero }}}{\partial \beta_{1}} \delta \beta_{1} \\
& +\frac{\partial \mathrm{Thrust}_{\text {aero }}}{\partial \mathrm{w}_{1}} \delta \mathrm{w}_{1}+\frac{\partial \mathrm{Thrust}_{\text {aero }}}{\partial \beta_{2}} \delta \beta_{2}+\frac{\partial \mathrm{Thrust}_{\text {aero }}}{\partial \mathrm{w}_{2}} \delta \mathrm{w}_{2}+\frac{\partial \mathrm{Thrust}_{\text {aero }}}{\partial \Omega} \delta \Omega .
\end{aligned}
$$

Let

$$
\frac{\partial \text { Thrust }_{\text {aero }}}{\partial \beta_{1}}=\zeta_{t_{1}}, \frac{\partial \text { Thrust }_{\text {aero }}}{\partial \beta_{2}}=\zeta_{t_{2}}, \frac{\partial \text { Thrust }_{\text {aero }}}{\partial \mathrm{w}_{1}}=\alpha_{t_{1}}, \frac{\partial \text { Thrust }_{\text {aero }}}{\partial \mathrm{w}_{2}}=\alpha_{t_{2}} .
$$

Then

$$
\begin{aligned}
& \delta \text { Thrust }_{\text {aero }}=\zeta_{t_{1}} \delta \beta_{1} \\
& +\alpha_{t_{1}} \delta \mathrm{w}_{1}+\zeta_{t_{2}} \delta \beta_{2}+\alpha_{t_{2}} \delta \mathrm{w}_{2}+\frac{\partial \mathrm{Thrust}_{\text {aero }}}{\partial \Omega} \delta \Omega .
\end{aligned}
$$

We will assume that the term $\frac{\partial \text { Thrust }_{\text {aero }}}{\partial \Omega} \delta \Omega$ goes back to the left hand side of the equation and is contained in the term $\mathrm{C}_{74} \delta \dot{\mathrm{q}}_{4}$, as part of the aerodynamic damping.

Let the states be defined as:

$\mathrm{x}_{1}=\delta \mathrm{q}_{1}$, perturbed blade-1 mode 1 flap displacement, $\mathrm{x}_{2}=\delta \dot{\mathrm{q}}_{1}$, perturbed blade-1 mode 1 flap velocity,

$\mathrm{x}_{3}=\delta \mathrm{q}_{2}$, perturbed blade- 2 mode 1 flap displacement $\mathrm{x}_{4}=\delta \dot{\mathrm{q}}_{2}$, perturbed blade- 2 mode 1 flap velocity,

$\mathrm{x}_{5}=\delta \dot{\mathrm{q}}_{4}$, perturbed rotor rotational speed,

$\mathrm{x}_{6}=\mathrm{K}_{\mathrm{d}}\left(\delta \mathrm{q}_{4}-\delta \mathrm{q}_{15}\right)$, perturbed drive-train torsional spring force,

$\mathrm{x}_{7}=\delta \dot{\mathrm{q}}_{15}$, perturbed generator rotational speed 
$\mathrm{x}_{8}=\delta \mathrm{q}_{7}$, perturbed tower first mode fore-aft deflection

$\mathrm{x}_{9}=\delta \dot{\mathrm{q}}_{7}$, perturbed tower first mode fore-aft velocity.

Then, the state space model is:

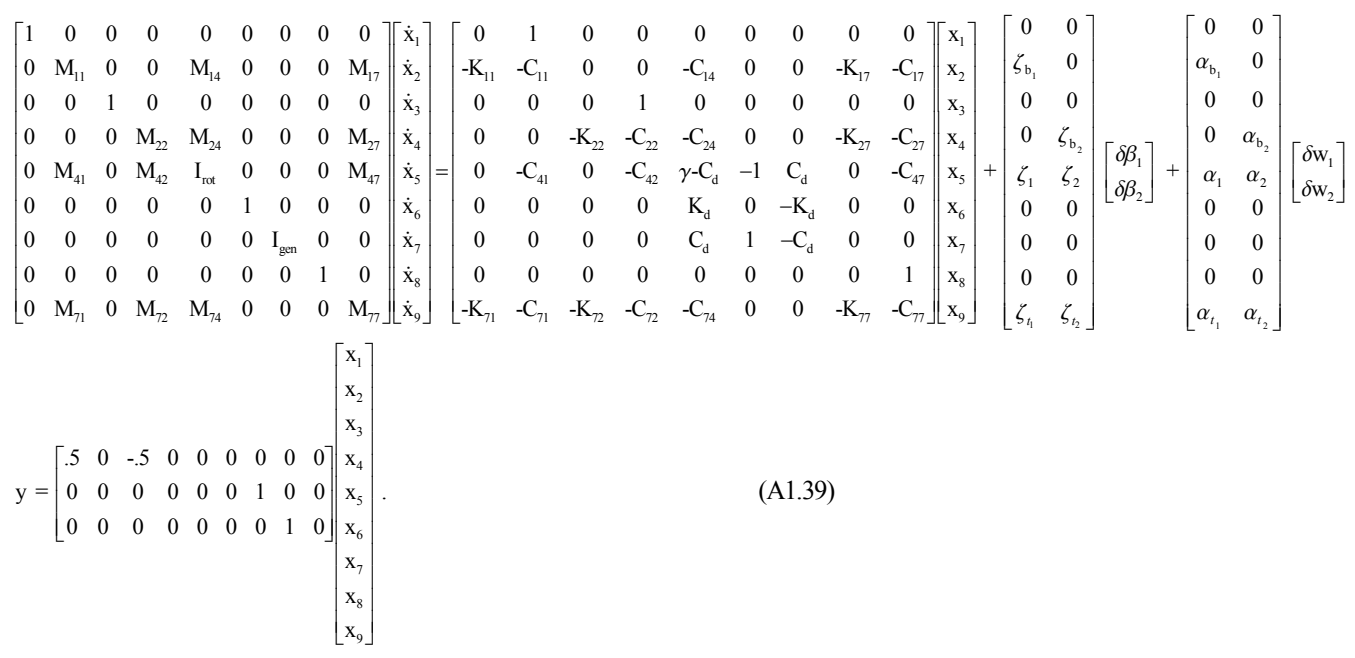

Here we assume that we measure perturbed generator speed $\mathrm{x}_{7}$, tower-top fore-aft deflection, and the rotor first asymmetric mode deflection $\frac{\mathrm{x}_{1}-\mathrm{x}_{3}}{2}$.

In the various chapters of this thesis, we will assume that the properties of blade-1 are the same as blade-2. This means that

$$
\begin{aligned}
& \zeta_{\mathrm{b}_{1}}=\zeta_{\mathrm{b}_{2}}=\zeta_{\mathrm{b}} ; \zeta_{1}=\zeta_{2}=\zeta ; \text { and } \zeta_{t_{1}}=\zeta_{t_{2}}=\zeta_{t} \\
& \text { also } \\
& \alpha_{\mathrm{b}_{1}}=\alpha_{\mathrm{b}_{2}}=\alpha_{\mathrm{b}} ; \alpha_{1}=\alpha_{2}=\alpha ; \text { and } \alpha_{t_{1}}=\alpha_{t_{2}}=\alpha_{t} .
\end{aligned}
$$

This is reflected in Eq. (2.8), in Chapter 2.

In Chapter 7 we first work with a model that includes only blade-1 and -2 flap and flap velocity, as well as rotor rotation (a model with 5 states). This model can be obtained from (A1.39) by eliminating the last 4 rows and columns. This model is: 


$$
\begin{aligned}
& {\left[\begin{array}{ccccc}
1 & 0 & 0 & 0 & 0 \\
0 & \mathrm{M}_{11} & 0 & 0 & \mathrm{M}_{14} \\
0 & 0 & 1 & 0 & 0 \\
0 & 0 & 0 & \mathrm{M}_{22} & \mathrm{M}_{24} \\
0 & \mathrm{M}_{41} & 0 & \mathrm{M}_{42} & \mathrm{I}_{\mathrm{rot}}+\mathrm{I}_{\mathrm{gen}}
\end{array}\right]\left[\begin{array}{c}
\dot{\mathrm{x}}_{1} \\
\dot{\mathrm{x}}_{2} \\
\dot{\mathrm{x}}_{3} \\
\dot{\mathrm{x}}_{4} \\
\dot{\mathrm{x}}_{5}
\end{array}\right]=\left[\begin{array}{ccccc}
0 & 1 & 0 & 0 & 0 \\
-\mathrm{K}_{11} & -\mathrm{C}_{11} & 0 & 0 & -\mathrm{C}_{14} \\
0 & 0 & 0 & 1 & 0 \\
0 & 0 & -\mathrm{K}_{22} & -\mathrm{C}_{22} & -\mathrm{C}_{24} \\
0 & -\mathrm{C}_{41} & 0 & -\mathrm{C}_{42} & \gamma
\end{array}\right]\left[\begin{array}{c}
\mathrm{x}_{1} \\
\mathrm{x}_{2} \\
\mathrm{x}_{3} \\
\mathrm{x}_{4} \\
\mathrm{x}_{5}
\end{array}\right]+\left[\begin{array}{cc}
0 & 0 \\
\zeta_{\mathrm{b}} & 0 \\
0 & 0 \\
0 & \zeta_{\mathrm{b}} \\
\zeta & \zeta
\end{array}\right]\left[\begin{array}{l}
\delta \beta_{1} \\
\delta \beta_{2}
\end{array}\right]+\left[\begin{array}{cc}
0 & 0 \\
\alpha_{\mathrm{b}} & 0 \\
0 & 0 \\
0 & \alpha_{\mathrm{b}} \\
\alpha & \alpha
\end{array}\right]\left[\begin{array}{l}
\delta \mathrm{w}_{1} \\
\delta \mathrm{w}_{2}
\end{array}\right]} \\
& \mathrm{y}=\left[\begin{array}{lllll}
0 & 0 & 0 & 0 & 1
\end{array}\right]\left[\begin{array}{l}
\mathrm{x}_{1} \\
\mathrm{x}_{2} \\
\mathrm{x}_{3} \\
\mathrm{x}_{4} \\
\mathrm{x}_{5}
\end{array}\right]
\end{aligned}
$$

A model that includes the drive-train torsion can be expressed as:

$$
\begin{aligned}
& {\left[\begin{array}{ccccccc}
1 & 0 & 0 & 0 & 0 & 0 & 0 \\
0 & \mathrm{M}_{11} & 0 & 0 & \mathrm{M}_{14} & 0 & 0 \\
0 & 0 & 1 & 0 & 0 & 0 & 0 \\
0 & 0 & 0 & \mathrm{M}_{22} & \mathrm{M}_{24} & 0 & 0 \\
0 & \mathrm{M}_{41} & 0 & \mathrm{M}_{42} & \mathrm{I}_{\text {rot }} & 0 & 0 \\
0 & 0 & 0 & 0 & 0 & 1 & 0 \\
0 & 0 & 0 & 0 & 0 & 0 & I_{\mathrm{gen}}
\end{array}\right]\left[\begin{array}{l}
\dot{\mathrm{x}}_{1} \\
\dot{\mathrm{x}}_{2} \\
\dot{\mathrm{x}}_{3} \\
\dot{\mathrm{x}}_{4} \\
\dot{\mathrm{x}}_{5} \\
\dot{\mathrm{x}}_{6}
\end{array}\right]=\left[\begin{array}{ccccccc}
0 & 1 & 0 & 0 & 0 & 0 & 0 \\
-\mathrm{K}_{11} & -\mathrm{C}_{11} & 0 & 0 & -\mathrm{C}_{14} & 0 & 0 \\
0 & 0 & 0 & 1 & 0 & 0 & 0 \\
0 & 0 & -\mathrm{K}_{22} & -\mathrm{C}_{22} & -\mathrm{C}_{24} & 0 & 0 \\
0 & -\mathrm{C}_{41} & 0 & -\mathrm{C}_{42} & \gamma_{-\mathrm{C}_{\mathrm{d}}} & -1 & \mathrm{C}_{\mathrm{d}} \\
0 & 0 & 0 & 0 & \mathrm{~K}_{\mathrm{d}} & 0 & -\mathrm{K}_{\mathrm{d}} \\
0 & 0 & 0 & 0 & \mathrm{C}_{\mathrm{d}} & 1 & -\mathrm{C}_{\mathrm{d}}
\end{array}\right]\left[\begin{array}{l}
\mathrm{x}_{1} \\
\mathrm{x}_{2} \\
\mathrm{x}_{3} \\
\mathrm{x}_{4} \\
\mathrm{x}_{5} \\
\mathrm{x}_{6} \\
\mathrm{x}_{7}
\end{array}\right]+\left[\begin{array}{cc}
0 & 0 \\
\zeta_{\mathrm{b}} & 0 \\
0 & 0 \\
0 & \zeta_{\mathrm{b}} \\
\zeta \\
\zeta & \zeta \\
0 & 0 \\
0 & 0
\end{array}\right]\left[\begin{array}{c}
\delta \beta_{1} \\
\delta \beta_{2}
\end{array}\right]+\left[\begin{array}{cc}
0 & 0 \\
\alpha_{\mathrm{b}} & 0 \\
0 & 0 \\
0 & \alpha_{\mathrm{b}} \\
\alpha & \alpha \\
0 & 0 \\
0 & 0
\end{array}\right]\left[\begin{array}{l}
\delta \mathrm{w}_{1} \\
\delta \mathrm{w}_{2}
\end{array}\right]} \\
& {\left[\begin{array}{l}
\mathrm{y}_{1} \\
\mathrm{y}_{2} \\
\mathrm{y}_{3}
\end{array}\right]=\left[\begin{array}{lllllll}
0 & 0 & 0 & 0 & 0 & 0 & 1 \\
1 & 0 & 0 & 0 & 0 & 0 & 0 \\
0 & 0 & 1 & 0 & 0 & 0 & 0
\end{array}\right]\left[\begin{array}{l}
\mathrm{x}_{1} \\
\mathrm{x}_{2} \\
\mathrm{x}_{3} \\
\mathrm{x}_{4} \\
\mathrm{x}_{5} \\
\mathrm{x}_{6} \\
\mathrm{x}_{7}
\end{array}\right] .}
\end{aligned}
$$

\section{A-1.4. Rotor and generator rotation, drive-train torsion, rotor first symmetric, asymmetric} flap, and tower first fore-aft mode model

We transform the blade flap states into rotor first symmetric and asymmetric flap, via:

$$
\begin{aligned}
& \bar{x}_{1}=\frac{1}{2}\left(\delta q_{1}+\delta q_{2}\right)=\frac{1}{2}\left(x_{1}+x_{3}\right) \\
& \bar{x}_{2}=\frac{1}{2}\left(\delta \dot{q}_{1}+\delta \dot{q}_{2}\right)=\frac{1}{2}\left(x_{2}+x_{4}\right) \\
& \bar{x}_{3}=\frac{1}{2}\left(\delta q_{1}-\delta q_{2}\right)=\frac{1}{2}\left(x_{1}-x_{3}\right) \\
& \bar{x}_{4}=\frac{1}{2}\left(\delta \dot{q}_{1}-\delta \dot{q}_{2}\right)=\frac{1}{2}\left(x_{2}-x_{4}\right) .
\end{aligned}
$$

We also keep the other 5 states in the last model:

$$
\begin{aligned}
& \overline{\mathrm{x}}_{5}=\delta \dot{\mathrm{q}}_{4}=\mathrm{x}_{5}, \\
& \overline{\mathrm{x}}_{6}=\mathrm{K}_{\mathrm{d}}\left(\delta \mathrm{q}_{4}-\delta \mathrm{q}_{15}\right)=\mathrm{x}_{6},
\end{aligned}
$$




$$
\begin{aligned}
& \overline{\mathrm{x}}_{7}=\delta \dot{\mathrm{q}}_{15}=\mathrm{x}_{7}, \\
& \overline{\mathrm{x}}_{8}=\delta \mathrm{q}_{7}, \text { perturbed generator rotational speed. } \\
& \overline{\mathrm{x}}_{9}=\delta \dot{\mathrm{q}}_{7}, \text { perturbed generator rotational speed. }
\end{aligned}
$$

Please note that the "barred" quantities are the transformed coordinates, and the "unbarred" quantities are the original blade coordinates, described in the last section.

We also transform blade 1 and 2 pitch and blade 1 and 2 wind speed:

$$
\begin{aligned}
& \delta \beta_{0}=\frac{1}{2}\left(\delta \beta_{1}+\delta \beta_{2}\right), \\
& \delta \beta_{c}=\frac{1}{2}\left(\delta \beta_{1}-\delta \beta_{2}\right), \\
& \delta \mathrm{w}_{0}=\frac{1}{2}\left(\delta \mathrm{w}_{1}+\delta \mathrm{w}_{2}\right), \\
& \delta \mathrm{w}_{\mathrm{c}}=\frac{1}{2}\left(\delta \mathrm{w}_{1}-\delta \mathrm{w}_{2}\right) .
\end{aligned}
$$

The pitch term $\delta \beta_{0}$ is called the rotor collective pitch, and the term $\delta \beta_{c}$ is called the rotor cyclic pitch component. The collective pitch input is the average pitch of both blades. The cyclic pitch input is proportional to the difference of the two pitch angles. Likewise, the "collective" wind component $\delta \mathrm{w}_{0}$ reflects an average of the wind seen on both blades, while the "cyclic" wind component $\delta \mathrm{w}_{\mathrm{c}}$ is proportional to the difference of the two wind speeds.

Now we add rows 2 and 4 of (A1.39), getting:

$$
\begin{aligned}
& \mathrm{M}_{11} \dot{\mathrm{x}}_{2}+\mathrm{M}_{14} \dot{\mathrm{x}}_{5}+\mathrm{M}_{22} \dot{\mathrm{x}}_{4}+\mathrm{M}_{24} \dot{\mathrm{x}}_{5}+\mathrm{M}_{17} \dot{\mathrm{x}}_{9}+\mathrm{M}_{27} \dot{\mathrm{x}}_{9}= \\
& -\mathrm{C}_{11} \mathrm{x}_{2}-\mathrm{C}_{22} \mathrm{x}_{4}-\mathrm{C}_{14} \mathrm{x}_{5}-\mathrm{C}_{24} \mathrm{x}_{5}-\mathrm{C}_{17} \mathrm{x}_{9}-\mathrm{C}_{27} \mathrm{x}_{9}-\mathrm{K}_{11} \mathrm{x}_{1}-\mathrm{K}_{22} \mathrm{x}_{3} \\
& -\mathrm{K}_{17} \mathrm{x}_{8}-\mathrm{K}_{27} \mathrm{x}_{8}+\zeta_{\mathrm{b}_{1}} \delta \beta_{1}+\alpha_{\mathrm{b}_{1}} \delta \mathrm{w}_{1}+\zeta_{\mathrm{b}_{2}} \delta \beta_{2}+\alpha_{\mathrm{b}_{2}} \delta \mathrm{w}_{2}
\end{aligned}
$$

Now we assume that blade 1 is identical to blade 2; this means that in Eq.s (A1.39),

$$
\begin{aligned}
& \mathrm{M}_{11}=\mathrm{M}_{22}, \mathrm{M}_{14}=\mathrm{M}_{24}, \mathrm{M}_{41}=\mathrm{M}_{42}, \mathrm{M}_{17}=\mathrm{M}_{27}, \mathrm{M}_{71}=\mathrm{M}_{72} ; \\
& \mathrm{C}_{11}=\mathrm{C}_{22}, \mathrm{C}_{14}=\mathrm{C}_{24}, \mathrm{C}_{41}=\mathrm{C}_{42}, \mathrm{C}_{17}=\mathrm{C}_{27}, \mathrm{C}_{71}=\mathrm{C}_{72}, \\
& \mathrm{~K}_{11}=\mathrm{K}_{22}, \mathrm{~K}_{17}=\mathrm{K}_{27}, \mathrm{~K}_{71}=\mathrm{K}_{72}, \\
& \zeta_{b_{2}}=\zeta_{b_{1}}=\zeta_{b}, \zeta_{t_{1}}=\zeta_{t_{2}}=\zeta_{t}, \text { and } \alpha_{t_{1}}=\alpha_{t_{2}}=\alpha_{t}, \alpha_{b_{2}}=\alpha_{b_{1}}=\alpha_{b} .
\end{aligned}
$$


Now (A1.42) becomes:

$$
\begin{aligned}
& \mathrm{M}_{11} \dot{\mathrm{x}}_{2}+\mathrm{M}_{14} \dot{\mathrm{x}}_{5}+\mathrm{M}_{17} \dot{\mathrm{x}}_{9}+\mathrm{M}_{22} \dot{\mathrm{x}}_{4}+\mathrm{M}_{24} \dot{\mathrm{x}}_{5}+\mathrm{M}_{27} \dot{\mathrm{x}}_{9}= \\
& -\mathrm{C}_{11} \mathrm{x}_{2}-\mathrm{C}_{22} \mathrm{x}_{4}-\mathrm{C}_{14} \mathrm{x}_{5}-\mathrm{C}_{17} \mathrm{x}_{9}-\mathrm{C}_{24} \mathrm{x}_{5}-\mathrm{C}_{27} \mathrm{x}_{9}-\mathrm{K}_{11} \mathrm{x}_{1}-\mathrm{K}_{22} \mathrm{x}_{3}-\mathrm{K}_{17} \mathrm{x}_{8} \\
& -\mathrm{K}_{27} \mathrm{x}_{8}+\zeta_{\mathrm{b}_{1}} \delta \beta_{1}+\alpha_{\mathrm{b}_{1}} \delta \mathrm{w}_{1}+\zeta_{\mathrm{b}_{2}} \delta \beta_{2}+\alpha_{\mathrm{b}_{2}} \delta \mathrm{w}_{2}
\end{aligned}
$$

or

$$
\begin{aligned}
& \mathrm{M}_{11}\left(\dot{\mathrm{x}}_{2}+\dot{\mathrm{x}}_{4}\right)+2 \mathrm{M}_{14} \dot{\mathrm{x}}_{5}+2 \mathrm{M}_{17} \dot{\mathrm{x}}_{9}= \\
& -\mathrm{C}_{11}\left(\mathrm{x}_{2}+\mathrm{x}_{4}\right)-2 \mathrm{C}_{14} \mathrm{x}_{5}-2 \mathrm{C}_{17} \mathrm{x}_{9}-\mathrm{K}_{11}\left(\mathrm{x}_{1}+\mathrm{x}_{3}\right)-2 \mathrm{~K}_{17} \mathrm{x}_{8} \\
& +\zeta_{\mathrm{b}_{1}}\left(\delta \beta_{1}+\delta \beta_{2}\right)+\alpha_{\mathrm{b}_{1}}\left(\delta \mathrm{w}_{1}+\delta \mathrm{w}_{2}\right) .
\end{aligned}
$$

Now, substituting the transformed coordinates, we get:

$$
\begin{aligned}
& 2 \mathrm{M}_{11} \dot{\overline{\mathrm{x}}}_{2}+2 \mathrm{M}_{14} \dot{\overline{\mathrm{x}}}_{5}+2 \mathrm{M}_{17} \dot{\overline{\mathrm{x}}}_{9}=-2 \mathrm{C}_{11} \overline{\mathrm{x}}_{2}-2 \mathrm{C}_{14} \overline{\mathrm{x}}_{5}-2 \mathrm{C}_{17} \overline{\mathrm{x}}_{9}-2 \mathrm{~K}_{11} \overline{\mathrm{x}}_{1} \\
& -2 \mathrm{~K}_{17} \overline{\mathrm{x}}_{8}+2 \zeta_{\mathrm{b}_{1}} \delta \beta_{0}+2 \alpha_{\mathrm{b}_{1}} \delta \mathrm{w}_{0} .
\end{aligned}
$$

This is the equation of motion for the rotor first symmetric flap mode.

Now we subtract row 4 from row 2 in (A1.39), getting:

$$
\begin{aligned}
& \mathrm{M}_{11}\left(\dot{\mathrm{x}}_{2}-\dot{\mathrm{x}}_{4}\right)=-\mathrm{C}_{11}\left(\mathrm{x}_{2}-\mathrm{x}_{4}\right)-\mathrm{K}_{11}\left(\mathrm{x}_{1}-\mathrm{x}_{3}\right) \\
& +\zeta_{\mathrm{b}_{1}}\left(\delta \beta_{1}-\delta \beta_{2}\right)+\alpha_{\mathrm{b}_{1}}\left(\delta \mathrm{w}_{1}+\delta \mathrm{w}_{2}\right) .
\end{aligned}
$$

Now writing in terms of transformed coordinates we get:

$$
2 \mathrm{M}_{11} \dot{\overline{\mathrm{x}}}_{4}=-2 \mathrm{C}_{11} \overline{\mathrm{x}}_{4}-2 \mathrm{~K}_{11} \overline{\mathrm{x}}_{3}+2 \zeta_{\mathrm{b}_{1}} \delta \beta_{\mathrm{c}}+2 \alpha_{\mathrm{b}_{1}} \delta \mathrm{w}_{\mathrm{c}} .
$$

This is the equation of motion for the rotor first asymmetric flap mode.

Now, looking at the rotor rotation equation of motion, we have

$$
\begin{aligned}
& 2 \mathrm{M}_{41} \dot{\overline{\mathrm{x}}}_{2}+\mathrm{I}_{r o t} \dot{\overline{\mathrm{x}}}_{5}+\mathrm{M}_{47} \dot{\overline{\mathrm{x}}}_{9}=-2 \mathrm{C}_{41} \overline{\mathrm{x}}_{2}-\mathrm{C}_{47} \overline{\mathrm{x}}_{9}+\left(\gamma-C_{d}\right) \overline{\mathrm{x}}_{5} \\
& -\overline{\mathrm{x}}_{6}+\mathrm{C}_{d} \overline{\mathrm{x}}_{7}+2 \zeta_{1} \delta \beta_{0}+2 \alpha_{1} \delta \mathrm{w}_{0} .
\end{aligned}
$$

The generator equation of motion is:

$$
\mathrm{I}_{\text {gen }} \dot{\overline{\mathrm{x}}}_{7}=\mathrm{C}_{\mathrm{d}} \overline{\mathrm{x}}_{5}+\overline{\mathrm{x}}_{6}-\mathrm{C}_{\mathrm{d}} \overline{\mathrm{x}}_{7} .
$$

Finally, the tower equation of motion is

$$
\begin{aligned}
& 2 \mathrm{M}_{71} \dot{\overline{\mathrm{x}}}_{2}+\mathrm{M}_{74} \dot{\overline{\mathrm{x}}}_{5}+\mathrm{M}_{77} \dot{\overline{\mathrm{x}}}_{9}=-2 \mathrm{~K}_{71} \overline{\mathrm{x}}_{1}-2 \mathrm{C}_{71} \overline{\mathrm{x}}_{2}-\mathrm{C}_{74} \overline{\mathrm{x}}_{5} \\
& -\mathrm{K}_{77} \overline{\mathrm{x}}_{8}-\mathrm{C}_{77} \overline{\mathrm{x}}_{9}+2 \zeta_{t_{1}} \delta \beta_{0}+2 \alpha_{t_{1}} \delta \mathrm{w}_{0} .
\end{aligned}
$$


Our state-space equation in these transformed coordinates can now be written:

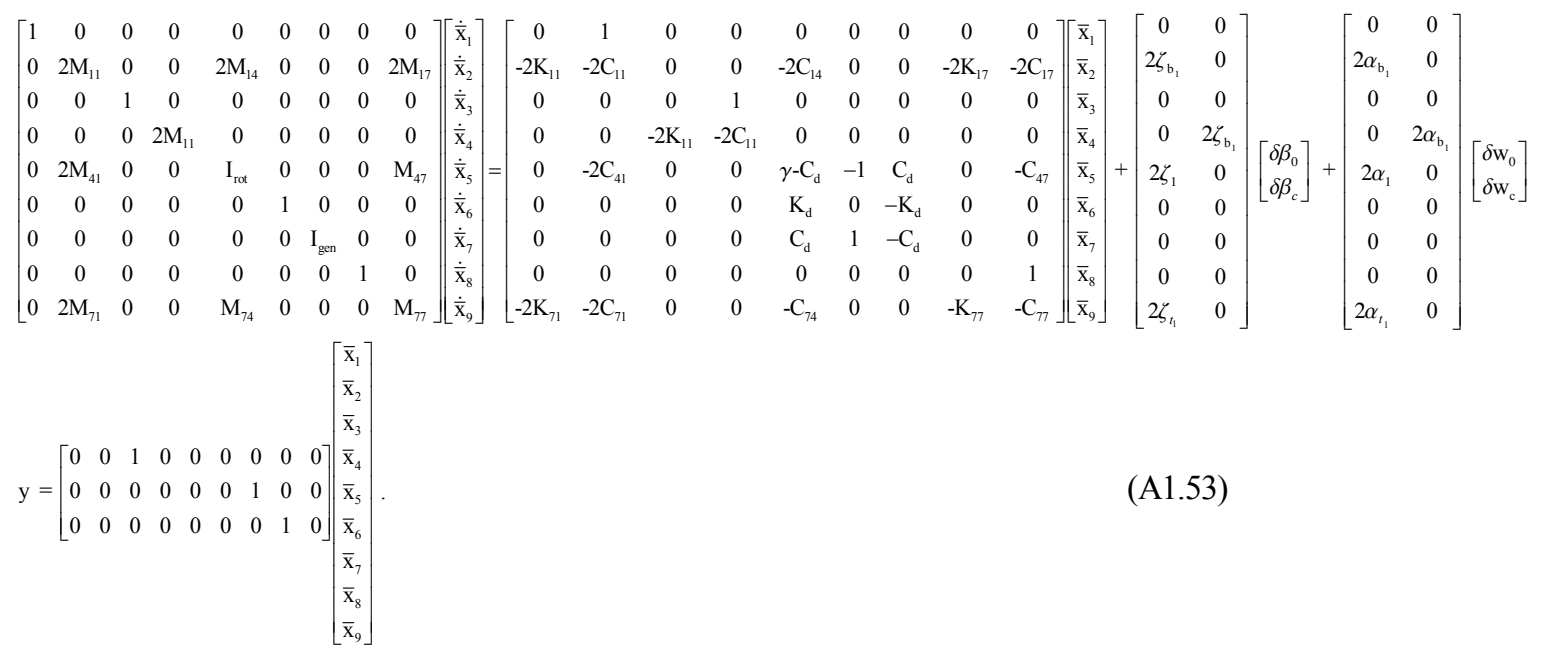

In the control algorithms described in Chapter 5 and 6 , we use only the rotor collective pitch input, assuming that the pitch of both blades is identical and equal to $\delta \beta$. The cyclic pitch becomes zero. Likewise, we assume that the wind input to the rotor is a constant over the rotor disk, equal to $\delta \mathrm{w}$. The cyclic wind component also becomes zero.

We also omit the equations for the two states describing the rotor first asymmetric mode (by deleting the $3^{\text {rd }}$ and $4^{\text {th }}$ rows and columns), since (as we will see in Chapter 5); this mode is uncontrollable, using rotor collective pitch. We thus obtain the state-space model:

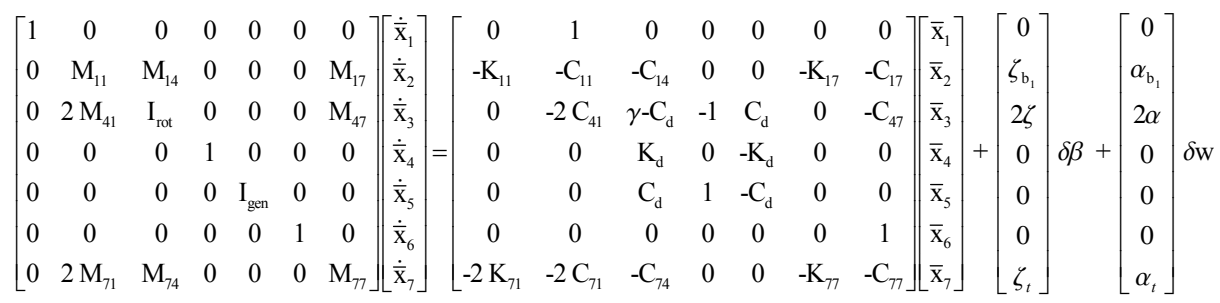

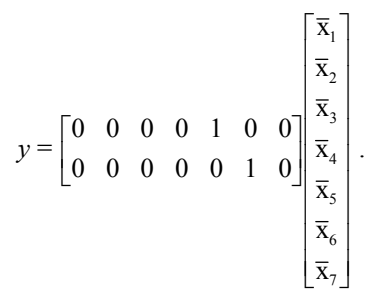

In this model, we measure the generator speed as well as the tower first fore-aft mode deflection. This is the state-space model we use in Chapters 5 and 6 using rotor collective pitch.

Another model that we use in Chapter 5 is the model without tower first fore-aft mode bending. If we omit columns and rows 6 and 7 from (A1.52) we get the 5-state model: 


$$
\begin{aligned}
& {\left[\begin{array}{ccccc}
1 & 0 & 0 & 0 & 0 \\
0 & \mathrm{M}_{11} & \mathrm{M}_{14} & 0 & 0 \\
0 & 2 \mathrm{M}_{41} & \mathrm{I}_{\text {rot }} & 0 & 0 \\
0 & 0 & 0 & 1 & 0 \\
0 & 0 & 0 & 0 & \mathrm{I}_{\text {gen }}
\end{array}\right]\left[\begin{array}{l}
\dot{\mathrm{x}}_{1} \\
\dot{\mathrm{x}}_{2} \\
\dot{\mathrm{x}}_{3} \\
\dot{\mathrm{x}}_{4} \\
\dot{\mathrm{x}}_{5}
\end{array}\right]=\left[\begin{array}{ccccc}
0 & 1 & 0 & 0 & 0 \\
-\mathrm{K}_{11} & -\mathrm{C}_{11} & -\mathrm{C}_{14} & 0 & 0 \\
0 & -2 \mathrm{C}_{41} & \gamma-\mathrm{C}_{\mathrm{d}} & -1 & \mathrm{C}_{\mathrm{d}} \\
0 & 0 & \mathrm{~K}_{\mathrm{d}} & 0 & -\mathrm{K}_{\mathrm{d}} \\
0 & 0 & \mathrm{C}_{\mathrm{d}} & 1 & -\mathrm{C}_{\mathrm{d}}
\end{array}\right]\left[\begin{array}{c}
\mathrm{x}_{1} \\
\mathrm{x}_{2} \\
\mathrm{x}_{3} \\
\mathrm{x}_{4} \\
\mathrm{x}_{5}
\end{array}\right]+\left[\begin{array}{c}
0 \\
\zeta_{\mathrm{b}_{1}} \\
2 \zeta \\
0 \\
0
\end{array}\right] \delta \beta+\left[\begin{array}{c}
0 \\
\alpha_{\mathrm{b}_{1}} \\
2 \alpha \\
0 \\
0
\end{array}\right] \delta \mathrm{w}}
\end{aligned}
$$

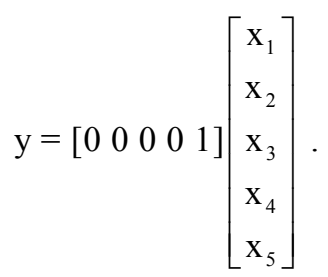




\section{A-2. Model Controllability and Observability}

We saw in Chapter 4 that several conditions were needed in order to design controllers using full state feedback, state estimation (without disturbance states), and DAC (which includes disturbance states). We needed controllability and observability. Let's look at the conditions for controllability and observability for the 3- and 5-state linear models. Those models have already been presented and won't be repeated here.

\section{A-2.1. Model controllability and observability for the 3-state model}

We now investigate model controllability and observability issues for the 3-state model derived in Section A1.2. We can gain insight into these issues by examining the determinant of the controllability and observability matrices. These systems are uncontrollable and unobservable when the determinant of these matrices becomes zero, indicating that the rank of the controllability or observability matrix is less than the order of the linear system. Much insight can be gained by deriving analytical expressions for the determinants of these matrices. First we look at the 3 -state linear model.

In all of this discussion we assume that the only control input is rotor collective pitch. The only measurement is generator speed.

\section{Controllability of $(A, B)$}

The 3-state linear model was described in Section A1.2. The determinant of the controllability matrix for the 3-state model is:

$$
\frac{K_{d}^{2} \zeta^{3}}{I_{g e n}}
$$

From this expression it can be seen that the controllability for this 3-state model depends upon the drive-train torsional spring stiffness $\mathrm{K}_{\mathrm{d}}$, the pitch input distribution gain $\zeta$, and the generator inertia $I_{\text {gen }}$.

The controllability of this system decreases (compared to a baseline case) when the spring stiffness and/or magnitude of the pitch gain $\zeta$ decrease, or if the generator inertia increases. As wind speed increases, the controllability of this system should increase, since the magnitude of the pitch gain increases and $K_{d}$ and $I_{\text {gen }}$ remain constant. Controllability then allows us to arbitrarily place the poles of the closed-loop system through full state feedback. We can see from this analysis which parameters affect system controllability.

Observability allows us to arbitrarily place the poles of the estimator states. First we look at the case when disturbances are ignored.

\section{Observability of $(A, C)$}

A symbolic expression for the determinant of the observability matrix of this system can be written:

$$
\frac{-K_{d}}{I_{\text {gen }}^{2}} \text {. }
$$

From this expression we can conclude that observability of this system decreases when the drive-train torsion spring constant decrease and the generator inertia increase relative to a baseline value. We now look at the observability of the augmented system which includes the wind disturbance state. 


\section{Observability of $(\bar{A}, \bar{C})$}

A symbolic expression for the determinant of the observability matrix of this system is:

$$
\frac{-K_{d}^{2} \alpha}{I_{g e n}{ }^{3}}
$$

From this expression we can conclude that observability of this system decreases when the drive-train torsion spring constant decreases or when the magnitude of the wind disturbance input gain $\alpha$ decreases relative to a baseline value. Observability decreases when the generator inertia increases relative to a baseline case.

\section{A-2.2. Model controllability and observability for the 5-state model}

Now lets look at controllability and observability of the 5-state model, listed in Section A1.3 (see Eq. [A1.53]).

\section{Controllability of $(A, B)$}

A symbolic expression for the determinant of the controllability matrix for this system contains many terms. To obtain useful information from this expression, we must make some simplifying assumptions. Let's first assume that the pitch input distribution gain related to the flap force $\zeta_{\mathrm{b}}$ (See Section A-1.3) is zero. With this assumption, the determinant of the controllability matrix can be written:

$$
\begin{aligned}
& \left(K _ { d } { } ^ { 2 } \left(I_{g e n}{ }^{2} K_{11}{ }^{2}+I_{g e n} K_{d} C_{11}{ }^{2}-2 K_{11} I_{g e n} K_{d} M_{11}-C_{11} C_{d}\left(I_{g e n} K_{11}+K_{d} M_{11}\right)\right.\right. \\
& \left.+M_{11}\left(C_{d}{ }^{2} K_{11}+K_{d}{ }^{2} M_{11}\right)\right)\left(-C_{14}{ }^{2} M_{11}\right. \\
& \left.\left.+C_{11} C_{14} M_{14}-K_{11} M_{14}{ }^{2}\right) \zeta^{5}\right) /\left(I_{g e n}{ }^{3}\left(I_{r o t} M_{11}-2 M_{14} M_{41}\right)^{5}\right)
\end{aligned}
$$

We can see that the controllability depends directly upon $K_{d}$ and $\zeta$. For decreased values of either of these parameters, controllability decreases. The controllability also depends upon various other mass, inertia, damping, and stiffness coefficients. It is important to note that the system is controllable even if $\zeta_{\mathrm{b}}=0$. Controllability is again decreased if the generator inertia Igen increases.

Now suppose the other pitch input distribution parameter $\zeta=0$. Then the determinant of the controllability matrix becomes:

$$
\begin{aligned}
& \left(8 K _ { d } { } ^ { 3 } \gamma \left(C_{41}{ }^{3} I_{g e n} I_{r o t}+K_{d} M_{41}{ }^{3} \gamma+C_{41} M_{41}{ }^{2}\left(I_{g e n} K_{d}+\right.\right.\right. \\
& \left.\left.\left.I_{r o t} K_{d}-C_{d} \gamma\right)-C_{41}{ }^{2} M_{41}\left(C_{d}\left(I_{g e n}+I_{r o t}\right)-I_{g e n} \gamma\right)\right) \zeta_{b 1}{ }^{5}\right) /\left(I_{g e n}{ }^{3}\left(I_{r o t} M_{11}-2 M_{14} M_{41}\right)^{5}\right)
\end{aligned}
$$

The controllability again depends directly upon $\mathrm{K}_{\mathrm{d}}$. It also depends upon $\zeta_{\mathrm{b}}$. If both $\zeta=0$ and $\zeta_{\mathrm{b}}=0$, then this determinant becomes 0 , and the system is uncontrollable. The controllability also depends upon the other mass, inertia, stiffness, and damping coefficients listed in the above expression.

Now we look at observability of $(A, C)$. 


\section{Observability of $(A, C)$}

A symbolic expression for the determinant of the observability matrix for this system contains many terms, and can be simplified to:

$$
\begin{aligned}
& -\left(4 K _ { 1 1 } ( C _ { 4 1 } { } ^ { 2 } M _ { 1 1 } - C _ { 1 1 } C _ { 4 1 } M _ { 4 1 } + K _ { 1 1 } M _ { 4 1 } ^ { 2 } ) \left(2 C_{41} C_{d} K_{d}\right.\right. \\
& \left(-C_{41} C_{d}+K_{d} M_{41}\right)+K_{d}^{3}\left(I_{r o t} M_{11}-2 M_{14} M_{41}\right) \\
& +C_{d}^{3} K_{11} \gamma+C_{d}{ }^{2} K_{d}\left(I_{r o t} K_{11}-C_{11} \gamma\right) \\
& \left.\left.+C_{d} K_{d}{ }^{2}\left(-C_{11} I_{r o t}+2 C_{41} M_{41}+M_{11} \gamma\right)\right)\right) /\left(I_{g e n}{ }^{4}\left(I_{r o t} M_{11}-2 M_{14} M_{41}\right)^{3}\right)
\end{aligned}
$$

The observability of this system depends directly upon the drive-train torsional stiffness $\mathrm{K}_{\mathrm{d}}$ and the blade first flap mode stiffness coefficient $\mathrm{K}_{11}$. The observability also depends directly upon various other mass and damping terms, such as $\mathrm{M}_{11}, \mathrm{M}_{41}, \mathrm{C}_{11}$, and $\mathrm{C}_{41}$, etc. It depends inversely upon the generator inertia.

Another interesting observation is that if the terms which couple the rotor first symmetric flap states to the other states of the system (such as $\mathrm{M}_{41}, \mathrm{M}_{14}, \mathrm{C}_{41}$, and $\mathrm{C}_{14}$ ) all become zero, then the system is unobservable. These coefficients decrease in magnitude as the blade pitch angle decreases. This is important because the presence of these coupling terms, for nonzero pitch angles, allows us to use state estimation when only measuring generator speed. The system becomes unobservable when these coupling terms become zero. This usually occurs for zero pitch angles (including zero blade pre-twist).

We can conclude that in general, as the inertia of the generator increases, observability decreases. In addition, as the drive-train torsional stiffness and blade flap stiffness decrease, observability decreases. It is interesting that observability of this system does not depend upon either the pitch gain $\zeta$ or disturbance gain $\alpha$. It depends only upon the mass, damping, and stiffness values of the modeled components.

Now we look at observability of $(\bar{A}, \bar{C})$, the system augmented with disturbance state.

\section{Observability of $(\bar{A}, \bar{C})$}

A symbolic expression for the determinant of the observability matrix for this augmented system contains many terms, and can be simplified to:

$$
\begin{aligned}
& -\left(4 K _ { 1 1 } { } ^ { 2 } K _ { d } ( C _ { 4 1 } { } ^ { 2 } M _ { 1 1 } - C _ { 1 1 } C _ { 4 1 } M _ { 4 1 } + K _ { 1 1 } M _ { 4 1 } { } ^ { 2 } ) \alpha \left(2 C_{41} C_{d} K_{d}\right.\right. \\
& \left(-C_{41} C_{d}+K_{d} M_{41}\right)+K_{d}{ }^{3}\left(I_{r o t} M_{11}-2 M_{14} M_{41}\right) \\
& +C_{d}^{3} K_{11} \gamma+C_{d}{ }^{2} K_{d}\left(I_{r o t} K_{11}-C_{11} \gamma\right) \\
& \left.\left.+C_{d} K_{d}{ }^{2}\left(-C_{11} I_{r o t}+2 C_{41} M_{41}+M_{11} \gamma\right)\right)\right) /\left(I_{g e n}{ }^{5}\left(I_{r o t} M_{11}-2 M_{14} M_{41}\right)^{4}\right)
\end{aligned}
$$

We can conclude many of the same observations as made for observability of $(A, C)$. Again, the presence of the terms which couple the rotor first symmetric flap mode with the other states in the system (such as $M_{41}, M_{14}, C_{41}$ ) permit us to use state estimation, because the system is then observable. In addition, the observability of this system is directly related to the wind disturbance gain $\alpha$, as well as drive-train stiffness $\mathrm{K}_{\mathrm{d}}$. If either one or both of these parameters is zero, then the system in unobservable. 
Again, if the terms which couple the rotor first symmetric flap states to the other states of the system (such as $\mathrm{M}_{41}$, $\mathrm{M}_{14}, \mathrm{C}_{41}$, and $\mathrm{C}_{14}$ ) all become zero, then the system is unobservable. These coefficients decrease in magnitude as the blade pitch angle decreases. This is important because the presence of these coupling terms, for nonzero pitch angles, allows us to use state estimation when only measuring generator speed. This usually occurs for zero pitch angles (including zero blade pre-twist).

For the models with additional states, symbolic expressions for the determinant of the controllability and observability matrices become too complicated for useful information. One can use other tools, such as controllability and observability grammians to assess the degree of controllability and observability. This concludes our discussion on observability and controllability of the systems modeled for rotor collective pitch. 


\section{Appendix B. Inputs to Control Design}

\section{B-1. Model Inputs for the CART}

\section{B-1.1 Turbine Components}

The Controls Advanced Research Turbine (CART) is a modified Westinghouse model WWG-0600 machine, with rated power of $600 \mathrm{~kW}$. The rotor is upwind of the tower and consists of two blades and a teetering hub. The turbine is shown in Figure 2-6. The following sections describe each major component in enough detail to enable readers to prepare a structural dynamic model of this machine. Further details regarding CART properties are provided in [Stol and Bir, 2003].

\section{Tower}

The tower is idealized as an elastic beam cantilevered to the ground. Table B-1.1 lists the distributed properties at 13 stations, referenced from the ground-attached coordinate system. The first station is at $\mathrm{x}=0.0 \mathrm{~m}$. (ground) and the last station as at $\mathrm{x}=34.862 \mathrm{~m}$. (tower-top). The mass and the mass moments of inertia, Iy and Iz, are all per unit length (y is the axis attached to the tower base, pointing to the side, while $\mathrm{z}$ is generally downwind). Attached to the tower top on the $\mathrm{x}$-axis is a concentrated mass of $1610 \mathrm{~kg}$. Further details regarding coordinate axes can be found in [Stol and Bir, 2003].

Table B-1.1. CART Tower Distributed Properties

\begin{tabular}{|c|c|c|c|c|c|c|c|}
\hline $\begin{array}{l}\text { Height, } \\
\text { (m) }\end{array}$ & $\begin{array}{l}\mathrm{m} / \mathrm{L} \\
(\mathrm{kg} / \mathrm{m})\end{array}$ & $\begin{array}{l}\text { ly/L } \\
\text { (kg-m) }\end{array}$ & $\begin{array}{l}\text { Iz/L } \\
\text { (kg-m) }\end{array}$ & $\begin{array}{l}G J \\
\left(N-m^{\wedge} 2\right)\end{array}$ & $\begin{array}{l}\text { EA } \\
\text { (N) }\end{array}$ & $\begin{array}{l}\text { Eiy } \\
\left(\mathrm{N}-\mathrm{m}^{\wedge} 2\right)\end{array}$ & $\begin{array}{l}\text { Eiz } \\
\left(\mathrm{N}-\mathrm{m}^{\wedge} 2\right)\end{array}$ \\
\hline 0 & 1548 & 3444 & 3444 & $3.06 E+10$ & $3.81 \mathrm{E}+10$ & $8.31 \mathrm{E}+10$ & $8.31 E+10$ \\
\hline 2.294 & 1361 & 2311 & 2311 & $2.05 E+10$ & $3.33 E+10$ & $5.58 \mathrm{E}+10$ & $5.58 \mathrm{E}+10$ \\
\hline 6.867 & 1428 & 1277 & 1277 & $1.13 E+10$ & $3.50 \mathrm{E}+10$ & $3.09 E+10$ & $3.09 E+10$ \\
\hline 9.145 & 1311 & 742 & 742 & $6.57 \mathrm{E}+09$ & $3.20 \mathrm{E}+10$ & $1.80 \mathrm{E}+10$ & $1.80 \mathrm{E}+10$ \\
\hline 11.481 & 1311 & 742 & 742 & $6.57 \mathrm{E}+09$ & $3.20 \mathrm{E}+10$ & $1.80 \mathrm{E}+10$ & $1.80 \mathrm{E}+10$ \\
\hline 14.986 & 1311 & 742 & 742 & $6.57 \mathrm{E}+09$ & $3.20 \mathrm{E}+10$ & $1.80 \mathrm{E}+10$ & $1.80 \mathrm{E}+10$ \\
\hline 17.909 & 878 & 482 & 482 & $4.28 \mathrm{E}+09$ & $2.11 \mathrm{E}+10$ & 1.17E+10 & 1.17E+10 \\
\hline 21.417 & 878 & 482 & 482 & $4.28 \mathrm{E}+09$ & $2.11 \mathrm{E}+10$ & 1.17E+10 & 1.17E+10 \\
\hline 24.339 & 878 & 482 & 482 & $4.28 E+09$ & $2.11 \mathrm{E}+10$ & $1.17 E+10$ & $1.17 E+10$ \\
\hline 27.248 & 599 & 317 & 317 & $2.81 \mathrm{E}+09$ & $1.39 \mathrm{E}+10$ & $7.65 \mathrm{E}+10$ & $7.65 E+10$ \\
\hline 30.727 & 599 & 317 & 317 & $2.81 \mathrm{E}+09$ & $1.39 \mathrm{E}+10$ & $7.65 \mathrm{E}+10$ & $7.65 \mathrm{E}+10$ \\
\hline 33.664 & 1311 & 742 & 742 & $6.57 E+09$ & $3.20 \mathrm{E}+10$ & $1.80 \mathrm{E}+10$ & $1.80 \mathrm{E}+10$ \\
\hline 34.862 & 1311 & 742 & 742 & $6.57 \mathrm{E}+09$ & $3.20 \mathrm{E}+10$ & $1.80 \mathrm{E}+10$ & $1.80 \mathrm{E}+10$ \\
\hline
\end{tabular}

\section{Nacelle}

The nacelle is assumed a rigid body attached to the tower-top with fixed tilt angle of 3.77 degrees (deg.). It is capable of yawing about the tower $\mathrm{x}$-axis, with positive yaw being clockwise when viewed from above. The mass and moments of inertia for all non-rotating parts are lumped to the nacelle, including the bedplate, shaft bearings, gearbox housing, and generator stator. In addition, the mass and lateral moments of inertia (Iy and Iz) of the high-speed shaft and generator rotor are included. The center-of-mass is located 
along the shaft axis upwind. Using the coordinate system attached to the nacelle in Figure B.1, the mass properties are as follows.

Mass:

Moments of inertia:
$23228 \mathrm{~kg}$

$$
\begin{aligned}
& I_{x}=1.2 \times 10^{3} \mathrm{~kg}-\mathrm{m}^{2} \\
& I_{y}=3.659 \times 10^{4} \mathrm{~kg}-\mathrm{m}^{2} \\
& I_{z}=3.659 \times 10^{4} \mathrm{~kg}-\mathrm{m}^{2}
\end{aligned}
$$

\section{High-Speed Shaft}

The high-speed shaft (HSS) is assumed a rigid body aligned with the shaft axis. Positive rotation is clockwise when viewed from upwind. The mass and lateral moments of inertia are lumped with the nacelle. The polar moment of inertia, which includes rotating components in the gearbox, is given by:

$$
\mathrm{J}=\mathrm{I}_{\mathrm{x}}=34.4 \mathrm{~kg}-\mathrm{m}^{2}
$$

\section{Gearbox}

The two-state gearbox is assumed $100 \%$ efficient, rotating the high-speed shaft in the same direction as the low-speed shaft. The gear ratio is 43.165. The gearbox housing and low-speed shaft bearings are coincident for modeling purposes, as shown in [Stol and Bir, 2003], and are rigidly attached to the nacelle.

\section{Low-Speed Shaft}

The low-speed shaft (LSS) is modeled as a massless cylindrical beam, cantilevered at the shaft bearings and rigidly attached to the hub yoke. The stiffness properties are as follows.

$$
\begin{aligned}
& \mathrm{EA}=1.519 \times 10^{10} \mathrm{~N} \\
& \mathrm{EI}=4.776 \times 10^{7} \mathrm{~N}-\mathrm{m}^{2} \\
& \mathrm{GJ}=1.851 \times 10^{7} \mathrm{~N}-\mathrm{m}^{2} \\
& \mathrm{GA}=5.889 \times 10^{9} \mathrm{~N}
\end{aligned}
$$

\section{Hub Yoke}

The yoke comprises the non-teetering components attached to the end of the low-speed shaft. It is idealized as a concentrated mass at the teeter pin.

$$
\text { Yoke Mass }=5885 \mathrm{~kg}
$$




\section{Hub Spindle}

The spindle behaves as a rigid body that teeters about a pin attached to the yoke. There is no delta-three angle or precone. The spindle center of mass is assumed to lie on the teeter pin with coordinate system as shown in [Stol and Bir, 2003]. The mass properties are:

Mass:

Moments of inertia:
$5852 \mathrm{~kg}$

$$
\begin{aligned}
& I_{x}=0 \mathrm{~kg}-\mathrm{m}^{2} \\
& I_{y}=1.5 \times 10^{4} \mathrm{~kg}-\mathrm{m}^{2} \\
& I_{z}=1.5 \times 10^{4} \mathrm{~kg}-\mathrm{m}^{2}
\end{aligned}
$$

\section{Blades}

Table B-1.2 lists properties for each blade at 18 stations, referenced from the spindle end. The first station is at $\mathrm{x}=0.0 \mathrm{~m}$ (blade root) and the last station is at $\mathrm{x}=19.995 \mathrm{~m}$ (blade tip). The reference $\mathrm{x}$-axis is the pitch axis, while the local y- and z-axes are aligned as shown in [Stol and Bir, 2003] for each station. The z-axis always points toward the leading edge of the airfoil along the chord line and the $y$-axis is generally pointing upwind. The cross-section center of mass is defined by the coordinates $\left\{y_{\mathrm{cg}}, z_{\mathrm{cg}}\right\}$ and the elastic center is defined by $\left\{y_{\text {elast }}, z_{\text {elast }}\right\}$, measured from the reference axes $\left\{y_{\text {ref }}, z_{\text {ref }}\right\}$. The aerodynamic center is located along the chord line at a distance of $z_{\text {ac }}$ from the reference x-axis. The structural twist, $\theta_{\text {elast }}$, representing the orientation of the principal elastic axes and principle moments of inertia, is defined as positive when the leading edge is rotated into the wind. The aerodynamic twist, $\theta_{\text {aero }}$, representing the orientation of the blade chord with respect to the plane of rotation, is defined the same way. The blades rotate clockwise if observed from upwind. Further details regarding CART properties are provided in [Stol and Bir, 2003]. 
Table B-1.2. CART Blade Distributed Properties

\begin{tabular}{|c|c|c|c|c|c|c|c|c|c|c|c|c|c|c|c|}
\hline $\begin{array}{c}\text { Dist. X } \\
\text { (m) }\end{array}$ & $\begin{array}{c}\mathrm{m} / \mathrm{L} \\
(\mathrm{kg} / \mathrm{m})\end{array}$ & $\begin{array}{c}\mathrm{ly} / \mathrm{L} \\
(\mathrm{kg}-\mathrm{m})\end{array}$ & $\begin{array}{c}\mathrm{Iz} / \mathrm{L} \\
(\mathrm{kg}-\mathrm{m})\end{array}$ & $\begin{array}{l}y c g \\
(m)\end{array}$ & $\begin{array}{l}\text { zcg } \\
(\mathrm{m})\end{array}$ & $\begin{array}{l}\text { yelast } \\
(\mathrm{m})\end{array}$ & $\begin{array}{l}\text { zelast } \\
\text { (m) }\end{array}$ & $\begin{array}{l}\text { Struct } \\
\text { twist } \\
\text { (deg) }\end{array}$ & $\begin{array}{c}\text { GJ } \\
\left(\mathrm{N}-\mathrm{m}^{\wedge} 2\right)\end{array}$ & $\begin{array}{l}\text { EA } \\
(N)\end{array}$ & $\begin{array}{c}\text { Ely } \\
\left(\mathrm{N}-\mathrm{m}^{\wedge} 2\right)\end{array}$ & $\begin{array}{c}\text { Elz } \\
\left(\mathrm{N}-\mathrm{m}^{\wedge} 2\right)\end{array}$ & $\begin{array}{l}\text { chord } \\
\text { (m) }\end{array}$ & $\begin{array}{l}\text { zac } \\
(\mathrm{m})\end{array}$ & $\begin{array}{l}\text { Aero } \\
\text { twist } \\
\text { (deg) }\end{array}$ \\
\hline 0 & 282.92 & 29.47 & 12.33 & 0 & -0.09 & 0 & 0.008 & 3.44 & $4.13 \mathrm{E}+07$ & $4.94 \mathrm{E}+09$ & $2.83 \mathrm{E}+08$ & $1.65 \mathrm{E}+08$ & 1.143 & -0.02 & 3.44 \\
\hline 0.4475 & 290.24 & 33.11 & 11.97 & 0 & -0.116 & 0 & -0.019 & 3.37 & $4.11 \mathrm{E}+07$ & $5.09 E+09$ & $3.18 E+08$ & $1.61 \mathrm{E}+08$ & 1.196 & -0.017 & 3.37 \\
\hline 1.0572 & 261.88 & 34.19 & 10.57 & 0 & -0.156 & 0 & -0.056 & 3.27 & $3.84 \mathrm{E}+07$ & $4.57 E+09$ & $3.28 \mathrm{E}+08$ & $1.42 \mathrm{E}+08$ & 1.268 & -0.014 & 3.27 \\
\hline 2.2764 & 201.28 & 31.97 & 7.35 & 0 & -0.238 & 0 & -0.131 & 3.08 & $2.86 \mathrm{E}+07$ & $3.45 E+09$ & $3.07 E+08$ & $9.87 E+07$ & 1.411 & -0.011 & 3.08 \\
\hline 3.4956 & 186.52 & 35.48 & 5.82 & 0 & -0.314 & 0 & -0.205 & 2.88 & $2.27 \mathrm{E}+07$ & $3.21 \mathrm{E}+09$ & $3.40 \mathrm{E}+08$ & $7.84 \mathrm{E}+07$ & 1.555 & -0.008 & 2.88 \\
\hline 4.7148 & 169.1 & 35.67 & 4.41 & 0 & -0.379 & 0 & -0.279 & 2.69 & $1.67 E+07$ & $2.93 E+09$ & $3.42 \mathrm{E}+08$ & $5.92 \mathrm{E}+07$ & 1.699 & -0.006 & 2.69 \\
\hline 5.9848 & 149.28 & 29.02 & 3.38 & 0 & -0.37 & 0 & -0.272 & 2.45 & $1.29 \mathrm{E}+07$ & $2.59 \mathrm{E}+09$ & $2.78 \mathrm{E}+08$ & $4.54 \mathrm{E}+07$ & 1.637 & -0.003 & 2.45 \\
\hline 7.2548 & 133.19 & 24.71 & 2.54 & 0 & -0.378 & 0 & -0.265 & 2.21 & $9.74 \mathrm{E}+06$ & $2.33 E+09$ & $2.37 \mathrm{E}+08$ & $3.41 \mathrm{E}+07$ & 1.575 & 0 & 2.21 \\
\hline 8.5248 & 111.74 & 17.58 & 1.86 & 0 & -0.341 & 0 & -0.249 & 1.91 & $7.24 \mathrm{E}+06$ & $1.96 \mathrm{E}+09$ & $1.69 \mathrm{E}+08$ & $2.50 \mathrm{E}+07$ & 1.494 & 0 & 1.91 \\
\hline 9.7948 & 96.86 & 14.34 & 1.33 & 0 & -0.338 & 0 & -0.234 & 1.61 & $5.29 \mathrm{E}+06$ & $1.73 E+09$ & $1.38 \mathrm{E}+08$ & $1.79 \mathrm{E}+07$ & 1.412 & 0 & 1.61 \\
\hline 11.0648 & 78.57 & 9.81 & 0.92 & 0 & -0.303 & 0 & -0.219 & 1.24 & $3.71 E+06$ & $1.42 E+09$ & $9.40 \mathrm{E}+07$ & $1.23 E+07$ & 1.331 & 0 & 1.24 \\
\hline 12.3348 & 65.03 & 7.54 & 0.61 & 0 & -0.296 & 0 & -0.204 & 0.86 & $2.53 E+06$ & $1.21 \mathrm{E}+09$ & $7.25 \mathrm{E}+07$ & $8.19 E+06$ & 1.25 & 0 & 0.86 \\
\hline 13.6048 & 49.68 & 4.87 & 0.38 & 0 & -0.265 & 0 & -0.189 & 0.38 & $1.63 E+06$ & $9.59 E+08$ & $4.67 \mathrm{E}+07$ & $5.14 \mathrm{E}+06$ & 1.168 & 0 & 0.38 \\
\hline 14.8748 & 37.59 & 3.4 & 0.23 & 0 & -0.257 & 0 & -0.174 & -0.11 & $1.01 \mathrm{E}+06$ & $7.74 \mathrm{E}+08$ & $3.26 \mathrm{E}+07$ & $3.02 E+06$ & 1.087 & 0 & -0.11 \\
\hline 16.1448 & 25.01 & 1.98 & 0.12 & 0 & -0.229 & 0 & -0.159 & -0.77 & $5.70 \mathrm{E}+05$ & $5.79 E+08$ & $1.90 \mathrm{E}+07$ & $1.62 E+06$ & 1.006 & 0 & -0.77 \\
\hline 17.4148 & 16.01 & 1.35 & 0.06 & 0 & -0.216 & 0 & -0.144 & -1.43 & $3.17 \mathrm{E}+05$ & $4.53 E+08$ & $1.30 \mathrm{E}+07$ & $8.68 E+05$ & 0.925 & 0 & -1.43 \\
\hline 18.6848 & 10.73 & 0.92 & 0.03 & 0 & -0.185 & 0 & -0.129 & -2.37 & $1.69 E+05$ & $3.98 E+08$ & $8.85 E+06$ & $4.68 \mathrm{E}+05$ & 0.843 & 0 & -2.37 \\
\hline 19.9548 & 6.02 & 0.71 & 0.02 & 0 & -0.172 & 0 & -0.114 & -3.31 & $7.58 \mathrm{E}+04$ & $3.53 E+08$ & $6.80 \mathrm{E}+06$ & $2.09 E+05$ & 0.762 & 0 & -3.31 \\
\hline
\end{tabular}




\title{
B-2. FAST and ADAMS Input Files
}

\section{B2.1 FAST Input File}

\author{
CARTNEW.FAD
}

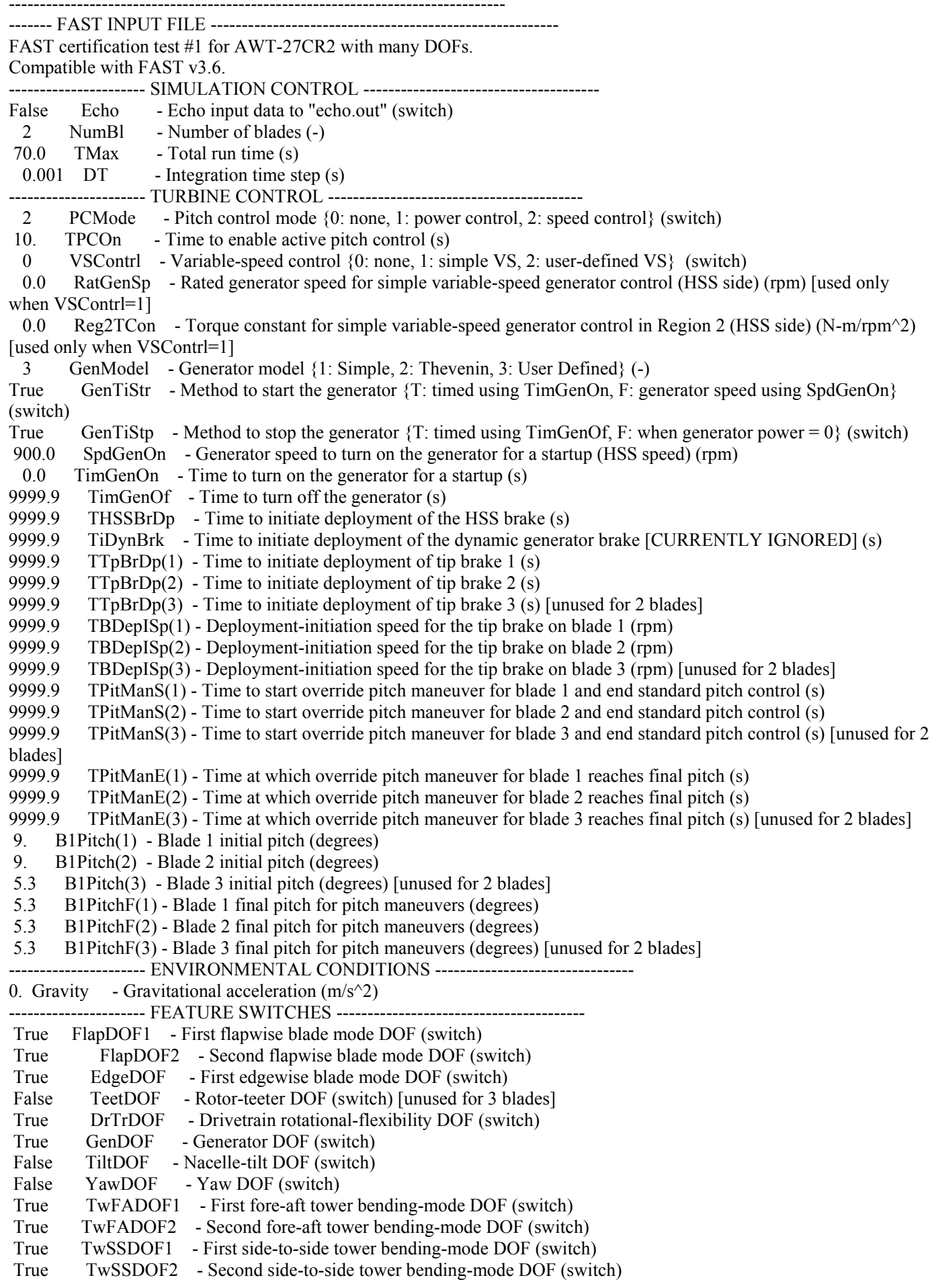




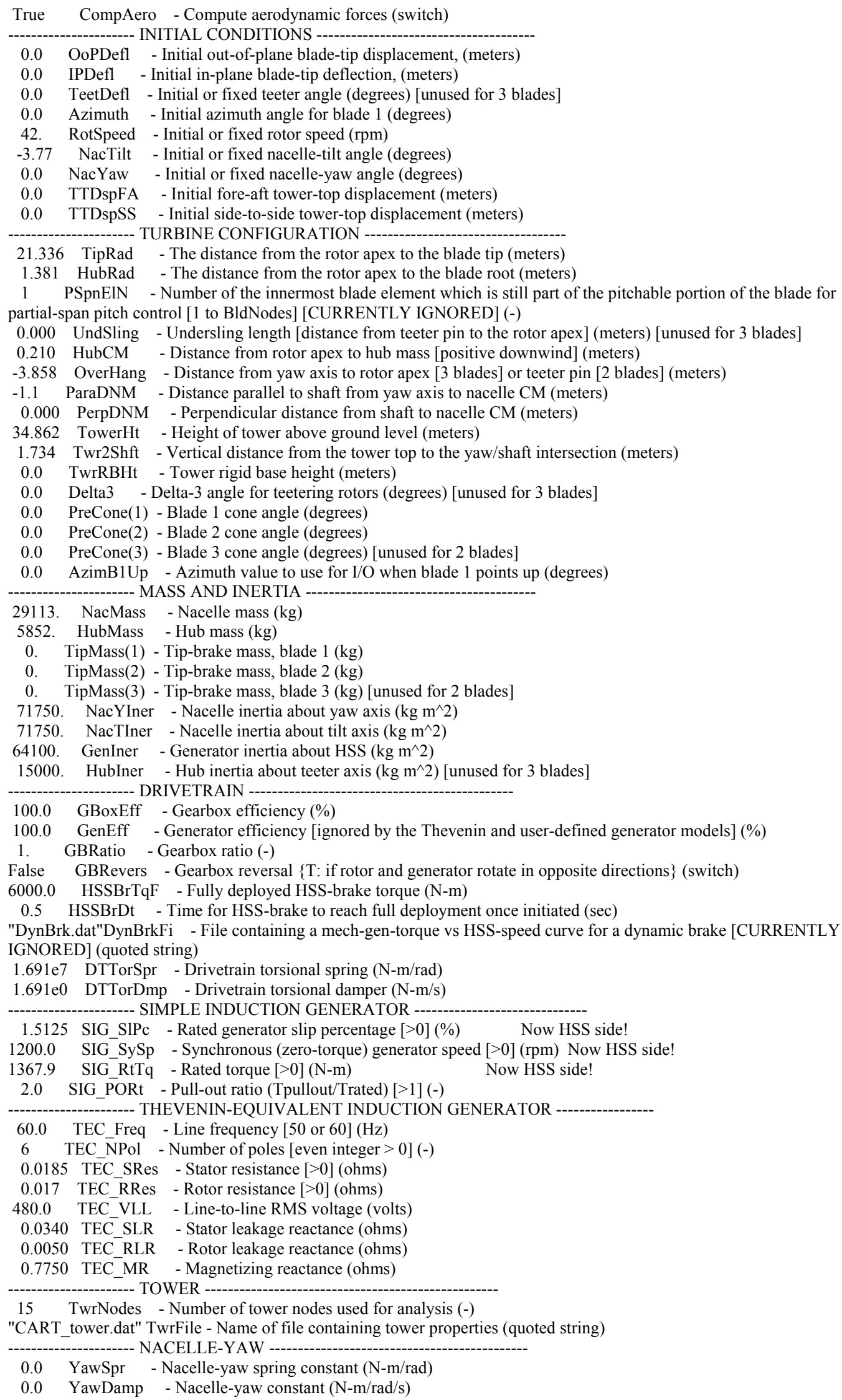




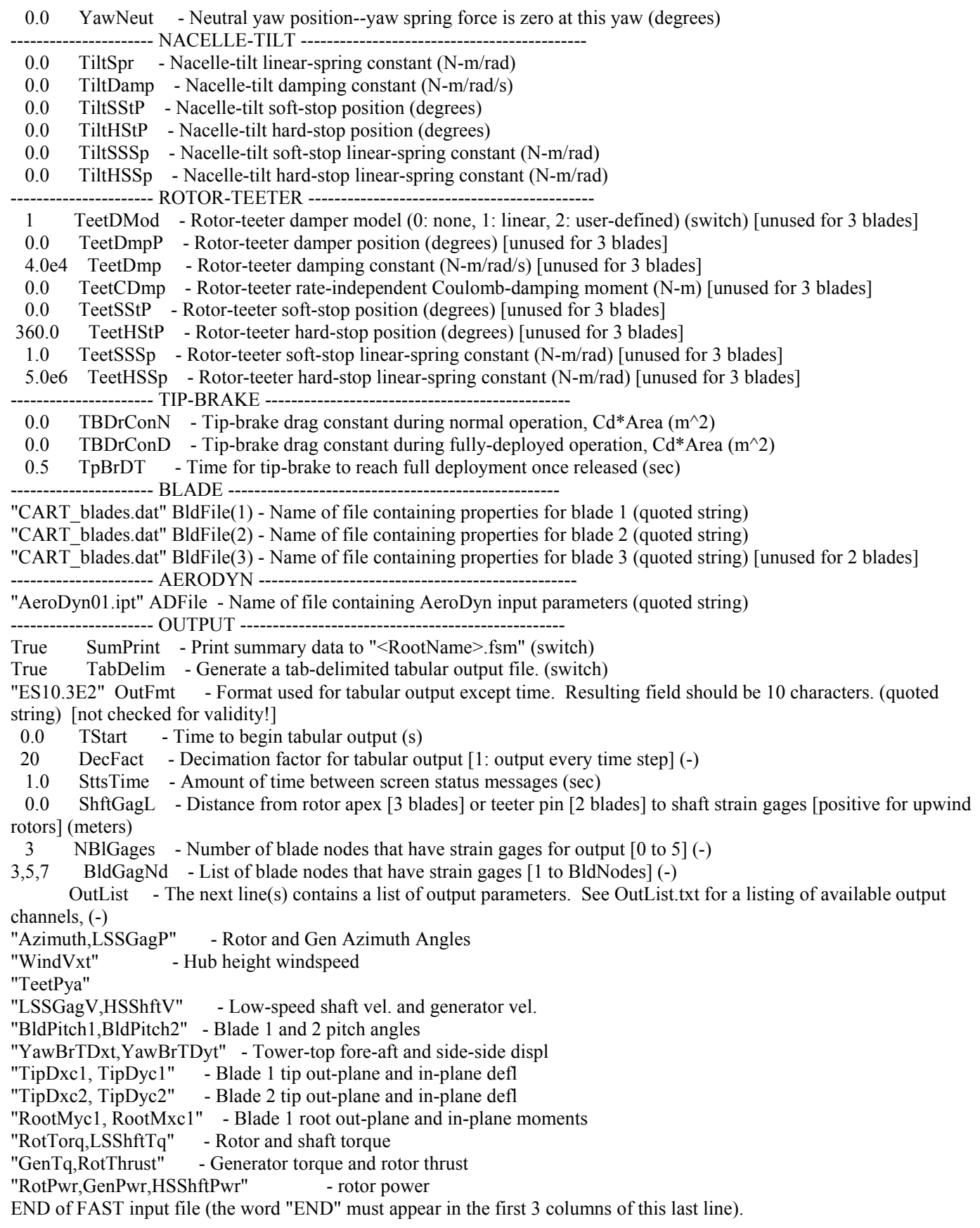

CART_Blades.dat

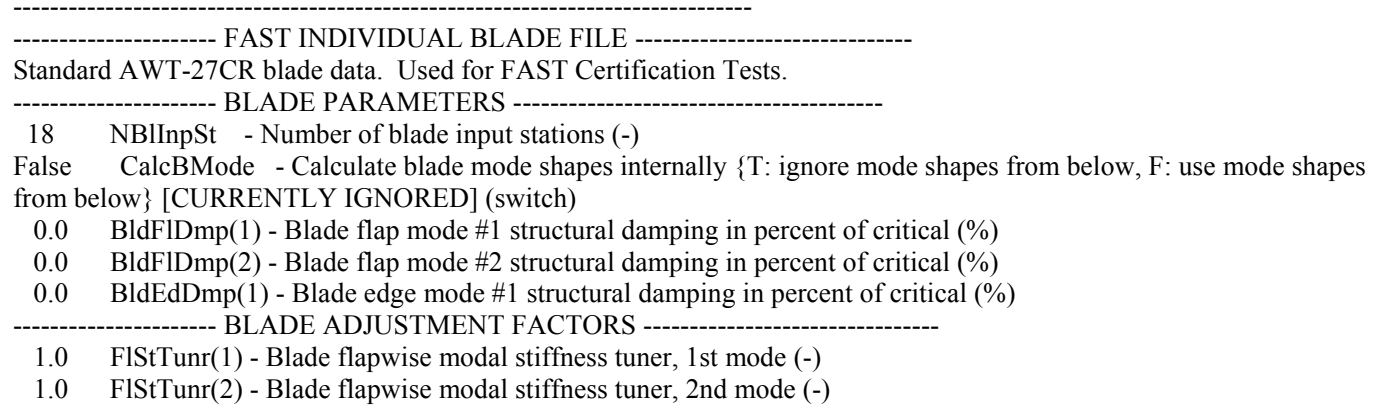




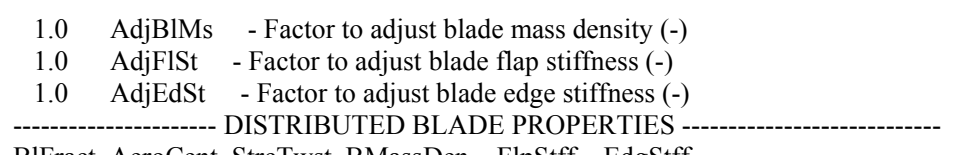

BlFract AeroCent StrcTwst BMassDen FlpStff EdgStff

$(-) \quad(-) \quad(\operatorname{deg}) \quad(\mathrm{kg} / \mathrm{m}) \quad\left(\mathrm{Nm}^{\wedge} 2\right) \quad\left(\mathrm{Nm}^{\wedge} 2\right)$

$\begin{array}{llllll}0.000 & 0.000 & 3.440 & 282.92 & 165000000 & 283000000\end{array}$

$\begin{array}{lllllll}0.022 & 0.000 & 3.370 & 290.24 & 161000000 & 318000000\end{array}$

$\begin{array}{llllll}0.053 & 0.000 & 3.270 & 261.88 & 142000000 & 328000000\end{array}$

$\begin{array}{llllll}0.114 & 0.000 & 3.080 & 201.28 & 98700000 & 307000000\end{array}$

$\begin{array}{llllll}0.175 & 0.000 & 2.880 & 186.52 & 78400000 & 340000000\end{array}$

$\begin{array}{lllllll}0.236 & 0.000 & 2.690 & 169.10 & 59200000 & 342000000\end{array}$

$\begin{array}{llllll}0.299 & 0.000 & 2.450 & 149.28 & 45400000 & 278000000\end{array}$

$\begin{array}{llllll}0.364 & 0.000 & 2.210 & 133.19 & 34100000 & 237000000\end{array}$

$\begin{array}{llllll}0.427 & 0.000 & 1.910 & 111.74 & 25000000 & 169000000\end{array}$

$\begin{array}{lllllll}0.491 & 0.000 & 1.610 & 96.86 & 17900000 & 138000000\end{array}$

$\begin{array}{lllllll}0.555 & 0.000 & 1.240 & 78.57 & 12300000 & 94000000\end{array}$

$\begin{array}{llllll}0.618 & 0.000 & 0.860 & 65.03 & 8190000 & 72500000\end{array}$

$\begin{array}{llllll}0.682 & 0.000 & 0.380 & 49.68 & 5140000 & 46700000\end{array}$

$\begin{array}{llllll}0.745 & 0.000 & -0.110 & 37.59 & 3020000 & 32600000\end{array}$

$\begin{array}{llllll}0.809 & 0.000 & -0.770 & 25.01 & 1620000 & 19000000\end{array}$

$\begin{array}{lllllll}0.873 & 0.000 & -1.430 & 16.01 & 868000 & 13000000\end{array}$

$\begin{array}{llllll}0.936 & 0.000 & -2.370 & 10.73 & 468000 & 8850000\end{array}$

$\begin{array}{llllll}1.000 & 0.000 & -3.310 & 6.02 & 209000 & 6800000\end{array}$

0.558 BldF11Sh(2) - Flap mode 1 , coeff of $\mathrm{x}^{\wedge} 2$

0.796 BldFl1Sh $(3)-\quad$, coeff of $x^{\wedge} 3$

-0.718 BldFl1Sh(4) - $\quad$, coeff of $x^{\wedge} 4$

0.877 BldF11Sh $(5)-\quad$, coeff of $x^{\wedge} 5$

-0.513 BldF11Sh $(6)-\quad$, coeff of $x^{\wedge} 6$

-1.198 BldFl2Sh(2) - Flap mode 2, coeff of $x^{\wedge} 2$

$0.805 \operatorname{BldF1} 2 \mathrm{Sh}(3)-\quad$, coeff of $\mathrm{x}^{\wedge} 3$

$-4.554 \operatorname{BldF} 2 \mathrm{Sh}(4)-\quad$, coeff of $\mathrm{x}^{\wedge} 4$

12.777 BldF12Sh(5) - , coeff of $x^{\wedge} 5$

-6.830 BldF12Sh $(6)-\quad$, coeff of $\mathrm{x}^{\wedge} 6$

1.778 BldEdgSh(2) - Edge mode 1, coeff of $x^{\wedge} 2$

-2.071 BldEdgSh $(3)-\quad$, coeff of $x^{\wedge} 3$

2.445 BldEdgSh $(4)-\quad$, coeff of $\mathrm{x}^{\wedge} 4$

-1.350 BldEdgSh $(5)-\quad, \quad$, coeff of $x^{\wedge} 5$

0.198 BldEdgSh $(6)-\quad$, coeff of $x^{\wedge} 6$

CART_Tower.dat

-

Standard AWT-27CR tower data. Used for FAST Certification Tests. - TOWER PARAMETERS

13 NTwInpSt - Number of input stations to specify tower geometry

False CalcTMode - Calculate tower mode shapes internally $\{\mathrm{T}$ : ignore mode shapes from below, F: use mode shapes from below $\}$ [CURRENTLY IGNORED] (switch)

0.0 TwrFADmp(1) - Tower 1st fore-aft mode structural damping ratio (\%)

0.0 TwrFADmp (2) - Tower 2nd fore-aft mode structural damping ratio (\%)

2.0 TwrSSDmp(1) - Tower 1st side-to-side mode structural damping ratio (\%)

0.0 TwrSSDmp(2) - Tower 2nd side-to-side mode structural damping ratio (\%)

TOWER ADJUSTMUNT FACTORS

1.0 FAStTunr(1) - Tower fore-aft modal stiffness tuner, 1st mode (-)

1.0 FAStTunr(2) - Tower fore-aft modal stiffness tuner, 2nd mode (-)

1.0 SSStTunr(1) - Tower side-to-side stiffness tuner, 1st mode (-)

1.0 SSStTunr(2) - Tower side-to-side stiffness tuner, 2nd mode (-)

1.0 AdjTwMa - Factor to adjust tower mass density (-)

1.0 AdjFASt - Factor to adjust tower fore-aft stiffness (-)

1.0 AdjSSSt - Factor to adjust tower side-to-side stiffness (-)

HtFract TMassDen TwFAStif TwSSStif

$(-) \quad(\mathrm{kg} / \mathrm{m}) \quad\left(\mathrm{Nm}^{\wedge} 2\right) \quad\left(\mathrm{Nm}^{\wedge} 2\right)$

$0.000 \quad 1548 . \quad 8.310 \mathrm{e} 10 \quad 8.310 \mathrm{e} 10$

0.0658 1361. $5.580 \mathrm{e} 10 \quad 5.580 \mathrm{e} 10$

$\begin{array}{llll}0.1970 & 1428 . & 3.09 \mathrm{e} 10 & 3.09 \mathrm{e} 10\end{array}$

0.2623 1311. $1.80 \mathrm{e} 10 \quad 1.80 \mathrm{e} 10$

0.3293 1311. $1.80 \mathrm{e} 10 \quad 1.80 \mathrm{e} 10$

0.4299 1311. $1.80 \mathrm{e} 10 \quad 1.80 \mathrm{e} 10$ 


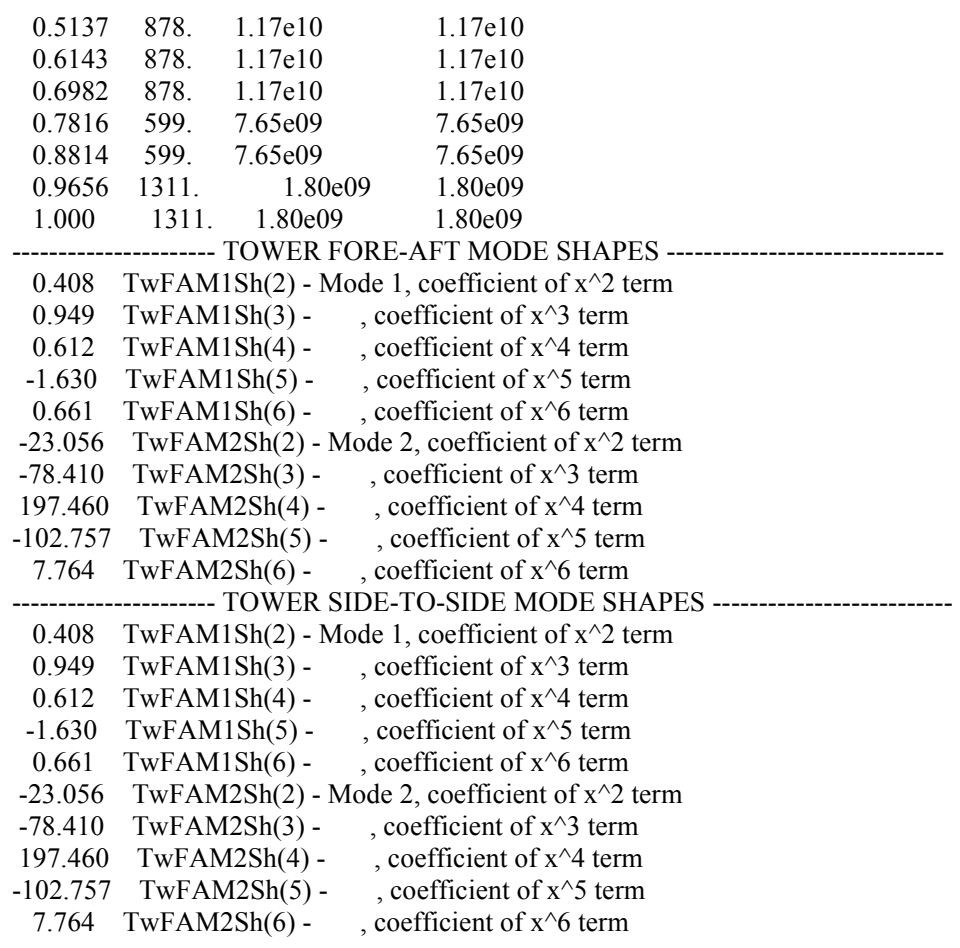

\section{AeroDyn01.ipt}

CART aerodynamic parameters for FAST.

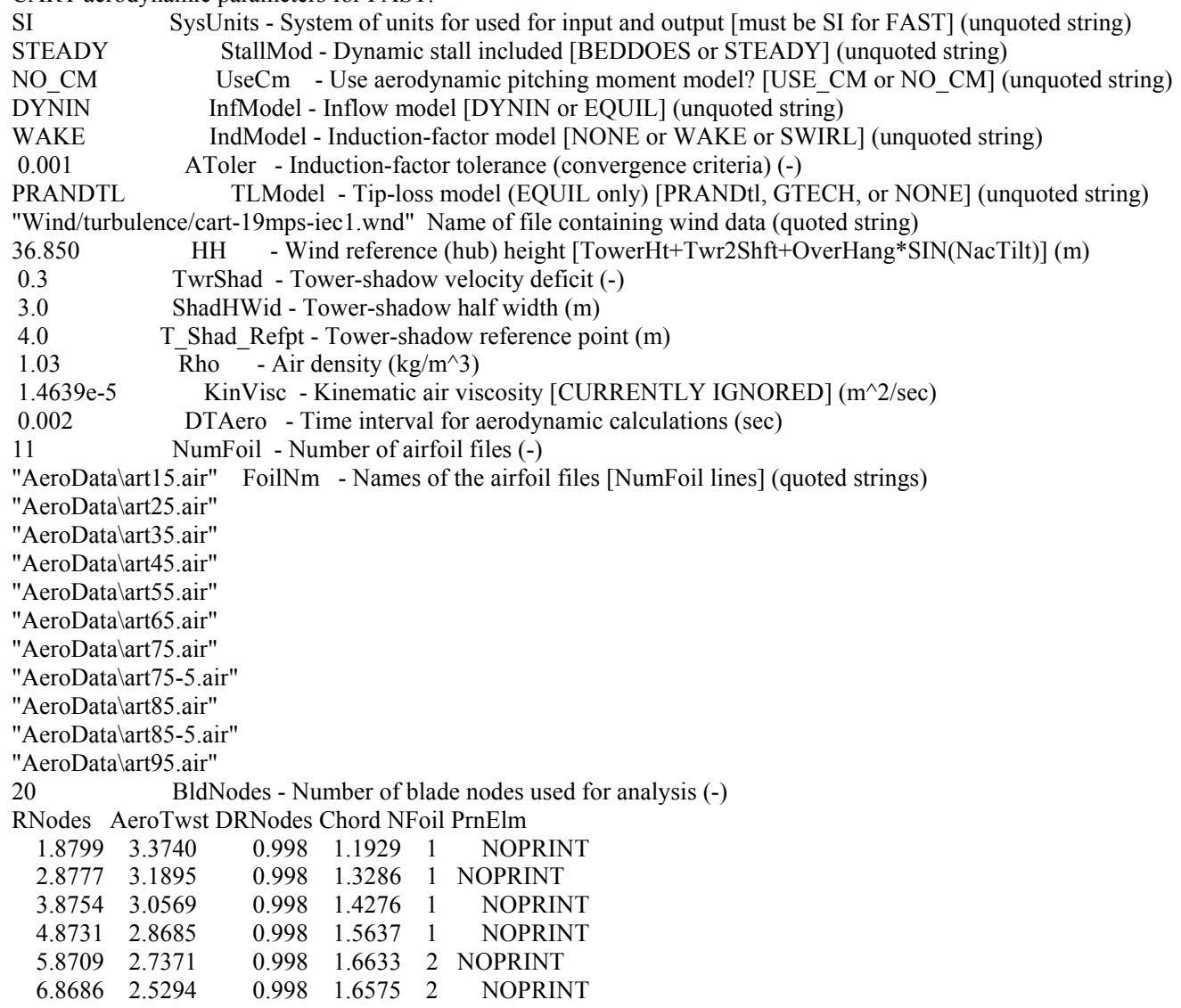




$\begin{array}{cccccc}7.8663 & 2.3700 & 0.998 & 1.6163 & 3 & \text { NOPRINT } \\ 8.8641 & 2.1379 & 0.998 & 1.5555 & 3 & \text { NOPRINT } \\ 9.8618 & 1.9386 & 0.998 & 1.5017 & 4 & \text { NOPRINT } \\ 10.8595 & 1.6665 & 0.998 & 1.4274 & 4 & \text { NOPRINT } \\ 11.8573 & 1.4339 & 0.998 & 1.3735 & 5 & \text { NOPRINT } \\ 12.8550 & 1.0945 & 0.998 & 1.3000 & 5 & \text { NOPRINT } \\ 13.8528 & 0.8374 & 0.998 & 1.2461 & 6 & \text { NOPRINT } \\ 14.8506 & 0.4020 & 0.998 & 1.1718 & 6 & \text { NOPRINT } \\ 15.8483 & 0.0770 & 0.998 & 1.1179 & 7 \text { NOPRINT } \\ 16.8460 & -0.4568 & 0.998 & 1.0444 & 7 \text { PRINT } \\ 17.8438 & -0.8951 & 0.998 & 0.9906 & 8 \text { NOPRINT } \\ 18.8416 & -1.5209 & 0.998 & 0.9171 & 9 \text { PRINT } \\ 19.8393 & -2.1452 & 0.998 & 0.8626 & 10 & \text { NOPRINT } \\ 20.8371 & -2.9979 & 0.998 & 0.7889 & 11 & \end{array}$

\section{CART ADAMS Input File}

MODEL \#8 OF CART (controls advanced research turbine, NWTC) with aero

! units: $\mathrm{m}, \mathrm{kg}, \mathrm{s}$, Newtons

! Received from Karl Stol on 4/11/02.

! Made the following changes after receipt from Karl:

! 1) decreased tower and blade field CRATIO statements to very small values.

! 2) decreased BEAM/3100 (drive-train torsion) CRATIO to value equivalent to damping

used in FAST

! 3) added MARKERS 2051 and 2051.

! 4) modified generator applied torque.

! Model has a difficult time running after decreasing blade and tower field CRATIOs.

! 5) Enabled drive-shaft fixed joint to replace BEAM/3100 to lock out drive-train torsion.

!!!!!!!NOTE: FREE TEETER 6/18/02: JOINT/4000.

! ground definition. global $\mathrm{z}$ axis vertical

PART/1,GROUND

MARKER $/ 1$, PART $=1 \quad$ IGRND

! marker oriented with $\mathrm{x}$ axis vertical

MARKER/11, PART $=1, \mathrm{QP}=0,0,0.0, \mathrm{REU}=90 \mathrm{D}, 90 \mathrm{D}, 90 \mathrm{D}$

$! \quad$ tower parts

! tower part \#1110

$\mathrm{PART} / 1110, \mathrm{MASS}=5101.1, \mathrm{CM}=1110$

, IP $=19123.3,14722.3,14722.3$

, $\mathrm{QG}=0,0,1.709, \mathrm{REU}=90 \mathrm{D}, 90 \mathrm{D}, 90 \mathrm{D}$

MARKER/1110, PART $=1110$

GRAPHIC/1110, OUTLINE $=11,1110$

FIELD $/ 1110, \mathrm{I}=1110, \mathrm{~J}=11, \mathrm{KMATRIX}=2.1242 \mathrm{e}+010$

$, 0.0000 \mathrm{e}+000,0.0000 \mathrm{e}+000,0.0000 \mathrm{e}+000,0.0000 \mathrm{e}+000,0.0000 \mathrm{e}+000$

$, 0.0000 \mathrm{e}+000,1.7519 \mathrm{e}+011,0.0000 \mathrm{e}+000,0.0000 \mathrm{e}+000,0.0000 \mathrm{e}+000,-1.4278 \mathrm{e}+011$

$, 0.0000 \mathrm{e}+000,0.0000 \mathrm{e}+000,1.7519 \mathrm{e}+011,0.0000 \mathrm{e}+000,1.4278 \mathrm{e}+011,0.0000 \mathrm{e}+000$

$, 0.0000 \mathrm{e}+000,0.0000 \mathrm{e}+000,0.0000 \mathrm{e}+000,1.5699 \mathrm{e}+010,0.0000 \mathrm{e}+000,0.0000 \mathrm{e}+000$

$, 0.0000 \mathrm{e}+000,0.0000 \mathrm{e}+000,1.4278 \mathrm{e}+011,0.0000 \mathrm{e}+000,1.5875 \mathrm{e}+011,0.0000 \mathrm{e}+000$

$, 0.0000 \mathrm{e}+000,-1.4278 \mathrm{e}+011,0.0000 \mathrm{e}+000,0.0000 \mathrm{e}+000,0.0000 \mathrm{e}+000,1.5875 \mathrm{e}+011$

,CMATRIX $=2.1242 \mathrm{e}+00$

$, 0.0000 \mathrm{e}+000,0.0000 \mathrm{e}+000,0.0000 \mathrm{e}+000,0.0000 \mathrm{e}+000,0.0000 \mathrm{e}+000$

$, 0.0000 \mathrm{e}+000,1.7519 \mathrm{e}+000,0.0000 \mathrm{e}+000,0.0000 \mathrm{e}+000,0.0000 \mathrm{e}+000,-1.4278 \mathrm{e}+000$

$, 0.0000 \mathrm{e}+000,0.0000 \mathrm{e}+000,1.7519 \mathrm{e}+00,0.0000 \mathrm{e}+000,1.4278 \mathrm{e}+00,0.0000 \mathrm{e}+000$

$, 0.0000 \mathrm{e}+000,0.0000 \mathrm{e}+000,0.0000 \mathrm{e}+000,1.5699 \mathrm{e}+00,0.0000 \mathrm{e}+000,0.0000 \mathrm{e}+000$

$, 0.0000 \mathrm{e}+000,0.0000 \mathrm{e}+000,1.4278 \mathrm{e}+00,0.0000 \mathrm{e}+000,1.5875 \mathrm{e}+00,0.0000 \mathrm{e}+000$

$, 0.0000 \mathrm{e}+000,-1.4278 \mathrm{e}+000,0.0000 \mathrm{e}+000,0.0000 \mathrm{e}+000,0.0000 \mathrm{e}+000,1.5875 \mathrm{e}+000$

,LENGTH $=1.709,0,0,0,0,0$

! tower part \#1120

$\mathrm{PART} / 1120, \mathrm{MASS}=4882.5, \mathrm{CM}=1120$ 
, IP $=11482.4,10685.8,10685.8$

, $\mathrm{QG}=0,0,5.238, \mathrm{REU}=90 \mathrm{D}, 90 \mathrm{D}, 90 \mathrm{D}$

MARKER/1120, PART $=1120$

GRAPHIC/1120, OUTLINE $=1110,1120$

FIELD $/ 1120, \mathrm{I}=1120, \mathrm{~J}=1110, \mathrm{KMATRIX}=9.7645 \mathrm{e}+009$

$, 0.0000 \mathrm{e}+000,0.0000 \mathrm{e}+000,0.0000 \mathrm{e}+000,0.0000 \mathrm{e}+000,0.0000 \mathrm{e}+000$

$, 0.0000 \mathrm{e}+000,1.3996 \mathrm{e}+010,0.0000 \mathrm{e}+000,0.0000 \mathrm{e}+000,0.0000 \mathrm{e}+000,-2.2851 \mathrm{e}+010$

$, 0.0000 \mathrm{e}+000,0.0000 \mathrm{e}+000,1.3996 \mathrm{e}+010,0.0000 \mathrm{e}+000,2.2851 \mathrm{e}+010,0.0000 \mathrm{e}+000$ $, 0.0000 \mathrm{e}+000,0.0000 \mathrm{e}+000,0.0000 \mathrm{e}+000,5.3344 \mathrm{e}+009,0.0000 \mathrm{e}+000,0.0000 \mathrm{e}+000$ $, 0.0000 \mathrm{e}+000,0.0000 \mathrm{e}+000,2.2851 \mathrm{e}+010,0.0000 \mathrm{e}+000,5.1589 \mathrm{e}+010,0.0000 \mathrm{e}+000$ $, 0.0000 \mathrm{e}+000,-2.2851 \mathrm{e}+010,0.0000 \mathrm{e}+000,0.0000 \mathrm{e}+000,0.0000 \mathrm{e}+000,5.1589 \mathrm{e}+010$ ,CMATRIX $=9.7645 \mathrm{e}+00$

$, 0.0000 \mathrm{e}+000,0.0000 \mathrm{e}+000,0.0000 \mathrm{e}+000,0.0000 \mathrm{e}+000,0.0000 \mathrm{e}+000$

$, 0.0000 \mathrm{e}+000,1.3996 \mathrm{e}+000,0.0000 \mathrm{e}+000,0.0000 \mathrm{e}+000,0.0000 \mathrm{e}+000,-2.2851 \mathrm{e}+000$ $, 0.0000 \mathrm{e}+000,0.0000 \mathrm{e}+000,1.3996 \mathrm{e}+00,0.0000 \mathrm{e}+000,2.2851 \mathrm{e}+00,0.0000 \mathrm{e}+000$

$, 0.0000 \mathrm{e}+000,0.0000 \mathrm{e}+000,0.0000 \mathrm{e}+000,5.3344 \mathrm{e}+000,0.0000 \mathrm{e}+000,0.0000 \mathrm{e}+000$

$, 0.0000 \mathrm{e}+000,0.0000 \mathrm{e}+000,2.2851 \mathrm{e}+00,0.0000 \mathrm{e}+000,5.1589 \mathrm{e}+00,0.0000 \mathrm{e}+000$

$, 0.0000 \mathrm{e}+000,-2.2851 \mathrm{e}+000,0.0000 \mathrm{e}+000,0.0000 \mathrm{e}+000,0.0000 \mathrm{e}+000,5.1589 \mathrm{e}+000$

,LENGTH= 3.529, 0, 0,0,0,0

! $\quad$ tower part \#1130

$\mathrm{PART} / 1130, \mathrm{MASS}=4764.9, \mathrm{CM}=1130$

, IP $=6952.3,8299.4,8299.4$

, $\mathrm{QG}=0,0,8.692, \mathrm{REU}=90 \mathrm{D}, 90 \mathrm{D}, 90 \mathrm{D}$

MARKER/1130, PART $=1130$

GRAPHIC/1130, OUTLINE=1120,1130

FIELD $/ 1130, \mathrm{I}=1130, \mathrm{~J}=1120, \mathrm{KMATRIX}=9.6995 \mathrm{e}+009$

$, 0.0000 \mathrm{e}+000,0.0000 \mathrm{e}+000,0.0000 \mathrm{e}+000,0.0000 \mathrm{e}+000,0.0000 \mathrm{e}+000$

$, 0.0000 \mathrm{e}+000,8.7902 \mathrm{e}+009,0.0000 \mathrm{e}+000,0.0000 \mathrm{e}+000,0.0000 \mathrm{e}+000,-1.3568 \mathrm{e}+010$ $, 0.0000 \mathrm{e}+000,0.0000 \mathrm{e}+000,8.7902 \mathrm{e}+009,0.0000 \mathrm{e}+000,1.3568 \mathrm{e}+010,0.0000 \mathrm{e}+000$ $, 0.0000 \mathrm{e}+000,0.0000 \mathrm{e}+000,0.0000 \mathrm{e}+000,3.1980 \mathrm{e}+009,0.0000 \mathrm{e}+000,0.0000 \mathrm{e}+000$ $, 0.0000 \mathrm{e}+000,0.0000 \mathrm{e}+000,1.3568 \mathrm{e}+010,0.0000 \mathrm{e}+000,2.9382 \mathrm{e}+010,0.0000 \mathrm{e}+000$ $, 0.0000 \mathrm{e}+000,-1.3568 \mathrm{e}+010,0.0000 \mathrm{e}+000,0.0000 \mathrm{e}+000,0.0000 \mathrm{e}+000,2.9382 \mathrm{e}+010$ , CMATRIX $=9.6995 \mathrm{e}+004$

$, 0.0000 \mathrm{e}+000,0.0000 \mathrm{e}+000,0.0000 \mathrm{e}+000,0.0000 \mathrm{e}+000,0.0000 \mathrm{e}+000$

$, 0.0000 \mathrm{e}+000,8.7902 \mathrm{e}+000,0.0000 \mathrm{e}+000,0.0000 \mathrm{e}+000,0.0000 \mathrm{e}+000,-1.3568 \mathrm{e}+000$ $, 0.0000 \mathrm{e}+000,0.0000 \mathrm{e}+000,8.7902 \mathrm{e}+000,0.0000 \mathrm{e}+000,1.3568 \mathrm{e}+00,0.0000 \mathrm{e}+000$

$, 0.0000 \mathrm{e}+000,0.0000 \mathrm{e}+000,0.0000 \mathrm{e}+000,3.1980 \mathrm{e}+000,0.0000 \mathrm{e}+000,0.0000 \mathrm{e}+000$ $, 0.0000 \mathrm{e}+000,0.0000 \mathrm{e}+000,1.3568 \mathrm{e}+00,0.0000 \mathrm{e}+000,2.9382 \mathrm{e}+00,0.0000 \mathrm{e}+000$

$, 0.0000 \mathrm{e}+000,-1.3568 \mathrm{e}+000,0.0000 \mathrm{e}+000,0.0000 \mathrm{e}+000,0.0000 \mathrm{e}+000,2.9382 \mathrm{e}+000$ ,LENGTH $=3.454,0,0,0,0,0$

! $\quad$ tower part \#1140

$\mathrm{PART} / 1140, \mathrm{MASS}=4570.4, \mathrm{CM}=1140$

, IP $=5173.5,7215.7,7215.7$

, $\mathrm{QG}=0,0,12.202, \mathrm{REU}=90 \mathrm{D}, 90 \mathrm{D}, 90 \mathrm{D}$

MARKER/1140, PART $=1140$

GRAPHIC/1140, OUTLINE $=1130,1140$

FIELD $/ 1140, \mathrm{I}=1140, \mathrm{~J}=1130, \mathrm{KMATRIX}=9.2020 \mathrm{e}+009$

$, 0.0000 \mathrm{e}+000,0.0000 \mathrm{e}+000,0.0000 \mathrm{e}+000,0.0000 \mathrm{e}+000,0.0000 \mathrm{e}+000$

$, 0.0000 \mathrm{e}+000,5.3514 \mathrm{e}+009,0.0000 \mathrm{e}+000,0.0000 \mathrm{e}+000,0.0000 \mathrm{e}+000,-9.1832 \mathrm{e}+009$ $, 0.0000 \mathrm{e}+000,0.0000 \mathrm{e}+000,5.3514 \mathrm{e}+009,0.0000 \mathrm{e}+000,9.1832 \mathrm{e}+009,0.0000 \mathrm{e}+000$ $, 0.0000 \mathrm{e}+000,0.0000 \mathrm{e}+000,0.0000 \mathrm{e}+000,2.0059 \mathrm{e}+009,0.0000 \mathrm{e}+000,0.0000 \mathrm{e}+000$ $, 0.0000 \mathrm{e}+000,0.0000 \mathrm{e}+000,9.1832 \mathrm{e}+009,0.0000 \mathrm{e}+000,2.1245 \mathrm{e}+010,0.0000 \mathrm{e}+000$ $, 0.0000 \mathrm{e}+000,-9.1832 \mathrm{e}+009,0.0000 \mathrm{e}+000,0.0000 \mathrm{e}+000,0.0000 \mathrm{e}+000,2.1245 \mathrm{e}+010$ , CMATRIX $=9.2020 \mathrm{e}+004$

$, 0.0000 \mathrm{e}+000,0.0000 \mathrm{e}+000,0.0000 \mathrm{e}+000,0.0000 \mathrm{e}+000,0.0000 \mathrm{e}+000$ $, 0.0000 \mathrm{e}+000,5.3514 \mathrm{e}+000,0.0000 \mathrm{e}+000,0.0000 \mathrm{e}+000,0.0000 \mathrm{e}+000,-9.1832 \mathrm{e}+000$ $, 0.0000 \mathrm{e}+000,0.0000 \mathrm{e}+000,5.3514 \mathrm{e}+000,0.0000 \mathrm{e}+000,9.1832 \mathrm{e}+000,0.0000 \mathrm{e}+000$ $, 0.0000 \mathrm{e}+000,0.0000 \mathrm{e}+000,0.0000 \mathrm{e}+000,2.0059 \mathrm{e}+000,0.0000 \mathrm{e}+000,0.0000 \mathrm{e}+000$ $, 0.0000 \mathrm{e}+000,0.0000 \mathrm{e}+000,9.1832 \mathrm{e}+000,0.0000 \mathrm{e}+000,2.1245 \mathrm{e}+00,0.0000 \mathrm{e}+000$ $, 0.0000 \mathrm{e}+000,-9.1832 \mathrm{e}+000,0.0000 \mathrm{e}+000,0.0000 \mathrm{e}+000,0.0000 \mathrm{e}+000,2.1245 \mathrm{e}+000$ ,LENGTH $=3.510,0,0,0,0,0$

! tower part \#1150 
PART $/ 1150$, MASS $=3939.1, \mathrm{CM}=1150$

, IP $=4415.3,6163.0,6163.0$

, $\mathrm{QG}=0,0,15.595, \mathrm{REU}=90 \mathrm{D}, 90 \mathrm{D}, 90 \mathrm{D}$

MARKER/1150, PART $=1150$

GRAPHIC/1150, OUTLINE=1140,1150

FIELD/1150, I=1150, J=1140, KMATRIX $=9.0964 \mathrm{e}+009$

$, 0.0000 \mathrm{e}+000,0.0000 \mathrm{e}+000,0.0000 \mathrm{e}+000,0.0000 \mathrm{e}+000,0.0000 \mathrm{e}+000$

$, 0.0000 \mathrm{e}+000,5.3278 \mathrm{e}+009,0.0000 \mathrm{e}+000,0.0000 \mathrm{e}+000,0.0000 \mathrm{e}+000,-8.9249 \mathrm{e}+009$

$, 0.0000 \mathrm{e}+000,0.0000 \mathrm{e}+000,5.3278 \mathrm{e}+009,0.0000 \mathrm{e}+000,8.9249 \mathrm{e}+009,0.0000 \mathrm{e}+000$ $, 0.0000 \mathrm{e}+000,0.0000 \mathrm{e}+000,0.0000 \mathrm{e}+000,1.8660 \mathrm{e}+009,0.0000 \mathrm{e}+000,0.0000 \mathrm{e}+000$ $, 0.0000 \mathrm{e}+000,0.0000 \mathrm{e}+000,8.9249 \mathrm{e}+009,0.0000 \mathrm{e}+000,2.0060 \mathrm{e}+010,0.0000 \mathrm{e}+000$ $, 0.0000 \mathrm{e}+000,-8.9249 \mathrm{e}+009,0.0000 \mathrm{e}+000,0.0000 \mathrm{e}+000,0.0000 \mathrm{e}+000,2.0060 \mathrm{e}+010$ ,CMATRIX $=9.0964 \mathrm{e}+004$

$, 0.0000 \mathrm{e}+000,0.0000 \mathrm{e}+000,0.0000 \mathrm{e}+000,0.0000 \mathrm{e}+000,0.0000 \mathrm{e}+000$

$, 0.0000 \mathrm{e}+000,5.3278 \mathrm{e}+000,0.0000 \mathrm{e}+000,0.0000 \mathrm{e}+000,0.0000 \mathrm{e}+000,-8.9249 \mathrm{e}+000$ $, 0.0000 \mathrm{e}+000,0.0000 \mathrm{e}+000,5.3278 \mathrm{e}+000,0.0000 \mathrm{e}+000,8.9249 \mathrm{e}+000,0.0000 \mathrm{e}+000$ $, 0.0000 \mathrm{e}+000,0.0000 \mathrm{e}+000,0.0000 \mathrm{e}+000,1.8660 \mathrm{e}+000,0.0000 \mathrm{e}+000,0.0000 \mathrm{e}+000$ $, 0.0000 \mathrm{e}+000,0.0000 \mathrm{e}+000,8.9249 \mathrm{e}+000,0.0000 \mathrm{e}+000,2.0060 \mathrm{e}+00,0.0000 \mathrm{e}+000$ $, 0.0000 \mathrm{e}+000,-8.9249 \mathrm{e}+000,0.0000 \mathrm{e}+000,0.0000 \mathrm{e}+000,0.0000 \mathrm{e}+000,2.0060 \mathrm{e}+000$ ,LENGTH= 3.393, $0,0,0,0,0$

! tower part \#1160

$\mathrm{PART} / 1160, \mathrm{MASS}=3184.3, \mathrm{CM}=1160$

, IP=3508.9, 4977.9, 4977.9

, $\mathrm{QG}=0,0,19.152, \mathrm{REU}=90 \mathrm{D}, 90 \mathrm{D}, 90 \mathrm{D}$

MARKER/1160, PART $=1160$

GRAPHIC/1160, OUTLINE=1150,1160

FIELD $/ 1160, \mathrm{I}=1160, \mathrm{~J}=1150, \mathrm{KMATRIX}=7.1454 \mathrm{e}+009$

$, 0.0000 \mathrm{e}+000,0.0000 \mathrm{e}+000,0.0000 \mathrm{e}+000,0.0000 \mathrm{e}+000,0.0000 \mathrm{e}+000$

$, 0.0000 \mathrm{e}+000,3.7853 \mathrm{e}+009,0.0000 \mathrm{e}+000,0.0000 \mathrm{e}+000,0.0000 \mathrm{e}+000,-6.3376 \mathrm{e}+009$ $, 0.0000 \mathrm{e}+000,0.0000 \mathrm{e}+000,3.7853 \mathrm{e}+009,0.0000 \mathrm{e}+000,6.3376 \mathrm{e}+009,0.0000 \mathrm{e}+000$ $, 0.0000 \mathrm{e}+000,0.0000 \mathrm{e}+000,0.0000 \mathrm{e}+000,1.4582 \mathrm{e}+009,0.0000 \mathrm{e}+000,0.0000 \mathrm{e}+000$ $, 0.0000 \mathrm{e}+000,0.0000 \mathrm{e}+000,6.3376 \mathrm{e}+009,0.0000 \mathrm{e}+000,1.4560 \mathrm{e}+010,0.0000 \mathrm{e}+000$ $, 0.0000 \mathrm{e}+000,-6.3376 \mathrm{e}+009,0.0000 \mathrm{e}+000,0.0000 \mathrm{e}+000,0.0000 \mathrm{e}+000,1.4560 \mathrm{e}+010$ , CMATRIX $=7.1454 \mathrm{e}+004$

$, 0.0000 \mathrm{e}+000,0.0000 \mathrm{e}+000,0.0000 \mathrm{e}+000,0.0000 \mathrm{e}+000,0.0000 \mathrm{e}+000$

$, 0.0000 \mathrm{e}+000,3.7853 \mathrm{e}+000,0.0000 \mathrm{e}+000,0.0000 \mathrm{e}+000,0.0000 \mathrm{e}+000,-6.3376 \mathrm{e}+000$ $, 0.0000 \mathrm{e}+000,0.0000 \mathrm{e}+000,3.7853 \mathrm{e}+000,0.0000 \mathrm{e}+000,6.3376 \mathrm{e}+000,0.0000 \mathrm{e}+000$ $, 0.0000 \mathrm{e}+000,0.0000 \mathrm{e}+000,0.0000 \mathrm{e}+000,1.4582 \mathrm{e}+000,0.0000 \mathrm{e}+000,0.0000 \mathrm{e}+000$ $, 0.0000 \mathrm{e}+000,0.0000 \mathrm{e}+000,6.3376 \mathrm{e}+000,0.0000 \mathrm{e}+000,1.4560 \mathrm{e}+00,0.0000 \mathrm{e}+000$ $, 0.0000 \mathrm{e}+000,-6.3376 \mathrm{e}+000,0.0000 \mathrm{e}+000,0.0000 \mathrm{e}+000,0.0000 \mathrm{e}+000,1.4560 \mathrm{e}+000$ ,LENGTH= 3.557,0,0,0,0,0

! tower part \#1170

$\mathrm{PART} / 1170, \mathrm{MASS}=3050.1, \mathrm{CM}=1170$

, IP=3348.0, 4763.1, 4763.1

, $\mathrm{QG}=0,0,22.658, \mathrm{REU}=90 \mathrm{D}, 90 \mathrm{D}, 90 \mathrm{D}$

MARKER/1170, PART $=1170$

GRAPHIC/1170, OUTLINE $=1160,1170$

FIELD $/ 1170, \mathrm{I}=1170, \mathrm{~J}=1160, \mathrm{KMATRIX}=6.0171 \mathrm{e}+009$

$, 0.0000 \mathrm{e}+000,0.0000 \mathrm{e}+000,0.0000 \mathrm{e}+000,0.0000 \mathrm{e}+000,0.0000 \mathrm{e}+000$

$, 0.0000 \mathrm{e}+000,3.2560 \mathrm{e}+009,0.0000 \mathrm{e}+000,0.0000 \mathrm{e}+000,0.0000 \mathrm{e}+000,-5.7088 \mathrm{e}+009$ $, 0.0000 \mathrm{e}+000,0.0000 \mathrm{e}+000,3.2560 \mathrm{e}+009,0.0000 \mathrm{e}+000,5.7088 \mathrm{e}+009,0.0000 \mathrm{e}+000$ $, 0.0000 \mathrm{e}+000,0.0000 \mathrm{e}+000,0.0000 \mathrm{e}+000,1.2205 \mathrm{e}+009,0.0000 \mathrm{e}+000,0.0000 \mathrm{e}+000$ $, 0.0000 \mathrm{e}+000,0.0000 \mathrm{e}+000,5.7088 \mathrm{e}+009,0.0000 \mathrm{e}+000,1.3346 \mathrm{e}+010,0.0000 \mathrm{e}+000$ $, 0.0000 \mathrm{e}+000,-5.7088 \mathrm{e}+009,0.0000 \mathrm{e}+000,0.0000 \mathrm{e}+000,0.0000 \mathrm{e}+000,1.3346 \mathrm{e}+010$ , $\mathrm{CMATRIX}=6.0171 \mathrm{e}+004$

$, 0.0000 \mathrm{e}+000,0.0000 \mathrm{e}+000,0.0000 \mathrm{e}+000,0.0000 \mathrm{e}+000,0.0000 \mathrm{e}+000$ $, 0.0000 \mathrm{e}+000,3.2560 \mathrm{e}+000,0.0000 \mathrm{e}+000,0.0000 \mathrm{e}+000,0.0000 \mathrm{e}+000,-5.7088 \mathrm{e}+000$ $, 0.0000 \mathrm{e}+000,0.0000 \mathrm{e}+000,3.2560 \mathrm{e}+000,0.0000 \mathrm{e}+000,5.7088 \mathrm{e}+000,0.0000 \mathrm{e}+000$ $, 0.0000 \mathrm{e}+000,0.0000 \mathrm{e}+000,0.0000 \mathrm{e}+000,1.2205 \mathrm{e}+000,0.0000 \mathrm{e}+000,0.0000 \mathrm{e}+000$ $, 0.0000 \mathrm{e}+000,0.0000 \mathrm{e}+000,5.7088 \mathrm{e}+000,0.0000 \mathrm{e}+000,1.3346 \mathrm{e}+00,0.0000 \mathrm{e}+000$ $, 0.0000 \mathrm{e}+000,-5.7088 \mathrm{e}+000,0.0000 \mathrm{e}+000,0.0000 \mathrm{e}+000,0.0000 \mathrm{e}+000,1.3346 \mathrm{e}+000$ ,LENGTH= 3.506, $0,0,0,0,0$ 
PART $/ 1180$, MASS $=2563.8, \mathrm{CM}=1180$

, IP $=2772.7,3953.2,3953.2$

, $\mathrm{QG}=0,0,26.039, \mathrm{REU}=90 \mathrm{D}, 90 \mathrm{D}, 90 \mathrm{D}$

MARKER/1180, PART $=1180$

GRAPHIC/1180, OUTLINE $=1170,1180$

FIELD $/ 1180, \mathrm{I}=1180, \mathrm{~J}=1170, \mathrm{KMATRIX}=5.6195 \mathrm{e}+009$

$, 0.0000 \mathrm{e}+000,0.0000 \mathrm{e}+000,0.0000 \mathrm{e}+000,0.0000 \mathrm{e}+000,0.0000 \mathrm{e}+000$

$, 0.0000 \mathrm{e}+000,3.2669 \mathrm{e}+009,0.0000 \mathrm{e}+000,0.0000 \mathrm{e}+000,0.0000 \mathrm{e}+000,-5.3147 \mathrm{e}+009$

$, 0.0000 \mathrm{e}+000,0.0000 \mathrm{e}+000,3.2669 \mathrm{e}+009,0.0000 \mathrm{e}+000,5.3147 \mathrm{e}+009,0.0000 \mathrm{e}+000$ $, 0.0000 \mathrm{e}+000,0.0000 \mathrm{e}+000,0.0000 \mathrm{e}+000,1.1391 \mathrm{e}+009,0.0000 \mathrm{e}+000,0.0000 \mathrm{e}+000$ $, 0.0000 \mathrm{e}+000,0.0000 \mathrm{e}+000,5.3147 \mathrm{e}+009,0.0000 \mathrm{e}+000,1.1744 \mathrm{e}+010,0.0000 \mathrm{e}+000$ $, 0.0000 \mathrm{e}+000,-5.3147 \mathrm{e}+009,0.0000 \mathrm{e}+000,0.0000 \mathrm{e}+000,0.0000 \mathrm{e}+000,1.1744 \mathrm{e}+010$ ,CMATRIX $=5.6195 \mathrm{e}+004$

$, 0.0000 \mathrm{e}+000,0.0000 \mathrm{e}+000,0.0000 \mathrm{e}+000,0.0000 \mathrm{e}+000,0.0000 \mathrm{e}+000$

$, 0.0000 \mathrm{e}+000,3.2669 \mathrm{e}+000,0.0000 \mathrm{e}+000,0.0000 \mathrm{e}+000,0.0000 \mathrm{e}+000,-5.3147 \mathrm{e}+000$ $, 0.0000 \mathrm{e}+000,0.0000 \mathrm{e}+000,3.2669 \mathrm{e}+000,0.0000 \mathrm{e}+000,5.3147 \mathrm{e}+000,0.0000 \mathrm{e}+000$ $, 0.0000 \mathrm{e}+000,0.0000 \mathrm{e}+000,0.0000 \mathrm{e}+000,1.1391 \mathrm{e}+000,0.0000 \mathrm{e}+000,0.0000 \mathrm{e}+000$ $, 0.0000 \mathrm{e}+000,0.0000 \mathrm{e}+000,5.3147 \mathrm{e}+000,0.0000 \mathrm{e}+000,1.1744 \mathrm{e}+00,0.0000 \mathrm{e}+000$ $, 0.0000 \mathrm{e}+000,-5.3147 \mathrm{e}+000,0.0000 \mathrm{e}+000,0.0000 \mathrm{e}+000,0.0000 \mathrm{e}+000,1.1744 \mathrm{e}+000$ ,LENGTH= 3.381,0,0,0,0,0

! tower part \#1190

PART/1190, MASS $=2362.4, \mathrm{CM}=1190$

, IP $=2537.6,3650.7,3650.7$

, $\mathrm{QG}=0,0,29.700, \mathrm{REU}=90 \mathrm{D}, 90 \mathrm{D}, 90 \mathrm{D}$

MARKER/1190, PART $=1190$

GRAPHIC/1190, OUTLINE=1180,1190

FIELD $/ 1190, \mathrm{I}=1190, \mathrm{~J}=1180, \mathrm{KMATRIX}=4.2051 \mathrm{e}+009$

$, 0.0000 \mathrm{e}+000,0.0000 \mathrm{e}+000,0.0000 \mathrm{e}+000,0.0000 \mathrm{e}+000,0.0000 \mathrm{e}+000$

$, 0.0000 \mathrm{e}+000,2.0760 \mathrm{e}+009,0.0000 \mathrm{e}+000,0.0000 \mathrm{e}+000,0.0000 \mathrm{e}+000,-3.6750 \mathrm{e}+009$ $, 0.0000 \mathrm{e}+000,0.0000 \mathrm{e}+000,2.0760 \mathrm{e}+009,0.0000 \mathrm{e}+000,3.6750 \mathrm{e}+009,0.0000 \mathrm{e}+000$ $, 0.0000 \mathrm{e}+000,0.0000 \mathrm{e}+000,0.0000 \mathrm{e}+000,8.5091 \mathrm{e}+008,0.0000 \mathrm{e}+000,0.0000 \mathrm{e}+000$ $, 0.0000 \mathrm{e}+000,0.0000 \mathrm{e}+000,3.6750 \mathrm{e}+009,0.0000 \mathrm{e}+000,8.8173 \mathrm{e}+009,0.0000 \mathrm{e}+000$ $, 0.0000 \mathrm{e}+000,-3.6750 \mathrm{e}+009,0.0000 \mathrm{e}+000,0.0000 \mathrm{e}+000,0.0000 \mathrm{e}+000,8.8173 \mathrm{e}+009$ ,CMATRIX $=4.2051 \mathrm{e}+004$

$, 0.0000 \mathrm{e}+000,0.0000 \mathrm{e}+000,0.0000 \mathrm{e}+000,0.0000 \mathrm{e}+000,0.0000 \mathrm{e}+000$

$, 0.0000 \mathrm{e}+000,2.0760 \mathrm{e}+000,0.0000 \mathrm{e}+000,0.0000 \mathrm{e}+000,0.0000 \mathrm{e}+000,-3.6750 \mathrm{e}+000$ $, 0.0000 \mathrm{e}+000,0.0000 \mathrm{e}+000,2.0760 \mathrm{e}+000,0.0000 \mathrm{e}+000,3.6750 \mathrm{e}+000,0.0000 \mathrm{e}+000$ $, 0.0000 \mathrm{e}+000,0.0000 \mathrm{e}+000,0.0000 \mathrm{e}+000,8.5091 \mathrm{e}+000,0.0000 \mathrm{e}+000,0.0000 \mathrm{e}+000$ $, 0.0000 \mathrm{e}+000,0.0000 \mathrm{e}+000,3.6750 \mathrm{e}+000,0.0000 \mathrm{e}+000,8.8173 \mathrm{e}+000,0.0000 \mathrm{e}+000$ $, 0.0000 \mathrm{e}+000,-3.6750 \mathrm{e}+000,0.0000 \mathrm{e}+000,0.0000 \mathrm{e}+000,0.0000 \mathrm{e}+000,8.8173 \mathrm{e}+000$ ,LENGTH $=3.661,0,0,0,0,0$

! $\quad$ tower part \#1200

$\mathrm{PART} / 1200, \mathrm{MASS}=3603.5, \mathrm{CM}=1200$

, IP $=4019.2,5571.6,5571.6$

, $\mathrm{QG}=0,0,33.275, \mathrm{REU}=90 \mathrm{D}, 90 \mathrm{D}, 90 \mathrm{D}$

MARKER/1200, PART $=1200$

GRAPHIC/1200, OUTLINE $=1190,1200$

FIELD $/ 1200, \mathrm{I}=1200, \mathrm{~J}=1190, \mathrm{KMATRIX}=6.0847 \mathrm{e}+009$

$, 0.0000 \mathrm{e}+000,0.0000 \mathrm{e}+000,0.0000 \mathrm{e}+000,0.0000 \mathrm{e}+000,0.0000 \mathrm{e}+000$

$, 0.0000 \mathrm{e}+000,3.1891 \mathrm{e}+009,0.0000 \mathrm{e}+000,0.0000 \mathrm{e}+000,0.0000 \mathrm{e}+000,-6.4026 \mathrm{e}+009$ $, 0.0000 \mathrm{e}+000,0.0000 \mathrm{e}+000,3.1891 \mathrm{e}+009,0.0000 \mathrm{e}+000,6.4026 \mathrm{e}+009,0.0000 \mathrm{e}+000$ $, 0.0000 \mathrm{e}+000,0.0000 \mathrm{e}+000,0.0000 \mathrm{e}+000,1.2423 \mathrm{e}+009,0.0000 \mathrm{e}+000,0.0000 \mathrm{e}+000$ $, 0.0000 \mathrm{e}+000,0.0000 \mathrm{e}+000,6.4026 \mathrm{e}+009,0.0000 \mathrm{e}+000,1.6095 \mathrm{e}+010,0.0000 \mathrm{e}+000$ $, 0.0000 \mathrm{e}+000,-6.4026 \mathrm{e}+009,0.0000 \mathrm{e}+000,0.0000 \mathrm{e}+000,0.0000 \mathrm{e}+000,1.6095 \mathrm{e}+010$ CMATRIX $=6.0847 \mathrm{e}+004$

$, 0.0000 \mathrm{e}+000,0.0000 \mathrm{e}+000,0.0000 \mathrm{e}+000,0.0000 \mathrm{e}+000,0.0000 \mathrm{e}+000$ $, 0.0000 \mathrm{e}+000,3.1891 \mathrm{e}+000,0.0000 \mathrm{e}+000,0.0000 \mathrm{e}+000,0.0000 \mathrm{e}+000,-6.4026 \mathrm{e}+000$ $, 0.0000 \mathrm{e}+000,0.0000 \mathrm{e}+000,3.1891 \mathrm{e}+000,0.0000 \mathrm{e}+000,6.4026 \mathrm{e}+000,0.0000 \mathrm{e}+000$ $, 0.0000 \mathrm{e}+000,0.0000 \mathrm{e}+000,0.0000 \mathrm{e}+000,1.2423 \mathrm{e}+000,0.0000 \mathrm{e}+000,0.0000 \mathrm{e}+000$ $, 0.0000 \mathrm{e}+000,0.0000 \mathrm{e}+000,6.4026 \mathrm{e}+000,0.0000 \mathrm{e}+000,1.6095 \mathrm{e}+00,0.0000 \mathrm{e}+000$ 


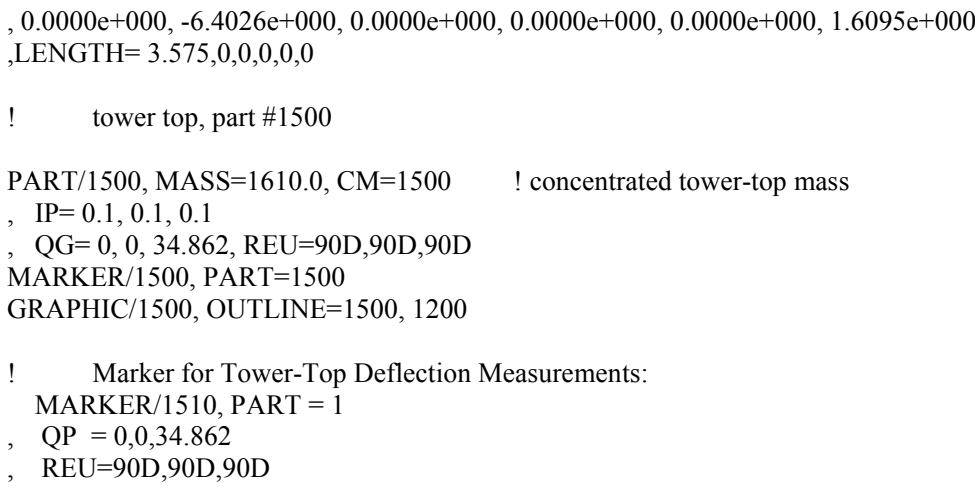




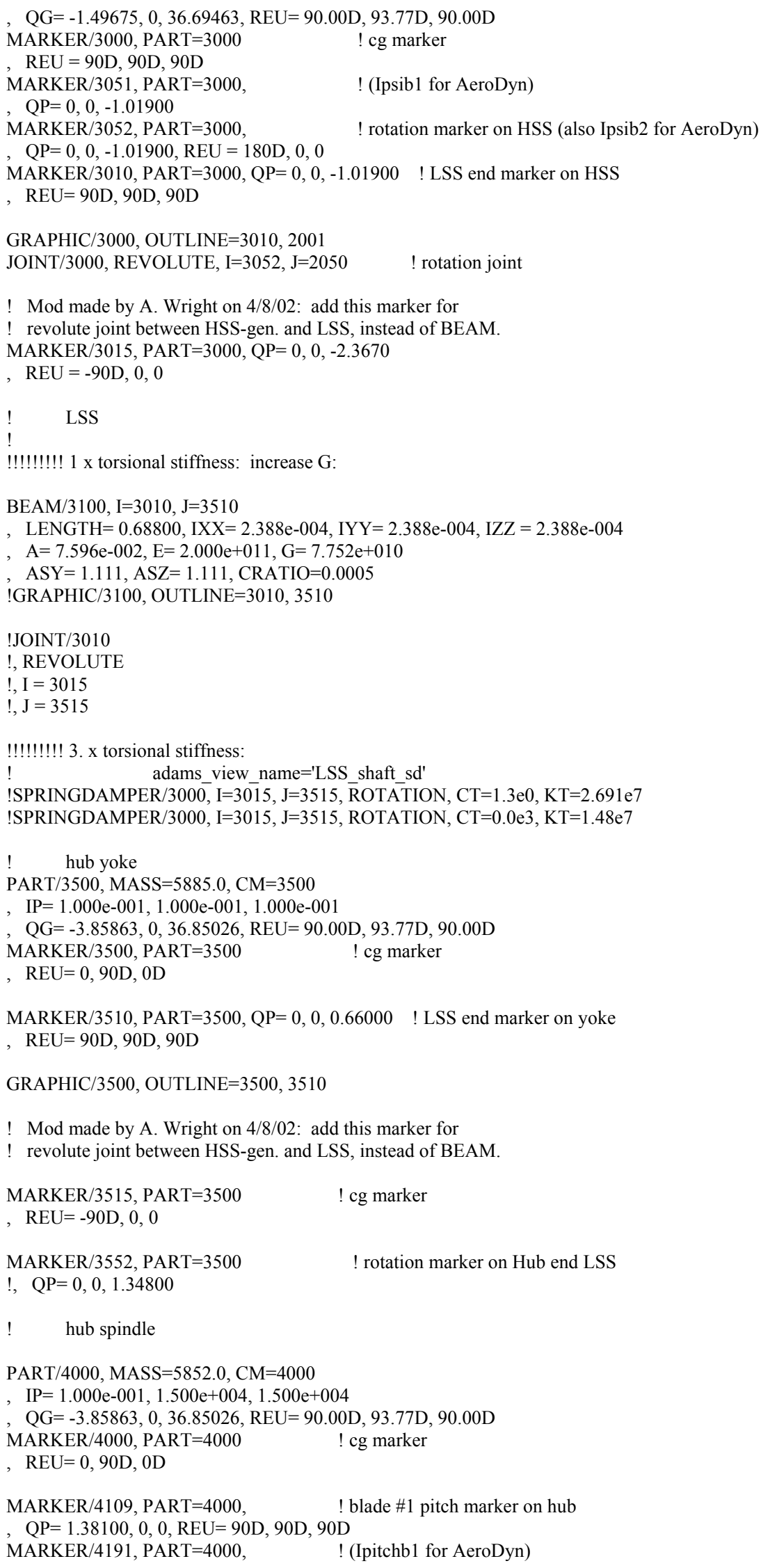




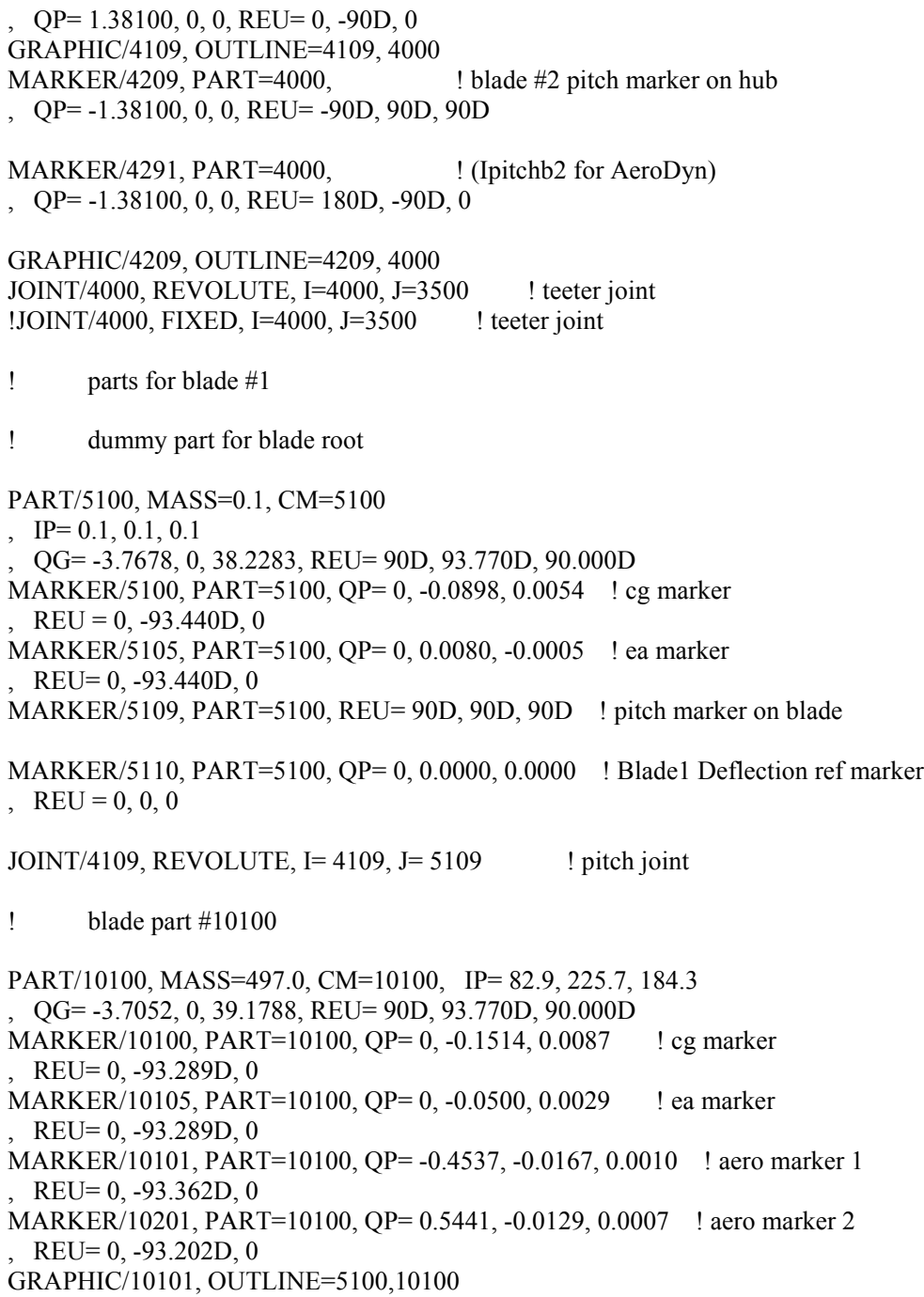


MARKER/10401, PART $=10200, \mathrm{QP}=0.5290,-0.0080,0.0004 \quad$ ! aero marker 2 , REU $=0,-92.881 \mathrm{D}, 0$

GRAPHIC/10201, OUTLINE=10100,10200

FIELD $/ 10200, \mathrm{I}=10205, \mathrm{~J}=10105, \mathrm{KMATRIX}=1.9831 \mathrm{e}+009$

$, 0.0000 \mathrm{e}+000,0.0000 \mathrm{e}+000,0.0000 \mathrm{e}+000,0.0000 \mathrm{e}+000,0.0000 \mathrm{e}+000$

$, 0.0000 \mathrm{e}+000,1.7168 \mathrm{e}+008,0.0000 \mathrm{e}+000,0.0000 \mathrm{e}+000,0.0000 \mathrm{e}+000,-1.5823 \mathrm{e}+008$ $, 0.0000 \mathrm{e}+000,0.0000 \mathrm{e}+000,4.8129 \mathrm{e}+008,0.0000 \mathrm{e}+000,4.8364 \mathrm{e}+008,0.0000 \mathrm{e}+000$ $, 0.0000 \mathrm{e}+000,0.0000 \mathrm{e}+000,0.0000 \mathrm{e}+000,1.5951 \mathrm{e}+007,0.0000 \mathrm{e}+000,0.0000 \mathrm{e}+000$ $, 0.0000 \mathrm{e}+000,0.0000 \mathrm{e}+000,4.8364 \mathrm{e}+008,0.0000 \mathrm{e}+000,6.4812 \mathrm{e}+008,0.0000 \mathrm{e}+000$ $, 0.0000 \mathrm{e}+000,-1.5823 \mathrm{e}+008,0.0000 \mathrm{e}+000,0.0000 \mathrm{e}+000,0.0000 \mathrm{e}+000,2.0247 \mathrm{e}+008$ CMATRIX $=1.9831 \mathrm{e}+004$

$, 0.0000 \mathrm{e}+000,0.0000 \mathrm{e}+000,0.0000 \mathrm{e}+000,0.0000 \mathrm{e}+000,0.0000 \mathrm{e}+000$

$, 0.0000 \mathrm{e}+000,1.7168 \mathrm{e}+000,0.0000 \mathrm{e}+000,0.0000 \mathrm{e}+000,0.0000 \mathrm{e}+000,-1.5823 \mathrm{e}+000$ $, 0.0000 \mathrm{e}+000,0.0000 \mathrm{e}+000,4.8129 \mathrm{e}+000,0.0000 \mathrm{e}+000,4.8364 \mathrm{e}+000,0.0000 \mathrm{e}+000$ $, 0.0000 \mathrm{e}+000,0.0000 \mathrm{e}+000,0.0000 \mathrm{e}+000,1.5951 \mathrm{e}+000,0.0000 \mathrm{e}+000,0.0000 \mathrm{e}+000$ $, 0.0000 \mathrm{e}+000,0.0000 \mathrm{e}+000,4.8364 \mathrm{e}+000,0.0000 \mathrm{e}+000,6.4812 \mathrm{e}+000,0.0000 \mathrm{e}+000$ $, 0.0000 \mathrm{e}+000,-1.5823 \mathrm{e}+000,0.0000 \mathrm{e}+000,0.0000 \mathrm{e}+000,0.0000 \mathrm{e}+000,2.0247 \mathrm{e}+000$ , $\quad$ LENGTH $=2.0100,0.0010,-0.1225,0.321 \mathrm{D}, 0,0$

! $\quad$ blade part $\# 10300$

$\mathrm{PART} / 10300, \mathrm{MASS}=328.0, \mathrm{CM}=10300, \mathrm{IP}=73.1,172.9,117.1$

, $\mathrm{QG}=-3.4418,0,43.1757, \mathrm{REU}=90 \mathrm{D}, 93.770 \mathrm{D}, 90.000 \mathrm{D}$

MARKER $/ 10300, \mathrm{PART}=10300, \mathrm{QP}=0,-0.3544,0.0163 \quad$ ! cg marker

, REU $=0,-92.632 \mathrm{D}, 0$

MARKER/10305, $\mathrm{PART}=10300, \mathrm{QP}=0,-0.2527,0.0116 \quad$ ! ea marker

, REU $=0,-92.632 \mathrm{D}, 0$

MARKER $/ 10501, \mathrm{PART}=10300, \mathrm{QP}=-0.4683,-0.0064,0.0003 \quad$ ! aero marker 1

, REU $=0,-92.725 \mathrm{D}, 0$

MARKER $/ 10601, \mathrm{PART}=10300, \mathrm{QP}=0.5294,-0.0042,0.0002 \quad$ ! aero marker 2

, REU $=0,-92.544 \mathrm{D}, 0$

GRAPHIC/10301, OUTLINE=10200,10300

FIELD $/ 10300, \mathrm{I}=10305, \mathrm{~J}=10205, \mathrm{KMATRIX}=1.5487 \mathrm{e}+009$

$, 0.0000 \mathrm{e}+000,0.0000 \mathrm{e}+000,0.0000 \mathrm{e}+000,0.0000 \mathrm{e}+000,0.0000 \mathrm{e}+000$

$, 0.0000 \mathrm{e}+000,1.0866 \mathrm{e}+008,0.0000 \mathrm{e}+000,0.0000 \mathrm{e}+000,0.0000 \mathrm{e}+000,-1.0068 \mathrm{e}+008$ $, 0.0000 \mathrm{e}+000,0.0000 \mathrm{e}+000,4.9512 \mathrm{e}+008,0.0000 \mathrm{e}+000,4.9495 \mathrm{e}+008,0.0000 \mathrm{e}+000$ $, 0.0000 \mathrm{e}+000,0.0000 \mathrm{e}+000,0.0000 \mathrm{e}+000,1.0338 \mathrm{e}+007,0.0000 \mathrm{e}+000,0.0000 \mathrm{e}+000$ $, 0.0000 \mathrm{e}+000,0.0000 \mathrm{e}+000,4.9495 \mathrm{e}+008,0.0000 \mathrm{e}+000,6.5901 \mathrm{e}+008,0.0000 \mathrm{e}+000$ $, 0.0000 \mathrm{e}+000,-1.0068 \mathrm{e}+008,0.0000 \mathrm{e}+000,0.0000 \mathrm{e}+000,0.0000 \mathrm{e}+000,1.2878 \mathrm{e}+008$ CMATRIX $=1.5487 \mathrm{e}+004$

$, 0.0000 \mathrm{e}+000,0.0000 \mathrm{e}+000,0.0000 \mathrm{e}+000,0.0000 \mathrm{e}+000,0.0000 \mathrm{e}+000$ $, 0.0000 \mathrm{e}+000,1.0866 \mathrm{e}+000,0.0000 \mathrm{e}+000,0.0000 \mathrm{e}+000,0.0000 \mathrm{e}+000,-1.0068 \mathrm{e}+000$ $, 0.0000 \mathrm{e}+000,0.0000 \mathrm{e}+000,4.9512 \mathrm{e}+000,0.0000 \mathrm{e}+000,4.9495 \mathrm{e}+000,0.0000 \mathrm{e}+000$ $, 0.0000 \mathrm{e}+000,0.0000 \mathrm{e}+000,0.0000 \mathrm{e}+000,1.0338 \mathrm{e}+000,0.0000 \mathrm{e}+000,0.0000 \mathrm{e}+000$ $, 0.0000 \mathrm{e}+000,0.0000 \mathrm{e}+000,4.9495 \mathrm{e}+000,0.0000 \mathrm{e}+000,6.5901 \mathrm{e}+000,0.0000 \mathrm{e}+000$ $, 0.0000 \mathrm{e}+000,-1.0068 \mathrm{e}+000,0.0000 \mathrm{e}+000,0.0000 \mathrm{e}+000,0.0000 \mathrm{e}+000,1.2878 \mathrm{e}+000$

, $\quad$ LENGTH $=1.9950,0.0015,-0.0804,0.336 \mathrm{D}, 0,0$

! $\quad$ blade part $\# 10400$

$\mathrm{PART} / 10400, \mathrm{MASS}=269.6, \mathrm{CM}=10400, \mathrm{IP}=55.1,138.6,94.6$

, $\mathrm{QG}=-3.3109,0,45.1625, \mathrm{REU}=90 \mathrm{D}, 93.770 \mathrm{D}, 90.000 \mathrm{D}$

MARKER/10400, $\mathrm{PART}=10400, \mathrm{QP}=0,-0.3634,0.0143 \quad$ ! cg marker

, REU $=0,-92.251 \mathrm{D}, 0$

MARKER/10405, PART $=10400, \mathrm{QP}=0,-0.2640,0.0104 \quad$ ! ea marker

, REU $=0,-92.251 \mathrm{D}, 0$

MARKER/10701, PART $=10400, \mathrm{QP}=-0.4640,-0.0018,0.0001 \quad$ ! aero marker 1

, REU $=0,-92.355 \mathrm{D}, 0$

MARKER $/ 10801, \mathrm{PART}=10400, \mathrm{QP}=0.5338,0.0000,0.0000 \quad !$ aero marker 2

, REU $=0,-92.156 \mathrm{D}, 0$

GRAPHIC/10401, OUTLINE=10300,10400

FIELD $/ 10400, \mathrm{I}=10405, \mathrm{~J}=10305, \mathrm{KMATRIX}=1.3202 \mathrm{e}+009$

$, 0.0000 \mathrm{e}+000,0.0000 \mathrm{e}+000,0.0000 \mathrm{e}+000,0.0000 \mathrm{e}+000,0.0000 \mathrm{e}+000$

$, 0.0000 \mathrm{e}+000,7.0972 \mathrm{e}+007,0.0000 \mathrm{e}+000,0.0000 \mathrm{e}+000,0.0000 \mathrm{e}+000,-6.5678 \mathrm{e}+007$ $, 0.0000 \mathrm{e}+000,0.0000 \mathrm{e}+000,4.3826 \mathrm{e}+008,0.0000 \mathrm{e}+000,4.1541 \mathrm{e}+008,0.0000 \mathrm{e}+000$ $, 0.0000 \mathrm{e}+000,0.0000 \mathrm{e}+000,0.0000 \mathrm{e}+000,6.6475 \mathrm{e}+006,0.0000 \mathrm{e}+000,0.0000 \mathrm{e}+000$

$, 0.0000 \mathrm{e}+000,0.0000 \mathrm{e}+000,4.1541 \mathrm{e}+008,0.0000 \mathrm{e}+000,5.3755 \mathrm{e}+008,0.0000 \mathrm{e}+000$ 
$, 0.0000 \mathrm{e}+000,-6.5678 \mathrm{e}+007,0.0000 \mathrm{e}+000,0.0000 \mathrm{e}+000,0.0000 \mathrm{e}+000,8.3878 \mathrm{e}+007$ ,CMATRIX $=5.0387 \mathrm{e}+004$

$, 0.0000 \mathrm{e}+000,0.0000 \mathrm{e}+000,0.0000 \mathrm{e}+000,0.0000 \mathrm{e}+000,0.0000 \mathrm{e}+000$

$, 0.0000 \mathrm{e}+000,2.1538 \mathrm{e}+000,0.0000 \mathrm{e}+000,0.0000 \mathrm{e}+000,0.0000 \mathrm{e}+000,-1.0041 \mathrm{e}+000$ $, 0.0000 \mathrm{e}+000,0.0000 \mathrm{e}+000,4.2296 \mathrm{e}+000,0.0000 \mathrm{e}+000,2.0622 \mathrm{e}+000,0.0000 \mathrm{e}+000$ $, 0.0000 \mathrm{e}+000,0.0000 \mathrm{e}+000,0.0000 \mathrm{e}+000,4.2078 \mathrm{e}+000,0.0000 \mathrm{e}+000,0.0000 \mathrm{e}+000$ $, 0.0000 \mathrm{e}+000,0.0000 \mathrm{e}+000,2.0622 \mathrm{e}+000,0.0000 \mathrm{e}+000,1.3247 \mathrm{e}+000,0.0000 \mathrm{e}+000$ $, 0.0000 \mathrm{e}+000,-1.0041 \mathrm{e}+000,0.0000 \mathrm{e}+000,0.0000 \mathrm{e}+000,0.0000 \mathrm{e}+000,6.3070 \mathrm{e}+000$ , $\mathrm{LENGTH}=1.9910,0.0018,-0.0112,0.380 \mathrm{D}, 0,0$

! $\quad$ blade part \#10500

$\mathrm{PART} / 10500, \mathrm{MASS}=214.7, \mathrm{CM}=10500, \mathrm{IP}=37.7,105.1,74.3$

, $\mathrm{QG}=-3.1801,0,47.1473, \mathrm{REU}=90 \mathrm{D}, 93.770 \mathrm{D}, 90.000 \mathrm{D}$

MARKER/10500, PART $=10500, \mathrm{QP}=0,-0.3452,0.0109 \quad$ ! cg marker

, REU $=0,-91.807 \mathrm{D}, 0$

MARKER/10505, PART $=10500, \mathrm{QP}=0,-0.2442,0.0077 \quad$ ! ea marker

, REU $=0,-91.807 \mathrm{D}, 0$

MARKER/10901, PART $=10500, \mathrm{QP}=-0.4576,0.0000,0.0000 \quad$ ! aero marker 1

, REU $=0,-91.920 \mathrm{D}, 0$

MARKER $/ 11001, \mathrm{PART}=10500, \mathrm{QP}=0.5401,0.0000,0.0000 \quad$ ! aero marker 2

, REU $=0,-91.685 \mathrm{D}, 0$

GRAPHIC/10501, OUTLINE $=10400,10500$

FIELD $/ 10500, \mathrm{I}=10505, \mathrm{~J}=10405, \mathrm{KMATRIX}=1.0753 \mathrm{e}+009$

$, 0.0000 \mathrm{e}+000,0.0000 \mathrm{e}+000,0.0000 \mathrm{e}+000,0.0000 \mathrm{e}+000,0.0000 \mathrm{e}+000$

$, 0.0000 \mathrm{e}+000,4.5365 \mathrm{e}+007,0.0000 \mathrm{e}+000,0.0000 \mathrm{e}+000,0.0000 \mathrm{e}+000,-4.1547 \mathrm{e}+007$ $, 0.0000 \mathrm{e}+000,0.0000 \mathrm{e}+000,3.0934 \mathrm{e}+008,0.0000 \mathrm{e}+000,2.8543 \mathrm{e}+008,0.0000 \mathrm{e}+000$ $, 0.0000 \mathrm{e}+000,0.0000 \mathrm{e}+000,0.0000 \mathrm{e}+000,4.2996 \mathrm{e}+006,0.0000 \mathrm{e}+000,0.0000 \mathrm{e}+000$ $, 0.0000 \mathrm{e}+000,0.0000 \mathrm{e}+000,2.8543 \mathrm{e}+008,0.0000 \mathrm{e}+000,3.6376 \mathrm{e}+008,0.0000 \mathrm{e}+000$ $, 0.0000 \mathrm{e}+000,-4.1547 \mathrm{e}+007,0.0000 \mathrm{e}+000,0.0000 \mathrm{e}+000,0.0000 \mathrm{e}+000,5.2727 \mathrm{e}+007$ ,CMATRIX $=1.0753 \mathrm{e}+004$

$, 0.0000 \mathrm{e}+000,0.0000 \mathrm{e}+000,0.0000 \mathrm{e}+000,0.0000 \mathrm{e}+000,0.0000 \mathrm{e}+000$

$, 0.0000 \mathrm{e}+000,4.5365 \mathrm{e}+000,0.0000 \mathrm{e}+000,0.0000 \mathrm{e}+000,0.0000 \mathrm{e}+000,-4.1547 \mathrm{e}+000$ $, 0.0000 \mathrm{e}+000,0.0000 \mathrm{e}+000,3.0934 \mathrm{e}+000,0.0000 \mathrm{e}+000,2.8543 \mathrm{e}+000,0.0000 \mathrm{e}+000$ $, 0.0000 \mathrm{e}+000,0.0000 \mathrm{e}+000,0.0000 \mathrm{e}+000,4.2996 \mathrm{e}+000,0.0000 \mathrm{e}+000,0.0000 \mathrm{e}+000$ $, 0.0000 \mathrm{e}+000,0.0000 \mathrm{e}+000,2.8543 \mathrm{e}+000,0.0000 \mathrm{e}+000,3.6376 \mathrm{e}+000,0.0000 \mathrm{e}+000$ $, 0.0000 \mathrm{e}+000,-4.1547 \mathrm{e}+000,0.0000 \mathrm{e}+000,0.0000 \mathrm{e}+000,0.0000 \mathrm{e}+000,5.2727 \mathrm{e}+000$ , LENGTH=1.9890,0.0019,0.0199, 0.444D, 0,0

! $\quad$ blade part \#10600

$\mathrm{PART} / 10600, \mathrm{MASS}=162.8, \mathrm{CM}=10600, \mathrm{IP}=23.8,75.4,55.5$

, $\mathrm{QG}=-3.0496,0,49.1281, \mathrm{REU}=90 \mathrm{D}, 93.770 \mathrm{D}, 90.000 \mathrm{D}$

MARKER/10600, PART $=10600, \mathrm{QP}=0,-0.3163,0.0071 \quad$ ! cg marker

, REU $=0,-91.278 \mathrm{D}, 0$

MARKER/10605, PART $=10600, \mathrm{QP}=0,-0.2206,0.0049 \quad$ ! ea marker

, REU $=0,-91.278 \mathrm{D}, 0$

MARKER/11101, PART $=10600, \mathrm{QP}=-0.4472,0.0000,0.0000 \quad$ ! aero marker 1

, REU $=0,-91.411 \mathrm{D}, 0$

MARKER $/ 11201, \mathrm{PART}=10600, \mathrm{QP}=0.5505,0.0000,0.0000 \quad$ ! aero marker 2

, REU $=0,-91.118 \mathrm{D}, 0$

GRAPHIC/10601, OUTLINE=10500,10600

FIELD/10600, $\mathrm{I}=10605, \mathrm{~J}=10505, \mathrm{KMATRIX}=8.4118 \mathrm{e}+008$

$, 0.0000 \mathrm{e}+000,0.0000 \mathrm{e}+000,0.0000 \mathrm{e}+000,0.0000 \mathrm{e}+000,0.0000 \mathrm{e}+000$

$, 0.0000 \mathrm{e}+000,2.7316 \mathrm{e}+007,0.0000 \mathrm{e}+000,0.0000 \mathrm{e}+000,0.0000 \mathrm{e}+000,-2.4634 \mathrm{e}+007$

$, 0.0000 \mathrm{e}+000,0.0000 \mathrm{e}+000,1.9775 \mathrm{e}+008,0.0000 \mathrm{e}+000,1.8104 \mathrm{e}+008,0.0000 \mathrm{e}+000$

$, 0.0000 \mathrm{e}+000,0.0000 \mathrm{e}+000,0.0000 \mathrm{e}+000,2.6424 \mathrm{e}+006,0.0000 \mathrm{e}+000,0.0000 \mathrm{e}+000$

$, 0.0000 \mathrm{e}+000,0.0000 \mathrm{e}+000,1.8104 \mathrm{e}+008,0.0000 \mathrm{e}+000,2.2951 \mathrm{e}+008,0.0000 \mathrm{e}+000$

$, 0.0000 \mathrm{e}+000,-2.4634 \mathrm{e}+007,0.0000 \mathrm{e}+000,0.0000 \mathrm{e}+000,0.0000 \mathrm{e}+000,3.0960 \mathrm{e}+007$

,CMATRIX $=8.4118 \mathrm{e}+003$

$, 0.0000 \mathrm{e}+000,0.0000 \mathrm{e}+000,0.0000 \mathrm{e}+000,0.0000 \mathrm{e}+000,0.0000 \mathrm{e}+000$

$, 0.0000 \mathrm{e}+000,2.7316 \mathrm{e}+000,0.0000 \mathrm{e}+000,0.0000 \mathrm{e}+000,0.0000 \mathrm{e}+000,-2.4634 \mathrm{e}+000$ $, 0.0000 \mathrm{e}+000,0.0000 \mathrm{e}+000,1.9775 \mathrm{e}+000,0.0000 \mathrm{e}+000,1.8104 \mathrm{e}+000,0.0000 \mathrm{e}+000$ $, 0.0000 \mathrm{e}+000,0.0000 \mathrm{e}+000,0.0000 \mathrm{e}+000,2.6424 \mathrm{e}+000,0.0000 \mathrm{e}+000,0.0000 \mathrm{e}+000$ $, 0.0000 \mathrm{e}+000,0.0000 \mathrm{e}+000,1.8104 \mathrm{e}+000,0.0000 \mathrm{e}+000,2.2951 \mathrm{e}+000,0.0000 \mathrm{e}+000$ $, 0.0000 \mathrm{e}+000,-2.4634 \mathrm{e}+000,0.0000 \mathrm{e}+000,0.0000 \mathrm{e}+000,0.0000 \mathrm{e}+000,3.0960 \mathrm{e}+000$ , $\quad \mathrm{LENGTH}=1.9850,0.0020,0.0237,0.530 \mathrm{D}, 0,0$ 
$\mathrm{PART} / 10700, \mathrm{MASS}=114.8, \mathrm{CM}=10700, \mathrm{IP}=13.7,50.2,38.6$

, $\mathrm{QG}=-2.9193,0,51.1055, \mathrm{REU}=90 \mathrm{D}, 93.770 \mathrm{D}, 90.000 \mathrm{D}$

MARKER $/ 10700, \mathrm{PART}=10700, \mathrm{QP}=0,-0.2815,0.0031 \quad$ ! cg marker

, REU $=0,-90.628 \mathrm{D}, 0$

MARKER $/ 10705, \mathrm{PART}=10700, \mathrm{QP}=0,-0.1973,0.0022 \quad$ ! ea marker

, REU $=0,-90.628 \mathrm{D}, 0$

MARKER/11301, PART $=10700, Q P=-0.4334,0.0000,0.0000 \quad$ ! aero marker 1

, REU $=0,-90.808 \mathrm{D}, 0$

MARKER/11401, PART $=10700, \mathrm{QP}=0.5644,0.0000,0.0000 \quad$ ! aero marker 2

, REU $=0,-90.431 \mathrm{D}, 0$

GRAPHIC/10701, OUTLINE=10600,10700

FIELD $/ 10700, \mathrm{I}=10705, \mathrm{~J}=10605, \mathrm{KMATRIX}=6.4385 \mathrm{e}+008$

$, 0.0000 \mathrm{e}+000,0.0000 \mathrm{e}+000,0.0000 \mathrm{e}+000,0.0000 \mathrm{e}+000,0.0000 \mathrm{e}+000$

$, 0.0000 \mathrm{e}+000,1.5223 \mathrm{e}+007,0.0000 \mathrm{e}+000,0.0000 \mathrm{e}+000,0.0000 \mathrm{e}+000,-1.3529 \mathrm{e}+007$

$, 0.0000 \mathrm{e}+000,0.0000 \mathrm{e}+000,1.2322 \mathrm{e}+008,0.0000 \mathrm{e}+000,1.1242 \mathrm{e}+008,0.0000 \mathrm{e}+000$

$, 0.0000 \mathrm{e}+000,0.0000 \mathrm{e}+000,0.0000 \mathrm{e}+000,1.5168 \mathrm{e}+006,0.0000 \mathrm{e}+000,0.0000 \mathrm{e}+000$

$, 0.0000 \mathrm{e}+000,0.0000 \mathrm{e}+000,1.1242 \mathrm{e}+008,0.0000 \mathrm{e}+000,1.4212 \mathrm{e}+008,0.0000 \mathrm{e}+000$

$, 0.0000 \mathrm{e}+000,-1.3529 \mathrm{e}+007,0.0000 \mathrm{e}+000,0.0000 \mathrm{e}+000,0.0000 \mathrm{e}+000,1.6847 \mathrm{e}+007$

,CMATRIX $=6.4385 \mathrm{e}+003$

$, 0.0000 \mathrm{e}+000,0.0000 \mathrm{e}+000,0.0000 \mathrm{e}+000,0.0000 \mathrm{e}+000,0.0000 \mathrm{e}+000$

$, 0.0000 \mathrm{e}+000,1.5223 \mathrm{e}+000,0.0000 \mathrm{e}+000,0.0000 \mathrm{e}+000,0.0000 \mathrm{e}+000,-1.3529 \mathrm{e}+000$ $, 0.0000 \mathrm{e}+000,0.0000 \mathrm{e}+000,1.2322 \mathrm{e}+000,0.0000 \mathrm{e}+000,1.1242 \mathrm{e}+000,0.0000 \mathrm{e}+000$ $, 0.0000 \mathrm{e}+000,0.0000 \mathrm{e}+000,0.0000 \mathrm{e}+000,1.5168 \mathrm{e}+000,0.0000 \mathrm{e}+000,0.0000 \mathrm{e}+000$ $, 0.0000 \mathrm{e}+000,0.0000 \mathrm{e}+000,1.1242 \mathrm{e}+000,0.0000 \mathrm{e}+000,1.4212 \mathrm{e}+000,0.0000 \mathrm{e}+000$ $, 0.0000 \mathrm{e}+000,-1.3529 \mathrm{e}+000,0.0000 \mathrm{e}+000,0.0000 \mathrm{e}+000,0.0000 \mathrm{e}+000,1.6847 \mathrm{e}+000$ , $\mathrm{LENGTH}=1.9820,0.0022,0.0234,0.650 \mathrm{D}, 0,0$

! blade part \#10800

PART $/ 10800$, MASS $=72.9, \mathrm{CM}=10800, \quad \mathrm{IP}=7.1,30.2,24.1$

, $\mathrm{QG}=-2.7896,0,53.0737, \mathrm{REU}=90 \mathrm{D}, 93.770 \mathrm{D}, 90.000 \mathrm{D}$

MARKER $/ 10800, \mathrm{PART}=10800, \mathrm{QP}=0,-0.2492,-0.0008 \quad$ ! cg marker

, $\mathrm{REU}=0,-89.822 \mathrm{D}, 0$

MARKER $/ 10805, \mathrm{PART}=10800, \mathrm{QP}=0,-0.1740,-0.0005 \quad$ ! ea marker

, $\mathrm{REU}=0,-89.822 \mathrm{D}, 0$

MARKER $/ 11501, \mathrm{PART}=10800, \mathrm{QP}=-0.4104,0.0000,0.0000 \quad$ ! aero marker 1

, REU $=0,-90.047 \mathrm{D}, 0$

MARKER $/ 11601, \mathrm{PART}=10800, \mathrm{QP}=0.5873,0.0000,0.0000 \quad$ ! aero marker 2

, REU $=0,-89.583 \mathrm{D}, 0$

GRAPHIC $/ 10801$, OUTLINE $=10700,10800$

FIELD $/ 10800, \mathrm{I}=10805, \mathrm{~J}=10705, \mathrm{KMATRIX}=4.7422 \mathrm{e}+008$

$, 0.0000 \mathrm{e}+000,0.0000 \mathrm{e}+000,0.0000 \mathrm{e}+000,0.0000 \mathrm{e}+000,0.0000 \mathrm{e}+000$

$, 0.0000 \mathrm{e}+000,7.6902 \mathrm{e}+006,0.0000 \mathrm{e}+000,0.0000 \mathrm{e}+000,0.0000 \mathrm{e}+000,-6.6072 \mathrm{e}+006$ $, 0.0000 \mathrm{e}+000,0.0000 \mathrm{e}+000,7.3081 \mathrm{e}+007,0.0000 \mathrm{e}+000,6.4793 \mathrm{e}+007,0.0000 \mathrm{e}+000$ $, 0.0000 \mathrm{e}+000,0.0000 \mathrm{e}+000,0.0000 \mathrm{e}+000,7.9462 \mathrm{e}+005,0.0000 \mathrm{e}+000,0.0000 \mathrm{e}+000$ $, 0.0000 \mathrm{e}+000,0.0000 \mathrm{e}+000,6.4793 \mathrm{e}+007,0.0000 \mathrm{e}+000,8.0415 \mathrm{e}+007,0.0000 \mathrm{e}+000$ $, 0.0000 \mathrm{e}+000,-6.6072 \mathrm{e}+006,0.0000 \mathrm{e}+000,0.0000 \mathrm{e}+000,0.0000 \mathrm{e}+000,8.0459 \mathrm{e}+006$ , $\mathrm{CMATRIX}=4.7422 \mathrm{e}+003$

$, 0.0000 \mathrm{e}+000,0.0000 \mathrm{e}+000,0.0000 \mathrm{e}+000,0.0000 \mathrm{e}+000,0.0000 \mathrm{e}+000$

$, 0.0000 \mathrm{e}+000,7.6902 \mathrm{e}+000,0.0000 \mathrm{e}+000,0.0000 \mathrm{e}+000,0.0000 \mathrm{e}+000,-6.6072 \mathrm{e}+000$ $, 0.0000 \mathrm{e}+000,0.0000 \mathrm{e}+000,7.3081 \mathrm{e}+000,0.0000 \mathrm{e}+000,6.4793 \mathrm{e}+000,0.0000 \mathrm{e}+000$ $, 0.0000 \mathrm{e}+000,0.0000 \mathrm{e}+000,0.0000 \mathrm{e}+000,7.9462 \mathrm{e}+000,0.0000 \mathrm{e}+000,0.0000 \mathrm{e}+000$ $, 0.0000 \mathrm{e}+000,0.0000 \mathrm{e}+000,6.4793 \mathrm{e}+000,0.0000 \mathrm{e}+000,8.0415 \mathrm{e}+000,0.0000 \mathrm{e}+000$ $, 0.0000 \mathrm{e}+000,-6.6072 \mathrm{e}+000,0.0000 \mathrm{e}+000,0.0000 \mathrm{e}+000,0.0000 \mathrm{e}+000,8.0459 \mathrm{e}+000$ , LENGTH $=1.9730,0.0024,0.0233,0.805 \mathrm{D}, 0,0$

! blade part \#10900

$\mathrm{PART} / 10900, \mathrm{MASS}=40.5, \mathrm{CM}=10900, \mathrm{IP}=3.5,16.3,13.1$

, $\mathrm{QG}=-2.6596,0,55.0463, \mathrm{REU}=90 \mathrm{D}, 93.770 \mathrm{D}, 90.000 \mathrm{D}$

MARKER $/ 10900, \mathrm{PART}=10900, \mathrm{QP}=0,-0.2194,-0.0046 \quad$ ! cg marker

, REU $=0,-88.808 \mathrm{D}, 0$

MARKER/10905, PART $=10900, \mathrm{QP}=0,-0.1506,-0.0031 \quad$ ! ea marker

, REU $=0,-88.808 \mathrm{D}, 0$

MARKER $/ 11701, \mathrm{PART}=10900, \mathrm{QP}=-0.3918,0.0000,0.0000 \quad$ ! aero marker 1

, $\mathrm{REU}=0,-89.065 \mathrm{D}, 0$

MARKER $/ 11801, \mathrm{PART}=10900, \mathrm{QP}=0.6060,0.0000,0.0000 \quad$ ! aero marker 2 
, REU $=0,-88.536 \mathrm{D}, 0$

GRAPHIC/10901, OUTLINE=10800,10900

FIELD $/ 10900, \mathrm{I}=10905, \mathrm{~J}=10805, \mathrm{KMATRIX}=3.2430 \mathrm{e}+008$

$, 0.0000 \mathrm{e}+000,0.0000 \mathrm{e}+000,0.0000 \mathrm{e}+000,0.0000 \mathrm{e}+000,0.0000 \mathrm{e}+000$

$, 0.0000 \mathrm{e}+000,3.2750 \mathrm{e}+006,0.0000 \mathrm{e}+000,0.0000 \mathrm{e}+000,0.0000 \mathrm{e}+000,-2.7721 \mathrm{e}+006$

$, 0.0000 \mathrm{e}+000,0.0000 \mathrm{e}+000,3.7449 \mathrm{e}+007,0.0000 \mathrm{e}+000,3.2685 \mathrm{e}+007,0.0000 \mathrm{e}+000$ $, 0.0000 \mathrm{e}+000,0.0000 \mathrm{e}+000,0.0000 \mathrm{e}+000,3.6362 \mathrm{e}+005,0.0000 \mathrm{e}+000,0.0000 \mathrm{e}+000$ $, 0.0000 \mathrm{e}+000,0.0000 \mathrm{e}+000,3.2685 \mathrm{e}+007,0.0000 \mathrm{e}+000,4.0221 \mathrm{e}+007,0.0000 \mathrm{e}+000$ $, 0.0000 \mathrm{e}+000,-2.7721 \mathrm{e}+006,0.0000 \mathrm{e}+000,0.0000 \mathrm{e}+000,0.0000 \mathrm{e}+000,3.3469 \mathrm{e}+006$ ,CMATRIX $=3.2430 \mathrm{e}+003$

$, 0.0000 \mathrm{e}+000,0.0000 \mathrm{e}+000,0.0000 \mathrm{e}+000,0.0000 \mathrm{e}+000,0.0000 \mathrm{e}+000$

$, 0.0000 \mathrm{e}+000,3.2750 \mathrm{e}+000,0.0000 \mathrm{e}+000,0.0000 \mathrm{e}+000,0.0000 \mathrm{e}+000,-2.7721 \mathrm{e}+000$ $, 0.0000 \mathrm{e}+000,0.0000 \mathrm{e}+000,3.7449 \mathrm{e}+000,0.0000 \mathrm{e}+000,3.2685 \mathrm{e}+000,0.0000 \mathrm{e}+000$ $, 0.0000 \mathrm{e}+000,0.0000 \mathrm{e}+000,0.0000 \mathrm{e}+000,3.6362 \mathrm{e}+000,0.0000 \mathrm{e}+000,0.0000 \mathrm{e}+000$ $, 0.0000 \mathrm{e}+000,0.0000 \mathrm{e}+000,3.2685 \mathrm{e}+000,0.0000 \mathrm{e}+000,4.0221 \mathrm{e}+000,0.0000 \mathrm{e}+000$ $, 0.0000 \mathrm{e}+000,-2.7721 \mathrm{e}+000,0.0000 \mathrm{e}+000,0.0000 \mathrm{e}+000,0.0000 \mathrm{e}+000,3.3469 \mathrm{e}+000$ , $\mathrm{LENGTH}=1.9760,0.0027,0.0234,1.015 \mathrm{D}, 0,0$

! $\quad$ blade part \#11000

$\mathrm{PART} / 11000, \mathrm{MASS}=19.7, \mathrm{CM}=11000, \mathrm{IP}=1.9,8.1,6.3$

, $\mathrm{QG}=-2.5299,0,57.0146, \mathrm{REU}=90 \mathrm{D}, 93.770 \mathrm{D}, 90.000 \mathrm{D}$

MARKER $/ 11000, \mathrm{PART}=11000, \mathrm{QP}=0,-0.1892,-0.0082 \quad$ ! cg marker

REU $=0,-87.525 \mathrm{D}, 0$

MARKER/11005, PART $=11000, \mathrm{QP}=0,-0.1272,-0.0055 \quad$ ! ea marker

, REU $=0,-87.525 \mathrm{D}, 0$

MARKER $/ 11901, \mathrm{PART}=11000, \mathrm{QP}=-0.3689,0.0000,0.0000 \quad$ ! aero marker 1

, REU $=0,-87.798 \mathrm{D}, 0$

MARKER $/ 12001, \mathrm{PART}=11000, \mathrm{QP}=0.6289,0.0000,0.0000 \quad$ ! aero marker 2

, REU $=0,-87.059 \mathrm{D}, 0$

GRAPHIC/11001, OUTLINE=10900,11000

FIELD $/ 11000, \mathrm{I}=11005, \mathrm{~J}=10905, \mathrm{KMATRIX}=2.2852 \mathrm{e}+008$

$, 0.0000 \mathrm{e}+000,0.0000 \mathrm{e}+000,0.0000 \mathrm{e}+000,0.0000 \mathrm{e}+000,0.0000 \mathrm{e}+000$

$, 0.0000 \mathrm{e}+000,1.2811 \mathrm{e}+006,0.0000 \mathrm{e}+000,0.0000 \mathrm{e}+000,0.0000 \mathrm{e}+000,-1.0680 \mathrm{e}+006$ $, 0.0000 \mathrm{e}+000,0.0000 \mathrm{e}+000,1.8971 \mathrm{e}+007,0.0000 \mathrm{e}+000,1.6904 \mathrm{e}+007,0.0000 \mathrm{e}+000$ $, 0.0000 \mathrm{e}+000,0.0000 \mathrm{e}+000,0.0000 \mathrm{e}+000,1.4884 \mathrm{e}+005,0.0000 \mathrm{e}+000,0.0000 \mathrm{e}+000$ $, 0.0000 \mathrm{e}+000,0.0000 \mathrm{e}+000,1.6904 \mathrm{e}+007,0.0000 \mathrm{e}+000,2.1043 \mathrm{e}+007,0.0000 \mathrm{e}+000$ $, 0.0000 \mathrm{e}+000,-1.0680 \mathrm{e}+006,0.0000 \mathrm{e}+000,0.0000 \mathrm{e}+000,0.0000 \mathrm{e}+000,1.2759 \mathrm{e}+006$ ,CMATRIX $=2.2852 \mathrm{e}+003$

$, 0.0000 \mathrm{e}+000,0.0000 \mathrm{e}+000,0.0000 \mathrm{e}+000,0.0000 \mathrm{e}+000,0.0000 \mathrm{e}+000$

$, 0.0000 \mathrm{e}+000,1.2811 \mathrm{e}+000,0.0000 \mathrm{e}+000,0.0000 \mathrm{e}+000,0.0000 \mathrm{e}+000,-1.0680 \mathrm{e}+000$ $, 0.0000 \mathrm{e}+000,0.0000 \mathrm{e}+000,1.8971 \mathrm{e}+000,0.0000 \mathrm{e}+000,1.6904 \mathrm{e}+000,0.0000 \mathrm{e}+000$ $, 0.0000 \mathrm{e}+000,0.0000 \mathrm{e}+000,0.0000 \mathrm{e}+000,1.4884 \mathrm{e}+000,0.0000 \mathrm{e}+000,0.0000 \mathrm{e}+000$ $, 0.0000 \mathrm{e}+000,0.0000 \mathrm{e}+000,1.6904 \mathrm{e}+000,0.0000 \mathrm{e}+000,2.1043 \mathrm{e}+000,0.0000 \mathrm{e}+000$ $, 0.0000 \mathrm{e}+000,-1.0680 \mathrm{e}+000,0.0000 \mathrm{e}+000,0.0000 \mathrm{e}+000,0.0000 \mathrm{e}+000,1.2759 \mathrm{e}+000$ , $\mathrm{LENGTH}=1.9730,0.0029,0.0233,1.283 \mathrm{D}, 0,0$

! Special Blade Tip Marker for Impact

MARKER/13001, PART $=11000, \mathrm{QP}=1.1277,-0.1718,-0.0074$

, $\mathrm{REU}=0,0,0$

GRAPHIC/13000, OUTLINE=13001,11000

parts for blade \#2

dummy part for blade root

$\mathrm{PART} / 5200, \mathrm{MASS}=0.1, \mathrm{CM}=5200$

, IP $=0.1,0.1,0.1$

, $\mathrm{QG}=-3.9494,0,35.4722, \mathrm{REU}=90 \mathrm{D}, 93.770 \mathrm{D},-90.000 \mathrm{D}$

MARKER/5200, PART $=5200, \mathrm{QP}=0,-0.0898,0.0054 \quad$ ! cg marker

, REU $=0,-93.440 \mathrm{D}, 0$

MARKER/5205, PART $=5200, \mathrm{QP}=0,0.0080,-0.0005 \quad$ ! ea marker

REU $=0,-93.440 \mathrm{D}, 0$

MARKER/5209, PART $=5200$, REU $=90 \mathrm{D}, 90 \mathrm{D}, 90 \mathrm{D} \quad$ ! pitch marker on blade

MARKER/5210, $\mathrm{PART}=5200, \mathrm{QP}=0,0.0000,0.0000 \quad$ ! Blade2 Deflection ref marker , $\mathrm{REU}=0,0,0$ 


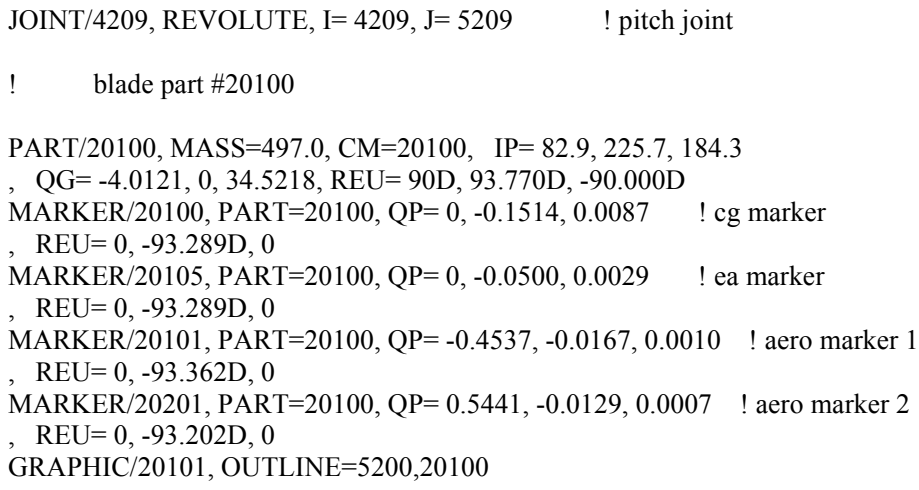

FIELD $/ 20100, \mathrm{I}=20105, \mathrm{~J}=5205, \mathrm{KMATRIX}=5.0387 \mathrm{e}+009$ $, 0.0000 \mathrm{e}+000,0.0000 \mathrm{e}+000,0.0000 \mathrm{e}+000,0.0000 \mathrm{e}+000,0.0000 \mathrm{e}+000$ $, 0.0000 \mathrm{e}+000,2.1538 \mathrm{e}+009,0.0000 \mathrm{e}+000,0.0000 \mathrm{e}+000,0.0000 \mathrm{e}+000,-1.0041 \mathrm{e}+009$ $, 0.0000 \mathrm{e}+000,0.0000 \mathrm{e}+000,4.2296 \mathrm{e}+009,0.0000 \mathrm{e}+000,2.0622 \mathrm{e}+009,0.0000 \mathrm{e}+000$ $, 0.0000 \mathrm{e}+000,0.0000 \mathrm{e}+000,0.0000 \mathrm{e}+000,4.2078 \mathrm{e}+007,0.0000 \mathrm{e}+000,0.0000 \mathrm{e}+000$ $, 0.0000 \mathrm{e}+000,0.0000 \mathrm{e}+000,2.0622 \mathrm{e}+009,0.0000 \mathrm{e}+000,1.3247 \mathrm{e}+009,0.0000 \mathrm{e}+000$ $, 0.0000 \mathrm{e}+000,-1.0041 \mathrm{e}+009,0.0000 \mathrm{e}+000,0.0000 \mathrm{e}+000,0.0000 \mathrm{e}+000,6.3070 \mathrm{e}+008$ ,CMATRIX $=5.0387 \mathrm{e}+000$

$, 0.0000 \mathrm{e}+000,0.0000 \mathrm{e}+000,0.0000 \mathrm{e}+000,0.0000 \mathrm{e}+000,0.0000 \mathrm{e}+000$

$, 0.0000 \mathrm{e}+000,2.1538 \mathrm{e}+000,0.0000 \mathrm{e}+000,0.0000 \mathrm{e}+000,0.0000 \mathrm{e}+000,-1.0041 \mathrm{e}+000$ $, 0.0000 \mathrm{e}+000,0.0000 \mathrm{e}+000,4.2296 \mathrm{e}+000,0.0000 \mathrm{e}+000,2.0622 \mathrm{e}+000,0.0000 \mathrm{e}+000$ $, 0.0000 \mathrm{e}+000,0.0000 \mathrm{e}+000,0.0000 \mathrm{e}+000,4.2078 \mathrm{e}+000,0.0000 \mathrm{e}+000,0.0000 \mathrm{e}+000$ $, 0.0000 \mathrm{e}+000,0.0000 \mathrm{e}+000,2.0622 \mathrm{e}+000,0.0000 \mathrm{e}+000,1.3247 \mathrm{e}+000,0.0000 \mathrm{e}+000$ $, 0.0000 \mathrm{e}+000,-1.0041 \mathrm{e}+000,0.0000 \mathrm{e}+000,0.0000 \mathrm{e}+000,0.0000 \mathrm{e}+000,6.3070 \mathrm{e}+000$ , $\mathrm{LENGTH}=0.9530,0.0001,-0.0581,0.151 \mathrm{D}, 0,0$

! $\quad$ blade part \#20200

$\mathrm{PART} / 20200, \mathrm{MASS}=393.8, \mathrm{CM}=20200, \quad \mathrm{IP}=81.7,198.2,143.6$

, $\mathrm{QG}=-4.1443,0,32.5156, \mathrm{REU}=90 \mathrm{D}, 93.770 \mathrm{D},-90.000 \mathrm{D}$

MARKER/20200, PART $=20200, Q P=0,-0.2776,0.0144 \quad$ ! cg marker

, REU $=0,-92.968 \mathrm{D}, 0$

MARKER/20205, PART $=20200, Q P=0,-0.1723,0.0089 \quad$ ! ea marker

, REU $=0,-92.968 \mathrm{D}, 0$

MARKER/20301, PART $=20200, \mathrm{QP}=-0.4687,-0.0104,0.0006 \quad$ ! aero marker 1

, REU $=0,-93.044 \mathrm{D}, 0$

MARKER/20401, $\mathrm{PART}=20200, \mathrm{QP}=0.5290,-0.0080,0.0004 \quad$ ! aero marker 2

, REU $=0,-92.881 \mathrm{D}, 0$

GRAPHIC/20201, OUTLINE=20100,20200

FIELD $/ 20200, \mathrm{I}=20205, \mathrm{~J}=20105, \mathrm{KMATRIX}=1.9831 \mathrm{e}+009$

$, 0.0000 \mathrm{e}+000,0.0000 \mathrm{e}+000,0.0000 \mathrm{e}+000,0.0000 \mathrm{e}+000,0.0000 \mathrm{e}+000$

$, 0.0000 \mathrm{e}+000,1.7168 \mathrm{e}+008,0.0000 \mathrm{e}+000,0.0000 \mathrm{e}+000,0.0000 \mathrm{e}+000,-1.5823 \mathrm{e}+008$ $, 0.0000 \mathrm{e}+000,0.0000 \mathrm{e}+000,4.8129 \mathrm{e}+008,0.0000 \mathrm{e}+000,4.8364 \mathrm{e}+008,0.0000 \mathrm{e}+000$ $, 0.0000 \mathrm{e}+000,0.0000 \mathrm{e}+000,0.0000 \mathrm{e}+000,1.5951 \mathrm{e}+007,0.0000 \mathrm{e}+000,0.0000 \mathrm{e}+000$ $, 0.0000 \mathrm{e}+000,0.0000 \mathrm{e}+000,4.8364 \mathrm{e}+008,0.0000 \mathrm{e}+000,6.4812 \mathrm{e}+008,0.0000 \mathrm{e}+000$ $, 0.0000 \mathrm{e}+000,-1.5823 \mathrm{e}+008,0.0000 \mathrm{e}+000,0.0000 \mathrm{e}+000,0.0000 \mathrm{e}+000,2.0247 \mathrm{e}+008$ , $\mathrm{CMATRIX}=1.9831 \mathrm{e}+004$

$, 0.0000 \mathrm{e}+000,0.0000 \mathrm{e}+000,0.0000 \mathrm{e}+000,0.0000 \mathrm{e}+000,0.0000 \mathrm{e}+000$

$, 0.0000 \mathrm{e}+000,1.7168 \mathrm{e}+000,0.0000 \mathrm{e}+000,0.0000 \mathrm{e}+000,0.0000 \mathrm{e}+000,-1.5823 \mathrm{e}+000$ $, 0.0000 \mathrm{e}+000,0.0000 \mathrm{e}+000,4.8129 \mathrm{e}+000,0.0000 \mathrm{e}+000,4.8364 \mathrm{e}+000,0.0000 \mathrm{e}+000$ $, 0.0000 \mathrm{e}+000,0.0000 \mathrm{e}+000,0.0000 \mathrm{e}+000,1.5951 \mathrm{e}+000,0.0000 \mathrm{e}+000,0.0000 \mathrm{e}+000$ $, 0.0000 \mathrm{e}+000,0.0000 \mathrm{e}+000,4.8364 \mathrm{e}+000,0.0000 \mathrm{e}+000,6.4812 \mathrm{e}+000,0.0000 \mathrm{e}+000$ $, 0.0000 \mathrm{e}+000,-1.5823 \mathrm{e}+000,0.0000 \mathrm{e}+000,0.0000 \mathrm{e}+000,0.0000 \mathrm{e}+000,2.0247 \mathrm{e}+000$ ， $\mathrm{LENGTH}=2.0100,0.0010,-0.1225,0.321 \mathrm{D}, 0,0$

! blade part \#20300

$\mathrm{PART} / 20300, \mathrm{MASS}=328.0, \mathrm{CM}=20300, \mathrm{IP}=73.1,172.9,117.1$

, $\mathrm{QG}=-4.2754,0,30.5248, \mathrm{REU}=90 \mathrm{D}, 93.770 \mathrm{D},-90.000 \mathrm{D}$

MARKER/20300, $\mathrm{PART}=20300, \mathrm{QP}=0,-0.3544,0.0163 \quad$ ! cg marker

, REU $=0,-92.632 \mathrm{D}, 0$

MARKER/20305, PART $=20300, \mathrm{QP}=0,-0.2527,0.0116 \quad$ ! ea marker

, REU $=0,-92.632 \mathrm{D}, 0$

MARKER/20501, PART $=20300, \mathrm{QP}=-0.4683,-0.0064,0.0003 \quad$ ! aero marker 1 
, REU $=0,-92.725 \mathrm{D}, 0$

MARKER/20601, PART $=20300, \mathrm{QP}=0.5294,-0.0042,0.0002 \quad$ ! aero marker 2

, REU $=0,-92.544 \mathrm{D}, 0$

GRAPHIC/20301, OUTLINE=20200,20300

FIELD/20300, $\mathrm{I}=20305, \mathrm{~J}=20205$, KMATRIX $=1.5487 \mathrm{e}+009$

$, 0.0000 \mathrm{e}+000,0.0000 \mathrm{e}+000,0.0000 \mathrm{e}+000,0.0000 \mathrm{e}+000,0.0000 \mathrm{e}+000$

$, 0.0000 \mathrm{e}+000,1.0866 \mathrm{e}+008,0.0000 \mathrm{e}+000,0.0000 \mathrm{e}+000,0.0000 \mathrm{e}+000,-1.0068 \mathrm{e}+008$ $, 0.0000 \mathrm{e}+000,0.0000 \mathrm{e}+000,4.9512 \mathrm{e}+008,0.0000 \mathrm{e}+000,4.9495 \mathrm{e}+008,0.0000 \mathrm{e}+000$ $, 0.0000 \mathrm{e}+000,0.0000 \mathrm{e}+000,0.0000 \mathrm{e}+000,1.0338 \mathrm{e}+007,0.0000 \mathrm{e}+000,0.0000 \mathrm{e}+000$ $, 0.0000 \mathrm{e}+000,0.0000 \mathrm{e}+000,4.9495 \mathrm{e}+008,0.0000 \mathrm{e}+000,6.5901 \mathrm{e}+008,0.0000 \mathrm{e}+000$ $, 0.0000 \mathrm{e}+000,-1.0068 \mathrm{e}+008,0.0000 \mathrm{e}+000,0.0000 \mathrm{e}+000,0.0000 \mathrm{e}+000,1.2878 \mathrm{e}+008$ ,CMATRIX $=1.5487 \mathrm{e}+004$

$, 0.0000 \mathrm{e}+000,0.0000 \mathrm{e}+000,0.0000 \mathrm{e}+000,0.0000 \mathrm{e}+000,0.0000 \mathrm{e}+000$

$, 0.0000 \mathrm{e}+000,1.0866 \mathrm{e}+000,0.0000 \mathrm{e}+000,0.0000 \mathrm{e}+000,0.0000 \mathrm{e}+000,-1.0068 \mathrm{e}+000$ $, 0.0000 \mathrm{e}+000,0.0000 \mathrm{e}+000,4.9512 \mathrm{e}+000,0.0000 \mathrm{e}+000,4.9495 \mathrm{e}+000,0.0000 \mathrm{e}+000$ $, 0.0000 \mathrm{e}+000,0.0000 \mathrm{e}+000,0.0000 \mathrm{e}+000,1.0338 \mathrm{e}+000,0.0000 \mathrm{e}+000,0.0000 \mathrm{e}+000$ $, 0.0000 \mathrm{e}+000,0.0000 \mathrm{e}+000,4.9495 \mathrm{e}+000,0.0000 \mathrm{e}+000,6.5901 \mathrm{e}+000,0.0000 \mathrm{e}+000$ $, 0.0000 \mathrm{e}+000,-1.0068 \mathrm{e}+000,0.0000 \mathrm{e}+000,0.0000 \mathrm{e}+000,0.0000 \mathrm{e}+000,1.2878 \mathrm{e}+000$ , LENGTH $=1.9950,0.0015,-0.0804,0.336 \mathrm{D}, 0,0$

! $\quad$ blade part $\# 20400$

$\mathrm{PART} / 20400, \mathrm{MASS}=269.6, \mathrm{CM}=20400, \quad \mathrm{IP}=55.1,138.6,94.6$

, $\mathrm{QG}=-4.4064,0,28.5380, \mathrm{REU}=90 \mathrm{D}, 93.770 \mathrm{D},-90.000 \mathrm{D}$

MARKER $/ 20400, \mathrm{PART}=20400, \mathrm{QP}=0,-0.3634,0.0143 \quad$ ! cg marker

, REU $=0,-92.251 \mathrm{D}, 0$

MARKER $/ 20405, \mathrm{PART}=20400, \mathrm{QP}=0,-0.2640,0.0104 \quad$ ! ea marker

, REU $=0,-92.251 \mathrm{D}, 0$

MARKER $/ 20701, \mathrm{PART}=20400, \mathrm{QP}=-0.4640,-0.0018,0.0001 \quad$ ! aero marker 1

, REU $=0,-92.355 \mathrm{D}, 0$

MARKER $/ 20801, \mathrm{PART}=20400, \mathrm{QP}=0.5338,0.0000,0.0000 \quad$ ! aero marker 2

, REU $=0,-92.156 \mathrm{D}, 0$

GRAPHIC/20401, OUTLINE=20300,20400

FIELD/20400, $\mathrm{I}=20405, \mathrm{~J}=20305$, KMATRIX $=1.3202 \mathrm{e}+009$

$, 0.0000 \mathrm{e}+000,0.0000 \mathrm{e}+000,0.0000 \mathrm{e}+000,0.0000 \mathrm{e}+000,0.0000 \mathrm{e}+000$

$, 0.0000 \mathrm{e}+000,7.0972 \mathrm{e}+007,0.0000 \mathrm{e}+000,0.0000 \mathrm{e}+000,0.0000 \mathrm{e}+000,-6.5678 \mathrm{e}+007$ $, 0.0000 \mathrm{e}+000,0.0000 \mathrm{e}+000,4.3826 \mathrm{e}+008,0.0000 \mathrm{e}+000,4.1541 \mathrm{e}+008,0.0000 \mathrm{e}+000$ $, 0.0000 \mathrm{e}+000,0.0000 \mathrm{e}+000,0.0000 \mathrm{e}+000,6.6475 \mathrm{e}+006,0.0000 \mathrm{e}+000,0.0000 \mathrm{e}+000$ $, 0.0000 \mathrm{e}+000,0.0000 \mathrm{e}+000,4.1541 \mathrm{e}+008,0.0000 \mathrm{e}+000,5.3755 \mathrm{e}+008,0.0000 \mathrm{e}+000$ $, 0.0000 \mathrm{e}+000,-6.5678 \mathrm{e}+007,0.0000 \mathrm{e}+000,0.0000 \mathrm{e}+000,0.0000 \mathrm{e}+000,8.3878 \mathrm{e}+007$ ,CMATRIX $=5.0387 \mathrm{e}+004$

$, 0.0000 \mathrm{e}+000,0.0000 \mathrm{e}+000,0.0000 \mathrm{e}+000,0.0000 \mathrm{e}+000,0.0000 \mathrm{e}+000$ $, 0.0000 \mathrm{e}+000,2.1538 \mathrm{e}+000,0.0000 \mathrm{e}+000,0.0000 \mathrm{e}+000,0.0000 \mathrm{e}+000,-1.0041 \mathrm{e}+000$ $, 0.0000 \mathrm{e}+000,0.0000 \mathrm{e}+000,4.2296 \mathrm{e}+000,0.0000 \mathrm{e}+000,2.0622 \mathrm{e}+000,0.0000 \mathrm{e}+000$ $, 0.0000 \mathrm{e}+000,0.0000 \mathrm{e}+000,0.0000 \mathrm{e}+000,4.2078 \mathrm{e}+000,0.0000 \mathrm{e}+000,0.0000 \mathrm{e}+000$ $, 0.0000 \mathrm{e}+000,0.0000 \mathrm{e}+000,2.0622 \mathrm{e}+000,0.0000 \mathrm{e}+000,1.3247 \mathrm{e}+000,0.0000 \mathrm{e}+000$ $, 0.0000 \mathrm{e}+000,-1.0041 \mathrm{e}+000,0.0000 \mathrm{e}+000,0.0000 \mathrm{e}+000,0.0000 \mathrm{e}+000,6.3070 \mathrm{e}+000$

, LENGTH $=1.9910,0.0018,-0.0112,0.380 \mathrm{D}, 0,0$

! blade part $\# 20500$

$\mathrm{PART} / 20500, \mathrm{MASS}=214.7, \mathrm{CM}=20500, \mathrm{IP}=37.7,105.1,74.3$

, $\mathrm{QG}=-4.5371,0,26.5532, \mathrm{REU}=90 \mathrm{D}, 93.770 \mathrm{D},-90.000 \mathrm{D}$

MARKER $/ 20500, \mathrm{PART}=20500, \mathrm{QP}=0,-0.3452,0.0109 \quad$ ! cg marker

, REU $=0,-91.807 \mathrm{D}, 0$

MARKER $/ 20505, \mathrm{PART}=20500, \mathrm{QP}=0,-0.2442,0.0077 \quad$ ! ea marker

, REU $=0,-91.807 \mathrm{D}, 0$

MARKER $/ 20901, \mathrm{PART}=20500, \mathrm{QP}=-0.4576,0.0000,0.0000 \quad$ ! aero marker 1

, REU $=0,-91.920 \mathrm{D}, 0$

MARKER $/ 21001, \mathrm{PART}=20500, \mathrm{QP}=0.5401,0.0000,0.0000 \quad$ ! aero marker 2

, REU $=0,-91.685 \mathrm{D}, 0$

GRAPHIC/20501, OUTLINE=20400,20500

FIELD $/ 20500, \mathrm{I}=20505, \mathrm{~J}=20405, \mathrm{KMATRIX}=1.0753 \mathrm{e}+009$

$, 0.0000 \mathrm{e}+000,0.0000 \mathrm{e}+000,0.0000 \mathrm{e}+000,0.0000 \mathrm{e}+000,0.0000 \mathrm{e}+000$

$, 0.0000 \mathrm{e}+000,4.5365 \mathrm{e}+007,0.0000 \mathrm{e}+000,0.0000 \mathrm{e}+000,0.0000 \mathrm{e}+000,-4.1547 \mathrm{e}+007$

$, 0.0000 \mathrm{e}+000,0.0000 \mathrm{e}+000,3.0934 \mathrm{e}+008,0.0000 \mathrm{e}+000,2.8543 \mathrm{e}+008,0.0000 \mathrm{e}+000$ 
$, 0.0000 \mathrm{e}+000,0.0000 \mathrm{e}+000,0.0000 \mathrm{e}+000,4.2996 \mathrm{e}+006,0.0000 \mathrm{e}+000,0.0000 \mathrm{e}+000$ $, 0.0000 \mathrm{e}+000,0.0000 \mathrm{e}+000,2.8543 \mathrm{e}+008,0.0000 \mathrm{e}+000,3.6376 \mathrm{e}+008,0.0000 \mathrm{e}+000$ $, 0.0000 \mathrm{e}+000,-4.1547 \mathrm{e}+007,0.0000 \mathrm{e}+000,0.0000 \mathrm{e}+000,0.0000 \mathrm{e}+000,5.2727 \mathrm{e}+007$ ,CMATRIX $=1.0753 \mathrm{e}+004$

$, 0.0000 \mathrm{e}+000,0.0000 \mathrm{e}+000,0.0000 \mathrm{e}+000,0.0000 \mathrm{e}+000,0.0000 \mathrm{e}+000$

$, 0.0000 \mathrm{e}+000,4.5365 \mathrm{e}+000,0.0000 \mathrm{e}+000,0.0000 \mathrm{e}+000,0.0000 \mathrm{e}+000,-4.1547 \mathrm{e}+000$ $, 0.0000 \mathrm{e}+000,0.0000 \mathrm{e}+000,3.0934 \mathrm{e}+000,0.0000 \mathrm{e}+000,2.8543 \mathrm{e}+000,0.0000 \mathrm{e}+000$ $, 0.0000 \mathrm{e}+000,0.0000 \mathrm{e}+000,0.0000 \mathrm{e}+000,4.2996 \mathrm{e}+000,0.0000 \mathrm{e}+000,0.0000 \mathrm{e}+000$ $, 0.0000 \mathrm{e}+000,0.0000 \mathrm{e}+000,2.8543 \mathrm{e}+000,0.0000 \mathrm{e}+000,3.6376 \mathrm{e}+000,0.0000 \mathrm{e}+000$ $, 0.0000 \mathrm{e}+000,-4.1547 \mathrm{e}+000,0.0000 \mathrm{e}+000,0.0000 \mathrm{e}+000,0.0000 \mathrm{e}+000,5.2727 \mathrm{e}+000$

, LENGTH $=1.9890,0.0019,0.0199,0.444 \mathrm{D}, 0,0$

! blade part \#20600

$\mathrm{PART} / 20600, \mathrm{MASS}=162.8, \mathrm{CM}=20600, \quad \mathrm{IP}=23.8,75.4,55.5$

, $\mathrm{QG}=-4.6677,0,24.5724, \mathrm{REU}=90 \mathrm{D}, 93.770 \mathrm{D},-90.000 \mathrm{D}$

MARKER $/ 20600, \mathrm{PART}=20600, \mathrm{QP}=0,-0.3163,0.0071 \quad$ ! cg marker

, REU $=0,-91.278 \mathrm{D}, 0$

MARKER $/ 20605, \mathrm{PART}=20600, \mathrm{QP}=0,-0.2206,0.0049 \quad$ ! ea marker

, REU $=0,-91.278 \mathrm{D}, 0$

MARKER/21101, PART $=20600, \mathrm{QP}=-0.4472,0.0000,0.0000 \quad$ ! aero marker 1

, REU $=0,-91.411 \mathrm{D}, 0$

MARKER $/ 21201, \mathrm{PART}=20600, \mathrm{QP}=0.5505,0.0000,0.0000 \quad$ ! aero marker 2

, REU $=0,-91.118 \mathrm{D}, 0$

GRAPHIC/20601, OUTLINE=20500,20600

FIELD $/ 20600, \mathrm{I}=20605, \mathrm{~J}=20505$, KMATRIX $=8.4118 \mathrm{e}+008$

$, 0.0000 \mathrm{e}+000,0.0000 \mathrm{e}+000,0.0000 \mathrm{e}+000,0.0000 \mathrm{e}+000,0.0000 \mathrm{e}+000$

$, 0.0000 \mathrm{e}+000,2.7316 \mathrm{e}+007,0.0000 \mathrm{e}+000,0.0000 \mathrm{e}+000,0.0000 \mathrm{e}+000,-2.4634 \mathrm{e}+007$ $, 0.0000 \mathrm{e}+000,0.0000 \mathrm{e}+000,1.9775 \mathrm{e}+008,0.0000 \mathrm{e}+000,1.8104 \mathrm{e}+008,0.0000 \mathrm{e}+000$ $, 0.0000 \mathrm{e}+000,0.0000 \mathrm{e}+000,0.0000 \mathrm{e}+000,2.6424 \mathrm{e}+006,0.0000 \mathrm{e}+000,0.0000 \mathrm{e}+000$ $, 0.0000 \mathrm{e}+000,0.0000 \mathrm{e}+000,1.8104 \mathrm{e}+008,0.0000 \mathrm{e}+000,2.2951 \mathrm{e}+008,0.0000 \mathrm{e}+000$ $, 0.0000 \mathrm{e}+000,-2.4634 \mathrm{e}+007,0.0000 \mathrm{e}+000,0.0000 \mathrm{e}+000,0.0000 \mathrm{e}+000,3.0960 \mathrm{e}+007$ ,CMATRIX $=8.4118 \mathrm{e}+003$

$, 0.0000 \mathrm{e}+000,0.0000 \mathrm{e}+000,0.0000 \mathrm{e}+000,0.0000 \mathrm{e}+000,0.0000 \mathrm{e}+000$

$, 0.0000 \mathrm{e}+000,2.7316 \mathrm{e}+000,0.0000 \mathrm{e}+000,0.0000 \mathrm{e}+000,0.0000 \mathrm{e}+000,-2.4634 \mathrm{e}+000$ $, 0.0000 \mathrm{e}+000,0.0000 \mathrm{e}+000,1.9775 \mathrm{e}+000,0.0000 \mathrm{e}+000,1.8104 \mathrm{e}+000,0.0000 \mathrm{e}+000$ $, 0.0000 \mathrm{e}+000,0.0000 \mathrm{e}+000,0.0000 \mathrm{e}+000,2.6424 \mathrm{e}+000,0.0000 \mathrm{e}+000,0.0000 \mathrm{e}+000$ $, 0.0000 \mathrm{e}+000,0.0000 \mathrm{e}+000,1.8104 \mathrm{e}+000,0.0000 \mathrm{e}+000,2.2951 \mathrm{e}+000,0.0000 \mathrm{e}+000$ $, 0.0000 \mathrm{e}+000,-2.4634 \mathrm{e}+000,0.0000 \mathrm{e}+000,0.0000 \mathrm{e}+000,0.0000 \mathrm{e}+000,3.0960 \mathrm{e}+000$

, LENGTH $=1.9850,0.0020,0.0237,0.530 \mathrm{D}, 0,0$

! $\quad$ blade part \#20700

$\mathrm{PART} / 20700, \mathrm{MASS}=114.8, \mathrm{CM}=20700, \quad \mathrm{IP}=13.7,50.2,38.6$

, $\mathrm{QG}=-4.7980,0,22.5951, \mathrm{REU}=90 \mathrm{D}, 93.770 \mathrm{D},-90.000 \mathrm{D}$

MARKER $/ 20700, \mathrm{PART}=20700, \mathrm{QP}=0,-0.2815,0.0031 \quad$ ! cg marker

, REU $=0,-90.628 \mathrm{D}, 0$

MARKER $/ 20705, \mathrm{PART}=20700, \mathrm{QP}=0,-0.1973,0.0022 \quad$ ! ea marker

, REU $=0,-90.628 \mathrm{D}, 0$

MARKER $/ 21301, \mathrm{PART}=20700, \mathrm{QP}=-0.4334,0.0000,0.0000 \quad$ ! aero marker 1

, REU $=0,-90.808 \mathrm{D}, 0$

MARKER $/ 21401, \mathrm{PART}=20700, \mathrm{QP}=0.5644,0.0000,0.0000 \quad$ ! aero marker 2

, REU $=0,-90.431 \mathrm{D}, 0$

GRAPHIC/20701, OUTLINE=20600,20700

FIELD $/ 20700, \mathrm{I}=20705, \mathrm{~J}=20605$, KMATRIX $=6.4385 \mathrm{e}+008$

$, 0.0000 \mathrm{e}+000,0.0000 \mathrm{e}+000,0.0000 \mathrm{e}+000,0.0000 \mathrm{e}+000,0.0000 \mathrm{e}+000$

$, 0.0000 \mathrm{e}+000,1.5223 \mathrm{e}+007,0.0000 \mathrm{e}+000,0.0000 \mathrm{e}+000,0.0000 \mathrm{e}+000,-1.3529 \mathrm{e}+007$ $, 0.0000 \mathrm{e}+000,0.0000 \mathrm{e}+000,1.2322 \mathrm{e}+008,0.0000 \mathrm{e}+000,1.1242 \mathrm{e}+008,0.0000 \mathrm{e}+000$ $, 0.0000 \mathrm{e}+000,0.0000 \mathrm{e}+000,0.0000 \mathrm{e}+000,1.5168 \mathrm{e}+006,0.0000 \mathrm{e}+000,0.0000 \mathrm{e}+000$ $, 0.0000 \mathrm{e}+000,0.0000 \mathrm{e}+000,1.1242 \mathrm{e}+008,0.0000 \mathrm{e}+000,1.4212 \mathrm{e}+008,0.0000 \mathrm{e}+000$ $, 0.0000 \mathrm{e}+000,-1.3529 \mathrm{e}+007,0.0000 \mathrm{e}+000,0.0000 \mathrm{e}+000,0.0000 \mathrm{e}+000,1.6847 \mathrm{e}+007$ ,CMATRIX $=6.4385 \mathrm{e}+003$

$, 0.0000 \mathrm{e}+000,0.0000 \mathrm{e}+000,0.0000 \mathrm{e}+000,0.0000 \mathrm{e}+000,0.0000 \mathrm{e}+000$ $, 0.0000 \mathrm{e}+000,1.5223 \mathrm{e}+000,0.0000 \mathrm{e}+000,0.0000 \mathrm{e}+000,0.0000 \mathrm{e}+000,-1.3529 \mathrm{e}+000$ $, 0.0000 \mathrm{e}+000,0.0000 \mathrm{e}+000,1.2322 \mathrm{e}+000,0.0000 \mathrm{e}+000,1.1242 \mathrm{e}+000,0.0000 \mathrm{e}+000$ $, 0.0000 \mathrm{e}+000,0.0000 \mathrm{e}+000,0.0000 \mathrm{e}+000,1.5168 \mathrm{e}+000,0.0000 \mathrm{e}+000,0.0000 \mathrm{e}+000$ $, 0.0000 \mathrm{e}+000,0.0000 \mathrm{e}+000,1.1242 \mathrm{e}+000,0.0000 \mathrm{e}+000,1.4212 \mathrm{e}+000,0.0000 \mathrm{e}+000$ 
$, 0.0000 \mathrm{e}+000,-1.3529 \mathrm{e}+000,0.0000 \mathrm{e}+000,0.0000 \mathrm{e}+000,0.0000 \mathrm{e}+000,1.6847 \mathrm{e}+000$

, $\mathrm{LENGTH}=1.9820,0.0022,0.0234,0.650 \mathrm{D}, 0,0$

! $\quad$ blade part $\# 20800$

PART/20800, $\mathrm{MASS}=72.9, \mathrm{CM}=20800, \mathrm{IP}=7.1,30.2,24.1$

, $\mathrm{QG}=-4.9277,0,20.6268, \mathrm{REU}=90 \mathrm{D}, 93.770 \mathrm{D},-90.000 \mathrm{D}$

MARKER/20800, PART $=20800, \mathrm{QP}=0,-0.2492,-0.0008 \quad$ ! cg marker

, REU $=0,-89.822 \mathrm{D}, 0$

MARKER/20805, $\mathrm{PART}=20800, \mathrm{QP}=0,-0.1740,-0.0005 \quad$ ! ea marker

, REU $=0,-89.822 \mathrm{D}, 0$

MARKER/21501, PART $=20800, \mathrm{QP}=-0.4104,0.0000,0.0000 \quad$ ! aero marker 1

, REU $=0,-90.047 \mathrm{D}, 0$

MARKER/21601, $\mathrm{PART}=20800, \mathrm{QP}=0.5873,0.0000,0.0000 \quad$ ! aero marker 2

, REU $=0,-89.583 \mathrm{D}, 0$

GRAPHIC/20801, OUTLINE=20700,20800

FIELD $/ 20800, \mathrm{I}=20805, \mathrm{~J}=20705, \mathrm{KMATRIX}=4.7422 \mathrm{e}+008$

$, 0.0000 \mathrm{e}+000,0.0000 \mathrm{e}+000,0.0000 \mathrm{e}+000,0.0000 \mathrm{e}+000,0.0000 \mathrm{e}+000$

$, 0.0000 \mathrm{e}+000,7.6902 \mathrm{e}+006,0.0000 \mathrm{e}+000,0.0000 \mathrm{e}+000,0.0000 \mathrm{e}+000,-6.6072 \mathrm{e}+006$ $, 0.0000 \mathrm{e}+000,0.0000 \mathrm{e}+000,7.3081 \mathrm{e}+007,0.0000 \mathrm{e}+000,6.4793 \mathrm{e}+007,0.0000 \mathrm{e}+000$ $, 0.0000 \mathrm{e}+000,0.0000 \mathrm{e}+000,0.0000 \mathrm{e}+000,7.9462 \mathrm{e}+005,0.0000 \mathrm{e}+000,0.0000 \mathrm{e}+000$ $, 0.0000 \mathrm{e}+000,0.0000 \mathrm{e}+000,6.4793 \mathrm{e}+007,0.0000 \mathrm{e}+000,8.0415 \mathrm{e}+007,0.0000 \mathrm{e}+000$ $, 0.0000 \mathrm{e}+000,-6.6072 \mathrm{e}+006,0.0000 \mathrm{e}+000,0.0000 \mathrm{e}+000,0.0000 \mathrm{e}+000,8.0459 \mathrm{e}+006$ , CMATRIX $=4.7422 \mathrm{e}+003$

$, 0.0000 \mathrm{e}+000,0.0000 \mathrm{e}+000,0.0000 \mathrm{e}+000,0.0000 \mathrm{e}+000,0.0000 \mathrm{e}+000$ $, 0.0000 \mathrm{e}+000,7.6902 \mathrm{e}+000,0.0000 \mathrm{e}+000,0.0000 \mathrm{e}+000,0.0000 \mathrm{e}+000,-6.6072 \mathrm{e}+000$ $, 0.0000 \mathrm{e}+000,0.0000 \mathrm{e}+000,7.3081 \mathrm{e}+000,0.0000 \mathrm{e}+000,6.4793 \mathrm{e}+000,0.0000 \mathrm{e}+000$ $, 0.0000 \mathrm{e}+000,0.0000 \mathrm{e}+000,0.0000 \mathrm{e}+000,7.9462 \mathrm{e}+000,0.0000 \mathrm{e}+000,0.0000 \mathrm{e}+000$ $, 0.0000 \mathrm{e}+000,0.0000 \mathrm{e}+000,6.4793 \mathrm{e}+000,0.0000 \mathrm{e}+000,8.0415 \mathrm{e}+000,0.0000 \mathrm{e}+000$ $, 0.0000 \mathrm{e}+000,-6.6072 \mathrm{e}+000,0.0000 \mathrm{e}+000,0.0000 \mathrm{e}+000,0.0000 \mathrm{e}+000,8.0459 \mathrm{e}+000$

, $\mathrm{LENGTH}=1.9730,0.0024,0.0233,0.805 \mathrm{D}, 0,0$

! $\quad$ blade part $\# 20900$

$\mathrm{PART} / 20900, \mathrm{MASS}=40.5, \mathrm{CM}=20900, \mathrm{IP}=3.5,16.3,13.1$

, $\mathrm{QG}=-5.0576,0,18.6542, \mathrm{REU}=90 \mathrm{D}, 93.770 \mathrm{D},-90.000 \mathrm{D}$

MARKER/20900, PART $=20900, \mathrm{QP}=0,-0.2194,-0.0046 \quad$ ! cg marker

, REU $=0,-88.808 \mathrm{D}, 0$

MARKER/20905, PART=20900, $\mathrm{QP}=0,-0.1506,-0.0031 \quad$ ! ea marker

REU $=0,-88.808 \mathrm{D}, 0$

MARKER/21701, $\mathrm{PART}=20900, \mathrm{QP}=-0.3918,0.0000,0.0000 \quad$ ! aero marker 1

, REU $=0,-89.065 \mathrm{D}, 0$

MARKER/21801, PART $=20900, \mathrm{QP}=0.6060,0.0000,0.0000 \quad$ ! aero marker 2

, REU $=0,-88.536 \mathrm{D}, 0$

GRAPHIC/20901, OUTLINE=20800,20900

FIELD $/ 20900, \mathrm{I}=20905, \mathrm{~J}=20805, \mathrm{KMATRIX}=3.2430 \mathrm{e}+008$

$, 0.0000 \mathrm{e}+000,0.0000 \mathrm{e}+000,0.0000 \mathrm{e}+000,0.0000 \mathrm{e}+000,0.0000 \mathrm{e}+000$

$, 0.0000 \mathrm{e}+000,3.2750 \mathrm{e}+006,0.0000 \mathrm{e}+000,0.0000 \mathrm{e}+000,0.0000 \mathrm{e}+000,-2.7721 \mathrm{e}+006$ $, 0.0000 \mathrm{e}+000,0.0000 \mathrm{e}+000,3.7449 \mathrm{e}+007,0.0000 \mathrm{e}+000,3.2685 \mathrm{e}+007,0.0000 \mathrm{e}+000$ $, 0.0000 \mathrm{e}+000,0.0000 \mathrm{e}+000,0.0000 \mathrm{e}+000,3.6362 \mathrm{e}+005,0.0000 \mathrm{e}+000,0.0000 \mathrm{e}+000$ $, 0.0000 \mathrm{e}+000,0.0000 \mathrm{e}+000,3.2685 \mathrm{e}+007,0.0000 \mathrm{e}+000,4.0221 \mathrm{e}+007,0.0000 \mathrm{e}+000$ $, 0.0000 \mathrm{e}+000,-2.7721 \mathrm{e}+006,0.0000 \mathrm{e}+000,0.0000 \mathrm{e}+000,0.0000 \mathrm{e}+000,3.3469 \mathrm{e}+006$ CMATRIX $=3.2430 \mathrm{e}+003$

$, 0.0000 \mathrm{e}+000,0.0000 \mathrm{e}+000,0.0000 \mathrm{e}+000,0.0000 \mathrm{e}+000,0.0000 \mathrm{e}+000$

$, 0.0000 \mathrm{e}+000,3.2750 \mathrm{e}+000,0.0000 \mathrm{e}+000,0.0000 \mathrm{e}+000,0.0000 \mathrm{e}+000,-2.7721 \mathrm{e}+000$ $, 0.0000 \mathrm{e}+000,0.0000 \mathrm{e}+000,3.7449 \mathrm{e}+000,0.0000 \mathrm{e}+000,3.2685 \mathrm{e}+000,0.0000 \mathrm{e}+000$ $, 0.0000 \mathrm{e}+000,0.0000 \mathrm{e}+000,0.0000 \mathrm{e}+000,3.6362 \mathrm{e}+000,0.0000 \mathrm{e}+000,0.0000 \mathrm{e}+000$ $, 0.0000 \mathrm{e}+000,0.0000 \mathrm{e}+000,3.2685 \mathrm{e}+000,0.0000 \mathrm{e}+000,4.0221 \mathrm{e}+000,0.0000 \mathrm{e}+000$ $, 0.0000 \mathrm{e}+000,-2.7721 \mathrm{e}+000,0.0000 \mathrm{e}+000,0.0000 \mathrm{e}+000,0.0000 \mathrm{e}+000,3.3469 \mathrm{e}+000$ , $\mathrm{LENGTH}=1.9760,0.0027,0.0234,1.015 \mathrm{D}, 0,0$

! $\quad$ blade part $\# 21000$

$\mathrm{PART} / 21000, \mathrm{MASS}=19.7, \mathrm{CM}=21000, \mathrm{IP}=1.9,8.1,6.3$

, $\mathrm{QG}=-5.1873,0,16.6859, \mathrm{REU}=90 \mathrm{D}, 93.770 \mathrm{D},-90.000 \mathrm{D}$

MARKER/21000, $\mathrm{PART}=21000, \mathrm{QP}=0,-0.1892,-0.0082 \quad$ ! cg marker

, REU $=0,-87.525 \mathrm{D}, 0$ 


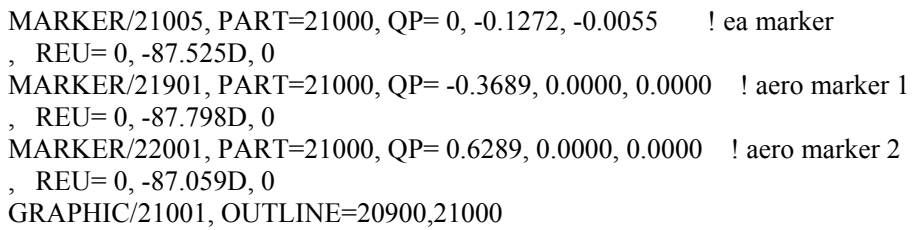


, FUNCTION $=$ USER $(1,8,0,10801)$

GFORCE $/ 10901, \mathrm{I}=10901, \mathrm{JFLOAT}=109, \mathrm{RM}=10901$

, FUNCTION = USER $(1,9,0,10901)$

GFORCE $/ 11001, \mathrm{I}=11001, \mathrm{JFLOAT}=110, \mathrm{RM}=11001$

, FUNCTION $=$ USER $(1,10,0,11001)$

GFORCE/11101, $\mathrm{I}=11101, \mathrm{JFLOAT}=111, \mathrm{RM}=11101$

, FUNCTION = USER $(1,11,0,11101)$

GFORCE/11201, $\mathrm{I}=11201, \mathrm{JFLOAT}=112, \mathrm{RM}=11201$

, FUNCTION $=$ USER $(1,12,0,11201)$

GFORCE/11301, $\mathrm{I}=11301, \mathrm{JFLOAT}=113, \mathrm{RM}=11301$

, FUNCTION $=$ USER $(1,13,0,11301)$

GFORCE/11401, $\mathrm{I}=11401, \mathrm{JFLOAT}=114, \mathrm{RM}=11401$

, FUNCTION $=$ USER $(1,14,0,11401)$

GFORCE $/ 11501, \mathrm{I}=11501, \mathrm{JFLOAT}=115, \mathrm{RM}=11501$

, FUNCTION $=\operatorname{USER}(1,15,0,11501)$

GFORCE $/ 11601, \mathrm{I}=11601, \mathrm{JFLOAT}=116, \mathrm{RM}=11601$

, FUNCTION $=$ USER $(1,16,0,11601)$

GFORCE $/ 11701, \mathrm{I}=11701, \mathrm{JFLOAT}=117, \mathrm{RM}=11701$

, FUNCTION $=$ USER $(1,17,0,11701)$

GFORCE $/ 11801, \mathrm{I}=11801, \mathrm{JFLOAT}=118, \mathrm{RM}=11801$

, FUNCTION $=$ USER $(1,18,0,11801)$

GFORCE/11901, I=11901, JFLOAT = 119, RM=11901

, FUNCTION = USER $(1,19,0,11901)$

GFORCE/12001, I=12001, JFLOAT $=120, \mathrm{RM}=12001$

, FUNCTION $=$ USER $(1,20,0,12001)$

! blade \#2 aero markers on ground

MARKER/ 201, PART $=1$, FLOATING

MARKER/ 202, PART $=1$, FLOATING

MARKER/ 203, PART $=1$, FLOATING

MARKER/ 204, PART $=1$, FLOATING

MARKER/ 205, PART $=1$, FLOATING

MARKER/ 206, PART $=1$, FLOATING

MARKER/ 207, PART $=1$, FLOATING

MARKER/ 208, PART $=1$, FLOATING

MARKER/ 209, PART $=1$, FLOATING

MARKER/ 210, PART $=1$, FLOATING

MARKER/ 211, PART $=1$, FLOATING

MARKER/ 212, PART $=1$, FLOATING

MARKER/ 213, PART $=1$, FLOATING

MARKER/ 214, PART $=1$, FLOATING

MARKER/ 215, PART $=1$, FLOATING

MARKER/ 216, PART $=1$, FLOATING

MARKER/ 217, PART $=1$, FLOATING

MARKER/ 218, PART $=1$, FLOATING

MARKER/ 219, PART $=1$, FLOATING

MARKER/ 220, PART $=1$, FLOATING

! blade \#2 calls to VFORCESUB

GFORCE $/ 20101, \mathrm{I}=20101, \mathrm{JFLOAT}=201, \mathrm{RM}=20101$

, FUNCTION $=$ USER(2, 1, 0,20101)

GFORCE $/ 20201, \mathrm{I}=20201, \mathrm{JFLOAT}=202, \mathrm{RM}=20201$

, FUNCTION $=$ USER(2, 2, 0,20201)

GFORCE/20301, $\mathrm{I}=20301, \mathrm{JFLOAT}=203, \mathrm{RM}=20301$

, FUNCTION $=$ USER(2, 3, 0,20301)

GFORCE/20401, I=20401, JFLOAT $=204, \mathrm{RM}=20401$

, FUNCTION $=$ USER $(2,4,0,20401)$

GFORCE/20501, I=20501, JFLOAT $=205, \mathrm{RM}=20501$

, FUNCTION $=$ USER(2, 5, 0,20501)

GFORCE/20601, I=20601, JFLOAT $=206, \mathrm{RM}=20601$ , FUNCTION $=$ USER $(2,6,0,20601)$

GFORCE/20701, I=20701, JFLOAT $=207, \mathrm{RM}=20701$ , FUNCTION $=$ USER $(2,7,0,20701)$

GFORCE/20801, I=20801, JFLOAT $=208, \mathrm{RM}=20801$ , FUNCTION $=$ USER(2, 8, 0,20801)

GFORCE/20901, I=20901, JFLOAT $=209, \mathrm{RM}=20901$ , FUNCTION $=\operatorname{USER}(2,9,0,20901)$

GFORCE $/ 21001, \mathrm{I}=21001, \mathrm{JFLOAT}=210, \mathrm{RM}=21001$ 


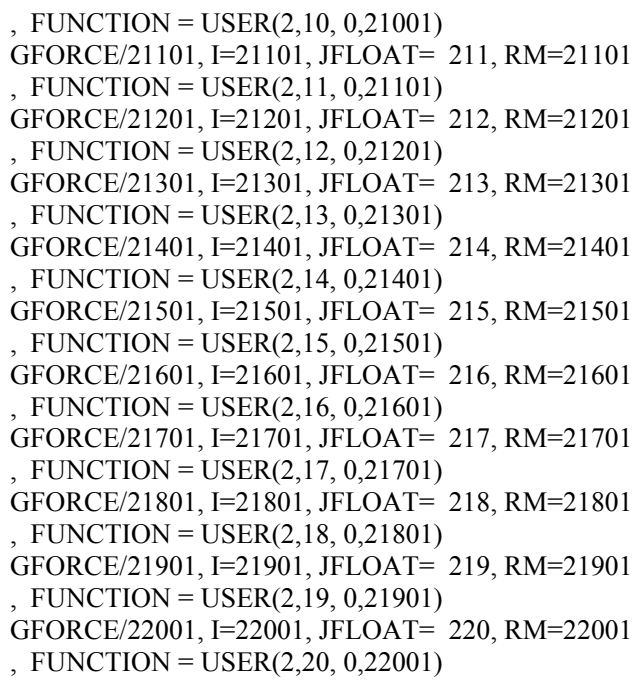




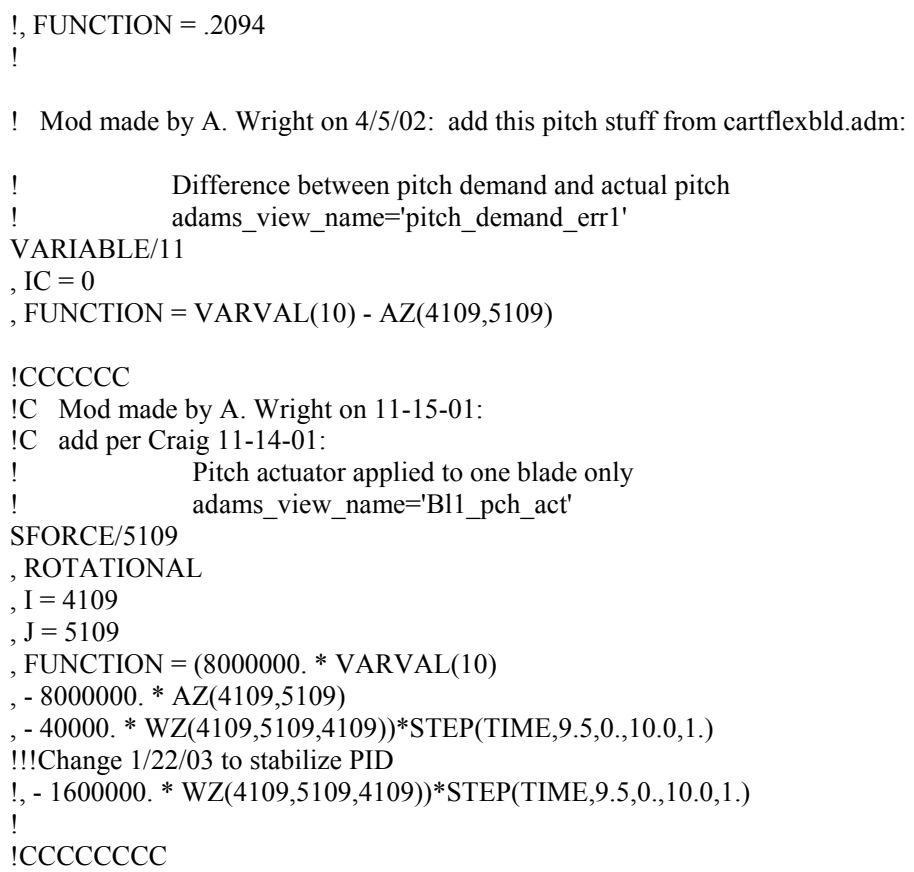

! Mod made by A. Wright on 4/5/02: add this output request info: !================================= OUTPUT

! Format for request is USER( T Start, AeroFlag, NumBlades, BladesFlag, HingeLoc ).

!! adams_view_name $=$ 'UserRequest'

REQUEST $/ 1$, FUNCTION $=\operatorname{USER}(1,0,0,1,0)$

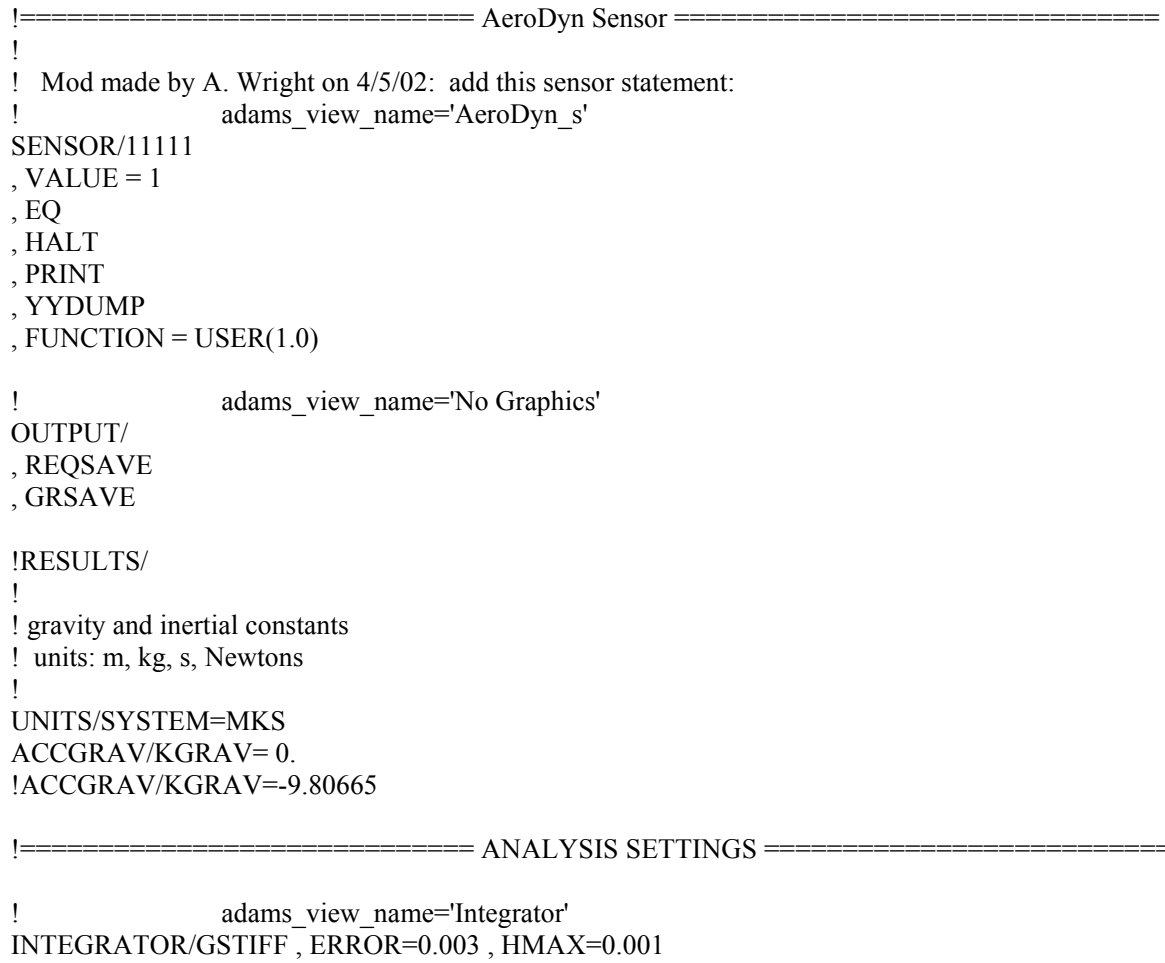

END 


\section{Appendix C. Control Modeling}

\section{C-1. Control Theory}

\section{C-1.1 Linear State-Space Descriptions}

Generally, a linear model of a wind turbine system will contain states to describe the most important turbine dynamics. It will also contain a control input such as rotor or blade pitch (either rotor collective pitch [the pitch is the same for each blade] or individual blade pitch) or generator torque (generator torque is held constant in region 3 but may be used as a control input to provide extra damping for the drive-train). It may also contain a disturbance input, which is the manner in which wind disturbances are accounted for in the model. The linear model will also contain an equation to describe the measured values, as a function of the turbine states.

$$
\begin{aligned}
& \underline{\dot{x}}=A \underline{x}+B \underline{u}+\Gamma \underline{u_{d}} \\
& \underline{y}=C \underline{x}+D \underline{u} .
\end{aligned}
$$

where $\underline{x} \in R^{N}$ is the state vector, $\underline{u} \in R^{M}$ is the control input vector, and $\underline{u}_{d} \in R^{O}$ is the disturbance input vector, $\underline{y} \in R^{P}$ is the control (or measured) output. $A \in R^{N x N}$ represents the state matrix, $B \in R^{N x M}$ the control gain matrix, and $\Gamma \in R^{N x O}$ the disturbance gain matrix. $C \in R^{P x N}$ relates the measured output $\underline{y}$ to the turbine states. $D \in R^{P x M}$ relates the output to the control input. We will usually be dealing with systems, in which $D$ is zero for the cases studied in this thesis.

We now describe the control theory used in this thesis.

\section{C-1.2. General State-Space Equation Solutions}

We are interested in solutions to the linear time-invariant first order state-space equation without disturbance input:

$$
\begin{aligned}
& \underline{\dot{x}}=A \underline{x}+B \underline{u} \\
& \underline{x}(0)=\underline{x}_{0}
\end{aligned}
$$

\section{C-1.2.1. Homogeneous equations-zero input case:}

We find solutions to the homogeneous case first, by setting $\underline{u}=\underline{0}$ :

$$
\begin{aligned}
& \underline{\dot{x}}=A \underline{x} \\
& \underline{x}(0)=\underline{x}_{0} .
\end{aligned}
$$

It can be shown that solutions to this equation are: 


$$
\underline{x}(t)=e^{A t} \underline{x}_{0}
$$

Where $e^{A t}$ is the matrix exponential, which can be calculated from knowledge of $A$.

\section{C-1.2.2 Stability}

Our interest is in the stability of the state-space system. How do solutions to (C1.3) behave as $t \rightarrow \infty$ ? It can be shown that

$$
\begin{aligned}
& \|\underline{x}(t)\|=\left\|e^{A t} \underline{x}_{0}\right\| \leq\left\|e^{A t}\right\|\left\|\underline{x}_{0}\right\|, \\
& \text { where }\|\| \text { is the 2-norm. }
\end{aligned}
$$

This means that $\|\underline{x}(t)\|$ is bounded by $\left\|e^{A t}\right\|$, since $\left\|\underline{x}_{0}\right\|$ is fixed for all t. Thus if we can determine the growth or decay properties of $e^{A t}$ then we can determine the behavior of $\underline{x}(t)$.

We state the following definition:

Definition C1.1: A stable means, $\operatorname{Real}\left(\lambda_{k}\right)<0$, where $\lambda_{k}: k=1, \ldots, N$ are the eigenvalues of $\mathrm{A}$.

An important theorem for determining stability of $\mathrm{C} 1.2$ is:

\section{Theorem C1.1:}

A is stable (this applies for the case where A has distinct eigenvalues) if and only if there exists $K \geq 1$ and $\sigma>0$ such that.

$$
\left\|e^{A t}\right\| \leq K e^{-\sigma t}
$$

for all $t>0$.

For a proof of this theorem, see [Kwakernaak and Sivan, 1972].

This tells us that if $\mathrm{A}$ is stable, then so are solutions to $\mathrm{C} 1.2$, since

$$
\|\underline{x}(t)\| \leq\left\|e^{A t} \underline{x}_{0}\right\| \leq\left\|e^{A t}\right\|\left\|\underline{x}_{0}\right\| \leq K e^{-\sigma t}\left\|\underline{x}_{0}\right\|
$$

thus

$$
\|\underline{x}(t)\| \leq K e^{-\sigma t}\left\|\underline{x}_{0}\right\|
$$

Thus if $e^{-\sigma t} \rightarrow 0$ as $t \rightarrow \infty$, then so does $\|\underline{x}(t)\|$. 
The key is determining the eigenvalues of the matrix A and determining if all eigenvalues have negative real parts. If at least one eigenvalue of $\mathrm{A}$ has a positive real part, then the system is unstable, and $\|\underline{x}(t)\| \rightarrow \infty$ as $t \rightarrow \infty$. For A to be stable, all of its eigenvalues must have negative real parts.

\section{C-1.2.3. Solving general state-space equations}

Now we will find the solution to the general state-space equation with input $\underline{u}$ :

$$
\begin{aligned}
& \underline{\dot{x}}=A \underline{x}+B \underline{u} \\
& \underline{y}=C \underline{x}+D \underline{u}
\end{aligned}
$$

It can be shown that the solution consists of two parts: the zero input response and the zero state response:

$$
\underline{x(t)}=e^{A t} \underline{x}_{0}+e^{A t} * B \underline{u(t)}
$$

The zero input response is $e^{A t} \underline{x}_{0}$, while the zero state response is $e^{A t} * B \underline{u(t)}$.

The zero state response can also be expressed in terms of the convolution integral:

$$
e^{A t} * B \underline{u(t)}=\int_{0}^{t}\left(e^{A(t-\tau)} B\right) \underline{u(\tau)} d \tau
$$

We can see that both parts of the solution depend upon $e^{A t}$. For a stable system, the zero input response will die out, leaving the zero state response as the persistent part of the solution.

Our measurement y can then be found from:

$$
\begin{aligned}
& \underline{y}=C \underline{x}+D \underline{u} \\
& =C\left(e^{A t} \underline{x}_{0}+e^{A t} * B \underline{u(t)}\right)+D \underline{u}(t) \\
& =C e^{A t} \underline{x}_{0}+C e^{A t} * B \underline{u(t)}+D \underline{u}(t)
\end{aligned}
$$

The solution can also be found in terms of transfer functions.

We assume zero initial conditions, i.e. $\underline{x}_{0}=\underline{0}$. Let $\mathrm{L}$ represent the Laplace Transform operator:

$$
\mathrm{L}[f(t)]=\int_{0}^{\infty} f(t) e^{-s t} d t=F(s)
$$


Then it can be shown that the transfer function between $\underline{y}$ and $\underline{u} \mathrm{u}$ is;

$$
T(s)=\left[C(s I-A)^{-1} B+D\right]
$$

Thus

$$
\underline{y}(t)=\mathrm{L}^{-1}\left(\left[C(s I-A)^{-1} B+D\right] U(s)\right)
$$

Where $L^{-1}$ is the inverse Laplace operator.

We now describe controllability and observability properties of linear systems.

\section{C-1.3. Controllability and Observability}

Controllability is fundamental to successfully determining control laws to meet our control objectives. It can be stated as:

Definition C1.2: (A,B) controllable means that for all $\underline{x}_{0} \in C^{N}, \underline{x}_{T} \in C^{N}$, and for all $T>0$, there exists a $\underline{u}(t) \in C^{M}$ so that

$$
\begin{aligned}
& \underline{x}(0)=\underline{x}_{0} \\
& \text { and } \\
& \underline{x}(T)=\underline{x}_{T} .
\end{aligned}
$$

Thus if a state-space system is controllable, then we can always find a function $\underline{u}(t)$ to take us from $\underline{x}_{0}$ to $\underline{x}_{T}$ in a finite time $T$. This does not mean that if a system is not controllable that a $\underline{u}(t)$ can not be found to go from $\underline{x}_{0}$ to $\underline{x}_{T}$ in some finite time T. It means that we have no guarentee that we can find such a $\underline{u}(t)$. Controllability assures us that for any $\underline{x}_{0}$ and $\underline{x}_{T}$ we can always find a $\underline{u}(t)$ that take us from $\underline{x}(0)=\underline{x}_{0}$ to $\underline{x}(T)=\underline{x}_{T}$. in any finite time $\mathrm{T}$.

It turns out that the controllability of a linear system can be determined by a simple matrix rank test:

\section{Theorem C-1.3: The system $(A, B)$ is controllable if and only if}

$\operatorname{Rank}\left[B \vdots A B \vdots A^{2} B \vdots \ldots A^{N-1} B\right]=N$

We form the matrix multiplications $A B, A^{2} B$, etc. and form a matrix whose columns consist of $B$, $A B$, etc. We then test the rank of this matrix. If the rank of this matrix is $\mathrm{N}$, then the system is controllable. A proof of this theorem is given in [Kwakernaak and Sivan, 1972].

The definition of observability is: 
Definition C-1.3: A system (A,C) is observable if for all $T>0$ and $y(t)$ given on the interval $[\mathbf{0 , T}]$ the states $\underline{x}(t)$ can be determined uniquely on $[0, T]$.

In otherwords: "If you look at ouputs over some finite interval from 0 to $\mathrm{T}$, you can recover the states of the system."

To determine observability of a system, another rank test can be performed

Theorem C1.4: $(A, C)$ is observable if and only if

$$
\operatorname{Rank}\left[\begin{array}{c}
C \\
C A \\
C A^{2} \\
\vdots \\
C A^{N-1}
\end{array}\right]=N
$$

The proof is given in [Kwakernaak and Sivan, 1972].

Observability is very important to us. It permits us to reduce the number of state measurements that we need in order to design control to meet the desired control objectives.

We now give some linear control design fundamentals.

\section{C-1.4. Full-State Feedback}

Suppose we have a linear plant, with input $\underline{u}(t)$, and output $\underline{y}(t)$. Suppose we feed back the states of the plant in a linear control law with gain G:

$$
\underline{u(t)}=G \underline{x(t)}
$$

Then we can form the closed-loop equation:

$$
\begin{aligned}
& \underline{\dot{x}}=A \underline{x}+B \underline{u}=A \underline{x}+B G \underline{x} \\
& =(A+B G) \underline{x} .
\end{aligned}
$$

A question we might ask is whether or not we can choose the gain matrix $\mathrm{G}$ to enhance stability of this closed loop system. This can be determined by examining the eigenvalues of the closed loop system: $A+B G$.

Controllability allows us to arbitrarily place the eigenvalues (pole placement) of the closed loop system $A+B G$. Once we specify the location of the poles of the system in the complex plane, we can determine the elements of the gain matrix G so that the closed loop system has those poles that we specify. This can be stated in a Theorem (Wonham, 1967):

\section{Theorem C1.5:}

The poles of the closed loop system $(\mathrm{A}+\mathrm{BG})$ can be arbitrarily placed if and only if $(\mathrm{A}, \mathrm{B})$ is controllable. 
The proof of this theorem is given in [Kwakernaak and Sivan, 1972].

Pole placement is of great value to us in the design of controls for wind turbines. It allows us to design controllers to not only regulate turbine speed or power, but to also add damping to those flexible modes that have very small amounts of damping. We do this by locating the real parts of those eigenvalues further to the left in the complex plane, so that they have higher amounts of damping.

It would be nice to be able to use Full-state feedback in wind turbine control. Unfortunately, this requires that all states in the feedback law $\underline{u(t)}=G \underline{x(t)}$ be available to the controller. This means that we must measure all of these states! This is not practical in the wind turbine industry. We must really use a method that allows us to use the fewest measurements possible. This means that we must estimate the plant states from a limited number of turbine measurements, using State Estimation. We now describe state estimation.

\section{C-1.5. State Estimation}

State estimation is a technique to estimate the states of a plant from a limited number of plant output measurements. State estimation is based upon a linear model of the plant.

Assume we have a state-space model of the plant, with given A, B, C, and D matrices. Let the linear turbine be governed by Eq. (C1.2) (we ignore the disturbance for now).

We now form the state estimates:

$$
\begin{aligned}
& \underline{\dot{\hat{x}}}(t)=A \underline{\hat{x}}(t)+B \underline{u}(t)+K(\underline{y}(t)-\underline{\hat{y}}(t)) \\
& \underline{\hat{y}}(t)=C \underline{\hat{x}}(t)+D \underline{u}(t) ; \underline{\hat{x}}(0)=\underline{0}
\end{aligned}
$$

where $\underline{\hat{x}}$ are the estimated plant states and $\underline{\hat{y}}$ are the estimated plant outputs.

There is an error between the actual plant state $\underline{x}$ and the estimated state $\underline{\hat{x}}$. This error term can be expressed:

$$
\underline{\hat{x}}(t)=\underline{x}(t)+e(t)
$$

or

$$
\underline{e}(t)=\underline{\hat{x}}(t)-\underline{x}(t)
$$

Note that the derivative of this error can be expressed: 


$$
\begin{aligned}
& \underline{\dot{e}}(t)=\underline{\dot{x}}(t)-\underline{\dot{x}}(t) \\
& =A \underline{\hat{x}}(t)+B \underline{u}(t)+K(\underline{y}(t)-\underline{\hat{y}}(t))-A \underline{x}(t)-B \underline{u}(t) \\
& =A(\underline{\hat{x}}(t)-\underline{x}(t))+K(\underline{y}(t)-\underline{\hat{y}}(t)) \\
& =A \underline{e}(t)+K(C \underline{x}(t)-C \underline{\hat{x}}(t)) \\
& =A \underline{e}(t)-K C \underline{e}(t) \\
& =(A-K C) \underline{e}(t)
\end{aligned}
$$

This is a first order linear differential equation. We know that solutions to this equation are of the form:

$$
\underline{e}(t)=e^{(A-K C) t} \underline{e}(0)
$$

We also know that $\underline{e}(0)=-\underline{x}_{0}$.

Thus

$$
\underline{e}(t)=-e^{(A-K C) t} \underline{x}_{0}
$$

If we can place the eigenvalues of A-KC so that all poles have negative real parts then: $\underline{e}(t) \rightarrow 0$ as $t \rightarrow \infty$. In fact if we place these poles to have negative real parts far to the left of the imaginary axis in the complex plane, then the state estimator errors will decay quickly to zero. The eigenvalues of (A-KC) can be arbitrarily placed if $(\mathrm{A}, \mathrm{C})$ is observable. This is stated in the following theorem.

Theorem C1.6: The eigenvalues of A-KC can be arbitrarily placed if and only if $(\mathrm{A}, \mathrm{C})$ is observable.

The proof of the theorem is given in [Kwakernaak and Sivan, 1972].

This allows us to do state estimation for wind turbines, estimating the states from perhaps just a single turbine output: generator rotational speed. If we can successfully perform state estimation than we can reduce the number of turbine measurements needed for our control systems. Some wind turbine measurements are very difficult to obtain, such as wind speed at the rotor disk, blade flapwise and edgewise deflections and velocities, etc.

Figure 4.7 (Chapter 4) shows a diagram of a state estimator controller for a plant. Note that the inputs to the state estimator are the measurement $\underline{y}$ and the control input $\underline{u}$. The output of the state estimator is the estimated states $\underline{\hat{x}}$, which are modified by a gain in the full-state feedback law. The final control output $\underline{u}$ can be expressed:

$$
\underline{u}(t)=G \underline{\hat{x}}(t)=G \underline{x}(t)+G \underline{e}(t)
$$

Thus

$$
\underline{\dot{e}}(t)=(A-K C) \underline{e}(t)
$$

We can thus form the augmented state-space equation: 


$$
\left[\begin{array}{c}
\underline{\dot{x}} \\
\dot{e}
\end{array}\right]=\left[\begin{array}{cc}
A+B G & B G \\
0 & A-K C
\end{array}\right]\left[\begin{array}{l}
\underline{x} \\
\underline{e}
\end{array}\right]
$$

This is the state space equation for our system augmented by the state estimator error.

A wonderful thing is the separation principle. It states that the closed loop eigenvalues for this system are the union of the eigenvalues of $\mathrm{A}+\mathrm{BG}$ and the eigenvalues of $\mathrm{A}-\mathrm{KC}$.

This means that we can design our control system in two parts. In part I we place the poles of the plant using $\mathrm{A}+\mathrm{BG}$, assuming that the system is controllable. If no state estimation is used, this means that all of the system states that are represented in the linear model must then be available to the controller (through accurate measurements). We really want to reduce the number of necessary measurements through the use of state estimation.

In part II, we select state estimator pole locations. If the system is observable, then we can place the state estimator poles using A-KC. The theorem then tells us that the poles of the closed loop system will be the union of the poles of $\mathrm{A}+\mathrm{BG}$ and the poles of A-KC. Moreover we are guaranteed a stable closed loop system by choosing the poles of $\mathrm{A}+\mathrm{BG}$ and $\mathrm{A}-\mathrm{KC}$ to have negative real parts. Sometimes the poles of the controller alone may not have negative real parts, i.e. the controller alone may be unstable. When we use the controller in closed loop operation, this theorem guarantees stable closed loop behavior!

Often it is desirable to determine an equivalent transfer function for the controller. Let's assume we have the system:

$$
\begin{aligned}
& \underline{\dot{x}}=A \underline{x}+B \underline{u} \\
& \underline{y}=C \underline{x}+D \underline{u} .
\end{aligned}
$$

with state estimator:

$$
\begin{aligned}
& \underline{\dot{\hat{x}}}(t)=A \underline{\hat{x}}(t)+B \underline{u}(t)+K(\underline{y}(t)-\underline{\hat{y}}(t)) \\
& \underline{\hat{y}}(t)=C \underline{\hat{x}}(t)+D \underline{u}(t)
\end{aligned}
$$

Form the feedback law:

$$
\underline{u}(t)=G \underline{\hat{x}}(t)
$$

then, 


$$
\begin{aligned}
& \underline{\dot{\hat{x}}}(t)=A \underline{\hat{x}}(t)+B G \underline{\hat{x}}(t)+K(\underline{y}(t)-\underline{\hat{y}}(t)) \\
& \underline{\hat{y}}(t)=C \underline{\hat{x}}(t)+D G \underline{\hat{x}}(t)=(C+D G) \underline{\hat{x}}(t) \\
& \text { thus } \\
& \underline{\dot{\hat{x}}}(t)=A \underline{\hat{x}}(t)+B G \underline{\hat{x}}(t)+K(\underline{y}(t)-(C+D G) \underline{\hat{x}}(t)) \\
& =(A+B G-K C-K D G) \underline{\hat{x}}(t)+K \underline{y}(t)
\end{aligned}
$$

The final system can be expressed as:

$$
\begin{aligned}
& \dot{\hat{x}}(t)=L \underline{\hat{x}}(t)+K \underline{y}(t) \\
& \underline{u}(t)=G \underline{\hat{x}}(t), \\
& \text { where } \\
& L=(A+B G-K C-K D G)
\end{aligned}
$$

We know that for the system:

$$
\begin{aligned}
& \underline{\dot{x}}=A \underline{x}+B \underline{u} \\
& \underline{y}=C \underline{x}
\end{aligned}
$$

the equivalent transfer function is:

$$
T(s)=C(s I-A)^{-1} B .
$$

But Eq. (C1.30) is exactly in this form, with

$$
\begin{aligned}
& A=L, \\
& C=G, \\
& \text { and } \\
& B=K .
\end{aligned}
$$

Thus the equivalent transfer function for $(\mathrm{C} 1.30)$ is

$$
T(s)=G(s I-L)^{-1} K .
$$

As we will see, Eq. (C1.19) neglects wind disturbances. One state-space method that can be used to account for wind disturbances is Disturbance Accommodating Control (DAC). DAC allows us to use state estimation to estimate the wind disturbance states, as well as the turbine states. As we will see, the performance of the controller will be improved if it can account for this wind speed disturbance. We now describe DAC. 


\section{C-1.6. Disturbance Accommodating Control}

We know that wind turbines must operate in the presence of a highly turbulent wind environment. Turbulent winds cause fluctuations in the blade aerodynamic forces, and thus influence the power, torque and cyclic loads of the machine. We must design controls to account for these disturbances. We need an approach to counteract or accommodate these disturbances while also permitting Full-state feedback and State Estimation.

An approach to the reduction or counteraction of persistent disturbances was developed by Johnson in [Johnson, 1976] for lumped parameter systems and extended by Balas in [Balas, 1980] and [Balas, 1990], for large-scale and distributed parameter systems via model reduction and residual mode filters. Balas applied this theory to wind turbine control in [Balas, et al., 1998]. The basic idea of Disturbance Accommodating Control (DAC) is the augmentation of the usual state-estimator-based controller to recreate disturbance states via an assumed-waveform model; these disturbance states are used as part of the feedback control to reduce ("accommodate") or counteract any persistent disturbance effects.

Please refer to Eq. (C1.1) for the plant model. Here the control inputs, outputs, and states are members of $R^{M}$, $R^{P}$, and $R^{N}$ respectively. The persistent disturbance inputs $\underline{u_{d}}(t)$ have dimension $M_{D}$ and are given by the following disturbance waveform generator:

$$
\begin{aligned}
& \underline{u_{D}}(t)=\theta \underline{z_{D}}(t) \\
& \underline{\dot{z}_{D}}(t)=F \quad \underline{z_{D}}(t) ; \underline{z_{D}}(0)=\underline{z^{0}} .
\end{aligned}
$$

Here the disturbance states $\underline{z_{D}}(t)$ have dimension $N_{D}$ and the matrices $\theta$ and $F$ are assumed known; however, the initial condition $z_{D}^{0}$ is not known. This is equivalent to saying: the disturbances have a known waveform but unknown amplitude [Johnson, 1976].

Examples of this are step disturbances where $\theta \equiv 1$ and $F \equiv 0$ and ramp disturbances where $\theta \equiv\left[\begin{array}{ll}1 & 0\end{array}\right]$, and $F \equiv\left[\begin{array}{ll}0 & 1 \\ 0 & 0\end{array}\right]$; of course, in any waveform disturbance, we must assume that $F$ is stable, but not exponentially stable.

\section{C-1.6.1. Disturbance estimation}

The most fundamental need is to estimate the disturbance states $\underline{z_{D}}(t)$ from the plant outputs $\underline{y}(t)$; this can be accomplished with an augmentation of the usual plant state estimator.

The plant state estimator will have the form:

$$
\begin{aligned}
& \underline{\dot{\hat{x}}}(t)=A \underline{\hat{x}}(t)+B \underline{u}(t)+\Gamma \underline{\hat{u}_{d}}(t)+K_{x}(\underline{y}(t)-\underline{\hat{y}}(t)) \\
& \underline{\hat{y}}(t)=C \underline{\hat{x}}(t) ; \quad \underline{\hat{x}}(0)=\underline{0}
\end{aligned}
$$

Since the actual disturbance inputs can't be directly measured (or if they can, the problem addressed here is trivial to solve), we append the following disturbance estimator: 


$$
\begin{aligned}
& \frac{\hat{u}_{D}}{\underline{\dot{\hat{z}}_{D}}}(t)=\theta \underline{\hat{z}_{D}}(t)=F \underline{\hat{z}_{D}}(t)+K_{D}(\underline{y}(t)-\underline{\hat{y}}(t)) ; \quad \underline{\hat{z}_{D}}(0)=\underline{0} .
\end{aligned}
$$

This is a model of the disturbance generator with feedback correction from the plant output error

$$
\underline{y}(t)-\underline{\hat{y}}(t) .
$$

The design of the gains $K_{x}$ and $K_{D}$ can be derived from the estimator error equation where

$$
\begin{aligned}
& \underline{e}_{x}(t)=\underline{x}(t)-\underline{\hat{x}}(t) \\
& \underline{e}_{D}(t)=\underline{z}_{D}(t)-\underline{\hat{z}}_{D}(t)
\end{aligned}
$$

and

$$
\underline{e}(t)=\left[\begin{array}{ll}
\underline{e}_{\mathrm{x}}{ }^{T} & \underline{e}_{\mathrm{D}}{ }^{T}
\end{array}\right]^{T}
$$

Now the equation for the error is:

$$
\underline{\dot{e}}(t)=(\bar{A}-\bar{K} \bar{C}) \underline{e}(t) .
$$

where $\bar{A}=\left[\begin{array}{cc}A & \Gamma \theta \\ 0 & F\end{array}\right], \bar{C}=\left[\begin{array}{ll}C & 0\end{array}\right]$, and $\bar{K}=\left[\begin{array}{c}K_{x} \\ K_{D}\end{array}\right]$.

We see that (C1.33) follows from (C1.29)-(C1.32) since $\bar{A}-\bar{K} \bar{C}=\left[\begin{array}{cc}A-K_{x} C & \Gamma \theta \\ -K_{D} C & F\end{array}\right]$.

Consequently, when $(\bar{A}, \bar{C})$ is observable, we can have arbitrary rates of convergence in the state estimates $\underline{\hat{x}}(t)=\underline{x}(t)+\underline{e}_{x}(t)$ and $\underline{\hat{z}}_{D}(t)=\underline{z}_{D}(t)+\underline{e}_{D}(t)$ by appropriate choices of the gains $K_{x}$ and $K_{D}$.

Thus in order to insure success of this control, all we need to do is to check observability of the augmented pair $(\bar{A}, \bar{C})$.

\section{C-1.6.2. Disturbance Accommodating Control}

The Ideal DAC Control Law is a superposition of the plant full-state feedback law plus a disturbance feedback law as follows:

$$
\underline{u}_{*}(t)=G \underline{x}(t)+G_{D} \underline{z}_{D}(t) .
$$


Insertion of (C1.40) into (C1.1) yields:

$$
\underline{\dot{x}}(t)=(A+B G) \underline{x}(t)+\left(B G_{D}+\Gamma \theta\right) \underline{z}_{D}(t) .
$$

When $(A, B)$ is controllable, the plant state gain $G$ is designed for appropriate transient behavior; however the choice of the disturbance state gain $G_{D}$ can be made independently, to mitigate the effect of input disturbances.

In the case of exact counteraction, we have

$$
B G_{D}+\Gamma \theta=0
$$

and the closed-loop becomes "disturbance free." Even when exact cancellation can't be achieved, $G_{D}$ can still be chosen to reduce the disturbance effects at their inputs to the plant.

Of course, $(\mathrm{C} 1.40)$ is not realizable since the plant and disturbance states are rarely directly measurable. Still, a Realizable DAC Law can be generated using the state estimators of Section C1.6.1:

$$
u(t)=G \underline{\hat{x}}(t)+G_{D} \underline{\hat{z}}_{D}(t)
$$

which produces

$$
u(t)=u_{*}(t)+\bar{G} e(t)
$$

where

$$
\bar{G}=\left[\begin{array}{ll}
G & G_{D}
\end{array}\right]
$$

and the error $e(t)$ dissipates exponentially via (C1.33).

Consequently, the closed-loop $\mathrm{C} 1.1, \mathrm{C} 1.26, \mathrm{C} 1.27$, and $\mathrm{C} 1.28$ produce

$$
\left[\begin{array}{c}
\underline{\dot{x}} \\
\dot{e}
\end{array}\right]=\left[\begin{array}{cc}
A+B G & B \bar{G} \\
0 & \bar{A}-\bar{K} \bar{C}
\end{array}\right]\left[\begin{array}{l}
\underline{x} \\
\underline{e}
\end{array}\right]+\left[\begin{array}{c}
\mathrm{BG}_{\mathrm{D}}+\Gamma \theta \\
0
\end{array}\right] \underline{z}_{D}
$$

where $\bar{G} \equiv\left[\begin{array}{ll}G & G_{D}\end{array}\right]$ and $\bar{K} \equiv\left[\begin{array}{c}K \\ K_{D}\end{array}\right]$ can be designed for appropriate transient stability when $(A, B)$ is controllable, and $(\bar{A}, \bar{C})$ is observable. If (C1.36) is satisfied, then the closed-loop is "disturbance-free"; otherwise, $G_{D}$ is chosen to mitigate the persistent disturbance effects to the lowest level possible. 
Here are the steps we will use to design control systems using the linear control method: Disturbance Accommodating Control (DAC).

1. Generate an appropriate linear model of the turbine at a specific operating point (the control design point). Evaluate the state matrices $A, B, C$, and $\Gamma$. Determine the open loop eigenvalues of the A matrix.

2. Assess controllability of the system $(A, B)$ in order to allow pole placement. If the system is controllable then choose system poles to enhance damping and improve system response as desired.

3. Calculate gains $G$ to give the desired poles that were chosen in Step 2).

4. Form the feedback law $\underline{u}_{*}(t)=G \underline{x}(t)+G_{D} z_{D}(t)$. Choose the wind disturbance state gain $G_{D}$ to exactly cancel wind speed disturbances if possible, or to minimize their effects. This can be done by choosing $G_{D}$ so that $B G_{D}+\Gamma \theta=0$. If this is not possible then we try and minimize the norm $\left\|B G_{D}+\Gamma \theta\right\|$. We now have numerical values for the vector $\bar{G} \equiv\left[\begin{array}{ll}G & G_{D}\end{array}\right]$.

5. Calculate the augmented state matrices $(\bar{A}, \bar{B}, \bar{C})$ and assess observability of $(\bar{A}, \bar{C})$. Recall that:

$$
\bar{A}=\left[\begin{array}{cc}
A & \Gamma \theta \\
0 & F
\end{array}\right], \bar{C}=\left[\begin{array}{ll}
C & 0
\end{array}\right], \text { and } \bar{B}=\left[\begin{array}{l}
B \\
0
\end{array}\right]
$$

1. If observability is achieved, choose the poles of the state estimators (including the wind state estimator) to achieve desired behavior (high damping for state estimator poles). We now have numerical values for the elements of the vector $\bar{K} \equiv\left[\begin{array}{c}K \\ K_{D}\end{array}\right]$.

2. Now that $\bar{G} \equiv\left[\begin{array}{ll}G & G_{D}\end{array}\right]$ and $\bar{K} \equiv\left[\begin{array}{c}K \\ K_{D}\end{array}\right]$ have been calculated, calculate the equivalent controller transfer function $T_{c}(s)=\overline{\mathrm{G}}(\mathrm{sI}-\overline{\mathrm{L}})^{-1} \bar{K}$, where $\mathrm{I}$ is the identity matrix. We will then incorporate this transfer function in the control subroutines used with ADAMS and FAST. We will simulate the closed loop system for various conditions to assess controller performance.

This allows us a simple way of incorporating this control into the simulation codes: by incorporating the coefficients of the numerator and denominator of this transfer function into a set of subroutines. These subroutines will convert this transfer function into a set of linear first order state-space equations. We now examine the form of $\overline{\mathrm{A}}$ for a system that includes measurement dependence on the control input $\underline{u}$ and disturbance input $\underline{u_{D}}$. 


\section{Examination of $\overline{\mathrm{L}}$.}

Suppose we have the system

$$
\begin{aligned}
& \underline{\dot{x}}=A \underline{x}+B \underline{u}+\Gamma \underline{u_{d}} \\
& \underline{y}=C \underline{x}+D \underline{u}+E_{e} \underline{u_{D}} .
\end{aligned}
$$

We want to determine the form of $\overline{\mathrm{L}}$ in the equivalent transfer function for the DAC, $T_{c}(s)=\overline{\mathrm{G}}(\mathrm{sI}-\overline{\mathrm{L}})^{-1} \bar{K}$.

The full-state feedback law can be expressed as:

$\underline{\underline{u}}=G \underline{\underline{\mathbf{x}}}+G_{D} \underline{\underline{z}}_{D}$

Expressing the plant state and output estimator, we have:

$\dot{\hat{\mathbf{x}}}=A \underline{\hat{x}}+B \underline{u}+K(\underline{\underline{Y}}-\underline{\hat{y}})+\Gamma \hat{u}_{D}$

$\underline{\hat{y}}=C \underline{\underline{\hat{x}}}+D \underline{u}+E e \hat{u}_{D}$

After substituting Eq. (C1.48) and Eq. (C1.46) into Eq. (C1.47) we get for the right hand side of (C1.47):

$\Gamma \Theta \underline{\underline{\hat{z}}}_{D}+A \underline{\underline{\hat{x}}}+B\left(G_{D} \underline{\underline{\underline{z}}}_{D}+G \underline{\underline{\hat{x}}}\right)+K\left(-E e \Theta \underline{\underline{\hat{z}}}_{D}+\underline{\underline{\underline{Y}}}-\mathrm{C} \hat{\underline{\hat{x}}}-D\left(G_{D} \underline{\underline{\underline{z}}}_{D}+G \underline{\underline{\hat{x}}}\right)\right)$

Expressing the disturbance state estimator, we have:

$\dot{\hat{\mathbf{z}}}_{D}=F \underline{\hat{z}}_{D}+K_{D}(\underline{\underline{Y}}-\underline{\hat{\underline{y}}})$

After substituting (C1.48) into (C1.50) we obtain the right hand side of (C1.50) as

$F \underline{\underline{\underline{z}}}_{D}+K_{D}\left(-E e \Theta \hat{\underline{\underline{z}}}_{D}+\underline{\underline{Y}}-C \hat{\underline{\hat{x}}}-D\left(G_{D} \hat{\underline{\underline{z}}}_{D}+G \hat{\underline{\hat{x}}}\right)\right):$

We can express equations (C1.49) and (C1.51) in the state-space form:

$$
\left[\begin{array}{c}
\underline{\dot{x}} \\
\dot{\underline{x}}_{D}
\end{array}\right]=\left[\begin{array}{cc}
A+B G-C K-D G K & -E_{e} K \theta+\Gamma \theta+B G_{d}-D K G_{d} \\
-C K_{d}-D G K_{d} & F-E_{e} \theta K_{d}-D G_{d} K_{d}
\end{array}\right]\left[\begin{array}{l}
\underline{x} \\
\underline{z}_{D}
\end{array}\right]+\left[\begin{array}{c}
K \\
K_{d}
\end{array}\right] \underline{y}
$$

Thus

$$
\bar{L}=\left[\begin{array}{cc}
A+B G-C K-D G K & -E_{e} K \theta+\Gamma \theta+B G_{d}-D K G_{d} \\
-C K_{d}-D G K_{d} & F-E_{e} \theta K_{d}-D G_{d} K_{d}
\end{array}\right]
$$

It is interesting to note what happens when 
$\underline{\dot{x}}=A \underline{x}+B \underline{u}+\Gamma \underline{u_{d}}$

$\underline{y}=C \underline{x}$

so that $D=0$, and $E_{e}=0$.

Then

$\bar{L}=\left[\begin{array}{cc}A+B G-C K & \Gamma \theta+B G_{d} \\ -C K_{d} & F\end{array}\right]$.

We will use the form for $\bar{L}$ shown in Eq. (C1.53) in Section 5.5.2 on measuring tower-top acceleration.

\section{C-1.7. Linear Quadratic Regulator Design}

LQR involves finding the linear feedback gain, $G$, that minimizes the following quadratic cost function:

$$
J=\int_{0}^{\infty}\left(\underline{x}^{T} Q \underline{x}+\underline{u}^{T} R \underline{u}\right) d t
$$

where $Q$ is the symmetric, positive semidefinite weighting on the states, and $R$ is the symmetric, positive definite weighting on the input $\underline{u}$.

In most cases, the quadratic cost function $J$, has no physical significance, but rather provides a means to trade-off opposing objectives; state regulation versus control usage. For a time invariant system, the solution to this optimization problem is related to the Algebraic Riccati Equation [Kwakernaak and Sivan, 1972].

This method provides us with a calculation of $G$, in which we then form the full-state feedback law $\underline{u}=G \underline{x}$. MATLAB [The Math Works, Inc., 2003] will be used to calculate $G$ using LQR as is illustrated in Chapter 4. We can also use this technique to calculate the state estimator gain matrix also.

\section{C-1.8. Including Actuator Dynamics in Linear Plant Models}

An approach to treat actuator dynamics is to think of the actuator and plant as one composite plant. Figure C-1.1 depicts this composite plant. The control input is the input entering the actuator $\underline{u}_{c}$. The composite plant also contains the actuator states $\underline{x}_{A}$, as well as the plant states $\underline{x}$. The control input to the turbine (or plant) $\underline{u}$ now becomes an internal state of this composite system, passing as output from the actuator model of the composite plant and entering as input to the plant model in the composite plant. For the actuator state-space model (assuming a first order model, as described in Chapter 3), we have: 


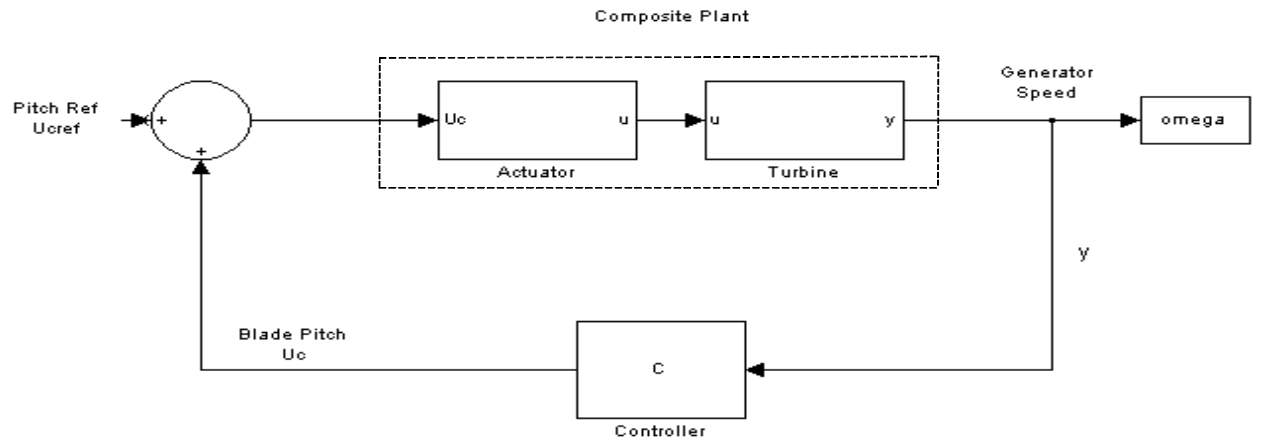

Figure C-1.1. Illustration of Composite Plant

$$
\begin{aligned}
& \underline{\dot{x}}_{A}=A_{A} \underline{x}_{A}+B_{A} \underline{u}_{C} \\
& \underline{u}=C_{A} \underline{x}_{\mathrm{A}}
\end{aligned}
$$

The state-space model for the plant becomes:

$$
\begin{aligned}
\underline{\dot{x}} & =A \underline{x}+B \underline{u}+\Gamma \underline{u_{d}} \\
& =A \underline{x}+B C_{A} \underline{x}_{A}+\Gamma \underline{u_{d}} \\
\underline{y} & =C \underline{x}+\mathrm{D}_{\underline{u}}+\mathrm{E}_{\mathrm{e}} \underline{u_{d}} \\
& =C \underline{x}+\mathrm{D}_{A} \underline{x}_{A}+\mathrm{E}_{\mathrm{e}} \underline{u_{d}}
\end{aligned}
$$

or

$$
\begin{aligned}
& {\left[\begin{array}{l}
\underline{\dot{x}} \\
\underline{\dot{x}}_{A}
\end{array}\right]=\left[\begin{array}{cc}
A & B C_{A} \\
0 & A_{A}
\end{array}\right]\left[\begin{array}{l}
\underline{x} \\
\underline{x}_{A}
\end{array}\right]+\left[\begin{array}{c}
0 \\
B_{A}
\end{array}\right] \underline{u}_{C}+\left[\begin{array}{l}
\Gamma \\
0
\end{array}\right] \underline{u}_{d}} \\
& \underline{y}=\left[\begin{array}{ll}
C & D C_{A}
\end{array}\right]\left[\begin{array}{l}
\underline{x}_{\underline{x}_{A}}
\end{array}\right]+\mathrm{E}_{\mathrm{e}} \underline{u_{d}}
\end{aligned}
$$

We can now consider this composite plant to be the plant model used for control design. If the resulting system is controllable, we can place poles arbitrarily, using full-state feedback (including the actuator pole). If the system is observable, we can use state estimation to estimate plant states. We can design DAC, as in chapters 5, 6, and 7, if the augmented system is observable.

This concludes the synopsis of the control theory used in this report. 


\section{Appendix D. Control Design Details}

D-1. Controls from Chapter 4

D-1.1. Full-State Feedback Design Using the 1-State Linear Model

Case I Plant pole at -2

$\mathbf{A}=-0.120533$

$\mathbf{B}=-2.8818$

plant poles -2

$\mathbf{G}=0.652185$

Case II Plant pole at -1

$\mathbf{A}=-0.120533$

$\mathbf{B}=-2.8818$

plant poles -1

$\mathbf{G}=0.305$

D-1.2 Full-State Feedback Design from the 3-State Linear Model

$$
\begin{aligned}
& A=\left(\begin{array}{ccc}
-0.144544 & -3.10781 \times 10^{-6} & 0 . \\
2.691 \times 10^{7} & 0 . & -2.691 \times 10^{7} \\
0 . & 0.0000156006 & 0 .
\end{array}\right) \\
& B=\left(\begin{array}{c}
-3.45588 \\
0 . \\
0
\end{array}\right) \\
& \text { Cont }=\left(\begin{array}{ccc}
-3.45588 & 0.499528 & 288.947 \\
0 . & -9.29979 \times 10^{7} & 1.34423 \times 10^{7} \\
0 . & 0 . & -1450.82
\end{array}\right) \\
& \operatorname{Det}[\text { Cont }]=-4.6628 \times 10^{11} \\
& \text { choose plant poles }=\{-2 .+22.4 \dot{i},-2 .-22.4 \dot{i},-2 .\} \\
& \mathrm{G}=\left(\begin{array}{lll}
1.69434 & 1.10928 \times 10^{-7} & -1.03897
\end{array}\right)
\end{aligned}
$$

D-1 


\section{D-1.3. State Estimator Design from the 3-State Linear Model}

Step 1: Generate an appropriate linear model of the turbine at a specific operating point (the control design point). Evaluate the state matrices $A, B, C$. Determine the open loop eigenvalues of the $A$ matrix.

$$
\begin{aligned}
& A=\left(\begin{array}{ccc}
-0.144544 & -3.10781 \times 10^{-6} & 0 . \\
2.691 \times 10^{7} & 0 . & -2.691 \times 10^{7} \\
0 . & 0.0000156006 & 0 .
\end{array}\right) \\
& B=\left(\begin{array}{c}
-3.45588 \\
0 . \\
0 .
\end{array}\right) \\
& C=\left(\begin{array}{lll}
0 & 0 & 1
\end{array}\right)
\end{aligned}
$$

Step 2: Assess controllability of the system $(A, B)$ in order to allow pole placement. If the system is controllable then choose plant poles to enhance damping and improve system response as desired.

$$
\begin{aligned}
& \text { Cont }=\left(\begin{array}{ccc}
-3.45588 & 0.499528 & 288.947 \\
0 . & -9.29979 \times 10^{7} & 1.34423 \times 10^{7} \\
0 . & 0 . & -1450.82
\end{array}\right) \\
& \text { Det }[\text { Cont }]=-4.6628 \times 10^{11} \\
& \text { choose plant poles }=\{-2 .+22.4 \text { i },-2 .-22.4 \dot{i},-2 .\}
\end{aligned}
$$

Step3: Calculate gains $G$ to give the desired poles that were chosen in Step 2

$$
\mathrm{G}=\left(\begin{array}{ll}
1.69434 & 1.10928 \times 10^{-7}-1.03897
\end{array}\right)
$$

Step 4: Assess observability of $(A, C)$.

$O b s v=\left(\begin{array}{c}C \\ C A \\ C A^{2}\end{array}\right)$

$$
\operatorname{Obsv}=\left(\begin{array}{ccc}
0 & 0 & 1 \\
0 . & 0.0000156006 & 0 . \\
419.813 & 0 . & -419.813
\end{array}\right)
$$

$\operatorname{Det}[$ Obsv $]=-0.00654934$

Choose: State Estimator Poles $=\{-16 .,-15 .,-14$.

Step 5: Calculate the gains in the state estimator gain matrix $K$ that give us the desired state estimator poles chosen in step 4.

$$
\text { State Estimator Gains: } K=\left(\begin{array}{c}
-1.13315 \\
1.0517 \times 10^{7} \\
44.8555
\end{array}\right)
$$


Step 6: Calculate equivalent controller transfer function $T_{c}(s)=G(s I-L)^{-1} K$ :

Controller Transfer Function : TC $(s)=\left(\frac{2102.76-369.692 s-47.3566 s^{2}}{5734.29+946.965 s+50.8554 s^{2}+s^{3}}\right)$

\section{D-1.4. DAC Design from the 3-State Linear Model}

Step 1: Generate an appropriate linear model of the turbine at a specific operating point (the control design point). Evaluate the state matrices $A, B, C$, and $\Gamma$. Determine the open loop eigenvalues of the $A$ matrix.

$$
\begin{aligned}
& A=\left(\begin{array}{ccc}
-0.144544 & -3.10781 \times 10^{-6} & 0 . \\
2.691 \times 10^{7} & 0 . & -2.691 \times 10^{7} \\
0 . & 0.0000156006 & 0 .
\end{array}\right) \\
& B=\left(\begin{array}{c}
-3.45588 \\
0 . \\
0 .
\end{array}\right) \\
& C=\left(\begin{array}{ll}
0 & 0 \\
1
\end{array}\right) \\
& \Gamma=\left(\begin{array}{c}
0.0789384 \\
0 . \\
0 .
\end{array}\right)
\end{aligned}
$$

Step 2: Assess controllability of the system $(A, B)$ in order to allow pole placement. If the system is controllable then choose plant poles to enhance damping and improve system response as desired.

$$
\begin{aligned}
& \text { Cont }=\left(\begin{array}{ccc}
-3.45588 & 0.499528 & 288.947 \\
0 . & -9.29979 \times 10^{7} & 1.34423 \times 10^{7} \\
0 . & 0 . & -1450.82
\end{array}\right) \\
& \text { Det }[\text { Cont }]=-4.6628 \times 10^{11} \\
& \text { choose plant poles }=\{-2 .+22.4 \text { i },-2 .-22.4 \dot{i},-2 .\}
\end{aligned}
$$

Step3: Calculate gains $G$ to give the desired poles that were chosen in Step 2

$$
\mathrm{G}=\left(\begin{array}{ll}
1.69434 & 1.10928 \times 10^{-7}-1.03897
\end{array}\right)
$$

Step4: Calculate gains $G_{d}$ to cancel or attenuate windspeed disturbances.

Disturbance Gain: $\mathrm{Gd}=0.0126012$

$$
\overline{\mathrm{G}}=\left(\begin{array}{llll}
1.69434 & 1.10928 \times 10^{-7} & -1.03897 & 0.0126012
\end{array}\right)
$$

Step5: Calculate the augmented state matrices $(\bar{A}, \bar{B}, \bar{C})$ and assess observability of $(\bar{A}, \bar{C})$.

$$
\overline{\mathrm{C}}=\left(\begin{array}{llll}
0 & 0 & 1 & 0
\end{array}\right)
$$




$$
\begin{aligned}
& \overline{\mathrm{A}}=\left(\begin{array}{cccc}
-0.144544 & -3.10781 \times 10^{-6} & 0 . & 0.0789384 \\
2.691 \times 10^{7} & 0 . & -2.691 \times 10^{7} & 0 . \\
0 . & 0.0000156006 & 0 . & 0 . \\
0 & 0 & 0 & 0
\end{array}\right) \\
& \overline{\mathrm{B}}=\left(\begin{array}{c}
-3.45588 \\
0 \\
0 \\
0
\end{array}\right) \\
& \text { Obsv }=\left(\begin{array}{c}
\bar{C} \\
\overline{C A} \\
\overline{C A}^{2} \\
\overline{C A}^{3}
\end{array}\right) \\
& \text { Obsv }=\left(\begin{array}{cccc}
0 & 0 & 1 & 0 \\
0 . & 0.0000156006 & 0 . & 0 . \\
419.813 & 0 . & -419.813 & 0 . \\
-60.6815 & -0.00785404 & 0 . & 33.1393
\end{array}\right) \\
& \text { Det }[\mathrm{Obsv}]=-0.217041
\end{aligned}
$$

Step 6: If observability is achieved, choose the poles of the state estimators (including the wind disturbance state estimator) to achieve desired behavior (high damping for state estimator poles).

$$
\begin{aligned}
& \text { State Estimator Poles }=\{-17.0008,-15.9979,-15.0019,-13.9994\} \\
& \text { State Estimator Gains : } \overline{\mathrm{K}}=\left(\begin{array}{c}
22.511 \\
5.93963 \times 10^{7} \\
61.8555 \\
1723.64
\end{array}\right)
\end{aligned}
$$

Step 7: Calculate equivalent controller transfer function $T_{c}(s)=\bar{G}(s I-\bar{L})^{-1} \bar{K}$ :

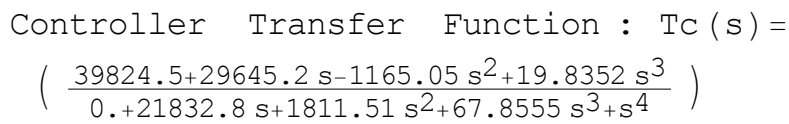

\section{D-2. Controls Designed from Chapter 5}

\section{D-2.1. DAC Controls Designed from the 1-State Linear Model}

Step 1: Generate an appropriate linear model of the turbine at a specific operating point (the control design point). Evaluate the state matrices $A, B, C$, and $\Gamma$. Determine the open loop eigenvalues of the $A$ matrix.

$A=-0.120533$ 
$B=-2.8818$

$\mathrm{C}=1$

$\Gamma=0.0658253$

Step 2: Assess controllability of the system $(A, B)$ in order to allow pole placement. If the system is controllable then choose plant poles to enhance damping and improve system response as desired.

Controllability automatically satisfied, since $\mathrm{B}=-2.88$, nonzero.

Choose plant pole at -1 .

Step 3: Calculate gains $G$ to give the desired poles that were chosen in Step 2

$G=0.652185$

Step 4: Calculate gains $G_{d}$ to cancel or attenuate windspeed disturbances.

$\mathrm{Gd}=0.0228417$

$\overline{\mathrm{G}}=\left(\begin{array}{ll}0.652185 & 0.0228417\end{array}\right)$

Step 5: Calculate the augmented state matrices $(\bar{A}, \bar{B}, \bar{C})$ and assess observability of $(\bar{A}, \bar{C})$.

$$
\begin{aligned}
& \overline{\mathrm{C}}=\left(\begin{array}{cc}
1 & 0
\end{array}\right) \\
& \overline{\mathrm{A}}=\left(\begin{array}{cc}
-0.120533 & 0.0658253 \\
0 & 0
\end{array}\right) \\
& \overline{\mathrm{B}}=\left(\begin{array}{c}
-2.8818 \\
0
\end{array}\right) \\
& \text { Obsv }=\left(\begin{array}{c}
\bar{C} \\
\bar{C} A
\end{array}\right) \\
& \text { Obsv }=\left(\begin{array}{c}
1 \\
-0.120533 \\
\text { Det }[\text { Obsv }]=0.0658253
\end{array}\right)
\end{aligned}
$$

Step 6: If observability is achieved, choose the poles of the state estimators (including the wind disturbance state estimator) to achieve desired behavior (high damping for state estimator poles).

$$
\begin{aligned}
& \text { State Estimator Poles }=\{-15,-14 .\} \\
& \text { State Estimator Gains: } \overline{\mathrm{K}}=\left(\begin{array}{l}
28.8795 \\
3190.26
\end{array}\right)
\end{aligned}
$$

Step 7: Calculate equivalent controller transfer function $T_{c}(s)=\bar{G}(s I-\bar{L})^{-1} \bar{K}$ : 
Controller Transfer Function : $\operatorname{TC}(s)=\left(\frac{145.742+91.7059 s}{0 .+30.8795 s+s^{2}}\right)$

\section{D-2.2. DAC Control Design from the 3-State Model}

Already shown above, in Section D1.5.

\section{D-2.3. Control Designs using a 5-State Model}

Step 1: Generate an appropriate linear model of the turbine at a specific operating point (the control design point). Evaluate the state matrices $A, B, C$, and $\Gamma$. Determine the open loop eigenvalues of the $A$ matrix.

$$
\begin{aligned}
& \mathrm{A}=\left(\begin{array}{ccccc}
0 . & 1 . & 0 . & 0 . & 0 . \\
-200.185 & -8.98988 & -20.0526 & 0.0000290587 & 0 . \\
1.03275 & 0.00784164 & -0.0410937 & -3.25772 \times 10^{-6} & 0 . \\
0 . & 0 . & 2.691 \times 10^{7} & 0 . & -2.691 \times 10^{7} \\
0 . & 0 . & 0 . & 0.0000156006 & 0 .
\end{array}\right) \\
& B=\left(\begin{array}{c}
0 . \\
-1120.92 \\
2.32689 \\
0 \\
0
\end{array}\right) \\
& \mathrm{C}=\left(\begin{array}{lllll}
0 & 0 & 0 & 0 & 1
\end{array}\right) \\
& \Gamma=\left(\begin{array}{c}
0 \\
14.1321 \\
0.00603125 \\
0 \\
0
\end{array}\right)
\end{aligned}
$$

Step 2: Assess controllability of the system $(A, B)$ in order to allow pole placement. If the system is controllable then choose plant poles to enhance damping and improve system response as desired.

$$
\begin{aligned}
& \text { Cont }=\left(\begin{array}{ccccc}
0 . & -1120.92 & 10030.3 & 136217 . & -3.21371 \times 10^{6} \\
-1120.92 & 10030.3 & 136217 . & -3.21371 \times 10^{6} & -390376 . \\
2.32689 & -8.88545 & -1282.59 & 12258.5 & 313048 . \\
0 . & 6.26165 \times 10^{7} & -2.39107 \times 10^{8} & -6.08017 \times 10^{10} & 4.30257 \times 10^{11} \\
0 . & 0 . & 976.856 & -3730.22 & -948545 .
\end{array}\right) \\
& \operatorname{Det}[\text { Cont }]=3.50695 \times 10^{25} \\
& \text { choose plant poles }=\{-2 .+22.6 \dot{\mathbb{1}},-2 .-22.6 \dot{\mathbb{1}},-4.4+13.3 \dot{\mathbb{1}},-4.4-13.3 \dot{\mathbb{1}},-2 .\}
\end{aligned}
$$

Step 3: Calculate gains $G$ to give the desired poles that were chosen in Step 2

$$
G=\left(\begin{array}{lllll}
0.0357243 & 0.0049286 & -0.105066 & -3.48875 \times 10^{-7} & 0.754115
\end{array}\right)
$$


Step 4: Calculate gains $G_{d}$ to cancel or attenuate windspeed disturbances.

Disturbance Gain: $\mathrm{Gd}=0.0126012$

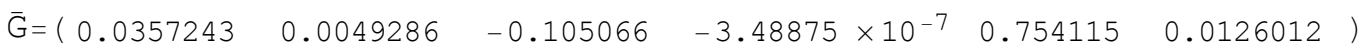

Step 5: Calculate the augmented state matrices $(\bar{A}, \bar{B}, \bar{C})$ and assess observability of $(\bar{A}, \bar{C})$.

$$
\begin{aligned}
& \overline{\mathrm{C}}=\left(\begin{array}{llllll}
0 & 0 & 0 & 0 & 1 & 0
\end{array}\right) \\
& \overline{\mathrm{A}}=\left(\begin{array}{cccccc}
0 . & 1 . & 0 . & 0 . & 0 . & 0 . \\
-200.185 & -8.98988 & -20.0526 & 0.0000290587 & 0 . & 14.1321 \\
1.03275 & 0.00784164 & -0.0410937 & -3.25772 \times 10^{-6} & 0 . & 0.00603125 \\
0 . & 0 . & 2.691 \times 10^{7} & 0 . & -2.691 \times 10^{7} & 0 . \\
0 . & 0 . & 0 . & 0.0000156006 & 0 . & 0 . \\
0 & 0 & 0 & 0 & 0 & 0
\end{array}\right) \\
& \overline{\mathrm{B}}=\left(\begin{array}{c}
0 \\
-1120.92 \\
2.32689 \\
0 . \\
0 \\
0
\end{array}\right)
\end{aligned}
$$$$
\text { Obsv }=\left(\begin{array}{c}
\bar{C} \\
\overline{C A} \\
\overline{C A}^{2} \\
\overline{C A}^{3} \\
\overline{C A}^{4} \\
\overline{C A}^{5}
\end{array}\right)
$$$$
\text { Obsv }=\left(\begin{array}{cccccc}
0 & 0 & 0 & 0 & 1 & 0 \\
0 . & 0 . & 0 . & 0.0000156006 & 0 . & 0 . \\
0 . & 0 . & 419.813 & 0 . & -419.813 & 0 . \\
433.56 & 3.29202 & -17.2517 & -0.00791698 & 0 . & 2.532 \\
-676.829 & 403.83 & -213111 . & 0.000151863 & 213046 . & 46.4192 \\
-300930 . & -5978.35 & 4746.33 & 4.02964 & -4086.63 & 4421.64
\end{array}\right)
$$

$\operatorname{Det}[$ Obsv $]=-7.70391 \times 10^{6}$

Step 6: If observability is achieved, choose the poles of the state estimators (including the wind disturbance state estimator) to achieve desired behavior (high damping for state estimator poles).

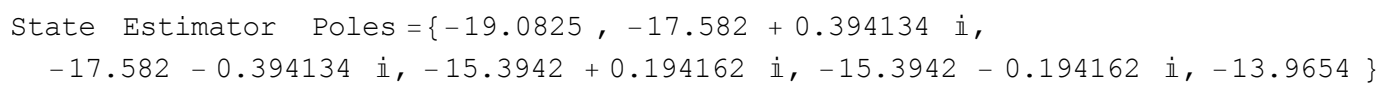


State Estimator Gains : $\overline{\mathrm{K}}=\left(\begin{array}{c}235.348 \\ -5798.26 \\ 84.9206 \\ 1.63733 \times 10^{8} \\ 89.9694 \\ 2944.78\end{array}\right)$

Step 7: Calculate equivalent controller transfer function $T_{c}(s)=\bar{G}(s I-\bar{L})^{-1} \bar{K}$ :

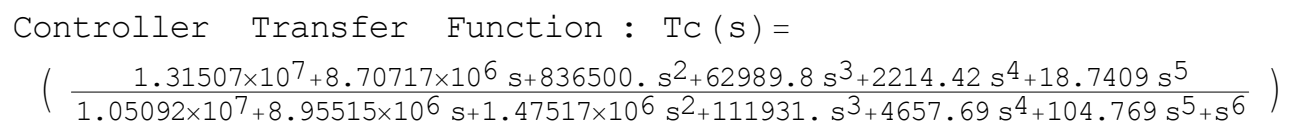

\section{D-2.4. Results from the 7-State Model (measuring tower-top fore-aft deflection)}

Step 1: Generate an appropriate linear model of the turbine at a specific operating point (the control design point). Evaluate the state matrices $A, B, C$, and $\Gamma$. Determine the open loop eigenvalues of the $A$ matrix.

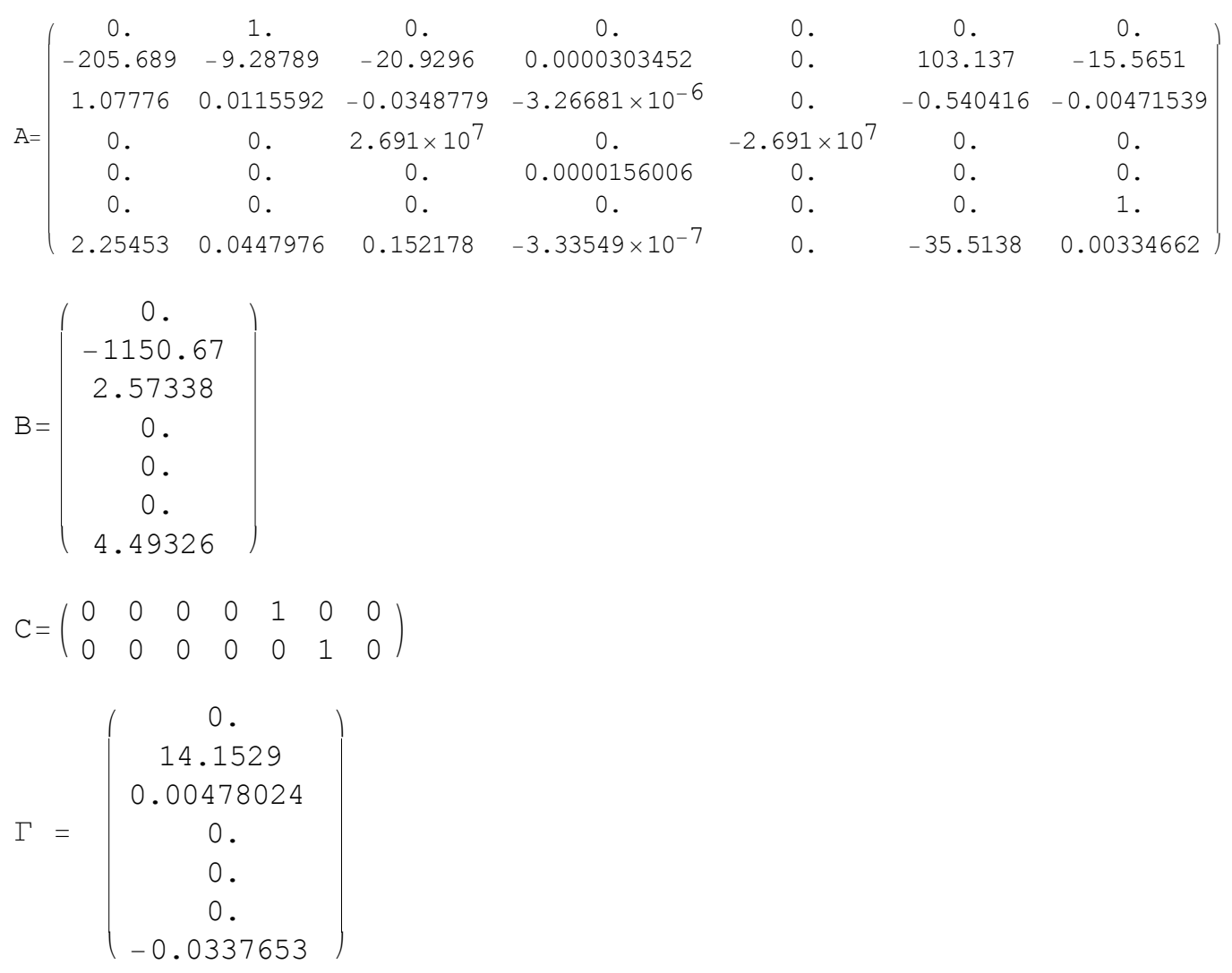

Step 2: Assess controllability of the system $(A, B)$ in order to allow pole placement. If the system is controllable then choose plant poles to enhance damping and improve system response as desired. 
$\operatorname{Cont}=\left(\begin{array}{ccccccc}0 . & -1150.67 & 10563.5 & 142209 . & -3.44579 \times 10^{6} & -261695 . & 7.19664 \times 10^{8} \\ -1150.67 & 10563.5 & 142209 . & -3.44579 \times 10^{6} & -261695 . & 7.19664 \times 10^{8} & -5.24738 \times 10^{9} \\ 2.57338 & -13.4118 & -1345.99 & 14293.3 & 327333 . & -5.49821 \times 10^{6} & -1.10197 \times 10^{8} \\ 0 . & 6.92497 \times 10^{7} & -3.60911 \times 10^{8} & -6.52926 \times 10^{10} & 5.36147 \times 10^{11} & 3.62192 \times 10^{13} & -3.73038 \times 10^{14} \\ 0 . & 0 . & 1080.34 & -5630.43 & -1.0186 \times 10^{6} & 8.36423 \times 10^{6} & 5.65042 \times 10^{8} \\ 0 . & 4.49326 & -51.1407 & -2305.89 & 31910.5 & 272203 . & -9.04172 \times 10^{6} \\ 4.49326 & -51.1407 & -2305.89 & 31910.5 & 272203 . & -9.04172 \times 10^{6} & 9.03442 \times 10^{6}\end{array}\right)$

Det $[$ Cont $]=-1.10272 \times 10^{39}$

choose plant poles $=\{-2 .+22.6 \dot{i},-2 .-22.6 \dot{i},-4.4+13.3 \dot{i},-4.4-13.3 \dot{i},-2 .+5.9 \dot{1},-2 .-5.9 \dot{i},-2$.

Step 3: Calculate gains $G$ to give the desired poles that were chosen in Step 2

$G=\left(\begin{array}{lllllll}0.0763604 & 0.00988856 & 0.0828986 & -4.271 \times 10^{-7} & 0.625768 & 0.436495 & 0.37492\end{array}\right)$

Step 4: Calculate gains $G_{d}$ to cancel or attenuate windspeed disturbances.

Disturbance Gain: $\mathrm{Gd}=0.0122856$

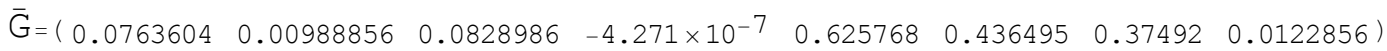

Step 5: Calculate the augmented state matrices $(\bar{A}, \bar{B}, \bar{C})$ and assess observability of $(\bar{A}, \bar{C})$.

$\overline{\mathbf{C}}=\left(\begin{array}{llllllll}0 & 0 & 0 & 0 & 1 & 0 & 0 & 0 \\ 0 & 0 & 0 & 0 & 0 & 1 & 0 & 0\end{array}\right)$

$\overline{\mathrm{A}}=\left(\begin{array}{cccccccc}0 . & 1 . & 0 . & 0 . & 0 . & 0 . & 0 . & 0 . \\ -205.689 & -9.28789 & -20.9296 & 0.0000303452 & 0 . & 103.137 & -15.5651 & 14.1529 \\ 1.07776 & 0.0115592 & -0.0348779 & -3.26681 \times 10^{-6} & 0 . & -0.540416 & -0.00471539 & 0.00478024 \\ 0 . & 0 . & 2.691 \times 10^{7} & 0 . & -2.691 \times 10^{7} & 0 . & 0 . & 0 . \\ 0 . & 0 . & 0 . & 0.0000156006 & 0 . & 0 . & 0 . & 0 . \\ 0 . & 0 . & 0 . & 0 . & 0 . & 0 . & 1 . & 0 . \\ 2.25453 & 0.0447976 & 0.152178 & -3.33549 \times 10^{-7} & 0 . & -35.5138 & 0.00334662 & -0.0337653 \\ 0 & 0 & 0 & 0 & 0 & 0 & 0 & 0\end{array}\right)$

$\bar{B}=\left(\begin{array}{c}0 . \\ -1150.67 \\ 2.57338 \\ 0 . \\ 0 . \\ 0 . \\ 4.49326 \\ 0\end{array}\right)$ 
Obsv $=\left(\begin{array}{c}\bar{C} \\ \overline{C A} \\ \overline{C A}^{2} \\ \overline{C A}^{3} \\ \overline{C A}^{4} \\ \overline{C A}^{5} \\ \overline{C A}^{6} \\ \overline{C A}^{7}\end{array}\right)$

Observability matrix not shown

From MATLAB: the rank of the observability matrix is 8 , thus this system is observable.

Step 6: If observability is achieved, choose the poles of the state estimators (including the wind disturbance state estimator) to achieve desired behavior (high damping for state estimator poles).

$$
\begin{aligned}
& \text { State Estimator Poles= } \\
& \{-12.3138,-11.987,-11.6577,-11.3543,-11.0179,-10.6655,-10.2949,-10.0089\} \\
& \text { State Estimator Gains : } \overline{\mathrm{K}}=\left(\begin{array}{cc}
102.963 & -615.044 \\
-61.0408 & -17296.1 \\
2.78732 & 223.162 \\
-1.74793 \times 10^{6} & 5.86193 \times 10^{8} \\
36.5777 & 203.609 \\
0.0817797 & 43.4029 \\
10.6247 & 447.332 \\
486.703 & 4411.44
\end{array}\right)
\end{aligned}
$$

Step 7: Calculate equivalent controller transfer function $T_{c}(s)=\bar{G}(s I-\bar{L})^{-1} \bar{K}$ :

\footnotetext{
Controller Transfer Function: $\operatorname{TCl}(s)=\frac{1.82311 \times 10^{8}+2.9456 \times 10^{8} s+9.8209 \times 10^{7} s^{2}+1.5063 \times 10^{7} s^{3}+1.31953 \times 10^{6} s^{4}+72756.4 s^{5}+2496.68 s^{6}+41.124 s^{7}}{3.28189 \times 10^{8}+3.01783 \times 10^{8} s+1.00036 \times 10^{8} s^{2}+1.72203 \times 10^{7} s^{3}+1.75469 \times 10^{6} s^{4}+111172 . s^{5}+4364.44 s^{6}+98.7806 s^{7}+s^{8}}$

Controller Transfer Function: Tc2 $(s)=\frac{-1.99802 \times 10^{9}-7.8056 \times 10^{8} s-1.53402 \times 10^{8} s^{2}-2.24645 \times 10^{7} s^{3}-2.44351 \times 10^{6} s^{4}-166063 . s^{5}-5919.07 s^{6}-81.5935 s^{7}}{3.28189 \times 10^{8}+3.01783 \times 10^{8} s+1.00036 \times 10^{8} s^{2}+1.72203 \times 10^{7} s^{3}+1.75469 \times 10^{6} s^{4}+111172 . s^{5}+4364.44 s^{6}+98.7806 s^{7}+s^{8}}$
} 


\section{D-2.5. Results from the 7-State Model (measuring tower-top fore-aft acceleration)}

Step 1: Generate an appropriate linear model of the turbine at a specific operating point (the control design point). Evaluate the state matrices $A, B, C$, and $\Gamma$. Determine the open loop eigenvalues of the $A$ matrix.

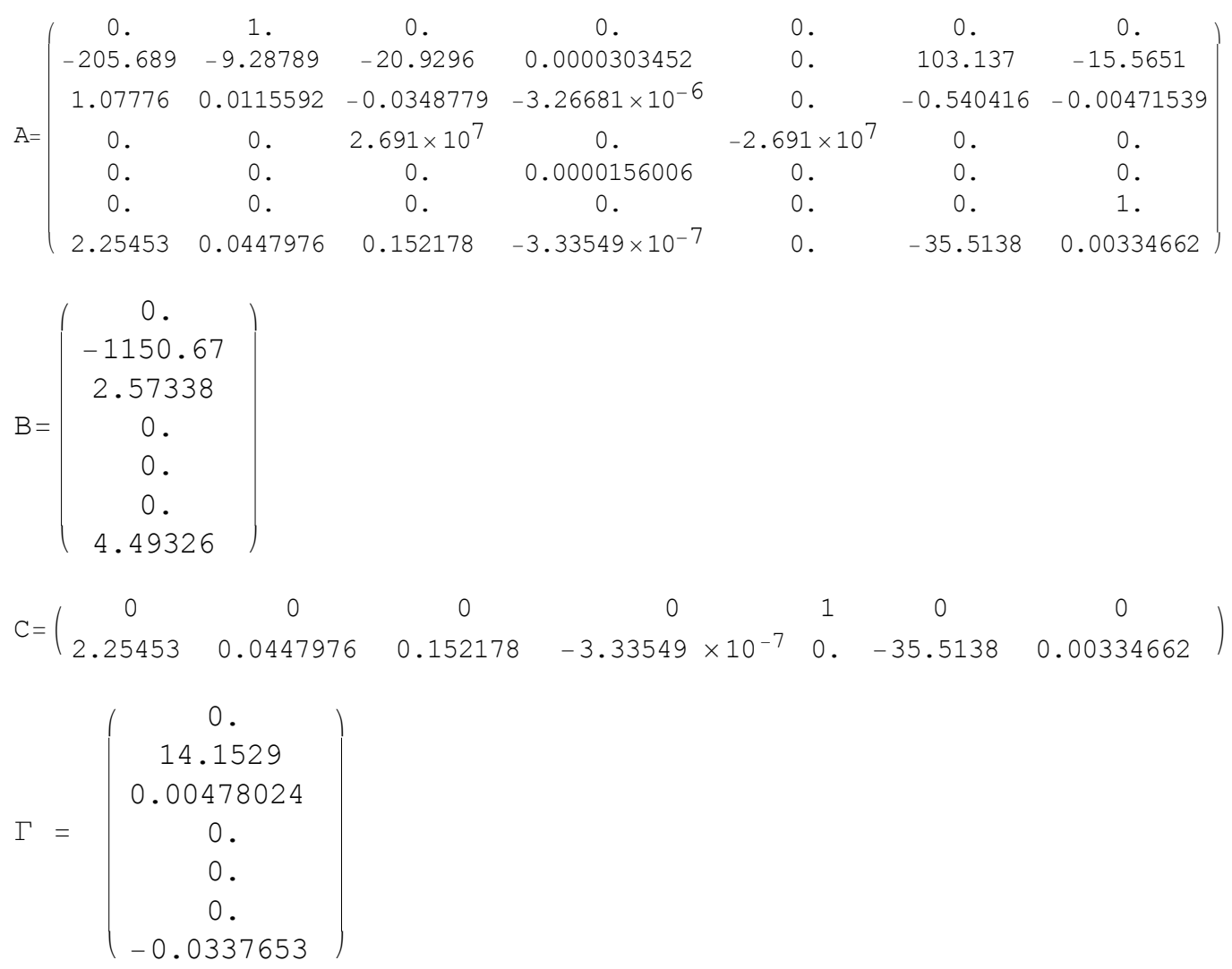

Step 2: Assess controllability of the system $(A, B)$ in order to allow pole placement. If the system is controllable then choose plant poles to enhance damping and improve system response as desired.

$$
\text { Cont }=\left(\begin{array}{ccccccc}
0 . & -1150.67 & 10563.5 & 142209 . & -3.44579 \times 10^{6} & -261695 . & 7.19664 \times 10^{8} \\
-1150.67 & 10563.5 & 142209 . & -3.44579 \times 10^{6} & -261695 . & 7.19664 \times 10^{8} & -5.24738 \times 10^{9} \\
2.57338 & -13.4118 & -1345.99 & 14293.3 & 327333 . & -5.49821 \times 10^{6} & -1.10197 \times 10^{8} \\
0 . & 6.92497 \times 10^{7} & -3.60911 \times 10^{8} & -6.52926 \times 10^{10} & 5.36147 \times 10^{11} & 3.62192 \times 10^{13} & -3.73038 \times 10^{14} \\
0 . & 0 . & 1080.34 & -5630.43 & -1.0186 \times 10^{6} & 8.36423 \times 10^{6} & 5.65042 \times 10^{8} \\
0 . & 4.49326 & -51.1407 & -2305.89 & 31910.5 & 272203 . & -9.04172 \times 10^{6} \\
4.49326 & -51.1407 & -2305.89 & 31910.5 & 272203 . & -9.04172 \times 10^{6} & 9.03442 \times 10^{6}
\end{array}\right)
$$

$\operatorname{Det}[$ Cont $]=-1.10272 \times 10^{39}$

choose plant poles $=\{-2 .+22.6 \dot{i},-2 .-22.6 \dot{i},-4.4+13.3 \dot{i},-4.4-13.3 \dot{i},-2 .+5.9 \dot{i},-2 .-5.9 \dot{i},-2$.

Step 3: Calculate gains $G$ to give the desired poles that were chosen in Step 2

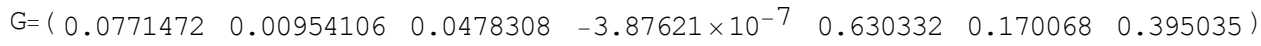

Step 4: Calculate gains $G_{d}$ to cancel or attenuate windspeed disturbances. 
Disturbance Gain: $\mathrm{Gd}=0.0122856$

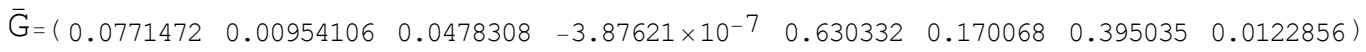

Step 5: Calculate the augmented state matrices $(\bar{A}, \bar{B}, \bar{C})$ and assess observability of $(\bar{A}, \bar{C})$.

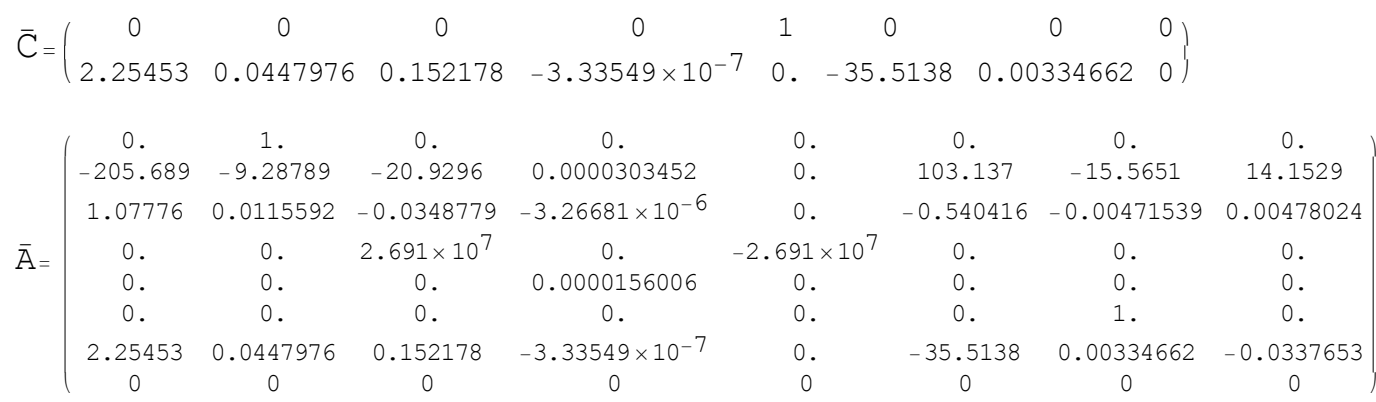

$\overline{\mathrm{B}}=\left(\begin{array}{c}0 . \\ -1150.67 \\ 2.57338 \\ 0 . \\ 0 . \\ 0 . \\ 4.49326 \\ 0\end{array}\right)$

Obsv $=\left(\begin{array}{c}\bar{C} \\ \overline{C A} \\ \overline{C A}^{2} \\ \overline{C A}^{3} \\ \overline{C A}^{4} \\ \overline{C A}^{5} \\ \overline{C A}^{6} \\ \overline{C A}^{7}\end{array}\right)$

Observability matrix not shown

From MATLAB: the rank of the observability matrix is 8 , thus this system is observable.

Step 6: If observability is achieved, choose the poles of the state estimators (including the wind disturbance state estimator) to achieve desired behavior (high damping for state estimator poles).

State Estimator Poles $=\{-12.3004,-11.999,-11.6991,-11.3028,-11.0007,-10.6975,-10.2998,-10.0007\}$ 


$$
\text { State Estimator Gains : } \overline{\mathrm{K}}=\left(\begin{array}{cc}
26.138 & 1.66978 \\
147.971 & 88.823 \\
2.80198 & -1.13566 \\
1.4369 \times 10^{7} & -2.72456 \times 10^{6} \\
44.5303 & -0.903837 \\
0.938853 & -0.759527 \\
-6.86833 & -0.915559 \\
430.399 & -25.065
\end{array}\right)
$$

Step 7: Calculate equivalent controller transfer function $T_{c}(s)=\bar{G}(s I-\bar{L})^{-1} \bar{K}$ :

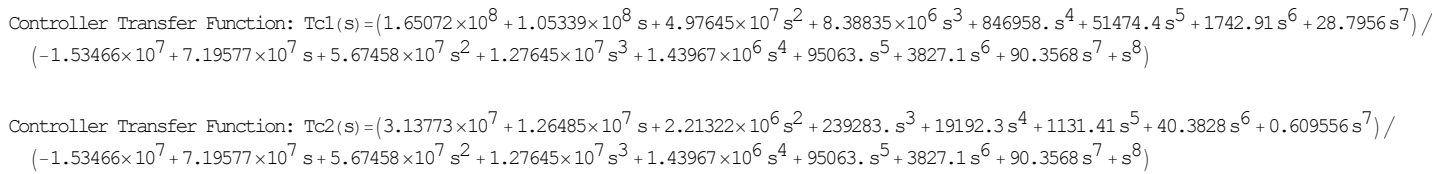

\section{D-3. Controls Designed from Chapter 6}

\section{D-3.1. Results from the 3-State Model (for generator torque control)}

Step 1: Generate an appropriate linear model of the turbine at a specific operating point (the control design point). Evaluate the state matrices $A, B, C$. Determine the open loop eigenvalues of the $A$ matrix.

$$
\begin{aligned}
& A=\left(\begin{array}{ccc}
-0.144544 & -3.10781 \times 10^{-6} & 0 . \\
2.691 \times 10^{7} & 0 . & -2.691 \times 10^{7} \\
0 . & 0.0000156006 & 0 .
\end{array}\right) \\
& B=\left(\begin{array}{c}
0 . \\
0 . \\
-0.0000156006
\end{array}\right) \\
& C=\left(\begin{array}{lll}
0 & 0 & 1
\end{array}\right)
\end{aligned}
$$

Step 2: Assess controllability of the system $(A, B)$ in order to allow pole placement. If the system is controllable then choose plant poles to enhance damping and improve system response as desired.

$$
\begin{aligned}
& \text { Cont }=\left(\begin{array}{ccc}
0 . & 0 . & -0.0013047 \\
0 . & 419.813 & 0 . \\
-0.0000156006 & 0 . & 0.00654934
\end{array}\right) \\
& \text { Det }[\text { Cont }]=-8.54492 \times 10^{-6} \\
& \text { choose plant poles }=\{-2 .+22.4 \dot{i},-2 .-22.4 \text { i },-0.12\}
\end{aligned}
$$

Step 3: Calculate gains $G$ to give the desired poles that were chosen in Step 2

$$
G=(-255065 . \quad-0.00529144 \quad 254827 .)
$$

Step 4: Assess observability of $(A, C)$. 
$O b s v=\left(\begin{array}{c}C \\ C A \\ C A^{2}\end{array}\right)$

$\operatorname{Obsv}=\left(\begin{array}{ccc}0 & 0 & 1 \\ 0 . & 0.0000156006 & 0 . \\ 419.813 & 0 . & -419.813\end{array}\right)$

Det $[$ Obsv $]=-0.00654934$

Choose: State Estimator Poles $=\{-16 .,-15 .,-14$.

Step 5: Calculate the gains in the state estimator gain matrix $K$ that give us the desired state estimator poles chosen in step 4.

State Estimator Gains : $\overline{\mathrm{K}}=\left(\begin{array}{c}-1.13315 \\ 1.0517 \times 10^{7} \\ 44.8555\end{array}\right)$

Step 6: Calculate equivalent controller transfer function $T_{c}(s)=G(s I-L)^{-1} K$ :

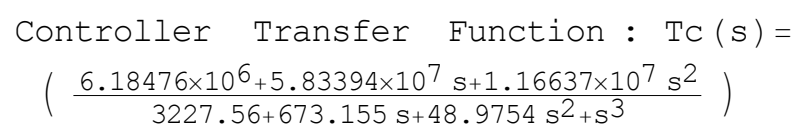

\section{D-3.2. Control Designs Using a 5-State Model (with $1^{\text {st }}$ drive-train torsion poles placed} at open-loop values)

Step 1: Generate an appropriate linear model of the turbine at a specific operating point (the control design point). Evaluate the state matrices $A, B, C$, and $\Gamma$. Determine the open loop eigenvalues of the $A$ matrix.

$$
\begin{aligned}
& \mathrm{A}=\left(\begin{array}{ccccc}
0 . & 1 . & 0 . & 0 . & 0 . \\
-200.185 & -8.98988 & -20.0526 & 0.0000290587 & 0 . \\
1.03275 & 0.00784164 & -0.0410937 & -3.25772 \times 10^{-6} & 0 . \\
0 . & 0 . & 2.691 \times 10^{7} & 0 . & -2.691 \times 10^{7} \\
0 . & 0 . & 0 . & 0.0000156006 & 0 .
\end{array}\right) \\
& B=\left(\begin{array}{c}
0 \\
-1120.92 \\
2.32689 \\
0 \\
0
\end{array}\right) \\
& \mathrm{C}=\left(\begin{array}{lllll}
0 & 0 & 0 & 0 & 1
\end{array}\right)
\end{aligned}
$$


$\Gamma=\left(\begin{array}{c}0 . \\ 14.1321 \\ 0.00603125 \\ 0 . \\ 0 .\end{array}\right)$

Step 2: Assess controllability of the system $(A, B)$ in order to allow pole placement. If the system is controllable then choose plant poles to enhance damping and improve system response as desired.

Cont $=\left(\begin{array}{ccccc}0 . & -1120.92 & 10030.3 & 136217 . & -3.21371 \times 10^{6} \\ -1120.92 & 10030.3 & 136217 . & -3.21371 \times 10^{6} & -390376 . \\ 2.32689 & -8.88545 & -1282.59 & 12258.5 & 313048 . \\ 0 . & 6.26165 \times 10^{7} & -2.39107 \times 10^{8} & -6.08017 \times 10^{10} & 4.30257 \times 10^{11} \\ 0 . & 0 . & 976.856 & -3730.22 & -948545 .\end{array}\right)$

$\operatorname{Det}[$ Cont $]=3.50695 \times 10^{25}$

choose plant poles $=$

$\{-0.033+22.6 \dot{\mathbf{i}},-0.033-22.6 \dot{\mathbf{i}},-4.4+13.3 \dot{\mathbf{i}},-4.4-13.3 \dot{\mathbf{i}},-2$.

Step 3: Calculate gains $G$ to give the desired poles that were chosen in Step 2

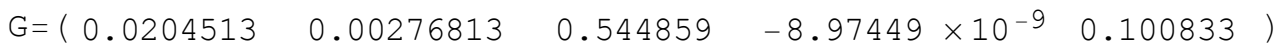

Step 4: Calculate gains $G_{d}$ to cancel or attenuate wind-speed disturbances.

Disturbance Gain: Gd=0.0126012

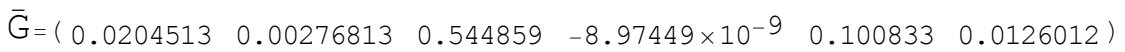

Step 5: Calculate the augmented state matrices $(\bar{A}, \bar{B}, \bar{C})$ and assess observability of $(\bar{A}, \bar{C})$.

$$
\begin{aligned}
& \overline{\mathrm{C}}=\left(\begin{array}{cccccccc}
0 & 0 & 0 & 0 & 1 & 0
\end{array}\right) \\
& \overline{\mathrm{A}}=\left(\begin{array}{cccccc}
0 . & 1 . & 0 . & 0 . & 0 . & 0 . \\
-200.185 & -8.98988 & -20.0526 & 0.0000290587 & 0 . & 14.1321 \\
1.03275 & 0.00784164 & -0.0410937 & -3.25772 \times 10^{-6} & 0 . & 0.00603125 \\
0 . & 0 . & 2.691 \times 10^{7} & 0 . & -2.691 \times 10^{7} & 0 . \\
0 . & 0 . & 0 . & 0.0000156006 & 0 . & 0 . \\
0 & 0 & 0 & 0 & 0 & 0 \\
\overline{\mathrm{B}} & =\left(\begin{array}{c}
0 . \\
-1120.92 \\
2.32689 \\
0 . \\
0 .
\end{array}\right)
\end{array}\right)
\end{aligned}
$$


Obsv $=\left(\begin{array}{c}\bar{C} \\ \overline{C A} \\ \overline{C A}^{2} \\ \overline{C A}^{3} \\ \overline{C A}^{4} \\ \overline{C A}^{5}\end{array}\right)$

$\mathrm{Obsv}=$

$$
\begin{aligned}
& \left(\begin{array}{cccccc}
0 & 0 & 0 & 0 & 1 & 0 \\
0 . & 0 . & 0 . & 0.0000156006 & 0 . & 0 . \\
0 . & 0 . & 419.813 & 0 . & -419.813 & 0 . \\
433.56 & 3.29202 & -17.2517 & -0.00791698 & 0 . & 2.532 \\
-676.829 & 403.83 & -213111 . & 0.000151863 & 213046 . & 46.4192 \\
-300930 . & -5978.35 & 4746.33 & 4.02964 & -4086.63 & 4421.64
\end{array}\right) \\
& \operatorname{Det}\left[\text { Obsv ] }=-7.70391 \times 10^{6}\right.
\end{aligned}
$$

Step 6: If observability is achieved, choose the poles of the state estimators (including the wind disturbance state estimator) to achieve desired behavior (high damping for state estimator poles).

$$
\begin{aligned}
& \text { State Estimator Poles }= \\
& \{-19.0825,-17.582+0.394134 \dot{1},-17.582-0.394134 \dot{1}, \\
& -15.3942+0.194162 \dot{\mathbb{1}},-15.3942-0.194162 \dot{\mathbb{1}},-13.9654\} \\
& \text { State Estimator Gains : } \overline{\mathrm{K}}=\left(\begin{array}{c}
235.348 \\
-5798.26 \\
84.9206 \\
1.63733 \times 10^{8} \\
89.9694 \\
2944.78
\end{array}\right)
\end{aligned}
$$

Step 7: Calculate equivalent controller transfer function $T_{c}(s)=\bar{G}(s I-\bar{L})^{-1} \bar{K}$ :

$$
\begin{aligned}
& \text { Controller Transfer Function: } \operatorname{TC}(s)= \\
& 1\left(\frac{1.30764 \times 10^{7}+8.73318 \times 10^{6} s+456579 . s^{2}+58077.4 s^{3}+822.69 s^{4}+79.7427 s^{5}}{9.7607 \times 10^{6}+9.73956 \times 10^{6} s+1.34238 \times 10^{6} s^{2}+96815.6 s^{3}+4257.26 s^{4}+100.835 s^{5}+s^{6}}\right)
\end{aligned}
$$

\section{D-3.3. Control Designs using a 7-State Model (with $1^{\text {st }}$ drive-train torsion poles placed at open-loop values)}

This is the same as the controller designed in Section D2.4 except that the $1^{\text {st }}$ drive-train torsion mode poles are place at their open-loop values. Steps not shown. 


\section{D-4. Controls Designed from Chapter 7}

\section{D-4.1 State Estimation Based On the 5-State Model}

Part I: State Estimation (neglecting disturbance states)

Step 1: Generate an appropriate linear model of the turbine at a specific operating point (the control design point). Evaluate the state matrices $A, B, C$, and $\Gamma$. Determine the open loop eigenvalues of the $A$ matrix.

$$
\begin{aligned}
& A=\left(\begin{array}{ccccc}
0 . & 1 . & 0 . & 0 . & 0 . \\
-194.783 & -8.94887 & -3.81034 & -0.0289319 & -20.1159 \\
0 . & 0 . & 0 . & 1 . & 0 . \\
-3.81034 & -0.0289319 & -194.783 & -8.94887 & -20.1159 \\
0.427171 & 0.00324351 & 0.427171 & 0.00324351 & -0.0339949
\end{array}\right) \\
& B=\left(\begin{array}{cc}
0 . & 0 . \\
-1108.75 & -8.5851 \\
0 . & 0 . \\
-8.5851 & -1108.75 \\
0.962462 & 0.962462
\end{array}\right) \\
& C=\left(\begin{array}{lllll}
0 & 0 & 0 & 0 & 1
\end{array}\right)
\end{aligned}
$$

Step 2: Assess controllability of the system $(A, B)$ in order to allow pole placement. If the system is controllable then choose plant poles to enhance damping and improve system response as desired.

$$
\text { Cont }=\left(\begin{array}{cccccccccc}
0 . & 0 . & -1108.75 & -8.5851 & 9902.91 & 89.5442 & 127449 . & 4882.66 & -3.06098 \times 10^{6} & -93610 . \\
-1108.75 & -8.5851 & 9902.91 & 89.5442 & 127449 . & 4882.66 & -3.06098 \times 10^{6} & -93610 . & 2.45673 \times 10^{6} & -605220 . \\
0 . & 0 . & -8.5851 & -1108.75 & 89.5442 & 9902.91 & 4882.66 & 127449 . & -93610 . & -3.06098 \times 10^{6} \\
-8.5851 & -1108.75 & 89.5442 & 9902.91 & 4882.66 & 127449 . & -93610 . & -3.06098 \times 10^{6} & -605220 . & 2.45673 \times 10^{6} \\
0.962462 & 0.962462 & -3.6568 & -3.6568 & -444.756 & -444.756 & 4712.82 & 4712.82 & 46136 . & 46136 .
\end{array}\right)
$$

Rank (Cont) $=5$, thus system is controllable.

$$
\begin{aligned}
& \text { plant poles }= \\
& \{-4.7+13.5 \dot{\mathbf{i}},-4.7-13.5 \dot{\mathbf{i}},-4.5+13.1 \dot{\mathbf{i}},-4.5-13.1 \dot{\mathbf{i}},-2 .\}
\end{aligned}
$$

Step 3: Calculate gains $G$ to give the desired poles that were chosen in Step 2

$$
G=\left(\begin{array}{ccccc}
0.0197711 & 0.00186392 & 0.00820158 & 0.00144438 & 0.670494 \\
0.00715175 & 0.00139498 & 0.00797024 & 0.00146644 & 0.626825
\end{array}\right)
$$

Step 4: Assess observability of $(A, C)$.

$$
C=\left(\begin{array}{ccccc}
0 & 0 & 0 & 0 & 1 \\
0.5 & 0 & -0.5 & 0 & 0
\end{array}\right)
$$


$O b s v=\left(\begin{array}{c}C \\ C A \\ C A^{2} \\ C A^{3} \\ C A^{4}\end{array}\right)$

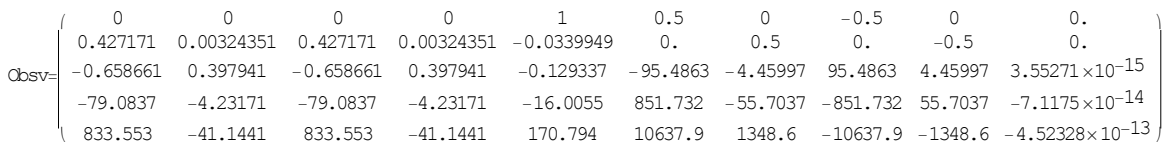

$\operatorname{Rank}(\mathrm{Obsv})=5$

Step 5: If observability is achieved, choose the poles of the state estimators (including the wind disturbance state estimator) to achieve desired behavior (high damping for state estimator poles).

State Estimator Poles $=\{-14 .,-11 .,-11 .,-10 .,-10$.

State Estimator Gains: $K=\left(\begin{array}{cc}-0.830393 & 12.0801 \\ -4260.26 & -188.726 \\ -0.830393 & -12.0801 \\ -4260.26 & 188.726 \\ 25.9882 & -4.74594 \times 10^{-10}\end{array}\right)$

equivalent controller transfer function $T_{c}(s)=G(s I-L)^{-1} K$ :

Controller Transfer Function: $\operatorname{TC}(s)=\left(\begin{array}{ll}\frac{110829 .+21806.7 s+1650.32 s^{2}+85.2949 s^{3}+3.3075 s^{4}}{282201 .+108441 . s+17161.2 s^{2}+1397.29 s^{3}+58.4683 s^{4}+s^{5}} & \frac{-3681.38-734.12 s-42.2842 s^{2}+0.274925 s^{3}+0.0605831 s^{4}}{282201 .+108441 . s+17161.2 s^{2}+1397.29 s^{3}+58.4683 s^{4}+s^{5}} \\ \frac{121424 .+29259.7 s+2869.09 s^{2}+151.033 s^{3}+4.08713 s^{4}}{282201 .+108441 . s+17161.2 s^{2}+1397.29 s^{3}+58.4683 s^{4}+s^{5}} & \frac{1114.01+159.443 s+9.86434 s^{2}+0.304306 s^{3}+0.00359828 s^{4}}{282201 .+108441 . s+17161.2 s^{2}+1397.29 s^{3}+58.4683 s^{4}+s^{5}}\end{array}\right)$

\section{D-4.2 DAC Based On the 5-State Model}

Step 1: Generate an appropriate linear model of the turbine at a specific operating point (the control design point). Evaluate the state matrices $A, B, C$, and $\Gamma$. Determine the open loop eigenvalues of the $A$ matrix.

$$
\begin{aligned}
& \mathrm{A}=\left(\begin{array}{ccccc}
0 . & 1 . & 0 . & 0 . & 0 . \\
-194.783 & -8.94887 & -3.81034 & -0.0289319 & -20.1159 \\
0 . & 0 . & 0 . & 1 . & 0 . \\
-3.81034 & -0.0289319 & -194.783 & -8.94887 & -20.1159 \\
0.427171 & 0.00324351 & 0.427171 & 0.00324351 & -0.0339949
\end{array}\right) \\
& \mathrm{B}=\left(\begin{array}{cc}
0 . & 0 . \\
-1108.75 & -8.5851 \\
0 . & 0 . \\
-8.5851 & -1108.75 \\
0.962462 & 0.962462
\end{array}\right)
\end{aligned}
$$


$C=\left(\begin{array}{cccccc}0 & 0 & 0 & 0 & 1 & 0 \\ 0.5 & 0 & -0.5 & 0 & 0 & 0\end{array}\right)$

$\Gamma=\left(\begin{array}{cc}0.1859 & 0 \\ 14.18 .1414 \\ 0.14 .1859 & 0.14 .1414 \\ 6.93889 \times 10^{-18} & 0.00498937\end{array}\right)$

Step 2: Assess controllability of the system $(A, B)$ in order to allow pole placement. If the system is controllable then choose plant poles to enhance damping and improve system response as desired.

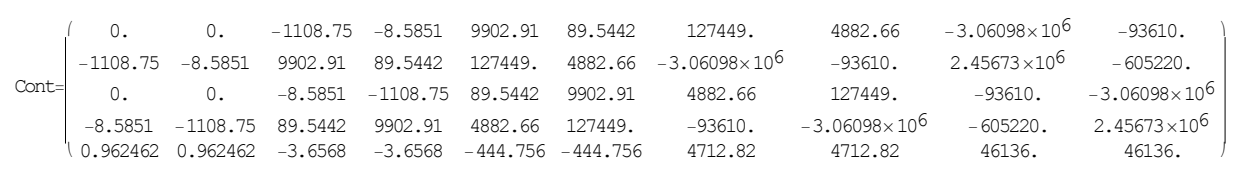

$\operatorname{Rank}($ cont $)=5$, thus system is controllable

$$
\begin{aligned}
& \text { choose plant poles }= \\
& \{-4.7+13.5 \dot{\mathbb{1}},-4.7-13.5 \dot{\mathbb{1}},-4.5+13.1 \dot{\mathbb{1}},-4.5-13.1 \dot{\mathbb{1}},-2 .\}
\end{aligned}
$$

Step 3: Calculate gains $G$ to give the desired poles that were chosen in Step 2

$$
\mathrm{G}=\left(\begin{array}{ccccc}
0.0197711 & 0.00186392 & 0.00820158 & 0.00144438 & 0.670494 \\
0.00715175 & 0.00139498 & 0.00797024 & 0.00146644 & 0.626825
\end{array}\right)
$$

Step 4: Calculate gains $G_{d}$ to cancel or attenuate windspeed disturbances.

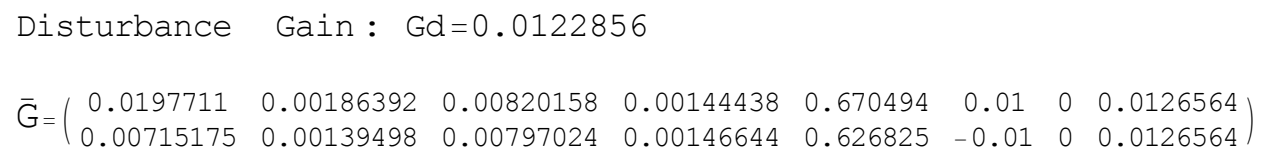

Step 5: Calculate the augmented state matrices $(\bar{A}, \bar{B}, \bar{C})$ and assess observability of $(\bar{A}, \bar{C})$.

$$
\begin{aligned}
& \overline{\mathrm{C}}=\left(\begin{array}{cccccccc}
0 & 0 & 0 & 0 & 1 & 0 & 0 & 0 \\
0.5 & 0 & -0.5 & 0 & 0 & 0 & 0 & 0
\end{array}\right) \\
& \overline{\mathrm{A}}=\left(\begin{array}{cccccccc}
0 . & 1 . & 0 . & 0 . & 0 . & 0 . & 0 . & 0 . \\
-194.783 & -8.94887 & -3.81034 & -0.0289319 & -20.1159 & 14.1859 & 0 . & 14.1414 \\
0 . & 0 . & 0 . & 1 . & 0 . & 0 . & 0 . & 0 . \\
-3.81034 & -0.0289319 & -194.783 & -8.94887 & -20.1159 & -14.1859 & 0 . & 14.1414 \\
0.427171 & 0.00324351 & 0.427171 & 0.00324351 & -0.0339949 & 6.93889 \times 10^{-18} & 0 . & 0.00498937 \\
0 & 0 & 0 & 0 & 0 & 0 & 1 & 0 \\
0 & 0 & 0 & 0 & 0 & -19.3442 & 0 & 0 \\
0 & 0 & 0 & 0 & 0 & 0 & 0 & 0
\end{array}\right) \\
& \overline{\mathrm{B}}=\left(\begin{array}{cc}
0 . & 0 . \\
-1108.75 & -8.5851 \\
0 . & 0 . \\
-8.5851 & -1108.75 \\
0.962462 & 0.962462 \\
0 & 0 \\
0 & 0 \\
0 & 0
\end{array}\right)
\end{aligned}
$$


Obsv $=\left(\begin{array}{c}\bar{C} \\ \overline{C A} \\ \overline{C A}^{2} \\ \overline{C A}^{3} \\ \overline{C A}^{4} \\ \overline{C A}^{5} \\ \overline{C A}^{6} \\ \overline{C A}^{7}\end{array}\right)$

Observability matrix not shown

From MATLAB: the rank of the observability matrix is 8 , thus this system is observable.

Step 6: If observability is achieved, choose the poles of the state estimators (including the wind disturbance state estimator) to achieve desired behavior (high damping for state estimator poles).

$$
\begin{aligned}
& \text { State Estimator Poles }=\{-11.0002,-10.9999,-10.0003, \\
& -9.99955,-9.00038,-8.99972,-8.00008,-7.9999\} \\
& \text { State Estimator Gains: } \overline{\mathrm{K}}=\left(\begin{array}{cc}
118.553 & 29.0801 \\
-3790.17 & 69.2909 \\
118.553 & -29.0801 \\
-3790.17 & -69.2909 \\
28.9882 & 2.66177 \times 10^{-11} \\
3.11389 \times 10^{-9} & 186.588 \\
3.0453 \times 10^{-9} & -150.312 \\
605.854 & 1.5923 \times 10^{-9}
\end{array}\right)
\end{aligned}
$$

Step 7: Calculate equivalent controller transfer function $T_{c}(s)=\bar{G}(s I-\bar{L})^{-1} \bar{K}$ :

Not shown 
D-4.3. DAC Based on the 9-State Model

Step 1: Generate an appropriate linear model of the turbine at a specific operating point (the control design point). Evaluate the state matrices $A, B, C$, and $\Gamma$. Determine the open loop eigenvalues of the $A$ matrix.

$$
\begin{aligned}
& \mathrm{A}=\left(\begin{array}{ccccccccc}
0 . & 1 . & 0 . & 0 . & 0 . & 0 . & 0 . & 0 . & 0 . \\
-197.716 & -9.18512 & -7.97263 & -0.11461 & -20.9296 & 0.0000303452 & 0 . & 103.135 & -15.5419 \\
0 . & 0 . & 0 . & 1 . & 0 . & 0 . & 0 . & 0 . & 0 . \\
-7.97263 & -0.11461 & -197.716 & -9.18512 & -20.9296 & 0.0000303452 & 0 . & 103.135 & -15.5419 \\
0.538881 & 0.00581059 & 0.538881 & 0.00581059 & -0.0348779 & -3.26681 \times 10^{-6} & 0 . & -0.540402 & -0.00483677 \\
0 . & 0 . & 0 . & 0 . & 2.691 \times 10^{7} & 0 . & -2.691 \times 10^{7} & 0 . & 0 . \\
0 . & 0 . & 0 . & 0 . & 0 . & 0.0000156006 & 0 . & 0 . & 0 . \\
0 . & 0 . & 0 . & 0 . & 0 . & 0 . & 0 . & 0 . & 1 . \\
1.12726 & 0.0225063 & 1.12726 & 0.0225063 & 0.152178 & -3.33549 \times 10^{-7} & 0 . & -35.513 & 0.00309199
\end{array}\right) \\
& B=\left(\begin{array}{cc}
0 & 0 \\
-1110.6 & -16.9062 \\
0 . & 0 . \\
-16.9062 & -1110.6 \\
1.226 & 1.226 \\
0 . & 0 . \\
0 . & 0 . \\
0 . & 0 . \\
2.11931 & 2.11931
\end{array}\right) \\
& \overline{\mathrm{C}}=\left(\begin{array}{cccccccccccc}
0.5 & 0 & -0.5 & 0 & 0 & 0 & 0 & 0 & 0 & 0 & 0 & 0 \\
0 & 0 & 0 & 0 & 0 & 0 & 1 & 0 & 0 & 0 & 0 & 0 \\
0 & 0 & 0 & 0 & 0 & 0 & 0 & 1 & 0 & 0 & 0 & 0
\end{array}\right) \\
& \Gamma=\left(\begin{array}{cc}
0 . & 0 . \\
14.1026 & 14.1529 \\
0 . & 0 . \\
-14.1026 & 14.1529 \\
6.93889 \times 10^{-18} & 0.00478024 \\
0 . & 0 . \\
0 . & 0 . \\
0 . & 0 . \\
1.38778 \times 10^{-17} & -0.0337653
\end{array}\right)
\end{aligned}
$$

Step 2: Assess controllability of the system $(A, B)$ in order to allow pole placement. If the system is controllable then choose plant poles to enhance damping and improve system response as desired.

Assessed Controllability with MATLAB

$$
\begin{aligned}
& \text { choose plant poles }= \\
& \begin{array}{l}
\{-2.47518+23.1319 \dot{\mathbb{1}},-2.47518-23.1319 \dot{\mathbb{1}}, \\
-5.29699+13.3396 \dot{\mathbb{1}},-5.29699-13.3396 \dot{\mathbb{1}}, \\
-4.53526+13.0067 \dot{\mathbb{1}},-4.53526-13.0067 \dot{\mathbb{1}}, \\
-1.9384+5.71752 \dot{\mathbb{1}},-1.9384-5.71752 \dot{\mathbb{1}},-1.78916\}
\end{array}
\end{aligned}
$$

based on LQR, with 


$Q=\left(\begin{array}{ccccccccc}0 . & 0 . & 0 . & 0 . & 0 . & 0 . & 0 . & 0 . & 0 . \\ 0 . & 0 . & 0 . & 0 . & 0 . & 0 . & 0 . & 0 . & 0 . \\ 0 . & 0 . & 0 . & 0 . & 0 . & 0 . & 0 . & 0 . & 0 . \\ 0 . & 0 . & 0 . & 0 . & 0 . & 0 . & 0 . & 0 . & 0 . \\ 0 . & 0 . & 0 . & 0 . & 0.0001 & 0 . & 0 . & 0 . & 0 . \\ 0 . & 0 . & 0 . & 0 . & 0 . & 1 . \times 10^{-12} & 0 . & 0 . & 0 . \\ 0 . & 0 . & 0 . & 0 . & 0 . & 0 . & 1 . & 0 . & 0 . \\ 0 . & 0 . & 0 . & 0 . & 0 . & 0 . & 0 . & 10 . & 0 . \\ 0 . & 0 . & 0 . & 0 . & 0 . & 0 . & 0 . & 0 . & 0.1\end{array}\right)$ and
$R=\left(\begin{array}{ll}1 . & 0 . \\ 0 . & 1 .\end{array}\right)$

Step 3: Calculate gains $G$ to give the desired poles that were chosen in Step 2

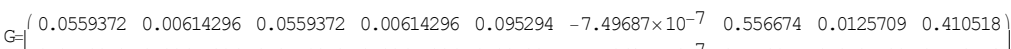

$G=\left(\begin{array}{lllllllll}0.0559372 & 0.00614296 & 0.0559372 & 0.00614296 & 0.095294 & -7.49687 \times 10^{-7} & 0.556674 & 0.0125709 & 0.410518\end{array}\right)$

Step 4: Calculate gains $G_{d}$ to cancel or attenuate windspeed disturbances.

Disturbance Gain: $\operatorname{Gd}=\left(\begin{array}{ccc}0.01 & 0 & 0.0125559 \\ -0.01 & 0 & 0.0125559\end{array}\right)$

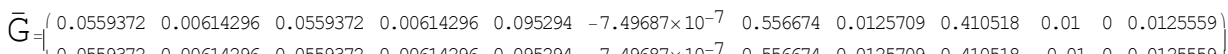

Step 5: Calculate the augmented state matrices $(\bar{A}, \bar{B}, \bar{C})$ and assess observability of $(\bar{A}, \bar{C})$.

$\overline{\mathrm{C}}=\left(\begin{array}{cccccccccccc}0.5 & 0 & -0.5 & 0 & 0 & 0 & 0 & 0 & 0 & 0 & 0 & 0 \\ 0 & 0 & 0 & 0 & 0 & 0 & 1 & 0 & 0 & 0 & 0 & 0 \\ 0 & 0 & 0 & 0 & 0 & 0 & 0 & 1 & 0 & 0 & 0 & 0\end{array}\right)$

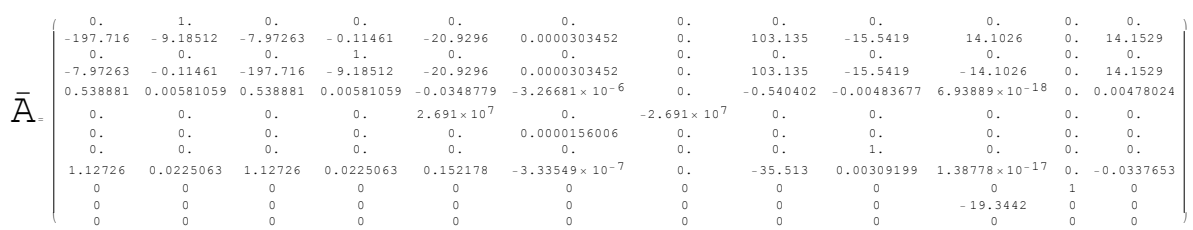

$\overline{\mathrm{B}}=\left(\begin{array}{cc}0 . & 0 \\ -1110.6 & -16.9062 \\ 0 . & 0 . \\ -16.9062 & -1110.6 \\ 1.226 & 1.226 \\ 0 . & 0 . \\ 0 . & 0 . \\ 0 . & 0 . \\ 2.11931 & 2.11931 \\ 0 & 0 \\ 0 & 0 \\ 0 & 0\end{array}\right)$ 
Obsv $=\left(\begin{array}{c}\bar{C} \\ \overline{C A} \\ \overline{C A}^{2} \\ \overline{C A}^{3} \\ \overline{C A}^{4} \\ \overline{C A}^{5} \\ \overline{C A}^{6} \\ \overline{C A}^{7}\end{array}\right)$

Observability matrix not shown-Assessed observability using MATLAB. From MATLAB: the rank of the observability matrix is 12 , thus this system is observable.

Step 6: If observability is achieved, choose the poles of the state estimators (including the wind disturbance state estimator) to achieve desired behavior (high damping for state estimator poles).

$$
\begin{aligned}
& \text { State Estimator Poles }= \\
& \begin{aligned}
\{-7.01307+22.0992 \dot{\mathbb{1}},-7.01307-22.0992 \dot{\mathbb{1}}, \\
-10.5884+15.1297 \dot{\mathbb{1}},-10.5884-15.1297 \dot{\mathbb{1}}, \\
-4.60133+13.6255 \dot{\mathbb{1}},-4.60133-13.6255 \dot{\mathbb{1}}, \\
-11.5255,-8.79675,-4.28496+6.52792 \dot{\mathbb{1}}, \\
-4.28496-6.52792 \dot{\mathbb{1}},-3.85353,-2.28286\} \text { Based on LQR for determining state }
\end{aligned}
\end{aligned}
$$

estimator gain matrix, with Qe $=$

$\left(\begin{array}{cccccccccccc}500 . & 0 . & 0 . & 0 . & 0 . & 0 . & 0 . & 0 . & 0 . & 0 . & 0 . & 0 . \\ 0 . & 5000 . & 0 . & 0 . & 0 . & 0 . & 0 . & 0 . & 0 . & 0 . & 0 . & 0 . \\ 0 . & 0 . & 50 . & 0 . & 0 . & 0 . & 0 . & 0 . & 0 . & 0 . & 0 . & 0 . \\ 0 . & 0 . & 0 . & 50000 . & 0 . & 0 . & 0 . & 0 . & 0 . & 0 . & 0 . & 0 . \\ 0 . & 0 . & 0 . & 0 . & 120 . & 0 . & 0 . & 0 . & 0 . & 0 . & 0 . & 0 . \\ 0 . & 0 . & 0 . & 0 . & 0 . & 800 . & 0 . & 0 . & 0 . & 0 . & 0 . & 0 . \\ 0 . & 0 . & 0 . & 0 . & 0 . & 0 . & 220 . & 0 . & 0 . & 0 . & 0 . & 0 . \\ 0 . & 0 . & 0 . & 0 . & 0 . & 0 . & 0 . & 20 . & 0 . & 0 . & 0 . & 0 . \\ 0 . & 0 . & 0 . & 0 . & 0 . & 0 . & 0 . & 0 . & 2500 . & 0 . & 0 . & 0 . \\ 0 . & 0 . & 0 . & 0 . & 0 . & 0 . & 0 . & 0 . & 0 . & 100000 . & 0 . & 0 . \\ 0 . & 0 . & 0 . & 0 . & 0 . & 0 . & 0 . & 0 . & 0 . & 0 . & 1 . \times 10^{6} & 0 . \\ 0 . & 0 . & 0 . & 0 . & 0 . & 0 . & 0 . & 0 . & 0 . & 0 . & 0 . & 100000 .),\end{array}\right.$,

and

$\operatorname{Re}=\left(\begin{array}{lll}1 . & 0 . & 0 . \\ 0 . & 1 . & 0 . \\ 0 . & 0 . & 1 .\end{array}\right)$. 
State Estimator Gains : $\overline{\mathrm{K}}=$

$\left(\begin{array}{ccc}27.4848 & 22.6384 & 6.01381 \\ 252.723 & -0.682216 & 24.0435 \\ -27.4859 & 22.6391 & 6.01383 \\ -252.722 & -0.651917 & 24.0442 \\ -0.0000235432 & 9.38751 & 0.383109 \\ -161.098 & 1.30173 \times 10^{7} & 396947 . \\ -0.000344049 & 25.0212 & 0.305797 \\ -0.0000104977 & 0.305797 & 8.52564 \\ -0.000119693 & 4.07662 & 26.3904 \\ 388.44 & -0.0216342 & -0.000553418 \\ 125.112 & -0.158275 & -0.00382363 \\ -0.00719364 & 302.853 & 91.2878\end{array}\right)$

Step 7: Calculate equivalent controller transfer function $T_{c}(s)=\bar{G}(s I-\bar{L})^{-1} \bar{K}$ :

Not Shown.

\section{D-5. Controls Designed from Chapter 8}

\section{D-5.1 DAC Based On the 7-State Model with Actuator Dynamics}

Step 1: Generate an appropriate linear model of the turbine at a specific operating point (the control design point). Evaluate the state matrices $A, B, C$, and $\Gamma$. Determine the open loop eigenvalues of the $A$ matrix.

$$
\begin{aligned}
& \mathrm{A}=\left(\begin{array}{cccccccc}
0 . & 1 . & 0 . & 0 . & 0 . & 0 . & 0 . & 0 . \\
-205.845 & -9.35289 & -21.0761 & 822.304 & 0 . & 103.859 & -15.674 & -1158.73 \\
1.07858 & 0.0118997 & -0.0341104 & -87.9398 & 0 . & -0.544198 & -0.00414463 & 2.61558 \\
0 . & 0 . & 1 . & 0 . & -1 . & 0 . & 0 . & 0 . \\
0 . & 0 . & 0 . & 419.813 & 0 . & 0 . & 0 . & 0 . \\
0 . & 0 . & 0 . & 0 . & 0 . & 0 . & 1 . & 0 . \\
2.25625 & 0.045512 & 0.153788 & -9.03861 & 0 . & -35.5218 & 0.00454393 & 4.58177 \\
0 . & 0 . & 0 . & 0 . & 0 . & 0 . & 0 . & -5 .
\end{array}\right) \\
& B=\left(\begin{array}{l}
0 . \\
0 . \\
0 . \\
0 . \\
0 . \\
0 . \\
0 . \\
5 .
\end{array}\right) \\
& C=\left(\begin{array}{llllllll}
0 & 0 & 0 & 0 & 1 & 0 & 0 & 0 \\
0 & 0 & 0 & 0 & 0 & 1 & 0 & 0
\end{array}\right)
\end{aligned}
$$


$\Gamma=\left(\begin{array}{c}0 \\ 14.252 \\ 0.00426126 \\ 0 \\ 0 \\ 0 \\ -0.034854 \\ 0\end{array}\right)$

Step 2: Assess controllability of the system $(A, B)$ in order to allow pole placement. If the system is controllable then choose plant poles to enhance damping and improve system response as desired.

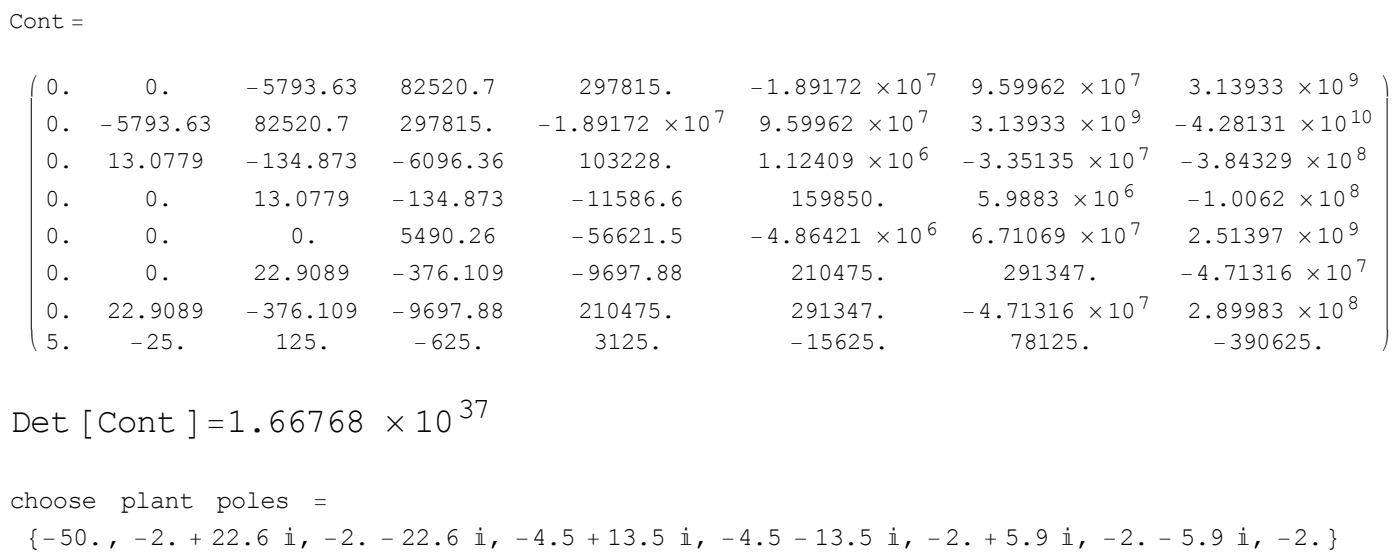

Step 3: Calculate gains $G$ to give the desired poles that were chosen in Step 2

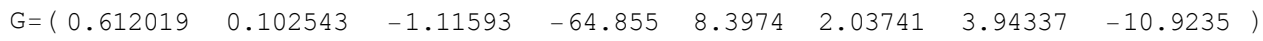

Step 4: Calculate gains $G_{d}$ to cancel or attenuate windspeed disturbances.

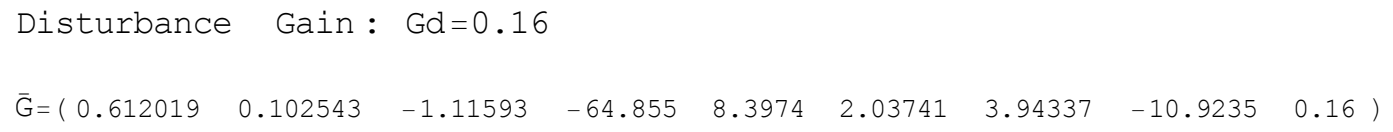

Step 5: Calculate the augmented state matrices $(\bar{A}, \bar{B}, \bar{C})$ and assess observability of $(\bar{A}, \bar{C})$.

$$
\overline{\mathrm{C}}=\left(\begin{array}{lllllllll}
0 & 0 & 0 & 0 & 1 & 0 & 0 & 0 & 0 \\
0 & 0 & 0 & 0 & 0 & 1 & 0 & 0 & 0
\end{array}\right)
$$$$
\overline{\mathrm{A}}=\left(\begin{array}{ccccccccc}
0 . & 1 . & 0 . & 0 . & 0 . & 0 . & 0 . & 0 . & 0 . \\
-205.845 & -9.35289 & -21.0761 & 822.304 & 0 . & 103.859 & -15.674 & -1158.73 & 14.252 \\
1.07858 & 0.0118997 & -0.0341104 & -87.9398 & 0 . & -0.544198 & -0.00414463 & 2.61558 & 0.00426126 \\
0 . & 0 . & 1 . & 0 . & -1 . & 0 . & 0 . & 0 . & 0 . \\
0 . & 0 . & 0 . & 419.813 & 0 . & 0 . & 0 . & 0 . & 0 . \\
0 . & 0 . & 0 . & 0 . & 0 . & 0 . & 1 . & 0 . & 0 . \\
2.25625 & 0.045512 & 0.153788 & -9.03861 & 0 . & -35.5218 & 0.00454393 & 4.58177 & -0.034854 \\
0 . & 0 . & 0 . & 0 . & 0 . & 0 . & 0 . & -5 . & 0 . \\
0 & 0 & 0 & 0 & 0 & 0 & 0 & 0 & 0
\end{array}\right)
$$ 
$\overline{\mathrm{B}}=\left(\begin{array}{c}0 . \\ 0 . \\ 0 . \\ 0 . \\ 0 . \\ 0 . \\ 0 . \\ 5 . \\ 0\end{array}\right)$

Obsv $=\left(\begin{array}{c}\bar{C} \\ \overline{C A} \\ \overline{C A}^{2} \\ \overline{C A}^{3} \\ \overline{C A}^{4} \\ \overline{C A}^{5} \\ \overline{C A}^{6} \\ \overline{C A}^{7} \\ \overline{C A}^{8}\end{array}\right)$

Observability matrix not shown

From MATLAB: the rank of the observability matrix is 9 , thus this system is observable.

Step 6: If observability is achieved, choose the poles of the state estimators (including the wind disturbance state estimator) to achieve desired behavior (high damping for state estimator poles).

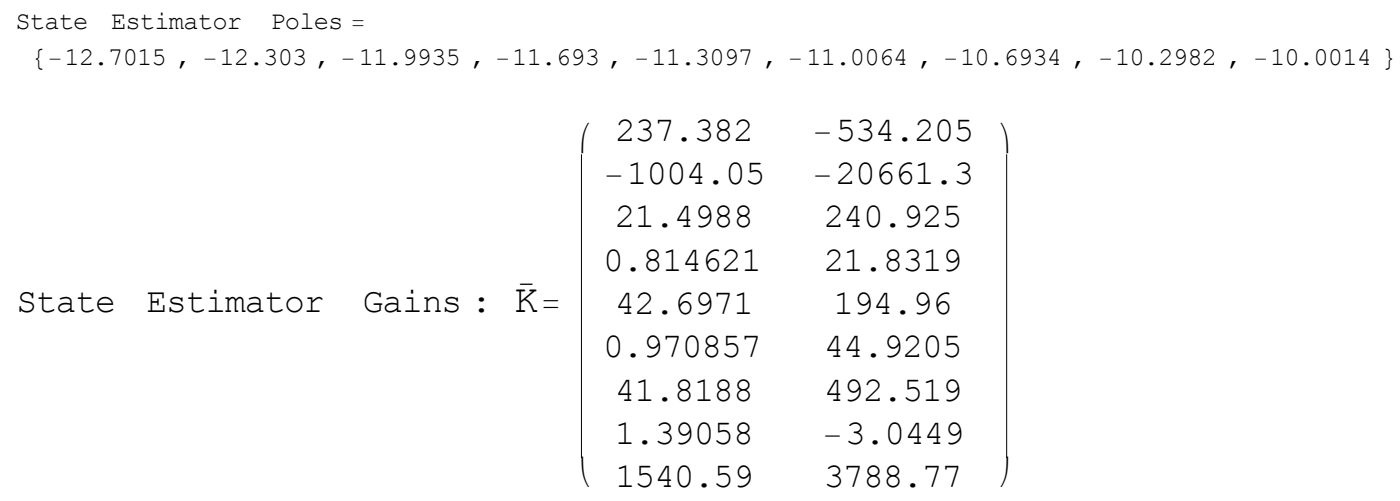

Step 7: Calculate equivalent controller transfer function $T_{c}(s)=\bar{G}(s I-\bar{L})^{-1} \bar{K}$ :

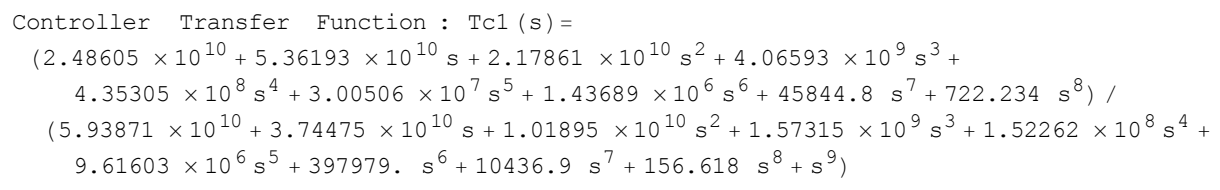




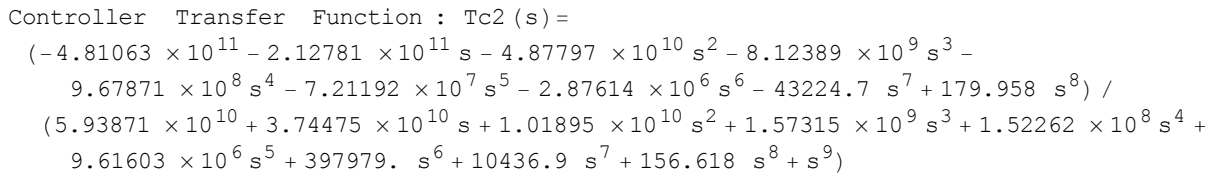




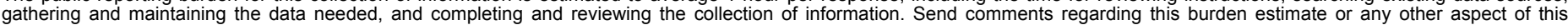

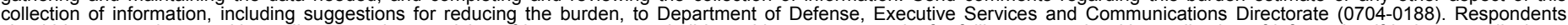

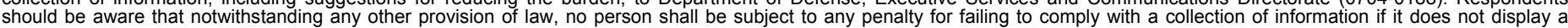

should be aware that notwithstanding

PLEASE DO NOT RETURN YOUR FORM TO THE ABOVE ORGANIZATION.

\begin{tabular}{ll|l}
\hline $\begin{array}{l}\text { 1. } \\
\text { REPORT DATE (DD-MM- J } 2004\end{array}$ & $\begin{array}{l}\text { REPORT TYPE } \\
\text { technical report }\end{array}$ \\
\hline 4. & TITLE AND SUBTITLE
\end{tabular}

Modern Control Design for Flexible Wind Turbines

3. DATES COVERED (From - To) June 2002 - June 2004

5a. CONTRACT NUMBER

DE-AC36-99-G010337

5b. GRANT NUMBER

5c. PROGRAM ELEMENT NUMBER

5d. PROJECT NUMBER

NREL/

Alan D. Wright

\section{5e. TASK NUMBER}

TP-500-35816

5f. WORK UNIT NUMBER
7. PERFORMING ORGANIZATION NAME(S) AND ADDRESS(ES)

National Renewable Energy Laboratory

1617 Cole Blvd.

Golden, CO 80401-3393

\section{PERFORMING ORGANIZATION REPORT NUMBER \\ NREL/TP-500-35816}

9. SPONSORING/MONITORING AGENCY NAME(S) AND ADDRESS(ES)

\section{SPONSOR/MONITOR'S ACRONYM(S)} NREL

11. SPONSORING/MONITORING AGENCY REPORT NUMBER

12. DISTRIBUTION AVAILABILITY STATEMENT

National Technical Information Service

U.S. Department of Commerce

5285 Port Royal Road

Springfield, VA 22161

13. SUPPLEMENTARY NOTES

14. ABSTRACT (Maximum 200 Words)

Control can improve energy capture and reduce dynamic loads in wind turbines. In the 1970s and 1980s, wind turbines used classical control designs to regulate power and speed. The methods used, however, were not always successful. Modern turbines are larger, mounted on taller towers, and more dynamically active than their predecessors. Control systems to regulate turbine power and maintain stable, closed-loop behavior in the presence of turbulent wind inflow will be critical for these designs. This report applies modern state-space control design methods to a two-bladed teetering hub upwind machine at the National Wind Technology Center (NWTC), which is managed by the U.S. Department of Energy's National Renewable Energy Laboratory (NREL) in Golden, Colorado. The design objective is to regulate turbine speed and enhance damping in several low-damped flexible modes of the turbine. Starting with simple control algorithms based on linear models, complexity is added incrementally until the desired performance is firmly established.

15. SUBJECT TERMS

wind turbine control systems; control design methods; National Wind Technology Center

\begin{tabular}{|c|c|c|c|c|}
\hline \multicolumn{3}{|c|}{ 16. SECURITY CLASSIFICATION OF: } & \multirow{2}{*}{$\begin{array}{l}\text { 17. LIMITATION } \\
\text { OF ABSTRACT } \\
\text { UL }\end{array}$} & \multirow{2}{*}{\begin{tabular}{|ll} 
18. & NUMBER \\
& OF PAGES
\end{tabular}} \\
\hline $\begin{array}{l}\text { a. REPORT } \\
\text { Unclassified }\end{array}$ & $\begin{array}{l}\text { b. ABSTRACT } \\
\text { Unclassified }\end{array}$ & $\begin{array}{l}\text { c. THIS PAGE } \\
\text { Unclassified }\end{array}$ & & \\
\hline
\end{tabular}

19a. NAME OF RESPONSIBLE PERSON

19b. TELEPONE NUMBER (Include area code) 